\title{
Comfort Climate Evaluation with Thermal Manikin Methods and Computer Simulation Models
}

\author{
Håkan O Nilsson
}

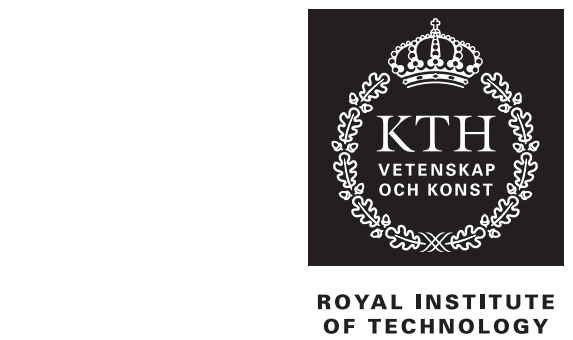

Department of Civil and Architectural Engineering

Royal Institute of Technology, Sweden

Department of Technology and Built Environment

University of Gävle, Sweden
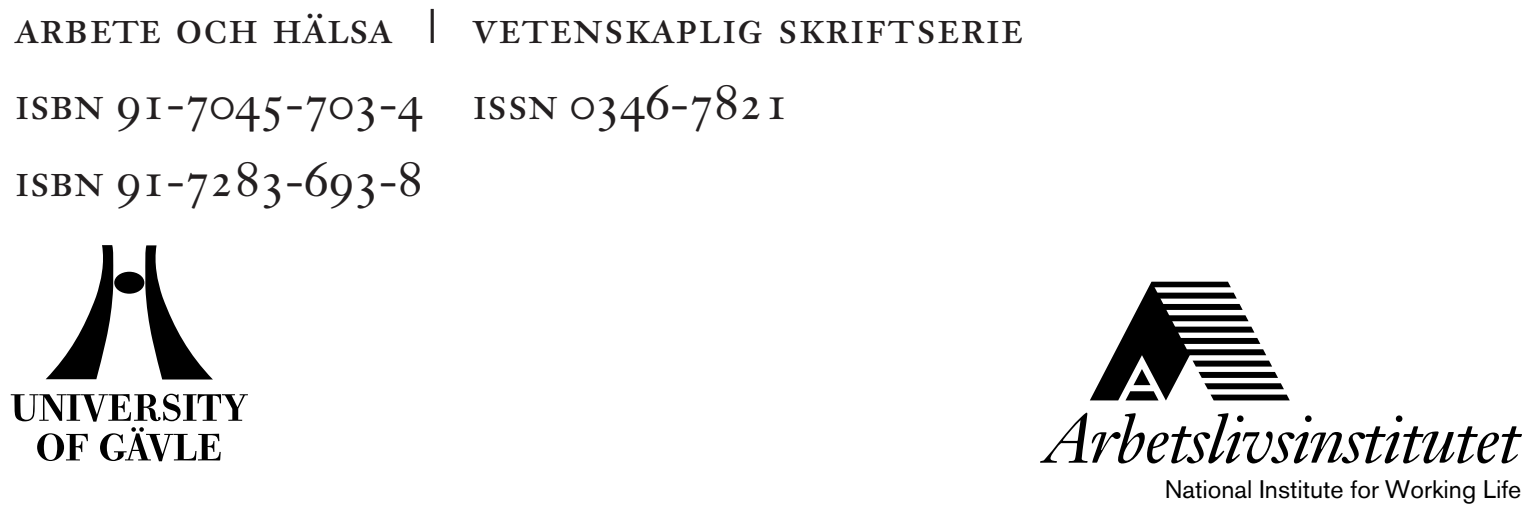


\section{Arbete och Hälsa}

Arbete och Hälsa (Work and Health) is a scientific report series published by the National Institute for Working Life. The series presents research by the Institute's own researchers as well as by others, both within and outside of Sweden. The series publishes scientific original works, dissertations, criteria documents and literature surveys.

Arbete och Hälsa has a broad targetgroup and welcomes articles in different areas. The language is most often English, but also Swedish manuscripts are welcome.

Summaries in Swedish and English as well as the complete original text are available at www.arbetslivsinstitutet.se/ as from I997.

ARBETE OCH HÄLSA

Editor-in-chief: Staffan Marklund

Co-editors: Marita Christmansson, Birgitta Meding,

Bo Melin and Ewa Wigaeus Tornqvist

(c) National Institut for Working Life \& authors 2004

National Institute for Working Life

S-113 91 Stockholm

Sweden

ISBN 91-7045-703-4

ISBN 91-7283-693-8

ISSN 0346-7821

http://www.arbetslivsinstitutet.se/

Printed at Elanders Gotab, Stockholm 


\section{Abstract}

Increasing concern about energy consumption and the simultaneous need for an acceptable thermal environment makes it necessary to estimate in advance what effect different thermal factors will have on the occupants. Temperature measurements alone do not account for all climate effects on the human body and especially not for local effects of convection and radiation. People as well as thermal manikins can detect heat loss changes on local body parts. This fact makes it appropriate to develop measurement methods and computer models with the corresponding working principles and levels of resolution. One purpose of this thesis is to link together results from these various investigation techniques with the aim of assessing different effects of the thermal climate on people. The results can be used to facilitate detailed evaluations of thermal influences both in indoor environments in buildings and in different types of vehicles.

This thesis presents a comprehensive and detailed description of the theories and methods behind full-scale measurements with thermal manikins. This is done with new, extended definitions of the concept of equivalent temperature, and new theories describing equivalent temperature as a vector-valued function. One specific advantage is that the locally measured or simulated results are presented with newly developed "comfort zone diagrams". These diagrams provide new ways of taking into consideration both seat zone qualities as well as the influence of different clothing types on the climate assessment with "clothing-independent" comfort zone diagrams.

Today, different types of computer programs such as CAD (Computer Aided Design) and CFD (Computational Fluid Dynamics) are used for product development, simulation and testing of, for instance, HVAC (Heating, Ventilation and Air Conditioning) systems, particularly in the building and vehicle industry. Three different climate evaluation methods are used and compared in this thesis: human subjective measurements, manikin measurements and computer modelling. A detailed description is presented of how developed simulation methods can be used to evaluate the influence of thermal climate in existing and planned environments. In different climate situations subjective human experiences are compared to heat loss measurements and simulations with thermal manikins. The calculation relationships developed in this research agree well with full-scale measurements and subject experiments in different thermal environments. The use of temperature and flow field data from CFD calculations as input produces acceptable results, especially in relatively homogeneous environments. In more heterogeneous environments the deviations are slightly larger. Possible reasons for this are presented along with suggestions for continued research, new relationships and computer codes.

Key-words: equivalent temperature, subject, thermal manikin, mannequin, thermal climate assessment, heat loss, office environment, cabin climate, ventilated seat, computer model, CFD, clothing-independent, comfort zone diagram. 


\section{Sammanfattning}

Ökat intresse för energiförbrukning och samtidiga krav på godtagbar termisk miljö gör det nödvändigt att i förväg kunna bedöma personpåverkan från olika kombinationer av klimatfaktorer. Enbart temperaturmätningar tar inte hänsyn till alla klimateffekter på en människa och speciellt inte för lokala effekter av luftrörelser och strålning. Eftersom såväl personer som termiska dockor (manikiner) kan känna av värmeförlustförändringar på enskilda kroppsdelar, är det lämpligt att mätmetoder och datormodeller med motsvarande arbetsprinciper och upplösningsnivåer utvecklas. Ett syfte med detta avhandlingsarbete är att sammanföra resultat från dessa olika typer av undersökningsteknik med målet att fastställa effekten av olika termiska klimat på människor. Resultatet kan användas till att göra detaljerade bedömningar av termisk klimatpåverkan $\mathrm{i}$ både inomhusmiljö $\mathrm{i}$ byggnader och i olika typer av fordon.

Avhandlingen presenterar en samlad och detaljerad beskrivning av de teorier och metoder som ligger bakom mätningar i full skala med termiska manikiner. Detta görs med nya utvidgade definitioner av begreppet ekvivalenttemperatur, samt nya teorier beskrivande ekvivalenttemperatur som en vektor värd funktion. En avgörande fördel är att presentationen av de lokalt uppmätta eller simulerade resultaten görs i nyutvecklade "komfortzondiagram". I dessa diagram tas på nya sätt hänsyn till både sittytornas egenskaper samt olika kläders påverkan på klimatbedömningen i "beklädnadsoberoende" komfortzondiagram.

Idag används ofta olika typer av datorprogram som CAD (Computer Aided Design) och CFD (Computational Fluid Dynamics) för produktutveckling, simulering och testning av bl.a. HVAC (Heating, Ventilation and Air Conditioning) system främst inom byggnads- och bilindustrin. Avhandlingen utnyttjar och jämför resultat från tre olika klimatbedömningsmetoder; personförsök, manikinmätningar och datormodeller. Det beskrivs utförligt hur utvecklade simuleringsmetoder kan användas för att bedöma klimatpåverkan i befintliga och planerade miljöer. I olika klimatsituationer har personupplevelser jämförts med värmeförlustmätningar och simuleringar med termiska manikiner. De beräkningssamband som tagits fram i detta arbete ger god överensstämmelse med mätningar och personförsök i full skala i olika termiska miljöer. Användning av temperatur- och flödesfältdata från CFD beräkningar som indata, ger godtagbara resultat speciellt i relativt homogena miljöer. I mer heterogena miljöer blir avvikelserna något större. Möjliga anledningar till detta presenteras tillsammans med förslag till fortsatt forskning, nya samband och programkoder.

Nyckelord: ekvivalenttemperatur, person, termisk docka, termisk manikin, klimatbedömning, värmeavgivning, kontorsmiljö, hyttklimat, ventilerad stol, datormodell, CFD, beklädnadsoberoende, komfortzondiagram. 


\section{Preface}

The work presented in this thesis has been carried out at the Department of Civil and Architectural Engineering, Royal Institute of Technology, the Department of Technology and Built Environment, University of Gävle and the Department for Work and Health, National Institute for Working Life.

I would like to express my sincere thanks to my supervisor Professor Mats Sandberg for all guidance and encouragement during the writing of this thesis. I also send many thanks to Professor Ingvar Holmér for giving me the opportunity to develop and work with thermal manikins and for his kind assistance in helping me getting this thesis together.

I am most grateful for all valuable comments I have received from Professor Anders Kjellberg. I would like to especially thank all researchers and students at KTH Research School at the Centre for Built Environment, for welcoming me in their team and for their kind help concerning my measurements. I am thankful to Professor Tor-Göran Malmström who has been my supervisor and contact at the Royal Institute of Techno$\log$.

I would like to thank all colleagues at the National Institute for Working Life for their kindness and helpfulness. My special tanks belongs to Professor Ewa Wigaeus Tornqvist, Désirée Gavhed, Sverker Grahn and Christer Dupuis for all your help and support over the years. It has been a great pleasure for me to work with you.

I highly appreciate the fact that different parts of this work have been made in collaboration with primarily Olle Norén, Mats Bohm and Jan Bergström at the Swedish Institute for Agricultural and Environmental Engineering. But also the Swedish Council for Work Life Research, Swedish Council for Building Research, Scania, Volvo Truck Corporation, Volvo Wheel Loaders, BG Industries, GE Plastic, Pilkington and ABB Ventilation Products.

I would like to take this opportunity to send my personal regards to all researchers forming The Thermal Manikin Network. I am always looking forward to see you at our biannual meetings.

Finally, my warmest thanks and gratitude to my beloved wife Lena and our sons Erik and Gustav.

Håkan O Nilsson

Stockholm March 2004 



\section{Contents}

Introduction and problem statement 1

Status of research 1

Research objectives 1

Thermal comfort 3

The human temperature regulation 3

Six major factors $\quad 4$

Two different ways of modelling thermal comfort 4

$\begin{array}{ll}\text { Summary thermal comfort } & 7\end{array}$

Scales of thermal comfort $\quad 8$

Comfort rating scales $\quad 8$

Ordinal and Cardinal scales $\quad 13$

$\begin{array}{ll}\text { Summary comfort scales } & 14\end{array}$

Indices for assessment of human climate comfort 16

$\begin{array}{ll}\text { Historical perspective } & 16\end{array}$

Equivalent temperature in the beginning $\quad 17$

Definition of equivalent temperature today $\quad 19$

$\begin{array}{ll}\text { Climates indices with similar principles } & 21\end{array}$

Other factors of importance $\quad 24$

Summary comfort indices $\quad 24$

$\begin{array}{ll}\text { Thermal manikin history } & 25\end{array}$

The first thermal manikins $\quad 25$

$\begin{array}{ll}\text { Thermal manikins in research } & 27\end{array}$

$\begin{array}{ll}\text { Application areas } & 28\end{array}$

$\begin{array}{ll}\text { Standardisation } & 29\end{array}$

$\begin{array}{ll}\text { Summary thermal manikins } & 30\end{array}$

Mathematical simulation models of the human body 31

Human thermophysiological modelling 31

Virtual manikins and CFD in research $\quad 32$

Summary mathematical simulation models $\quad 35$

Definitions and calculations of equivalent temperature $\quad 37$

$\begin{array}{ll}\text { General definition } & 37\end{array}$

Determination of $t_{e q}$ with equations for convection and radiation $\quad 38$

Determination based on complete heat balance equations $\quad 39$

Equivalent temperature from empirical equations $\quad 39$

Extended definitions $\quad 42$

Equivalent temperature vector theories $\quad 42$

Summary equivalent temperature $\quad 46$

Comparison with subjective responses $\quad 48$

$\begin{array}{ll}\text { Correlation with subjective ratings } & 48\end{array}$

Percentage dissatisfied $\quad 53$

Comparison with subjective responses in warm conditions $\quad 58$

$\begin{array}{ll}\text { Summary comparison with subjective responses } & 60\end{array}$ 
Clothing-independent comfort diagrams $\quad 62$

The concept of independent comfort diagrams $\quad 62$

Summary clothing-independent comfort zone diagrams 65

Measurements with thermal manikins $\quad 66$

The development of the manikin CLOUSSEAU 66

The development and construction of MANIKIN1 and MANIKIN2 67

Measurements of equivalent temperature with thermal manikins $\quad 68$

Summary thermal manikin measurements $\quad 69$

$\begin{array}{ll}\text { Simulations with the virtual manikin } & 70\end{array}$

$\begin{array}{ll}\text { The construction of MANIKIN3 } & 70\end{array}$

Modelling the environment with CFD codes $\quad 71$

Calculation procedures, definitions and orders of magnitude $\quad 72$

$\begin{array}{ll}\text { Boundary conditions } & 77\end{array}$

Summary virtual manikin methods $\quad 85$

$\begin{array}{ll}\text { Thermal insulation for seated persons } & 87\end{array}$

$\begin{array}{ll}\text { Introduction to the seat insulation study } & 87\end{array}$

$\begin{array}{ll}\text { Methods for seat measurements } & 88\end{array}$

Total seat insulation results $\quad 88$

$\begin{array}{lr}\text { Summary seat insulation } & 90\end{array}$

Impact of ventilated seats on thermal comfort $\quad 92$

Introduction to ventilated seat comfort research $\quad 92$

Experimental set-up and climatic chambers $\quad 93$

Measurement conditions $\quad 94$

Results with the ventilated seat $\quad 97$

New seat zone comfort diagrams 103

$\begin{array}{ll}\text { Summary seat comfort } & 108\end{array}$

$\begin{array}{ll}\text { Evaluation with full-scale experimental series } & 109\end{array}$

Case 1: The climatic chamber 110

$\begin{array}{ll}\text { Introduction } & 110\end{array}$

$\begin{array}{ll}\text { Real and virtual chamber } & 110\end{array}$

Calculation procedure $\quad 111$

Results from the climatic chamber (1:st case) 112

Summary the climatic chamber $\quad 115$

$\begin{array}{ll}\text { Case 2: The office environment } & 117\end{array}$

$\begin{array}{ll}\text { Introduction } & 117\end{array}$

$\begin{array}{ll}\text { Real and virtual office } & 117\end{array}$

Calculation Procedure $\quad 119$

Results office environment (2:nd case) 120

Summary office environment $\quad 125$

$\begin{array}{ll}\text { Case 3: The cabin simulator } & 127\end{array}$

Introduction $\quad 127$

$\begin{array}{ll}\text { Real and virtual cabin } & 127\end{array}$

$\begin{array}{ll}\text { Calculation procedure } & 128\end{array}$

Results from the cabin simulator (3:rd case) 129

Summary the cabin simulator $\quad 133$

$\begin{array}{ll}\text { Discussion and conclusions } & 134\end{array}$ 
Measurements, calculations and CFD

Future manikin research

Main findings and conclusions

Original articles

References

Nomenclature

Appendix A

Design and dimensions of the thermal MANIKIN2 155

Accuracy, reproducability and repeatability 161

Field operation 163

$\begin{array}{ll}\text { Appendix B } & 166\end{array}$

Design and dimensions of the virtual MANIKIN3 166

Surface temperature user subroutines $\quad 167$

$\begin{array}{ll}\text { Appendix C } & 172\end{array}$

Geometries and problem specification summaries for the cases 172

Virtual calibration $\quad 172$

Case $1 \quad 174$

$\begin{array}{ll}\text { Case } 2 & 176\end{array}$

Case $3 \quad 178$

$\begin{array}{lr}\text { Appendix D } & 180\end{array}$

Linear regressions of MTV against equivalent temperature. 180

$\begin{array}{ll}\text { Winter } & 180\end{array}$

Summer $\quad 189$

Ventilated seat 199

$\begin{array}{ll}\text { Warm conditions } & 200\end{array}$ 



\title{
Introduction and problem statement
}

\author{
Status of research
}

Today's demands for better energy performance have produced an increased interest for the requirements of good indoor and vehicle environments. Consequently, it is necessary even in the construction phase to have the ability to estimate the effects different climatic and ventilation solutions will have on people. Computational models and manikin methods provide a new approach to analysing the environmental effects on humans in both construction and evaluation stages.

The thermal environment is sometimes very complex. Convection, radiation and conduction are the common means of heat exchange and they vary independently over time and location. The final effects on the surface heat exchange of the human body are important factors for heat balance and for perception of the thermal conditions. Assessment of the thermal environment in a modern office or a car can create difficulties due to the complex interaction of the ventilation system with the situation close to the person and the external, environmental factors (e.g. radiation, air temperature and air movements). Furthermore, measurements in reality, as well as in the laboratory, contain various methodological problems.

Thermal sensation ratings provided by panels of subjects are probably still the best way of getting information about the effects of different thermal climate situations. However, subjective panels are expensive, time consuming and difficult to standardise and handle in terms of system specifications. Discrete measurements of relevant parameters around the human being are less expensive, repeatable and commonly practised. But, single measurements do not account for all the effects on a heated body, or independent effects of convection and radiation, as different types of sensors are required for their determination.

One approach is to use a full-size, man-shaped manikin with the surface covered by heating wires and temperature sensors, in order to measure in a realistic way the heat exchange over the whole body (Wyon et al., 1985, Elnäs, 1988, Nilsson et al., 1993, Bohm, 1999). The heat flow sensors are built into the manikin and measures local heat fluxes of defined areas of the surface. The manikin method has been used in different ways for several years now.

A more recent approach is to use simulations with mathematical models in virtual environments (Murakami et al., 1997, 1998). The increased calculation capacity of commonly used personal computers makes these methods more attractive as an analysing tool. However, values from manikins or models are of limited use if they are not converted into something that corresponds to human reactions.

\section{Research objectives}

One purpose of this thesis is to link together results of different investigation techniques of the thermal climate that have an impact on human beings. Results from human 
experiments and thermal manikins are used to develop a methodology based on a virtual manikin positioned in a CFD (Computational Fluid Dynamics) simulated environment. The advantages are that the results are presented not only as whole body influence, but also as local information on how the thermal climate varies over the human body. The method also produces comparable results and creates a presentation of an analysis in no more than a couple of hours. With computer simulation methods and controlled fullscale measurements with thermal manikins and persons the thermal effects on people in different environments are studied. The method uses a virtual thermal manikin in a virtual copy of the real or intended room. With this computational manikin, the effects of the environment on the human comfort experience are simulated and visualised.

The thesis has three major objectives:

A. Describe the theories behind physical thermal manikin measurement technology. Discuss the advantages and disadvantages with manikin measurements of equivalent temperature $\left(t_{e q}\right)$. Provide new information about how to define and work with the concept of equivalent temperature.

B. Present new ideas on the use of thermal comfort diagrams showing local as well as whole body influences on humans. Discuss the development of these diagrams with the background of several studies with human subjects and the complete theories behind the comfort zone diagrams. Show extensive research regarding considerations about these diagrams and how they can be further improved.

C. Show how the use of a newly developed virtual manikin can be used to calculate comfort zone diagrams with CFD simulated data as input. Present results from several cases with the proposed prediction model.

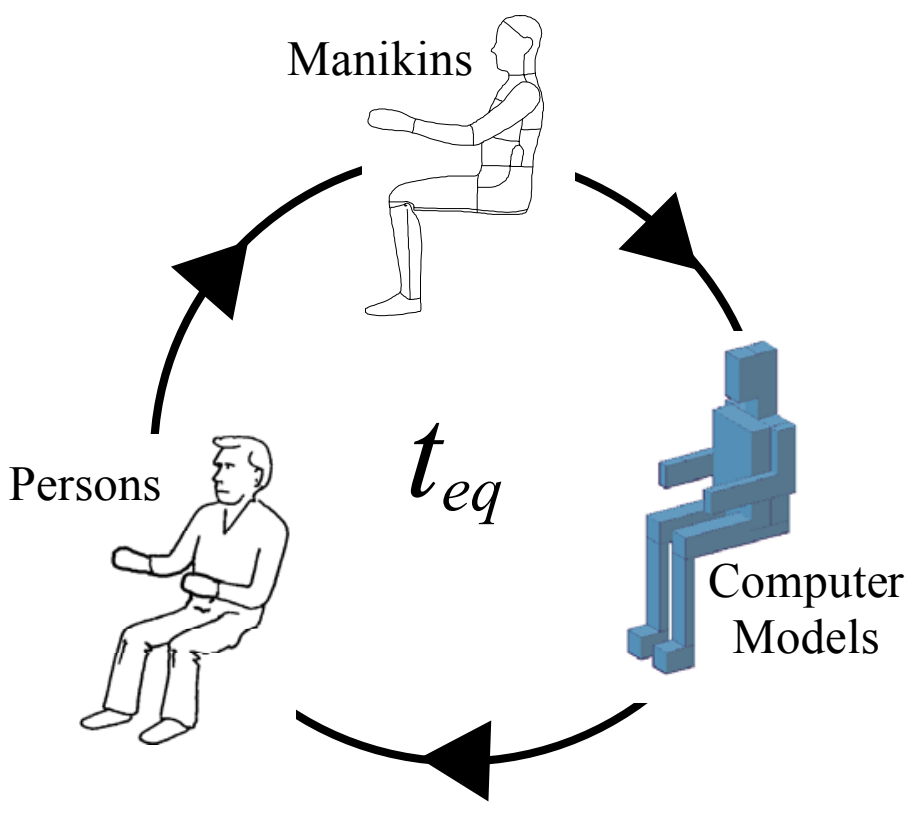

Figure 1. Proposed relationships between the investigated methods and equivalent temperature $-t_{e q}$. 


\section{Thermal comfort}

Comfortable climate is usually defined as "the condition of mind that expresses satisfaction with the thermal environment" (ASHRAE 55, 1992). The human climate experience is essentially an interaction between six parameters, air temperature, mean radiant temperature, relative air movements, air humidity, activity level and thermal properties of clothing and seat. It is also possible to further divide the physical comfort sensation in two categories, local and whole body thermal comfort. The whole body value only consists of a mean value, while the local values takes into consideration effects on different body parts.

\section{The human temperature regulation}

The human temperature regulation system allows physiological adjustment to a thermal stress and the provision of thermal comfort of the body under a variety of conditions. The heat is primarily produced by metabolism, which emanates from digestion and muscle exercise. In normal conditions this results in an average temperature of the deep body of about $37^{\circ} \mathrm{C}$. Inside a certain interval the human temperature control system strives to maintain this temperature when internal or external thermal disturbances arise. The effects resulting from a change in climatic conditions produces a reaction within the body concerning the physiological autonomous responses but also activates behavioural regulation. The autonomic regulation is controlled by the hypothalamus, controlling the different avenues of heat loss with the skin blood, sweating and respiration. Behavioural regulation can be active body movements and adjustment of clothing.

The human temperature-regulating centre is located in the hypothalamus. It works similar to a thermostat. The temperature set point may change during different physiological conditions. Thermosensitive receptors nerve endings, sends signals to the regulation centre. In a cool environment stimulation of the cold receptors may lower the set point, and the heat loss can decrease by means of vasoconstriction and shivering. In a warm environment, on the other hand, the set point becomes elevated, and the heat loss can increase by means of vasodilatation in the skin and sweating. The temperature regulatory centre is connected with receptors in the skin as well as the deep core and the brain itself. These receptors consist of a net of fine nerve endings that are specifically activated by heat or cold stimuli. These temperature receptors are especially sensitive to rapid changes in temperature and are highly susceptible to adaptation. The number of active receptors determine to some extent the sensation of temperature (Åstrand et al., 1986).

A person at rest produces approximately $100 \mathrm{~W}$ of heat. If clothing and ambient conditions are suitable the same amount of heat is lost to the environment. Whole body heat balance is adequate and the person feels thermally neutral. With increasing ambient temperature, convection and radiation diminish. Sweat evaporation has to compensate for this in order to balance the heat production of $100 \mathrm{~W}$. Sweating is associated with a sensation of warmth and eventually discomfort. In a cooler environment, convection and 
radiation increase, making total heat losses greater than $100 \mathrm{~W}$. The physiological response is to reduce skin and extremity blood flow in order to lower the external temperature gradient. In this case the person feels cool or cold and uncomfortable. A normal response is to add or remove clothing. In other words, for comfortable climatic conditions the dry heat loss can only vary within a certain narrow interval. In a similar way the heat balance of a skin segment can be analysed. Only for a certain narrow range of convective, radiative and conductive heat losses, an optimal local heat balance and a "comfortable" skin temperature maintained. Higher local heat losses will be felt as cool or cold and lower local heat losses as warm.

\section{Six major factors}

The human response to the thermal environment can be found to depend mainly on six factors (Fanger, 1970), (Parsons, 1993)):

- Air temperature

- Mean radiant temperature

- Air velocity

- Relative humidity

- Physical activity

- Clothing thermal resistance

These factors are the critical ones in steady state conditions. If the exposure is short or intermittent, the length of the exposure can also have an impact. The final decision whether a combination of the factors listed above, are representing a comfort situation or not, depends on the sensory reception of the climate. Hensel, 1979 states that the human sensory reception depends on the thermal comfort results from the integrated signals from various internal and external thermosensors. Warm discomfort is related to decreased heat loss initiated with warming detected by warm receptors. Cold discomfort is a response to the lowered temperature of the skin, as detected by the cold receptive nerve endings in the skin. In order to make correlations between this human thermosensation and different climatic disturbances it must be assumed that the human can not differentiate between the heat loss sensation of draft, radiation or conduction.

\section{Two different ways of modelling thermal comfort}

Two different types of models are most widely known for predicting steady state thermal comfort. One model is based on the heat balance of the human body. Another approach assumes an adaptation to the thermal environment to a certain degree.

- The Predicted Mean Vote (PMV) model; a method for the calculation of steady state thermal comfort index derived from the heat balance calculations and climate chamber studies. The model assumes a relation between optimal thermal conditions, using the steady state heat balance equation for the human body and thermal comfort ratings from panels of subjects. 
- The adaptive model; a relation for steady state thermal comfort obtained from large field studies. The model assumes that people will adapt to the thermal conditions, using only indoor and outdoor temperatures as dependent variables.

\section{The predicted mean vote}

The model is in essence a regression equation that relates PMV, on the 7-point thermal sensation scale of a group of people exposed to a certain environment to the calculated result of the basic heat balance equation (Fanger, 1970). This equation uses the heat balance for the human body and assumes a connection between the deviation from optimal thermal balance and thermal comfort vote. The greater the deviation, the more the comfort vote departs from zero. Fanger concludes that the PMV equation also can be applied to conditions with slow fluctuations. Fanger also found that unexpected changes in the temperature are corrected for by the regulatory mechanism. The PMV equation is primarily a steady-state model. It is a semi-empirical equation for predicting the mean rating on an ordinal rating scale of thermal comfort for a group of people. PMV is the most widely used thermal comfort index today. The ISO Standard 7730 (ISO 7730, 1995), uses limits of PMV and PPD as well as local recommendations as definitions of a comfort zone.

One difference compared to the adaptive model is that PMV sometimes overestimates the thermal sensation of warmth for occupants in non-air-conditioned buildings in warm climates. In these climates people are expected to adapt to a higher indoor temperatures and not ask for lower temperatures. Fanger corrects the PMV index with a expectancy factor, e, to be multiplied with PMV to reach the mean thermal sensation vote of the occupants of the actual non-air-conditioned building in a warm climate (Fanger et al., 2001). The factor e may vary between 1 for air-conditioned buildings to 0.5 for non-airconditioned buildings.

Table 1. Expectancy factors for non-air-conditioned buildings in warm climates. (Fanger \& Toftum, 2001)

\begin{tabular}{lll}
\hline Expectation & Classification of buildings & Expectancy factor, e \\
\hline High & $\begin{array}{l}\text { Non-air-conditioned buildings located in regions where } \\
\text { air-conditioned buildings are common. Warm periods } \\
\text { occurring briefly during the summer season. }\end{array}$ & $0.9-1.0$ \\
Moderate & $\begin{array}{l}\text { Non-air-conditioned buildings located in regions with } \\
\text { some air-conditioned buildings. Warm summer season. }\end{array}$ & $0.7-0.9$ \\
Low & $\begin{array}{l}\text { Non-air-conditioned buildings located in regions with } \\
\text { few air-conditioned buildings. Warm weather during all } \\
\text { seasons. }\end{array}$ & $0.5-0.7$ \\
\hline
\end{tabular}

To examine these hypotheses further, Fanger downloaded data from the database of thermal comfort field experiments (de Dear, 1998). The resulting PMV values were then multiplied with the expectancy factors estimated to be 0.9 for Brisbane, 0.7 for Athens and Singapore and 0.6 for Bangkok. The new extension of the PMV model for non-airconditioned buildings in warm climates was found to predict the actual votes well. 


\section{The adaptive principle}

The assumption of the adaptive approach is expressed by the adaptive principle:

If a change occurs such as to produce discomfort, people react in ways, which tend to restore their comfort.

The adaptive principle consequently suggests that people will adapt to certain climatic conditions. For instance in warmer climate, when the environmental thermal comfort parameters points at a higher PMV, people will become adapted to the higher temperatures and still feel comfortable. For analogy a corresponding "PMV principle" could be written as:

No change should occur that produces discomfort, in a properly designed environment will people maintain their comfort.

The adaptive principle (Nicol et al., 2001) is supported by field comfort studies in many different environments. From these global field studies of thermal comfort ratings and temperatures, Humphreys, 1976 found that the comfort temperature differed between groups of people feeling thermally comfortable. This means that comfort conditions calculated with the heat balance models did not fully agree with the comfort conditions found in the field. By linking the comfort rating to human behaviour the adaptive principle links the comfort temperature to the climatic situations in which persons find themselves. The comfort temperature is a result of the interaction between the subjects and the thermal environment. Nicol and Humphreys also conclude that people with more opportunities to adapt themselves to the environment will be less likely to suffer discomfort.

The adaptive model is essentially a regression equation that relates the desired temperature indoors to the monthly average temperature outdoors. The only input variable used is the average outdoor temperature, which has an indirect impact on the human heat balance. Consequently the adaptive model does not include six classical thermal parameters that have an impact on the human heat balance and therefore on the thermal sensation.

Nicol and Humphreys have also presented data showing that the mean comfort rating changed less with indoor temperature from climate to climate than might be expected. Humphreys, 1976 confirmed this from a number of different climate situations. The rate of change of comfort rating with temperature was shown to be much lower from one survey to another than within any particular survey. 


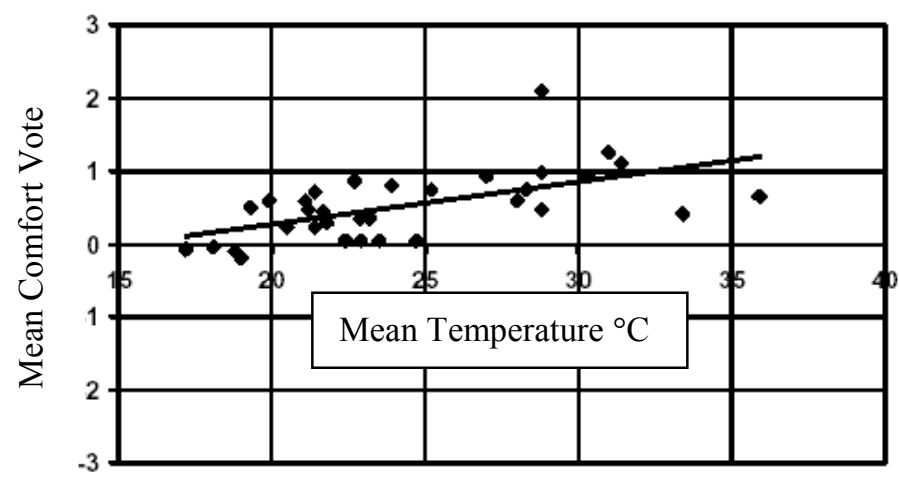

Figure 2. The variations of mean comfort rating with mean indoor temperature. Each point is the mean value from a comfort survey (redrawn from Humphreys 1976).

\section{Summary thermal comfort}

Thermal comfort is by definition a subjective sensation. However, the use of standardised scales for collection of ratings of thermal sensation for large groups of individuals, has enabled researchers to define the essential components of the thermal environment and their interaction in determining the average response of the population. The result is available as a standard method SS-EN ISO 7730.

Whole body heat balance is consequently determined by the overall climatic and clothing conditions. The preservation of a "comfortable" local skin temperature is the primary component. In the office or driving situation this is true when the physical activity of the person is low and the ventilation system provides sufficient heating as well as cooling to ensure an optimal whole body heat balance.

In practise the indoor climate is far from uniform. Shielding effects from furniture, cold draft from windows, supply of air by different ventilation systems contribute to the creation of various thermal asymmetries. These asymmetries are a result of less optimal overall heating and cooling and a disregard of the requirement for acceptable climate at all parts of the body surface.

For engineering purposes the "comfort" sensation has to be converted to and expressed in measurable, physical quantities. For the whole body sensation this can be done with well known standards with reasonable accuracy for relatively homogenous, indoor environments. In many cases the climate is far from uniform and considerable local thermal effects must be visualised and evaluated. This can readily be done using the concept of equivalent temperature. 


\section{Scales of thermal comfort}

This chapter describes different comfort scales for studies of thermal comfort and discuss the ideas behind them.

\section{Comfort rating scales}

Subjective methods, like the use of rating scales of thermal comfort, have the advantage of being relatively easy to use. They can also successfully be applied when the contributing factors to a response are not fully known. These comfort scales have, however, some disadvantages. The precise wordings at different levels of these scales can be fairly difficult to design as well as having a number of likely methodological problems. Thermal comfort is indeed a subjective quantity, different people will express different preferences. This means that this type of subjective methods require the use of a representative sample of the user population being exposed to the environment of interest, and hence can become quite costly. Thermal sensation ratings given by panels of subjects are indicative, but these panels are expensive and demand relatively large work effort in order to get repeatable results.

\section{Bedford scale}

Bedford, 1936 investigated the comfort of persons engaged in very light industrial work. Wide differences in the estimates of comfort were found. Large numbers of workers were questioned about their feelings of thermal comfort (warmth), and the actual climate was measured. The responses of the workers were classified with this sevenpoint scale:

$\begin{array}{ll}\text { Much too warm } & 1 \\ \text { Too warm } & 2 \\ \text { Comfortably warm } & 3 \\ \text { Comfortable } & 4 \\ \text { Comfortably cool } & 5 \\ \text { Too cool } & 6 \\ \text { Much too cool } & 7\end{array}$

The investigation was carried out in many different kinds of factory buildings. The observations were limited to winter months when the heating was in use. A total of 3,085 sets of observations were made. Most of the observations were made on women and girls. A majority of the subjects were seated at their work, but they had to leave their seats every now and then, in order to obtain new material. The subjects were tested one, two or three times. The observations were made on nearly 2,000 different persons. To be able to use statistics on the data numerical values to the different levels of the sensation scale were assigned. Bedford himself remarks that it probably would have 
been better to mark "comfortable" as zero, with positive numbers indicating increasing warmth and negative numbers increasing chilliness.

In the statistical treatment of these data it has to be kept in mind that this sensation scale is an ordinal scale. It can not be automatically assumed that the steps in this sensation scale necessarily indicate equal intervals of sensation. This point was examined already by Bedford, and he found that the use of such a scale gave results that did not differ significantly from those obtained with the scale described above. This indicates that this ordinal scale seems to have an approximately linear relationship to equivalent temperature, at least in the comfort region and hence can be treated as a cardinal scale. More explanations around this at an individual level later.

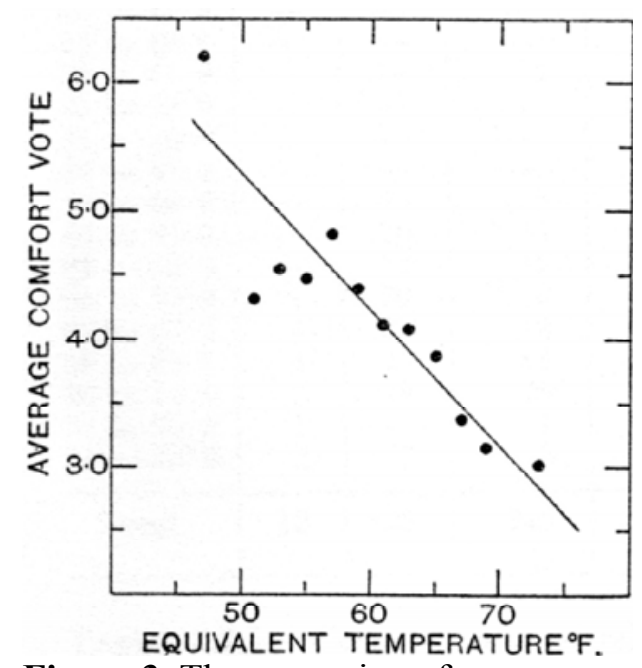

Figure 3. The regression of average comfort vote on equivalent temperature in ${ }^{\circ} \mathrm{F}$. (Bedford 1936)

Bedford also concludes that the use of equivalent temperature $\left(t_{e q}\right)$ gives the best correlation to his scale, and that $t_{e q}$ can be used both at low air speed as well as in considerable air movement. Equivalent temperature also takes full account for influence to the effects of radiant heat. He also concludes that $t_{e q}$ makes no allowance for the effects of humidity, but that this has only minor influence on the voting at temperatures below $23.8^{\circ} \mathrm{C}\left(75^{\circ} \mathrm{F}\right)$. At this stage it has to be emphasised that the comfort rating as well as $t_{e q}$ is not a measure of ordinary air temperature, nor corresponds to the centre point of any scale necessarily the preferred air temperature.

Bedford moreover states that conditions should be used which as many persons as possible will find comfortable. This means adopting a rather narrow comfort zone for practical purposes. Bedford suggested that a comfort zone should be chosen in which more than $70 \%$ the subjects were " comfortable ", and that in this range at least $86 \%$ of the votes recorded ranged from "comfortable cool" to "comfortably warm".

He did not, with this comfort zone, consider that in the summer people will commonly become more acclimatised to higher temperatures and wear less clothing than in the winter. Temperatures that would be found uncomfortably warm in winter could sometimes be accepted as pleasant in summer. He also proposed two more ways constructing these comfort zones. One was to use those conditions in which not less than $50 \%$ of votes ranged from "comfortably warm" to "comfortably cool". The second 
way of designing the comfort zone, was to take the whole range of temperature in which votes of "comfortably cool" or " comfortably warm ", were recorded.

\section{ASHRAE scale}

In studies made by Rohles et al., 1971 on 1,600 college students, correlations between comfort level, temperature, humidity, sex, and length of exposure were presented. The thermal sensation scale developed for these studies is called the ASHRAE (American Society of Heating, Refrigerating and Air-conditioning Engineers) thermal sensation scale:

$\begin{array}{ll}\text { Hot } & +3 \\ \text { Warm } & +2 \\ \text { Slightly warm } & +1 \\ \text { Neutral } & 0 \\ \text { Slightly cool } & -1 \\ \text { Cool } & -2 \\ \text { Cold } & -3\end{array}$

The numerical values in this scale are now changed compared to the Bedford scale, so that the scale ranges from -3 to +3 instead of from 1 to 7 . This with the intention that the scale should be easier to remember, as it is symmetrical around the zero point, so that a positive value corresponds to the warm side and a negative value to the cold side of neutral.

The American standard (ASHRAE 55, 1992) specifies comfort zones where $80 \%$ of sedentary or slightly active persons find the environment thermally acceptable. Because people change their clothing with the weather and the season, ASHRAE 55 specifies summer and winter comfort zones appropriate for clothing insulation levels of 0.5 and 0.9 clo $\left(0.078\right.$ and $0.14 \mathrm{~m}^{2} \mathrm{~K} / \mathrm{W}$, figure 4$)$.

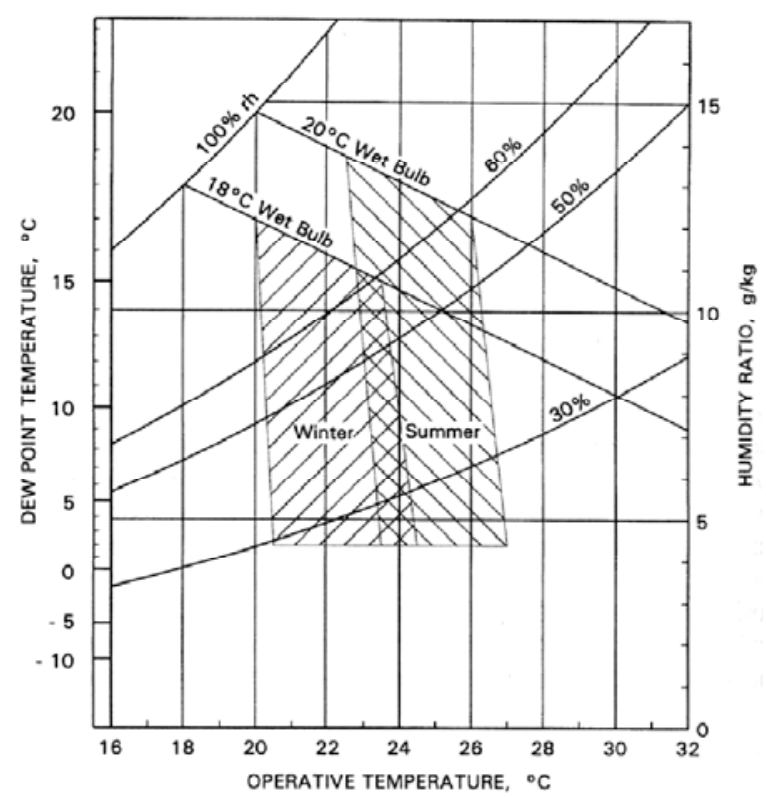

Figure 4. ASHRAE summer and winter comfort zones.

(ANSI/ASHRAE 55) 
In addition to independent environmental and personal variables influencing thermal response and comfort, other factors may also have some effect. These factors, such as non-uniformity of the environment, visual stimuli, noise, age, and outdoor climate are generally considered secondary factors.

\section{PMV scale}

The previously mentioned PMV scale, predicts the mean value of the votes of a large group of persons on the same psycho-physical thermal sensation scale as the ASHRAE 55. The theory is based on heat balance of the human body (Fanger 1970). The human being is in thermal balance when the internal heat production in the body is equal to the loss of heat to the environment.

In the PMV index the physiological response of the thermo-regulatory system has been related statistically to thermal sensation votes collected from more than 1,300 subjects. The index is derived for steady-state conditions, but can be applied with good approximation to minor fluctuations of one or more of the variables, provided that timeweighted averages of the variables during the previous 1-hour period are applied. It is recommended to use the PMV index only when main parameters are inside certain specified intervals.

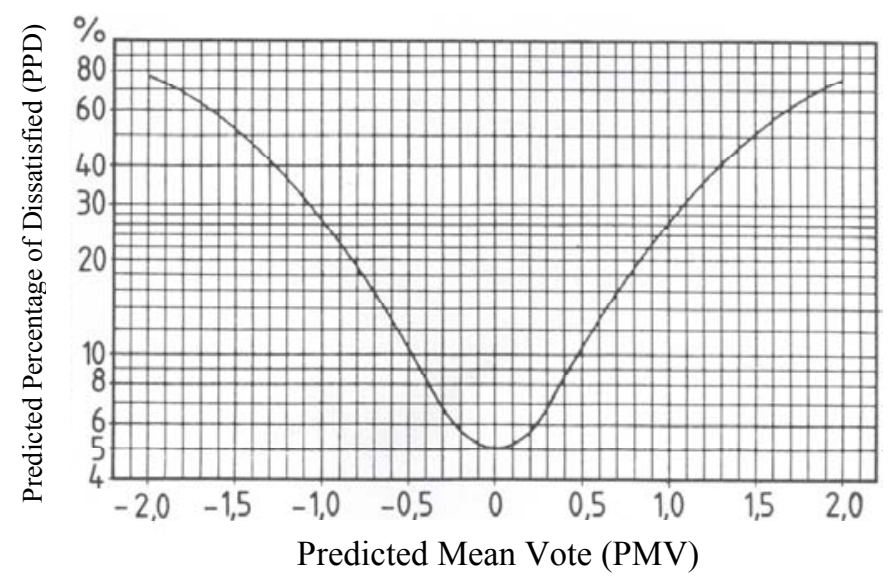

Figure 5. PPD as a function of PMV. (redrawn from SS-EN ISO 7730)

The PMV index predicts the mean value of the thermal votes of a large group of people exposed to the same environment. But individual votes are scattered around this mean value and it is consequently useful to predict the number of people likely to feel uncomfortably warm or cool. The PPD index (Predicted Percentage Dissatisfied) calculates a prediction of the number of thermally dissatisfied people (see figure 5). The PPD predicts the percentage of a large group of people likely to feel too warm or cool, i.e. voting hot $(+3)$, warm $(+2)$, cool $(-2)$ or cold $(-3)$ on the 7-point thermal sensation scale.

The PMV index gives the predicted mean vote of a large group of persons exposed to a given combination of the variables. This "mean vote" is indeed an expression for the general degree of discomfort for the group as a whole. This makes it difficult to interpret what the value of the PMV, determined in a practical case, can imply on the comfort for a single person. What does it mean when PMV is calculated to be -0.25 , a value 
between neutral and slightly cool? Is this acceptable? If all persons were corresponding to an "average person", the answer would be yes. It could then be expected that all would be comfortable enough not to complain. However all people are different. There will naturally be a certain variance in the thermal sensations of a group of persons exposed to the same environment. The persons of particular interest will be those who are decidedly uncomfortable, since it is these dissatisfied persons whom will be likely to complain about the environment.

Another objection to PMV is that those subjects voting -1 or +1 were not included as uncomfortable. This based on findings by Gagge et al., 1967 showing that "real" discomfort is expressed by those voting higher than +2 or lower than -2 . It was therefore decided to describe as dissatisfied, only those persons who feel decided discomfort according to the last definition. These considerations would slightly change the PPD index if a different definition had been chosen instead.

\section{MTV scale}

In series of experiments total and local heat fluxes from thermal manikins were determined for 30 different climatic conditions and compared with thermal sensation votes from 20 subjects exposed to the same conditions. The individual votes were averaged for each condition and reported as a Mean Thermal Vote (MTV). MTV and $t_{e q}$ for all conditions was subjected to a linear regression analysis. High correlation coefficients $(0.83$ and 0.92$)$ were found for segment heat flux and mean thermal vote of subjects for the same body segments. The procedure was repeated for all 16 different body segments for which subjective votes were obtained. The sets of low and high equivalent temperatures are plotted as two profile limits. In this way a comfort profile for the climate over the whole body surface is obtained.

$\begin{array}{lc}\text { Much too hot } & +3 \\ \text { Too hot } & +2 \\ \text { Hot but comfortable } & +1 \\ \text { Neutral } & 0 \\ \text { Cold but comfortable } & -1 \\ \text { Too cold } & -2 \\ \text { Much too cold } & -3\end{array}$

Mean thermal vote (MTV) refers to the mean value that is derived if the Bedford scale is weighted linearly with neutral as 0 , "Much too hot" as +3 and "Much to cold" as -3 . A regression of MTV against equivalent temperature is consequently linear in and around the comfort zone (Wyon et al., 1989, Nilsson et al., 1997).

As a consequence of language translation difficulties the meaning of the different levels, in the MTV scale, as well as other scales, can vary slightly. Nevertheless, the main objective has always been, to make it clear for the subjects that $-1,0$ and +1 is acceptable (comfortable) ratings, while -2 and +2 was "Too cold" respectively "Too hot", i.e. not acceptable (uncomfortable).

This is not the case with the ASHRAE (PMV) scale, were afterwards an interpretation of the facts that "cold" and "hot" are not acceptable while "cool" and "warm" are 
acceptable, have to be made. The same information can be derived from the combination of the ASHRAE scale with a "Scale of acceptability" that clearly asks the subject to chose between acceptable and not acceptable by choosing one of two scale halves without possibility to chose the sign. This scale goes from "Clearly acceptable" $(+1)$ to "Just acceptable" $(+0)$ in one half and continues in the other half from "Just not acceptable" (-0) to "Clearly unacceptable" (-1). By relating votes from the PMV or ASHRAE scale to an acceptability scale, the same goal is achieved as with the MTVmethod.

\section{Ordinal and Cardinal scales}

Ordinal scales involve the ranking along the continuum of the characteristic being scaled. An ordinal scale gives the order of preference but nothing about how much more one rating is preferred to another, that is if there is no information about the interval between any two ratings. Only positional statistics such as the median, quartile and percentile can be determined.
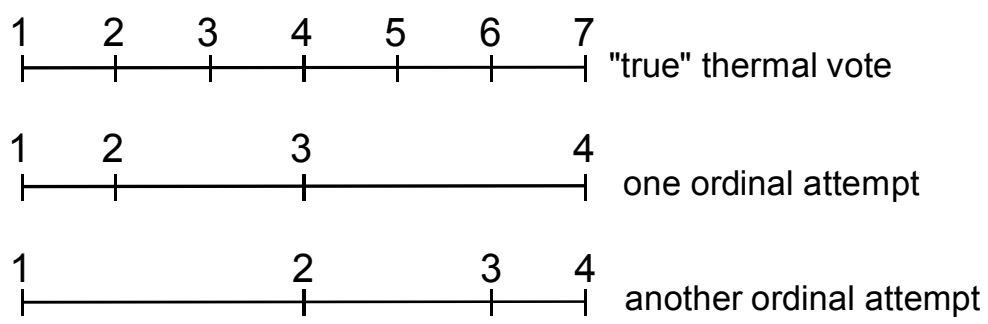

Figure 6. The upper scale showing the "true" thermal rating as a cardinal scale.

Below two ordinal attempts to correctly position the ratings.

It is only with interval scaled data it is possible to use the arithmetic mean as the measure of average. The interval or cardinal scale has equal units of measurement, thus making it possible to interpret not only the order of scale ratings but also the distance between them. The condition for a conversion between ordinal and cardinal scales is that the data forms an approximately linear relationship between MTV and $t_{e q}$ also at the individual level.
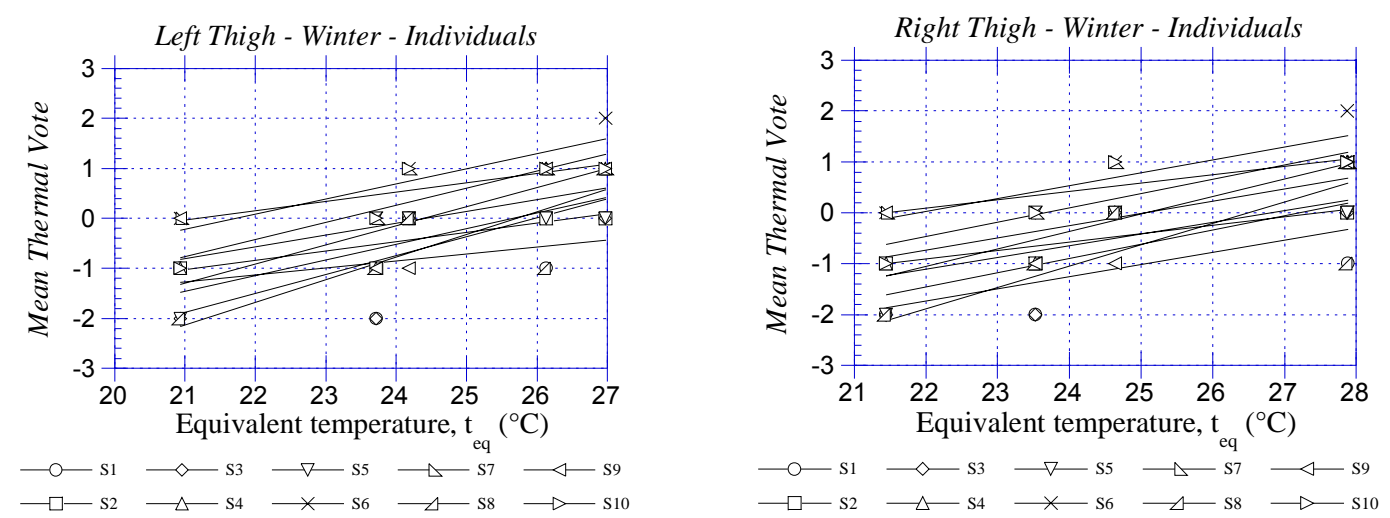

Figure 7. Regression of MTV for 10 individuals on equivalent temperature for the right and left thighs. The abbreviation S refers to Subject in the first of two series. 


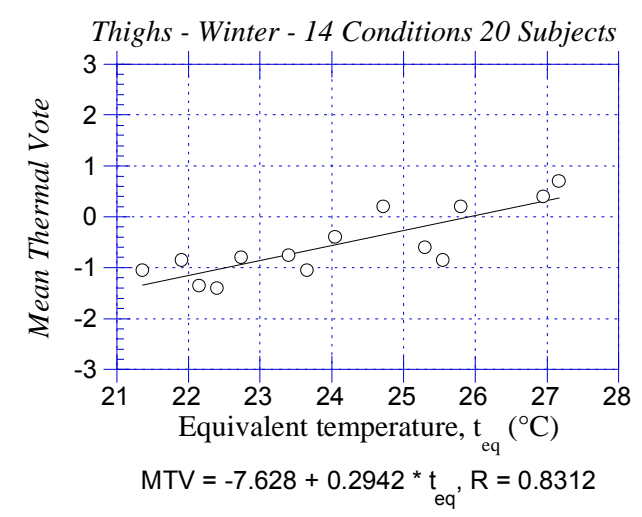

Figure 8. Regression of mean values of Mean Thermal Vote on equivalent temperature from both thighs and all conditions.

In figure 6 the relationship between MTV and $t_{e q}$ for the left and right thigh is plotted for the winter conditions. A linear relationship is found, with correlation coefficients between 0.62 to 0.99 . This is interpreted as an ability of the subjects to sense the thermal load irrespective of whether it was caused by warmth, cold or asymmetrical combinations of both. This constitutes the use of mean values for the evaluation of the thermal climate with the MTV scale. The figure 8 shows one of the final relationships calculated from mean values based on 20 individuals in 14 different conditions and two independent experimental series. This relationship should mainly be used for values of MTV between -2 and +2 . It will later be shown that the validity range can be extended to the warmer side.

\section{Summary comfort scales}

It is evident that the subjective heat and cold perception of thermal climate inside the comfort zone grows linearly with stimulus intensity, also at individual level, at least when measured with the proposed category scale. This justifies statistical treatment of ordinal scales as cardinal scales within that region. However, the feeling of comfort is for many people undeveloped and variable. A "mean rating" is still an expression for the general degree of discomfort for a group of people as a whole, and it is still impossible to predict the comfort of a single person.

Scales like the ASHRAE/PMV scale have to be related to an acceptability scale in order to achieve the same goal as with the MTV scale, were acceptability is included. By relating votes from the PMV or ASHRAE scale to an acceptability scale, a similar result is achieved as with the MTV-method. The use of multiple scales can however, be quite laborious when several parts of the body has to be rated for at the same time. The MTV scale consequently makes a good choice, especially when many local ratings have to be collected.

Some considerations also have to be taken to that, as a consequence of local behaviour and language translation, the meaning of the different levels can vary slightly in different countries. It is, still clear that the we cannot escape the fact that when persons are uncomfortable they tend to complain, so if satisfactory installations should be designed, attention still has to made to personal feelings of thermal comfort as well 
as possibilities to individual variation. The proposed relationship should mainly be used for values of MTV between -2 and +2 . 


\section{Indices for assessment of human climate comfort}

The ultimate purpose of the HVAC (Heating Ventilation and Air Conditioning) -system is to provide comfortable thermal conditions, irrespective of the environmental climatic conditions. Since several climatic factors play a role for the final heat exchange, an integrated measure of these factors, representing their relative importance, significantly reduces the need for comprehensive sets of measurements and more easily allow for comparisons of different thermal environments. Such an index would also be required for the assessment of the quality of the thermal environment from a user point of view. Air temperature for example is just one component of climate influence from the environment, and can hence not be expected to correlate well with climate sensations in all situations. Many attempts have been made produce a comfort index that would describe all feasible comfort conditions. The work has mostly been aiming at combining the fundamental climate factors in order to get a single number for estimation of climate comfort.

\section{Historical perspective}

One of the first persons attempting to measure human climate comfort was Hill et al., 1916 developing the heated kata thermometer (see figure 9). The cooling speed of the thermometer was assumed to have a connection with corresponding effects on a human body. However it was found that the thermometer was too sensitive to air movements in order to be useful as a climate comfort instrument. The kata thermometer is still today some times used as an anemometer. When the kata thermometer is used as an anemometer the heated bulb of the thermometer is dried and the rate of heat loss is measured, known as the dry kata "cooling power". Hill also describes the wet kata cooling power, which adds evaporation to the measurement.

Several efforts have been made to correlate the globe temperature measured with a globe thermometer to different comfort ratings. Globe temperature $\left(t_{g}\right)$ is measured with a non heated instrument that mainly considers the air temperature and the mean radiant temperature. The globe temperature is consequently defined for the actual air velocities, whereas the other indices usually are defined for a "standard" air velocity, often still air or air movement below $0.15 \mathrm{~m} / \mathrm{s}$. Bedford et al., 1934 showed that the globe thermometer, by itself, is not an adequate index of thermal comfort. 


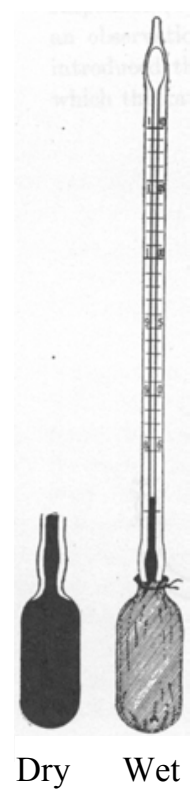

Figure 9. The Kata thermometer is an alcohol thermometer that is used to measure low air velocities. By heating the large glass bulb of the thermometer above $38^{\circ} \mathrm{C}\left(100^{\circ} \mathrm{F}\right)$ and note the time it takes to cool from 38 to $35^{\circ} \mathrm{C}\left(100\right.$ to $\left.95^{\circ} \mathrm{F}\right)$, or some other interval above ambient temperature. The cooling time is a measure of the air movements around the thermometer. (redrawn from Hill, 1916)

\section{Equivalent temperature in the beginning}

Dufton, 1929 developed a black copper cylinder in order to imitate the human body heat loss (eupatheostat), and he termed the measured variable 'equivalent temperature' $\left(t_{e q}\right)$. Later he constructed the eupatheoscope (Mark I) for recording equivalent temperature. The equivalent temperature was at this time defined as "that temperature of a uniform enclosure in which, in still air, a sizeable black body at $24^{\circ} \mathrm{C}\left(75^{\circ} \mathrm{F}\right)$ would lose heat at the same rate as in the environment". Later he developed the instrument so that the surface temperature became a precise function of the environment and corresponded to that of standard clothing (Dufton, 1936).

A new instrument was made, eupatheoscope Mark II (see figure 10 and 11). The adoption of this new instrument necessitated a slight modification of the definition of the equivalent temperature. This because the copper cylinder no longer had a surface temperature of $24^{\circ} \mathrm{C}$. The definition at this time was "the equivalent temperature of an environment is that temperature of a uniform enclosure in which, in still air, a black body of sufficient size would lose heat at the same rate as in the environment, the surface temperature of the body being one third of the way between the temperature of an enclosure and $38^{\circ} \mathrm{C}\left(100^{\circ} \mathrm{F}\right) "$.

The eupatheoscope was developed primarily as a laboratory instrument. Making measurements outside the laboratory with the original eupatheoscope was difficult due to the lack of electricity. For this purpose did Dufton utilises two special kata thermometers with a modified cooling range. One of the thermometers has a silver bulb and the other a black bulb. Over a wide range of conditions Dufton found that the 
equivalent temperature estimated with the portable kata thermometers had a mean error of less than $0.46^{\circ} \mathrm{C}\left(0.82^{\circ} \mathrm{F}\right)$, compared the eupatheoscope.

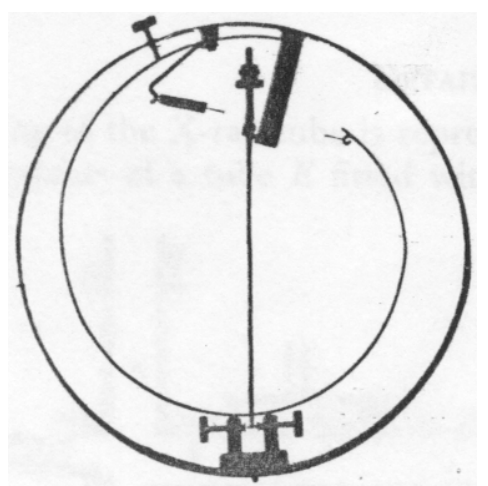

Figure 10. The eupatheoscope is heated by two 240 -volt electric lamps and the surface temperature is regulated by a thermostatic element consisting of a bimetallic thermostat and an electrical relay (Dufton, 1936).

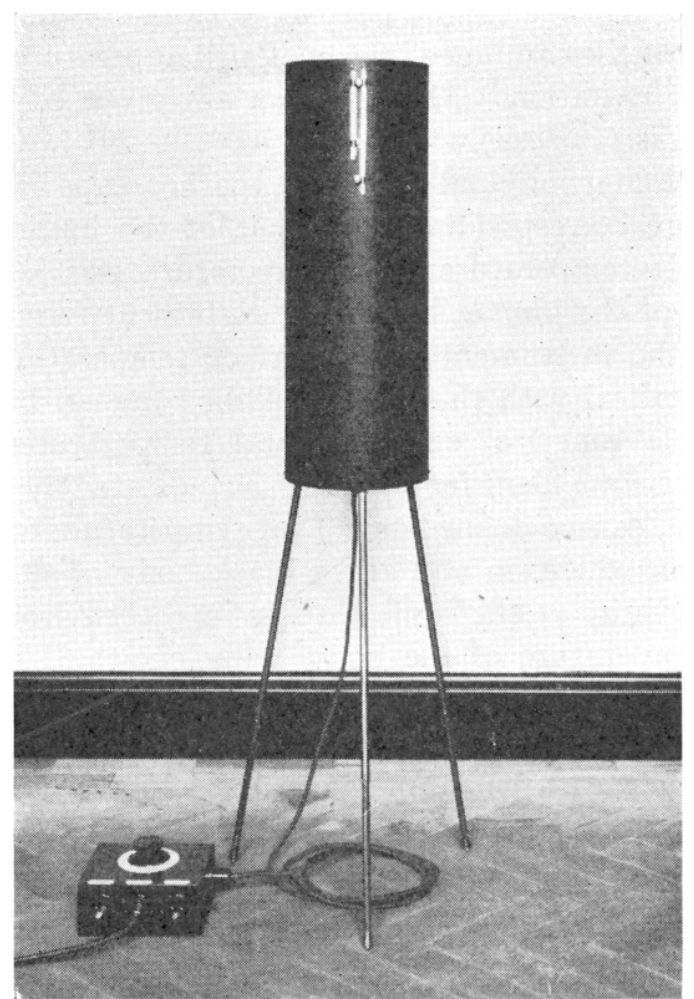

Figure 11. The eupatheoscope Mark II (Dufton, 1936).

The eupatheoscope has variable heat input, while its surface temperature is kept almost constant. Winslow et al., 1935 first described an instrument with the reversed principle of constant heat input and variable surface temperature. The Thermo-Integrator is, like the eupatheoscope, a hollow copper cylinder, electrically heated with constant heat input. The surface temperature of the instrument is recorded by means of thermocouples distributed over its periphery and connected to an automatic recorder.

Other normated temperatures using the same idea but with different names are Equivalent Homogeneous Temperature (EHT) (Wyon et al., 1989, Bohm et al., 1990). An alternative method for the determination of "equal thermal environments" is the RST or Resultant Surface Temperature and the associated "equivalent temperature" (Mayer et 
al., 1993). A constant surface heat loss is assumed and the temperature of the surface segment (RST-value) that solves the heat balance equation, for inhomogeneous conditions is used as a measure of the climatic influence from the environment.

\section{Definition of equivalent temperature today}

The definition today (ASHRAE 62, 1989) reads "the equivalent temperature $\left(t_{e q}\right)$ is the temperature of an imaginary enclosure with the mean radiant temperature equal to air temperature and still air in which a person has the same heat exchange by convection and radiation as in the actual conditions".

The equivalent temperature is a recognised measure of the effects of non-evaporative heat loss from the human body (Madsen et al., 1984, SAE J2234, 1993, Nilsson et al., 1999a). It is particularly useful whenever complex interactions of various heat fluxes are present. The equivalent temperature is derived from the operative temperature by the inclusion of the effect of air velocity on a heated body.

The well-known operative temperature only considers the air temperature and the mean radiant temperature and is defined for the actual air velocity, whereas the equivalent temperature $\left(t_{e q}\right)$ is defined for a standard low air velocity.

One advantage of $t_{e q}$ is that it expresses the effects of combined thermal influences in a single figure, easy to interpret and explain. It is particularly useful for differential assessment of the climatic conditions. However, the underlying hypothesis is that the $t_{e q}$ value always represents the same "subjective" response irrespective of the kind of combinations of heat losses. Today this seems to be true, at least for conditions close to thermal neutrality $( \pm 2 \mathrm{MTV})$ and within limited variations of the climatic factors (Bohm et al., 1990, Schwab et al., 1999).

\section{Measurements of equivalent temperature with different sensors today}

Today the equivalent temperature is measured with various instruments and devices. The most developed method is the use of a man-sized heated manikin, which simulates in a realistic way the three-dimensional heat exchange of a human.

\section{Measurement of basic climatic parameters}

With measurements of air temperature, mean radiant temperature and air velocity, radiant and convective heat losses can be calculated and $t_{e q}$ determined using appropriate equations described later.

\section{Measurement of convective and radiative heat losses with heated sensors}

Heated sensors can be used for determination of body surface heat losses by convection and radiation.

\section{Ellipsoid sensors}

A heated, ellipsoid sensor may serve as a representative physical model of the human body (Brüel \& Kjær, 1982, Madsen et al., 1986). The heat exchange of the sensor is assumed to correspond to the total (and uniform) convective and radiative heat 
exchanges of the human body surface. The instrument directly calculates the equivalent temperature.

\section{Flat surface sensor}

Heated, flat surface sensors give reliable and relevant estimates of dry heat losses. The 3-dimensional nature of human heat transfer requires special arrangements of several flat sensors or measurements on several locations, for example on the surface of a body shaped dummy. In order to provide a single figure for the overall $t_{e q}$ values, the individual values are added and area weighted in a representative way (Mayer \& Schwab, 1993).

\section{Local discomfort meter}

A double-sided heated skin element has been constructed (Madsen et al., 1992). The difference in mean heat flux (MHF) from the two opposite elements can directly be transformed to a thermal asymmetry. The electrical signals received from the skin element are transformed by a microprocessor into a value called Perceived Heat Flux (PHF) which is equivalent to the sensation of local thermal discomfort. The calibration of the instrument is done in the same way as for the manikins.

\section{Measurement of equivalent temperature with thermal manikins}

A heated thermal manikin represents the ultimate heat flux transducer. The whole manikin surface is heated to and controlled at the same "skin" temperature as the human body surface. The early manikins comprised only one zone. Today's manikins provide $16,20,35$ or even more individually heated and controlled zones. To get a single figure for $t_{e q}$ the local heat losses are added and the total value divided by the total body surface area gives the dry heat loss to be used for the $t_{e q}$-computation.

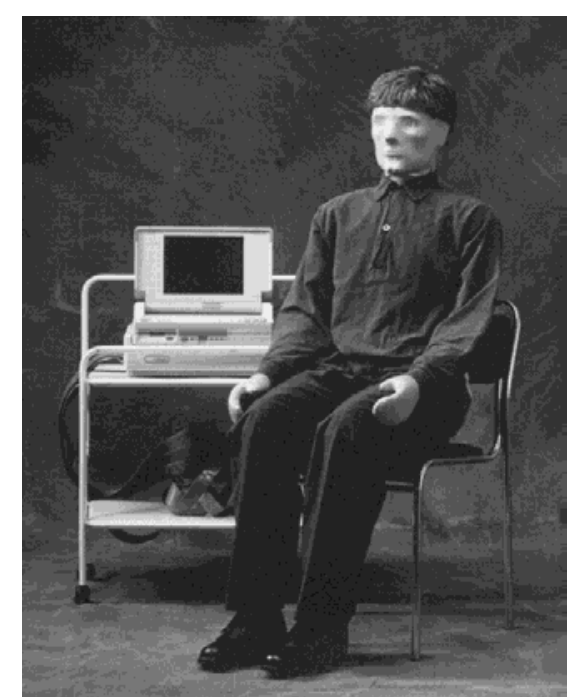

Figure 12. The heated manikin MANIKIN2 with 33 individually controlled zones. This manikin was especially constructed for climate evaluation in 1991.

Manikin with constant surface temperature

The surface temperature of the manikin is uniform and constant (viz. $34{ }^{\circ} \mathrm{C}$ ) over all zones. 


\section{Manikin with distributed surface temperature}

The manikin has a distributed temperature over the zones. Usually the temperature declines from the torso to the extremities (Bischof et al., 1991).

\section{Manikin with constant heat loss}

This manikin type operates with constant heat loss from the different zones. Small radial as well as inwards axial heat losses may influence the measurements by this technique.

\section{Manikin with adaptable surface temperature}

Surface temperature of the manikin is allowed to change as function of dry heat loss using an expression derived on the basis of Fanger's comfort criteria (Madsen et al., 1986).

$t_{s}=36.4-0.054 \cdot Q_{t} \quad$ (nude)

$t_{\text {eq }}=t_{s}-0.155 \cdot I_{t} \cdot Q_{t} \quad($ clothed $)$

\section{Equation 1a and 1b}

In a cold environment the manikin will produce a higher heat loss. This is interpreted as a request for a lower skin temperature. However, this lower skin temperature corresponds to thermal neutrality only for a person in thermal balance, matching the higher heat loss by an increased activity level. In other words, this method always determines equivalent temperature under conditions of thermal neutrality. This additional requirement is then required as part of the definition.

\section{Manikin with heated sensors}

This method measures the surface temperature of the sensors at constant heat loss with 18 small sensors on a manikin body, each containing two heated surfaces with two different power levels. The surface temperature of the two surfaces with known heat loss is measured and a linear model is used to calculate the surface temperature for an unheated surface, i.e. $t_{e q}$ (Palazzetti et al., 1996).

\section{Climates indices with similar principles}

A number of indexes have been developed, all according to similar methods, by normation of the "real" environment to a "standard" environment which gives the same thermal climate experience.

\section{Effective temperature}

Effective Temperature (ET) is an index developed in collaboration with ASHRAE (American Society of Heating and Air Conditioning Engineers). It is based on experiments in which both dressed and undressed subjects were moved between two rooms with different combinations of climate factors (Houghten et al., 1923). The conditions in one of the rooms were adjusted until the same climate sensations were reached in both rooms. 
The ET index uses the concept "temperature in standard environment" as index value. ET is originally defined as "the temperature in a standard environment ( $r h=100 \%$, $t_{a}=\bar{t}_{r}, v<0.15 \mathrm{~m} / \mathrm{s}$ ) that gives the same experience of warmth as in the real environment". The person in the standard environment should have the same clothing and activity level as in the real environment. A serious problem with this definition of effective temperature is that it makes no special allowance for radiation. As long as $t_{a}=t_{r}$ the scales below are applicable, otherwise not.

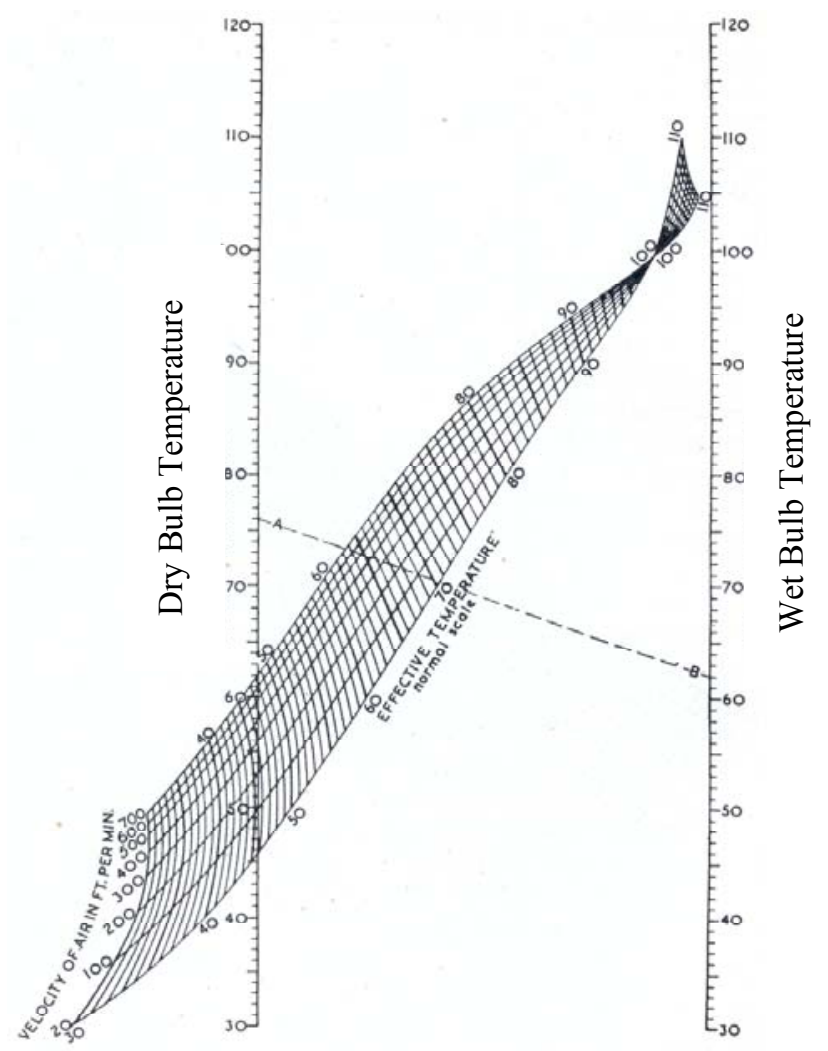

Figure 13. ET chart (Normal scale) (redrawn from Bedford 1936).

Two charts for calculation of ET was published, one for each clothing level. Charts for a person without clothes basic effective temperature (BET) and another for normally dressed persons normal effective temperature (NET). The use of these ET charts makes it possible to determine an ET value with help of the dry and wet temperature and the air velocity. All combinations of these factors that gives the same ET value, shall according to the definition be equally comfortable. The original ET had no correction for radiation. One way to take mean radiant temperature into account is to exchange the dry temperature with globe temperature.

This study contained however uncertainties. The subjects went between two chambers and compared different combinations of air temperature and moisture in order to give a subjective experience of the climate. The subjects gave their experience, which largely is determined by effects from immediate thermal sensation caused by temperature and, eventually condensation and evaporation of moisture from skin and clothes. The results can therefore only be used for studies of transient effects and over estimate effects of moisture when looking on steady state. 
After the establishment of ET as defined by Houghten \& Yagloglou, 1923, a 'comfort zone' and several conditions for climate comfort was developed. The comfort zone was defined as the ET values at which more than $50 \%$ of the subjects was comfortable with the climate.

\section{The comfort equation}

Fanger 1970 describes the conditions for climate comfort and methods and principles for evaluation and analysis of different environments out of a climate comfort point of view, with the indices Predicted Mean Vote (PMV) and Predicted Percentage Dissatisfied (PPD). Fanger defines conditions that must be fulfilled in order for a person to be in whole body climate comfort. An additional condition is the absence of any local climate discomfort. The intention was to develop a comfort equation where just the above mentioned six factors are needed, in order to calculate in which thermal state a normal person is. These calculation- and estimations methods are now both Swedish and European as well as International standards (ISO 7730, 1995) and are used all over the world.

\section{Further development of these climate indices}

A number of methods for correction of different indexes have proposed in order to allow that, for instance, radiation will be taken in to consideration. Missenard, 1959 suggested a number of methods in order to calculate a climate index, which he called resultant temperature $(R T)$. He derived an equation for effective temperature and introduced a radiation term. By using a function named "fictitious air velocity" instead of the real air velocity. He could use ET charts for calculations of RT. He was also one of many scientists that has used wet and dry globe thermometers in order to imitate the thermal climate influence on the human body. Another method was to replace the drybulb temperature with the globe temperature (Vernon et al., 1932) this index was called corrected effective temperature (CET).

In the 70-ies a lot of work was carried out in the USA at "J. B. Pierce Foundation Laboratory" in order to develop improved versions of different climate comfort indices. Gagge et al., 1971 suggested a new effective temperature (ET*) (E-T-star) that described the real environment in terms of the temperature in a standard environment $(r h=50 \%$, $\left.t_{a}=\bar{t}_{r}, v<0.15 \mathrm{~m} / \mathrm{s}\right)$. It is based on the condition that a person with accordingly experience would have the same skin wetness $(w)$, the same skin temperature $\left(t_{s k}\right)$ and the same heat loss from the skin $\left(H_{s k}\right)$ as in the real environment. ET* is foremost intended for persons with sitting work and lighter clothing. The definition of ET* in ASHRAE 55 has evolved to "the operative temperature $\left(t_{o}\right)$ of an enclosure at $50 \%$ relative humidity that would cause the same sensible plus latent heat exchange from a person as would the actual environment".

Gagge et al., 1971 also developed ET to also include the influence of activity- and clothing is called standard effective temperature (SET). SET in the real environment is defined as the temperature in a standard environment $\left(r h=50 \%, t_{a}=\bar{t}_{r}, v<0.15 \mathrm{~m} / \mathrm{s}\right)$, in which a person with a standardised clothing would have the same heat loss and the same skin wetness $(w)$, the same skin temperature $\left(t_{s k}\right)$ like in the real environment. ET* 
is of course equal to SET for still jobs and light clothing. The activity assumed to be the same in both environments. The clothing chosen depends on the activity level.

Gagge meant that the PMV index underestimated effects of moisture, and suggested a new index, $P M V^{*}(P-M-V$-star) (Gagge et al., 1986). Identical with PMV, apart from that the operative temperature $\left(t_{o}\right)$ is replaced with ET* in PMV equation. Fanger usually claims that PMV defines conditions of climate comfort, and that effects of evaporation and moisture are minimal in that situation.

\section{Other factors of importance}

Body build, sex, age, geographical differences, food and beverage plus several other environmental factors also influence perception of climate comfort. The effect of these components is not taken into consideration in any of the above indices. They may have effect on the perception of thermal comfort and contributes eventually, to the sometimes-significant individual variation in responses, seen in practice.

\section{Summary comfort indices}

Presently the three most popular, and widely used, comfort indices are PMV, ET* and $t_{e q}$. But the search after the ultimate climate comfort index continues! Today there is a competition between two modelling schools. One model is the previously mentioned, internationally standardised PMV model; based on the heat balance of the human body. The other model is the Adaptive model; assuming a human adaptation to the thermal environment to a certain degree. Also in this field does the fast personal computer development speeding up the process.

Climate models can be used, not just to calculate different indices, but also in order to predict different types of reactions from an average person. However, the computer development have not changed principles behind climate comfort theory, only just provided us with better tools. Although this area still is undergoing some development, there already exist models and computer systems that can be used in construction and evaluation of new and existing climate environments. They all provide a rational description of the physical heat exchange of humans with relations to the perception of thermal comfort. Further refinement of equations with results from human experiments as well as manikin measurements and simulation methods are needed. 


\section{Thermal manikin history}

\section{The first thermal manikins}

The word manikin is believed to originate from the Dutch word "Manneken", a small man. In French "Mannequin" could mean a display mannequin or a jointed wooden dummy. The first manikin "Manichino" did historically appear in Florence Italy around the end of the 15th century. That manikin was used as a reference tool to learn the basics of figure drawing. One of the first using these art manikins was the Italian high renaissance painter Frà Bartolomeo.

The first thermal manikin was a one-segment copper manikin made for the US Army in the 40's (Belding, 1949). Belding first build a headless and armless manikin from pipes and metal sheets. The manikin had an internal heater and fan to distribute the heat. In 1942, Belding collaborated with engineers at the General Electric Co., and build a new thermal manikin. This manikin was made out of an electroplated copper shell with electrical circuits that uniformly heated the surface. The manikin was made with a feature to change the surface temperature of the hands and feet independent of the surface temperature of the rest of the manikins body. Several of this kind were manufactured and also used for indoor climate (HVAC) research. The reliability and robust construction is some of the reasons that some of them are still in use.

The need for more detailed information brought forward the construction of manikins with several, independently controlled segments over the body surface. Almost all manikins today provide for more than 15 segments. To reduce costs and weight other materials have been used and many of the modern manikins are made of aluminium and plastic. Another significant step forward was taken with the introduction of digital regulation techniques. This allowed for more flexible protocols and accurate measurements.

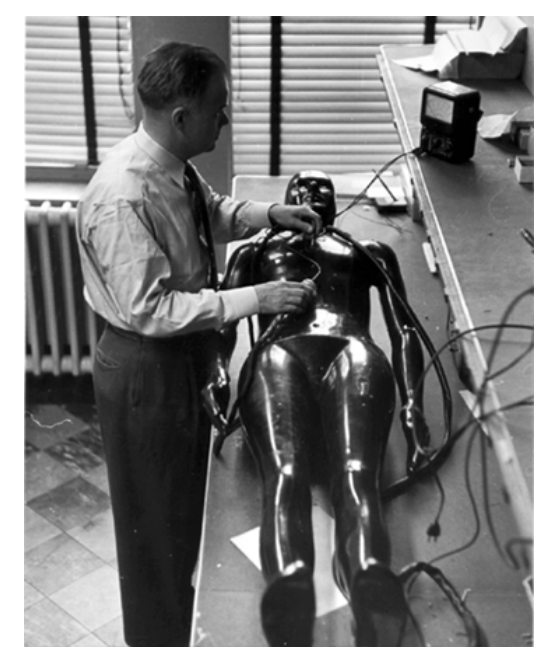

Figure 14. Copper manikin used in studies on body temperature. Photo: H. Corsini. June 1951. Oakland: School of Public Health, University of Pittsburgh. From the Collections of the Pennsylvania Department, The Carnegie Library of Pittsburgh. 
It was early recognised that a static, standing thermal manikin provided clothing test values with limited relevance to actual user conditions. Manikins were hence constructed with joints that allowed the manikin to be seated. With more robust constructions manikins could even be constantly moveable, i.e. perform "walking" or "cycling" movements. Most of these manikins are used for clothing evaluation. Clothing for protection against cold water required a special type of thermal manikin to be developed.

Table 2. Milestones in the development of human shaped thermal manikins (extended and modified from (Wyon, 1989) and (Holmer, 1999).

\begin{tabular}{lllllll}
\hline Type & Segmentation & Series & Material & Regulation & Posture & Country \\
\hline A & 1-segment & SAM & copper & analogue & standing & USA 1942 \\
B & 11-segments & ALMANKIN & aluminium & analogue & standing & UK 1964 \\
C & radiation manikin & CEPAT400 & aluminium & analogue & standing & France 1972 \\
D & 16-segments & HENRIK2 & plastic & analogue & moveable & Denmark 1973 \\
E & 16-segments & CHARLIE & plastic & analogue & moveable & Germany 1978 \\
F & 16-segments & SIBMAN & plastic & digital & sit, stand & Sweden 1980 \\
G & 19-segments & VOLTMAN & plastic & digital & sitting & Sweden 1982 \\
H & 36-segments & ASSMAN & plastic & digital & sitting & Sweden 1983 \\
& & (MANIKIN1) & & & & \\
I & 19-segments & TORE & plastic & digital & moveable & Sweden 1984 \\
J & 7-segments & CLOUSSEAU & plastic & analogue & standing & Sweden 1987 \\
K & sweating manikin & COPELIUS & plastic & digital & moveable & Finland 1988 \\
L & female manikin & NILLE & plastic & comfort & moveable & Denmark 1989 \\
M & 33+3-segments & HEATMAN & plastic & multi & sitting & Sweden 1991 \\
& & (MANIKIN2) & & & & \\
N & 1-segment sweating & WALTER & fabric & water & moveable & Hong Kong 1991 \\
O & 36-segments & HEATMAN & plastic & digital & moveable & France 1995 \\
P & breathing manikin & NILLE & plastic & multi & moveable & Denmark 1996 \\
Q & sweating manikin & SAM & plastic & digital & moveable & Switzerland 2001 \\
R & 26-segments & TOM & copper & digital & moveable & USA 2003 \\
S & 126-segments & ADAM & composite & model & moveable & USA 2003 \\
\hline & & & & & & \\
\hline
\end{tabular}

The number of manikins has considerably increased and may count more than a hounded in use worldwide. Table 2 presents a list of milestones in the development of thermal manikins. Each new example represents a significant improvement in the technique. Country of development and the approximate year of construction are indicated. References below are not necessarily the first, but provide information about the different manikins.

A complete understanding of human heat exchange requires not only convective, conductive and radiative heat losses to be measured. In warm environments the main mechanism for heat loss is sweat evaporation. An increasing number of manikins in operation can simulate human sweating and provide valuable information about heat exchange by evaporation (Burke et al., 1994, Dozen, 1989, Meinander, 1992, Fan et al., 1991, Lebbin et al., 2003, Burke et al., 2003). 


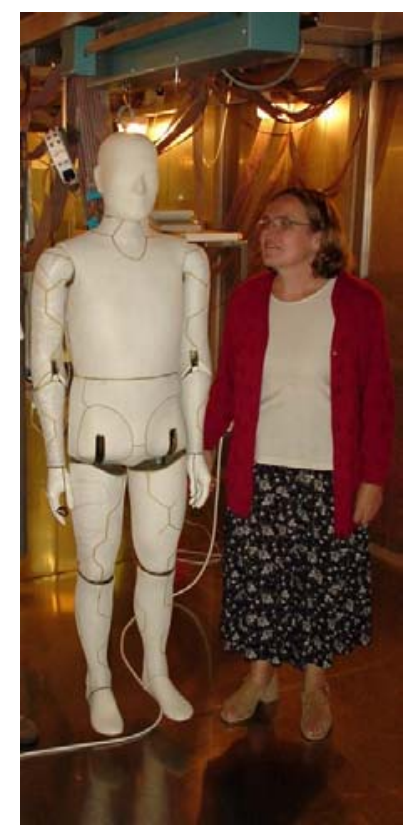

Figure 15. The Finnish sweating Manikin Copelius made during a Nordforsk project developing the TORE series in the 80-ties. (To the right Dr Harriet Meinander, VTT, Finland).

\section{Thermal manikins in research}

Manikins are complex, delicate and expensive instruments with many advanced and useful features. The list below provides important arguments for the use of thermal manikins. A human shaped thermal manikin measures convective, radiative and conductive heat losses over the whole surface and in all directions. Depending on number of segments of the manikins surface the spatial resolution can be high. Many manikins in use have more than 100 individually regulated segments. By summing up the area weighted values, a value for whole body heat loss is determined.

\section{Significant performance features of thermal manikins:}

- relevant simulation of human body heat exchange, whole body and local

- measurement of 3-dimensional heat exchange

- integration of dry heat losses in a realistic manner

- objective method for measurement of clothing thermal insulation

- quick, accurate and repeatable

- cost-effective instrument for comparative measurements and product development

- provide values for prediction models; clothing insulation and evaporative resistance, heat losses

For the same exposure conditions, a thermal manikin measures heat losses in a relevant, reliable and accurate way. The method is quick and easily standardised and repeatable. Due to the nature of the method, values obtained can serve directly as input for mathematical models for prediction of thermal responses (see standardisation below). 


\section{Application areas}

When the clo-value was defined for thermal insulation of whole clothing ensembles in the early 40-ies, a method was needed for its determination. The first thermal manikins were constructed for this purpose. Extensive clothing research with manikins has been carried out by USARIEM Natick Laboratories (Goldman, 1983), Kansas State University (McCullough et al., 1983, McCullough et al., 1985) and the Technical university of Denmark (Olesen, 1982) and also by the Hohenstein group (Umbach, 1988). In recent years more clothing studies are done in Scandinavia (Wyon et al., 1986, Holmér, 1995, Meinander, 2000, Holand, 2000), as well as Poland (Soltynski, 2000), Japan (Tamura et al., 1994) and China (Zhai et al., 2001).

It was early recognised that a heated thermal manikin could also be used for evaluation of the microclimate conditions caused by different ventilation systems (HVAC) (ASHRAE 62, 1989). This application has increased in recent years, in particular within the automobile industry (Wyon et al., 1989, Olesen, 1992, Palazzetti et al., 1996, Nilsson et al., 1997). European research project (With the acronym EQUIV) analysed and proposed the use of thermal manikins for assessment of vehicle climate. This research was reported at the ATA conference in Florence in November 1999 (EQUIV, 1999).

Similar measurement principles can also be used for detailed analysis of indoor air evaluation (Nielsen et al., 1978, de Dear et al., 1993, Nilsson et al., 2000). Manikins can simulate any skin temperature distribution, thereby simulating specific thermal conditions of the human body. In this way accurate and precise measurements can be made of total and local heat losses under the given conditions (Lotens, 1993, Wang et al., 1992, Ichihara et al., 1996, de Dear et al., 1997).

Main application fields for thermal manikins:

- evaluation of HVAC-systems

- control and construction of buildings

- control and construction of vehicles

- control and construction of incubators

- evaluation of indoor air quality

- simulation of human occupancy

- testing of thermal properties

- testing of protection equipment

- evaluation of clothing design

- physiological simulations

All manikins so far had been men and the first female manikin appeared in the middle of the 80s (Madsen et al., 1986). This manikin also provided a new technique for heating and measuring as well as a new regulation concept. A special technique is used to have a single wire for both heating and measuring of each zone. The regulation program uses "comfort" algorithms for the control of the different body segments. A significant improvement was the simulation of breathing (Nielsen, 2000, Melikov, 2003). This feature is particularly useful in ventilation research. 
Another important innovation is the "Walter"-sweating fabric manikin (Fan \& Keighley, 1991, Fan, 2003). Walter is equipped with simulation of "walking motion", automated water supply and real time measurement of evaporative water loss. The surface temperature is regulated through the regulation of the pumps inside the manikin body.

Thermal Observation Manikins (TOM) (Lebbin et al., 2003) were designed and built for use in automobile applications incorporating heat flux transducers (HFT) to measure heat gain when exposed to external heating conditions. These manikins will incorporate a subjective model of thermal comfort along with a human thermal physiological model to produce a thermal sensation vote based on a combination of heat flux transducers, temperature sensors, and heater power measurements.

A new type of thermal manikin has been developed as part of a suite of tools for evaluation of occupant thermal comfort in automotive cabins (Burke et al., 2003). ADAM has 126 separate metal-skinned composite regions with independent control of surface temperature and sweat rate plus a measure of transient heat flux. The manikin is designed for tethered or wireless operation with an internal battery pack and transceiver for real-time data reporting and setpoint adjustment. An internal air pump simulates breathing for realistic airflow patterns. ADAM also couples to an external real-time numerical model predicting human thermoregulatory response and perceived comfort.

\section{Standardisation}

The use of thermal manikins in research and standards has significantly increased in recent years. New fields of application such as evaluation of HVAC-systems in rooms and vehicles have grown. Thermal manikins have found their application not only in research but also in test houses and industrial test laboratories. For research purposes a thermal manikin must provide relevant, reliable and accurate measurements. However, the specific aims and needs of the research problem may require specific design and performance features. The manikins do not necessarily need to be compatible and exactly comparable with other manikins. For testing purposes the same conditions apply, if the manikin is used for in-house development work. However, as soon as test values need to be compared with values from other laboratories or test houses, the manikin, methods and procedures need to be standardised.

Recent studies by Topp et al., 2003a, 2003b show, when examining the overall airflow and temperatures in a room, that flow around a thermal manikin with a simple geometry can be sufficient. A more detailed geometry is proposed when local conditions close to the occupant are to be studied. Values obtained with different manikins in different test houses must be comparable and similar within defined limits for the same test conditions. Standardisation work in this area are being done in several work groups (WGs) ISO TC92 WG17 (clothing testing), ISO/TC152 SC5 WG1 (vehicle testing) as well as ISO/TC159 SC5 WG1 (thermal comfort) (Olesen et al., 2002). In order to not exclude any of the more than 100 manikins built around the world, no standardised ISOmanikin has yet been defined. 


\section{Summary thermal manikins}

Human shaped thermal manikins measures convective, radiative and conductive heat losses over the whole surface and in all directions. Depending on number of surface segments the resolution can be adjusted to be sufficiently high to complete the measurement task. Some manikins in use today have only 1 zone others more than 100 individually regulated segments. By summing up the area weighted heat loss values from the manikin, a total value for whole body heat loss is determined.

Some performance features of the most commonly used thermal manikins are; simulation of human body heat exchange, measurement of 3-dimensional heat exchange, integration of dry heat losses, measurement of clothing thermal insulation, product development and providing values for prediction models.

The important calibration is carried out with the manikin dressed in standard clothing. Clothing affects the value of the heat transfer coefficient. The less insulation clothing provides, the more sensitive will the manikin segments be to thermal influences. It is important to specify the clothing used, whenever data are compared from different investigations and manikins. This comparison can be facilitated with the use of "clothing-independent" comfort zone diagrams.

Values obtained with different manikins in different test houses should be comparable and similar within defined limits for the same test conditions. The conditions and requirements for comparable measurements with different manikins and methods must be laid down in standards. There is an ongoing work to standardise these methods within the international standardisation organisation, ISO. However, the question of a standardised "ISO-manikin" might be difficult to obtain, having not to exclude too many of the more than 100 manikins built and used around the world today. 


\title{
Mathematical simulation models of the human body
}

\author{
Human thermophysiological modelling
}

A number of models have been developed during the past years in order to describe human thermal response to various conditions. Although the scientists and engineers who developed these models use many different approaches, all of them can be characterised in terms of the following factors:

- The amount of details provided about the temperature field within the body

- The way thermoregulatory responses are handled

- The treatment of garments and boundary conditions

- The possibilities to correlate with relevant measured data

- The amount of computational effort and usability

The way, in which these factors are handled in each model, determines the capability for simulating various conditions within the range of possible human experiences. Some models attempt to provide a detailed description of the temperature field within the body, while others define only central and mean skin temperatures. The amount of detail incorporated into a model is generally limited by the scientific effort and the speed and amount of memory provided by the computer on which the model runs.

Developing descriptions of physiological phenomena is much more difficult than computing temperatures. Three major processes are involved in thermoregulation: cardiovascular responses, sweating and shivering. It is not surprising that they have been incorporated into various mathematical models in different ways, and therefore these form differences between models. Sweating and shivering are essentially pure thermal responses whose intensities are determined by the magnitude of different signals generated by central and peripheral thermoreceptors.

Clothing plays a very important role in determining human thermal response and must be described in a mathematical model. The thermal properties of garments are characterised primarily by two factors; one is the resistance to sensible heat transfer and the other is resistance to water transport through the garment. These clothing properties are normally measured with thermal manikins. 
Table 3. Human thermal mathematical models (extended from Wissler 1985)

\begin{tabular}{|c|c|c|}
\hline Author & Description & Computer \\
\hline Stolwijk, 1971 & $\begin{array}{l}\text { Physiological } \\
\text { Validated for hot environments; not } \\
\text { cold exposure }\end{array}$ & $\begin{array}{l}\text { Personal computer } \\
\text { Often used in CFD calculations }\end{array}$ \\
\hline Givoni et al., 1971 & $\begin{array}{l}\text { Empirical } \\
\text { Validated for hot environments; not } \\
\text { cold exposure }\end{array}$ & Pocket calculator \\
\hline Gagge et al., 1971 & $\begin{array}{l}\text { Physiological } \\
\text { Two-node model }\end{array}$ & $\begin{array}{l}\text { Personal computer } \\
\text { Often used in CFD } \\
\text { calculations }\end{array}$ \\
\hline Wissler, 1988 & $\begin{array}{l}\text { Physiological } \\
\text { Validated for hot and cold } \\
\text { environments }\end{array}$ & Mainframe \\
\hline Werner et al., 1993) & $\begin{array}{l}\text { Physiological } \\
\text { Six-cylinder model. } \\
\text { Steady-state }\end{array}$ & Personal computer \\
\hline Piniec et al., 1997 & $\begin{array}{l}\text { Physiological MATHER } \\
\text { Steady state and transient } \\
\text { cold, neutral and hot conditions }\end{array}$ & Personal computer \\
\hline Fiala et al., 1999 & $\begin{array}{l}\text { Physiological } \\
\text { Steady state and transient } \\
\text { cold, neutral and hot conditions }\end{array}$ & Personal computer \\
\hline Huizenga et al., 2001 & $\begin{array}{l}\text { Physiological } \\
\text { Steady state and cold as well as hot } \\
\text { transients }\end{array}$ & Personal computer \\
\hline $\begin{array}{l}\text { Kohri et al., 2002; } \\
\text { Kohri et al., } 2003\end{array}$ & $\begin{array}{l}\text { Physiological dispersed two-node } \\
\text { Steady state comfort climate } \\
\text { Correlation with regional SET* }\end{array}$ & Personal computer \\
\hline
\end{tabular}

To summarise, one or more of the following factors often limit the validity of mathematical models currently available for analysing human responses to different environmental factors:

- Too low resolution to describe the temperature field within the body

- Inadequate description of one or more aspects of thermoregulatory responses

- Insufficient information about the clothing worn by the subject

- Lack of agreement between computed and measured results

- The need for use of special computers and long calculation times

Since several experimental studies and extensive computation would be required to determine exact probabilities for even a single case, the complexity of the simulation often determines the extent to which models have been validated for either the specific or closely related conditions.

\section{Virtual manikins and CFD in research}

The interest in using virtual thermal manikins in research has grown in recent years. This is seen in the number of manikins being described in the literature as well as the organisation of international meetings addressing virtual manikin applications. 
One of the first researchers to use Computational Fluid Dynamics (CFD) for numerical predictions of the indoor environment was Peter V Nielsen in his thesis (Nielsen, 1974). He did not at that time use virtual thermal manikins. Early use of "numerical thermal occupants" for assessment of indoor thermal comfort was made by Gan, 1994. Gan and colleagues developed a CFD code for evaluation the indoor environment in ventilated rooms. The program produces thermal sensation indices, the PMV and PPD for evaluation. Further development of the program was made to enable both thermal sensation and draught risk indices to be predicted. As a turbulence model he used standard $k-\varepsilon$ model with a SIMPLE algorithm and QUICK and UPC differencing schemes. The time to solve the airflow equations together with the comfort equation was 35 hours for 800 iterations with a grid $38 \times 36 \times 30$ on a Sun Sparc 1.

Later Brohus and Nielsen (Brohus et al., 1996) presented three different CFD models of a person. The models were evaluated by comparison with measurements using a fullscale breathing thermal manikin standing in a channel. The three CFD models were made with a rectangular geometry. One manikin was a heated cuboid with the same surface area and heat flux as human being. Another more complex manikin included "legs" and "head". The models were evaluated with steady-state CFD simulations of personal exposure to a contaminant source in a uniform velocity field. The height and the surface area of the computer-simulated person (CSP) corresponded to an average sized woman with a clothing insulation of 0.8 clo. The convective heat flux was fixed at $25 \mathrm{~W} / \mathrm{m}^{2}$.

In a paper by Kato, 1996 the flow and the temperature fields around a computational manikin are analysed by CFD. The manikin is placed in a air conditioned room with displacement ventilation system. Using CFD the age of the supplied air and the residual lifetime were analysed. The contaminant distribution in the room was simulated with three different positions of the contaminant generation. They used low-Reynolds number $k-\varepsilon$ model with the manikin heated uniformly with $20 \mathrm{~W} / \mathrm{m}^{2}$.

For the computation of thermal comfort of occupants in a vehicle compartment Maué et al., 1997 at Daimler-Benz AG used three different programs to calculate PMV and $t_{e q}$. For calculation of velocity and temperature distribution in the passenger compartment, the CFD-code STAR-CD ${ }^{\circledR}$ was used. To determine the heat flux by convection, radiation (thermal radiation, solar radiation) and conduction into the compartment they used the in-house code SWF. In order to calculate the thermal state of an occupant in the vehicle they used the ThermophysIological Model of a passenger simulation program TIM. The highly turbulent nature of the flow was calculated with a standard $k$ - $\varepsilon$ model using logarithmic law of the wall.

Murakami et al., 1997 and 1998 developed a system that is called; the "computational thermal manikin" for predicting human thermal comfort. They modelled the different thermal transport processes in a coupled manner, by simulating the combined effects of airflow, radiation, moisture transport, etc. They described the development of the computational thermal manikin in two stages. The first stage (Part 1), was the heat transfer interaction between a nude manikin and its surrounding flow was analysed with CFD. The convective heat transfer coefficients between the nude manikin and its environment was calculated. In Part 2 they used the manikin to analyse ventilation efficiency indices, the relationship between the concentration distribution and the age of supply air as well as the residual lifetime of air. They used low-Reynolds number $k-\varepsilon$ 
model with a fine grid near the manikin. The computation for each case took about 100 hours on a supercomputer with a speed of 8 GFLOPS (FLOPS $=$ floating point operations per second).

Han et al., 2001 has developed virtual methods of predicting occupant thermal comfort to support automotive climate control systems. They used a model of the human thermal regulatory system based on a 16 segment Stolwijk model. This comfort model had the ability to predict local thermal comfort level of an occupant in a non-uniform thermal environment as a function of air temperature, surrounding surface temperatures, air velocity, humidity, direct solar flux, as well as the level of activity and clothing type used. They call this technique Virtual Thermal Comfort Engineering (VTCE). The FLUENT CFD-code was used together with the UC Berkeley physiological model in order to calculate $t_{e q}$ and PMV.

Bjørn, 2000 uses CFD to simulate the effects of respiration measured in displacement ventilated rooms. The concept behind the physical manikin was to make a geometry with well-defined geometrical primitives, easy to convert into an accurate virtual geometry for CFD simulations. He simulated the geometry of the manikins with a body fitted, unstructured grid. The grid contained around 640,000 cells. Only half of the room and the manikin were simulated. He uses the RNG $k-\varepsilon$ turbulence model with buoyancy effects and second-order discretisation scheme.

Kang, 2002 presents a CFD analysis of the WBGT index in a fully occupied space with mechanical ventilation. They use a standard high Reynolds number $k-\varepsilon$ turbulence model. A 15-segment thermoregulation model with 61 computational nodes was coupled to the CFD model. An iterative algorithm was developed for the WBGT estimation. The WBGT distribution in the occupied space was calculated according to the predicted parameters by the CFD model.

Tanabe, 2002 has developed a 65-node thermoregulation model based on the Stolwijk model. The model has 16 body segments corresponding to the physical thermal manikin, each consisting of 4 layers for core, muscle, fat, and skin. The 65th node in the model is the central blood compartment, which exchanges convective heat with all other nodes via the blood flow. Convective and radiant heat transfer coefficients and clothing insulation were derived from thermal manikin experiments. The model combined with a radiation exchange model and CFD calculations. The model uses anthropometric data of an averaged man with the body weight of $74,430 \mathrm{~kg}$ and the body surface area of $1,870 \mathrm{~m}^{2}$. They use a detailed human body model with 4,396 surfaces for radiation analysis, and a simplified model with 1,542 surfaces for CFD analyses, maintaining sufficient accuracy.

Buxon et al., 2003 is developing a "Virtual Manikin" modelling system, based on CFD to investigate protective clothing and workspace design. They use the "Virtual Manikin" as an additional layer of analysis within the "Integated Performance Modelling Environment" capability, that links environmental stressors to the task being performed and the impact on task performance. They use the commercial CFD-code PHOENICS.

The continuous development of computer capacity has also improved the possibilities of use for this type of simulations. However there are still many unexplained differences in the results within and in between simulation methods, pointing out several limitations of the currently available CFD-methods. This shows the need for validation of CFDresults with real life measurements with human beings. 
Table 4. A summary of the virtual thermal manikin methods described above.

\begin{tabular}{|c|c|c|}
\hline Author & Physiological modelling and results & Turbulence modelling and codes \\
\hline Gan (1994) & $\begin{array}{l}\text { Passive heating } \\
\text { Steady state } \\
\text { Air flow predictions }\end{array}$ & $\begin{array}{l}\text { Two-equation k- } \varepsilon \text { model } \\
35 \text { hours on a } \operatorname{Sun}^{\circledR} \text { Sparc } 1\end{array}$ \\
\hline Brohus et. al. (1996) & $\begin{array}{l}\text { Passive heating }\left(25 \mathrm{~W} / \mathrm{m}^{2}\right) \\
\text { Steady state } \\
\text { Air flow predictions }\end{array}$ & $\begin{array}{l}\text { Two-equation k- } \varepsilon \text { model } \\
\text { FLOVENT }^{\circledR} \text { special-purpose CFD } \\
\text { program. }\end{array}$ \\
\hline Kato et. al. (1996) & $\begin{array}{l}\text { Passive heating }\left(20 \mathrm{~W} / \mathrm{m}^{2}\right) \\
\text { Steady state } \\
\text { Contaminant flow field analyses }\end{array}$ & Low-Re k- $\varepsilon$ model \\
\hline Maue et. al. (1997) & $\begin{array}{l}\text { Physiological model } \\
\text { Steady state } \\
\text { PMV and } t_{\text {eq }}(8 \text { zones })\end{array}$ & $\begin{array}{l}\text { Three different codes, single or } \\
\text { coupled use. Star-CD }{ }^{\circledR} \text {, in-house } \\
\text { code SWF, phys. program TIM }\end{array}$ \\
\hline $\begin{array}{l}\text { Murakami et. al. } \\
(1997,1998)\end{array}$ & $\begin{array}{l}\text { Physiological model } \\
\text { Gagge 2-node } \\
\text { Air temperature, humidity, radiation }\end{array}$ & $\begin{array}{l}\text { Low-Re k- } \varepsilon \text { model } \\
\text { Several codes, iterative process } \\
\text { Coupled simulations }\end{array}$ \\
\hline Bjørn et. al. (2000) & $\begin{array}{l}\text { Passive heating }\left(34.7 \mathrm{~W} / \mathrm{m}^{2}\right) \\
\text { Steady state } \\
\text { Respiration effects }\end{array}$ & RNG k- $\varepsilon$ model \\
\hline $\begin{array}{l}\text { Huizenga et. al. } \\
(2001)\end{array}$ & $\begin{array}{l}\text { Physiological model } \\
\text { Stolwijk } 25 \text {-node } \\
\text { PMV and EHT ( } 16 \text { zones) }\end{array}$ & $\begin{array}{l}\text { Several codes } \\
\text { Coupled simulations } \\
\text { The Berkeley Comfort Model }\end{array}$ \\
\hline Han et. al. (2001) & $\begin{array}{l}\text { Physiological model } \\
\text { Stolwijk model } \\
\text { PMV and } t_{e q}\end{array}$ & $\begin{array}{l}\text { FLUENT }^{\circledR} \\
\text { The Berkeley Comfort Model }\end{array}$ \\
\hline Kang et. al. (2002) & $\begin{array}{l}\text { Physiological model } \\
\text { Stolwijk 61-node } \\
\text { Steady state WBGT }\end{array}$ & $\begin{array}{l}\text { High-Re k- } \varepsilon \text { model with wall } \\
\text { functions. } \\
\text { Coupled simulations }\end{array}$ \\
\hline Tanabe et. al. (2002) & $\begin{array}{l}\text { Physiological model } \\
\text { Stolwijk } 65 \text {-node } \\
\text { (16 zones) }\end{array}$ & $\begin{array}{l}\text { Physiological model combined with } \\
\text { radiation exchange model and CFD } \\
\text { code }\end{array}$ \\
\hline Buxton et. al. (2003) & $\begin{array}{l}\text { Physiological model } \\
\text { Gagge 2-node model } \\
\text { Several physiological parameters }\end{array}$ & $\begin{array}{l}\text { Coupled simulations } \\
\text { IMAP and PHOENICS }^{\circledR}\end{array}$ \\
\hline Nilsson (2004) & $\begin{array}{l}\text { Subject and manikin correlated model } \\
\text { Steady state } \\
\text { Comfort zone diagram, } t_{e q}(18 \text { zones })\end{array}$ & $\begin{array}{l}\text { Zero-equation with dynamic } \\
\text { boundaries. One code, integrated } \\
\text { use. CFX }{ }^{\circledR}, \text { Star-CD }{ }^{\circledR}, \text { MANIKIN3 }\end{array}$ \\
\hline
\end{tabular}

\section{Summary mathematical simulation models}

Many factors often limit mathematical models currently available for analysing human responses to environmental stress and exercise; too low resolution, inadequate description, insufficient clothing information and sometimes disagreements between computed and measured results. The models are furthermore complicated to link to available CFD packages. These facts make the search for other empirical solutions interesting.

Early use of "numerical thermal occupants", like computational or virtual manikins, for assessment of indoor thermal comfort was made in the mid 90's. The CFD models were made with a mostly rectangular geometry. Some of the models where calculating PMV others were used to evaluate personal exposure to a contaminant source in a velocity field. The virtual manikin heat flux was often fixed. In the late 90's the models became more articulated. The modelling also becomes more advanced, with physiological modelling of the different thermal transport processes in a coupled 
manner, by simulating the combined effects of airflow, radiation, moisture transport, etc.

Today these calculation methods have developed further and a growing field of research verifies the suitability of CFD for simulation of the human thermal environment. The continuous development of computer capacity has also improved the possibilities for this type of simulations. Despite an increased accuracy, there are still some differences in the results within and in between simulation methods. This under lines the need for a continued validation of CFD-results with full-scale measurements and human beings. 


\title{
Definitions and calculations of equivalent temperature
}

\author{
General definition
}

The equivalent temperature $\left(\mathrm{t}_{\mathrm{eq}}\right)$ is defined as:

The temperature of an imaginary enclosure with the mean radiant temperature equal to air temperature and still air in which a person has the same heat exchange by convection and radiation as in the actual conditions.

The equivalent temperature is a recognised integrated measure of the effects of nonevaporative heat loss from the human body (Dufton, 1936, Madsen et al., 1984, Nilsson et al., 1999a). This definition is related to the "whole body" of a human being. Later this definition will be extended to include sensors and body part influences.

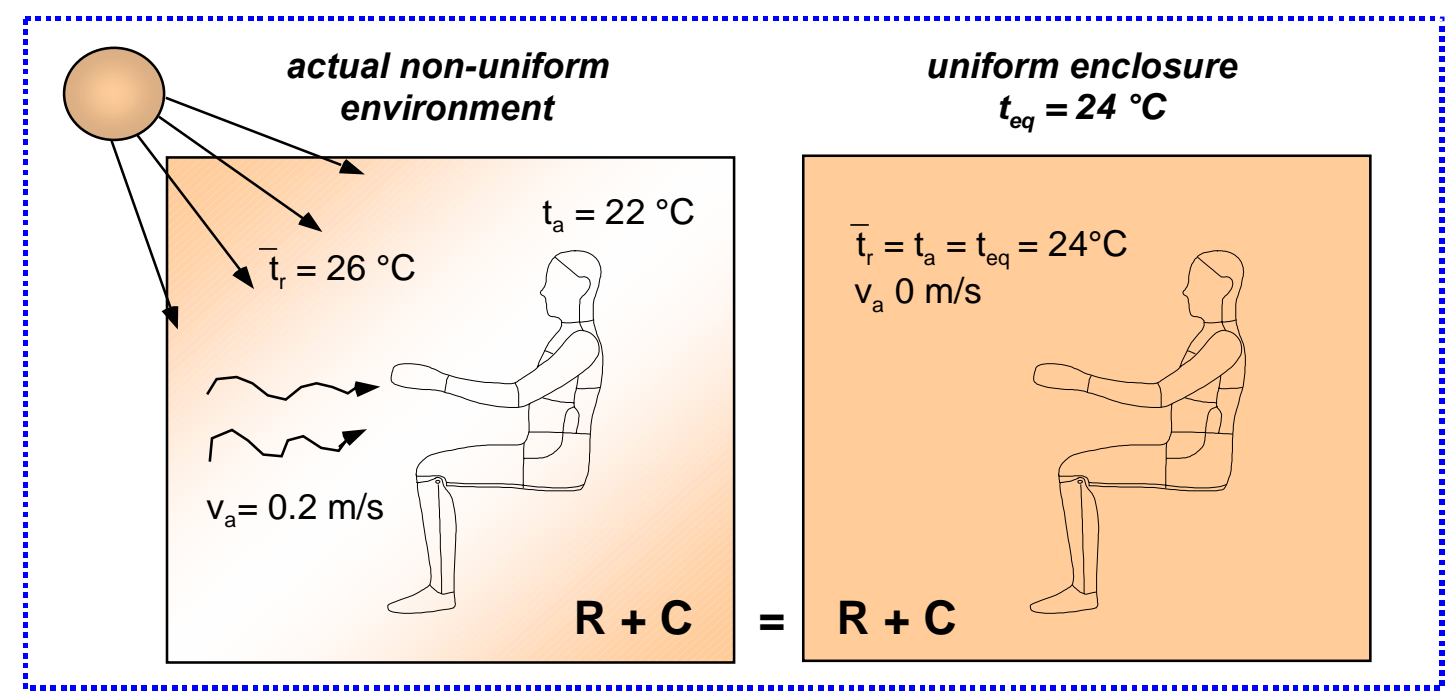

Figure 16. Illustration of the definition of equivalent temperature.

In the figure 16 a thermal manikin is exposed to two different environments, one actual with non-uniform and one "imaginary" with uniform climatic conditions. It is assumed that the posture, the activity level and the clothing are the same in both environments. The dry heat exchange, as well as the equivalent temperature, becomes the same in both environments. The heat exchange can of course be negative in warm environments and positive in cold environments. Table 5 shows the description of the dependencies between the measured climatic parameters and equivalent temperature $\left(t_{e q}\right)$. 
Table 5. Description of the connection between the measured quantities and equivalent temperature.

\begin{tabular}{rc}
\hline Action & Influence \\
\hline Increased air speed $\uparrow$ & $\downarrow$ Lower $t_{e q}$ \\
Decreased air temperature $\downarrow$ & $\downarrow$ Lower $t_{e q}$ \\
Decreased mean radiant temperature $\downarrow$ & $\downarrow$ Lower $t_{e q}$ \\
Decreased air speed $\downarrow$ & $\uparrow$ Higher $t_{e q}$ \\
Increased air temperature $\uparrow$ & $\uparrow$ Higher $t_{e q}$ \\
Increased mean radiant temperature $\uparrow$ & $\uparrow$ Higher $t_{e q}$ \\
\hline
\end{tabular}

\section{Determination of $t_{e q}$ with equations for convection and radiation}

Equations for convective and radiative heat transfer for clothed situations.

For unclothed situations $F_{c l}=f_{c l}=1$.

$$
\begin{array}{ll}
R=F_{c l} \cdot f_{c l} \cdot h_{r} \cdot\left(t_{s}-\bar{t}_{r}\right) & \text { Equation 2 } \\
C=F_{c l} \cdot f_{c l} \cdot h_{c} \cdot\left(t_{s}-t_{a}\right) & \text { Equation 3 }
\end{array}
$$

Where

$t_{a} \quad$ is the ambient air temperature, ${ }^{\circ} \mathrm{C}$

$\bar{t}_{r} \quad$ is the mean radiant temperature of the environment, ${ }^{\circ} \mathrm{C}$

$t_{s} \quad$ surface temperature, ${ }^{\circ} \mathrm{C}$

$F_{c l} \quad$ reduction factor for sensible heat exchange due to wearing clothes, ND

$f_{c l} \quad$ Clothing area factor, $A_{c l} / A_{u}$ (n.d.)

$h_{c} \quad$ convective heat transfer coefficient, $\mathrm{W} / \mathrm{m}^{2 \circ} \mathrm{C}$

$h_{r} \quad$ radiation heat transfer coefficient, $\mathrm{W} / \mathrm{m}^{2}{ }^{\circ} \mathrm{C}$

By assigning different values for $h_{r}$ and $h_{c}$ representative for the "homogenous" condition with zero air velocity, the equivalent temperature $\left(t_{e q}=\bar{t}_{r}=t_{a}\right)$ can be calculated, that gives the same $R+C$ as in the actual situation, with no influence of the humidity.

The dependence between $h_{r}$ and $h_{c}$ at different temperature differences and air velocities may be considered. This could lead to, presumably small, deviations between $t_{e q}$ at calibration and measured $t_{e q}$. This and the use of clothing should be taken into consideration during calibration. Often heat transfer coefficients, $h_{r}$ and $h_{c}$, are assumed to be constant. In reality, in homogenous climate, they are depending on the difference between the surface temperature and $t_{e q}$. Consequently $F_{c l}, f_{c l}$ and $h_{r}, h_{c}$ is supposed to be the same in both environments the following formula can be derived.

The total heat loss is:

$$
R+C=h_{r} \cdot\left(t_{s}-\bar{t}_{r}\right)+h_{c} \cdot\left(t_{s}-t_{a}\right)
$$


By definition of equivalent temperature we have:

$R+C=h_{r} \cdot\left(t_{s}-t_{e q}\right)+h_{c} \cdot\left(t_{s}-t_{e q}\right)$

Equation 5

Using Equation 4 and 5 or just 5 and solving for $t_{e q}$ gives:

$t_{e q}=\frac{h_{r} \cdot \bar{t}_{r}+h_{c} \cdot t_{a}}{\left(h_{r}+h_{c}\right)}=t_{s}-\frac{R+C}{\left(h_{r}+h_{c}\right)}$

Equation 6

This equation 6 is a fundamental relationship for the calculation of $t_{e q}$ and can be found in different calculation and calibration situations in this thesis.

\section{Determination based on complete heat balance equations}

The criterion applied here is that $t_{e q}$ is the temperature, replacing $\bar{t}_{r}$ and $t_{a}$, that with zero air velocity gives the same PMV-value as in the actual condition (Fanger, 1970). Analysing conditions for thermal neutrality requires PMV-values $=0$.

$$
\begin{aligned}
& P M V=\left(0.303 \cdot e^{-0.036 \cdot M}+0.028\right) \cdot\left\{(M-W)-3.05 \cdot 10^{-3} \cdot[5733-6.99 \cdot(M-W)\right. \\
& \left.-p_{a}\right]-0.42 \cdot[(M-W)-58.15]-1.7 \cdot 10^{-5} \cdot M \cdot\left(5867-p_{a}\right)-0.0014 \cdot M \cdot\left(34-t_{a}\right)- \\
& \left.3.96 \cdot 10^{-8} \cdot f_{c l} \cdot\left[\left(t_{c l}+273\right)^{4}-\left(\bar{t}_{r}+273\right)^{4}\right]-f_{c l} \cdot h_{c} \cdot\left(t_{c l}-t_{a}\right)\right\} \\
& \text { where } \\
& t_{c l}=35.7-0.028 \cdot(M-W)-I_{c l} \cdot\left\{3.96 \cdot 10^{-8} \cdot f_{c l} \cdot\left[\left(t_{c l}+273\right)^{4}-\left(\bar{t}_{r}+273\right)^{4}\right]\right. \\
& \left.+f_{c l} \cdot h_{c} \cdot\left(t_{c l}-t_{a}\right)\right\}
\end{aligned}
$$

Equation 7

Other existing models and equations are rather similar - the PMV-equation is just an example. The use of heat balance equations for determining $t_{e q}$ is not unequivocal, since a change in the relation between $\bar{t}_{r}$ and $t_{a}$ also affects other avenues of heat loss for example by respiration and evaporation. In other words, dry heat loss may be equal according to equations 2 and 3, but the total heat balance may not be equal because respiratory and evaporative heat transfer will change depending on ambient water vapour pressure and air temperature, unless it is assumed that this is constant. Consequently, extending the definition of $t_{e q}$ to include respiratory and evaporative heat loss will alter the heat balance, although only by a small fraction. In order to calculate $t_{e q}$ the heat balance equation used also requires a constant ambient vapour pressure (changing relative humidity), otherwise balance may be achieved for several $t_{e q}$ values.

\section{Equivalent temperature from empirical equations}

Bedford, (1936) did a study that deals with climate effects on people with light industry work. With help of these data Bedford did derive two equations for equivalent temperature: 


$$
t_{e q}=0.522 \cdot t_{a}+0.478 \cdot t_{w}-0.01474 \cdot \sqrt{v} \cdot\left(100-t_{a}\right)
$$

\section{Equation 8}

Where

$t_{a} \quad$ is the air temperature in ${ }^{\circ} \mathrm{F}$

$t_{w} \quad$ is the mean temperature of the surroundings in ${ }^{\circ} \mathrm{F}$

$v \quad$ is the air speed in feet/minute.

He also derived an equation using the globe temperature $t_{g}$ in ${ }^{\circ} \mathrm{F}$

$$
t_{e q}=0.522 \cdot t_{a}+0.478 \cdot t_{g}+\sqrt{v} \cdot\left(0.0808 \cdot t_{g}-0.0661 \cdot t_{a}-1.474\right) \quad \text { Equation } 9
$$

Similar equations for calculation of $t_{e q}$ was suggested by (Madsen et al., 1984):

$$
\begin{array}{lll}
\text { for } v_{a} \leq 0.1 \mathrm{~m} / \mathrm{s} \quad t_{e q}=t_{o}=0.5 \cdot\left(t_{a}+\bar{t}_{r}\right) & \text { Equation 10a } \\
\text { for } v_{a}>0.1 \mathrm{~m} / \mathrm{s} & t_{e q}=0.55 \cdot t_{a}+0.45 \cdot \bar{t}_{r}+\frac{0.24-0.75 \cdot \sqrt{v_{a}}}{1+I_{c l}}\left(36.5-t_{a}\right) & \text { Equation 10b }
\end{array}
$$

Equation $10 \mathrm{~b}$ contains all the important elements affecting the $t_{e q}$ value. Apart from the air temperature, mean radiant temperature and air velocity it also shows the influence of clothing. The equation, however, refers only to sedentary conditions (energy metabolism $\approx 70 \mathrm{~W} / \mathrm{m}^{2}$ ). For resting conditions and light clothing this formula predicts values very close to the more complex calculation (see below).

In table 6, calculations have been made according to equation 7, the PMV equation with $v_{a}=0$, PMV equation with $p_{a}=$ const. and the empirical equation 10 (emp.) for eight different homogeneous conditions. PMV is calculated according to EN-ISO-7730 (1995), which have been checked with the example in the standard. For these conditions the deviations between the different calculation methods are only minor.

These comparative calculations show that we could expect equivalent temperatures within a range from approx. 13 to $28^{\circ} \mathrm{C}$ corresponding to $P M V$ values from -8.3 to 1.3 . The dry heat loss values $(R+C)$ should according to the calculations be within the region of approx. 22 to $166 \mathrm{~W} / \mathrm{m}^{2}$. The results from the equivalent temperature calculations are rather similar except for the empirical formula at higher metabolism, since that is not accounted for in the formula.

It is the authors opinion that the calculation with constant water vapour pressure ( $p_{a}=$ const.) is the most reasonable, because the humidity is omitted in the same intention as in the definition. Notice specially that the results in condition 1 correspond to the conditions for the practical calibration. 
Table 6. Comparative calculations made with different methods. PMV equation with ( $P M V$ $\mathrm{va}=0)$, PMV equation with $\left(P M V \mathrm{p}_{\mathrm{a}}=\right.$ const.) and the empirical equation (emp.) for eight different homogeneous conditions. The relative air velocity is estimated to be zero for $M<58$ $\mathrm{W} / \mathrm{m}^{2}$ and $v_{a r}=5.17 \cdot 10^{-3} \cdot(M-58)$ for $M>58 \mathrm{~W} / \mathrm{m}^{2}$.

\begin{tabular}{|c|c|c|c|c|c|c|c|c|c|}
\hline Conditions & 1 & 2 & 3 & 4 & 5 & 6 & 7 & 8 & Unit \\
\hline$M$ & 58.0 & 58.0 & 58.0 & 58.0 & 58.0 & 58.0 & 58.0 & 58.0 & $W / m^{2}$ \\
\hline$I_{c l}$ & 0.0 & 0.0 & 0.0 & 0.0 & 0.0 & 0.0 & 0.0 & 0.0 & clo \\
\hline$t_{a}$ & 24.0 & 24.0 & 24.0 & 24.0 & 24.0 & 19.0 & 29.0 & 24.0 & ${ }^{\circ} \mathrm{C}$ \\
\hline$t_{r}$ & 24.0 & 24.0 & 24.0 & 29.0 & 34.0 & 19.0 & 29.0 & 21.0 & ${ }^{\circ} \mathrm{C}$ \\
\hline$v_{a}$ & 0.00 & 0.30 & 1.00 & 0.30 & 0.30 & 0.30 & 0.30 & 0.30 & $\mathrm{~m} / \mathrm{s}$ \\
\hline$R H\left(p_{a}=\right.$ const.$)$ & 40 & 46 & 65 & 41 & 35 & 48 & 44 & 50 & $\%$ \\
\hline$p_{a}$ & 1.19 & 1.19 & 1.19 & 1.19 & 1.19 & 0.88 & 1.60 & 1.19 & $\mathrm{kPa}$ \\
\hline$p_{a}\left(v_{a}=0\right)$ & 1.19 & 1.03 & 0.73 & 1.17 & 1.34 & 0.73 & 1.45 & 0.95 & $\mathrm{kPa}$ \\
\hline$t_{e q}\left(P M V v_{a}=0\right)$ & 24.0 & 21.6 & 16.1 & 23.7 & 26.0 & 16.1 & 27.3 & 20.3 & ${ }^{\circ} \mathrm{C}$ \\
\hline$t_{e q}\left(P M V p_{a}=\right.$ const. $)$ & 24.0 & 21.5 & 15.9 & 23.7 & 26.1 & 16.1 & 27.3 & 20.3 & ${ }^{\circ} \mathrm{C}$ \\
\hline$t_{e q}($ emp. $)$ & 24.0 & 21.9 & 17.6 & 24.1 & 26.4 & 16.0 & 27.7 & 20.5 & ${ }^{\circ} \mathrm{C}$ \\
\hline$R+C$ & 87 & 111 & 166 & 89 & 67 & 164 & 56 & 123 & $\mathrm{~W} / \mathrm{m}^{2}$ \\
\hline$P M V$ & -3.1 & -4.6 & -8.3 & -3.2 & -1.8 & -8.2 & -0.9 & -5.4 & ND \\
\hline Higher M & 1 & 2 & 3 & 4 & 5 & 6 & 7 & 8 & Unit \\
\hline$M$ & 91.4 & 91.4 & 91.4 & 91.4 & 91.4 & 91.4 & 91.4 & 91.4 & $W / m^{2}$ \\
\hline$I_{c l}$ & 0.0 & 0.0 & 0.0 & 0.0 & 0.0 & 0.0 & 0.0 & 0.0 & clo \\
\hline$R H\left(p_{a}=\right.$ const. $)$ & 40 & 46 & 63 & 40 & 35 & 48 & 43 & 49 & $\%$ \\
\hline$p_{a}$ & 1.19 & 1.19 & 1.19 & 1.19 & 1.19 & 0.88 & 1.60 & 1.19 & $\mathrm{kPa}$ \\
\hline$p_{a}\left(v_{a}=0\right)$ & 1.19 & 0.98 & 0.73 & 1.12 & 1.30 & 0.63 & 1.48 & 0.91 & $\mathrm{kPa}$ \\
\hline$t_{e q}\left(P M V v_{a}=0\right)$ & 24.0 & 20.8 & 16.0 & 23.0 & 25.4 & 13.9 & 27.6 & 19.5 & ${ }^{\circ} \mathrm{C}$ \\
\hline$t_{e q}\left(P M V p_{a}=\right.$ const. $)$ & 24.0 & 20.7 & 15.8 & 23.0 & 25.4 & 13.8 & 27.5 & 19.3 & ${ }^{\circ} \mathrm{C}$ \\
\hline$t_{e q}(e m p)$. & 24.0 & 21.9 & 17.6 & 24.1 & 26.4 & 16.0 & 27.7 & 20.5 & ${ }^{\circ} \mathrm{C}$ \\
\hline$R+C$ & 86 & 116 & 159 & 94 & 72 & 178 & 53 & 128 & $\mathrm{~W} / \mathrm{m}^{2}$ \\
\hline$P M V$ & -1.1 & -2.3 & -4.0 & -1.5 & -0.6 & -4.8 & 0.3 & -2.8 & ND \\
\hline Higher $I_{c l}$ & 1 & 2 & 3 & 4 & 5 & 6 & 7 & 8 & Unit \\
\hline$M$ & 58.0 & 58.0 & 58.0 & 58.0 & 58.0 & 58.0 & 58.0 & 58.0 & $W / m^{2}$ \\
\hline$I_{c l}$ & 1.0 & 1.0 & 1.0 & 1.0 & 1.0 & 1.0 & 1.0 & 1.0 & clo \\
\hline$R H\left(p_{a}=\right.$ const. $)$ & 40 & 43 & 47 & 38 & 34 & 45 & 42 & 46 & $\%$ \\
\hline$p_{a}$ & 1.19 & 1.19 & 1.19 & 1.19 & 1.19 & 0.88 & 1.60 & 1.19 & $\mathrm{kPa}$ \\
\hline$p_{a}\left(v_{a}=0\right)$ & 1.19 & 1.10 & 1.02 & 1.24 & 1.41 & 0.79 & 1.53 & 1.03 & $\mathrm{kPa}$ \\
\hline$t_{e q}\left(P M V v_{a}=0\right)$ & 24.0 & 22.7 & 21.4 & 24.7 & 26.8 & 17.3 & 28.2 & 21.6 & ${ }^{\circ} \mathrm{C}$ \\
\hline$t_{e q}\left(P M V p_{a}=\right.$ const. $)$ & 24.0 & 22.6 & 21.2 & 24.7 & 27.0 & 17.2 & 28.2 & 21.4 & ${ }^{\circ} \mathrm{C}$ \\
\hline$t_{e q}($ emp.) & 24.0 & 22.9 & 20.8 & 25.2 & 27.4 & 17.5 & 28.4 & 21.6 & ${ }^{\circ} \mathrm{C}$ \\
\hline$R+C$ & 38 & 43 & 48 & 35 & 26 & 64 & 22 & 48 & $\mathrm{~W} / \mathrm{m}^{2}$ \\
\hline$P M V$ & 0.2 & -0.2 & -0.5 & 0.4 & 0.9 & -1.7 & 1.3 & -0.5 & ND \\
\hline
\end{tabular}

In order to emphasise the importance of defined conditions regarding metabolism and clothing, two more sets of calculations have been made. The first one with a higher $M$ (91.4 met) and the other one with a clothing insulation $\left(I_{c l}\right)$ of 1 clo. The results show, 
as expected, that a higher metabolism results in a slightly higher heat loss and increased clothing insulation in a much lower heat loss, respectively. This is also reflected in changes of several degrees in equivalent temperature.

\section{Extended definitions}

The equivalent temperature is a pure physical quantity, which in a physically sound way integrates the independent effects of convection and radiation on human body heat loss. This physical value of $t_{e q}$ does not take into account human perception and sensation or other subjective aspects. The measured equivalent temperature always depends on the geometry of the sensor. It is consequently quite difficult to make correct comparisons of measured equivalent temperature between instruments with large geometrical and functional differences. In terms of mathematical definitions several expressions have been defined (Nilsson et al., 1999a). When reading the definitions below, please keep in mind that $t_{e q}$ can be described as a vector originating from every point of the measuring geometry, defined by magnitude and direction.

\section{Local equivalent temperature}

Refers to the heat exchange between one side of an open surface and the surroundings. It can be measured by a flat or curved directional sensor. Local equivalent temperature is the $t_{e q}$ that is related to a body segment or an open surface sensor.

\section{Whole body equivalent temperature}

This is the $t_{e q}$ detected with a closed surface body shape. It can be measured by an omnidirectional sensor measuring in all directions or calculated from the local equivalent temperatures, measured in all directions. Whole body equivalent temperature is the $t_{e q}$ related to the whole body of a human being or a closed surface sensor.

\section{Equivalent temperature vector theories}

Variables such as surface temperatures and heat transfer coefficients are indeed scalar quantities. However as a result of the radiative heat transfer component, the full description of equivalent temperature contains not only its magnitude but also the direction, emanating from the interaction between the ambience and the sensor geometry used. According to the definition the heat transfer consists of two parts, one scalar convective part and one vector radiative part. These two together forms a heat transfer vector in every point of the surface geometry. Equivalent temperature can accordingly be defined as a vector quantity.

\section{The vector-valued equivalent temperature}

The surface integration of vector-valued functions is defined in terms of integration over regions of the surface. The heat flow through a surface element is considered 
mathematically the same for any vector field. Consequently $\boldsymbol{t}_{e q}(\boldsymbol{r})$, were $\boldsymbol{r}$ is the radius vector defined over the surface area $\mathrm{S}$, can be described as a vector-valued function in every point of $S$ (All vectors are presented in bold type face). It refers to the heat exchange between the geometry of the surface and the geometry of the surroundings.

With this definition is it obvious that the vector $\boldsymbol{t}_{e q}(\boldsymbol{r})$ can take a multitude of directions given by the geometry of the sensor surface as well as the surrounding surfaces geometry. It can be measured by a sensor, with certain geometry, measuring in one or several directions or calculated with integration over the same geometry and directions.

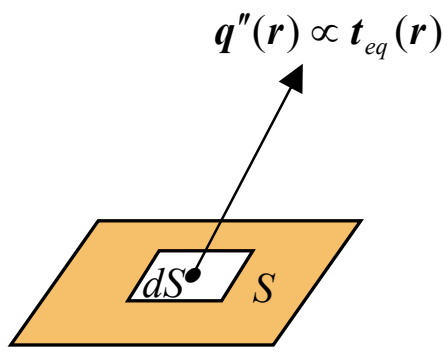

Figure 17. The $\boldsymbol{t}_{e q}(\boldsymbol{r})$ described as a vector-valued function dependent on all parameters in the definition for the magnitude and the radiation field for the direction.

If $\boldsymbol{q}^{\prime \prime}(\boldsymbol{r})$ is the heat loss $\left(\mathrm{W} / \mathrm{m}^{2}\right)$ from the surface $\mathrm{S}\left(\mathrm{m}^{2}\right)$ with a surface temperature $t_{s}$ $\left({ }^{\circ} \mathrm{C}\right)$ and a heat transfer coefficient $h\left(\mathrm{Wm}^{-2} \mathrm{~K}^{-1}\right)$, in the figure 17. The heat flux $q^{\prime \prime}(\boldsymbol{r})$ has the direction (unit vector) $\hat{q}^{\prime \prime}(\boldsymbol{r})$ and the magnitude $\boldsymbol{q}^{\prime \prime}(\boldsymbol{r})$. Similar to Equation 11, the vector valued equivalent temperature can be defined as:

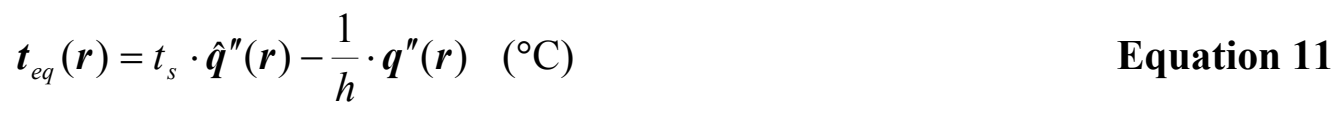

Consider the heat flux $\boldsymbol{q}^{\prime \prime}(\boldsymbol{r})$ from a surface $S$ to the environment (see figure 17). In the same way the flux due to the equivalent temperature flow between a differential surface element $d S$ and the ambience, is directed from or to $d S$, and is proportional to the scalar value of the equivalent temperature at $d S$. Hence the surface averaged equivalent temperature vector, here denoted by $\widetilde{\boldsymbol{t}}_{e q}(S)$, for the local equivalent temperature is given by:

$\widetilde{\boldsymbol{t}}_{e q}(S)=\frac{\iint_{S} \boldsymbol{t}_{e q}(\boldsymbol{r}) d S}{|S|}\left({ }^{\circ} \mathrm{C}\right)$
where $|S|=\iint_{S} d S$ is the total open surface area.

A similar equation can consequently be written for the whole body equivalent temperature:

\section{Equation 12}

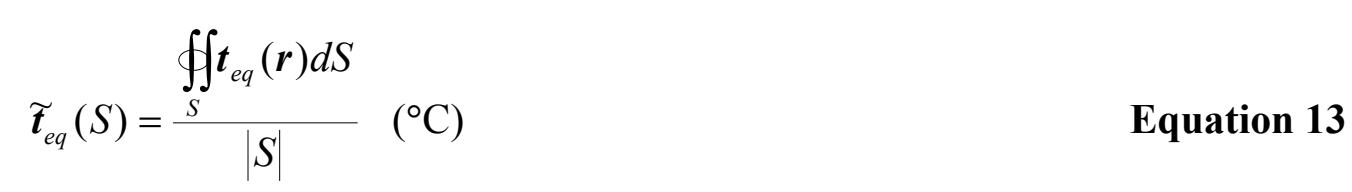


where $|S|=\oiiint_{S} d S$ is the total closed surface area.

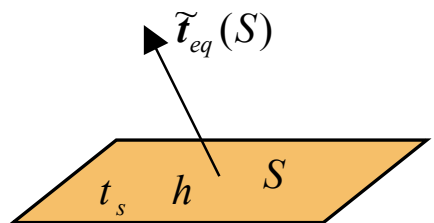

Figure 18. The surface integrated mean equivalent temperature vector.

With this method he total flux due to the entire surface $S$ is thus a vector, $\widetilde{t}_{e q}(S)$, having a magnitude and direction obtained by surface integration of the contributions of all elements $d S$ over the open surface $S$ :

$\widetilde{\boldsymbol{t}}_{\text {eq }}(S) \cdot|S|=t_{s} \cdot \iint_{S} \hat{\boldsymbol{q}}^{\prime \prime}(\boldsymbol{r}) d S-\frac{1}{h} \cdot \iint_{S} \boldsymbol{q}^{\prime \prime}(\boldsymbol{r}) d S \quad\left({ }^{\circ} \mathrm{C}\right) \quad$ Equation 14

Below an illustration of a graphical computation of the equivalent temperature vector for a three dimensional system is shown in figure 19.

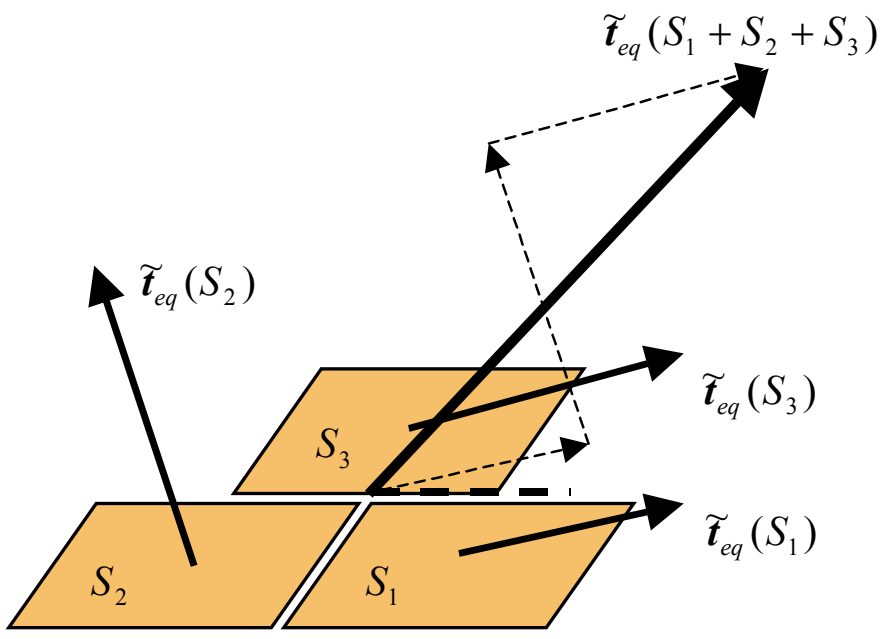

Figure 19. Graphical summation of a vector originating from three different surfaces (zones).

The mathematical summation of all the contributions from $S_{1}, S_{2}, S_{3}$ then becomes:

$\widetilde{\boldsymbol{t}}_{e q}\left(S_{1}+S_{2}+S_{3}\right)=\frac{\widetilde{\boldsymbol{t}}_{e q}\left(S_{1}\right) \cdot\left|S_{1}\right|+\widetilde{\boldsymbol{t}}_{e q}\left(S_{2}\right) \cdot\left|S_{2}\right|+\widetilde{\boldsymbol{t}}_{e q}\left(S_{3}\right) \cdot\left|S_{3}\right|}{\left|S_{1}\right|+\left|S_{2}\right|+\left|S_{3}\right|}$

$\left({ }^{\circ} \mathrm{C}\right) \quad$ Equation 15

Another illustration of particular interest, is that of a small cube centred inside substantially larger imaginary room with heat exchange only between the cube and the left wall of the room. 


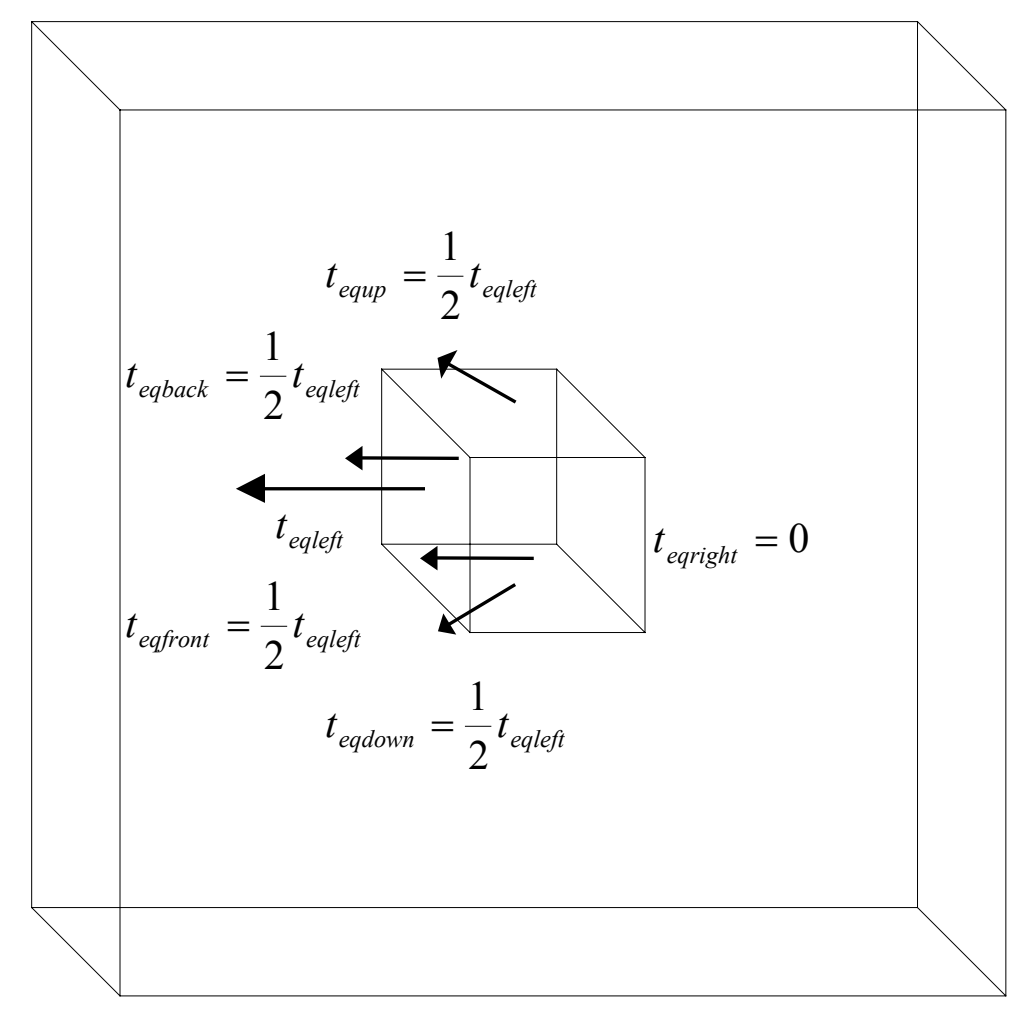

Figure 20. A small cube inside a much larger room where heat transfer takes place between the cube and the left wall.

Assume those four surfaces of the cube that are affected only partly of the heat transfer between the left wall and the cube. Thus having only half the equivalent temperature flux compared to the left side of the cube facing the whole left wall having full impact on the heat transfer. The angle is set to 45 degrees to simplify the example.

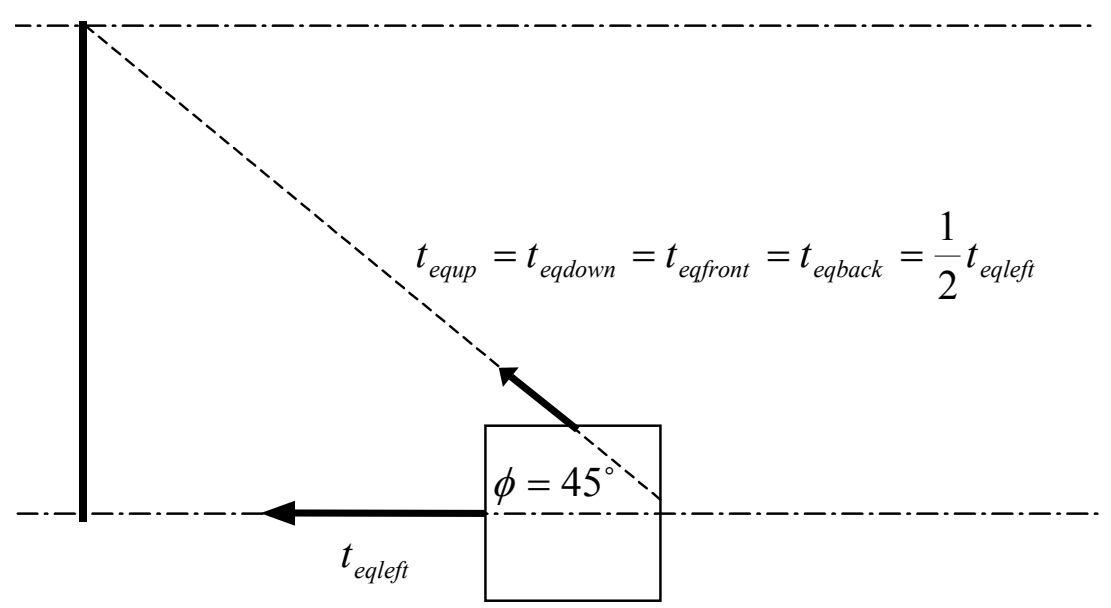

Figure 21. Two-dimensional enlargement of the infinitesimal cube in prior figure.

The heat transfer coefficient $h$ is held constant all over the cube and $t_{s}$, equal for all surfaces $\left(S=1 \mathrm{~m}^{2}\right)$, and set to $0^{\circ} \mathrm{C}$ in this case. All projections of heat transfer along the surfaces of the cube are consequently eliminated. Hence the only vectors of interest are the composants parallel to the surface. In this cube case equation 15 gives: 
$\widetilde{\boldsymbol{t}}_{e q}($ cube $)=\frac{\widetilde{\boldsymbol{t}}_{\text {eq }}(\text { left }) \cdot 1+4 \cdot \frac{1}{\sqrt{2}} \widetilde{\boldsymbol{t}}_{\text {eq }}(\text { left }) \cdot 1+0}{|1+1+1+1+1+1|+1 \mid}=\left(\frac{1}{6}+\frac{1}{3 \sqrt{2}}\right) \cdot \widetilde{\boldsymbol{t}}_{e q}($ left $)\left({ }^{\circ} \mathrm{C}\right)$ Equation 16

With a resulting direction parallel to $t_{\text {eqleft }}$. The vector treatment makes easier calculations and better understanding of the concept of equivalent temperature. We also have a direction of the resultant $t_{e q}$ presenting us more information of the current climate situation.

This equivalent temperature vector theory can be developed one step further. It is possible to make a Gauss map (Morgan, 1998) of the surface using a vector addition method based on the following assumption: For a given flux vector $q$ is the heat loss per unit area at any point on the surface only dependant on the direction of the tangent plane to the surface at that point.

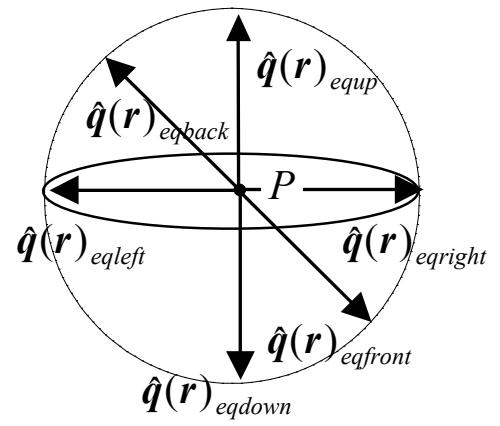

Figure 22. The unity vectors normal to all planes always forms a sphere, here the cube case.

To each plane in space (zone or surface) we can associate a unit vector, $\boldsymbol{q}(\boldsymbol{r})$, which is perpendicular to the plane. Now imagine at a point $P$, fixed in space, and then without changing the direction of each unit vector, move the vectors so that their tails are fixed to $P$. The set of all unit vector tips depicts a sphere in space. If we then inflate these unit vectors with their scalar values we get the same result as with the cube case, after the usual vector addition.

In the future these theories can be used to facilitate the computational calculations of equivalent temperature and better visualise the effects of different thermal environmental solutions. The use of vector-valued functions are often seen in finite element calculations, and could become a valuable instrument also in computational fluid dynamics calculations of surface averaged equivalent temperatures.

\section{Summary equivalent temperature}

The equivalent temperature is a recognised measure of the effects of non-evaporative heat loss from the human body. It is particularly useful in the confined spaces due to the complex interaction between different types of heat fluxes. The equivalent temperature is derived from the operative temperature by the inclusion of the effect of air velocity on a heated body. The operative temperature only considers the air temperature and the 
mean radiant temperature and is defined for the actual air velocity, whereas the equivalent temperature is defined for a standard low air velocity. The major advantage is that it expresses the combined effects of thermal influences in a single figure, easy to interpret and explain. It is consequently particularly useful for local assessment of climatic conditions.

A variable such as surface temperature is considered a scalar quantity. However as a result of the radiative heat transfer component, the full description of equivalent temperature contains not only its magnitude but also the direction, emanating from the interaction between the ambience and the sensor geometry used. Equivalent temperature can hence be defined as a vector-valued quantity. 


\section{Comparison with subjective responses}

The manikin technique has been validated in tests with subjects. The heat losses of the manikins as well as the subjective reactions of the two panels of subjects were obtained for more than 30 sets of climatic conditions (Holmér et al., 1992, Nilsson et al., 1997).

The experiments were performed in a small climatic test chamber that allowed asymmetries as well as the general temperature level to be controlled. The conditions comprised various asymmetrical thermal loads creating different levels of whole body heat loss.

Asymmetries were produced by vertical air temperature gradients and solar radiation. Two different clothing insulation levels where used, on both manikins and subjects, to be able to investigate the effects of summer and winter clothing. Totally 20 male subjects participated in the studies and were exposed to all the different sets of conditions. The subjects sat in the test cabin for 60 minutes. Subjective responses were recorded at the end of the first and second 30-min period. Thermal sensation was recorded using the MTV-scale (see figure 23)

\begin{tabular}{|c|l|}
\hline Thermal Vote & \multicolumn{1}{|c|}{ Interpretation } \\
\hline+3 & much too hot \\
+2 & too hot \\
+1 & hot but comfortable \\
0 & neutral \\
-1 & cold but comfortable \\
-2 & too cold \\
-3 & much too cold \\
\hline
\end{tabular}

Figure 23. MTV-scale for rating of thermal sensation.

Subjects rated their thermal sensation for different body segments and for the body as a whole. The segments corresponded to individual or combinations of segments of the thermal manikins.

\section{Correlation with subjective ratings}

In two series of experiments total and local heat fluxes, converted to equivalent temperatures $\left(t_{e q}\right)$, from the two manikins (MANIKIN1 and MANIKIN2) were determined for in total 30 different climatic conditions (14 winter and 16 summer). These equivalent temperatures were then compared with thermal sensation ratings of the $20(10+10)$ subjects exposed to the same conditions. The measurements during winter conditions were made using an extra cardigan giving a total insulation of 1.6 clo and during summer conditions without cardigan 1.3 clo in total insulation value. The 
subjects as well as both manikins were dressed in identical clothing, with a multitude of sizes chosen to give similar fit to all different body shapes.

Table 7. The 30 different conditions examined with both manikins and subjects. Abbreviations: (- NEGATIVE $4^{\circ} \mathrm{C}$ colder at head level compared to foot level, = NEUTRAL equal temperature at all levels, + POSITIVE $6^{\circ} \mathrm{C}$ warmer at head level compared to foot level. 0 No sun 0 $\mathrm{W} / \mathrm{m}^{2}, 5$ Sun $500 \mathrm{~W} / \mathrm{m}^{2}, 7$ Sun $700 \mathrm{~W} / \mathrm{m}^{2}, 8$ Sun $800 \mathrm{~W} / \mathrm{m}^{2}$. C Cold, H Hot, N Neutral, X Extra, G special glazing)

\begin{tabular}{|c|c|c|c|c|c|}
\hline Conditions & $\begin{array}{l}\text { Heat loss } \\
\left(\mathrm{W} / \mathrm{m}^{2}\right)\end{array}$ & $\begin{array}{r}\text { Air temp. } \\
\text { gradient }\end{array}$ & $\begin{array}{c}\text { Sun } \\
\text { radiation }\end{array}$ & $\begin{array}{l}\text { Air temp. } \\
\text { inside }\end{array}$ & $\begin{array}{c}\text { Air temp. } \\
\text { outside }\end{array}$ \\
\hline$\overline{\mathrm{W}-\mathrm{C}}$ & 56 & - & $\overline{0}$ & 24.4 & -20 \\
\hline W-N & 48 & - & 0 & 26.5 & -20 \\
\hline W-H & 40 & - & 0 & 28.6 & -20 \\
\hline $\mathbf{W}=\mathbf{C}$ & 56 & $=$ & 0 & 24.5 & -20 \\
\hline $\mathbf{W}=\mathbf{N}$ & 48 & $=$ & 0 & 26.4 & -20 \\
\hline $\mathbf{W}=\mathbf{H}$ & 40 & $=$ & 0 & 28.4 & -20 \\
\hline $\mathbf{W}+\mathbf{C}$ & 56 & + & 0 & 24.3 & -20 \\
\hline $\mathbf{W}+\mathbf{N}$ & 48 & + & 0 & 26.5 & -20 \\
\hline $\mathbf{W}+\mathbf{H}$ & 40 & + & 0 & 28.5 & -20 \\
\hline $\mathbf{W}=\mathbf{C}$ & 57 & $=$ & 0 & 22.0 & -20 \\
\hline $\mathbf{W}=\mathbf{N}$ & 46 & $=$ & 0 & 25.4 & -20 \\
\hline $\mathbf{W}=\mathbf{H}$ & 37 & $=$ & 0 & 27.1 & -20 \\
\hline $\mathbf{W}=\mathbf{C X}$ & 60 & $=$ & 0 & 21.9 & -20 \\
\hline $\mathbf{W}=\mathbf{H X}$ & 34 & $=$ & 0 & 29.0 & -20 \\
\hline S-CO & 56 & - & 0 & 19.8 & 35 \\
\hline S-NO & 48 & - & 0 & 21.4 & 35 \\
\hline S-HO & 40 & - & 0 & 23.1 & 35 \\
\hline S-HXO & 32 & - & 0 & 25.0 & 35 \\
\hline S-C5 & 56 & - & 5 & 19.8 & 30 \\
\hline S-N5 & 48 & - & 5 & 20.7 & 30 \\
\hline S-H5 & 40 & - & 5 & 21.7 & 30 \\
\hline S-C7 & 56 & - & 7 & 18.2 & 30 \\
\hline S-N7 & 48 & - & 7 & 19.6 & 30 \\
\hline S-H7 & 40 & - & 7 & 20.7 & 30 \\
\hline S-HX7 & 32 & - & 7 & 22.2 & 30 \\
\hline $\mathbf{S}=\mathbf{C G}$ & 55 & $=$ & 8 & 21.2 & 30 \\
\hline$S=N G$ & 39 & $=$ & 8 & 24.1 & 30 \\
\hline $\mathrm{S}=\mathbf{H G}$ & 25 & $=$ & 8 & 26.5 & 30 \\
\hline$S=C$ & 65 & $=$ & 8 & 20.2 & 30 \\
\hline $\mathbf{S}=\mathbf{H}$ & 37 & $=$ & 8 & 21.5 & 30 \\
\hline
\end{tabular}




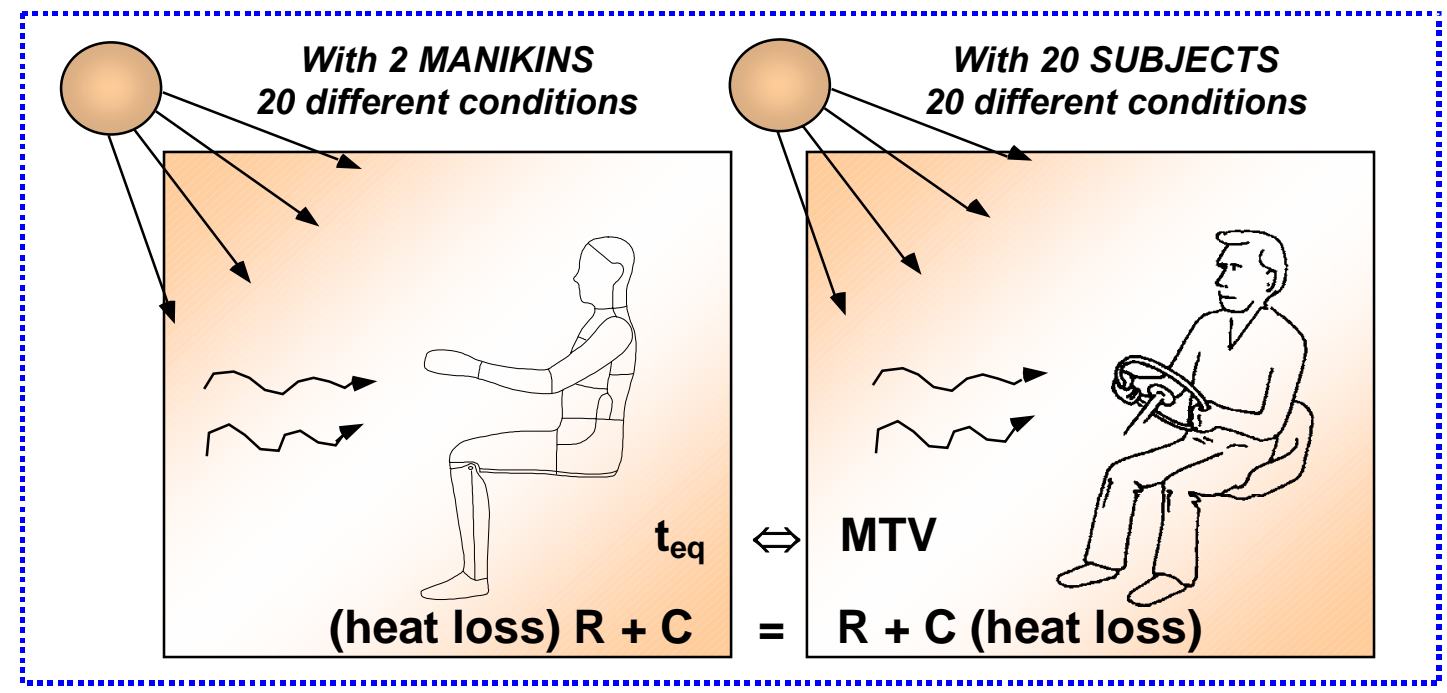

Figure 24. The manikins and subjects were exposed to 20 identical asymmetrical climate conditions. MTV and $t_{e q}$ was obtained for all sets of conditions.

MTV and $t_{e q}$ for all conditions were subjected to a linear regression analysis. High correlation coefficients were found for segmental heat flux and mean thermal rating of subjects for the same body segments (zones). The procedure was repeated for all different body segments for which subjective ratings were obtained.

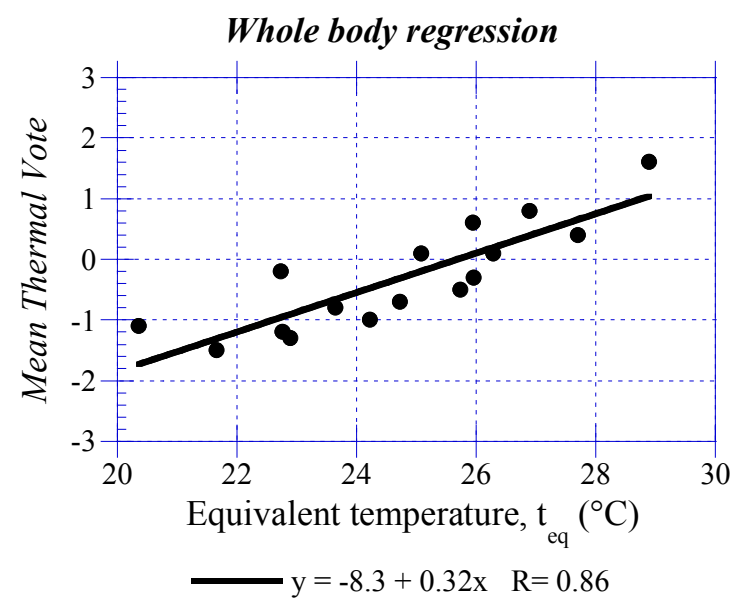

Figure 25. Regression of MTV on $t_{\mathrm{eq}}$ with measurements made with both manikins.

The sets of low and high equivalent temperatures are plotted as two profile limits. In this way a comfort profile for the climate over the whole body surface is obtained. This diagram shows the range of $t_{e q}$ values inside which test values should be positioned to be acceptable. Values closer to the centred optimal profile $(\mathrm{OP}=\mathrm{MTV}=0)$ mean better thermal conditions. 


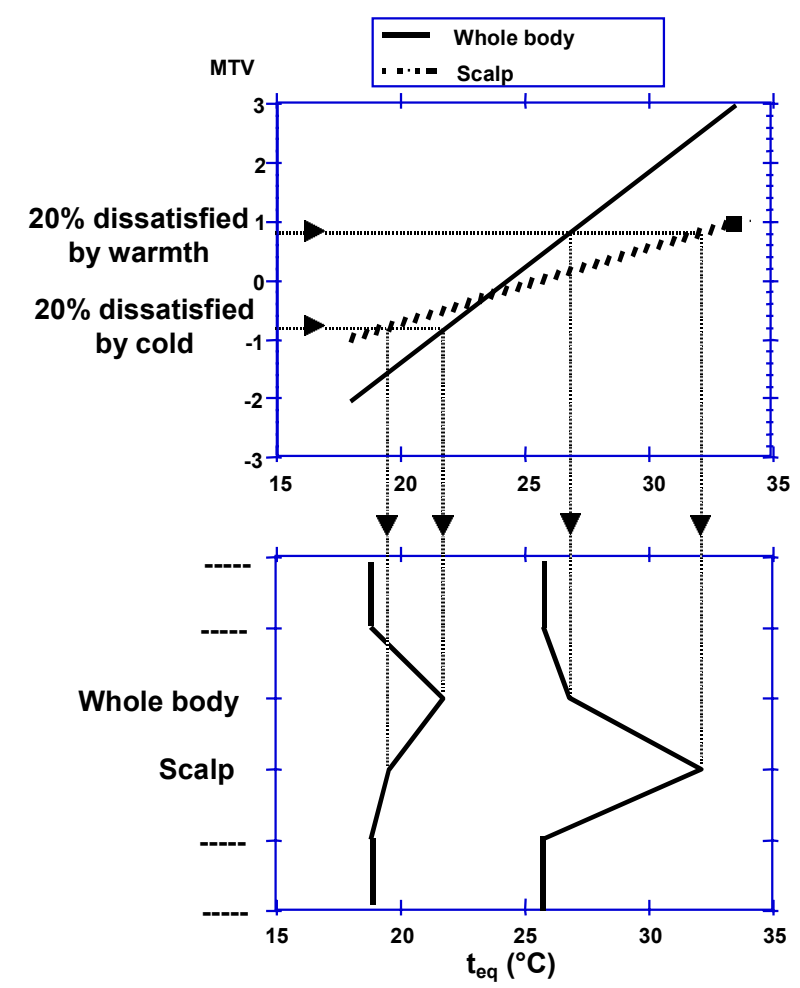

Figure 26. The construction of comfort profile.

Ideally, the intersection of each line with the value of MTV $=0$ (neutral thermal sensation) would indicate the optimal climate conditions. To use one centre line as a base for evaluation is not realistic due to practical and economical reasons. A more reasonable way to evaluate the thermal climate conditions is to give at least two limit lines of $t_{e q}$ values. Indicating in between what limits that a certain percentage of the subjects would be satisfied. The level chosen at this time is \pm 0.8 in MTV-value, that is the level where $80 \%$ of the persons are predicted to be satisfied between the lines. For each regression of MTV on $t_{e q}$ it is possible to derive this $t_{e q}$-range. The low and high $t_{e q}$ values obtained in this manner were then used to draw the $t_{e q}$-profile in the figure 26.

When using this approach the limit lines differ at some points when different panels and manikins are used. The largest differences can be seen at the hands in winter conditions and at head level in summer conditions. Therefore positioning of the nude hands and head is very important for the acquired result. Similar differences could be found between other manikins and panels in the same situations. Also consider two values close to the limit line but on different sides, one will be regarded as "acceptable" but the other will be "not acceptable". For relative measurements with one and the same manikin, the absolute limits described by this particular manikin could be used.

However, for comparative purposes between manikins and for the presentation of absolute values, as well as a result of methodological and individual variations, averaged "zones" should be defined. Showing that within a certain range the subjects would make a rating according to the MTV-scale. The zones are constructed to be \pm 0.5 MTV around each rating. In the same way as for the limit lines is it possible to derive such a zone for each regression of MTV on $t_{e q}$. The $t_{e q}$ zones obtained in this manner were then used to draw the $t_{e q}$-profile in figure 27 and also presented in the tables 8 and 9 . 

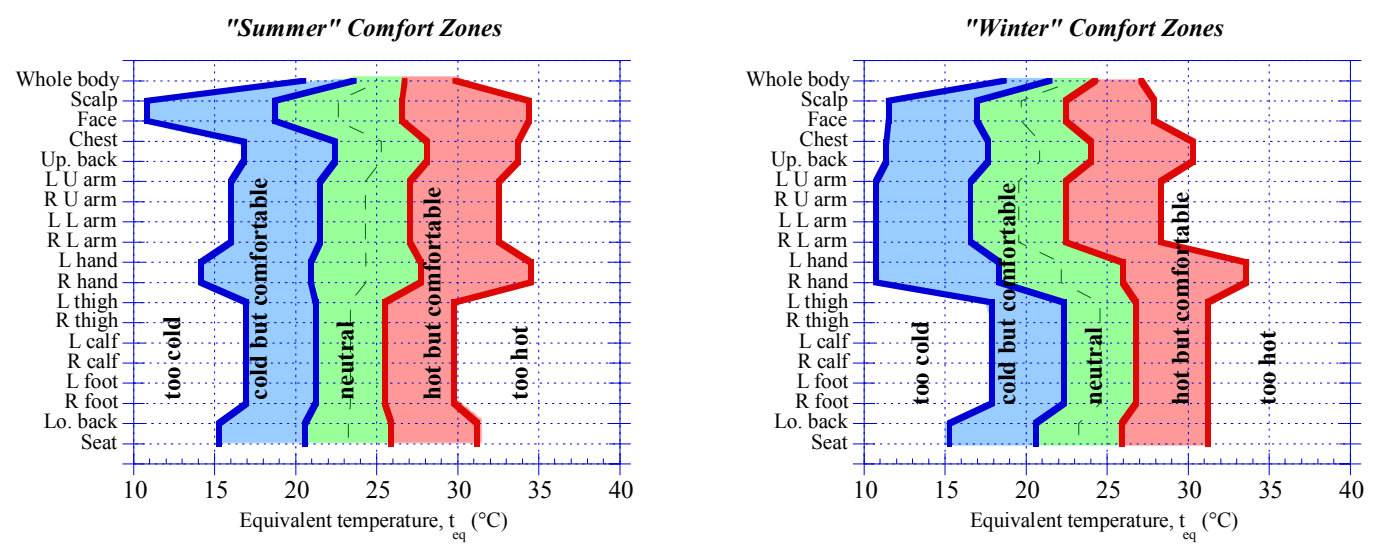

Figure 27. Comfort zones for 16 segments of the body. Abbreviations refer to $\mathrm{L}=$ left, $\mathrm{R}=$ right, $\mathrm{U}=$ upper. Comfort zone diagrams derived for the summer and winter clothing ensembles used in the subjective as well as manikin correlation studies. Notice the shift of the comfort zones towards the cold side for manikin zones with increased insulation.

In figure 27 at least two evaluations are considered, whole body and local distribution of $t_{e q}$. Both criteria have to be met. If, for instance, all $t_{e q}$ values are inside any of the "but comfortable" zones, this will generally not result in a whole body $t_{e q}$ acceptance. It has to be remembered that the whole body value is the weighted sum of the local values. Whole body acceptance can be satisfied in primarily two ways; all local values more or less equal to the whole body value or locally cold parts compensated with locally warm parts within the zones. However, local "too hot" or "too cold" values will of course render complaints, even if the whole body-value are within the neutral zone.

It can also be seen that the increased insulation on the upper body of the manikins and subjects in the winter situations, with increased insulation on the upper body, results in broader zones shifted to the cold side, as could be expected.

If comfort zones are introduced the differences between the series and manikins are marginal. This adjustment makes a more realistic base for judgement of complicated climate situations, as it opens up for a general profile usable with different manikins in different situations. More data from validation experiments with subjects and different manikins will contribute to the development of this more general evaluation criteria. 
Table 8. Comfort zones with summer clothing with total insulation of 1.3 clo.

\begin{tabular}{|c|c|c|c|c|c|c|c|c|c|c|}
\hline Zone & too cold & $\begin{array}{l}\text { cold } \\
\text { com- } \\
\text { fort }\end{array}$ & neutral & $\begin{array}{c}\text { warm } \\
\text { com- } \\
\text { fort }\end{array}$ & $\begin{array}{c}\text { too } \\
\text { warm }\end{array}$ & $\begin{array}{c}\text { S } 20 \% \\
\text { cold }\end{array}$ & $\begin{array}{l}\text { S } 20 \% \\
\text { warm }\end{array}$ & $\overline{\mathbf{R}_{\mathrm{T}}}$ & $\begin{array}{l}q^{\prime \prime} T \\
\text { cold } \\
20 \%\end{array}$ & $\begin{array}{c}q^{\prime \prime} T \\
\text { warm } \\
20 \%\end{array}$ \\
\hline Whole body & 20.5 & 23.6 & 25.2 & 26.7 & 29.9 & 22.7 & 27.7 & 0.206 & 55 & 31 \\
\hline Scalp & 10.9 & 18.7 & 22.6 & 26.6 & 34.4 & 16.4 & 28.9 & 0.199 & 89 & 26 \\
\hline Face & 10.9 & 18.7 & 22.6 & 26.6 & 34.4 & 16.4 & 28.9 & 0.199 & 89 & 26 \\
\hline Chest & 16.8 & 22.4 & 25.3 & 28.1 & 33.7 & 20.8 & 29.8 & 0.229 & 58 & 18 \\
\hline Up. back & 16.8 & 22.4 & 25.3 & 28.1 & 33.7 & 20.8 & 29.8 & 0.229 & 58 & 18 \\
\hline $\mathbf{L} \mathbf{U}$ arm & 16.0 & 21.5 & 24.3 & 27.0 & 32.5 & 19.9 & 28.7 & 0.215 & 66 & 25 \\
\hline R U arm & 16.0 & 21.5 & 24.3 & 27.0 & 32.5 & 19.9 & 28.7 & 0.215 & 66 & 25 \\
\hline L L arm & 16.0 & 21.5 & 24.3 & 27.0 & 32.5 & 19.9 & 28.7 & 0.215 & 66 & 25 \\
\hline R L arm & 16.0 & 21.5 & 24.3 & 27.0 & 32.5 & 19.9 & 28.7 & 0.215 & 66 & 25 \\
\hline L hand & 14.1 & 20.9 & 24.3 & 27.7 & 34.5 & 18.9 & 29.8 & 0.109 & 139 & 39 \\
\hline$R$ hand & 14.1 & 20.9 & 24.3 & 27.7 & 34.5 & 18.9 & 29.8 & 0.109 & 139 & 39 \\
\hline L thigh & 17.0 & 21.2 & 23.4 & 25.5 & 29.8 & 20.0 & 26.8 & 0.215 & 65 & 34 \\
\hline R thigh & 17.0 & 21.2 & 23.4 & 25.5 & 29.8 & 20.0 & 26.8 & 0.215 & 65 & 34 \\
\hline L calf & 17.0 & 21.2 & 23.4 & 25.5 & 29.8 & 20.0 & 26.8 & 0.215 & 65 & 34 \\
\hline$R$ calf & 17.0 & 21.2 & 23.4 & 25.5 & 29.8 & 20.0 & 26.8 & 0.215 & 65 & 34 \\
\hline$L$ foot & 17.0 & 21.2 & 23.4 & 25.5 & 29.8 & 20.0 & 26.8 & 0.215 & 65 & 34 \\
\hline$R$ foot & 17.0 & 21.2 & 23.4 & 25.5 & 29.8 & 20.0 & 26.8 & 0.215 & 65 & 34 \\
\hline Lo. back & 15.3 & 20.6 & 23.3 & 25.9 & 31.2 & 19.0 & 27.5 & 0.247 & 61 & 26 \\
\hline Seat & 15.3 & 20.6 & 23.3 & 25.9 & 31.2 & 19.0 & 27.5 & 0.247 & 61 & 26 \\
\hline
\end{tabular}

Table 9. Comfort zones with winter clothing with total insulation of 1.6 clo.

\begin{tabular}{ccccccccccc}
\hline Whole body & 18.6 & 21.4 & 22.9 & 24.3 & 27.1 & 20.6 & 25.1 & 0.250 & 54 & 36 \\
Scalp & 11.5 & 17.0 & 19.7 & 22.4 & 27.9 & 15.3 & 24.1 & 0.193 & 97 & 51 \\
Face & 11.5 & 17.0 & 19.7 & 22.4 & 27.9 & 15.3 & 24.1 & 0.193 & 97 & 51 \\
Chest & 11.4 & 17.7 & 20.8 & 24.0 & 30.3 & 15.8 & 25.9 & 0.387 & 47 & 21 \\
Up. back & 11.4 & 17.7 & 20.8 & 24.0 & 30.3 & 15.8 & 25.9 & 0.387 & 47 & 21 \\
L U arm & 10.8 & 16.6 & 19.5 & 22.4 & 28.3 & 14.9 & 24.2 & 0.354 & 54 & 28 \\
R U arm & 10.8 & 16.6 & 19.5 & 22.4 & 28.3 & 14.9 & 24.2 & 0.354 & 54 & 28 \\
L L arm & 10.8 & 16.6 & 19.5 & 22.4 & 28.3 & 14.9 & 24.2 & 0.354 & 54 & 28 \\
R L arm & 10.8 & 16.6 & 19.5 & 22.4 & 28.3 & 14.9 & 24.2 & 0.354 & 54 & 28 \\
L hand & 10.8 & 18.4 & 22.2 & 26.0 & 33.6 & 16.1 & 28.3 & 0.146 & 123 & 39 \\
R hand & 10.8 & 18.4 & 22.2 & 26.0 & 33.6 & 16.1 & 28.3 & 0.146 & 123 & 39 \\
L thigh & 17.9 & 22.3 & 24.6 & 26.8 & 31.2 & 21.0 & 28.1 & 0.215 & 60 & 27 \\
R thigh & 17.9 & 22.3 & 24.6 & 26.8 & 31.2 & 21.0 & 28.1 & 0.215 & 60 & 27 \\
L calf & 17.9 & 22.3 & 24.6 & 26.8 & 31.2 & 21.0 & 28.1 & 0.215 & 60 & 27 \\
R calf & 17.9 & 22.3 & 24.6 & 26.8 & 31.2 & 21.0 & 28.1 & 0.215 & 60 & 27 \\
L foot & 17.9 & 22.3 & 24.6 & 26.8 & 31.2 & 21.0 & 28.1 & 0.215 & 60 & 27 \\
R foot & 17.9 & 22.3 & 24.6 & 26.8 & 31.2 & 21.0 & 28.1 & 0.215 & 60 & 27 \\
Lo. back & 15.3 & 20.6 & 23.3 & 25.9 & 31.2 & 19.0 & 27.5 & 0.304 & 49 & 21 \\
Seat & 15.3 & 20.6 & 23.3 & 25.9 & 31.2 & 19.0 & 27.5 & 0.304 & 49 & 21 \\
\hline
\end{tabular}

\section{Percentage dissatisfied}

The degree of thermal discomfort can also be expressed as "percentage dissatisfied" PD: the percentage of subjects who find the thermal situation unacceptable. From the database of MTV, the PD can be determined. The construction of the MTV scale has made it possible for the subjects that with a vote on $-1,0$ and +1 show that the climate is 
acceptable (comfortable). While -2 and +2 was "too cold" respectively "too hot", i.e. not acceptable (uncomfortable).

Relationship between MTV and PD

$n=530$
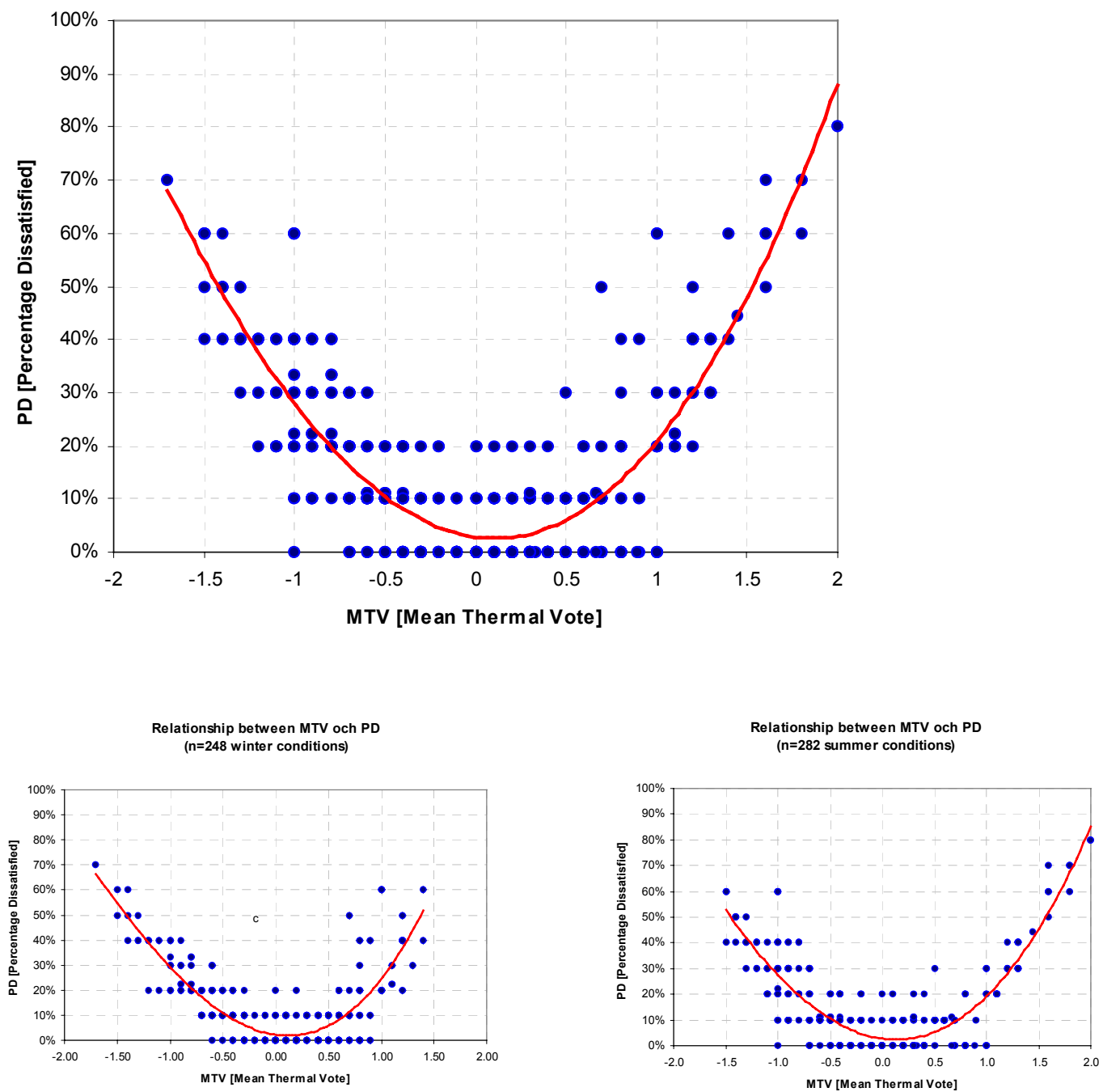

Figure 28. The figures above shows the measured number of subjects dissatisfied with the different climate conditions, that is according to the idea of the MTV-scale persons voting either $-2,-3$ or $+2,+3$ and consequently do not regard the climate as comfortable. The small graphs are showing the winter and summer results separated.

The maximum number of people dissatisfied with their thermal comfort conditions is $100 \%$ and the minimum number, even in what would be considered perfectly comfortable conditions, are in these tests $2 \% ; 1$ percent are too cold and 1 percent are too warm. This shows that it is not possible to satisfy everyone, even within a perfectly controlled climate environment.

Figure 28 shows that the relationship is validated for MTV values between -1.7 and +2 , that corresponds to $70 \%$ respectively $80 \%$ dissatisfied people. Correlation studies on the warm side have shown that the relationship seems to be valid up to values of at least +4 . Which of course is outside of the original Bedford and MTV scales. These cases are however of little interest from a comfort point of view. 
The u-shaped graphs above also shows the connection between PD and the construction of the comfort zones. It can easily be seen that the $20 \%$ limit value discussed above corresponds to a MTV of -0.8 and +1 . This asymmetry is another reason why it is better to work with comfort zones compared to limits.

\section{Normally distributed data}

A histogram of the standardised residuals with a normal curve superimposed, as shown in the figures below. A histogram of in total 530 observations is presumed to be randomly sampled from the normal distribution. Below the histogram of the MTV data.

\section{Histogram of MTV, with Normal Curve}

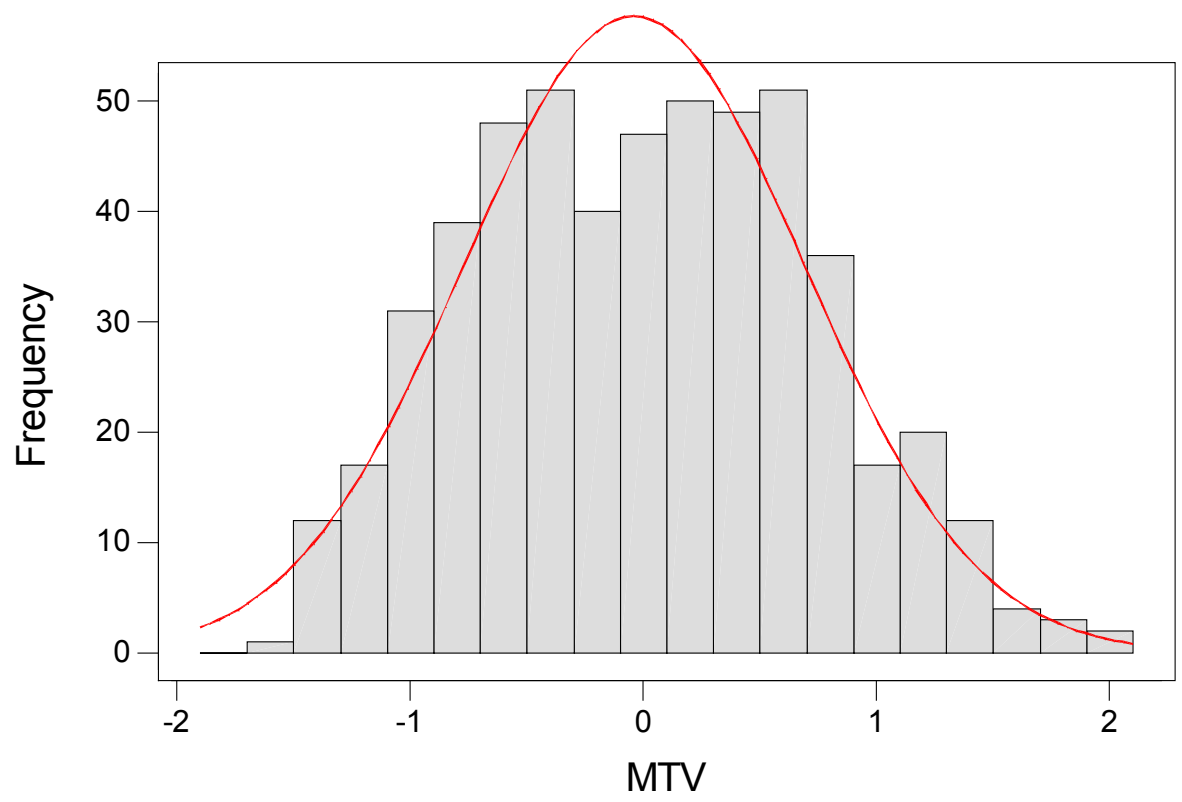

Histogram of MTV winter, with Normal Curve

Histogram of MTV summer, with Normal Curve
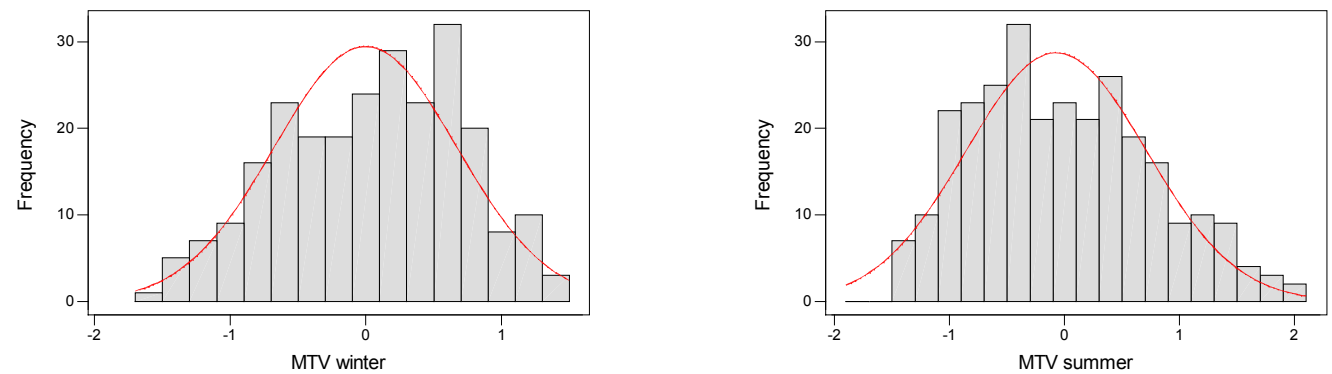

Figure 29. Three histograms of the standardised residuals for 530 MTV-values With normal curves superimposed. 


\section{Histogram of teq, with Normal Curve}

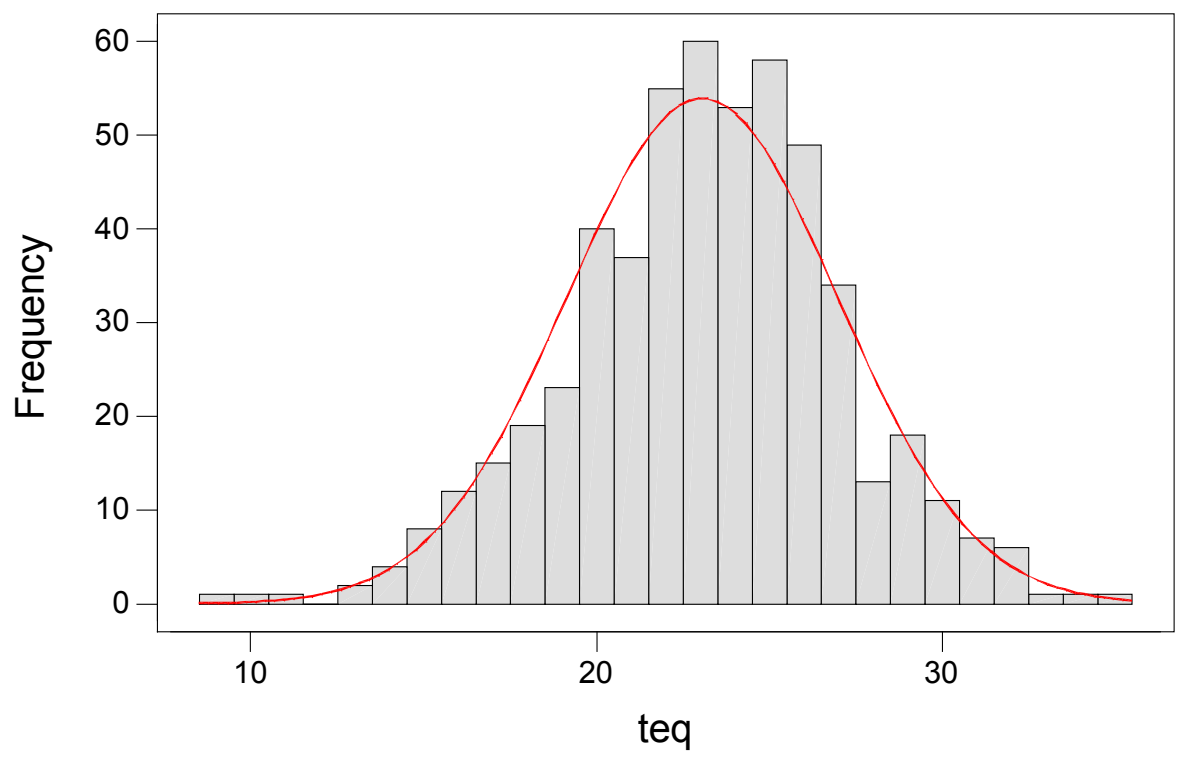

Histogram of teq winter, with Normal Curve

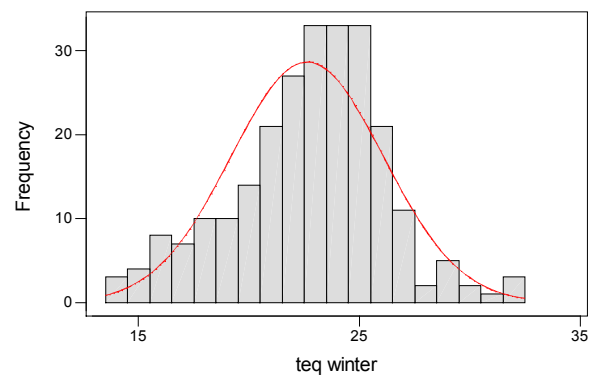

Histogram of teq summer, with Normal Curve

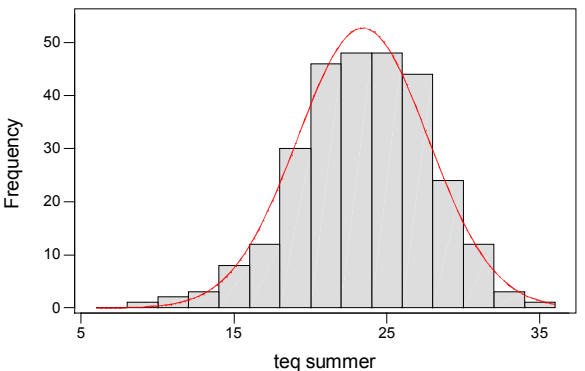

Figure 30. Three histograms of the standardised residuals for $530 t_{e q}$ values with normal curves superimposed.

\section{Normal probability plot}

The plot below compares the MTV and $t_{e q}$ data with what would be expected of data that is normally distributed. When the normal probability plot is linear the data are sampled from a normal distribution. Below you see the normal probability plot of the data. 


\section{Normal Probability Plot}

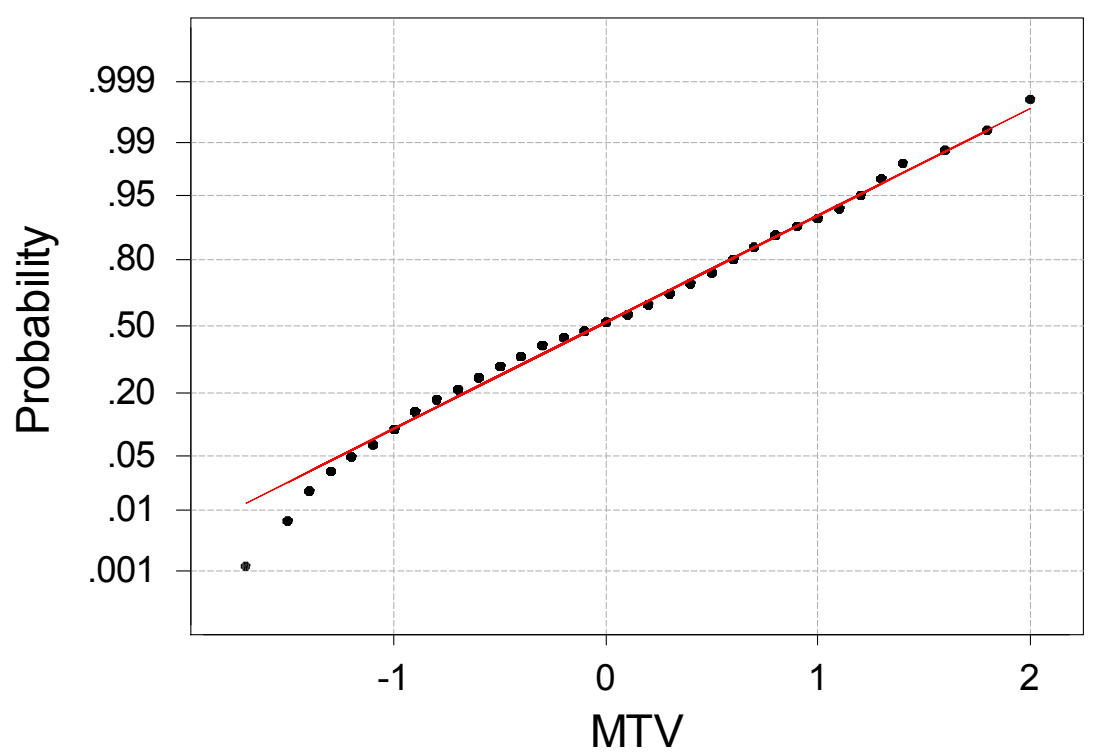

Average: -0.0386792

Kolmogorov-Smirnov Normality Test

StDev: 0.733350

D+: 0.040 D-: 0.026 D: 0.040

$\mathrm{N}: 530$

Approximate P-Value: 0.042

Normal Probability Plot

Normal Probability Plot

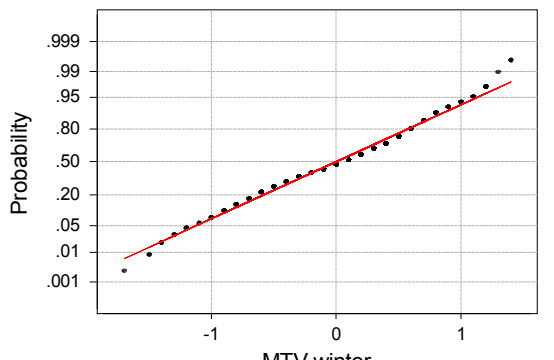

Average: -0.002016
StDev: 0.0671601
N. 248

Kolmogorov-Smirnov Normality Test
$D+0.035$ D: $0.052 \mathrm{D}: 0.052$

Dito 0.035 D-: 0.052 D: 0.052

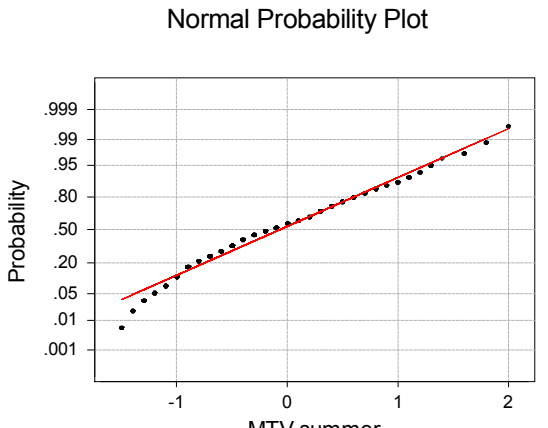

Average: 0.00709220
Siteve: 0.783417

D+: 0.060 D: 0.031 D: 0.060

Figure 31. The normal probability plots of 530 random MTV-values. As the plotted points form a diagonal line, MTV seems to be normally distributed with a probability of 0.042 . 


\section{Normal Probability Plot}

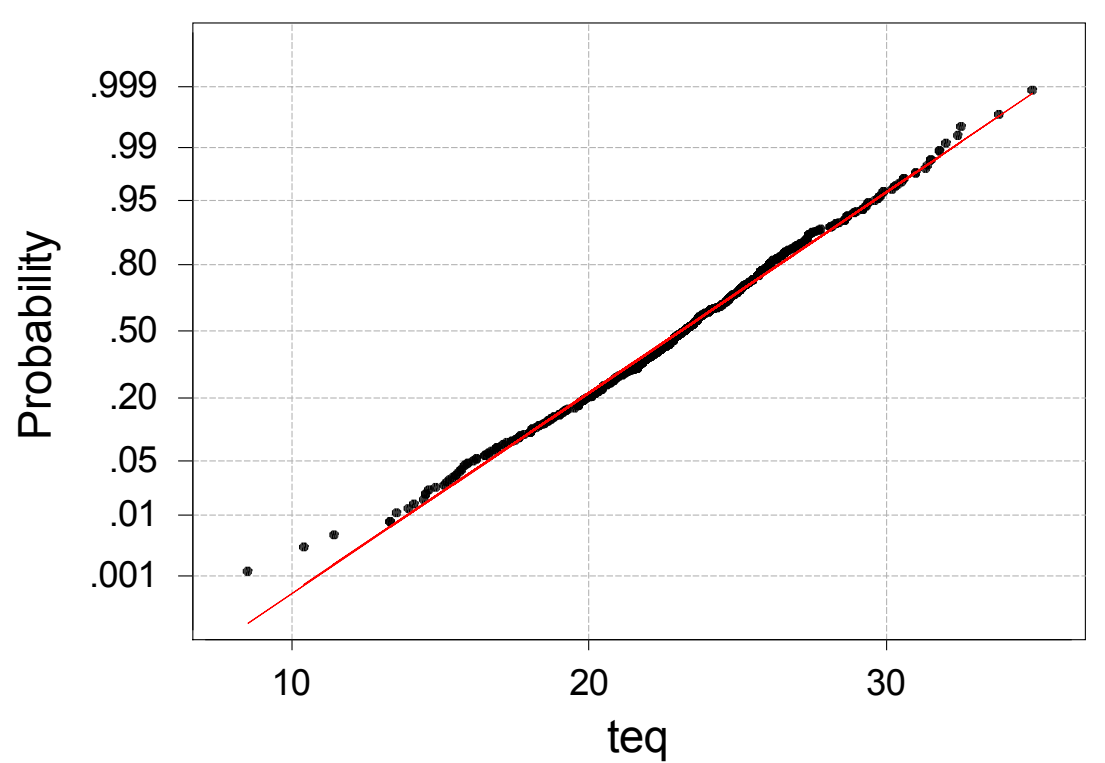

Average: 23.0625

StDev: 3.92447

N: 530

Kolmogorov-Smirnov Normality Test $\mathrm{D}+: 0.026 \mathrm{D}$-: $0.037 \mathrm{D}: 0.037$ Approximate P-Value: 0.081

Normal Probability Plot

Normal Probability Plot
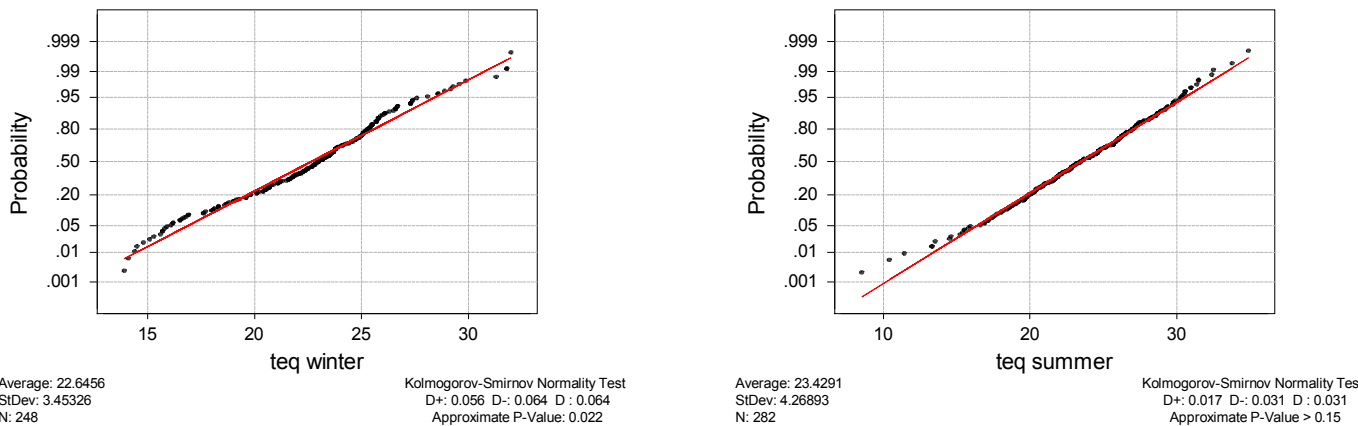

Figure 32. The normal probability plots of 530 random $t_{e q}$ values. As the plotted points forma diagonal line, $t_{e q}$ seems to be normally distributed with a probability of 0.081 and a standard deviation for the subject measurements always larger then $3^{\circ} \mathrm{C}$

\section{Comparison with subjective responses in warm conditions}

The objective of this study was to investigate how well $t_{e q}$ is correlated to human subjective responses to the thermal environment in warm situations above the set point $\left(34^{\circ} \mathrm{C}\right)$ of the surface temperature of MANIKIN2. The regulation program of MANIKIN2 is made to switch over to surface temperature as soon as zero heat flux is detected. The experiments were carried out at the Fraunhofer-Institut für Bauphysik (IBP) in Holzkirchen, Germany. Existing human subjective data from five different thermal environments, originating from previous laboratory experiments (Schwab et al., 1993), were used for the analyses. 


\section{Experimental conditions}

The experiments were carried out in the climatic chamber at IBP. Inside the chamber a cabin was located equipped with ventilation system and car seats. A sun simulator, installed outside the cabin, produced radiation asymmetries created in the experiments. The subjective experiments was made using the Local Mean Vote scale which is different from the MTV scale, thus giving interesting information regarding manikin measurements and subjective responses to warmth.

The Local Mean Vote (LMV) scale used in these experiments. (Schwab et al., 1999) not acceptably cold -5

$\begin{array}{ll}\text { very cold } & -4 \\ \text { cold } & -3 \\ \text { cool } & -2 \\ \text { slightly cool } & -1 \\ \text { neutral } & 0 \\ \text { slightly warm } & 1 \\ \text { warm } & 2 \\ \text { hot } & 3 \\ \text { very hot } & 4 \\ \text { not acceptably hot } & 5\end{array}$

Table 10. The five warm conditions examined with MANIKIN2 and subjects. Abbreviations: No sun $<15 \mathrm{~W} / \mathrm{m}^{2},+$ Sun $800 \mathrm{~W} / \mathrm{m}^{2}$.

\begin{tabular}{lccccc}
\hline & \multicolumn{5}{c}{ Condition } \\
Sun radiation $\left(\mathbf{W} / \mathbf{m}^{2}\right)$ & $\mathbf{1}$ & $\mathbf{2}$ & $\mathbf{3}$ & $\mathbf{4}$ & $\mathbf{5}$ \\
\hline Radiation on Head & $\mathbf{8 0 0}$ & $\mathbf{8 0 0}$ & $\mathbf{8 0 0}$ & $<\mathbf{1 5}$ & $<\mathbf{1 5}$ \\
Radiation on Chest & - & - & - & - & - \\
Radiation on Thighs & - & + & + & - & - \\
Ventilation rate $\left(\mathrm{m}^{3} / \mathrm{h}\right)$ & + & + & + & - & - \\
Inlet temperature $\left({ }^{\circ} \mathrm{C}\right)$ & 150 & 65 & 210 & 65 & 65 \\
\hline
\end{tabular}

Three hot summer conditions (1,2 and 3) and two moderate conditions without sun (4 and 5) were investigated. MANIKIN2 was seated at the right front seat in the simulation car cabin. The five conditions described above were reconstructed with the same settings of sun radiation, ventilation rates, inlet temperatures and radiant temperatures in the cabin, as in the already measured subject experiments. 


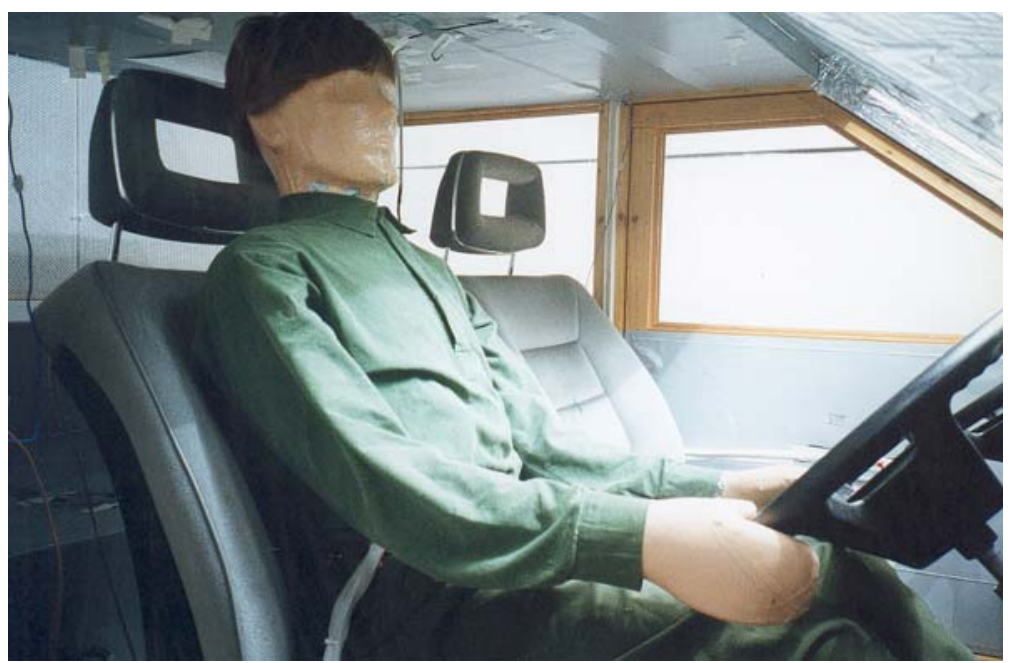

Figure 33. MANIKIN2 inside the test cabin for the warm condition tests.

\section{Correlation results}

The comparison between the measured $t_{e q}$ values and the LMV, coming from the ratings of about 50 subjects in a prior experiment (Schwab et al., 1989). From this data the correlation between LMV and $t_{e q}$ was calculated. The result for MANIKIN2 is shown in table 11.

Table 11. Comparison of the Mean Vote (LMV), found with 50 subjects with the measured equivalent temperature from MANIKIN2. For regression diagrams see Appendix D.

\begin{tabular}{|c|c|c|c|c|c|c|c|c|c|c|c|c|}
\hline Zone & Head & & Chest & & L arm & & $\mathrm{R}$ arm & & L leg & & R leg & \\
\hline Condition & $t_{e q}$ & LMV & $t_{e q}$ & LMV & $t_{e q}$ & LMV & $t_{e q}$ & LMV & $t_{e q}$ & LMV & $t_{e q}$ & LMV \\
\hline 1 & 36.7 & 1.8 & 37.2 & 1.8 & 37.5 & 1.5 & 38.4 & 1.5 & 39.7 & 1.6 & 40.8 & 1.6 \\
\hline 2 & 38.6 & 2.6 & 45.3 & 2.3 & 44.1 & 2 & 46.6 & 2 & 39.7 & 1.9 & 40.6 & 1.8 \\
\hline 3 & 35.7 & 1.4 & 39.6 & 1.2 & 39.6 & 1 & 42.9 & 1 & 39.1 & 1.2 & 40.3 & 1.1 \\
\hline 4 & 23.1 & -0.1 & 21.5 & -0.1 & 24.3 & -0.8 & 24.2 & -1 & 24.4 & -0.4 & 26.8 & -0.6 \\
\hline 5 & 27.5 & 0.2 & 25.2 & 0.2 & 26.8 & -0.2 & 28.4 & -0.3 & 27.5 & -0.2 & 30.4 & -0.2 \\
\hline Correlation $r^{2}$ & 0.93 & & 0.91 & & 0.94 & & 0.91 & & 0.95 & & 0.95 & \\
\hline
\end{tabular}

The maximal number of zones were further reduced, for comparative reasons, to six body parts. As expected, did MANIKIN2 show $t_{e q}$ values equal to the surface temperature with a corresponding zero heat flux for some or all zones, at the hot summer conditions. In spite of measuring equivalent temperature with a zero or negative heat flux at condition 1 to 3 , the correlation with the local mean votes was good.

\section{Summary comparison with subjective responses}

To use comfort diagrams with limit lines as a base for climate evaluation is, as a result of methodological and individual variations, not the optimal solution. A more reasonable way to evaluate the thermal climate conditions is to give a range of $t_{e q}$ values. If comfort zones are introduced the differences between measurements with different manikins, like MANIKIN1 and MANIKIN2, become marginal. This improvement 
makes a more realistic base for judgement of complicated local thermal climate, and opens up for a general profile usable with different manikins, possibly also different methods, in various environments. However, more data from validation experiments with subjects and different methods will contribute to the development of this more general evaluation criteria.

The degree of thermal discomfort can also be expressed as "percentage dissatisfied" PD: the percentage of subjects who find the thermal situation unacceptable. From the database of MTV, the PD has been determined. The construction of the MTV scale has made it possible for the subjects, with a rating on $-1,0$ and +1 show that the climate is acceptable (comfortable). The maximum number of people dissatisfied with their thermal comfort conditions is $100 \%$ and the minimum number, even in what would be considered perfectly comfortable conditions, are in these tests $2 \%$.

The number of subjects dissatisfied with the different climate conditions were shown to be normally distributed. The measured values were also checked for normality by plotting standardised residuals with a normal curve superimposed. Histograms of 530 MTV and $t_{e q}$ observations show that the data are randomly sampled from a normal distribution. All normal probability plots are linear as well; showing once more that the data are sampled from normal distributions. The distribution asymmetry between the summer and winter tests, shown in this thesis, is still another reason why it is better to work with comfort zones compared to limit lines.

A comparison between $t_{e q}$ values measured with MANIKIN2 and the Local Mean Vote (LMV), coming from 50 subjects in warm to hot conditions gave good correlation even when measuring equivalent temperature as the surface temperature with a zero or negative heat flux at the warmest conditions. 


\section{Clothing-independent comfort diagrams}

\section{The concept of independent comfort diagrams}

The development of new "comfort zones" is one step towards a more general evaluation criteria applicable to different manikins and maybe also different instruments. Another important task is to make these zones change with the change of clothing worn by the subjects as well as the manikins. The clothing that manikins use today makes the measurement method sensitive, difficult to handle and interpretation of results mostly troublesome. When the manikin is moved between locations, it happens that shape and positioning of the clothing are altered and therefore changing the insulation. Even a small change in the clothing insulation, and consequently the heat transfer coefficient, have significant influence on the final results as the equivalent temperature $\left(t_{e q}\right)$ is calculated according to:

$t_{\text {eq }}=t_{s}-\frac{q_{T}^{\prime \prime}}{h_{\text {teq }}}=t_{s}-R_{T} \cdot q_{T}^{\prime \prime}$

\section{Equation 17}

Where

$q_{T}^{\prime \prime} \quad$ measured manikin heat loss during the actual conditions $\left(\mathrm{W} / \mathrm{m}^{2}\right)$

$h_{\text {teq }}$ dry heat transfer coefficient, including clothing, determined during calibration $\left(\mathrm{W} / \mathrm{m}^{2} \mathrm{~K}\right)$

$R_{T} \quad$ total insulation, seated, winter/summer clothing $\left(\mathrm{m}^{2} \mathrm{~K} / \mathrm{W}\right)$

$t_{s} \quad$ manikin surface temperature $\left({ }^{\circ} \mathrm{C}\right)$

$t_{e q} \quad$ equivalent temperature of the uniform, homogenous environment. $\left({ }^{\circ} \mathrm{C}\right)$

In order to make the manikin method easy to handle in the calibration and measurement situation, as well as increase the repeatability, the manikin should wear more tight clothes with good fitting, or no clothing at all. This would minimise the possibilities for insulation changes during transport and measurement. According to the definition the $t_{e q}$ calibration should correct for the deviation that another clothing brings. That is for a given temperature difference $\left(t_{s}-t_{\text {eq }}\right)$ the term $\left(q_{T}^{\prime \prime} / h_{\text {teq }}\right)$ will still be the same. The heat loss from the clothed zones is of course changed but corrected with the calibration. It is further more practical to be able to calculate the effects of the clothing on the measurement.

The comfort zones used up till today are only valid for the clothes worn at the tests (summer clothing 1.3 clo and winter clothing 1.6 clo, total insulation), or similar clothing. For comparison and versatility reasons it is important to be able to use different comfort zones for evaluation depending on the clothing used. This does not influence measured $t_{e q}$ values, but more insulative clothing should imply that the persons using it would accept a broader interval of $t_{e q}$. These results can then be used to derive comfort zones for different clothing insulation values. In the comfort zone diagrams below it can easily be seen that the zones with slightly higher insulation on the upper body, with the winter clothing, is shifted towards the colder side of the diagram. 
The concept of clothing independence assumes that the human being is equally sensitive to different heat losses independent of the insulation of the clothing worn. This might not be true, especially at the borders of no clothing or heavy clothing. In the figures 33 and 34 as well as the tables 12, 13 and 14, equation 17 has been used to calculate a mean acceptable heat loss. This heat loss has then been used to calculate how the diagrams should look like if clothing is used. Garment descriptions: "no clothing" (just air layer, total insulation 0.9 clo or $0.134 \mathrm{~m}^{2} \mathrm{~K} / \mathrm{W}$ ), "light summer" (long sleeve shirt, shorts, shoes 1.1 clo or $0.167 \mathrm{~m}^{2} \mathrm{~K} / \mathrm{W}$ ) and "medium winter" (cap, winter jacket, trousers, gloves, winter shoes 2.3 clo or $0.350 \mathrm{~m}^{2} \mathrm{~K} / \mathrm{W}$ )

\section{Comfort diagrams for "no clothing", "light summer" and "medium winter"}

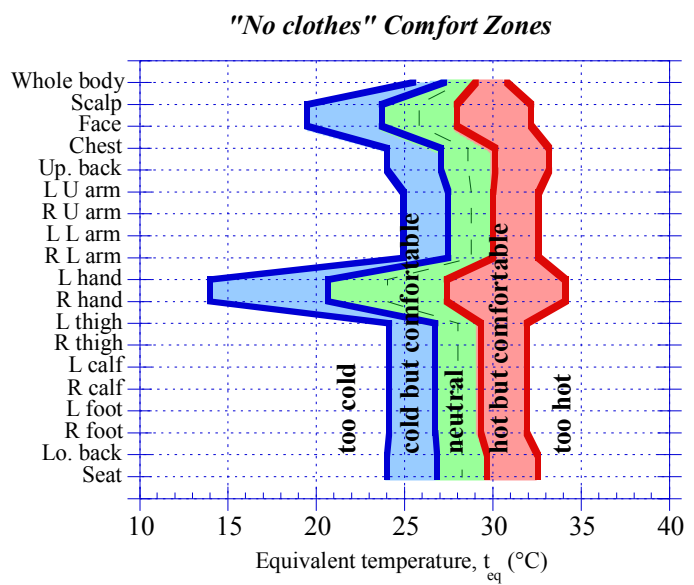

Figure 34. Comfort zone diagram derived for the case where no clothing is used. Notice that the comfort zones of acceptance has become significantly more narrow except for the "less sensitive" face and hands. The neutral $t_{e q}$ for the unclothed manikin are for the other zones around $28{ }^{\circ} \mathrm{C}$, which corresponds well with the neutral temp for a nude person.
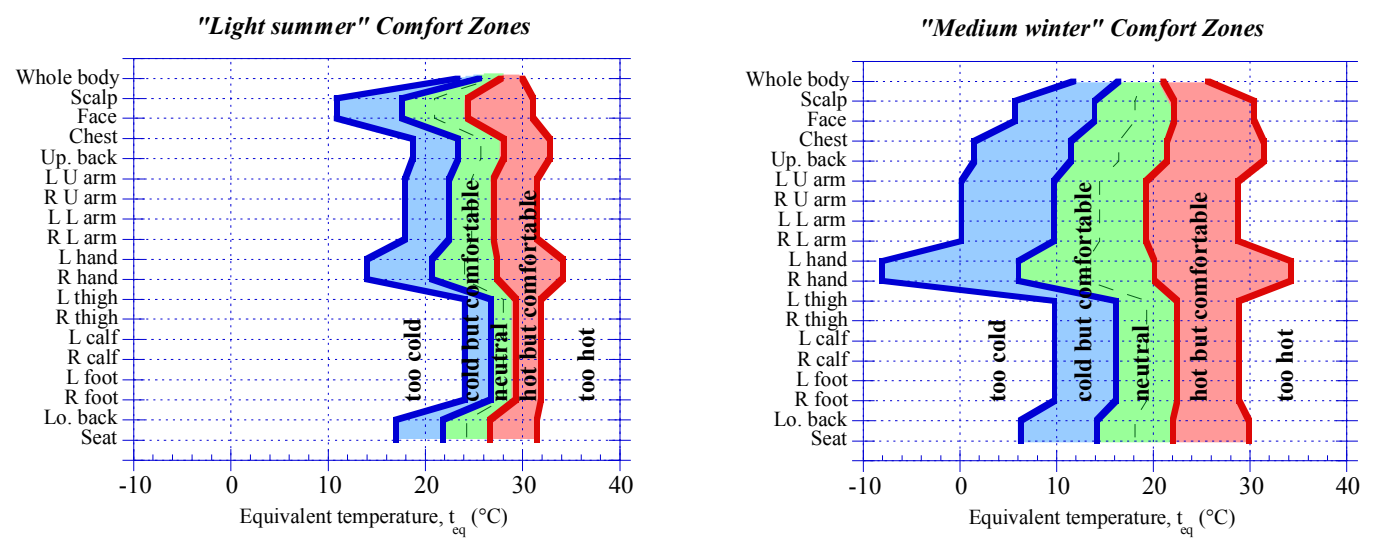

Figure 35. Notice the scale! Only used in these diagrams. Comfort zone diagrams derived for new different "light summer" and "medium winter" clothing ensembles. Notice again the shift of the comfort zones towards the cold side for manikin zones with increased insulation as well as the narrowing of the zones when the insulation is small or none. 
Table 12. Comfort zones with no clothing and a total insulation of 0.9 clo.

\begin{tabular}{|c|c|c|c|c|c|c|c|c|}
\hline$\overline{\text { Zone }}$ & too cold & $\begin{array}{c}\text { cold but } \\
\text { com- } \\
\text { fortable }\end{array}$ & neutral & $\begin{array}{l}\text { warm } \\
\text { but com- } \\
\text { fortable }\end{array}$ & $\begin{array}{c}\text { too } \\
\text { warm }\end{array}$ & $\begin{array}{c}\mathbf{R}_{\mathrm{T}} \\
\left(\mathrm{m}^{2} \mathrm{~K} / \mathrm{W}\right)\end{array}$ & $\begin{array}{l}q^{\prime \prime} T \\
\text { cold } \\
20 \%\end{array}$ & $\begin{array}{l}q^{q^{\prime \prime}} \mathbf{T} \\
\text { warm } \\
\mathbf{2 0 \%}\end{array}$ \\
\hline Whole body & 25.5 & 27.3 & 28.1 & 29.0 & 30.8 & 0.134 & 54 & 33 \\
\hline Scalp & 19.5 & 23.7 & 25.8 & 27.9 & 32.2 & 0.125 & 93 & 38 \\
\hline Face & 19.5 & 23.7 & 25.8 & 27.9 & 32.2 & 0.125 & 93 & 38 \\
\hline Chest & 24.0 & 27.1 & 28.6 & 30.1 & 33.2 & 0.149 & 52 & 20 \\
\hline Up. back & 24.0 & 27.1 & 28.6 & 30.1 & 33.2 & 0.149 & 52 & 20 \\
\hline L U arm & 24.9 & 27.5 & 28.8 & 30.0 & 32.6 & 0.122 & 60 & 26 \\
\hline R U arm & 24.9 & 27.5 & 28.8 & 30.0 & 32.6 & 0.122 & 60 & 26 \\
\hline L L arm & 24.9 & 27.5 & 28.8 & 30.0 & 32.6 & 0.122 & 60 & 26 \\
\hline R L arm & 24.9 & 27.5 & 28.8 & 30.0 & 32.6 & 0.122 & 60 & 26 \\
\hline L hand & 14.0 & 20.7 & 24.0 & 27.4 & 34.1 & 0.117 & 131 & 39 \\
\hline$R$ hand & 14.0 & 20.7 & 24.0 & 27.4 & 34.1 & 0.117 & 131 & 39 \\
\hline L thigh & 24.1 & 26.7 & 28.0 & 29.3 & 31.9 & 0.128 & 63 & 30 \\
\hline R thigh & 24.1 & 26.7 & 28.0 & 29.3 & 31.9 & 0.128 & 63 & 30 \\
\hline$L$ calf & 24.1 & 26.7 & 28.0 & 29.3 & 31.9 & 0.128 & 63 & 30 \\
\hline$R$ calf & 24.1 & 26.7 & 28.0 & 29.3 & 31.9 & 0.128 & 63 & 30 \\
\hline$L$ foot & 24.1 & 26.7 & 28.0 & 29.3 & 31.9 & 0.128 & 63 & 30 \\
\hline$R$ foot & 24.1 & 26.7 & 28.0 & 29.3 & 31.9 & 0.128 & 63 & 30 \\
\hline Lo. back & 24.0 & 26.8 & 28.3 & 29.7 & 32.5 & 0.145 & 55 & 24 \\
\hline Seat & 24.0 & 26.8 & 28.3 & 29.7 & 32.5 & 0.145 & 55 & 24 \\
\hline
\end{tabular}

Table 13. Comfort zones with light summer clothing with total insulation of 1.1 clo.

\begin{tabular}{|c|c|c|c|c|c|c|c|c|}
\hline Zone & too cold & $\begin{array}{c}\text { cold but } \\
\text { com- } \\
\text { fortable }\end{array}$ & neutral & $\begin{array}{c}\text { warm } \\
\text { but com- } \\
\text { fortable }\end{array}$ & $\begin{array}{c}\text { too } \\
\text { warm }\end{array}$ & $\underset{\left(\mathbf{m}^{2} \mathbf{K} / \mathbf{W}\right)}{\mathbf{R}_{\mathrm{T}}}$ & $\begin{array}{c}q^{\prime \prime} T \\
\text { cold } \\
20 \%\end{array}$ & $\begin{array}{c}q^{\prime \prime} T \\
\text { warm } \\
20 \%\end{array}$ \\
\hline Whole body & 23.3 & 25.6 & 26.7 & 27.8 & 30.0 & 0.167 & 54 & 33 \\
\hline Scalp & 10.9 & 17.6 & 21.0 & 24.3 & 31.1 & 0.199 & 93 & 38 \\
\hline Face & 10.9 & 17.6 & 21.0 & 24.3 & 31.1 & 0.199 & 93 & 38 \\
\hline Chest & 18.7 & 23.4 & 25.7 & 28.1 & 32.8 & 0.229 & 52 & 20 \\
\hline Up. back & 18.7 & 23.4 & 25.7 & 28.1 & 32.8 & 0.229 & 52 & 20 \\
\hline $\mathbf{L} \mathbf{U}$ arm & 17.9 & 22.5 & 24.7 & 27.0 & 31.5 & 0.215 & 60 & 26 \\
\hline $\mathbf{R} \mathbf{U} \mathbf{a r m}$ & 17.9 & 22.5 & 24.7 & 27.0 & 31.5 & 0.215 & 60 & 26 \\
\hline $\mathbf{L} \mathbf{L}$ arm & 17.9 & 22.5 & 24.7 & 27.0 & 31.5 & 0.215 & 60 & 26 \\
\hline R L arm & 17.9 & 22.5 & 24.7 & 27.0 & 31.5 & 0.215 & 60 & 26 \\
\hline$L$ hand & 14.0 & 20.7 & 24.0 & 27.4 & 34.1 & 0.117 & 131 & 39 \\
\hline R hand & 14.0 & 20.7 & 24.0 & 27.4 & 34.1 & 0.117 & 131 & 39 \\
\hline L thigh & 24.1 & 26.7 & 28.0 & 29.3 & 31.9 & 0.128 & 63 & 30 \\
\hline R thigh & 24.1 & 26.7 & 28.0 & 29.3 & 31.9 & 0.128 & 63 & 30 \\
\hline L calf & 24.1 & 26.7 & 28.0 & 29.3 & 31.9 & 0.128 & 63 & 30 \\
\hline$R$ calf & 24.1 & 26.7 & 28.0 & 29.3 & 31.9 & 0.128 & 63 & 30 \\
\hline$L$ foot & 24.1 & 26.7 & 28.0 & 29.3 & 31.9 & 0.128 & 63 & 30 \\
\hline$R$ foot & 24.1 & 26.7 & 28.0 & 29.3 & 31.9 & 0.128 & 63 & 30 \\
\hline Lo. back & 17.0 & 21.9 & 24.3 & 26.7 & 31.5 & 0.247 & 55 & 24 \\
\hline Seat & 17.0 & 21.9 & 24.3 & 26.7 & 31.5 & 0.247 & 55 & 24 \\
\hline
\end{tabular}


Table 14. Comfort zones with medium winter clothing with total insulation of 2.3 clo.

\begin{tabular}{|c|c|c|c|c|c|c|c|c|}
\hline$\overline{\text { Zone }}$ & too cold & $\begin{array}{c}\text { cold but } \\
\text { com- } \\
\text { fortable }\end{array}$ & neutral & $\begin{array}{c}\text { warm } \\
\text { but com- } \\
\text { fortable }\end{array}$ & $\begin{array}{c}\text { too } \\
\text { warm }\end{array}$ & $\underset{\left(\mathbf{m}^{2} \mathbf{K} / \mathbf{W}\right)}{\mathbf{R}_{\mathrm{T}}}$ & $\begin{array}{c}q^{\prime \prime} T \\
\text { cold } \\
20 \%\end{array}$ & $\begin{array}{c}\text { q"T }^{\prime \prime} \\
\text { warm } \\
20 \%\end{array}$ \\
\hline Whole body & 11.7 & 16.4 & 18.7 & 21.0 & 25.6 & 0.350 & 54 & 33 \\
\hline Scalp & 5.7 & 13.9 & 18.0 & 22.2 & 30.4 & 0.243 & 93 & 38 \\
\hline Face & 5.7 & 13.9 & 18.0 & 22.2 & 30.4 & 0.243 & 93 & 38 \\
\hline Chest & 1.5 & 11.5 & 16.4 & 21.4 & 31.4 & 0.487 & 52 & 20 \\
\hline Up. back & 1.5 & 11.5 & 16.4 & 21.4 & 31.4 & 0.487 & 52 & 20 \\
\hline L U arm & 0.1 & 9.7 & 14.5 & 19.2 & 28.8 & 0.454 & 60 & 26 \\
\hline $\mathbf{R} \mathbf{U}$ arm & 0.1 & 9.7 & 14.5 & 19.2 & 28.8 & 0.454 & 60 & 26 \\
\hline L L arm & 0.1 & 9.7 & 14.5 & 19.2 & 28.8 & 0.454 & 60 & 26 \\
\hline R L arm & 0.1 & 9.7 & 14.5 & 19.2 & 28.8 & 0.454 & 60 & 26 \\
\hline L hand & -8.0 & 6.1 & 13.1 & 20.1 & 34.2 & 0.246 & 131 & 39 \\
\hline $\mathbf{R}$ hand & -8.0 & 6.1 & 13.1 & 20.1 & 34.2 & 0.246 & 131 & 39 \\
\hline L thigh & 9.7 & 16.1 & 19.3 & 22.5 & 28.9 & 0.315 & 63 & 30 \\
\hline R thigh & 9.7 & 16.1 & 19.3 & 22.5 & 28.9 & 0.315 & 63 & 30 \\
\hline L calf & 9.7 & 16.1 & 19.3 & 22.5 & 28.9 & 0.315 & 63 & 30 \\
\hline$R$ calf & 9.7 & 16.1 & 19.3 & 22.5 & 28.9 & 0.315 & 63 & 30 \\
\hline$L$ foot & 9.7 & 16.1 & 19.3 & 22.5 & 28.9 & 0.315 & 63 & 30 \\
\hline$R$ foot & 9.7 & 16.1 & 19.3 & 22.5 & 28.9 & 0.315 & 63 & 30 \\
\hline Lo. back & 6.2 & 14.1 & 18.1 & 22.0 & 29.9 & 0.404 & 55 & 24 \\
\hline Seat & 6.2 & 14.1 & 18.1 & 22.0 & 29.9 & 0.404 & 55 & 24 \\
\hline
\end{tabular}

\section{Summary clothing-independent comfort zone diagrams}

The development of new "comfort zone diagrams" is one step towards a more general evaluation criteria applicable to different manikins and maybe also different instruments. Another important task is to make these zones change with the change of clothing worn by the subjects as well as the manikins. The clothing that manikins use today makes the measurement method sensitive, difficult to handle and comparative interpretation of results from different manikins/methods mostly impossible. When the manikin is moved between locations, does it regularly happen, that shape and positioning of the clothing worn is altered, hence changing the total insulation.

It is therefore suggested that the comfort zone diagrams not only should be manikin and method independent but also clothing-independent. The concept of clothing independence assumes that the human being is equally sensitive to different heat losses independent of the insulation of the clothing worn. The new clothing-independent comfort zone diagrams has been constructed by calculating a mean acceptable heat loss. This heat loss has then been used to calculate how the diagrams should look like if "no clothing" (only air layer, 0.9 clo), "light summer" (1.1 clo) and "medium winter" (2.3 clo) clothing is used.

As expected, does the diagram with less clothing indicate increased sensitivity on all zones, except the normally unclothed face and hands. The opposite, decreased sensitivity, can be observed for the diagram with increased clothing insulation. 


\section{Measurements with thermal manikins}

\section{The development of the manikin CLOUSSEAU}

My interest for thermal manikins started in 1987 when I was working with my final exam at the Royal Institute of Technology (KTH). We used an uncomplicated approach with a display manikin filled with high power resistances and aluminium foil for heat distribution purposes. The manikin named "Clousseau" had no regulation of the seven segments. The power input was controlled by means of several turntable transformers. The report, published only in Swedish, is called "En enkel termisk docka" (Johansson et al., 1987) directly translated "A simple thermal manikin".

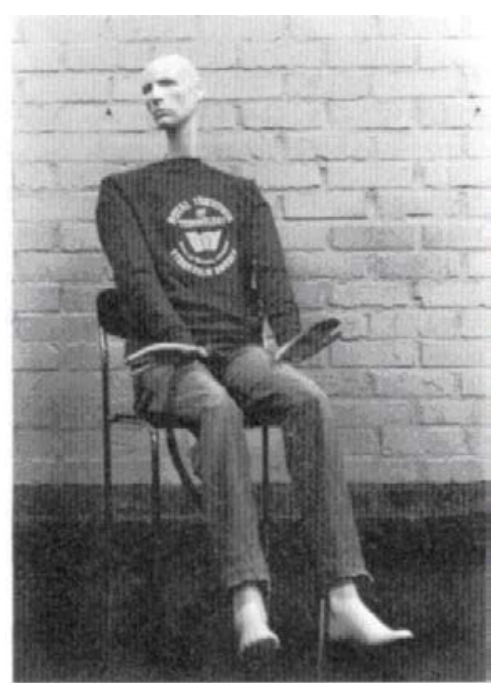

Figure 36. The manikin "Clousseau" used to determine convective heat transfer coefficients.

This manikin was later used in a study of thermal comfort and sensation under transient conditions (Wang, 1994).

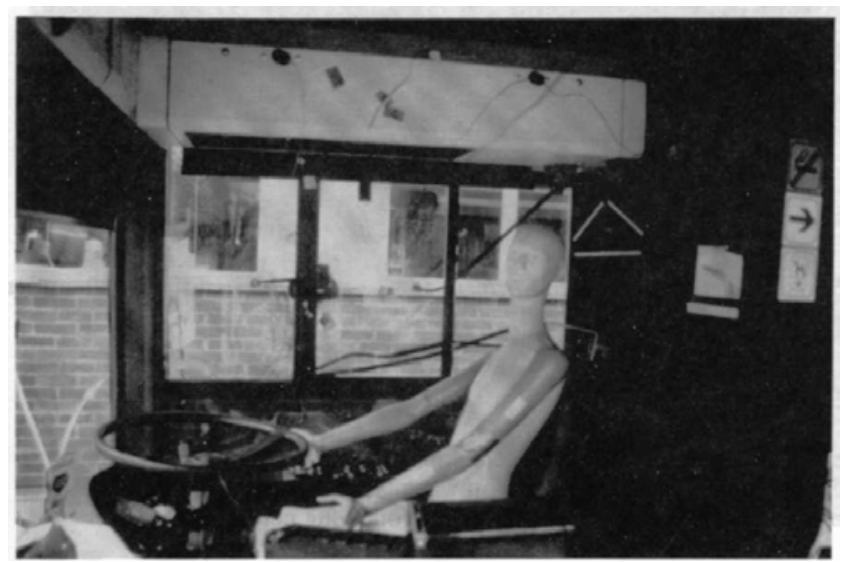

Figure 37. The manikin "Clousseau" in the drivers seat of a bus. Photo: X. Wang. 
Clousseau was once more used to determine convective heat transfer coefficients for various parts of the human body. The experimental technique with the manikin was used as basis to choose the most suitable ventilation system for bus drivers.

\section{The development and construction of MANIKIN1 and MANIKIN2}

For the purpose of the research reported here two slightly different manikins have been used MANIKIN1 from 1983 and the MANIKIN2 developed in 1991. The complete development process of MANIKIN2 is described in appendix A. Both manikins are a sitting thermal manikins constructed of plastic foam. The manikin surface is divided into independently temperature-regulated segments. Each zone on the surface layer of the manikin is densely covered with resistance wires, embedded in a hard plastic shell, on which surface temperature sensors are positioned. Every zone is regulated to a constant temperature $\left(34.0 \pm 0.1^{\circ} \mathrm{C}\right)$ and the power required is recorded with a personal computer.

During steady state this power input is equal to the power output, i.e. the dry heat loss. This heat loss measurement takes several factors into account like air temperature, air speed, radiation and clothing in the estimation of the climate. Once heated, the manikin responds to a step change and equilibrates at the new power consumption within approx. 20 minutes. The variation between double determinations in percentage of their average value is less than a few percent.

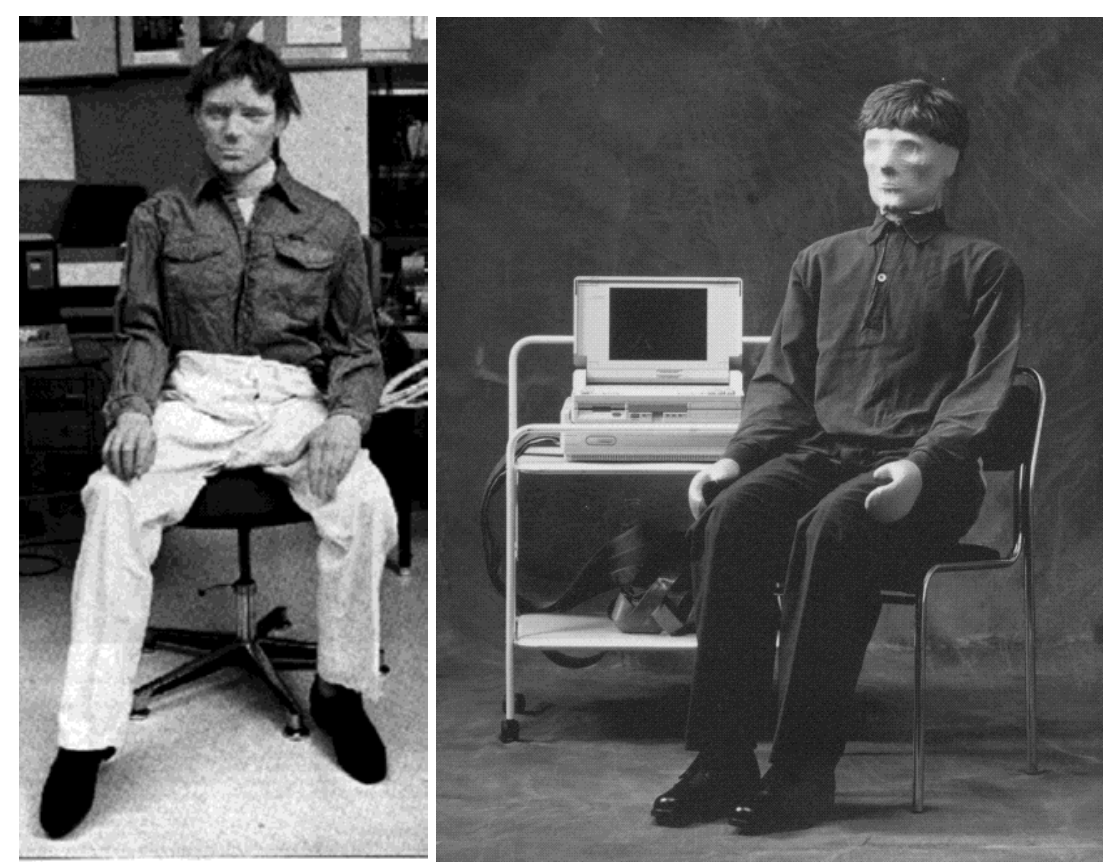

Figure 38. The thermal manikins used in this study. To the left MANIKIN1 from 1983 and to the right MANIKIN2 from 1991. On MANIKIN2 seat and thighs are adjusted to mimic the compressed shape during sitting conditions. MANIKIN2 also has a built in possibility to increase the weight by the insertion of several lead weights.

A thermal manikin measures the dry heat loss. In conditions where humans sweat it consequently gives an underestimation of the total heat loss. However, the research 
presented here deals with problems inside or close to the thermo neutral zone, where evaporative heat exchange represents a minor part that is not taken into account here. To facilitate the interpretation of results the heat flow data are recalculated and expressed as an equivalent temperature, ( $t_{e q}$ value), which serves as a standardised expression of the thermal load. This can be simplified with the use of the concept of "clothing-independent" comfort zone diagrams, described earlier.

\section{Measurements of equivalent temperature with thermal manikins}

Measurements with a thermal manikin produce both integrated and detailed information about thermal effects (Madsen et al., 1986, Wyon, 1989). Measurements with a thermal manikin yield a more complete, integrated and detailed information about thermal effects. The manikin called MANIKIN2 is a man-sized, sitting thermal manikin made of plastic foam The surface layer of the manikin is densely covered with resistance wires, embedded in a hard plastic shell. Manikin surface is divided into 18 independently controlled segments. Once heated the manikin responds to a step change and equilibrates at the new power consumption within approx. 20 minutes. The variation between double determinations in percent of their average value is less than a few percent. More information in Appendix A. The results are presented in a comfort diagram as variation of equivalent temperature described earlier (Nilsson et al., 1999a).

\section{Real $t_{e q}$-calibration}

Equivalent temperature defined earlier to be the temperature of a room with air temperature $\left(t_{a}\right)$ equal to mean radiant temperature $\left(\bar{t}_{r}\right.$, for calibration usually 21 or $24^{\circ} \mathrm{C}$ ) and low air velocity $\left(v_{a}\right)$ normally about $0.03 \mathrm{~m} / \mathrm{s}$. The heat transfer coefficient in this homogeneous environment can then be calculated as:

$$
h_{c a l}=\frac{q_{T, c a l}^{\prime \prime}}{\left(t_{s}-t_{a}\right)}
$$

\section{Equation 18}

gives in the assessed environment $\left(h_{c a l}\right.$ saved as $\left.h_{\text {teq }}\right)$

$$
t_{e q}=t_{s}-\frac{q_{T}^{\prime \prime}}{h_{t e q}}
$$

\section{Equation 19}

Where $q_{T}^{\prime \prime}$ is the measured manikin heat loss during the actual conditions, $q_{T, c a l}^{\prime \prime}$ is the dry heat loss for the homogenous, standard environment, $h_{c a l}$ is the dry heat transfer coefficient, determined during calibration in a standard environment, $t_{s}$ is the manikin surface temperature (normally $34^{\circ} \mathrm{C}$ ) and $t_{e q}$ is the temperature of the uniform, homogenous environment.

Calibration is carried out with the manikin dressed in standard clothing. Clothing affects the value of $h_{c a l}$ and, therefore, has to be defined properly and used throughout the series of measurements. The less insulation clothing provides, the more sensitive 
will the manikin segments be to thermal influences. It is important to specify the clothing used, whenever data are compared from different investigations and manikins.

\section{Measurement procedure}

The manikin is positioned in the environment that should be assessed. Heat flow in $\mathrm{W} / \mathrm{m}^{2}$ from the different segments of the manikin surface was measured and controlled. Data for the actual conditions were recorded when manikin had reached heat equilibrium with the environment

\section{Summary thermal manikin measurements}

For the purpose of the research in these thesis two slightly different manikins has been used MANIKIN1 from 1983 and the MANIKIN2 developed in 1991. The complete development process of MANIKN2 is described in appendix A. Both manikins are a sitting thermal manikins constructed of plastic foam.

The manikin surface is divided into 18 (max 33 ) independently controlled segments. Each zone on the surface layer of the manikin is densely covered with resistance wires, embedded in a hard plastic shell, on which surface temperature sensors are positioned. Every zone is regulated to a constant temperature $\left(34.0 \pm 0.1^{\circ} \mathrm{C}\right)$ and the power required is recorded with a personal computer.

During steady state is this power input is equal to the power output, i.e. the dry heat loss. This heat loss measurement takes several factors into account like air temperature, air speed, radiation and clothing in the estimation of the climate. The air temperature is always measured simultaneously with 3 radiation-shielded sensors at prescribed levels.

Once heated the manikin responds to a step change and equilibrates at the new power consumption within approx. 20 minutes. The variation between double determinations in percent of their average value is less than a few percent. The measured results are continuously presented in real time as a comfort zone diagram. The method presents a quick, accurate and reproducible technique for reliable assessment of many of the complex details of the climate and estimations of their integrated effects on humans. 


\section{Simulations with the virtual manikin}

\section{The construction of MANIKIN3}

MANIKIN3 is a virtual manikin built inside the computer with a heat flow interface to a CFD code (Appendix B). MANIKIN3 makes measurements in virtual environments before or after they are considered build environments.
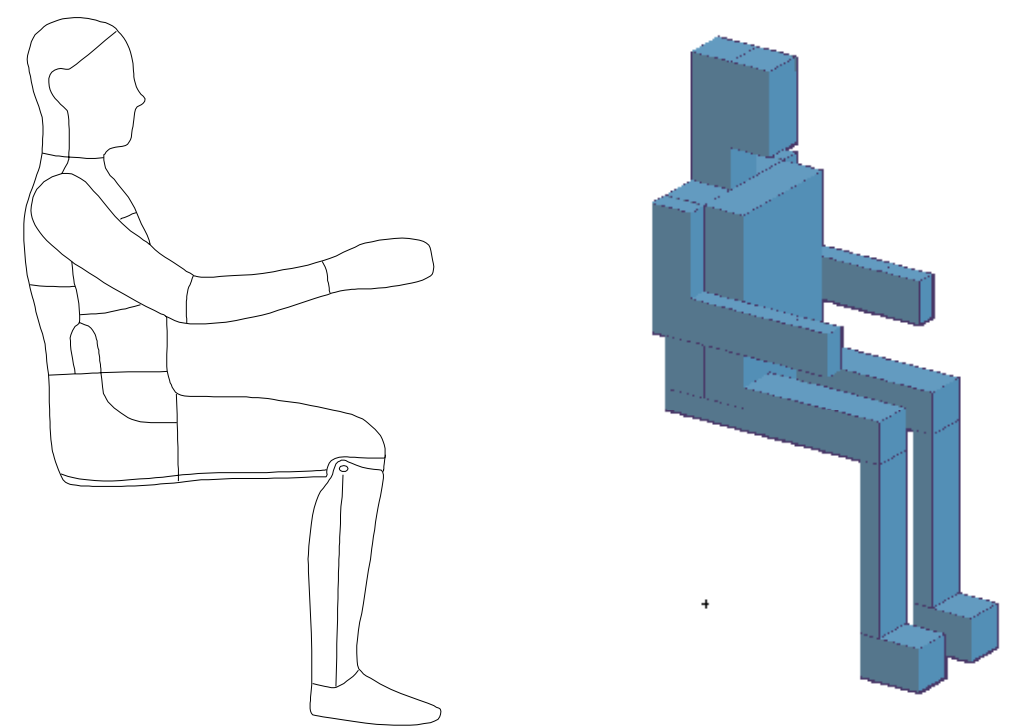

Figure 39. The geometry of the real MANIKIN2 (left) and the computational MANIKIN3 (right) built in CFX-Build with 18 zones at the same locations.

The virtual manikin is of cubical shape and formed with, as much as possible, the same size, areas and number of zones as the real MANIKIN2. MANIKIN3 as well as the MANIKIN2 has a free surface area of $1.6 \mathrm{~m}^{2}$, giving the same constant heat flux to the surrounding air. MANIKIN3 does not have the highest detail in body shape reconstruction.

However from measurements with MANIKIN1 and MANIKIN2 it is found that an important factor for the measurements is to have the body parts at the same position. MANIKIN3 corresponds well with the two other manikins in that respect. The way the surface temperature of MANIKIN3 is regulated/calculated, with the use of prescribed boundary conditions to the first grid cell, also makes the interface between MANIKIN3 and the air a little bit fuzzy. This fact is considered to even out some of the geometrical shape differences.

Moreover, a virtual manikin with thousands of patches, uses considerably more computer power to calculate the equivalent temperatures, a fact that has to be avoided at this stage. 
Table 15. The zone areas for MANIKIN2 and MANIKIN3

$\mathrm{L}=$ Left, $\mathrm{R}=$ Right, $\mathrm{U}=$ Upper, $\mathrm{L}=$ Lower, $\mathrm{O}=$ Outside, $\mathrm{I}=$ Inside, $\mathrm{B}=\mathrm{Back}, \mathrm{F}=$ Front

\begin{tabular}{cccc}
\hline $\begin{array}{c}\text { Zones } \\
\text { No }\end{array}$ & Name & $\begin{array}{c}\text { MANIKIN2 } \\
\mathbf{A}\left(\mathbf{m}^{2}\right)\end{array}$ & $\begin{array}{c}\text { MANIKIN3 } \\
\mathbf{A}\left(\mathbf{m}^{\mathbf{2}}\right)\end{array}$ \\
\hline 1 & Scalp & 0.0743 & 0.100 \\
2 & Face & 0.0654 & 0.080 \\
3 & Chest & 0.1838 & 0.225 \\
4 & BackU & 0.0754 & 0.065 \\
5 & ArmLU & 0.0811 & 0.065 \\
6 & ArmRU & 0.0805 & 0.065 \\
7 & ArmLL & 0.0545 & 0.070 \\
8 & ArmRL & 0.0596 & 0.070 \\
9 & HandL & 0.0439 & 0.035 \\
10 & HandR & 0.0449 & 0.035 \\
11 & ThighL & 0.1487 & 0.140 \\
12 & ThighR & 0.1465 & 0.140 \\
13 & CalfL & 0.1017 & 0.080 \\
14 & CalfR & 0.1052 & 0.080 \\
15 & FootL & 0.0526 & 0.070 \\
16 & FootR & 0.0528 & 0.070 \\
17 & BackL & 0.1788 & 0.120 \\
18 & Seat & 0.0422 & 0.095 \\
Total & & 1.5919 & 1.605 \\
\hline
\end{tabular}
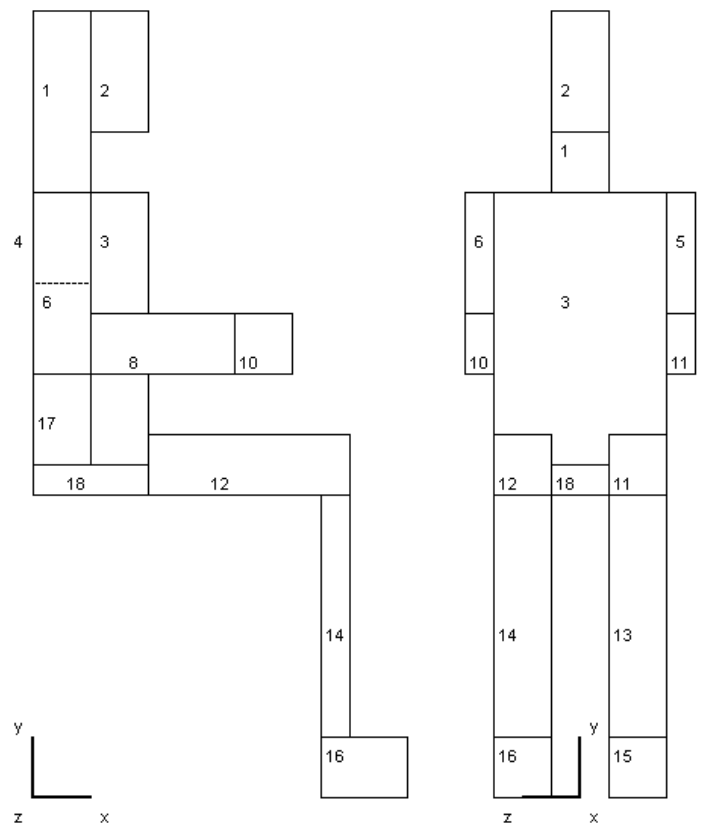

Figure 40. The geometry of the computational MANIKIN3 viewed from the right and front, with 18 numbered zones.

\section{Modelling the environment with CFD codes}

Today many commercial CFD codes are available on the open market. Some of them are highly specialised for a particular area of use as turbo machinery, chemical processes and sometimes indoor airflow. Others are more multipurpose. One important part of this 
work is to explore the possibilities to modify and control the boundary layers of the virtual manikin. This requires some kind of user programming possibilities. This leaves the choice of code to basically three CFD packages; FLUENT ${ }^{\circledR}$ (Fluent Inc. Fluent 5.4, 1998), STAR-CD ${ }^{\circledR}$ (Computational Dynamics Star CD 3.1B, 2001) and CFX ${ }^{\circledR}$ (Ansys Inc. CFX 5.4, 2000).

These 3 packages (suites) are today quite similar. First they use a pre-processor to set up the problem (FLUENT-Gambit, STAR-CD-Prostar and CFX-Build). This program contains tools for creating the computational mesh to represent the flow domain, as well as specifying the thermophysical properties of the fluid. It also specifies the different boundary conditions and finally writes all of this to appropriate data files

They secondly have a flow analysis part (FLUENT-Fluent, STAR-CD-Star and CFXSolver). This code consists of means for reading the input data previously created and also ways for judging the quality of the progress of the run by monitoring and analysing various output data and solution statistics.

Finally they have a post-processing part to visualise and analyse the calculated results (FLUENT-Fluent, STAR-CD-Prostar and CFX-Visualise). This involves the display and manipulation of output data created by the flow analysing code, using facilities for a multitude of different plot options with vectors, fields and tracks as well as movies illustrating different flow phenomenons.

All of these codes should be able to handle the calculations made in this thesis. The early work has been done with CFX Version 4.2 and 5.4 and the final work with STARCD Version 3.100B.

\section{Calculation procedures, definitions and orders of magnitude}

Full-scale subjective and manikin studies are both complicated, expensive and time consuming. The use of CFD makes it possible to solve the fluid flow as well as heat transfer and get satisfactory simulation results for most indoor airflows. In order to design a comfortable indoor environment with MANIKIN3, it is important to get relevant input data of the airflow pattern, velocity, and temperature around the virtual manikin. The majority of flows in the indoor environment are turbulent. Unfortunately no turbulence model exists for general use. Every model must be employed with care and its results treated with caution (Sørensen et al., 2003). A short description of some models used, beginning in the most detailed end of the turbulence simulation methods:

\section{DNS, LES, VLES and RANS}

Direct Numerical Simulations (DNS) and Large Eddy Simulations (LES), are computational tools with which almost all scales of turbulence can be simulated. (DNS) simulates all the scales of turbulence at the highest level of detail, at the expense of huge computer costs. LES computes only the large or very large (VLES) scales of turbulent fluid flow. At a slightly less complex level there is also Reynolds Averaged NavierStokes (RANS) methods that solve time-averaged equations, trying to model all the scales of turbulence in order to solve flows over more complex geometries at higher Reynolds numbers. 
Drawbacks of DNS and LES is that they are limited to fairly simple geometries such as channel flows, simple boundary layer flows and the use of large number of cells and many time steps that are well beyond current personal computational capabilities used by engineers today. For engineering use is it important to use a more simple turbulence model for the simulation of the indoor environment, that could calculate the airflow, velocity and temperature fields in an adequately correct way.

\section{Standard k- $\varepsilon$ model}

The 'standard' model in which the high (turbulent) Reynolds number forms of the $k$ and $\varepsilon$ equations are used in together with algebraic 'law of the wall' representations of flow, heat and mass transfer for the near-wall region (Launder et al., 1974). The standard $k-\varepsilon$ model is consequently often called a two-equation model. The standard k- $\varepsilon$ is appropriate for fully turbulent flows where the Reynolds number $(\mathrm{Re})$ is high.

\section{Low-Re number k- $\varepsilon$ model}

The low Reynolds numbers model in which general transport equations for $k$ and $\varepsilon$ are solved everywhere, including the near-wall regions. 'Law of the wall' functions are therefore not required. For low Reynolds number flows the standard k- $\varepsilon$ model overestimates the turbulent diffusivity. The standard $k-\varepsilon$ model has been modified to give better results with these flows. In low-Reynolds number turbulence models, correction functions are introduced in the calculation.

\section{RNG-k- $\varepsilon$ model}

The 'ReNormalisation Group' (RNG) version of the $k-\varepsilon$ model is denoted as RNG $k-\varepsilon$. This is used in high Reynolds number form together with 'law of the wall' functions. From a comparison by Chen, 1995, the $R N G k-\varepsilon$ model was recommended for the simulation of indoor environments. The RNG- $k-\varepsilon$ model was better than the standard $\mathrm{k}-\varepsilon$ model for mixed convection flow and impinging jets.

\section{Zero-equation turbulence model}

When the details of the turbulence are not so important, rather the general mixing behaviour, then it is often possible to use a constant turbulent (eddy) viscosity $\mu_{t}$ in stead of the molecular viscosity (Nielsen, 1998). This turbulence model is called the zero-equation model and uses a constant or an algebraic function to express the turbulent viscosity. It does not require the solution of any additional differential equations beyond the Navier-Stokes equations. This turbulence model calculates the turbulent viscosity empirically by: 
Where

$\mu_{t} \quad$ turbulent (eddy) viscosity (Pa s)

Fluid density $\left(\mathrm{kg} / \mathrm{m}^{3}\right)$

$u_{0} \quad$ Characteristic velocity, inlet velocity $(\mathrm{m} / \mathrm{s})$

$H \quad$ Characteristic length, inlet min length (m)

The length scale is a characteristic length, in this thesis the case specific min length of the inlet is used. In the same way the inlet velocity is used as the characteristic velocity for each case. The empirical constant suitable for different indoor airflows is a number between 0.038 and 0.040 . This model is often sufficient for predicting the total characteristic of a turbulent flow; it may not always be suitable for predicting local details. One benefit of this method is that the time used for calculations with the zeroequation model is much less compared to the more complicated models. Further more the use of this turbulence model does not need extensive grid refinement or the use of special wall functions, two factors that significantly speeds up the working process.

Consequently the computer power needed to calculate indoor airflow is less and can be realised with an ordinary personal computer.

The next level of complexity with the zero-equation models is to compute an effective viscosity that is a function of local conditions. This is made on the basis of Prandtl's mixing-length hypothesis, which gives that the viscosity is proportional to the local shear rate. Results calculated by Chen et al., 1998 shows that the accuracy of the zero-equation model is acceptable for indoor airflow design purpose. More experiments have been done to validate the zero-equation model ability to predict indoor airflow (Srebric et al., 1999). The results from these studies show that zero-equation model predicts the main flow reasonably well even with thicker boundary layers, due to the larger cell size used. Although there are some differences, the important air velocity and temperature profile results are good.

In this thesis such a simple model for indoor airflow simulation is used; the zeroequation turbulence model. Below table 16 with the turbulence viscosities used.

Table 16. Turbulence viscosities used in the 3 validation cases.

\begin{tabular}{|c|c|c|c|c|c|c|c|}
\hline \multicolumn{8}{|l|}{ Zero-equation model } \\
\hline$\overline{\text { Density }\left(\mathrm{kg} / \mathrm{m}^{3}\right)}$ & 1.205 & 1.205 & 1.205 & 1.205 & 1.205 & 1.205 & 1.205 \\
\hline Inlet velocity $(\mathrm{m} / \mathrm{s})$ & 0.03 & 0.03 & 0.08 & 0.17 & 0.25 & 3.38 & 0.15 \\
\hline Inlet characteristic min length (m) & 0.50 & 0.50 & 0.20 & 0.20 & 0.20 & 0.10 & 0.30 \\
\hline Constant (ND) & 0.04 & 0.04 & 0.04 & 0.04 & 0.04 & 0.04 & 0.04 \\
\hline Turbulence viscosity ( $\mathrm{Pa} \mathrm{s}$ ) & 0.00072 & 0.00072 & 0.00077 & 0.00164 & 0.00241 & 0.016 & 0.0022 \\
\hline Turbulence viscosity $\mathrm{k}-\varepsilon$ model typical ( $\mathrm{Pa} \mathrm{s})$ & 0.00047 & 0.00047 & 0.00034 & 0.00034 & 0.00034 & 0.015 & 0.0019 \\
\hline case & odis40 & odis55 & cclearg & cclearp & ccolop & creflg & \\
\hline$\overline{\text { Density }\left(\mathrm{kg} / \mathrm{m}^{3}\right)}$ & 1.205 & 1.205 & 1.205 & 1.205 & 1.205 & 1.205 & \\
\hline Inlet velocity $(\mathrm{m} / \mathrm{s})$ & 0.27 & 0.37 & 4.40 & 4.40 & 4.40 & 4.40 & \\
\hline Inlet characteristic min length (m) & 0.30 & 0.30 & 0.05 & 0.05 & 0.05 & 0.05 & \\
\hline Constant (ND) & 0.04 & 0.04 & 0.04 & 0.04 & 0.04 & 0.04 & \\
\hline Turbulence viscosity ( $\mathrm{Pa} \mathrm{s}$ ) & 0.0039 & 0.0054 & 0.011 & 0.011 & 0.011 & 0.011 & \\
\hline Turbulence viscosity $\mathrm{k}-\varepsilon$ model typical ( $\mathrm{Pa} \mathrm{s})$ & 0.0019 & 0.0019 & 0.011 & 0.011 & 0.011 & 0.011 & \\
\hline
\end{tabular}




\section{General conservation equations}

The conservation of mass, momentum and energy can describe the airflow field. If the boundary conditions are known, the flow pattern can be determined by solving the combined Navier-Stokes and energy equations. STAR-CD solves the general mass, momentum and scalar conservation equations, with different meanings of $\phi$ :

$$
\frac{\partial(\rho \phi)}{\partial t}=\frac{\partial}{\partial x_{j}}\left(\Gamma_{\phi} \frac{\partial \phi}{\partial x_{j}}-\rho u_{j} \phi\right)+S_{\phi}
$$

\section{Equation 21}

Where

$t \quad$ Time (s)

$\rho \quad$ Fluid density $\left(\mathrm{kg} / \mathrm{m}^{3}\right)$

$\phi \quad$ Represents any mean scalar variable

$u_{j} \quad$ Fluid velocity component in direction $x_{j}(\mathrm{~m} / \mathrm{s})$

$S_{\phi} \quad$ Source term

$\Gamma_{\phi} \quad$ Diffusion coefficient

The term to the left of the equal sign is the convection term, the first term to the right is the diffusion term and $\left(S_{\phi}\right)$ is the source term.

\section{Numerical solution algorithms and discretisation}

STAR-CD permits three different solution algorithms:

- SIMPLE method

- PISO method.

- SIMPISO algorithm (combines elements of both the other methods).

The solution is complicated by the pressure term in the momentum equation. One way to overcome this is a procedure in which the pressure field is obtained via the continuity equation, the Semi-Implicit Method for Pressure-Linked Equations (SIMPLE) (Patankar 1980). Given an initial pressure field, the momentum equations are solved. A pressure correction is obtained from the revised continuity equation and the velocity component values are corrected.

After calculation of the flow field variables, temperature and turbulent values, the calculated pressure is used as the new pressure field and the calculation is repeated until the solution is converged. SIMPLE differs from the PISO algorithm in two principal respects; it is mainly suitable for steady-state calculations and it employs only one corrector stage. Under-relaxation is required in iterative steady-flow calculations with SIMPLE; it is therefore essential for stability reasons, to under-relax velocity and pressure as well as other variables, such as the turbulence parameters.

\section{Buoyancy-driven flows and natural convection}

STAR-CD contains built-in functions for different kinds of body forces, including buoyancy. To analyse problems with buoyancy is it necessary to specify the pressure and gravity forces in the momentum equation. A correction term for the buoyancy force is 
then incorporated in the revised continuity equation (Computational Dynamics 1999). This feature is used in all calculations since effects of buoyancy can be expected.

\section{Differencing schemes}

Discretisation in space requires the flow field to be divided in small control volumes. First-order schemes select the nearest upwind neighbour value. This form of interpolation preserves the correct physical bounds on under all conditions, but can lead to numerical diffusion. This is way only second order schemes are used in the following calculations.

MARS (Monotone Advection and Reconstruction Scheme) is a multidimensional second-order scheme that works in two steps. MARS does not rely on any problem dependent parameters to work properly. The user can control the ability of the advection scheme to accurately capture sharp discontinuities in the flow by setting the scheme's compression level to a value between 0 and 1 . Low values for this parameter result in a computationally efficient scheme at the expense of sharpness of resolution. High values improve the resolution but result in an increased number of iterations when steady flows are computed. The default value for this parameter is 0.5 that is the best compromise between accuracy and convergence rate.

\section{Convergence scheme}

In order to avoid numerical instability, under-relaxation is introduced. A relaxation factor controls the change of a variable as calculated at each iteration. The solution for the selected variable(s) is taken as a weighted mean of the previous and current iteration. In principle, under-relaxation may be used on any of the dependent variables as well as quantities like density and viscosity. The convergence is checked by several criteria: the mass and heat conservation should be balanced; the residuals of the discretisised conservation equations must steadily decrease; and the change in field values between two iterations should be very small, below 0.01 .

\section{Grid technology}

CFD calculations are performed on a grid that fills the experimental volume, in the three dimensional case. The shape of the grid, over which the equations are solved, is very important. The simplest grid is called the Cartesian grid, in the 3-D case the cells are cubes. When a coarse grid is used, the cubical cells sometimes are severely deformed. A grid with smaller cells will solve this problem to some extent, but will on the other hand make the processing time longer.

One commonly used solution is to refine the grid only in areas where it is absolutely necessary. That is close to the manikin, walls and in/outlets. Another alternative is to use a grid based on tetrahedron cells. This solves some of the problem with distorted cubical cells. To take the best from these two grid solutions sometimes a combination of these two types is used. To avoid any prolongation of the calculation time the standard Cartesian 3-D grid used in the following analyses. 


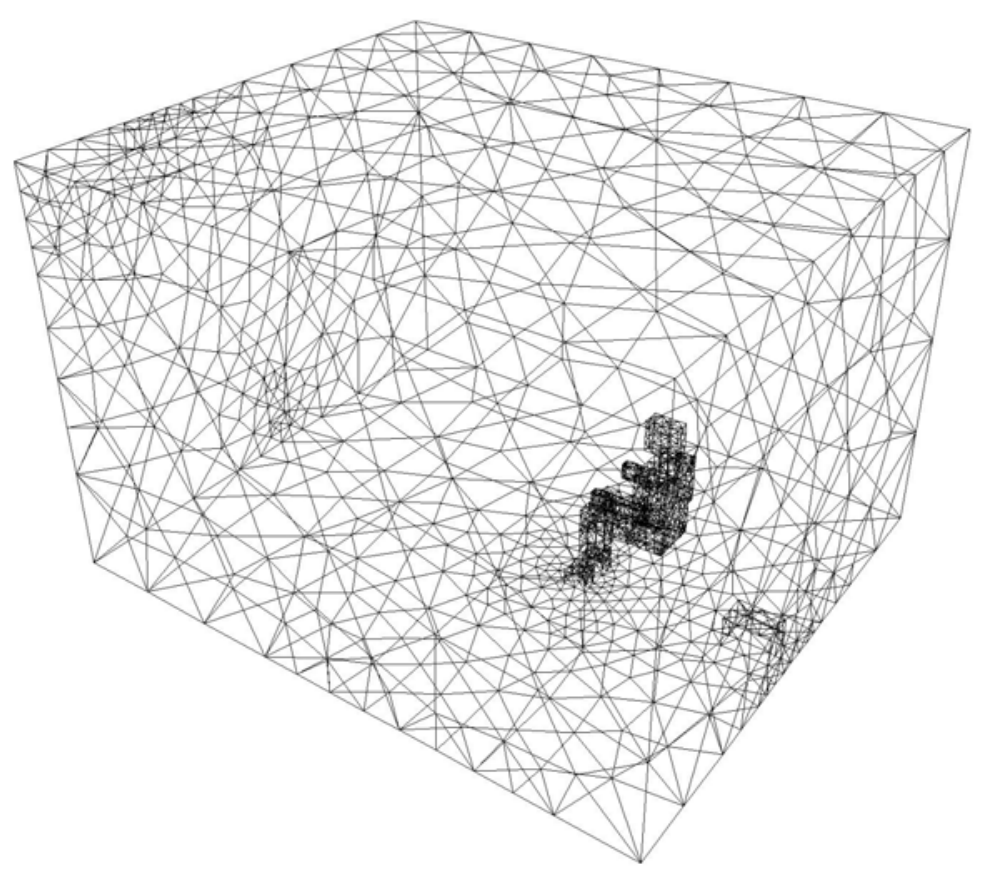

Figure 41. A tetrahedron surface grid for the first case, made with CFX-Build 5.4. The inlet and outlet is positioned on the left wall opposite the manikin. The manikin is sitting on a net chair with the power supply behind. In the final calculations it was decided to use a standard cartesian grid topology.

When a virtual thermal manikin is placed in a room it usually requires grid refinement close to the manikin surface. Fine grids are sometimes required to determine the flow field up to the smallest length scale close to the body (Murakami et al., 1989). These requirements sometimes create difficulties to reach convergence of the calculation domain as well as requiring high computational power.

Methods of creating a fine mesh by internal subdivision of a coarser mesh are usually called "embedded mesh refinement methods". Adaptation can result in a grid that is refined or coarsened when applying user-defined adaptation parameters is also solution dependent. In this thesis it was decided that no grid adaptation should be used, as this mostly increases the number of cells and hence the calculation time.

\section{Boundary conditions}

The success or failure of a fluid simulation depends not only on the code capabilities. For a simulation to have any chance of success all input information should be physically realistic and correctly presented to the analysis code. The process of defining boundary conditions for a finite element model can be divided into two major steps:

Identify the location of individual boundaries.

Specify the conditions at the boundaries.

It is of the great importance that boundaries are chosen and implemented correctly, since the outcome of any analysis depends on them. The use of turbulence models requires 
imposition of the appropriate boundary conditions. The types of boundaries requiring particular attention are walls and inflow streams. In this thesis the treatment of the wall boundaries are of particular interest since the outcome of the CFD analyses depends on them. The section below describes the treatment of the wall boundaries.

\section{Prescribed wall heat transfer}

In order to get good results from the numerical simulation the knowledge of the wall heat transfer from the calibrations with no clothes were used. In the CFD-simulations of the experimental cases the available wall heat transfer coefficients, an agreement between measurement and simulation was obtained. User code for modification of the manikin wall boundary conditions was developed to extend applicability of CFD calculations for indoor environment simulation. It is also possible in the future research to use the results from these test cases to further validate and develop wall functions and dynamic boundary prescription methods.

The near surface flow field in a room or around a heated body is characterised by a combination of natural, free and forced convection, developing boundary layers. The restricted validity of the heat transfer models often used originates from the assumptions that have been made to solve special boundary layer flows, for instance in pipes, (Schlichting, 1960). These assumptions are consequently not valid for boundary layer flows that can be commonly found in the indoor environment.

Hatton et al., (1996) present several empirical wall functions for natural and a mixing convection. They see the need for an accurate experimental method to calculate heat transfer coefficients for both natural and mixed convection for the different heated surfaces in the indoor environment. They also say that both thermal and CFD models require correct heat transfer coefficients for the calculations used to simulate the conditions in a room and recognises most existing computer models use coefficients derived for other circumstances.

One way of achieving correct convective heat transfer is described by Brohus, 1997. In 2D CFD calculations of the convective heat transfer, the node distance in the grid layout is adapted; in order to get the same convective heat transfer as in a test case. This method gives a grid node distance of $0.005 \mathrm{~m}$.

In results described by Loomans, 1997 the wall heat transfer was adjusted by adaptation of the thermal conductivity and the dynamic viscosity at the first grid cell. A higher conductivity increased the wall heat transfer indirectly. This approach indicated an improved agreement of simulation results with measurement results. However the modification of the dynamic viscosity changed the near wall flow properties unrealistically.

Further results presented by Loomans, 1998 show simulation of the flow pattern applying wall functions lead to significant deviations in the calculated temperature profile compared to measured profiles. The temperature gradient is significantly overestimated. The method has also the obvious drawback that the heat transfer characteristics should be known in advance. In these calculations a grid node distance of $0.01 \mathrm{~m}$ is used.

de Dear et al., (1997) makes a series of tests with a thermal manikin in order to find radiative heat transfer coefficients as well as natural- and forced-mode convective 
coefficients for the human body. The tests were conducted across a range of wind speeds from still air to $5.0 \mathrm{~m} / \mathrm{s}$, representing conditions typical of both indoors and outdoors. Standing and seated postures were investigated.

Silva, (2002) determines the convection coefficients of the different parts of the human body. The tests are performed in a wind tunnel with a thermal manikin for different flow angles and postures. The best fit of the experimental results was obtained with linear regression, instead of a power function as previously presented by other authors.

The results from manikin runs with MANIKIN2 in homogeneous climate at three different temperatures $\left(19,24\right.$ and $\left.28^{\circ} \mathrm{C}\right)$ are used to develop new convective heat transfer functions between the manikin and the environment.

\section{Clothed manikin heat transfer model}

In the general case where convective $\left(q^{\prime \prime} c\right)$ and radiative $\left(q^{\prime \prime} r\right)$ heat transfer modes are present simultaneously, the total zone heat flux $\left(q^{\prime \prime} T\right)$ is the sum of components:

$$
q_{T}^{\prime \prime}=q_{c}^{\prime \prime}+q_{r}^{\prime \prime}=f_{c l} \cdot h_{c} \cdot\left(t_{c l}-t_{a}\right)+f_{c l} \cdot h_{r} \cdot\left(t_{c l}-\bar{t}_{r}\right)=h_{c a l} \cdot\left(t_{s}-t_{e q}\right) \text { Equation } 22
$$

Where:

$q^{\prime \prime}{ }_{T} \quad$ Total heat transfer $\left(\mathrm{W} / \mathrm{m}^{2}\right)$

$q^{\prime \prime}{ }_{c} \quad$ Convective heat transfer $\left(\mathrm{W} / \mathrm{m}^{2}\right)$

$q^{\prime \prime}{ }_{r} \quad$ Radiative heat transfer $\left(\mathrm{W} / \mathrm{m}^{2}\right)$

$h_{c} \quad$ Convective heat transfer coefficient $\left(\mathrm{W} / \mathrm{m}^{2} \mathrm{~K}\right)$

$h_{r} \quad$ Radiative heat transfer coefficient $\left(\mathrm{W} / \mathrm{m}^{2} \mathrm{~K}\right)$

$h_{\text {cal }}$ Calibration heat transfer coefficient $\left(\mathrm{W} / \mathrm{m}^{2} \mathrm{~K}\right)$

$t_{a} \quad$ Ambient air (adjacent fluid) temperature $\left({ }^{\circ} \mathrm{C}\right)$

$\bar{t}_{r} \quad$ Mean radiant temperature $\left({ }^{\circ} \mathrm{C}\right)$

$t_{s} \quad$ Manikin surface temperature $\left({ }^{\circ} \mathrm{C}\right)$

$t_{e q} \quad$ Equivalent temperature $\left({ }^{\circ} \mathrm{C}\right)$

$A_{u} \quad$ Surface area unclothed manikin $\left(\mathrm{m}^{2}\right)$

$A_{c l} \quad$ Surface area clothed manikin $\left(\mathrm{m}^{2}\right)$

$f_{c l} \quad$ Clothing area factor, $A_{c l} / A_{u}$ (n.d.)

The clothing area factor (ISO 9920, 1993) is defined as the surface area of the clothed body divided with the area of the unclothed body and can be estimated as:

$$
f_{c l}=1+1.97 \cdot R_{c l}=1+0.3 \cdot I_{c l}
$$

Where

$I_{c l} \quad$ Intrinsic clothing insulation (clo)

$R_{c l} \quad$ Intrinsic clothing insulation $\left(\mathrm{m}^{2} \mathrm{~K} / \mathrm{W}\right)$

$R_{c l}=0.155 \cdot I_{c l}$ 
Clothing insulation is expressed in clo units when the symbol $I$ is used and in SI units $\left(\mathrm{m}^{2} \mathrm{~K} / \mathrm{W}\right)$ when the symbol $R$ is used. To avoid confusion is only $R$-values used from now on.

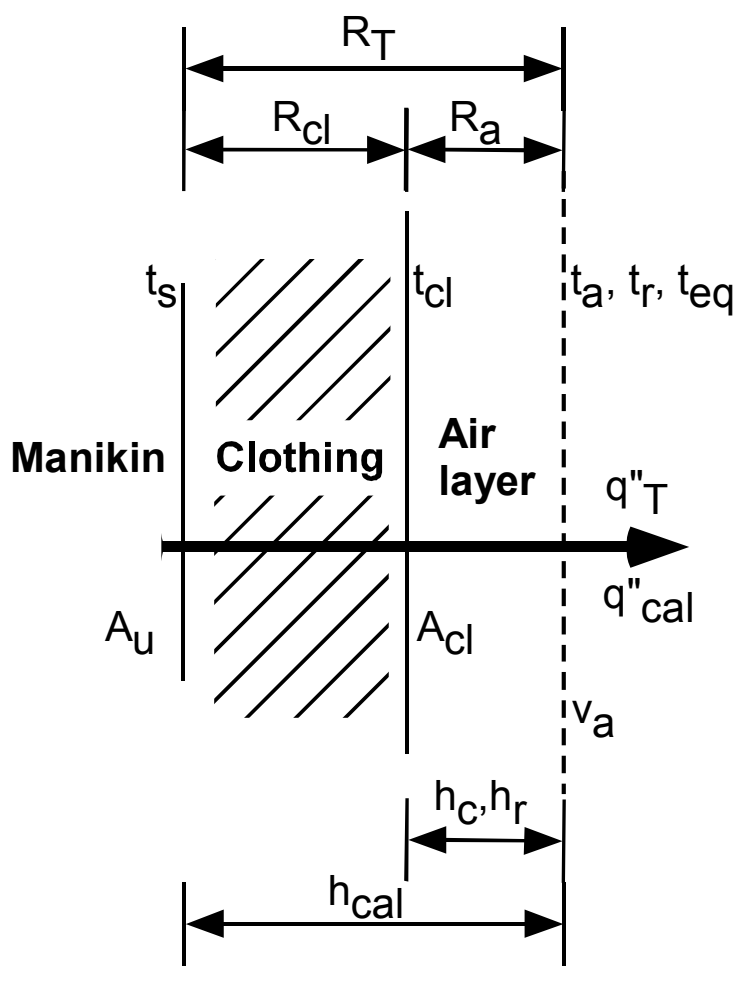

Figure 42. The model of the heat transfer from the manikin surface to the surroundings.

Here the convective heat transfer coefficient $h_{c}$ determining the heat flow between the wall and the ambience is given by:

$$
h_{c}=\frac{q_{c}^{\prime \prime}}{f_{c l}\left(t_{c l}-t_{a}\right)}=A \cdot v_{a}+C \cdot\left(t_{c l}-t_{a}\right)+D
$$

\section{Equation 24}

Where:

$f_{c l} \quad$ seated, calibration winter/summer clothing (@ 21, $\left.25\left({ }^{\circ} \mathrm{C}\right)\right)$ (n.d.)

A seated, constant from Silva et al. $2002\left(\mathrm{~W} / \mathrm{m}^{2} \mathrm{~K}\right)$

C, D seated, constants from unclothed calibration (@ 19, 24, 28 $\left.\left({ }^{\circ} \mathrm{C}\right)\right)\left(\mathrm{W} / \mathrm{m}^{2} \mathrm{~K}\right)$

$v_{a} \quad$ air velocity $(\mathrm{m} / \mathrm{s})$

The radiation area factor $f_{r}$, are derived from seated values suggested by de Dear et al., (1997). The linear dependency of air velocity constant A, comes from measurements reported by Silva et al., (2002), for seated posture. The temperature difference dependence constants are calculated with linear regression from unclothed seated calibration with MANIKIN2 in ambient temperatures 19,24 and $28\left({ }^{\circ} \mathrm{C}\right)$. 
Table 17. Values of the constants used in the modified manikin wall function are shown in the table. $\mathrm{s}=$ summer clothing, $\mathrm{w}=$ winter clothing

\begin{tabular}{|c|c|c|c|c|c|c|c|c|c|c|c|}
\hline $\mathrm{Nr}$ & Zone & $\begin{array}{c}h_{r} \\
\text { deDear } \\
1997\end{array}$ & $\begin{array}{c}f_{r} \\
\text { deDear } \\
1997\end{array}$ & $\begin{array}{c}A \\
\text { Silva et. } \\
\text { al. } 2002\end{array}$ & $\begin{array}{c}C \\
\text { slope }\end{array}$ & $\begin{array}{c}D \\
\text { intercept }\end{array}$ & $r^{2}$ & $\begin{array}{c}R_{c l} \\
\text { meas } \\
\mathrm{S}\end{array}$ & $\begin{array}{c}R_{c l} \\
\text { meas } \\
\text { W }\end{array}$ & $\begin{array}{c}f_{c l} \\
\text { meas } \\
\text { s }\end{array}$ & $\begin{array}{c}f_{c l} \\
\text { meas } \\
\mathrm{w}\end{array}$ \\
\hline 0 & Total & 4.5 & 0.76 & 4.2 & 0.140 & 1.952 & 1.00 & 0.096 & 0.146 & 1.188 & 1.288 \\
\hline 1 & Scalp & 3.9 & 0.66 & 2.9 & 0.189 & 2.161 & 1.00 & 0.178 & 0.160 & 1.350 & 1.315 \\
\hline 2 & Face & 3.9 & 0.66 & 2.9 & 0.124 & 3.544 & 0.96 & 0.009 & 0.013 & 1.019 & 1.025 \\
\hline 3 & Chest & 3.4 & 0.58 & 4.1 & 0.138 & 1.606 & 1.00 & 0.130 & 0.380 & 1.255 & 1.749 \\
\hline 4 & BackU & 4.6 & 0.78 & 4.1 & 0.140 & 2.563 & 0.96 & 0.077 & 0.205 & 1.152 & 1.404 \\
\hline 5 & ArmLU & 4.8 & 0.82 & 5.1 & 0.128 & 2.925 & 1.00 & 0.142 & 0.353 & 1.280 & 1.695 \\
\hline 6 & ArmRU & 4.8 & 0.82 & 5.1 & 0.125 & 2.201 & 0.99 & 0.127 & 0.333 & 1.251 & 1.655 \\
\hline 7 & ArmLL & 5.2 & 0.88 & 5.4 & 0.117 & 2.663 & 1.00 & 0.096 & 0.207 & 1.188 & 1.407 \\
\hline 8 & ArmRL & 5.2 & 0.88 & 5.4 & 0.149 & 1.851 & 0.99 & 0.094 & 0.206 & 1.184 & 1.406 \\
\hline 9 & HandL & 3.9 & 0.66 & 3.7 & 0.113 & 3.286 & 0.92 & 0.000 & 0.032 & 1.000 & 1.062 \\
\hline 10 & HandR & 3.9 & 0.66 & 3.7 & 0.123 & 3.470 & 0.99 & 0.000 & 0.042 & 1.000 & 1.083 \\
\hline 11 & ThighL & 4.6 & 0.78 & 4.3 & 0.154 & 1.608 & 1.00 & 0.088 & 0.101 & 1.174 & 1.199 \\
\hline 12 & ThighR & 4.6 & 0.78 & 4.3 & 0.139 & 1.787 & 0.99 & 0.093 & 0.100 & 1.182 & 1.196 \\
\hline 13 & CalfL & 5.4 & 0.92 & 4.9 & 0.194 & 0.938 & 1.00 & 0.089 & 0.094 & 1.175 & 1.186 \\
\hline 14 & CalfR & 5.4 & 0.92 & 4.9 & 0.174 & 0.750 & 1.00 & 0.089 & 0.093 & 1.176 & 1.184 \\
\hline 15 & FootL & 4.2 & 0.71 & 6.1 & 0.134 & 2.927 & 0.99 & 0.170 & 0.135 & 1.335 & 1.266 \\
\hline 16 & FootR & 4.2 & 0.71 & 6.1 & 0.139 & 2.914 & 1.00 & 0.154 & 0.133 & 1.303 & 1.262 \\
\hline 17 & BackL & 4.6 & 0.78 & 4.1 & 0.107 & 0.924 & 1.00 & 0.185 & 0.310 & 1.364 & 1.610 \\
\hline 18 & Seat & 4.8 & 0.82 & 4.0 & 0.083 & 2.086 & 0.96 & 0.107 & 0.088 & 1.211 & 1.172 \\
\hline
\end{tabular}

Unfortunately the radiation information generated with PROSTAR are not readily available in POSDAT at the moment. The internal patch numbers used in STAR do not correspond to PROSTAR patch numbers. Two reasons for that are; different numbering in STAR (like the cell numbering), and the fact that fictitious patches are assigned by PROSTAR to open boundaries like inlets and outlets. Consequently the following radiation calculations are used instead.

The linearised radiation heat transfer coefficient $h_{r}$ determining the radiative heat flow between the wall and the ambience is calculated as:

$h_{r}=\frac{q_{r}^{\prime \prime}}{f_{c l}\left(t_{c l}-\bar{t}_{r}\right)}=4 \cdot \varepsilon \cdot \sigma \cdot f_{r} \cdot\left(273.15+\frac{t_{c l}-\bar{t}_{r}}{2}\right)^{3}$

\section{Equation 25}

where:

$\varepsilon \quad$ the surface emissivity (0.95) (n.d.)

$\sigma \quad$ Stefan-Boltzmann constant $\left(5.669 * 10^{-8}\right)\left(\mathrm{W} / \mathrm{m}^{2} \mathrm{~K}^{4}\right)$

$A_{r} \quad$ Effective radiation area of manikin $\left(\mathrm{m}^{2}\right)$

$f_{r} \quad$ Radiation area factor, $A_{r} / A_{u}$, seated, de Dear et al. 1997 (n.d.)

Combining the above equations gives the following set of equations, used to calculate the manikin heat flow, and by iteration find the corresponding clothing or surface temperature (see also the Fortran code in appendix B):

$t_{c l}=t_{s}-R_{c l} \cdot q_{T}^{\prime \prime}$

Equation 26 
$t_{e q}=t_{s}-\frac{q_{T}^{\prime \prime}}{h_{t e q}}$

\section{Equation 27}

Where:

$R_{c l} \quad$ seated, calibration winter/summer clothing (@21, $\left.25\left({ }^{\circ} \mathrm{C}\right)\right)\left(\mathrm{m}^{2} \mathrm{~K} / \mathrm{W}\right)$

$h_{\text {teq }}$ seated, calibration winter/summer clothing $\left(@ 21,25\left({ }^{\circ} \mathrm{C}\right)\right)\left(\mathrm{W} / \mathrm{m}^{2} \mathrm{~K}\right)$

$h_{\text {cal }}$ seated, virtual calibration, saved as $h_{\text {teq }}\left(\mathrm{W} / \mathrm{m}^{2} \mathrm{~K}\right)$

As STAR-CD permits introduction of modified wall boundary conditions during the iteration procedure these functions are then introduced in the CFD calculations with the results of the flow field as input. A subroutine for calculation of new surface temperature of MANIKIN3 has been written calculating new surface temperatures depending on the present heat flow from each of the zones of the manikin. (Appendix B).

\section{Virtual $t_{e q}$-calibration}

In a similar manner as with the full-scale heat transfer coefficient calibration (" $t_{e q}$ calibration"), virtual manikin also needs adjustment of the heat transfer coefficients. The method is based on the fact that equivalent temperature is defined to be the temperature of a room with air temperature $\left(t_{a}\right)$ equal to mean radiant temperature $\left(\bar{t}_{r}\right.$, usually 21 or $24^{\circ} \mathrm{C}$ ) and low air velocity $\left(v_{a}\right)$ (normally about $0.03 \mathrm{~m} / \mathrm{s}$ ). The heat transfer coefficient in the homogeneous environment is given by the following relationships:

$h_{c a l}=\frac{q_{T, c a l}^{\prime \prime}}{\left(t_{s}-t_{a}\right)}$

Equation 28

gives in the assessed environment $\left(h_{c a l}\right.$ saved as $\left.h_{\text {teq }}\right)$

$t_{e q}=t_{s}-\frac{q_{T}^{\prime \prime}}{h_{\text {teq }}}$

\section{Equation 29}

Where

$q_{T}^{\prime \prime} \quad$ calculated manikin heat loss during the actual conditions $\left(\mathrm{W} / \mathrm{m}^{2}\right)$

$q_{T, c a l}^{\prime \prime} \quad$ dry heat loss for the homogenous, standard environment $\left(\mathrm{W} / \mathrm{m}^{2}\right)$

$h_{c a l} \quad$ dry heat transfer coefficient, determined during calibration in a standard environment $\left(\mathrm{W} / \mathrm{m}^{2} \mathrm{~K}\right)$

$t_{s} \quad$ manikin surface temperature $\left({ }^{\circ} \mathrm{C}\right)$

$t_{e q} \quad$ equivalent temperature of the uniform, homogenous environment. $\left({ }^{\circ} \mathrm{C}\right)$

The virtual calibration is carried out with the computational MANIKIN3 positioned a fictitious calibration chamber with the dimensions of $2 \times 2 \times 2 \mathrm{~m}$. The incoming air at 0.03 $\mathrm{m} / \mathrm{s}$ is entering through the floor, except right under the manikin (centered $0.7 \times 0.95 \mathrm{~m}$, $0.5 \mathrm{~m}$ from the back wall), and exits through the full roof area. This arrangement gives an ideal calibration environment, where the heat from the manikin is removed swiftly, with as little as possible influence on the calibration itself. The sensor tree is always positioned at the specified levels $(0.1,0.6$, and $1.1 \mathrm{~m})$ to the right of MANIKIN3. 

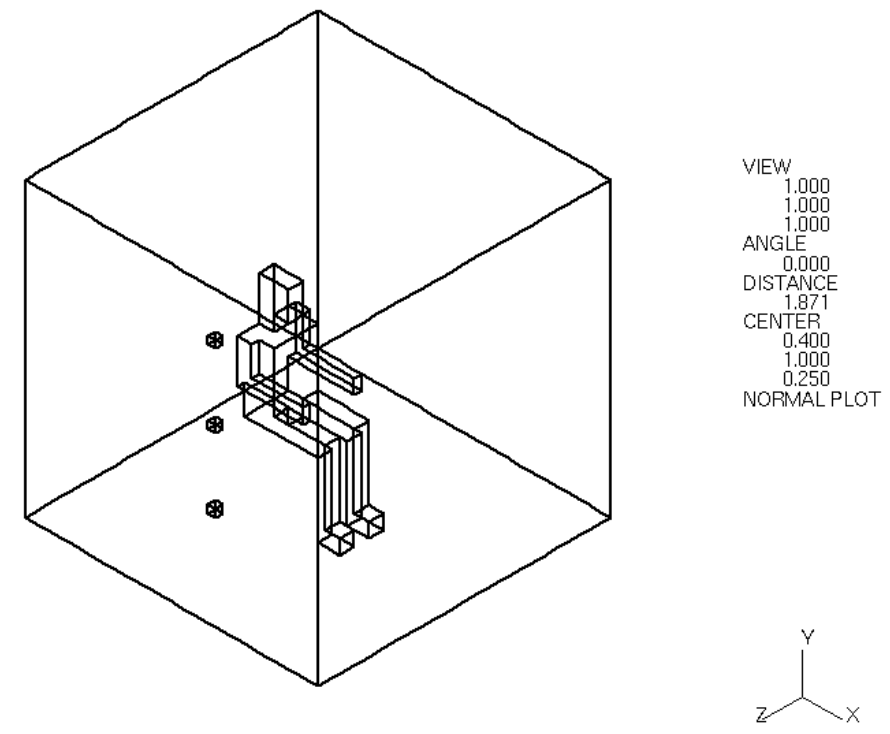

Figure 43. MANIKIN3 and sensor tree inside the virtual calibration cube.

Dressed in the same winter and summer clothing as MANIKIN2.

The unclothed calibrations of MANIKIN3 forming the basis for the heat transfer equations give excellent results in this environment, as well as in reality with MANIKIN2. The calibrations made are consequently the situations when MANIKIN3 are clothed in the same summer and winter clothing that was used for the real measurements with MANIKIN2. This is made in order to get the right computational $h_{\text {teq }}$ for the runs of the virtual cases described later. The results from calibrations with summer (s) and winter (w) clothing are shown in table 18.
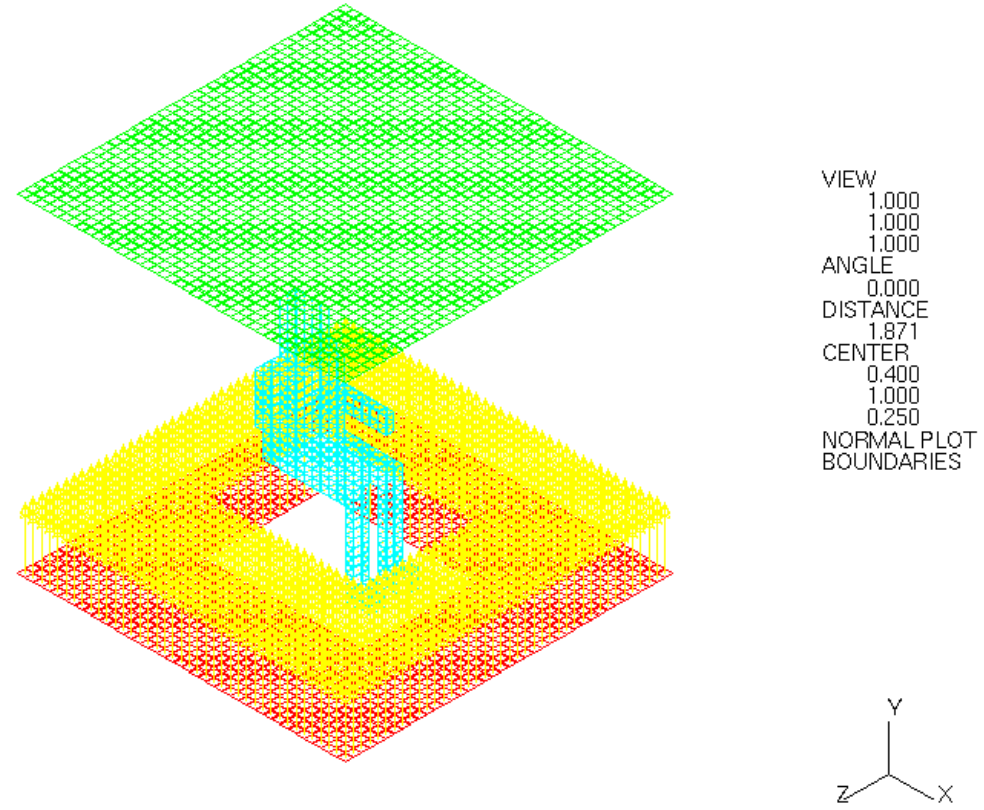

Figure 44. The boundaries of MANIKIN3 in the incoming air flow at $0.03 \mathrm{~m} / \mathrm{s}$ from the floor, exiting through the full roof area.

Table 18. The heat transfer coefficient in the homogeneous environment calculated with 
the new manikin wall functions are shown in the table. meas = measured with MANIKIN2, calc $=$ calculated with measured ambience data input, $\mathbf{c f d}=$ calculated with CFD simulated data input. ( $\mathrm{s}=$ summer clothing, $\mathrm{w}=$ winter clothing)

\begin{tabular}{lcccccccc}
\hline $\begin{array}{l}\text { Heat transfer coefficient }\left(\boldsymbol{h}_{\text {teq }}\right) \\
\left(\mathrm{W} / \mathrm{m}^{2} \mathrm{~K}\right)\end{array}$ & $\begin{array}{c}\mathrm{s} \\
\text { meas }\end{array}$ & $\begin{array}{c}\mathrm{W} \\
\text { meas }\end{array}$ & $\begin{array}{c}\mathrm{s} \\
\mathrm{calc}\end{array}$ & $\begin{array}{c}\mathrm{W} \\
\mathrm{calc}\end{array}$ & $\begin{array}{c}\mathrm{s} \\
\mathrm{cfd}\end{array}$ & $\begin{array}{c}\mathrm{W} \\
\text { cfd }\end{array}$ & $\begin{array}{c}\text { calc } \\
\text { mean } \\
\text { diff. }\end{array}$ & $\begin{array}{c}\text { cfd } \\
\text { mean } \\
\text { diff. }\end{array}$ \\
\hline Scalp & & & & & & & & \\
Face & 3.49 & 3.78 & 3.39 & 3.6 & 3.47 & 3.71 & $\mathbf{0 . 1}$ & $\mathbf{0 . 0}$ \\
Chest & 3.25 & 8.19 & 8.25 & 8.17 & 8.12 & 8.12 & $\mathbf{0 . 0}$ & $\mathbf{0 . 1}$ \\
BackU & 5.79 & 2.08 & 3.69 & 1.94 & 3.72 & 2.05 & $\mathbf{0 . 2}$ & $\mathbf{0 . 1}$ \\
ArmLU & 4.26 & 2.4 & 5.38 & 3.22 & 5.38 & 3.35 & $\mathbf{0 . 3}$ & $\mathbf{0 . 2}$ \\
ArmRU & 4.69 & 2.44 & 4.11 & 2.24 & 4.23 & 2.34 & $\mathbf{0 . 1}$ & $\mathbf{0 . 0}$ \\
ArmLL & 5.22 & 3.47 & 5.07 & 3.27 & 5.12 & 3.4 & $\mathbf{0 . 2}$ & $\mathbf{0 . 1}$ \\
ArmRL & 5.13 & 3.4 & 4.93 & 3.19 & 4.96 & 3.33 & $\mathbf{0 . 2}$ & $\mathbf{0 . 1}$ \\
HandL & 8.6 & 6.98 & 8.55 & 6.91 & 8.35 & 6.87 & $\mathbf{0 . 1}$ & $\mathbf{0 . 2}$ \\
HandR & 8.49 & 6.7 & 8.84 & 6.61 & 8.62 & 6.62 & $\mathbf{0 . 2}$ & $\mathbf{0 . 1}$ \\
ThighL & 4.96 & 4.71 & 4.78 & 4.53 & 4.8 & 4.6 & $\mathbf{0 . 2}$ & $\mathbf{0 . 1}$ \\
ThighR & 4.82 & 4.76 & 4.72 & 4.58 & 4.76 & 4.65 & $\mathbf{0 . 1}$ & $\mathbf{0 . 1}$ \\
CalfL & 5.11 & 4.98 & 4.88 & 4.79 & 4.92 & 4.88 & $\mathbf{0 . 2}$ & $\mathbf{0 . 1}$ \\
CalfR & 4.83 & 4.9 & 4.78 & 4.71 & 4.82 & 4.8 & $\mathbf{0 . 1}$ & $\mathbf{0 . 1}$ \\
FootL & 3.63 & 4.32 & 3.67 & 4.17 & 3.73 & 4.24 & $\mathbf{0 . 1}$ & $\mathbf{0 . 1}$ \\
FootR & 3.82 & 4.35 & 3.88 & 4.18 & 3.94 & 4.27 & $\mathbf{0 . 1}$ & $\mathbf{0 . 1}$ \\
BackL & 3.11 & 2.44 & 3.17 & 2.27 & 3.24 & 2.38 & $\mathbf{0 . 1}$ & $\mathbf{0 . 1}$ \\
Seat & 4.59 & 5.05 & 4.49 & 4.89 & 4.56 & 4.94 & $\mathbf{0 . 1}$ & $\mathbf{0 . 1}$ \\
\hline
\end{tabular}
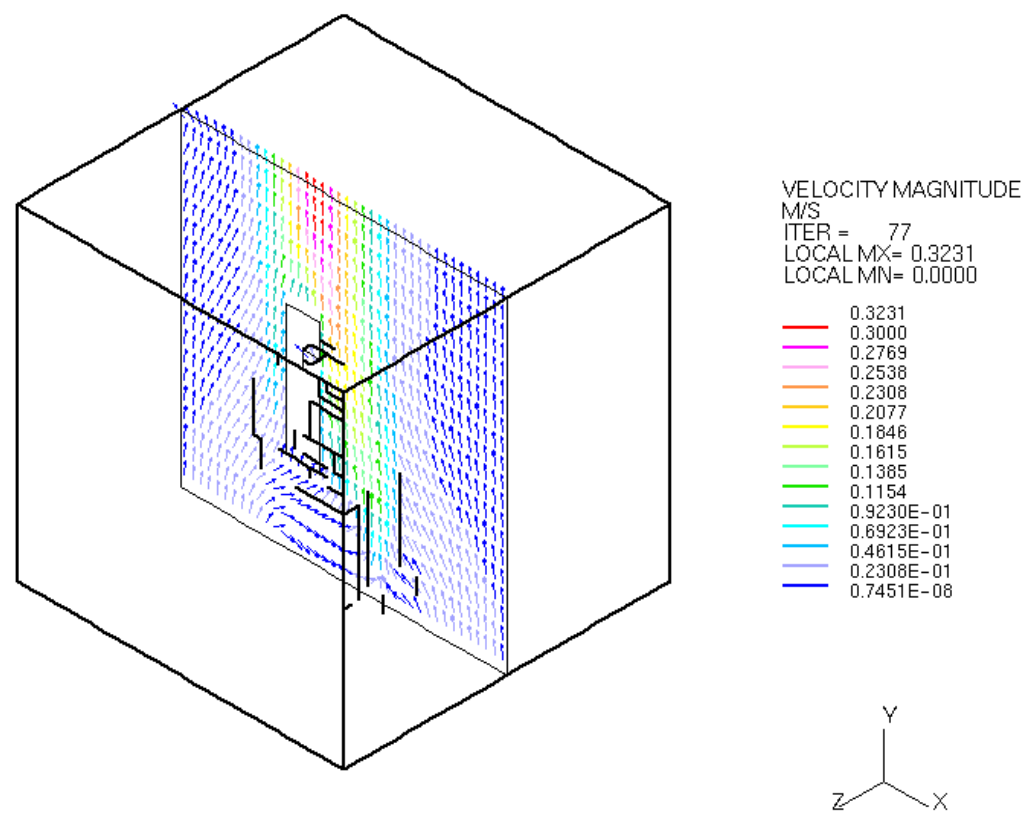

Figure 45. Velocity vector field at the virtual calibration with summer clothing. Low velocity field in the room, only buoyancy driven flow around the manikin. 


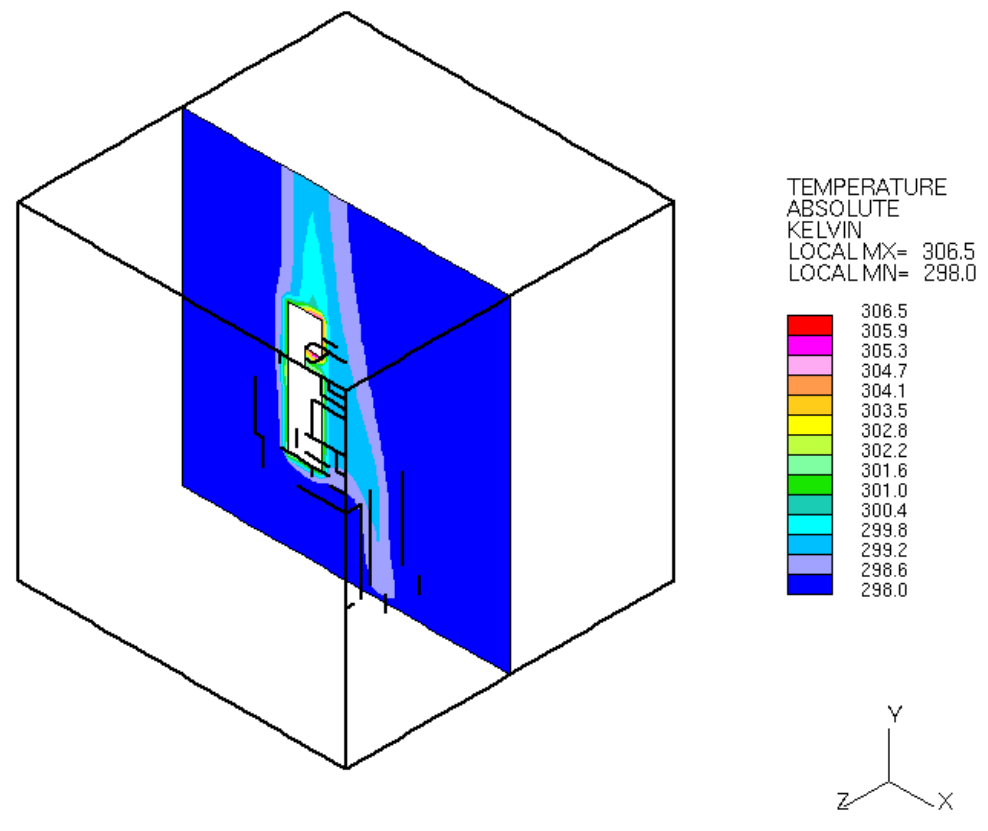

Figure 46. The temperatures of the plume around themanikin at the virtual calibration with summer clothing. Notice the increased temperatures around the face and hands.

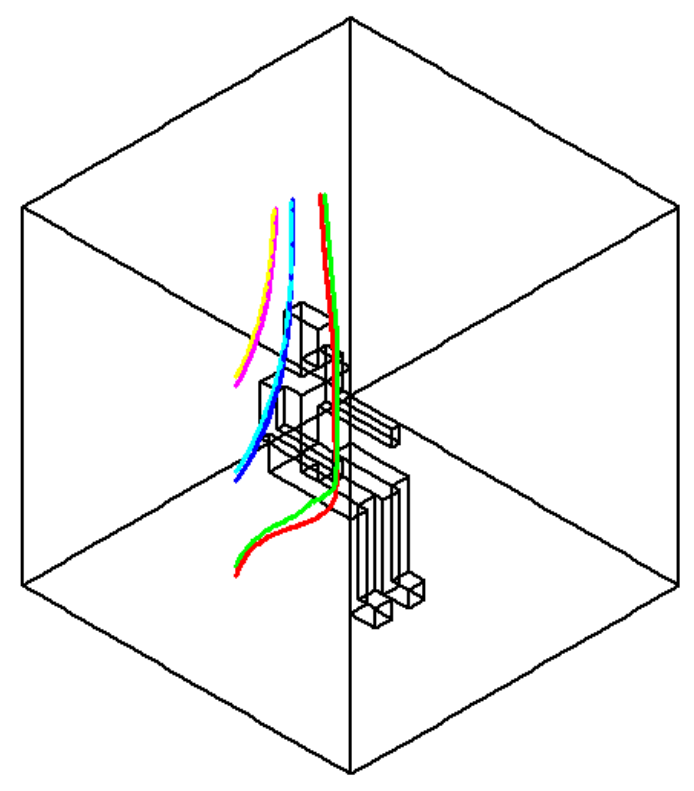

Figure 47. Flow tracks originating from the sensors,

showing the good nature of the flow at the virtual calibration.

\section{Summary virtual manikin methods}

A virtual MANIKIN3 of cubical shape was formed with the corresponding size, areas and number of zones as the real MANIKIN2. MANIKIN3 as well as MANIKIN2 has a free surface area of $1.6 \mathrm{~m}^{2}$, and the same constant the surface temperature modified by the clothing used and surrounding environment. MANIKIN3 is built inside the computer 
with the heat flow interface to a commercial CFD code. The aim for MANIKIN3 is to make simulations of virtual environments before and/or after they are made into built environments.

The modelling has steady state characteristics and is aimed at the assessment of human thermal comfort. Several commercial CFD codes should be able to handle the proposed calculations. The CFD calculations use the zero-equation model. Underrelaxation is used in the iterative steady-flow calculations with SIMPLE solution algorithms. Due to the buoyant flows involved the correction term for the buoyancy force is applied. In this work the MARS second order scheme are used (Monotone Advection and Reconstruction Scheme). It was decided that no grid adaptation should be used, as this mostly increases the number of cells and hence the calculation time.

The calibrations made are the situations when MANIKIN3 are clothed in the same summer and winter clothing that was used for the real measurements with the physical MANIKIN2. This is done to get the right computational $h_{\text {teq }}$ for the runs of the three virtual cases described later. Results from calibrations with summer (s) and winter (w) clothing show very consistent results. 


\section{Thermal insulation for seated persons}

Several predictive models describe overall thermal sensation as function of at least six thermal factors. Two important factors are the activity of the person and the insulation of the clothing. For a seated person the chair may have considerable impact on the heat exchange and thereby affect thermal sensation. Despite this fact, only limited information can be found in the literature about the influence of different seats on thermal insulation (McCullough et al., 1994).

\section{Introduction to the seat insulation study}

This chapter presents results from a study aimed at determining the insulation of 11 different types of commonly used chairs and seats. In this study two different types of thermal manikins were used. The sitting thermal manikin MANIKIN2, se appendix A. The standing/walking thermal manikin is one in the TORE-series and has been described detail elsewhere (Nilsson et al., 1992).

In short an external, pneumatically driven system applies a swinging movement to arms and legs. The action can be controlled to allow the manikin to perform realistic walking movements at different step rates. Measurements were carried out in a climatic chamber at an air velocity of about $0.12 \mathrm{~m} / \mathrm{s}$. Repeatability with the applied procedure for measuring insulation was high; difference between values in double determinations was always lesser than $6 \%$ of the average of the two.

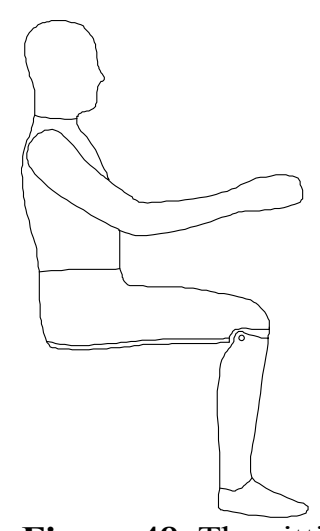

Figure 48. The sitting thermal manikin, MANIKIN2

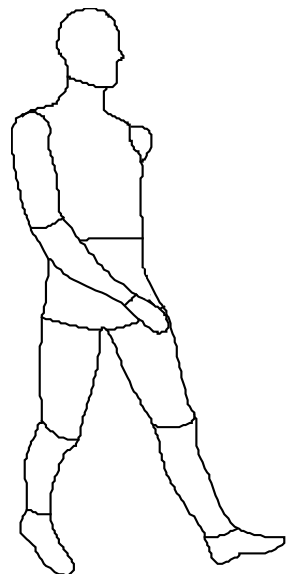

Figure 49. The standing walking manikin, TORE

The chairs selected for the measurement were two car seats, five commonly used office chairs and some additional seats like balance chair without back, 3-seat sofa, net chair and a wooden stool. 


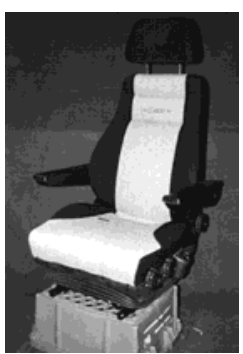

Ventilated car seat

Car seat

) (Volvo)
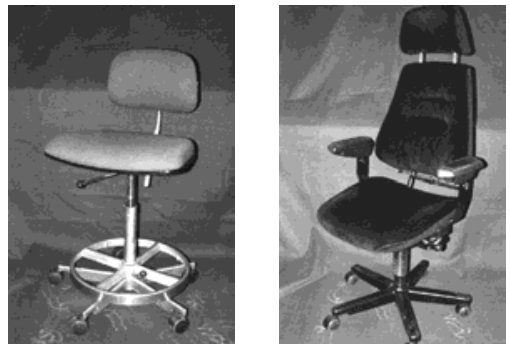

Office chair w. Office w head- HÅG Balance o. arms (KEVI) rest (Nyström) (No back)

Figure 50. Pictures of some of the seats.

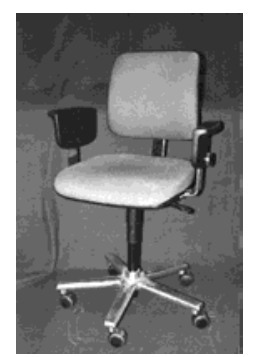

Office chair w. Office chair w.

armr. (Nomi3) armr. (Edsbyn)
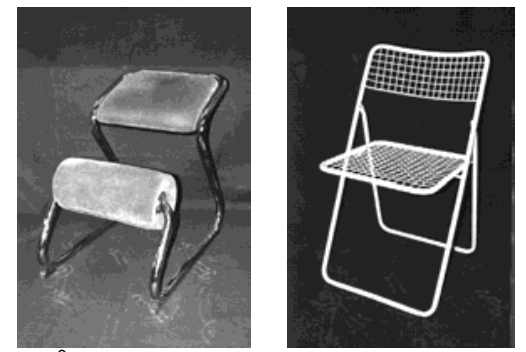

Net chair

(metal) (wood)

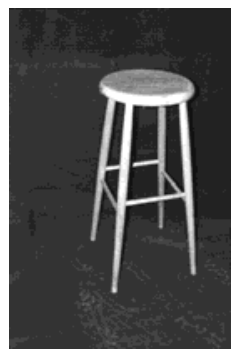

Stool

\section{Methods for seat measurements}

All the different seats were positioned in a climatic chamber. This chamber had a working range of +5 to $+40{ }^{\circ} \mathrm{C}, 10-90 \% \mathrm{RH}$ with an accuracy better than $\pm 0.5^{\circ} \mathrm{C}$ and $\pm 1 \% \mathrm{RH}$. The air speed during the experiments was less than $0.15 \mathrm{~m} / \mathrm{s}$ and the temperature was $20.8 \pm 0.2{ }^{\circ} \mathrm{C}$. MANIKIN2 wore Swedish Army cotton summer clothing as described before, consisting of trousers, shirt, shorts, socks and shoes with a total insulation of 1.34 clo and in size 50.

Following that some of the seats were measured with TORE in another climatic chamber. This chamber had a working range of +10 to $+40{ }^{\circ} \mathrm{C}, 20-80 \% \mathrm{RH}$ with an accuracy better than $\pm 1.0^{\circ} \mathrm{C}$ and $\pm 2 \% \mathrm{RH}$. The air speed during the experiments was less than $0.15 \mathrm{~m} / \mathrm{s}$ and the temperature was $13.1 \pm 0.6^{\circ} \mathrm{C}$. TORE wore similar Swedish Army cotton summer clothing as MANIKIN2 but in size 52 .

Both manikins were positioned in one seat at the time, placed in the respective climatic chamber. The heat flow from the different segments of the manikin surface was measured and controlled by the computerised system (described in Appendix A). Data for the actual conditions were recorded when the manikin had reached heat equilibrium with the environment.

\section{Total seat insulation results}

Results are given as total insulation values $\left(I_{T}\right)$, which are with the surrounding air layer included. In other words, values presented are directly measured with the manikin and require no measurements of external air layer insulation and clothing area factor. The 
results show no difference between the two manikins in the "No seat" and "BeGe fan 3" conditions, regardless of the different make and size of the manikins. This conforms well to earlier findings of small differences between manikins of different make and size (Wyon 1986) .

Table 19. Results from seat insulation study.

\begin{tabular}{|c|c|c|c|c|c|}
\hline Type of seat & Manikin & Position & $\begin{array}{c}\text { Total } \\
\text { insulation } \\
\text { (clo) }\end{array}$ & $\begin{array}{c}\text { Diff } \\
\text { from } \\
\text { No seat } \\
\text { (clo) }\end{array}$ & $\begin{array}{c}\text { Diff } \\
\text { from } \\
\text { No seat } \\
(\%)\end{array}$ \\
\hline No seat (net cage) & MANIKIN2 & Sitting & 1.34 & 0.00 & $0 \%$ \\
\hline BeGe ventilated car seat (fan 0 ) & MANIKIN2 & Sitting & 1.47 & 0.13 & $9 \%$ \\
\hline BeGe ventilated car seat (fan 1 ) & MANIKIN2 & Sitting & 1.38 & 0.04 & $3 \%$ \\
\hline BeGe ventilated car seat (fan 3 ) & MANIKIN2 & Sitting & 1.32 & -0.02 & $-2 \%$ \\
\hline Car seat (Volvo) & MANIKIN2 & Sitting & 1.61 & 0.27 & $17 \%$ \\
\hline Office chair w/o. armr. (KEVI) & MANIKIN2 & Sitting & 1.40 & 0.06 & $4 \%$ \\
\hline Office chair w. armr. (Nomi3) & MANIKIN2 & Sitting & 1.49 & 0.15 & $10 \%$ \\
\hline Office chair w. armr. (Edsbyn) & MANIKIN2 & Sitting & 1.45 & 0.11 & $8 \%$ \\
\hline Office chair w. wood armr. (Kinnarp) & MANIKIN2 & Sitting & 1.51 & 0.17 & $11 \%$ \\
\hline Office w arm- and headrest (Nyström) & MANIKIN2 & Sitting & 1.53 & 0.19 & $12 \%$ \\
\hline 3-seat sofa occupants on both sides & MANIKIN2 & Sitting & 1.69 & 0.35 & $21 \%$ \\
\hline 3-seat sofa manikin alone & MANIKIN2 & Sitting & 1.57 & 0.23 & $15 \%$ \\
\hline HÅG Balance (No back) & MANIKIN2 & Sitting & 1.46 & 0.12 & $8 \%$ \\
\hline Net chair (metal) & MANIKIN2 & Sitting & 1.33 & -0.01 & $-1 \%$ \\
\hline Stool (wood) & MANIKIN2 & Sitting & 1.34 & 0.00 & $0 \%$ \\
\hline No seat (hanging) & TORE & Sitting & 1.34 & 0.00 & $0 \%$ \\
\hline BeGe ventilated car seat (fan 3 ) & TORE & Sitting & 1.32 & -0.02 & $-2 \%$ \\
\hline No seat walking $0.80 \mathrm{~m} / \mathrm{s}$ & TORE & Walking & 0.98 & -0.36 & $-37 \%$ \\
\hline
\end{tabular}

Two car seats where tested, a standard seat (Volvo) increased the insulation with 0.27 clo $(1.61 \mathrm{clo})$. The ventilated seat $(\mathrm{BeGe})$ gave only half the increase, $0.13 \mathrm{clo}$, in "No fan" position. Ventilation of the seat gave the same insulation as the "No seat" condition, at maximum fan speed. This shows how ventilation makes the seat heat loss transparent for the user. The fan is working like a transporter of heat trough the seat (Nilsson \& Holmér, 1993).

Office chairs showed an increased insulation of 0.06-0.19 clo depending on the height of the backs and the thickness of the seats. A net chair and a wooden stool gave the same or slightly less insulation, relatively to the "No seat" condition. This can be explained by the fact that they are acting as cooling flanges and dissipating the heat with their larger conductivity. A three-seat sofa had an insulation increase of 0.23 clo with the manikin alone in the middle. With occupants on both sides the additional insulation became 0.35 clo.

Table 20. ISO 7733 calculations.

\begin{tabular}{llcccc}
\hline Type of seat & Manikin & $\begin{array}{c}\text { Total } \\
\text { insulation }\end{array}$ & PMV & PPD & $\begin{array}{c}\text { Preferred } \\
\text { operative } \\
\text { temperature } \\
\left({ }^{\circ} \mathrm{C}\right)\end{array}$ \\
\hline No seat (net cage) & MANIKIN2 & 1.34 & 0.4 & 92 & 22.0 \\
3-seat sofa occupants on both sides & MANIKIN2 & 1.69 & 0.7 & 85 & 22.0 \\
3-seat sofa occupants on both sides & MANIKIN2 & 1.69 & 0.4 & 91 & 20.5 \\
\hline
\end{tabular}


This case of sofa sitting has been overlooked in many cases using the ISO 7730 standard. Some calculations were made in order to predict a possible error due to increased insulation. The original conditions where operative temperature of $22^{\circ} \mathrm{C} \mathrm{a}$ relative humidity of $50 \%$ with an air velocity of $0.1 \mathrm{~m} / \mathrm{s}$ and a metabolism of $1.2 \mathrm{Met}$. This gave for the "No seat" condition a PMV of 0.4. In order to get the same PMV in the occupied 3-seat sofa the operative temperature would have to be $1.5^{\circ} \mathrm{C}$ lower (see also table 21).

Table 21. Local clothing insulation $\left(R_{c l, s}\right)$ results from seat study. To be used in modelling and calculations. Only zones that show increased insulation are presented.

\begin{tabular}{lcccccccccc}
\hline Rcl,s (W/m $\mathbf{2})$ & $\begin{array}{c}\text { Up. } \\
\text { back }\end{array}$ & $\begin{array}{c}\text { L U } \\
\text { arm }\end{array}$ & $\begin{array}{c}\text { R U } \\
\text { arm }\end{array}$ & $\begin{array}{c}\text { L } \\
\text { thigh }\end{array}$ & $\begin{array}{c}\text { R } \\
\text { thigh }\end{array}$ & $\begin{array}{c}\text { L } \\
\text { calf }\end{array}$ & $\begin{array}{c}\text { R } \\
\text { calf }\end{array}$ & $\begin{array}{c}\text { Lo. } \\
\text { Back }\end{array}$ & Seat & Air \\
Type of seat & $\mathbf{4}$ & $\mathbf{5}$ & $\mathbf{6}$ & $\mathbf{1 1}$ & $\mathbf{1 2}$ & $\mathbf{1 3}$ & $\mathbf{1 4}$ & $\mathbf{1 7}$ & $\mathbf{1 8}$ & $\mathbf{1 9}$ \\
\hline No seat (net cage) & 0.077 & 0.142 & 0.127 & 0.088 & 0.092 & 0.089 & 0.089 & 0.185 & 0.107 & 20.8 \\
BeGe ventilated car seat (fan 0) & 0.077 & 0.142 & 0.127 & 0.088 & 0.092 & 0.089 & 0.089 & $\mathbf{0 . 5 7 9}$ & $\mathbf{0 . 7 6 1}$ & 20.7 \\
BeGe ventilated car seat (fan 1) & 0.077 & 0.142 & 0.127 & 0.088 & 0.092 & 0.089 & 0.089 & $\mathbf{0 . 3 3 1}$ & 0.143 & 20.6 \\
BeGe ventilated car seat (fan 3) & 0.077 & 0.142 & 0.127 & 0.088 & 0.092 & 0.089 & 0.089 & $\mathbf{0 . 2 4 0}$ & 0.051 & 20.7 \\
Car seat (Volvo) & $\mathbf{0 . 3 1 3}$ & 0.142 & 0.127 & $\mathbf{0 . 1 8 5}$ & $\mathbf{0 . 2 0 7}$ & 0.089 & 0.089 & $\mathbf{0 . 5 8 2}$ & $\mathbf{0 . 6 5 8}$ & 20.8 \\
Office chair w/o. armr. (KEVI) & 0.077 & 0.142 & 0.127 & 0.088 & 0.092 & 0.089 & 0.089 & $\mathbf{0 . 3 6 6}$ & $\mathbf{0 . 5 9 5}$ & 20.8 \\
Office chair w. armr. (Nomi3) & $\mathbf{0 . 1 5 4}$ & 0.142 & 0.127 & $\mathbf{0 . 1 5 2}$ & $\mathbf{0 . 1 6 0}$ & 0.089 & 0.089 & $\mathbf{0 . 5 2 4}$ & $\mathbf{0 . 9 4 3}$ & 20.8 \\
Office chair w. armr. (Edsbyn) & 0.077 & 0.142 & 0.127 & 0.088 & 0.092 & 0.089 & 0.089 & $\mathbf{0 . 4 3 8}$ & $\mathbf{0 . 5 7 1}$ & 20.7 \\
Office chair w wood armr. (Kinnarp) & 0.077 & 0.142 & 0.127 & $\mathbf{0 . 1 8 1}$ & $\mathbf{0 . 1 8 7}$ & 0.089 & 0.089 & $\mathbf{0 . 5 0 0}$ & $\mathbf{0 . 8 2 9}$ & 21.0 \\
Office w arm- and headrest (Nyström) & $\mathbf{0 . 1 7 2}$ & 0.142 & 0.127 & $\mathbf{0 . 1 5 9}$ & $\mathbf{0 . 1 5 8}$ & 0.089 & 0.089 & $\mathbf{0 . 5 8 8}$ & $\mathbf{0 . 8 6 9}$ & 20.8 \\
3-seat sofa occupants on both sides & $\mathbf{0 . 3 9 5}$ & $\mathbf{0 . 1 9 6}$ & $\mathbf{0 . 1 9 8}$ & $\mathbf{0 . 1 9 0}$ & $\mathbf{0 . 1 6 8}$ & 0.089 & 0.089 & $\mathbf{1 . 5 0 8}$ & $\mathbf{1 . 0 5 7}$ & 21.1 \\
3-seat sofa manikin alone & $\mathbf{0 . 4 0 1}$ & 0.142 & 0.127 & $\mathbf{0 . 1 5 5}$ & $\mathbf{0 . 1 5 0}$ & 0.089 & 0.089 & $\mathbf{1 . 3 3 3}$ & $\mathbf{1 . 1 5 4}$ & 20.8 \\
HÅG Balance (No back) & 0.077 & 0.142 & 0.127 & 0.088 & 0.092 & $\mathbf{0 . 1 5 2}$ & $\mathbf{0 . 1 5 7}$ & 0.185 & $\mathbf{0 . 4 3 1}$ & 20.8 \\
Net chair (metal) & 0.077 & 0.142 & 0.127 & 0.088 & 0.092 & 0.089 & 0.089 & 0.185 & 0.107 & 21.2 \\
Stool (wood) & 0.077 & 0.142 & 0.127 & 0.088 & 0.092 & 0.089 & 0.089 & 0.185 & $\mathbf{0 . 1 7 1}$ & 21.0 \\
\hline
\end{tabular}

\section{Summary seat insulation}

The total insulation results show no difference between the manikins in the "No seat" and "BeGe fan 3" conditions. This in spite the different make and size of the manikins. This conforms well to earlier findings of small differences between manikins of different make and size.

Two car seats where tested, a standard seat increased the insulation with 0.27 clo. The ventilated seat gave only half the increase, $0.13 \mathrm{clo}$, in "No fan" position. Ventilation of the seat gave the same insulation as the "No seat" condition, at maximum fan speed. This shows how ventilation makes the seat transparent to heat. The fan is working like a transporter of heat trough the seat.

Office chairs showed an increased insulation of 0.06-0.19 clo depending on the height of the backs and the thickness of the seats. A net chair and a wooden stool gave the same or slightly less insulation, relatively to the "No seat" condition. This can be explained by the fact that they are acting as cooling flanges and dissipating the heat with their larger conductivity. A three-seat sofa had an insulation increase of 0.23 clo with the manikin alone in the middle. With occupants on both sides the additional insulation became 0.35 clo. 
The sofa sitting has been overlooked in many cases using the SS-EN ISO 7730 standard. Some calculations were made in order to predict a possible error due to increased insulation. The conditions where operative temperature of $22{ }^{\circ} \mathrm{C}$ a relative humidity of $50 \%$ with an air velocity of $0.1 \mathrm{~m} / \mathrm{s}$ and a metabolism of 1.2 Met. This gave for the "No seat" condition a PMV of 0.4. In order to get the same PMV in the occupied 3 -seat sofa the operative temperature would have to be $1.5^{\circ} \mathrm{C}$ lower. 


\section{Impact of ventilated seats on thermal comfort}

This chapter presents results from a study investigating the heat exchange between a person and a ventilated seat. Many car manufacturers today offers a ventilated seat option in order to keep the driver cool and dry. One question that often comes up is, how much does the ventilated seat help to increase and redistribute the human heat loss?

\section{Introduction to ventilated seat comfort research}

A great number of people work in an environmental shield like a cabin of any kind. Climate conditions in these cabins could often reach extreme levels compared to ordinary indoor climate (Nilsson \& Holmér, 1993, Fung et al., 1996, Bröde et al., 2003). One of the sources to these climate problems is the great cooling power needed for air conditioning, which causes draft.

Another problem is the seat since it is very well insulated and sometimes impermeable. This makes the seat climate usually too hot, as it has little influence from the ambience. One way to enhance the seat climate and decrease the required cooling power is to ventilate the seat. The points below identify several problems that have to be answered:

- Sunshine gives large radiation loads.

- In the process of compensating for the radiation, cool air with low temperatures and high velocities may cause draft problems that can cause medical problems. That is especially true for shaded areas.

- It is often difficult to get an optimal temperature distribution in the cabin.

- Existing air distribution systems often give high air speeds because of the high power consumption and air volume needed both for heating and cooling of a cabin.

- The seat becomes a barrier both for heat- and moisture transfer especially in summer climate, which leads to increased sweating and discomfort.

To remove the necessary energy large air volumes are demanded which creates many problems. All ways of lowering the power needed and thereby also the air volumes are favourable. One possibility is to use a ventilated seat and increase the heat loss from the part of the body that today is covered by the insulating seat.

The purpose of this study that was to compare results of experiments with subjects experiencing different seat climate with measurements made with a thermal manikin. In order to directly be able to compare measured values from the manikin with ratings from the subjects, a consolidation of closely positioned zones on the manikin was required. Since the climate was considered as symmetrical from left to right the 33 zones of MANIKIN2 was reduced to 16 according to the following table: 
Table 22. Zones for voting and regressions.

\begin{tabular}{ll}
\hline Body part/Zone & Body part/Zone \\
\hline Whole body & Right thigh, outside \\
Head & Left thigh, outside \\
Back, upper & Right thigh, seat side \\
Chest, stomach & Left thigh, seat side \\
Upper arms & Back, lower \\
Lower arms & Seat \\
Hands & Lower legs \\
Right thigh, inside & Feet \\
Left thigh, inside & \\
\hline
\end{tabular}

\section{Experimental set-up and climatic chambers}

Two ventilated seats were positioned in the same climatic chamber as before. The air speed during the experiments was controlled to be less than $0.1 \mathrm{~m} / \mathrm{s}$. The height of the seats could be adjusted individually to fit the subjects. The seat fans were supplied with a constant voltage of $27.6 \mathrm{~V}$ from two Switchbox ${ }^{\circledR} 60-5$ stabilised power supply, which could keep the voltage constantly switching with an accuracy better than $\pm 0.05 \mathrm{~V}$.

A wall was placed between the two subjects and they where told to:

- not change seat positioning

- have their arms on the arm rests

- have their back against seat back

- have their feet on the markings

- sense after at the first signal

- give their ratings at the second signal

- not talk with each other

Chamber temperature and relative humidity were measured with a Protimeter ${ }^{\circledR}$ DP989M with an accuracy of $\pm 0.25^{\circ} \mathrm{C}$ and $\pm 1 \%$. Eight external thermistors were positioned on the side of the seat at the heights $0.1,0.6,1.1 \mathrm{~m}$ and on the seat, $100 \mathrm{~mm}$ from the right side and $150 \mathrm{~mm}$ from the back of the seat. These thermistors are calibrated to accuracy better than $\pm 0.1^{\circ} \mathrm{C}$ and are collected on file by a personal computer that also gave the signals at the rating periods. The length of the experiments was 40 minutes with ratings on minute $10,20,30$ and 40 with a signal to sense the climate 1 minute before giving there rating.

In order to get access to a large number of different sizes did the subjects and MANIKIN2 did wear the same type of clothing (Swedish army cotton clothing) which consisted of trousers, shirt, shorts, socks and shoes with a total insulation of 1.3 clo. The clothing was stored in a room outside the chambers, which during the experiments had a temperature of $20 \pm 1{ }^{\circ} \mathrm{C}$ and relative humidity $35 \pm 5 \% \mathrm{RH}$. 

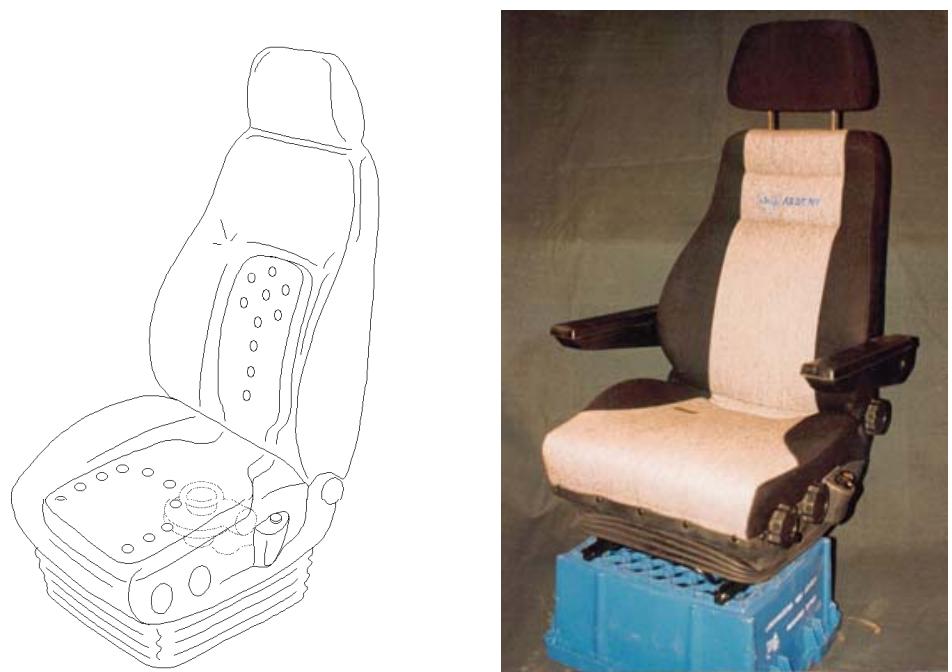

Figure 51. The ventilated seat showed see through view as well as a photo. The fan was positioned in the seat sucking air through channels in the seat and lower backrest.

\section{Measurement conditions}

The heat loss levels in the chamber were chosen to $32,40,48,56$ and $64 \mathrm{~W} / \mathrm{m}^{2}$. During the experiments the subjects were sitting at rest. This corresponds to a metabolism of approximately $60 \mathrm{~W} / \mathrm{m}^{2}$. The dry heat loss with this metabolism is approximately $48 \mathrm{~W} / \mathrm{m}^{2}$. These measurements were carried out in $50 \% \mathrm{RH}$. The heat loss level in the chamber for the wet conditions was chosen to $48 \mathrm{~W} / \mathrm{m}^{2}$ (see table 23 ).

Table 23. The investigated conditions.

\begin{tabular}{ccccc}
\hline $\begin{array}{c}\text { Chamber } \\
\text { emp. }\left({ }^{\circ} \mathbf{C}\right)\end{array}$ & $\begin{array}{c}\text { Relative } \\
\text { humidity } \\
(\mathbf{\%})\end{array}$ & $\begin{array}{c}\text { Fan } \\
\text { speed }\end{array}$ & $\begin{array}{c}\text { Seat exp. } \\
\text { code }\end{array}$ & $\begin{array}{c}\text { Heat loss } \\
\left(\mathbf{W} / \mathbf{m}^{2}\right)\end{array}$ \\
\hline 19.3 & 50 & 0 & 1640 & 65 \\
21.2 & 50 & 3 & 1643 & 64 \\
21.1 & 50 & 0 & 1560 & 56 \\
22.0 & 50 & 1 & 1561 & 56 \\
22.1 & 50 & 2 & 1562 & 56 \\
22.4 & 50 & 3 & 1563 & 56 \\
22.7 & 50 & 0 & 1480 & 47 \\
23.6 & 50 & 1 & 1483 & 49 \\
23.8 & 50 & 2 & 1482 & 48 \\
24.1 & 50 & 3 & 1483 & 48 \\
24.3 & 50 & 0 & 1400 & 40 \\
25.5 & 50 & $(4)$ & 1404 & 39 \\
25.6 & 35 & 1 & 140135 & 40 \\
25.5 & 70 & 1 & 140170 & 39 \\
25.7 & 50 & 2 & 1402 & 40 \\
25.9 & 50 & 3 & 1403 & 39 \\
26.2 & 50 & 0 & 1320 & 32 \\
27.7 & 50 & 3 & 1323 & 32 \\
\hline
\end{tabular}


On the basis of both manikin measurements and subject tests it was in found the condition adjustment phase that the difference between conditions were very small. Therefore the number of conditions was increased with a cooler, $64 \mathrm{~W} / \mathrm{m}^{2}$, and a warmer, $32 \mathrm{~W} / \mathrm{m}^{2}$, condition.

The difference in heat loss between the different fan speeds was also detected as small, see table 23. That resulted in that measurements with fan speed 2 were excluded. All these measurements were made at $50 \% \mathrm{RH}$, but two measurements were made at 35 respective $70 \% \mathrm{RH}$. Since the relative humidity only to a very little extent affects the dry heat loss, no difference in heat loss was measured with higher or lower humidity (see table 23).

The temperature levels, in order to receive desired heat loss at the different conditions, was adjusted with MANIKIN2 and documented for both seats. The subjects were exposed two by two to the different conditions. The order in which the conditions appeared was randomised so that the subjects did not know which temperature level or fan speed they were exposed to. They performed the experiments at the same time of the day, each day, in 14 consecutive working days. The first experiment was used as a "test", in order to let the subjects get accustomed to the experimental set-up and the procedures with the rating scales etc. That experiment was consequently run two times.

The subjects were 10 males with the age 20 to 50 year (see table 24) They were not allowed to smoke, snuff, drink coffee or eat within an hour before the experiment. The subjects voted for the 16 different body zones plus the total experience (Whole body). Each experiment lasted 60 minutes of which the efficient measurement time was 40 minutes with 10 minutes of preparation before and after the experiment. Four comfort ratings were carried out during each experiment, at minute 10, 20, 30 and 40 (see figure 52). The ratings were made in a protocol, where a new page was used for each rating in order to avoid direct transcript. The subjects had also an opportunity to annotate if anything special had happened at each rating.

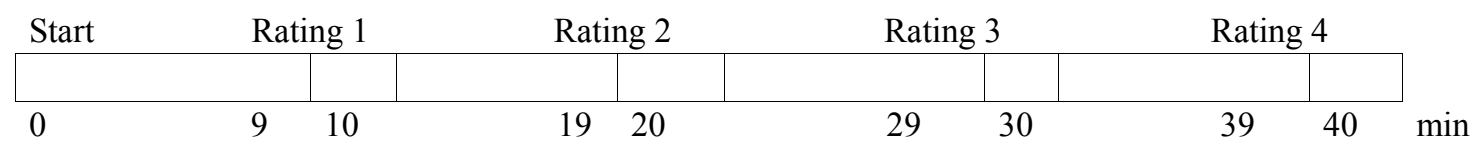

Figure 52. Experimental protocol

The length, weight plus some characteristic clothing measurements for the subjects were measured at the first experiment. The height of the seat was also measured for each subject so that the same height could be adjusted at each experiment. The chamber temperatures, humidity plus seat temperature was recorded each minute and could continuously be monitored throughout the experiment. The comfort ratings were made according to the 7-grade MTV scale (see chapter on comfort scales). 
Table 24. Subject data

\begin{tabular}{ccccc}
\hline Subject no & Height $(\mathbf{m})$ & Weight $(\mathbf{k g})$ & Age (year) & Seat height $\mathbf{( c m )}$ \\
\hline 1 & 1.75 & 82 & 41 & 45 \\
2 & 1.72 & 77.2 & 42 & 47.5 \\
3 & 1.72 & 62 & 46 & 43 \\
4 & 1.76 & 88.9 & 45 & 44 \\
5 & 1.94 & 96.5 & 23 & 51.5 \\
6 & 1.69 & 65.9 & 44 & 45.5 \\
7 & 1.86 & 82.7 & 47 & 47 \\
8 & 1.84 & 68.5 & 47 & 48 \\
9 & 1.79 & 74.2 & 33 & 45.5 \\
10 & 1.74 & 67.5 & 30 & 46.5 \\
\hline Mean & 1.78 & 76.5 & 40 & 46.4 \\
S.D. & 0.077 & 11.03 & 8.3 & 2.38 \\
Min & 1.69 & 62.0 & 23 & 43.0 \\
Max & 1.94 & 96.5 & 47 & 51.5 \\
\hline
\end{tabular}

\section{Conditions with simulated perspiration}

A total of 10 climate conditions have first been adjusted and then measured with MANIKIN2 (Total heat loss $48 \mathrm{~W} / \mathrm{m}^{2}$, fan speed 0, 1 and 3, 3 with manufacturing seat respective 3 with prototype seat plus 4 with wet cloth). The relative humidity was $50 \%$ for all wet conditions. The temperature levels to maintain different heat loss levels were measured with the manikin and documented for both seats with a maximum deviation of $\pm 1 \mathrm{~W} / \mathrm{m}^{2}$, which is marginal. The obtained chamber temperatures were then used also for the conditions so that comparisons should be possible. The cotton cloth was wetted to the same weight for all experiments. In conditions with simulated sweating, a cotton cloth $(9 \times 5 \mathrm{dm})$ was positioned seat zone at the lower ventilated part of the back.

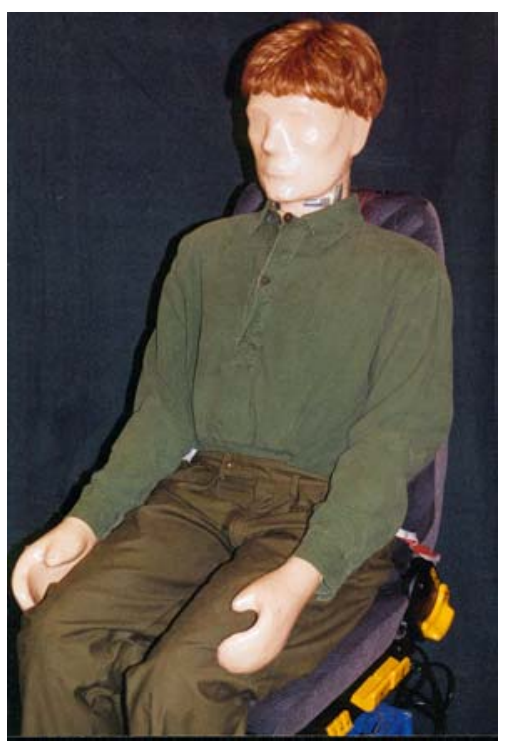

Figure 53. MANIKIN2 positioned in the ventilated seat.

The temperature levels to receive the required heat loss at the 6 the first conditions was measured with help of MANIKIN2 and documented for both seats with a highest discrepancy on $\pm 1 \mathrm{~W} / \mathrm{m}^{2}$ which is regarded as marginal. The chamber temperatures that 
had been received were then used in the experiments with wet cloth in order to do comparisons between the conditions. At the wet conditions the manikin was allowed to get a stable heat loss before it was positioned on the wetted cloth and the measurement began. The cloth was wetted to the same weight at all experiment. The weight before and after the measurement was registered.

\section{Results with the ventilated seat}

The results are shown as comfort zone profiles. The decrease in heat loss from zones in contact with the ambience is approximately $5 \mathrm{~W} / \mathrm{m}^{2}$ while zones with seat contact increase the heat loss with $10-20 \mathrm{~W} / \mathrm{m}^{2}$ with a total heat loss of $32 \mathrm{~W} / \mathrm{m}^{2}$. Correspondent values with a total heat loss of $64 \mathrm{~W} / \mathrm{m}^{2}$ become $10 \mathrm{~W} / \mathrm{m}^{2}$ and $30-50 \mathrm{~W} / \mathrm{m}^{2}$ respectively. The seat temperatures during subject experiments were also measured. For every condition without fan the seat temperature was $34.0 \pm 0.3{ }^{\circ} \mathrm{C}$ with a minimum value of 33.5 and a maximum value of $34.4{ }^{\circ} \mathrm{C}$. Corresponding values for maximum fan speed were $32.5 \pm 0.8^{\circ} \mathrm{C}$ with a minimum value of 31.4 and a maximum value of 33.6 ${ }^{\circ} \mathrm{C}$.

In order to study stability in the chamber temperatures and the humidity during the experiments an evaluation of the humidity, air temperature and air speed at ventilated seats was made. The collected values from the last 10 minutes of the experiment have been used. The deviation in humidity between experiments in the same condition was less than $\pm 0.9 \% \mathrm{RH}$ for all conditions. Accordingly, all air set point temperatures deviated less than $\pm 0.05^{\circ} \mathrm{C}$ (see the following table).

Table 25. Chamber air temperatures between the different conditions.

\begin{tabular}{lccccccccccccc}
\hline Conditions & $\mathbf{3 2 0}$ & $\mathbf{4 0 0}$ & $\mathbf{4 8 0}$ & $\mathbf{5 6 0}$ & $\mathbf{6 4 0}$ & $\mathbf{4 8 1}$ & $\mathbf{5 6 1}$ & $\mathbf{3 2 3}$ & $\mathbf{4 0 3}$ & $\mathbf{4 8 3}$ & $\mathbf{5 6 3}$ & $\mathbf{6 4 3}$ & $\mathbf{4 0 4}$ \\
\hline Chamber temp. $\left({ }^{\circ} \mathrm{C}\right)$ & 26.0 & 24.4 & 22.6 & 20.9 & 18.9 & 23.4 & 21.7 & 27.4 & 26.0 & 24.0 & 22.3 & 20.9 & 26.2 \\
S.D. & 0.02 & 0.02 & 0.02 & 0.03 & 0.03 & 0.04 & 0.05 & 0.03 & 0.03 & 0.03 & 0.02 & 0.03 & 0.04 \\
\hline
\end{tabular}

The gradient between 0.1 and $1.1 \mathrm{~m}$ was less than $0.3{ }^{\circ} \mathrm{C}$ at all conditions. The three minute mean value of the air speed, measured at the levels $0.1,0.6$ and $1.1 \mathrm{~m}$ over the floor, was $0.070 \pm 0.017 \mathrm{~m} / \mathrm{s}$ for both seats. As a reference to the subjective ratings and manikin measurements the temperature between test subject and seat have been studied. For all conditions without fan the contact temperature was $34.0 \pm 0.3{ }^{\circ} \mathrm{C}$ with a min value of 33.5 plus a max value of 34.4. While corresponding rates at max fan speed (mode 3 ) was $32.5 \pm 0.8^{\circ} \mathrm{C}$ with a min value of 31.4 plus a max value of $33.6^{\circ} \mathrm{C}$. The fan can consequently give a lowering of the seat contact temperature of in between 2.6 and $0.4{ }^{\circ} \mathrm{C}$ (see figure 54). 


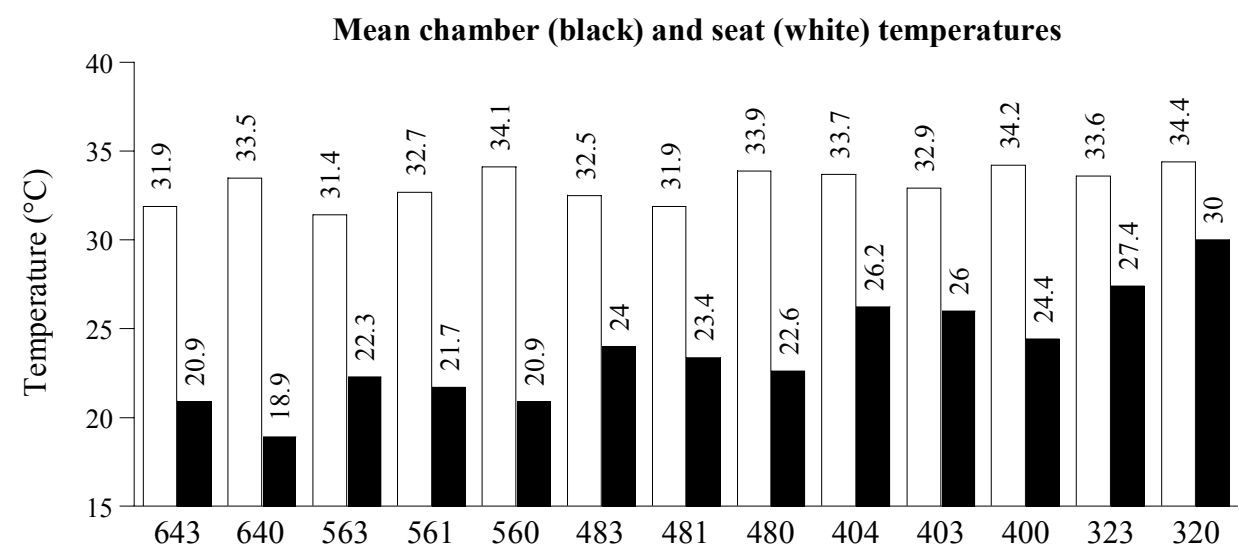

Figure 54. Chamber and seat temperatures at the different conditions.

As a control of the zone temperatures and $t_{e q}$-calibrations a comparison between the condition $48 \mathrm{~W} / \mathrm{m}^{2}$ was made, the fan speed 1 and the same configuration as 4 months before. Even after measurement wire changes and transports it was excellent correspondence between the two measurements.

\section{Control measurement}

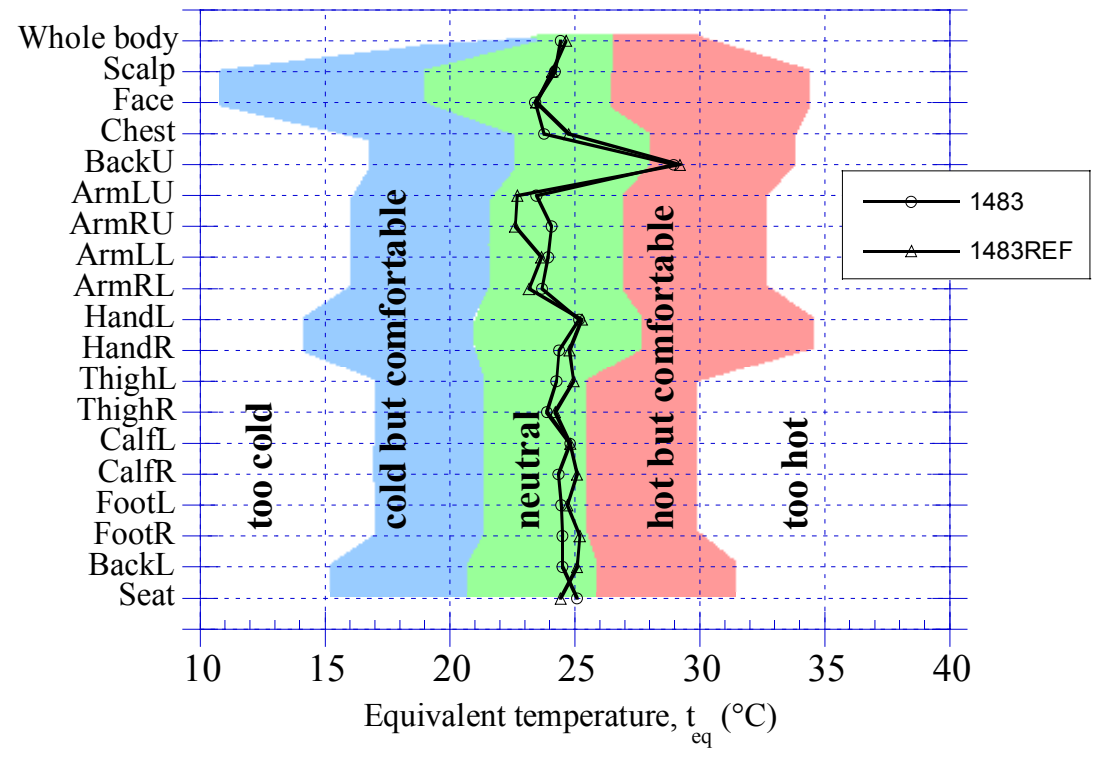

Figure 55. Control measurement with 4 months in between. Heat loss $49 \mathrm{~W} / \mathrm{m}^{2}$, triangle - fan speed 1 (prod. second) amb. temp $23.4^{\circ} \mathrm{C}$, Heat loss $48 \mathrm{~W} / \mathrm{m}^{2}$, ring - fan speed 1 (prod. first) amb. temp $23.6^{\circ} \mathrm{C}$, 
Fan speed 0, 1, 2 and 3

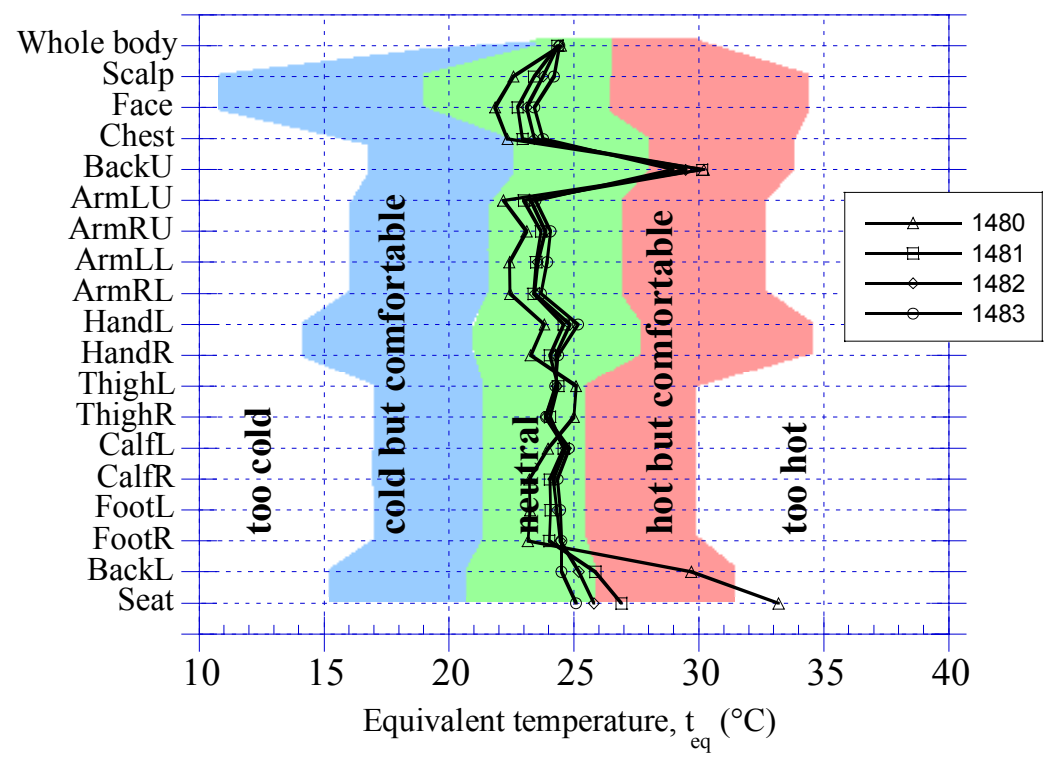

Figure 56. Heat loss $48 \mathrm{~W} / \mathrm{m}^{2}$, triangle - fan speed 0 , square - fan speed 1, rhomb - fan speed 2 , ring - fan speed 3,

\section{Different ambient relative humidity}

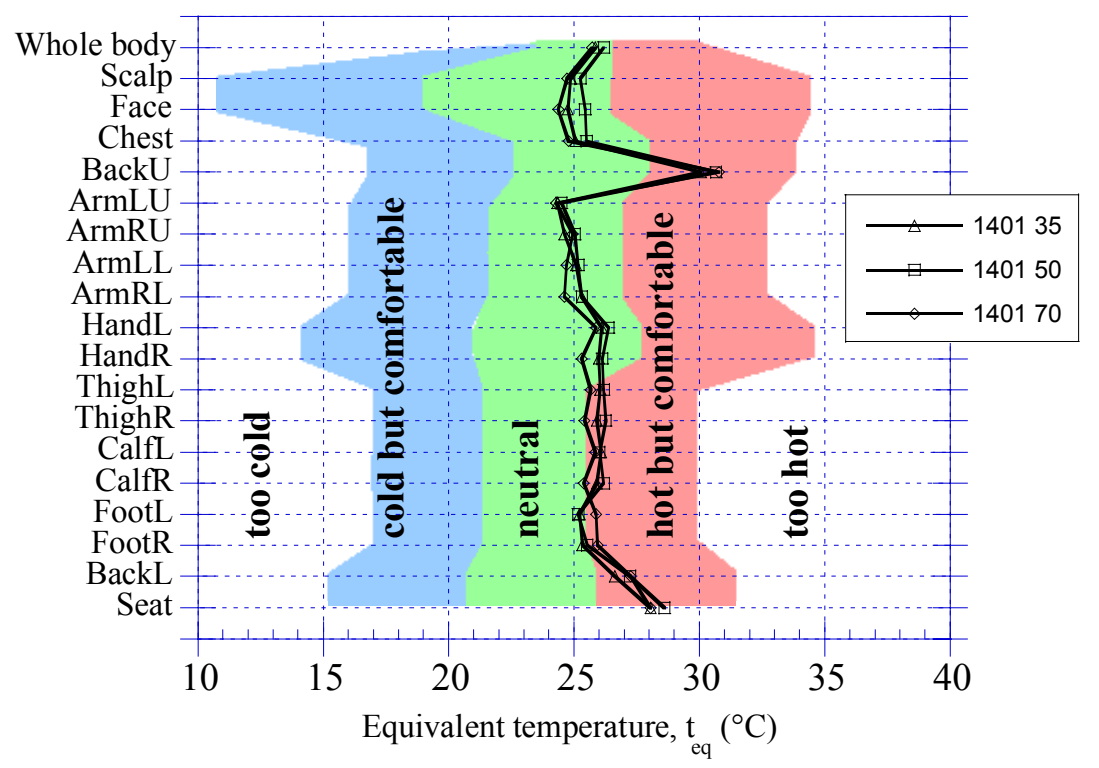

Figure 57. Heat loss $40 \mathrm{~W} / \mathrm{m}^{2}$, fan speed 1, triangle - 35\%RH, square - 50\%RH, rhomb $70 \%$ RH

The measurements were now made without the extra load of lead weights that increased the total weight to $68.3 \mathrm{~kg}$. In the early measurements it was discovered that the weights placed in the manikin stomach gave an unnatural weight distribution that influenced the fan, so that the flow decreased. The use of straps to give the manikin a more natural 
pressure downward in the seat was also tested. But this method showed not to give any change in fan flow or heat loss. The strapping was made with the straps positioned around the thighs and the breast of the manikin, and the straps were drawn just until they were tightened. Then the pneumatic air cylinder of the seat was used to increase the down pressure from 0 to 1.5 and finally to maximal $3 \mathrm{~cm}$. This was more than the weight of any of the subjects, since the air cylinder of the seat easily could lift every one of them.

In order to additionally examine this noticed decrease in flow, an anemometer was positioned in the fan exhaust and the seat was loaded with a person with a weight of 67 $\mathrm{kg}$, the legs not counted for (approx. $20 \mathrm{~kg}$ ). Measurements was made with the seat unloaded, the person plus the person and an additional weight of $20 \mathrm{~kg}$. Results are shown in table 26. The flow is calculated over an area of $40 \times 40 \mathrm{~mm}$. An increase in subject weight of $20 \mathrm{~kg}$ gives only minor decrease in the flow compared to the difference between the fan speeds.

\section{Different seat down pressure}

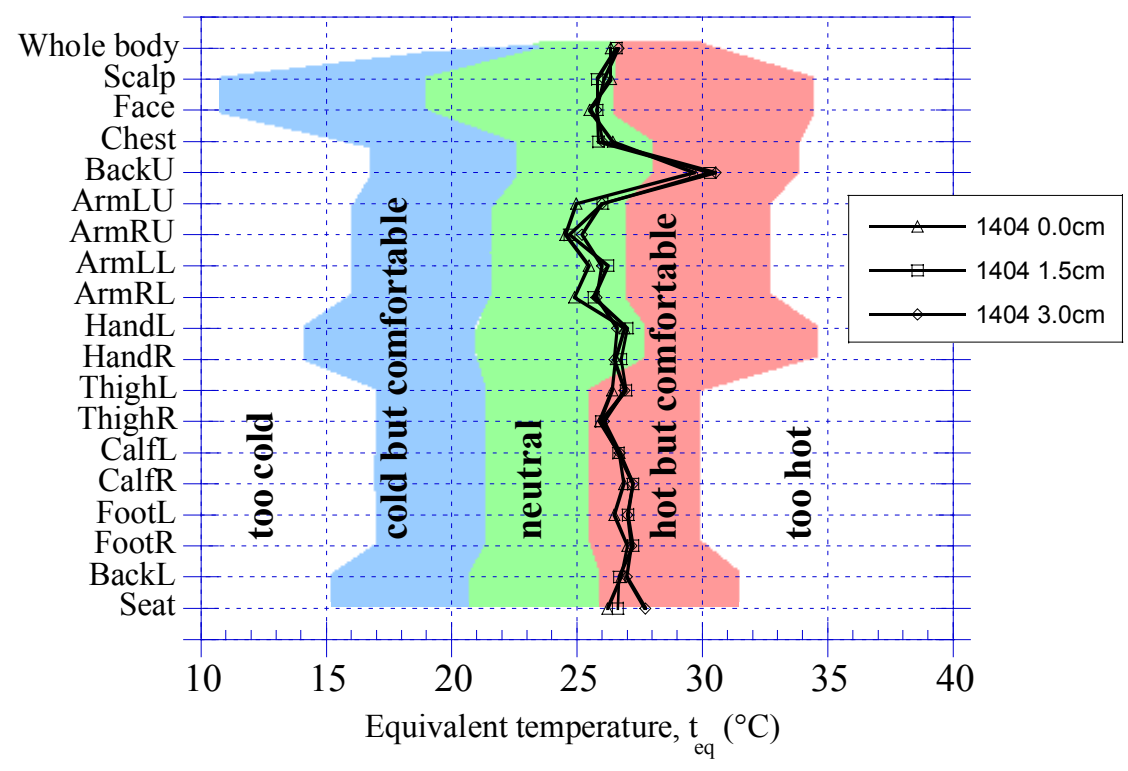

Figure 58. Heat loss $40 \mathrm{~W} / \mathrm{m}^{2}$, fan speed 4, Different down pressure; triangle $0 \mathrm{~cm}$, square $1.5 \mathrm{~cm}$, rhomb $-3 \mathrm{~cm}$

Table 26. Fan flow as a fiction of subject body weight.

\begin{tabular}{lccc}
$\begin{array}{l}\text { Subject weight (kg) } \\
\text { Fan speed }\end{array}$ & $\mathbf{0}$ & $\begin{array}{c}\mathbf{6 7} \\
\text { Air speed in } \\
\text { exhaust (m/s) }\end{array}$ & $\mathbf{8 7}$ \\
\hline 1 & 4.8 & 4.2 & 4.0 \\
2 & 7.0 & 6.3 & 6.1 \\
3 & 8.5 & 7.5 & 7.2 \\
\hline \multicolumn{4}{c}{$\begin{array}{c}\text { Flow in exhaust } \\
\left(\mathbf{m}^{\mathbf{3}} / \mathbf{h}\right)\end{array}$} \\
\hline 1 & 24.2 \\
2 & 27.6 & 36.3 & 23.0 \\
3 & 40.3 & 43.2 & 35.1 \\
\hline
\end{tabular}


Since the maximum fan speed sometimes was experienced as too low. One of experimental conditions changed to include also a "fourth" fan speed. Increased fan airflow was created with an increased voltage to the fan from 27.6 to $32.6 \mathrm{~V}$, which gave an equal step increase as between earlier fan speeds.

\section{Images on the both condition extremes.}

\section{Different seat down pressure}

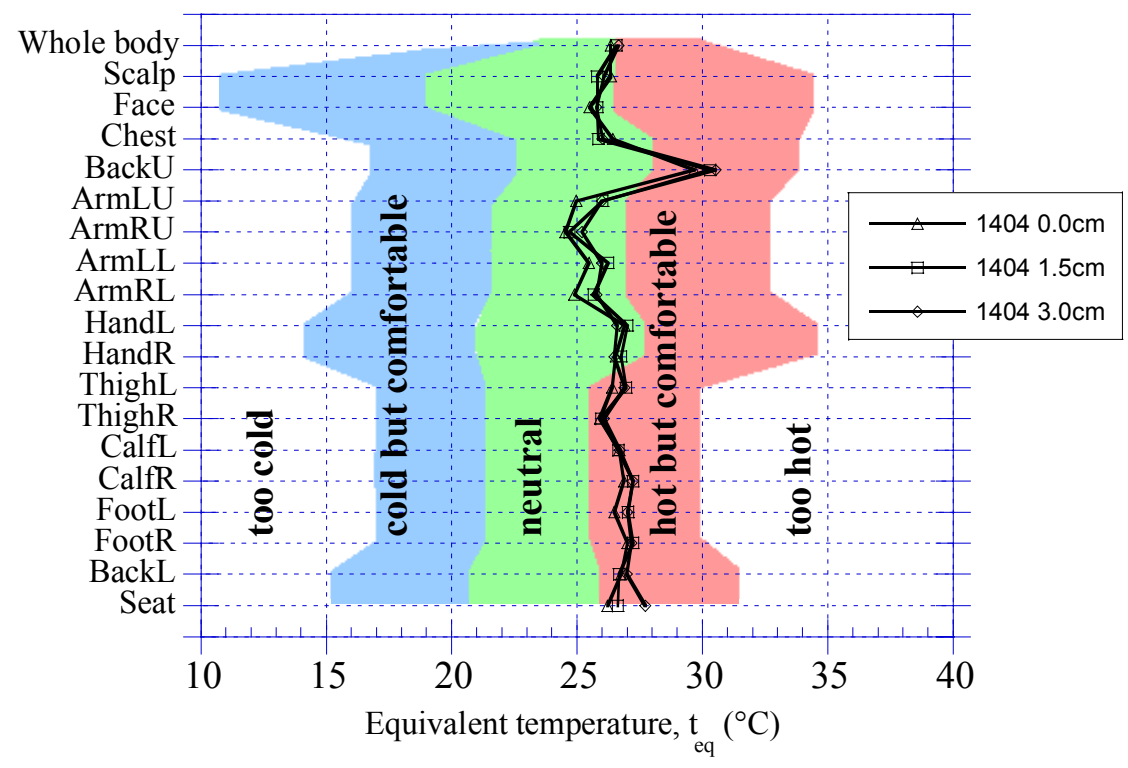

Figure 59. Heat loss $32 \mathrm{~W} / \mathrm{m}^{2}$, triangle - fan speed 0 , square - fan speed 3

\section{Condition extreme - high heat loss}

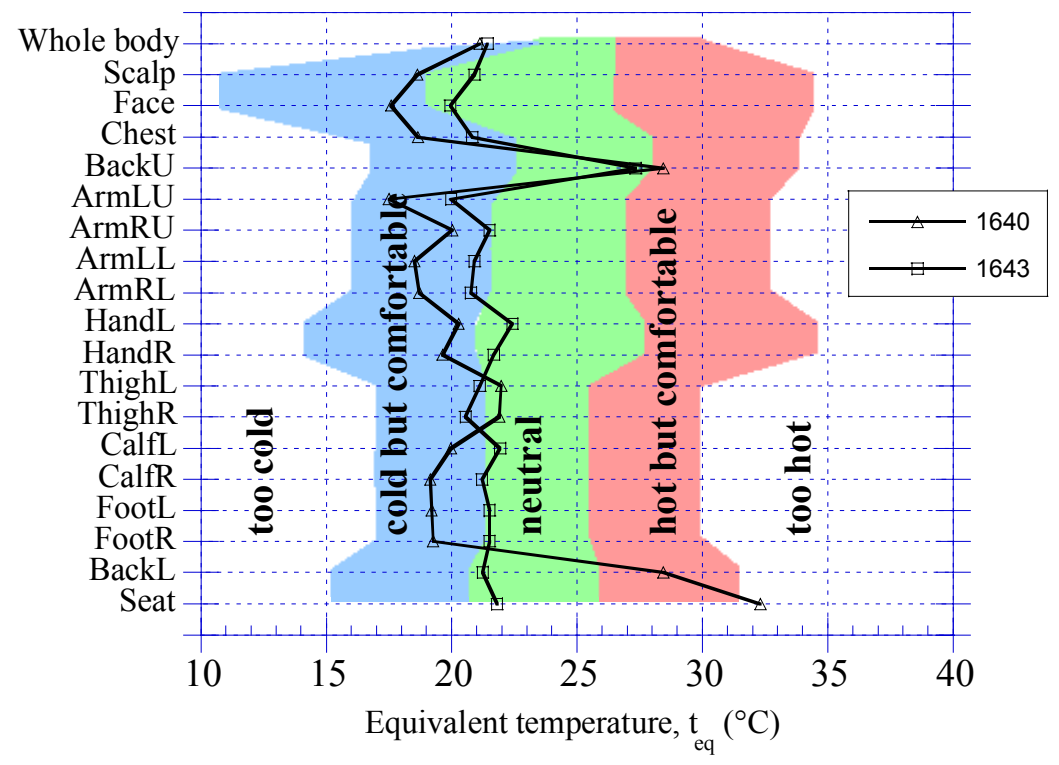

Figure 60. Heat loss $64 \mathrm{~W} / \mathrm{m}^{2}$, triangle - fan speed 0 , square - fan speed 3 


\section{The subjective comments}

The subjects had a possibility to, at each rating, make any comments. The following table displays these comments grouped and ranked in frequency of occurrence in the different conditions.

Table 27. Comments from the subjects

\begin{tabular}{|c|c|c|c|c|c|c|c|c|c|c|c|c|c|c|c|}
\hline & 400 & 480 & 560 & 481 & 561 & 640 & 563 & 403 & 403 & 483 & 320 & 404 & 323 & 643 & \\
\hline Comment & $A$ & $\boldsymbol{B}$ & $C$ & $D$ & $E$ & $\boldsymbol{F}$ & $G$ & $\boldsymbol{H}$ & $H F$ & $J$ & $K$ & $L$ & $M$ & $N$ & $\Sigma$ \\
\hline Sleepy, tired & 1 & 2 & & 1 & $\overline{1}$ & & $\overline{1}$ & $\overline{1}$ & $\overline{1}$ & 1 & 1 & 1 & 1 & 1 & 13 \\
\hline Stiff legs & 1 & 1 & 2 & 2 & 1 & & 2 & 1 & 1 & 1 & & & & & 12 \\
\hline Slightly humid air & 2 & 1 & & & 1 & & 1 & & 4 & & & & 2 & & 11 \\
\hline Cool air & & 1 & & 1 & & 4 & 1 & & & & & & & 3 & 10 \\
\hline Vibrations & & & & & & & & & & 2 & & 2 & 2 & 2 & 8 \\
\hline Warm air & & & & & & & & 4 & & & & & 3 & & 7 \\
\hline varm ai & 1 & & & & 3 & & & & & 3 & & & & & 7 \\
\hline ble, good & 1 & & & & & & & 2 & & & 2 & & 2 & & 7 \\
\hline Humid air & & & & & & & & 4 & & & 2 & & & & 6 \\
\hline ations, no sound & & 1 & 1 & & & & 2 & & & & & & & & 4 \\
\hline Annoying sound & & & & 1 & & & & 2 & & & & 1 & & & 4 \\
\hline Dry air & & 1 & & & 2 & & & & & & & & & & 3 \\
\hline Pain in the neck, back & 1 & & & & & & & & 1 & & & & & & 2 \\
\hline Slight perspiration & & & & & & & & & & & 1 & & & & 1 \\
\hline
\end{tabular}

Noticeable is that the subjects thought that the air was both cool and warm, plus that somebody even perspired a little in the warmest condition. Sleepiness and stiffness maybe do not occur if the seat is placed in a vehicle. Neither the vibrations, nor the sounds from the fan, that many commented on, will probably be heard or felt through the background noise and vibrations from a vehicle. A way to show the seat effect, apart from in comfort diagrams, is to study the heat loss from MANIKIN2 at some different conditions. The two extreme conditions have been selected in order to show a span between coolness and warmth. 
Table 28. Heat losses from the zones at highest and lowest total heat loss.

\begin{tabular}{lcccccc}
\hline Zone & $\begin{array}{c}\text { Heat loss } \\
\text { fan speed } 0 \\
\left(\mathrm{~W} / \mathrm{m}^{2}\right)\end{array}$ & $\begin{array}{c}\text { Heat loss } \\
\text { fan speed } \\
\left(\mathrm{W} / \mathrm{m}^{2}\right)\end{array}$ & $\begin{array}{c}\text { Diff. } \\
\text { Whole body }\end{array}$ & $\begin{array}{c}\text { Heat loss } \\
\text { fan speed } 0 \\
\left(\mathrm{~W} / \mathrm{m}^{2}\right)\end{array}$ & $\begin{array}{c}\text { Heat loss } \\
\text { fan speed } \\
\left(\mathrm{W} / \mathrm{m}^{2}\right)\end{array}$ & Diff. \\
\hline Head & 48 & 31 & -1 & 65 & 63 & -2 \\
Back, upper & 13 & 19 & -7 & 94 & 81 & -14 \\
Chest, stomach & 33 & 28 & -5 & 63 & 37 & 6 \\
Upper arms & 36 & 31 & -5 & 67 & 59 & -9 \\
Lower arms & 38 & 32 & -6 & 76 & 65 & -11 \\
Hands & 63 & 53 & -10 & 124 & 106 & -18 \\
Right thigh, inside & 36 & 37 & 1 & 72 & 74 & -2 \\
Left thigh, inside & 33 & 33 & 0 & 67 & 66 & -1 \\
Right thigh, outside & 40 & 36 & -4 & 80 & 73 & -8 \\
Left thigh, outside & 45 & 39 & -6 & 90 & 81 & -9 \\
Right thigh, lower side & 5 & 23 & 17 & 13 & 57 & 44 \\
Left thigh, lower side & 9 & 28 & 20 & 16 & 59 & 43 \\
Back, lower & 10 & 20 & 10 & 19 & 44 & 25 \\
Seat zone & 2 & 22 & 20 & 8 & 58 & 50 \\
Lower legs & 37 & 32 & -6 & 77 & 67 & -11 \\
Feet & 32 & 27 & -5 & 67 & 56 & -10 \\
Air temperature $\left({ }^{\circ} \boldsymbol{C}\right)$ & $\mathbf{2 6 . 2}$ & $\mathbf{2 7 . 7}$ & $\mathbf{1 . 5}$ & $\mathbf{1 9 . 3}$ & $\mathbf{2 1 . 2}$ & $\mathbf{1 . 9}$ \\
\hline
\end{tabular}

In table 28 can it be seen that the ventilated seat increases the possible ambient temperature with about 1.5 to $1.9^{\circ} \mathrm{C}$. This occurs through redistribution of heat losses from the zones in connection with ambient air to zones with seat contact. The heat loss decrease from zones with ambient air contact is in the region of $5 \mathrm{~W} / \mathrm{m}^{2}$. This compared to seat contact zones with an increase of $10-20 \mathrm{~W} / \mathrm{m}^{2}$, at a total heat loss of $32 \mathrm{~W} / \mathrm{m}^{2}$.

The same figures with a total heat loss of $64 \mathrm{~W} / \mathrm{m}^{2}$ become $10 \mathrm{~W} / \mathrm{m}^{2}$ respective $30-50$ $\mathrm{W} / \mathrm{m}^{2}$. The differences come from that the cooling ambient air jet that is sucked into the seat has a lower temperature in the last case. Accordingly the effect of the increase in dry heat loss will decrease at increased ambient temperature while factors as perspiration and consequently the evaporative heat loss will increase.

\section{New seat zone comfort diagrams}

The results has been compiled into comfort zone diagrams, and then adjusted for comfort zone limits for zones in connection with the seat. The measured values from the manikin shall be in side the comfort zone in the diagram for the climate to be considered as acceptable. 
Table 29. New suggested zone limits for the seat zones.

\begin{tabular}{lccccc}
\hline \multicolumn{1}{c}{ Zone } & too cold & $\begin{array}{c}\text { cold but } \\
\text { comfortable }\end{array}$ & neutral & $\begin{array}{c}\text { warm but } \\
\text { comfortable }\end{array}$ & too warm \\
\hline $\begin{array}{l}\text { Back, } \\
\text { upper }\end{array}$ & 16.8 & 22.4 & 25.3 & 28.1 & 33.7 \\
$\begin{array}{l}\text { Back, } \\
\text { lower }\end{array}$ & 15.3 & 20.6 & 23.3 & 25.9 & 31.2 \\
Seat & 15.3 & 20.6 & 23.3 & 25.9 & 31.2 \\
\hline
\end{tabular}

\section{The correlation between MTV and $t_{e q}$}

Within and in near proximity to the inner comfort zone, a linearly relationship between temperature experience (MTV) and the physical climate conditions can be expressed in $t_{e q}$. Consequently a linear regression analyses was conducted according to least square method with MTV that function of $t_{e q}$ (see figures below). The regression was done for all rated zones as well as for whole body. As a measure of quality of the regressions the regression constants $(\mathrm{a}, \mathrm{b})$ standard deviation $(\mathrm{SDa}, \mathrm{SDb})$ and residual square sum $\left(\mathrm{Q}_{0}\right)$ and correlation coefficients $\left(\mathrm{r}^{2}\right)$ are used. The results are shown in the following table.

Table 30. Results from regression analyses.

\begin{tabular}{lllllll}
\hline With and with out fan & $\mathbf{a}$ & $\mathbf{b}$ & SDa & SDb & $\mathbf{Q}_{\mathbf{0}}$ & $\mathbf{r}^{2}$ \\
\hline Whole body & -6.60 & 0.28 & 0.67 & 0.03 & 0.49 & 0.90 \\
Head & -3.55 & 0.17 & 0.51 & 0.02 & 0.47 & 0.84 \\
Back, upper & -10.78 & 0.37 & 2.91 & 0.10 & 1.49 & 0.57 \\
Chest, stomach & -5.05 & 0.22 & 0.70 & 0.03 & 0.81 & 0.83 \\
Upper arms & -4.80 & 0.21 & 0.66 & 0.03 & 0.48 & 0.84 \\
Lower arms & -4.26 & 0.19 & 0.49 & 0.02 & 0.39 & 0.88 \\
Hands & -3.97 & 0.17 & 0.53 & 0.02 & 0.43 & 0.85 \\
Right thigh, inside & -4.63 & 0.21 & 0.48 & 0.02 & 0.41 & 0.90 \\
Left thigh, inside & -4.37 & 0.20 & 0.43 & 0.02 & 0.34 & 0.91 \\
Right thigh, outside & -5.17 & 0.22 & 0.48 & 0.02 & 0.36 & 0.91 \\
Left thigh, outside & -5.93 & 0.24 & 0.66 & 0.03 & 0.51 & 0.88 \\
Right thigh, lower side & -1.71 & 0.08 & 1.18 & 0.04 & 1.60 & 0.25 \\
Left thigh, lower side & -1.28 & 0.06 & 1.16 & 0.04 & 1.89 & 0.18 \\
Back, lower & -1.99 & 0.09 & 1.12 & 0.04 & 1.85 & 0.32 \\
Seat zone & -0.71 & 0.05 & 0.81 & 0.03 & 1.90 & 0.20 \\
Lower legs & -5.41 & 0.22 & 0.59 & 0.02 & 0.50 & 0.89 \\
Feet & -3.95 & 0.19 & 0.53 & 0.02 & 0.46 & 0.87 \\
\hline
\end{tabular}




\section{Three examples of regression lines}

\section{Whole body}

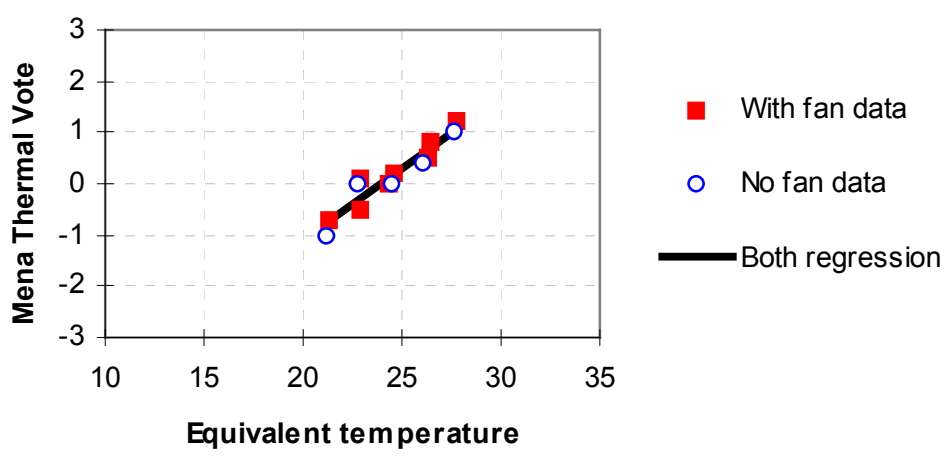

Figure 61. Solid line - with and without fan, $\mathrm{r}^{2}=0.90$. Long dashed line - with fan, $\mathrm{r}^{2}=0.91$. Short dashed line - without fan, $r^{2}=0.91$

\section{Chest, stomach}

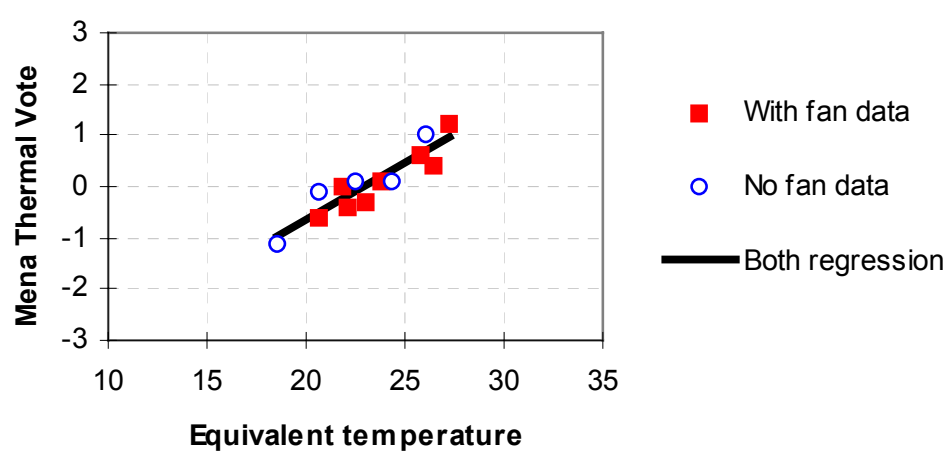

Figure 62. Solid line - with and without fan, $\mathrm{r}^{2}=0.83$. Long dashed line - with fan, $\mathrm{r}^{2}=0.85$. Short dashed line - without fan, $r^{2}=0.87$

\section{Back, lower}

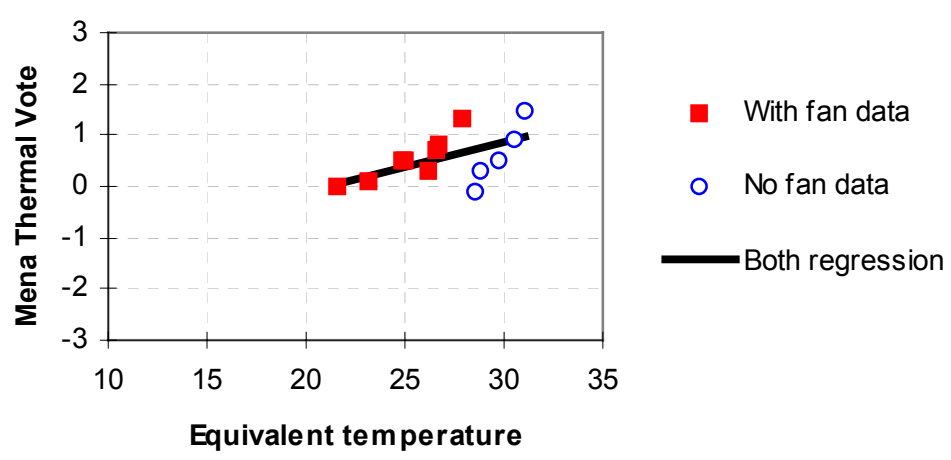

Figure 63. Solid line - with and without fan, $\mathrm{r}^{2}=0.32$. Long dashed line - with fan, $\mathrm{r}^{2}=0.78$. Short dashed line - without fan, $r^{2}=0.93$ 


\section{Analyses of the relationships}

Earlier seat temperature measurements on the subjects, shows a relatively high contact temperature, $34.0 \pm 0.3{ }^{\circ} \mathrm{C}$. The manikin as well, shows high $t_{e q}, 31$ to $33{ }^{\circ} \mathrm{C}$. The subjects have, on the other hand, not rated as high MTV as could be assumed. Rather they lowered their rating from approximately 2 to 0 when ambient air temperature was lowered from 26.0 to $18.9^{\circ} \mathrm{C}$. This can be explained by some type of transferring effect, from the experience of the ambience to the well insulated surfaces.

\section{Simulated sweating}

To measure evaporative heat loss may create some problems because MANIKIN2 is dimensioned to keep a very constant heat loss inside the comfort zone. The maximal power for the different zones, especially the seat zone, is therefore relatively low. This means that the zone in contact with the wet cloth quickly goes to maximum power output. This has been the case for all wet conditions except without fan where the water was heated up to $34^{\circ} \mathrm{C}$. Table 31 shows chamber temperatures, the weight change of the cloth and the heat loss levels for the respective conditions.

Table 31. Results from wet cloth experimental conditions

\begin{tabular}{lccccccc}
\hline $\begin{array}{l}\text { Chamber } \\
\text { temp. }\left({ }^{\circ} \mathbf{C}\right)\end{array}$ & $\begin{array}{c}\text { Fan } \\
\text { speed }\end{array}$ & $\begin{array}{c}\text { Code } \\
\text { prod. }\end{array}$ & $\begin{array}{c}\text { Weight of } \\
\text { cloth }(\mathbf{g}) \\
\text { before } / \mathbf{a f t e r}\end{array}$ & $\begin{array}{c}\text { Heat loss } \\
\left(\mathbf{W} / \mathbf{m}^{2}\right) \text { prod. } \\
\text { seat }\end{array}$ & $\begin{array}{c}\text { Code } \\
\text { proto. }\end{array}$ & $\begin{array}{c}\text { Weight of } \\
\text { cloth }(\mathbf{g}) \\
\text { before/after }\end{array}$ & $\begin{array}{c}\text { Heat loss } \\
\left(\mathbf{W} / \mathbf{m}^{2}\right) \\
\text { prod. seat }\end{array}$ \\
\hline $22.8 / 22.2$ & 0 & $1480 \mathrm{X}$ & - & 48 & $2480 \mathrm{X}$ & - & 49 \\
$23.4 / 23.4$ & 1 & $1481 \mathrm{X}$ & - & 49 & $2481 \mathrm{X}$ & - & 47 \\
$24.1 / 24.0$ & 3 & $1483 \mathrm{X}$ & - & 48 & $2483 \mathrm{X}$ & - & 47 \\
$-/ 22.2$ & 0 & 3480 & - & - & 4480 & $337 /-$ & 52 \\
$23.4 / 23.4$ & 1 & 3481 & $338 / 198$ & 62 & 4481 & $339 / 241$ & 59 \\
$-/ 24.0$ & 3 & 3483 & - & - & 4483 & $-/ 207$ & 56 \\
\hline
\end{tabular}

A continuous acquisition was made, to study how the manikin regulation system reacts on a change from no fan to fan speed 3. When the manikin was stabilised, the fan was switched on and the zone went to maximum power almost immediately. Even maximum power was not sufficient to raise the temperature to the set point, $34{ }^{\circ} \mathrm{C}$. As a consequence of this, it was decided, that measurements only should be done at the lowest fan speed. 
Manikin heat loss wet conditions with/without fan

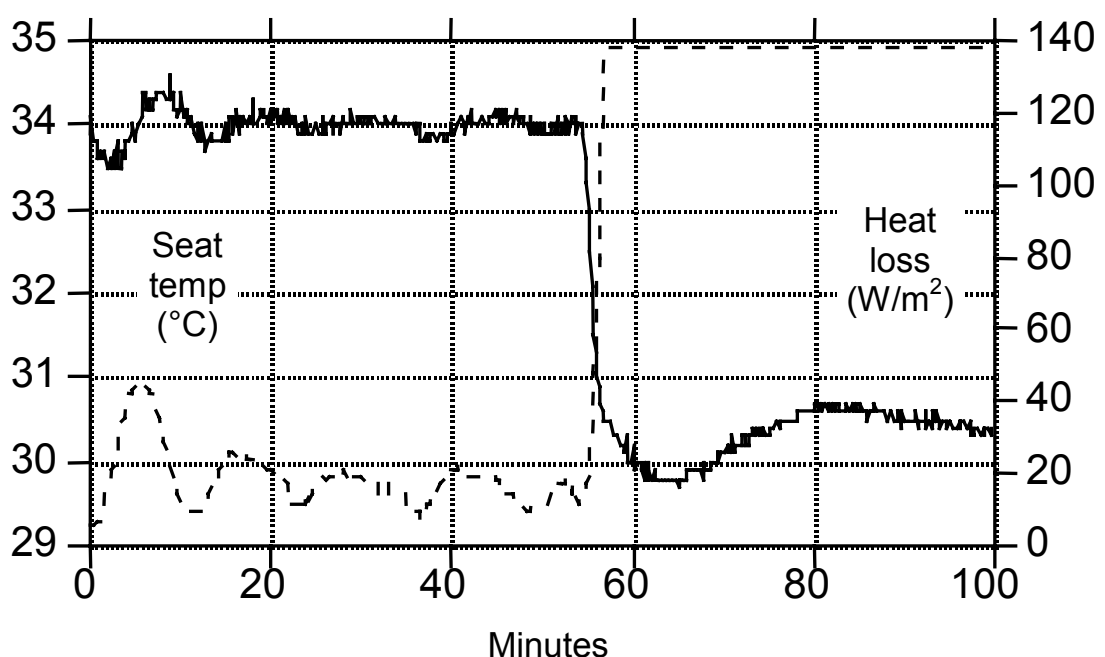

Figure 64. Temperature (dashed) and heat loss during the wet measurement with a change from fan speed 0 to 3 .

The results from the measurements with and without wet cloth must be treated with certain caution, since seat zones also at fan speed 1 was running at max heat loss. An equivalent temperature as low as $12{ }^{\circ} \mathrm{C}$ was measured for the seat in these humid conditions. The theoretically lowest temperature that can be achieved at $50 \%$ relative humidity and a ambient air temperature interval of $22-24{ }^{\circ} \mathrm{C}$ is approx. $15-17^{\circ} \mathrm{C}$ which equals the wet temperature of a psychrometer measurement. In these cases however the manikin surface temperatures, and thereby also contact temperatures, have been $30-33^{\circ} \mathrm{C}$.

The author also tried to sit in the seats during the same conditions. Already after a few minutes the seat zones felt unbearably cold. This can be used as a motivation for the low fan speeds, which inside the comfort zone was experienced as too low. In order to eliminate transferring effects and make larger temperature asymmetries between seat zones and other zones, a new study of seat zones in different climates is suggested. Since ventilated seats have the largest effect outside the comfort zone when perspiration has started it was of interest to try to show these effects on the heat loss.

The results point to the conclusion that the fan speeds that outside the comfort area seemed too low, in the sweating condition are more than sufficient. In situations where the ambient temperature is high enough for sweating to occur on body surfaces in contact with the ambience, and thereby cross the hot zone. At the same time as body surfaces in contact with the seat with the fan on could cut the cold zone.

\section{The relationship between MTV and $t_{e q}$}

In and in immediate surroundings of the comfort zone a linear relationship is expected between the perceived temperature feeling (MTV) and the physical climatic conditions expressed in $t_{e q}$. The relationship between subjective ratings and $t_{e q}$, for zones in contact with the ambient air, are very strong as in earlier experiments. The seat contact zones on the other hand are parted into two groups with strong relationships, one group for with the fan on regardless of fan speed and one for conditions with out fan. 
The seat temperature measurements are recorded as relatively high, $34.0 \pm 0 .{ }^{\circ} \mathrm{C}$, the manikin did also record high $t_{e q}$ values, 31 till $33^{\circ} \mathrm{C}$. The test panel on the other hand did not rate high MTV-values rather lowered the rating from 3 to 0 when the ambient temperature decreased from 26.0 to $18.9^{\circ} \mathrm{C}$. This phenomenon could perhaps be explained as some kind of transferring effect from the ambience to the seat surfaces.

\section{Summary seat comfort}

The investigation of ventilated seat comfort started with a correlation series where a relationship between 14 conditions set and measured with MANIKIN2 and then rated by a panel of 10 subjects.

One way of showing the effect of increased seat ventilation, is by reporting the temperature decrease on the seat fabric, of between 0.4 and $2.6^{\circ} \mathrm{C}$. Another way of showing the effects is to study the heat losses from different zones during the different conditions. In between the two extreme conditions the possible increase in ambient temperature with preserved heat loss is 1.5 to $1.9^{\circ} \mathrm{C}$. The heat loss is then redistributed from zones in contact with the ambient air to zones with seat contact.

To investigate the influence of sweating on the ventilation effect, tests with and without fan and wetted cotton cloth were conducted. The results from these measurements have to be handled with some care as the seat zones even at low fan speed reached maximum heat loss. Equivalent temperature values as low as $12^{\circ} \mathrm{C}$ were registered for the seat. Although the simulated sweating was quite large these tests show the validity for the low fan speeds which inside the comfort zone were regarded as too low. Further investigations about responses for zones in contact with the seat vs. zones in contact with the ambient air are needed.

Some kind of transfer effect, between zones in contact with the seat respective in contact with ambient air, can be the reason for correlation differences. Since the ambient air temperature affects the cooling capacity of the seat ventilation, the influence on the larger portion of body surface is turned towards the ambience. Also having in mind the human habit to accept elevated seat zone temperatures, points towards the conclusion that the heat loss changes from the seat zones have been suppressed. Since several subjects were complaining of difficulties when rating with the relatively coarse MTVscale maybe "half steps" should be introduced. 


\section{Evaluation with full-scale experimental series}

The objective of this work is to use and develop computer-based methods to visualise, analyse, and evaluate in order to improve the climate and ventilation conditions.

Computer simulations should complement difficult, time-consuming and expensive experimental investigations in order to evaluate thermal comfort. The method may be used to find useful system solutions that provide improved ventilation and indoor climate conditions in case-specific environments, e.g. schools, offices, hospitals, trucks, trains, working machines.

To develop these methods, a series of reference measurements has been carried out for validation of airflow patterns and temperatures. In order to investigate whether CFD calculations can predict the climate in the same way as measurements with a thermal manikin, 3 series of numerical calculations as well as full-scale measurements were carried out. These studies are all steps towards making computer simulations that predicts the effects of local climate disturbances correlated with the thermal sensation experienced by subjects.

The cases are ordered in complexity, starting with the relatively simple climatic chamber displacement ventilation situation. Then the office environment with both mixing and displacement ventilation. The last example is a complicated cabin with increased air speeds as well as radiation. The results are shown in three version, first the data measured (meas) with MANIKIN2, then the results from calculations (calc) done with the equations derived above with the measured data as input. Finally CFD (cfd) runs continuously generating new input data to the calculations of the MANIKIN3 boundary conditions. More results and geometrical specifications can be found in the appendices.

All cases were first simulated with standard k- $\varepsilon$ turbulence modelling. Due to problems both with the long calculation times as well as the poor results, the two equation modelling was changed to a zero-equation model. These simulations were found to both be faster and give better agreement with measured results. In the summaries are the results from k- $\varepsilon$ turbulence model presented within parenthesis. 


\section{Case 1: The climatic chamber}

\section{Introduction}

This investigation was a part of a project that investigated possible reason to why houses and working places may be a source for unhealth. The background is defective designed or constructed buildings that cause diseases, poor environments and often consume much energy. The project is a co-operation between the National Institute for Working Life, the Centre for Built Environment at the Royal Institute of Technology and ABB Ventilation Products. To achieve the goal, to improve the indoor environment, computerised tools are used and developed for assessment of good ventilation and indoor climate. The purpose was to investigate how well numerical CFD calculations as well as full scale measurements with a thermal manikin could predict the perceived climate in an uncomplicated chamber environment.

\section{Real and virtual chamber}

The manikin was positioned in the environment that should be assessed. In this study a displacement ventilated empty climatically controlled chamber, at the Centre for Built Environment in Gävle was used (Nilsson et al., 1999c). The room could be controlled to have air and wall temperatures close to $20^{\circ} \mathrm{C}$ (data see table).

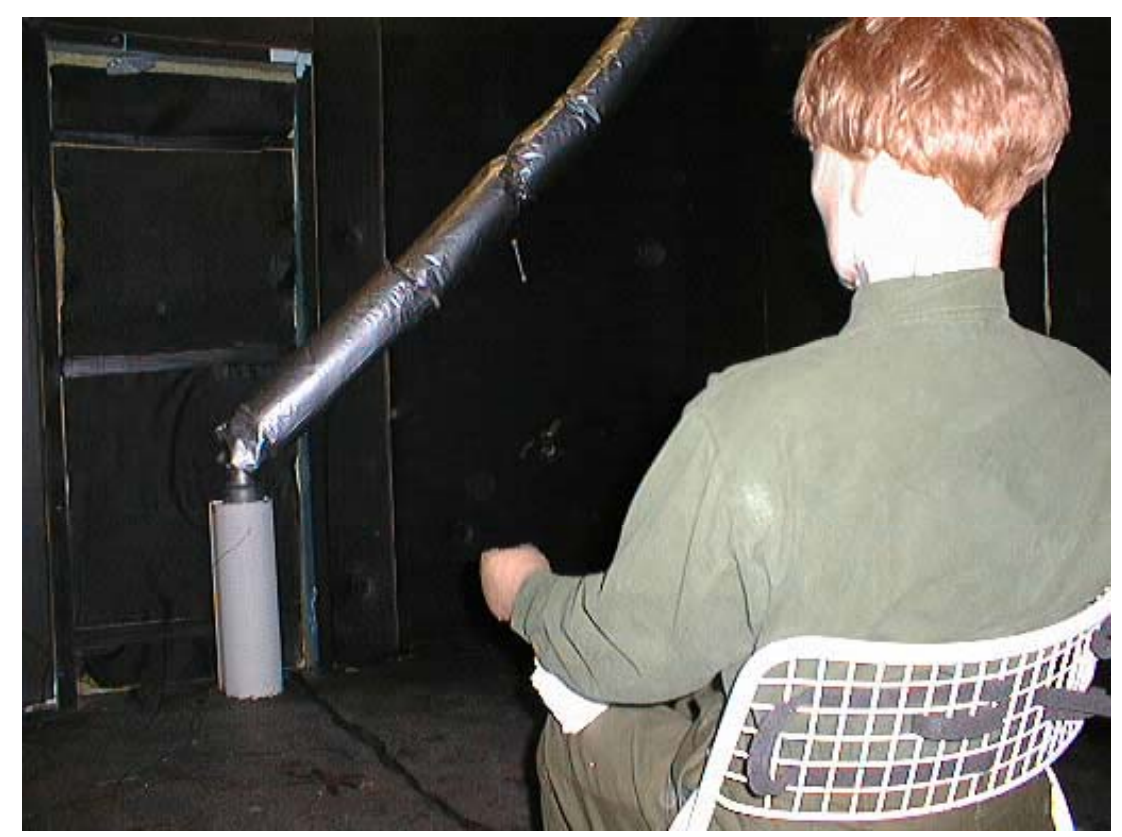

Figure 65. The thermal manikin MANIKIN2 inside the climatic room. 


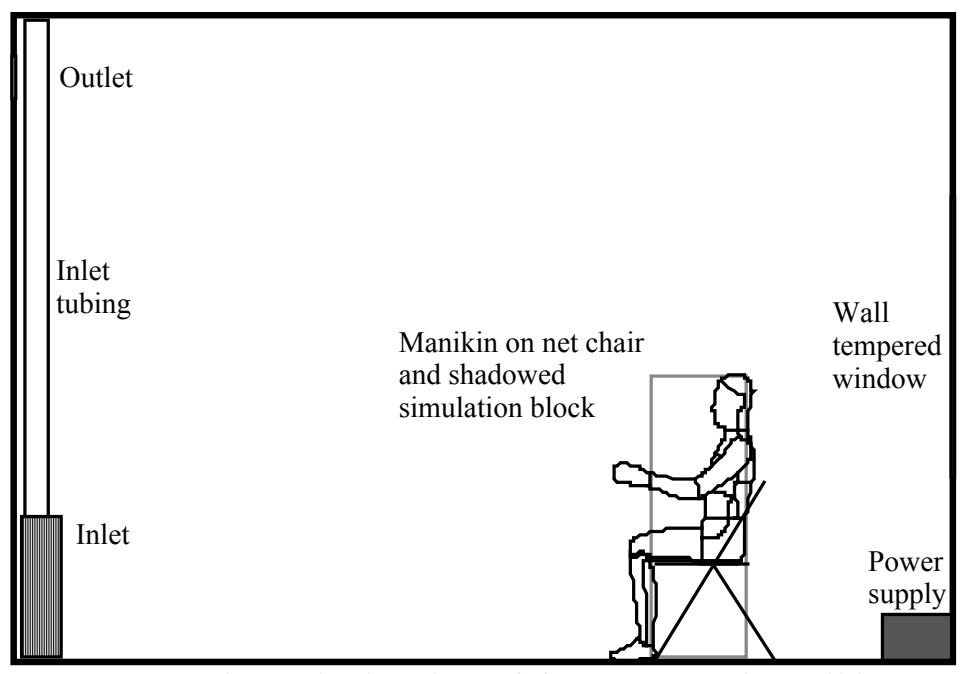

Figure 66. Schematic drawing of the segmented manikin inside the climatic room with deplacement ventilation.

Heat flow in $\mathrm{W} / \mathrm{m}^{2}$ from the different segments of the manikin surface was measured and controlled by a computerised system (Appendix A). Data for the actual conditions were recorded when manikin has reached heat equilibrium with the environment.

\section{Calculation procedure}

The manikin was positioned in front of the window with the same temperature as the walls. The manikin power boxes $\left(0.3 \times 0.2 \times 0.2 \mathrm{~m}^{3}\right)$ were positioned under the window and gave $10 \mathrm{~W} / \mathrm{m}^{2}$ heat load to the room. The incoming air supply rate was kept constant at 3 different levels 10, 20 and 30 1/s. The supply air was distributed through a displacement air terminal $\left(\right.$ EMTEK $\left.^{\circledR}\right)$. The supply-air temperature and normal velocity at the inlet was measured and set according to the measured values. The outlet $(0.5 \times 0.2$ $\mathrm{m}^{2}$ ) was positioned at the same wall as the inlet but with the centre $2.5 \mathrm{~m}$ above the floor, see figure 66. Boundary conditions for the CFD calculations are shown in the table 32. The flow field was calculated with three-dimensional CFD. 


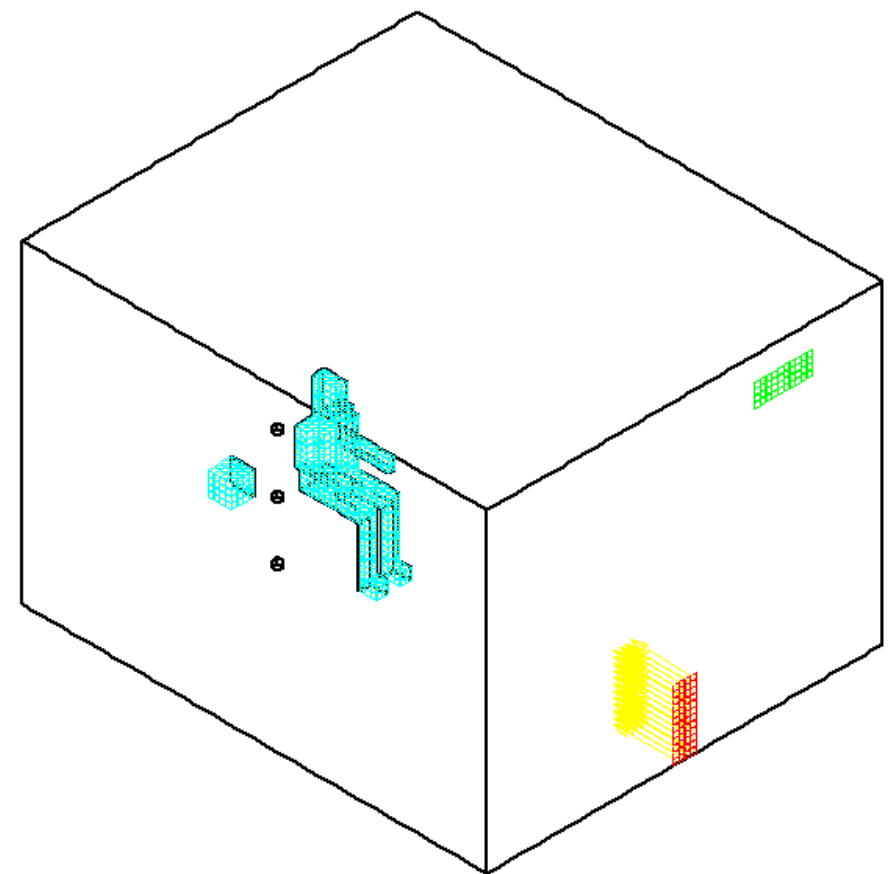

Figure 67. The geometry of the climatic room. The inlet and outlet is positioned on the left wall opposite the manikin. The manikin is sitting on a net chair with the power supply in front.

\section{Results from the climatic chamber (1:st case)}

In this first study a displacement ventilated empty room with air and wall temperatures close to $20^{\circ} \mathrm{C}$ was used. Three different cases have been studied both with the manikin and numerical simulations. Temperatures in the middle of the room as well as at the inlet were measured continuously during the measurements. The total heat loss from the manikin was measured for the three flow conditions.

rdis10: Displacement ventilation with $10 \mathrm{l} / \mathrm{s}$ air flow from the supply. rdis20: Displacement ventilation with $20 \mathrm{l} / \mathrm{s}$ air flow from the supply. rdis30: Displacement ventilation with $30 \mathrm{l} / \mathrm{s}$ air flow from the supply

Table 32. Measured temperature data together with data from the CFD calculations.

\begin{tabular}{lccccccccccc}
\hline $\begin{array}{l}\text { Temperature } \\
\left({ }^{\circ} \mathrm{C}\right)\end{array}$ & $\begin{array}{c}\text { rdis10 } \\
\text { meas }\end{array}$ & $\begin{array}{c}\text { rdis20 } \\
\text { meas }\end{array}$ & $\begin{array}{c}\text { rdis30 } \\
\text { meas }\end{array}$ & $\begin{array}{c}\text { rdis10 } \\
\text { calc }\end{array}$ & $\begin{array}{c}\text { rdis20 } \\
\text { calc }\end{array}$ & $\begin{array}{c}\text { rdis30 } \\
\text { calc }\end{array}$ & $\begin{array}{c}\text { rdis10 } \\
\text { cfd }\end{array}$ & $\begin{array}{c}\text { rdis20 } \\
\text { cfd }\end{array}$ & $\begin{array}{c}\text { rdis30 } \\
\text { cfd }\end{array}$ & $\begin{array}{c}\text { calc } \\
\text { mean } \\
\text { diff. }\end{array}$ & $\begin{array}{c}\text { cfd } \\
\text { mean } \\
\text { diff. }\end{array}$ \\
\hline Inlet & 16.6 & 17.1 & 17.8 & 16.6 & 17.1 & 17.8 & 16.6 & 17.1 & 17.8 & 0.0 & 0.0 \\
Air 0.1 & 19.0 & 18.9 & 19.2 & 19.0 & 18.9 & 19.2 & 19.8 & 19.4 & 19.2 & 0.0 & 0.4 \\
Air 0.6 & 20.0 & 20.3 & 20.4 & 20.0 & 20.3 & 20.4 & 19.8 & 19.4 & 19.2 & 0.0 & 0.7 \\
Air 1.1 & 20.8 & 20.6 & 20.8 & 20.8 & 20.6 & 20.8 & 19.9 & 19.5 & 19.3 & 0.0 & 1.2 \\
\hline
\end{tabular}


Table 33. Heat loss data $\left(\mathrm{W} / \mathrm{m}^{2}\right)$ from a selection of manikin segments during the exposure to the three different cases. $(\mathrm{L}=\mathrm{Left}, \mathrm{R}=\mathrm{Right})$

\begin{tabular}{lccccccccccc}
\hline $\begin{array}{l}\text { Heat loss } \\
\text { (W/m2) }\end{array}$ & $\begin{array}{c}\text { rdis10 } \\
\text { Zone }\end{array}$ & $\begin{array}{c}\text { rdis20 } \\
\text { meas }\end{array}$ & $\begin{array}{c}\text { rdis30 } \\
\text { meas }\end{array}$ & $\begin{array}{c}\text { rdis10 } \\
\text { calc }\end{array}$ & $\begin{array}{c}\text { rdis20 } \\
\text { calc }\end{array}$ & $\begin{array}{c}\text { rdis30 } \\
\text { calc }\end{array}$ & $\begin{array}{c}\text { rdis10 } \\
\text { cfd }\end{array}$ & $\begin{array}{c}\text { rdis20 } \\
\text { cfd }\end{array}$ & $\begin{array}{c}\text { rdis30 } \\
\text { cfd }\end{array}$ & $\begin{array}{c}\text { calc } \\
\text { mean } \\
\text { diff. }\end{array}$ & $\begin{array}{c}\text { cfd } \\
\text { mean } \\
\text { diff. }\end{array}$ \\
\hline Whole & 66 & 68 & 69 & 67 & 69 & 69 & 68 & 70 & 71 & 1 & 1 \\
body & & & & & & & & & & & \\
Scalp & 50 & 51 & 52 & 47 & 48 & 48 & 50 & 51 & 52 & 4 & 0 \\
Face & 107 & 110 & 112 & 113 & 117 & 118 & 120 & 124 & 126 & 7 & 14 \\
Chest & 52 & 55 & 53 & 51 & 53 & 53 & 54 & 55 & 56 & 1 & 2 \\
BackU & 68 & 70 & 70 & 73 & 76 & 77 & 77 & 80 & 81 & 6 & 10 \\
ArmLU & 55 & 57 & 58 & 57 & 58 & 59 & 60 & 62 & 62 & 1 & 5 \\
ArmRU & 58 & 61 & 62 & 58 & 60 & 60 & 61 & 63 & 64 & 1 & 2 \\
ArmLL & 66 & 71 & 73 & 74 & 74 & 75 & 73 & 75 & 76 & 4 & 5 \\
ArmRL & 67 & 71 & 71 & 72 & 72 & 74 & 71 & 73 & 74 & 3 & 3 \\
HandL & 115 & 122 & 130 & 126 & 128 & 131 & 125 & 129 & 131 & 6 & 6 \\
HandR & 114 & 120 & 122 & 131 & 132 & 135 & 130 & 134 & 136 & 14 & 15 \\
ThighL & 72 & 74 & 75 & 70 & 70 & 71 & 70 & 72 & 73 & 3 & 2 \\
ThighR & 64 & 66 & 65 & 69 & 69 & 70 & 69 & 71 & 72 & 4 & 5 \\
CalfL & 76 & 80 & 83 & 77 & 80 & 80 & 71 & 74 & 75 & 1 & 7 \\
CalfR & 69 & 71 & 72 & 75 & 78 & 78 & 70 & 72 & 73 & 7 & 1 \\
FootL & 64 & 63 & 63 & 57 & 59 & 59 & 53 & 55 & 55 & 5 & 9 \\
FootR & 64 & 64 & 63 & 61 & 62 & 63 & 56 & 58 & 59 & 1 & 6 \\
BackL & 46 & 47 & 46 & 46 & 46 & 47 & 46 & 47 & 48 & 0 & 1 \\
Seat & 70 & 70 & 70 & 65 & 65 & 66 & 65 & 67 & 68 & 5 & 3 \\
\hline
\end{tabular}

\section{Results from the 1:st case}

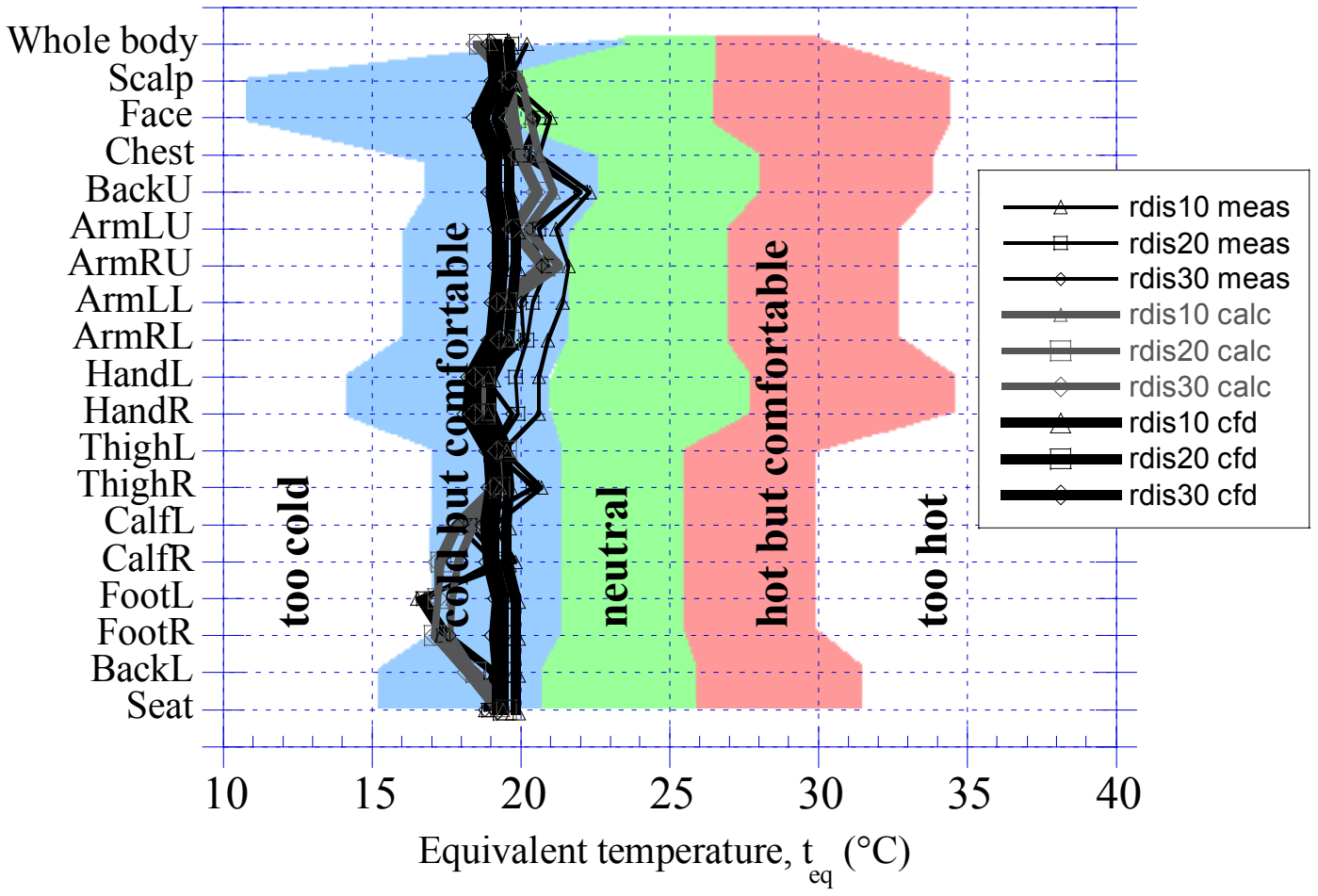

Figure 68. Data from the manikin agrees with the results above, showing the effects of a rather low ambient temperature and increased heat loss with increased cool air flow, especially at the lower legs and feet as well as the non insulated seat zones. 


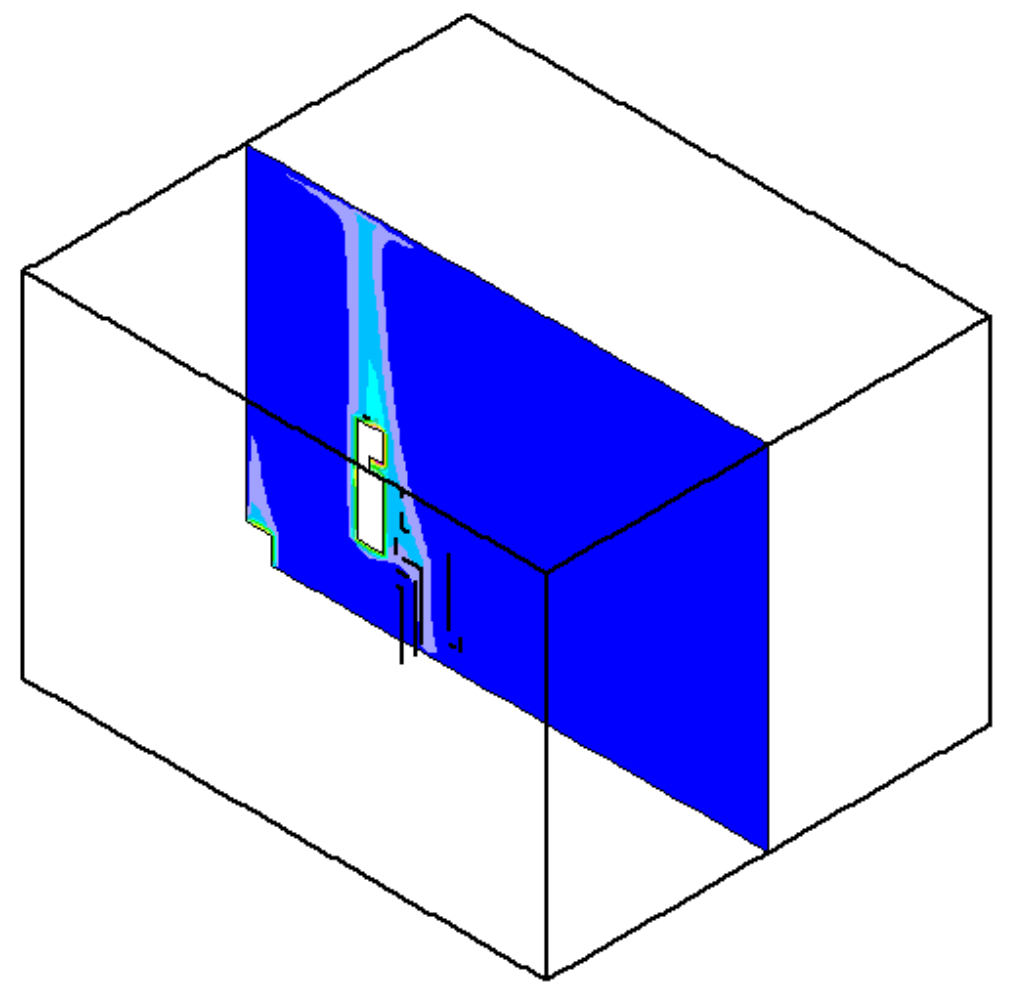

TEMPERATURE ABSOLUTE KELVIN

LOCALMX $=306.1$

LOCALMNN $=292.9$
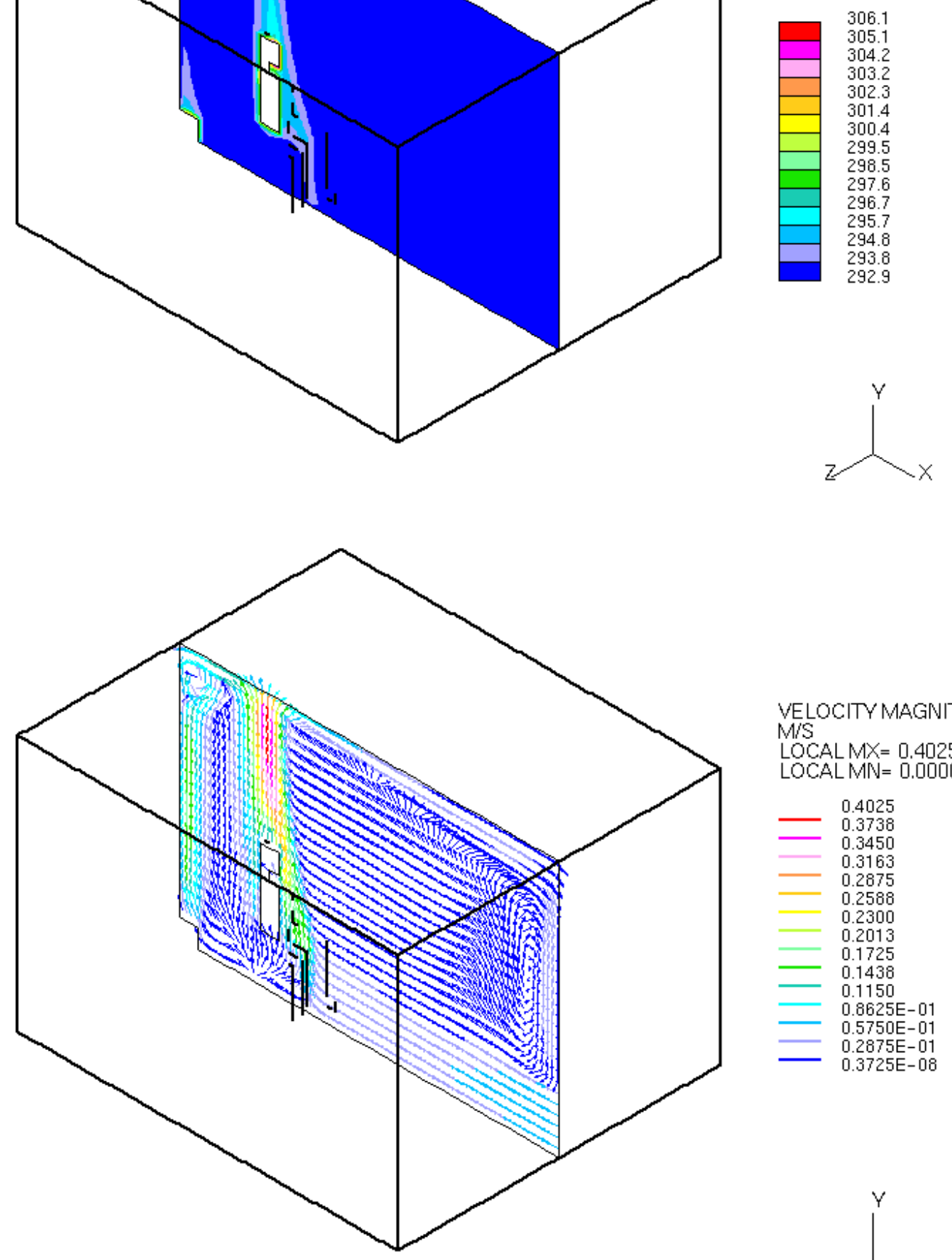
VELOCITY MAGNITUDE

LOCALMX $=0.4025$

LOCALMN= 0.0000

0.4025
$-\quad 0.3738$

$-\quad 0.3738$ 0.3463 $\begin{array}{r}0.3163 \\ -\quad 0.2875 \\ \hline\end{array}$

$\begin{array}{r}0.2588 \\ -\quad 0.2300 \\ \hline\end{array}$

- 0.2013

0.1725
-

- 0.1438

- $0.8625 \mathrm{E}-01$

- $0.5750 \mathrm{E}-01$

$\longrightarrow \quad \begin{aligned} & 0.2875 \mathrm{E}-01 \\ & 0.3725 \mathrm{E}-08\end{aligned}$

Figure 69. The temperature and velocity fields in a plane through the inlet and the manikin. Notice the air supply and the plumes from at the manikin and the power box. 
Table 34. Measured air speed (m/s) compared to data calculated with the CFD program.

\begin{tabular}{|c|c|c|c|c|c|c|c|c|c|c|c|}
\hline $\begin{array}{l}\text { Air speed } \\
(\mathrm{m} / \mathrm{s}) \\
\text { Position }\end{array}$ & $\begin{array}{c}\text { rdis } 10 \\
\text { meas }\end{array}$ & $\begin{array}{c}\text { rdis20 } \\
\text { meas }\end{array}$ & $\begin{array}{c}\text { rdis30 } \\
\text { meas }\end{array}$ & $\begin{array}{c}\text { rdis10 } \\
\text { calc }\end{array}$ & $\begin{array}{l}\text { rdis } 20 \\
\text { calc }\end{array}$ & $\begin{array}{l}\text { rdis30 } \\
\text { calc }\end{array}$ & $\begin{array}{l}\text { rdis10 } \\
\text { cfd }\end{array}$ & $\begin{array}{l}\text { rdis20 } \\
\text { cfd }\end{array}$ & $\begin{array}{c}\text { rdis30 } \\
\text { cfd }\end{array}$ & $\begin{array}{c}\text { calc } \\
\text { mean } \\
\text { diff. }\end{array}$ & $\begin{array}{c}\text { cfd } \\
\text { mean } \\
\text { diff. }\end{array}$ \\
\hline Air 0.1 & - & 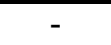 & - & 0.10 & 0.10 & 0.20 & 0.02 & 0.02 & 0.02 & - & - \\
\hline Air 0.6 & 0.10 & 0.10 & 0.20 & 0.10 & 0.10 & 0.20 & 0.03 & 0.03 & 0.03 & 0.00 & 0.11 \\
\hline Air 1.1 & - & - & - & 0.10 & 0.10 & 0.20 & 0.03 & 0.03 & 0.03 & - & - \\
\hline
\end{tabular}

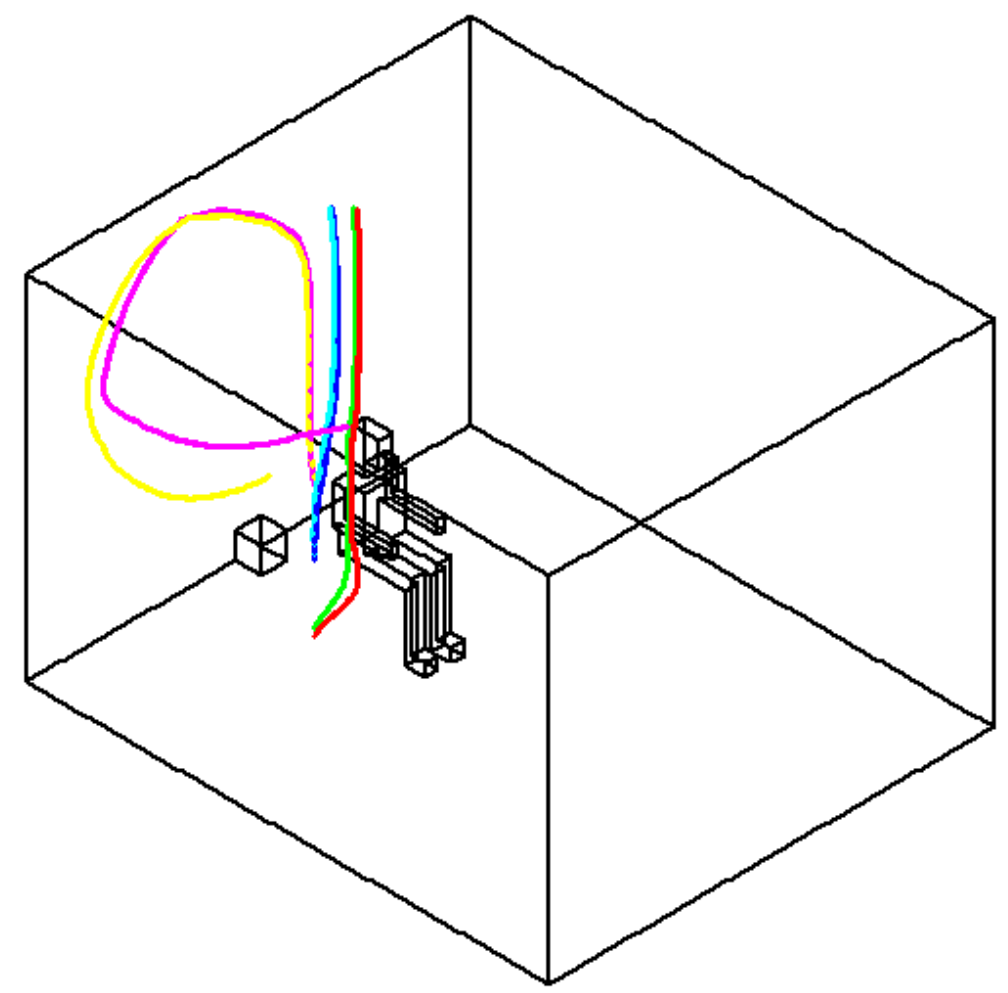

Figure 70. Air flow tracks for the rdis10 case, showing the buoyant air stream around the manikin.

\section{Summary the climatic chamber}

In this first case a displacement ventilated empty room with air and wall temperatures close to $20^{\circ} \mathrm{C}$ was used. Three different conditions have been studied both with MANIKIN2 and numerical simulations with MANIKIN3. Temperatures with the sensor tree close to MANIKIN2, as well as in the inlet air stream were measured continuously during the measurements. The total heat loss from the manikin was measured for the three flow conditions. The calculated and simulated results from this study show good agreement with the measurements made in the real environment. In homogeneous environments, like in this case, the simulations with MANIKIN3 give good predictions of how a human will perceive the thermal climate. The mean difference in $t_{e q}$ is less than the maximum deviation of $3.3^{\circ} \mathrm{C}$ that could be expected between calibrations of MANIKIN2 (Appendix A). The calculated data (calc) gave slightly smaller differences 
compared to measured data (meas) and data with CFD (cfd) simulation input. The air speeds are very low, close to the measurement limit of the measuring air speed sensor.

Table 35. Summary of calculation and simulation differences compared to measurements for case 1. (k- $\varepsilon$ model runs within in parenthesis.)

\begin{tabular}{lccclccc}
\hline Heat loss & case 1 & case 1 & & & $\begin{array}{l}\text { Equivalent } \\
\text { temperature }\end{array}$ & case 1 & case 1 \\
$\left(\mathrm{~W} / \mathrm{m}^{2}\right)$ & $\begin{array}{c}\text { calc } \\
\text { mean diff. }\end{array}$ & $\begin{array}{c}\text { cfd } \\
\text { mean diff. }\end{array}$ & & $\begin{array}{l}\left({ }^{\circ} \mathrm{C}\right) \\
\text { Zone }\end{array}$ & $\begin{array}{c}\text { calc } \\
\text { mean diff. }\end{array}$ & $\begin{array}{c}\text { cfd } \\
\text { mean diff. }\end{array}$ \\
\hline Mane & $\mathbf{1 4}$ & $\mathbf{1 5}(\mathbf{2 0})$ & & Max & $\mathbf{1 . 9}$ & $\mathbf{2 . 8 ( 3 . 1 )}$ \\
Mean & $\mathbf{4}$ & $\mathbf{5}(\mathbf{6})$ & & Mean & $\mathbf{0 . 8}$ & $\mathbf{1 . 8}(\mathbf{1 . 4})$ \\
Min & $\mathbf{0}$ & $\mathbf{0}(\mathbf{1})$ & & Min & $\mathbf{0 . 1}$ & $\mathbf{0 . 2}(\mathbf{0 . 1})$ \\
\hline
\end{tabular}




\section{Case 2: The office environment}

\section{Introduction}

This investigation was a part of the Swedish project (The Healthy Building) that investigated how the use of modern calculation tools can improve the thermal climate in buildings. This investigation has been performed in a real office unit at the National Institute of Working Life, and can therefore to some extent be regarded as a field investigation. The purpose was to investigate how well the standard office room concept of mixing or displacement ventilation, evaluated with numerical CFD methods calculations and measurements with a thermal manikin, can enable calculations of perceived thermal comfort.

\section{Real and virtual office}

This second investigation has been performed in a real office unit (Nilsson et al., 2000). The room normally has mixing ventilation but this room was equipped with displacement ventilation for study purpose. Temperatures at the centre of the room as well as at the different supply inlets and exhaust outlets were measured continuously during the measurements. MANIKIN2 was positioned in the office room. Heat flow from the different segments of the manikin surface was measured and controlled by a computerised system. Data for the actual conditions where recorded when the manikin had reached heat equilibrium with the environment. The following four cases where studied:

omix: Mixing ventilation with $135 \mathrm{l} / \mathrm{s}$ air flow inlet and outlet $2.9 \mathrm{~m}$ above the floor. odis23: Displacement ventilation with $23 \mathrm{l} / \mathrm{s}$ air flow from the supply. odis40: Displacement ventilation with $401 / \mathrm{s}$ air flow from the supply. odis50: Displacement ventilation with $55 \mathrm{l} / \mathrm{s}$ air flow from the supply.

The diffuser choice was made with the ABB company program WinDon ${ }^{\circledR}$ - Version 1.031. The program recommended a Floormaster ${ }^{\circledR}$ FMC-603 with an air flow of $48 \mathrm{1} / \mathrm{s}$ at an room air temp of $24{ }^{\circ} \mathrm{C}$ and an inlet temperature of $19.4^{\circ} \mathrm{C}$. Supply air was distributed from a cooler located in the ceiling of the corridor. Temperature and air speed measurements were made with two Brüel \& $\mathrm{Kjær}^{\circledR}$ Indoor Climate Analyzer 1213. The radiators were turned off, and windows and radiators where covered with 50 $\mathrm{mm}$ foam plastic, in order to minimise radiation influence. 


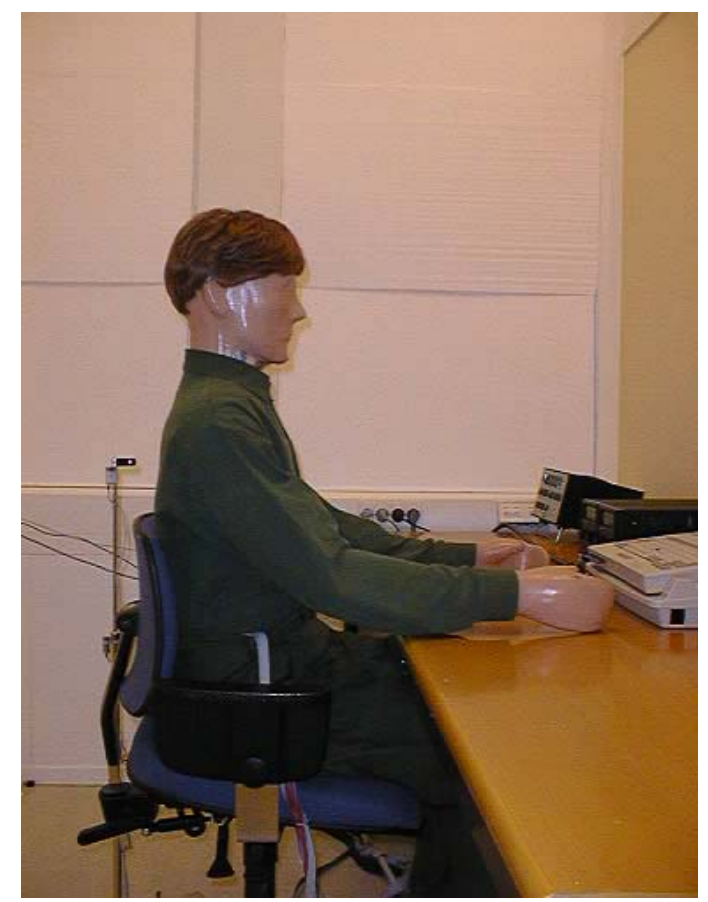

Figure 71. Picture showing manikin and computer in the office room.
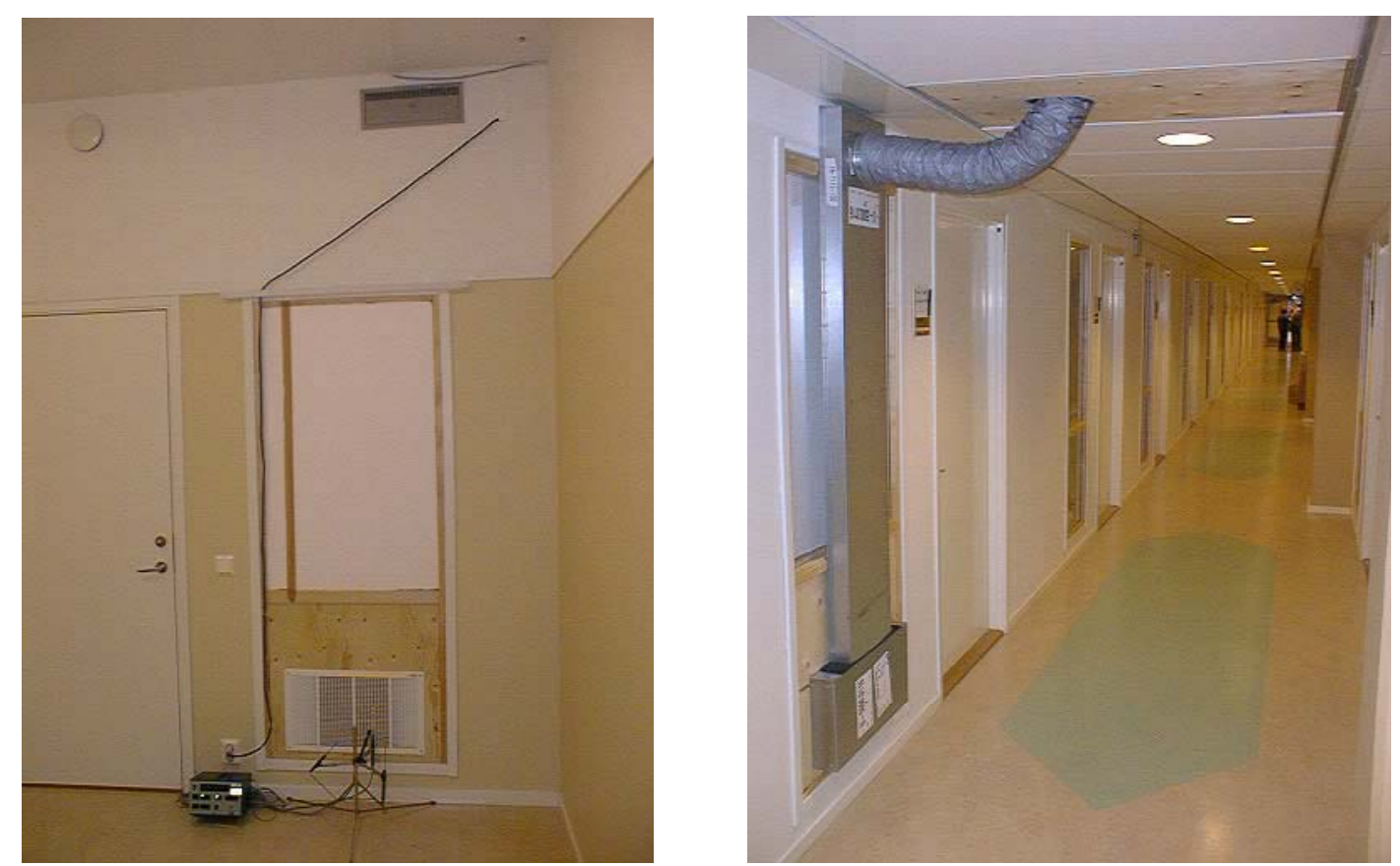

Figure 72. In the left picture the white diffuser at $0.2 \mathrm{~m}$ height and the grey squared mixing grill to the right side at $2.9 \mathrm{~m}$ and the white round exhaust at $2.9 \mathrm{~m}$ to the left. The right picture shows the air supply to the room from the corridor side. 


\section{Calculation Procedure}

The virtual manikin was positioned in geometry of the numerical test room $(2.9,3.0,3.8$ $\mathrm{m})$ that simulates a real office room. Ventilation air was supplied from a simulated FMC-603 diffuser with a supply area of $0.15 \mathrm{~m}^{2}$. The exhaust outlet was on the same wall, and had an area of $0.04 \mathrm{~m}^{2}$. The outlet was positioned at the back wall, se figure (middle) and appendix C. Boundary conditions for the CFD calculations are shown in table 36.

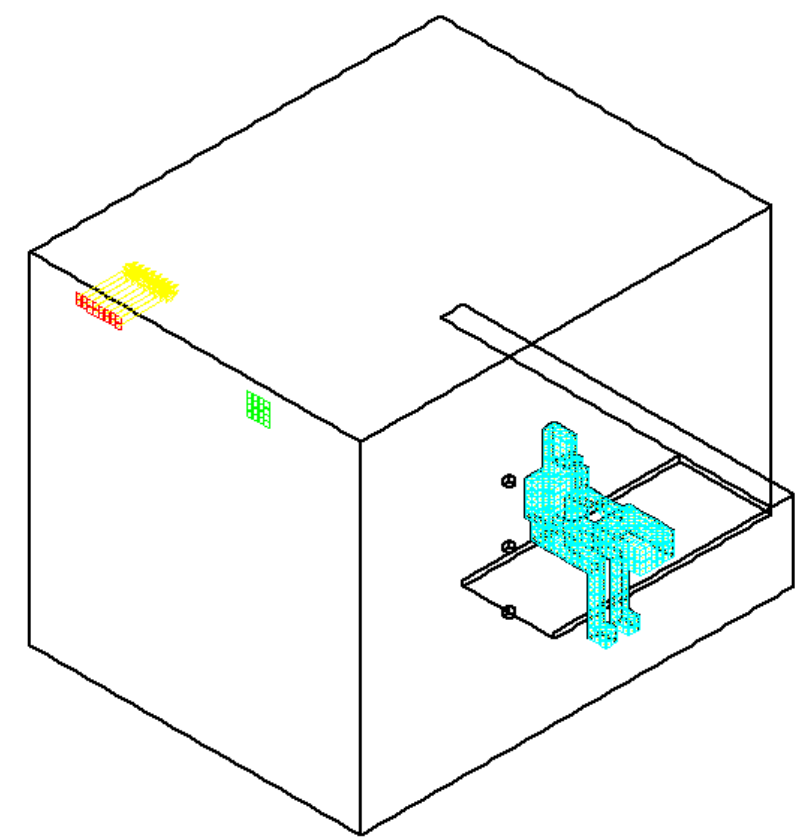

Figure 73. The virtual room with mixing ventilation.

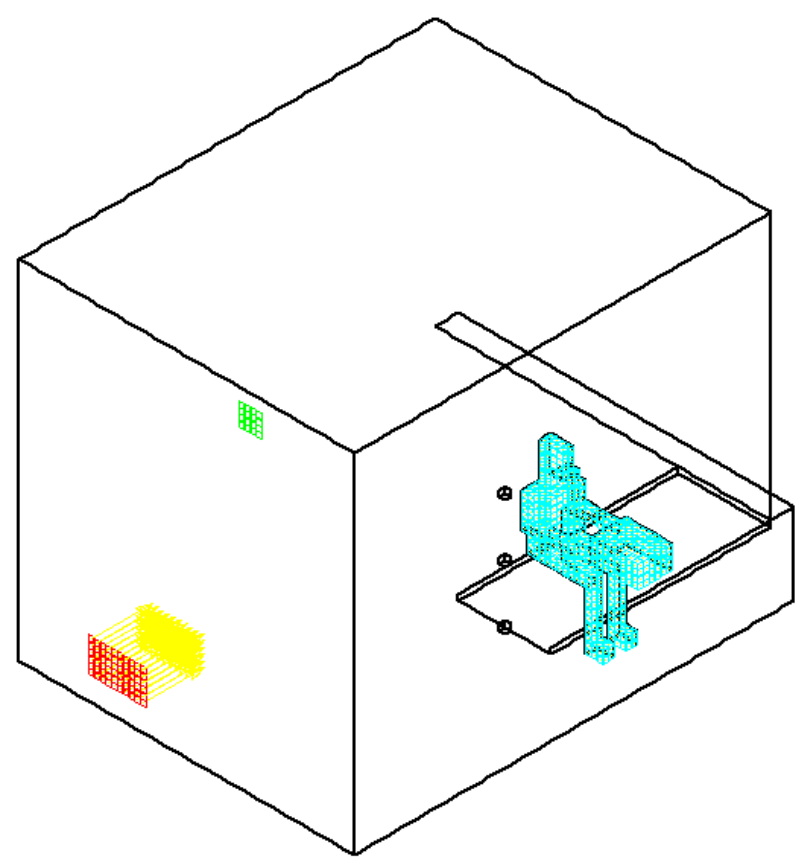

Figure 74. The same room but with displacement ventilation. 


\section{Results office environment (2:nd case)}

Four different conditions have been studied both with the manikin measurements and numerical simulations. The results from the numerical simulations are shown below. The heat loss from the manikin zones was measured and calculated for all conditions. Temperatures at the centre of the office room as well as at the different supply inlets and exhaust outlets were measured continuously during the measurements.

Table 36. Measured temperature data together with data from the CFD calculations.

\begin{tabular}{|c|c|c|c|c|c|c|c|c|c|c|c|c|c|c|}
\hline $\begin{array}{l}\text { Temperature } \\
\left({ }^{\circ} \mathrm{C}\right)\end{array}$ & omix & $\begin{array}{c}\text { odis } \\
23\end{array}$ & $\begin{array}{c}\text { odis } \\
40\end{array}$ & $\begin{array}{c}\text { odis } \\
55\end{array}$ & omix & $\begin{array}{c}\text { odis } \\
23\end{array}$ & $\begin{array}{c}\text { odis } \\
40\end{array}$ & $\begin{array}{c}\text { odis } \\
55\end{array}$ & omix & $\begin{array}{c}\text { odis } \\
23\end{array}$ & $\begin{array}{c}\text { odis } \\
40\end{array}$ & $\begin{array}{c}\text { odis } \\
55\end{array}$ & calc & cfd \\
\hline Position & meas & meas & meas & meas & calc & calc & calc & calc & cfd & $\mathrm{cfd}$ & cfd & cfd & $\begin{array}{c}\text { mean } \\
\text { diff. }\end{array}$ & $\begin{array}{c}\text { mean } \\
\text { diff. }\end{array}$ \\
\hline$\overline{\text { Inlet }}$ & 21.2 & 14.1 & 15.1 & 16.4 & 21.2 & 14.1 & 15.1 & 16.4 & 21.2 & 14.1 & 15.1 & 16.4 & 0.0 & 0.0 \\
\hline Air 0.1 & 23.3 & 21.0 & 19.8 & 19.7 & 23.3 & 21.0 & 19.8 & 19.7 & 24.1 & 21.4 & 19.9 & 19.5 & 0.0 & 0.4 \\
\hline Air 0.6 & 24.7 & 23.0 & 21.1 & 20.9 & 24.7 & 23.0 & 21.1 & 20.9 & 24.7 & 21.8 & 20.3 & 19.9 & 0.0 & 0.8 \\
\hline Air 1.1 & 23.8 & 23.0 & 22.2 & 21.8 & 23.8 & 23.0 & 22.2 & 21.8 & 25.4 & 21.5 & 20.0 & 19.6 & 0.0 & 1.9 \\
\hline
\end{tabular}

Table 37. Heat loss data $\left(\mathrm{W} / \mathrm{m}^{2}\right)$ from a selection of manikin segments during the exposure to the four different cases. ( $\mathrm{L}=\mathrm{Left}, \mathrm{R}=\mathrm{Right})$.

\begin{tabular}{lcccccccccccccc}
\hline $\begin{array}{l}\text { Heat loss } \\
\text { (W/m2) }\end{array}$ & omix & odis & odis & odis & omix & odis & odis & odis & omix & odis & odis & odis & calc & cfd \\
& & 23 & 40 & 55 & & 23 & 40 & 55 & & 23 & 40 & 55 & calc \\
Zone & meas & meas & meas & meas & calc & calc & calc & calc & cfd & cfd & cfd & $\begin{array}{c}\text { mean } \\
\text { diff. }\end{array}$ & $\begin{array}{c}\text { mean } \\
\text { diff. }\end{array}$ \\
\hline Whole & 45 & 50 & 55 & 57 & 44 & 50 & 57 & 59 & 41 & 55 & 62 & 64 & 1 & 6 \\
body & & & & & & & & & & & & & & \\
Scalp & 38 & 40 & 42 & 43 & 36 & 38 & 41 & 43 & 30 & 44 & 49 & 51 & 1 & 7 \\
Face & 78 & 86 & 91 & 99 & 84 & 91 & 99 & 104 & 71 & 106 & 120 & 124 & 6 & 20 \\
Chest & 35 & 37 & 41 & 43 & 38 & 41 & 45 & 47 & 33 & 48 & 53 & 55 & 4 & 9 \\
BackU & 59 & 63 & 68 & 71 & 56 & 60 & 65 & 68 & 47 & 69 & 77 & 79 & 3 & 9 \\
ArmLU & 45 & 47 & 52 & 54 & 43 & 47 & 50 & 52 & 37 & 53 & 60 & 61 & 2 & 7 \\
ArmRU & 45 & 49 & 54 & 55 & 44 & 47 & 51 & 54 & 38 & 54 & 61 & 63 & 1 & 7 \\
ArmLL & 53 & 54 & 60 & 65 & 48 & 57 & 67 & 69 & 49 & 63 & 71 & 73 & 5 & 8 \\
ArmRL & 54 & 57 & 63 & 64 & 47 & 55 & 66 & 67 & 47 & 62 & 69 & 72 & 3 & 6 \\
HandL & 85 & 81 & 91 & 95 & 78 & 95 & 114 & 118 & 81 & 107 & 122 & 126 & 16 & 23 \\
HandR & 87 & 90 & 100 & 103 & 81 & 98 & 118 & 122 & 83 & 111 & 126 & 130 & 13 & 20 \\
ThighL & 45 & 51 & 56 & 57 & 45 & 53 & 64 & 65 & 46 & 60 & 68 & 70 & 5 & 9 \\
ThighR & 39 & 47 & 53 & 54 & 44 & 53 & 63 & 64 & 45 & 59 & 67 & 69 & 8 & 12 \\
CalfL & 52 & 62 & 69 & 69 & 53 & 66 & 72 & 74 & 50 & 63 & 71 & 73 & 3 \\
CalfR & 50 & 62 & 68 & 70 & 52 & 64 & 71 & 72 & 49 & 62 & 70 & 72 & 2 \\
FootL & 45 & 53 & 57 & 59 & 40 & 49 & 54 & 55 & 38 & 47 & 53 & 54 & 4 \\
FootR & 43 & 54 & 57 & 58 & 43 & 52 & 57 & 58 & 40 & 50 & 56 & 58 & 1 & 2 \\
BackL & 23 & 26 & 30 & 32 & 15 & 18 & 21 & 21 & 15 & 20 & 22 & 23 & 9 \\
Seat & 6 & 9 & 11 & 10 & 10 & 11 & 13 & 13 & 9 & 12 & 14 & 14 & 3 \\
\hline
\end{tabular}


“dis23” Temperature field

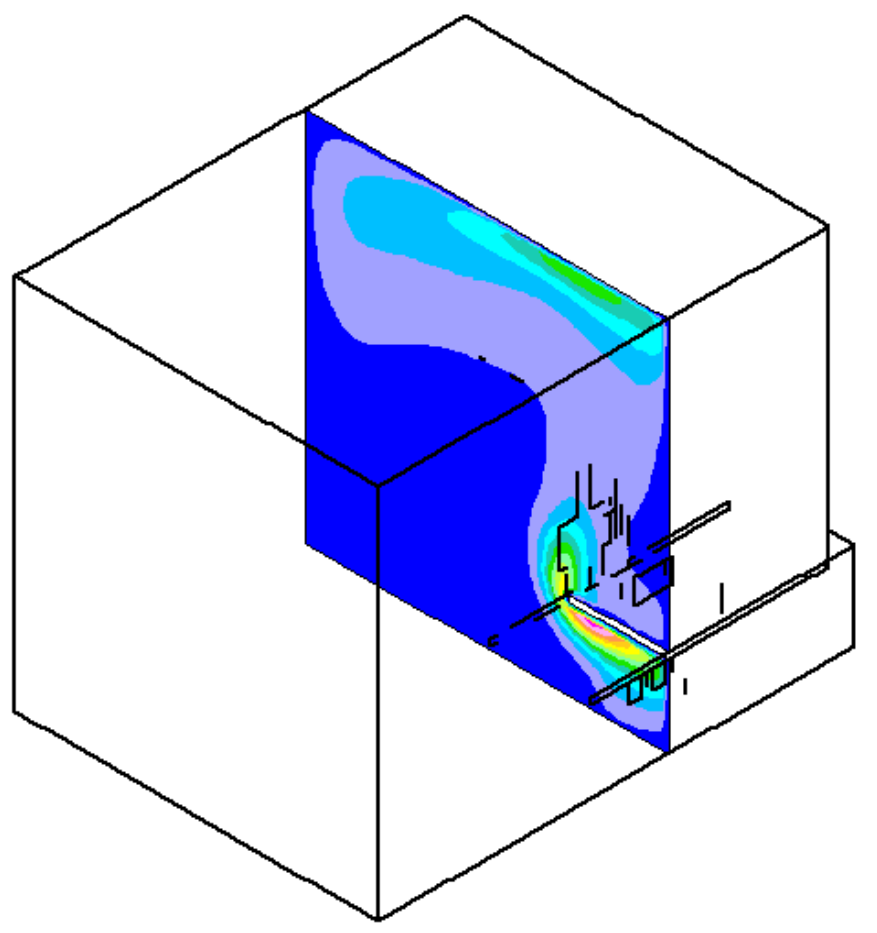

TEMPERATURE

ABSOLUT

KELV

LOCALMX $=296.2$

LOCALMN= 294.5

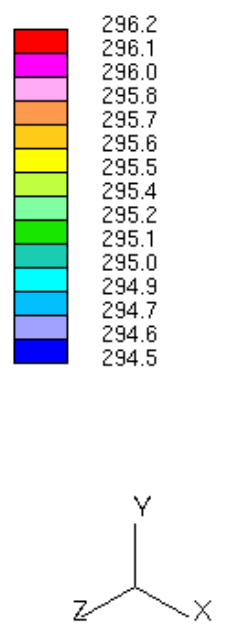

“mix" Temperature field

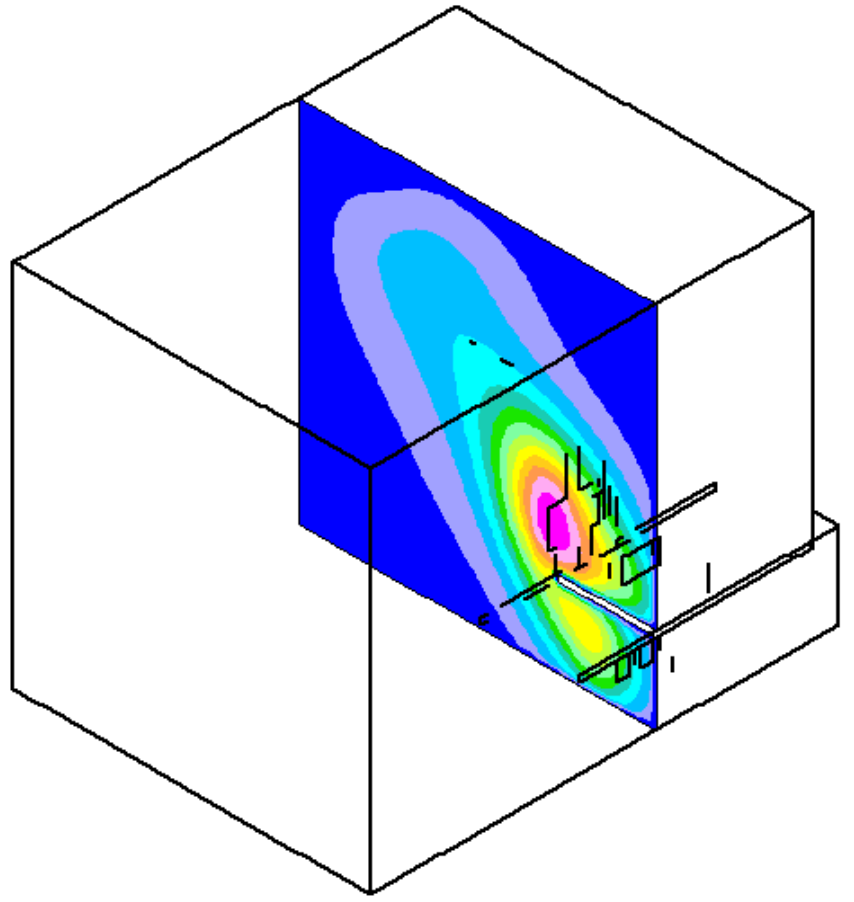

\section{TEMPERATURE ABSOLUTE \\ LOCALMX $=299.2$ \\ LOCALMN $=296.8$}

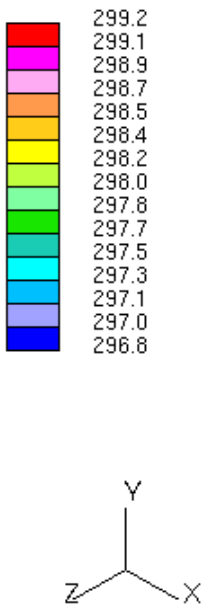

Figure 75a. In the first two figures above (DIS23 Temperature field and MIX Temperature field), the cooled air from the supply is distributed over the floor (left) and reaches the manikin and computer where a plume is formed (right) and the air is elevated to the exhaust level. 

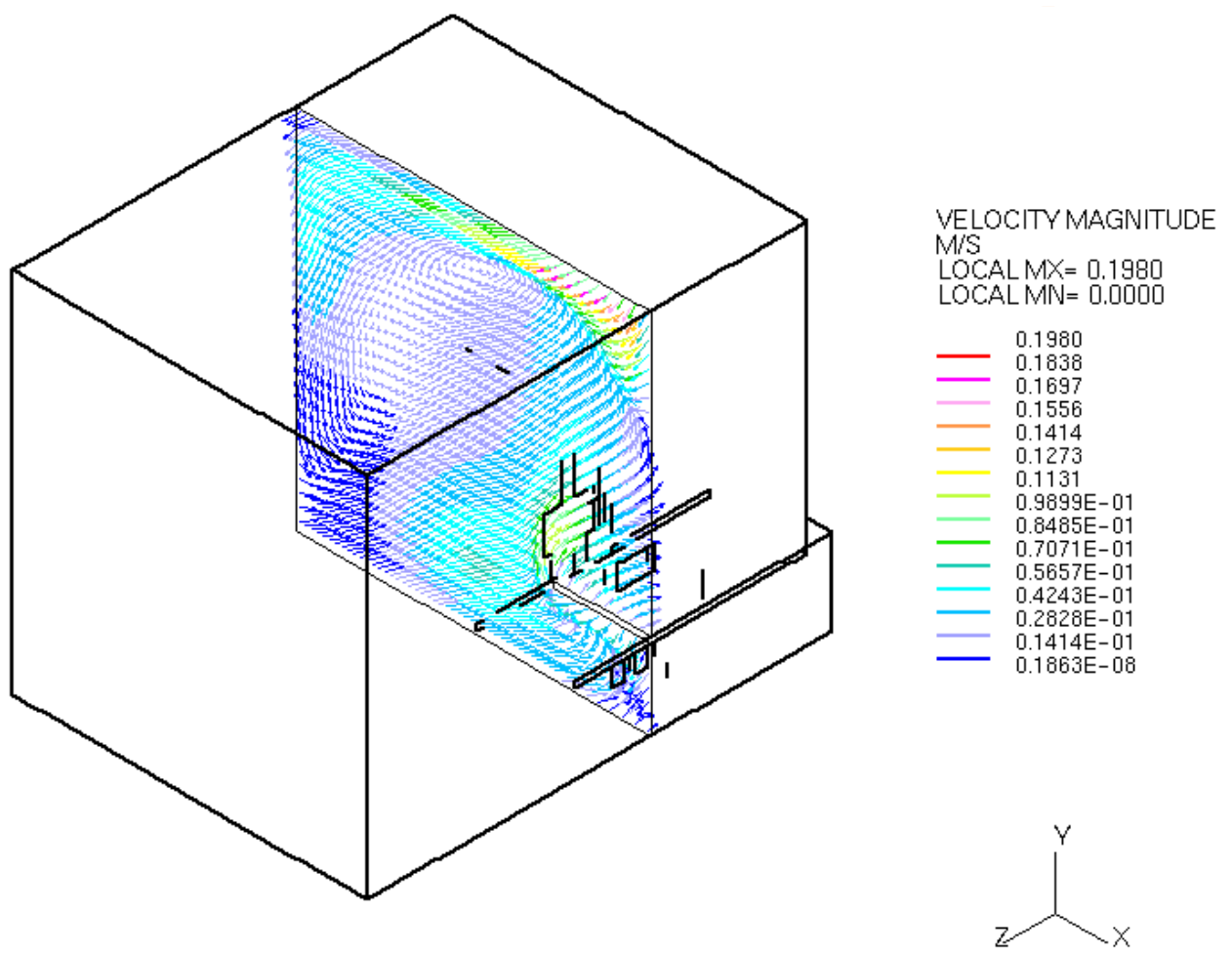

“mix" Velocity field
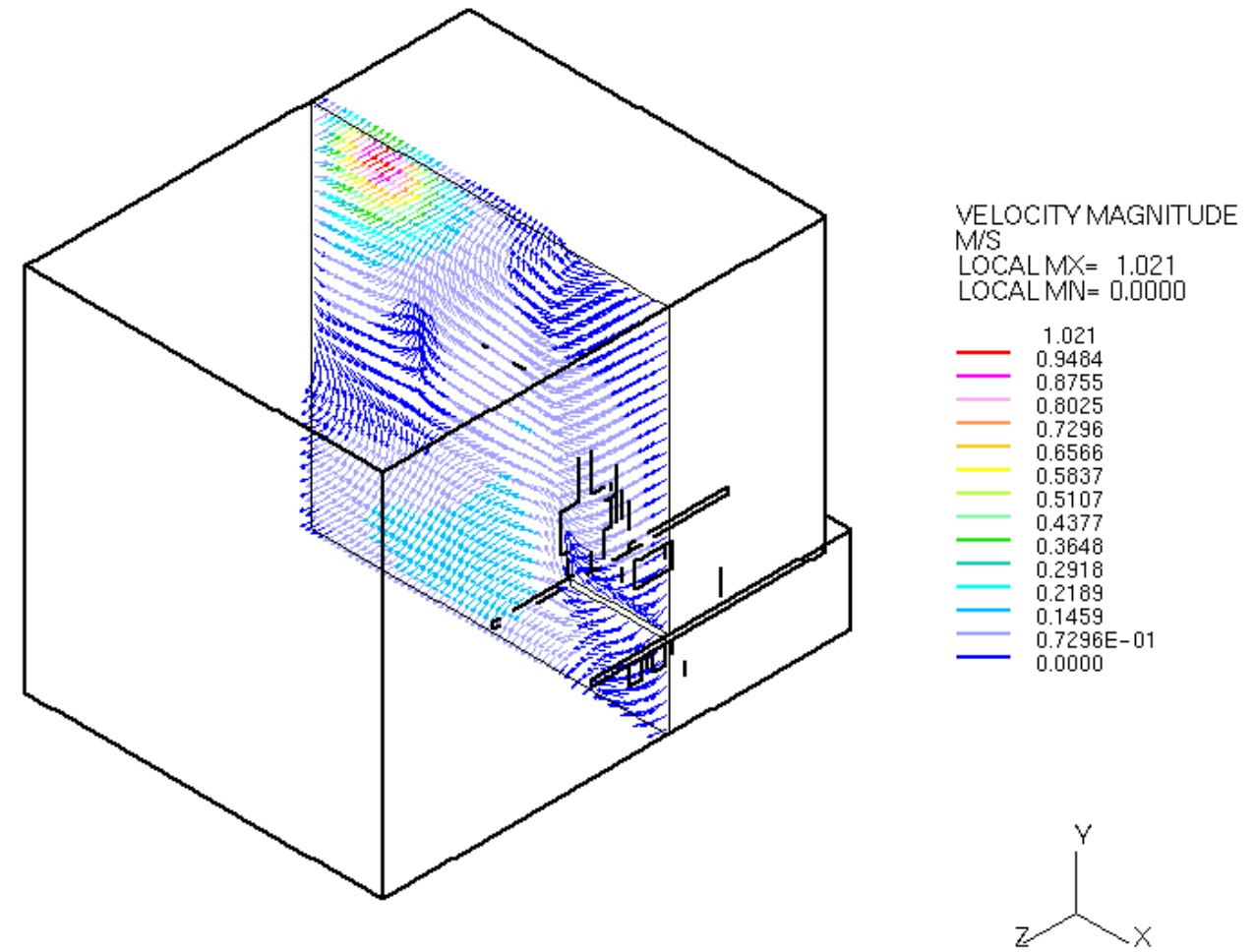

Figure 75b. These two pictures (DIS23 Velocity field and MIX Velocity field), show flow patterns in the office room as velocity vectors and contours in a plane at the manikin centre. With mixing ventilation air is blown right across the room at supply level and breaks down to the floor level at the opposite wall, then slowly elevate passing the manikin through the room up to the exhaust. 
Table 38. Measured air velocities and temperatures compared to CFD calculated values.

\begin{tabular}{lcccccccccccccc}
\hline $\begin{array}{l}\text { Air speed } \\
(\mathrm{m} / \mathrm{s})\end{array}$ & omix & $\begin{array}{c}\text { odis } \\
23\end{array}$ & $\begin{array}{c}\text { odis } \\
40\end{array}$ & $\begin{array}{c}\text { odis } \\
55\end{array}$ & omix & $\begin{array}{c}\text { odis } \\
23\end{array}$ & $\begin{array}{c}\text { odis } \\
40\end{array}$ & $\begin{array}{c}\text { odis } \\
55\end{array}$ & omix & $\begin{array}{c}\text { odis } \\
23\end{array}$ & $\begin{array}{c}\text { odis } \\
40\end{array}$ & $\begin{array}{c}\text { odis } \\
55\end{array}$ & calc & cfd \\
Position & meas & meas & meas & meas & calc & calc & calc & calc & cfd & cfd & cfd & $\begin{array}{c}\text { cfd } \\
\text { mean } \\
\text { diff. }\end{array}$ & $\begin{array}{c}\text { mean } \\
\text { diff. }\end{array}$ \\
\hline Air 0.1 & - & - & - & - & 0.04 & 0.04 & 0.06 & 0.09 & 0.09 & 0.04 & 0.04 & 0.04 & - & - \\
Air 0.6 & 0.04 & 0.04 & 0.06 & 0.09 & 0.04 & 0.04 & 0.06 & 0.09 & 0.12 & 0.06 & 0.06 & 0.06 & 0.00 & 0.03 \\
Air 1.1 & - & - & - & - & 0.04 & 0.04 & 0.06 & 0.09 & 0.12 & 0.05 & 0.05 & 0.05 & - & - \\
\hline
\end{tabular}

Displacement ventilation shows how the air is elevated to the ceiling and mostly removed by the outlet. In the case of mixing ventilation the swirling effects is clearly shown at the window wall. With mixing ventilation air is blown right across the room at supply level and breaks down to the floor level at the opposite wall, then passing the manikin circulating through the room up to the exhaust.

The data in the comfort zone diagram below shows that increased flow rates combined with low inlet temperatures are well detected by the manikin. With displacement ventilation the values for the calves and feet are decreasing to finally reach into the zone where the occupant would feel cold. 
“dis23" Tracks

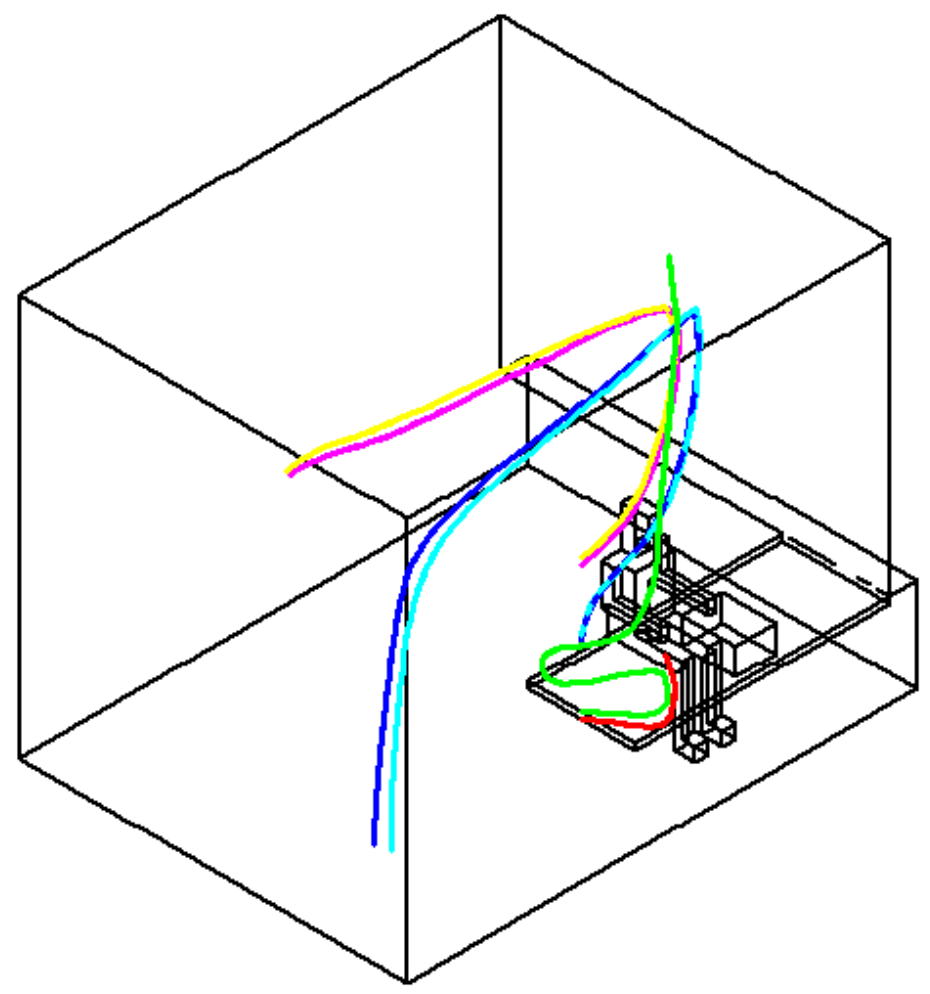

"mix" Tracks

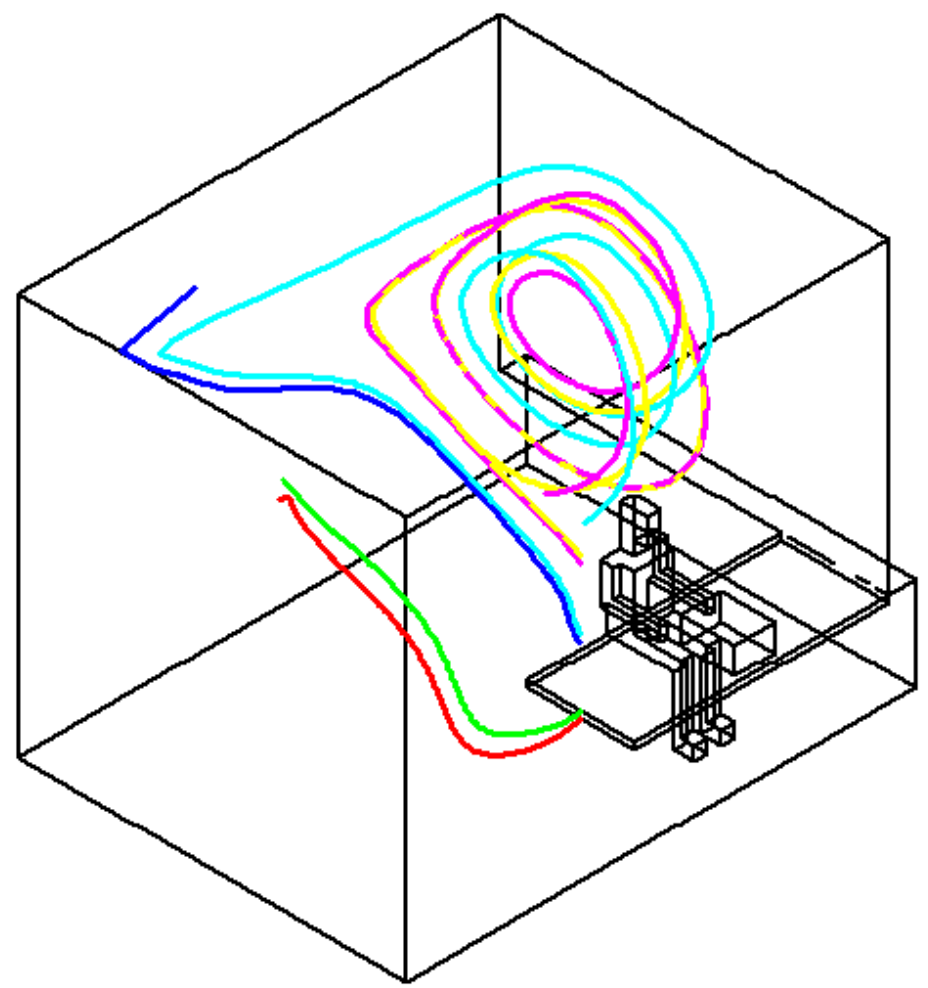

Figure 76a and b. Air flow tracks in the office room with displacement and mixing ventilation. 


\section{Results from the 2:nd case}

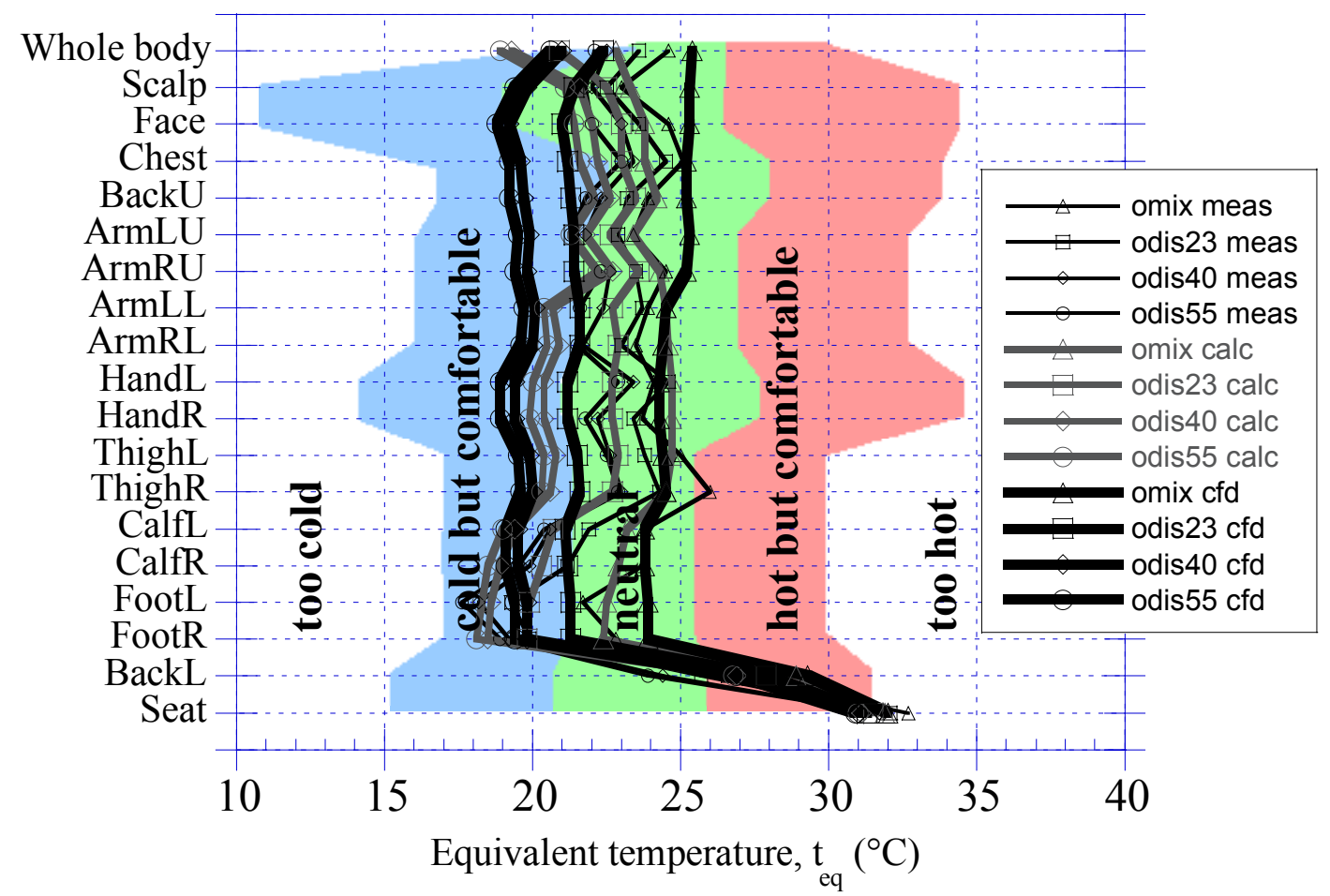

Figure 77. Comfort diagram with all four cases in the office series.

\section{Summary office environment}

In the second case a mixing-ventilated office room has been equipped with displacement-ventilation and evaluated by numerical simulations and supporting measurements with MANIKIN2. The objective of this study was to look at interactions between air movements and thermal comfort conditions in an actual office room.

Calculated results (calc) show good agreement with measurements made in the real environment with a mean deviation of $5 \mathrm{~W} / \mathrm{m}^{2}$. CFD simulations (cfd) on the other hand produces generally higher heat loss values resulting in lower equivalent temperatures, especially on nude body parts as face and hands, this due to a lower simulated air temperature.

MANIKIN3 has no problem simulating the increased insulation of the seat zones supplied by the virtual office chair. The calculated data gave smaller differences compared to data with CFD simulation input. The maximum heat loss deviations for the calculated data where found at the hands, the other zones had good agreement. The mean difference in $t_{e q}$ is still less than the MANIKIN2 maximum calibration deviation (appendix A) even for simulated data. The air speeds show good agreement except for the "omix cfd" case where higher values were simulated. 
Table 39. Summary of calculation and simulation differences compared to measurements for case 2. (k-E model runs within parenthesis.)

\begin{tabular}{|c|c|c|c|c|c|}
\hline $\begin{array}{l}\text { Heat loss } \\
\left(\mathrm{W} / \mathrm{m}^{2}\right) \\
\text { Zone }\end{array}$ & $\begin{array}{c}\text { case } 2 \\
\text { calc } \\
\text { mean diff. }\end{array}$ & $\begin{array}{c}\text { case } 2 \\
\text { cfd } \\
\text { mean diff. }\end{array}$ & $\begin{array}{l}\text { Equivalent } \\
\text { temperature } \\
\left({ }^{\circ} \mathrm{C}\right) \\
\text { Zone }\end{array}$ & $\begin{array}{c}\text { case } 2 \\
\text { calc } \\
\text { mean diff. }\end{array}$ & $\begin{array}{c}\text { case } 2 \\
\text { cfd } \\
\text { mean diff. }\end{array}$ \\
\hline Max & 16 & $23(54)$ & $\overline{M a x}$ & 2.7 & 2.9 (11.9) \\
\hline Mean & 5 & $9(16)$ & Mean & 1.1 & $1.8(3.3)$ \\
\hline Min & 1 & 1 (2) & Min & 0.1 & $0.2(0.3)$ \\
\hline
\end{tabular}




\section{Case 3: The cabin simulator}

\section{Introduction}

This investigation was a part of a research project that investigated how the use of special glazing can improve the thermal climate in a vehicle cabin (Nilsson et al., 1999b, Bohm et al., 2002). The two main reasons for thermal asymmetries in the cabin are the windows and high heating or cooling power required by the HVAC-system to obtain the right cab temperature. In sunny summer conditions the drivers are often exposed to severe radiant loads from the sun, which have to be compensated for with low air temperature in the cab. In winter conditions surface temperatures on the inner side of the glass is low, causing high radiant heat loss from the driver. These heat losses have to be compensated by high air temperatures in the cab. The high power required to heat or cool the cab air to optimal temperature considerably increases the asymmetries. This combined with the asymmetric radiant load on the driver from the sun or cold windows; the situation can become very complex. The purpose of this investigation was to make CFD calculations as well as full scale measurements with a thermal manikin and try to predict the perceived climate in the very complex thermal environment of vehicle cabin.

\section{Real and virtual cabin}

In this study a small climatic test cabin placed in a larger climatic chamber was used. Four different conditions have been studied both with the manikin and numerical simulations.
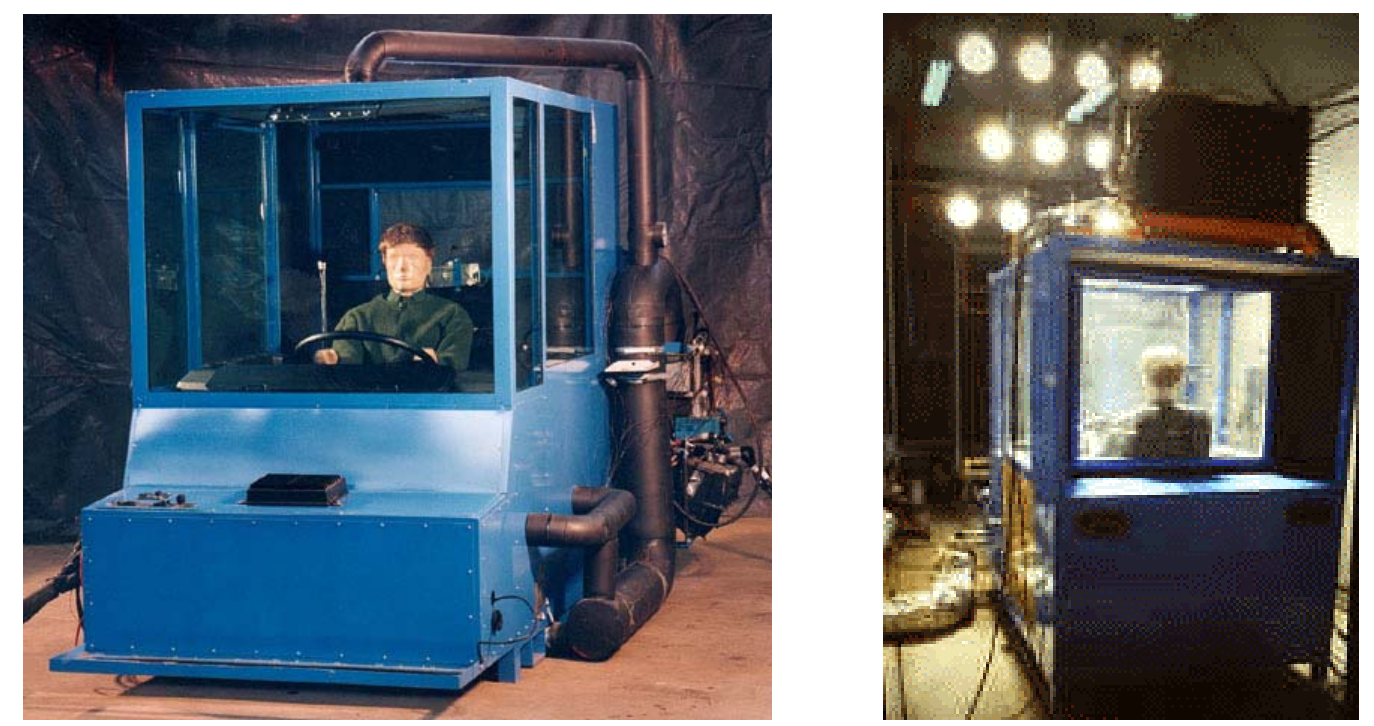

Figure 78. MANIKIN2 inside the cabin simulator exposed to the artificial sun. The two black outlets are visible on the lower back wall. 
The manikin is positioned in the cabin simulator inside a climatic chamber. Heat flow in $\mathrm{W} / \mathrm{m}^{2}$ from the different segments of the manikin surface is measured and controlled. Data for the conditions are recorded when manikin has reached heat equilibrium with the environment.
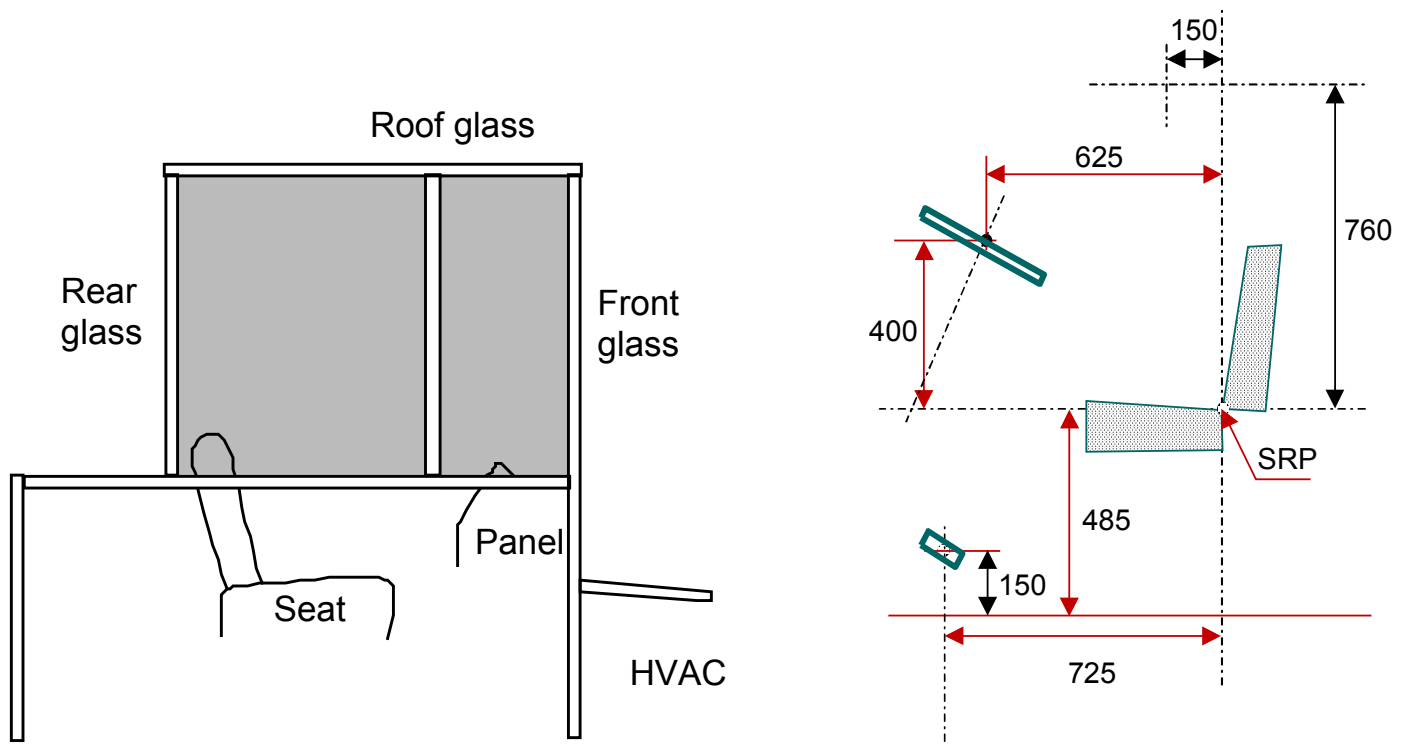

Figure 79. Schematic drawing of the cabin simulator to the left. To the right the measurement points inside the simulator. Seat, wheel and pedals according to ISO 4253. Eye level according to ISO 5721.

\section{Calculation procedure}

The virtual manikin was positioned in geometry of the cabin simulator. The incoming solar radiation varied with the glazing used, and was applied to the roof and front window. The supplied air was distributed through the defroster and panel inlets in the front and at the doors.

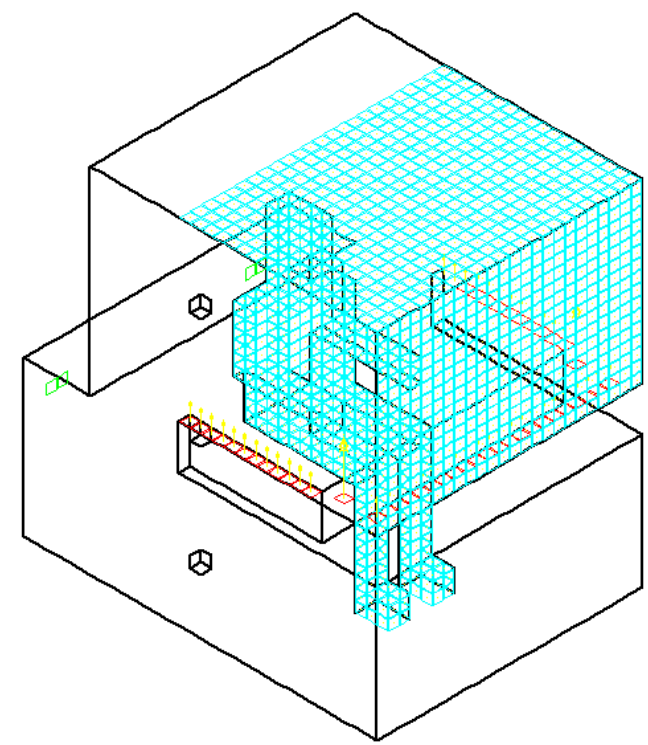

Figure 80. The geometry of the virtual cabin. The panel inlets at the front left and right, and the defroster inlets at the front and doors. The front and roof windows have elevated temperatures due to solar heat absorption. 
The supplied air temperature and normal velocity at the inlets was measured and set according to the measured data. The two outlets were positioned at the back wall, se figure. Boundary conditions for the CFD calculations are shown in the tables 40, 41 and 42 below.

\section{Results from the cabin simulator (3:rd case)}

Temperatures at the centre of the cabin as well as at the different inlets and outlets were measured continuously during the measurements:

cclearg: Clear glass, no sun radiation.

cclearp: Clear polycarbonate, sun radiation.

ccolop: Coloured polycarbonate, sun radiation.

creflg: Glass with reflective sunscreen inside the glass, sun radiation.

Table 40. Heat loss data $\left(\mathrm{W} / \mathrm{m}^{2}\right)$ from a selection of manikin segments

during the exposure to the four different cases. $(\mathrm{L}=\mathrm{Left}, \mathrm{R}=\mathrm{Right})$

\begin{tabular}{|c|c|c|c|c|c|c|c|c|c|c|c|c|c|c|}
\hline $\begin{array}{l}\text { Heat loss } \\
(\mathrm{W} / \mathrm{m} 2) \\
\text { Zone }\end{array}$ & $\begin{array}{c}\text { clearg } \\
\text { meas }\end{array}$ & $\begin{array}{c}\text { clearp } \\
\text { meas }\end{array}$ & $\begin{array}{l}\text { colop } \\
\text { meas }\end{array}$ & $\begin{array}{l}\text { reflg } \\
\text { meas }\end{array}$ & $\begin{array}{l}\text { clearg } \\
\text { calc }\end{array}$ & $\begin{array}{c}\text { clearp } \\
\text { calc }\end{array}$ & $\begin{array}{c}\text { colop } \\
\text { calc }\end{array}$ & $\begin{array}{c}\text { reflg } \\
\text { calc }\end{array}$ & $\begin{array}{c}\text { clearg } \\
\text { cfd }\end{array}$ & $\begin{array}{l}\text { clearp } \\
\text { cfd }\end{array}$ & $\begin{array}{l}\text { colop } \\
\text { cfd }\end{array}$ & $\begin{array}{c}\text { creflg } \\
\text { cfd }\end{array}$ & $\begin{array}{c}\text { calc } \\
\text { mean } \\
\text { diff. }\end{array}$ & $\begin{array}{c}\text { cfd } \\
\text { mean } \\
\text { diff. }\end{array}$ \\
\hline $\begin{array}{l}\text { Whole } \\
\text { body }\end{array}$ & 45 & 44 & 46 & 46 & 41 & 44 & 47 & 47 & 41 & 47 & 32 & 33 & 1 & 8 \\
\hline Scalp & 36 & 16 & 16 & 16 & 30 & 19 & 22 & 22 & 28 & 31 & 20 & 21 & 6 & 8 \\
\hline Face & 86 & 63 & 77 & 87 & 77 & 50 & 63 & 62 & 74 & 86 & 57 & 60 & 15 & 20 \\
\hline Chest & 42 & 20 & 20 & 20 & 35 & 23 & 27 & 27 & 34 & 38 & 25 & 26 & 6 & 9 \\
\hline BackU & 31 & 48 & 47 & 42 & 49 & 32 & 36 & 37 & 48 & 52 & 33 & 35 & 12 & 10 \\
\hline ArmLU & 34 & 24 & 29 & 30 & 38 & 25 & 28 & 28 & 36 & 40 & 26 & 27 & 2 & 6 \\
\hline ArmRU & 36 & 16 & 30 & 28 & 39 & 26 & 27 & 28 & 38 & 41 & 25 & 28 & 4 & 8 \\
\hline ArmLL & 50 & 27 & 38 & 42 & 44 & 57 & 46 & 46 & 44 & 46 & 27 & 30 & 12 & 12 \\
\hline ArmRL & 47 & 19 & 31 & 37 & 44 & 55 & 44 & 44 & 43 & 45 & 25 & 28 & 15 & 11 \\
\hline HandL & 100 & 122 & 116 & 120 & 79 & 112 & 96 & 95 & 78 & 87 & 55 & 59 & 19 & 45 \\
\hline HandR & 96 & 101 & 99 & 106 & 81 & 116 & 100 & 99 & 80 & 90 & 57 & 61 & 9 & 28 \\
\hline ThighL & 50 & 55 & 56 & 57 & 42 & 54 & 43 & 43 & 42 & 43 & 24 & 27 & 9 & 21 \\
\hline ThighR & 53 & 54 & 57 & 57 & 41 & 53 & 42 & 42 & 41 & 42 & 24 & 27 & 11 & 22 \\
\hline CalfL & 46 & 74 & 68 & 65 & 42 & 65 & 61 & 57 & 42 & 62 & 59 & 55 & 7 & 9 \\
\hline CalfR & 35 & 53 & 53 & 49 & 41 & 63 & 60 & 56 & 42 & 61 & 58 & 54 & 7 & 6 \\
\hline FootL & 36 & 54 & 48 & 45 & 32 & 48 & 46 & 43 & 31 & 46 & 43 & 40 & 4 & 6 \\
\hline FootR & 35 & 50 & 46 & 42 & 34 & 51 & 48 & 45 & 33 & 48 & 46 & 43 & 2 & 1 \\
\hline BackL & 24 & 34 & 32 & 31 & 21 & 21 & 13 & 13 & 23 & 22 & 11 & 13 & 13 & 13 \\
\hline Seat & 28 & 40 & 38 & 36 & 42 & 46 & 30 & 31 & 52 & 52 & 28 & 32 & 8 & 12 \\
\hline
\end{tabular}

Table 41. Measured air velocities and temperatures compared to CFD calculated values.

\begin{tabular}{|c|c|c|c|c|c|c|c|c|c|c|c|c|c|c|}
\hline $\begin{array}{l}\text { Air speed } \\
(\mathrm{m} / \mathrm{s}) \\
\text { Position }\end{array}$ & $\begin{array}{c}\text { clearg } \\
\text { meas }\end{array}$ & $\begin{array}{c}\text { clearp } \\
\text { meas }\end{array}$ & $\begin{array}{l}\text { colop } \\
\text { meas }\end{array}$ & $\begin{array}{l}\text { reflg } \\
\text { meas }\end{array}$ & $\begin{array}{c}\text { clearg } \\
\text { calc }\end{array}$ & $\begin{array}{l}\text { clearp } \\
\text { calc }\end{array}$ & $\begin{array}{c}\text { colop } \\
\text { calc }\end{array}$ & $\begin{array}{l}\text { reflg } \\
\text { calc }\end{array}$ & $\begin{array}{c}\text { clearg } \\
\text { cfd }\end{array}$ & $\begin{array}{c}\text { clearp } \\
\text { cfd }\end{array}$ & $\begin{array}{c}\text { colop } \\
\text { cfd }\end{array}$ & $\begin{array}{c}\text { creflg } \\
\text { cfd }\end{array}$ & $\begin{array}{c}\text { calc } \\
\text { mean } \\
\text { diff. }\end{array}$ & $\begin{array}{c}\text { cfd } \\
\text { mean } \\
\text { diff. }\end{array}$ \\
\hline$\overline{\text { Air } 0.1}$ & 0.20 & 0.20 & 0.20 & 0.20 & 0.20 & 0.20 & 0.20 & 0.20 & 0.24 & 0.26 & 0.27 & 0.26 & 0.00 & 0.06 \\
\hline Air 0.6 & 0.30 & 0.30 & 0.30 & 0.30 & 0.30 & 0.30 & 0.30 & 0.30 & 0.66 & 0.66 & 0.66 & 0.67 & 0.00 & 0.36 \\
\hline Air 1.1 & 0.60 & 0.60 & 0.60 & 0.60 & 0.60 & 0.60 & 0.60 & 0.60 & 0.69 & 0.69 & 0.69 & 0.69 & 0.00 & 0.09 \\
\hline
\end{tabular}


“cclearg" Temperature field

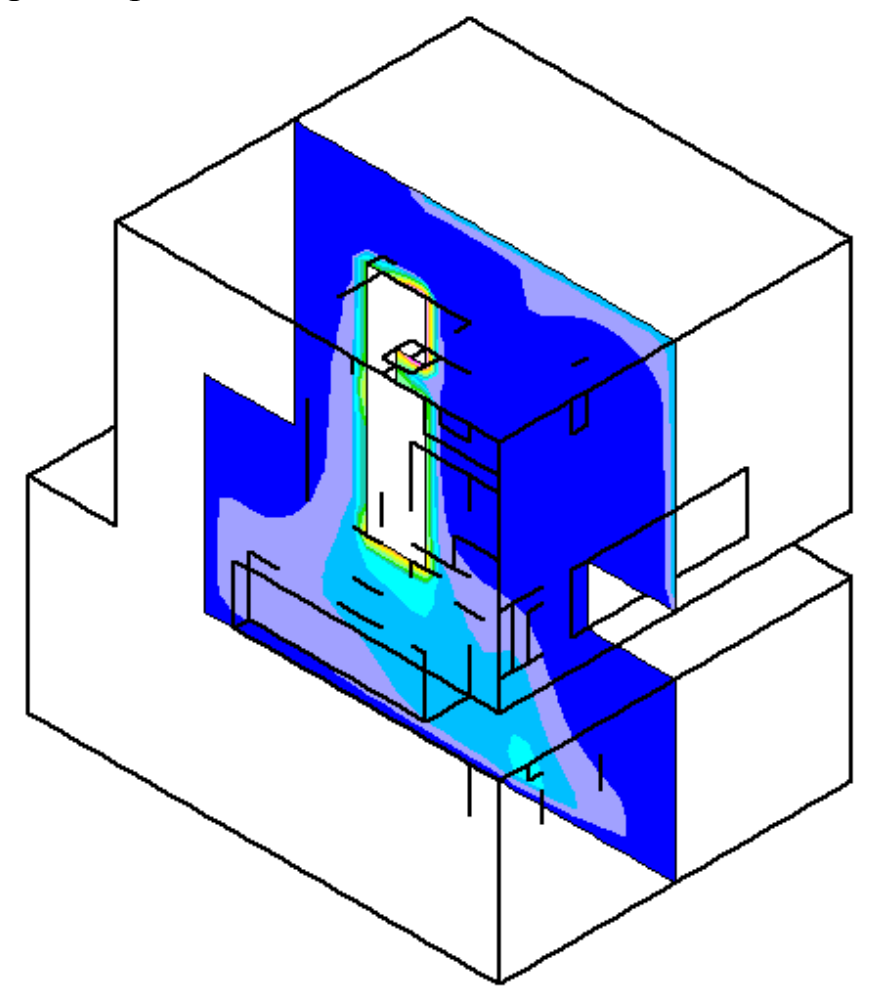

TEMPERATURE

ABSOLUTE

KELVIN

LOCALMX $=306.5$
LOCALMN $=298.9$

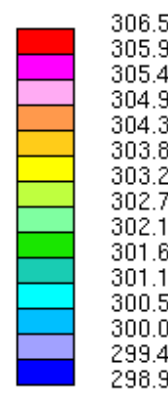

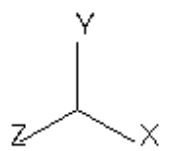

“creflg" Temperature field

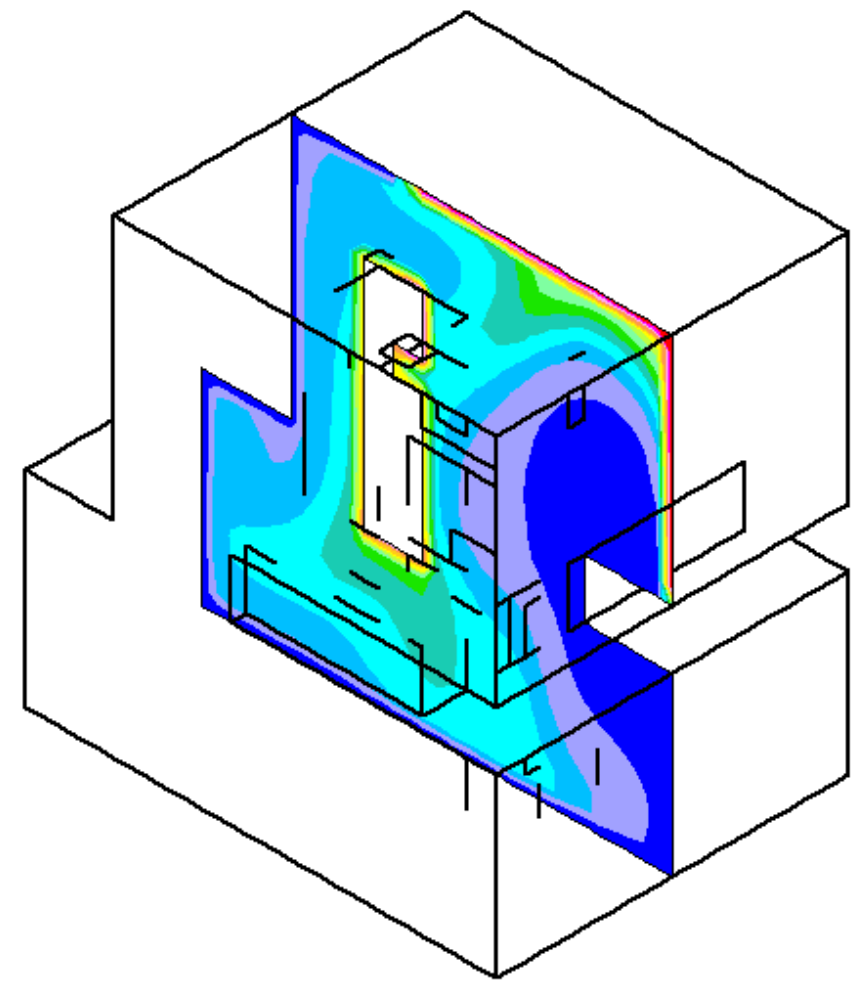

TEMPERATURE

KELVIN

LOCALMX $=307.3$

LOCALMN $=296.3$
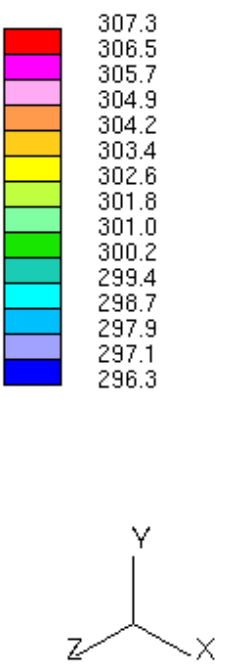

Figure 81. The temperature patterns in the cabin for the "cclearg" case with no sun and "creflg" case with sun. Note the heating effects from the windows. 


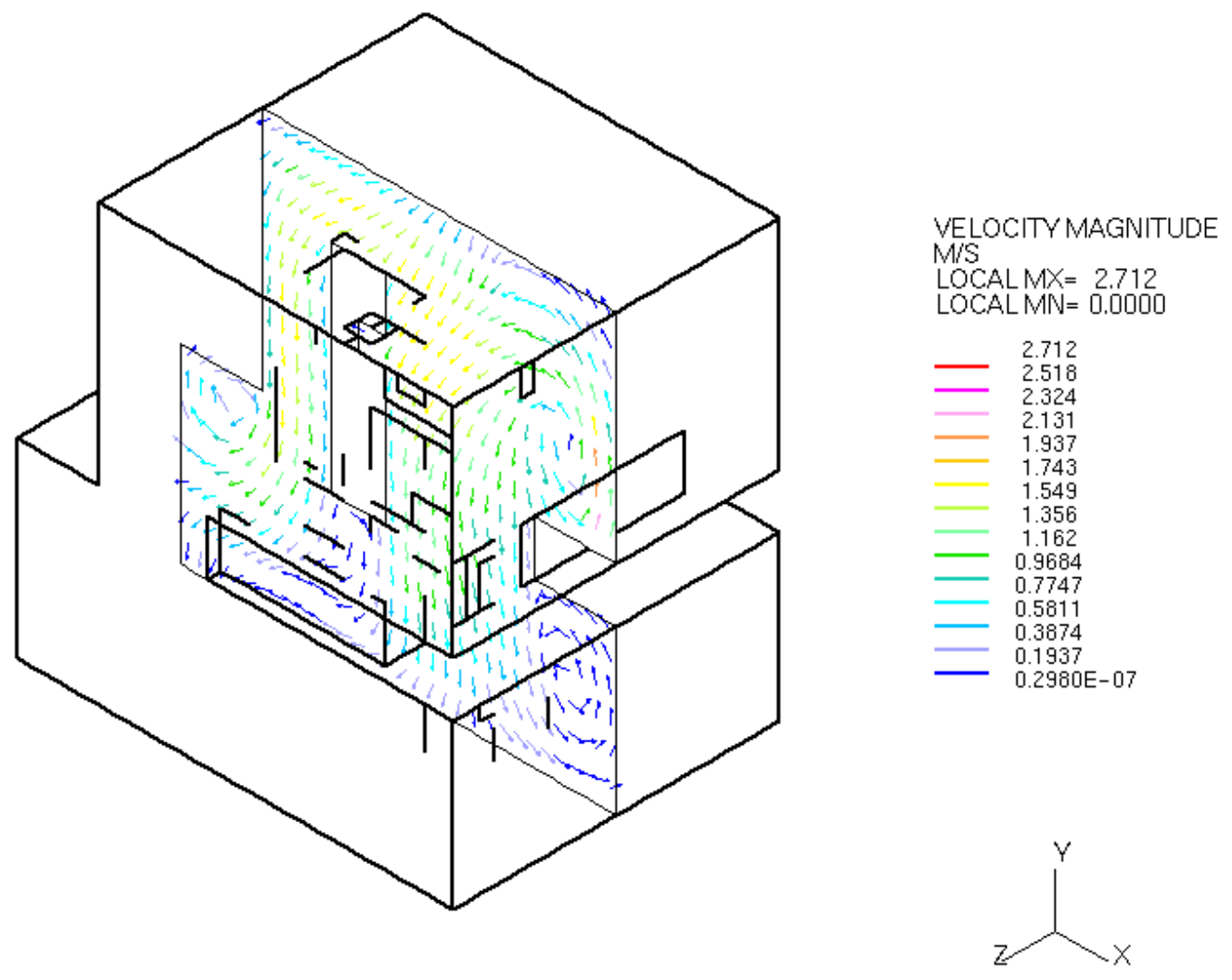

Figure 82. The flow patterns in the cabin shown as velocity vectors in a plane at the manikin centre for the "cclearg" case.

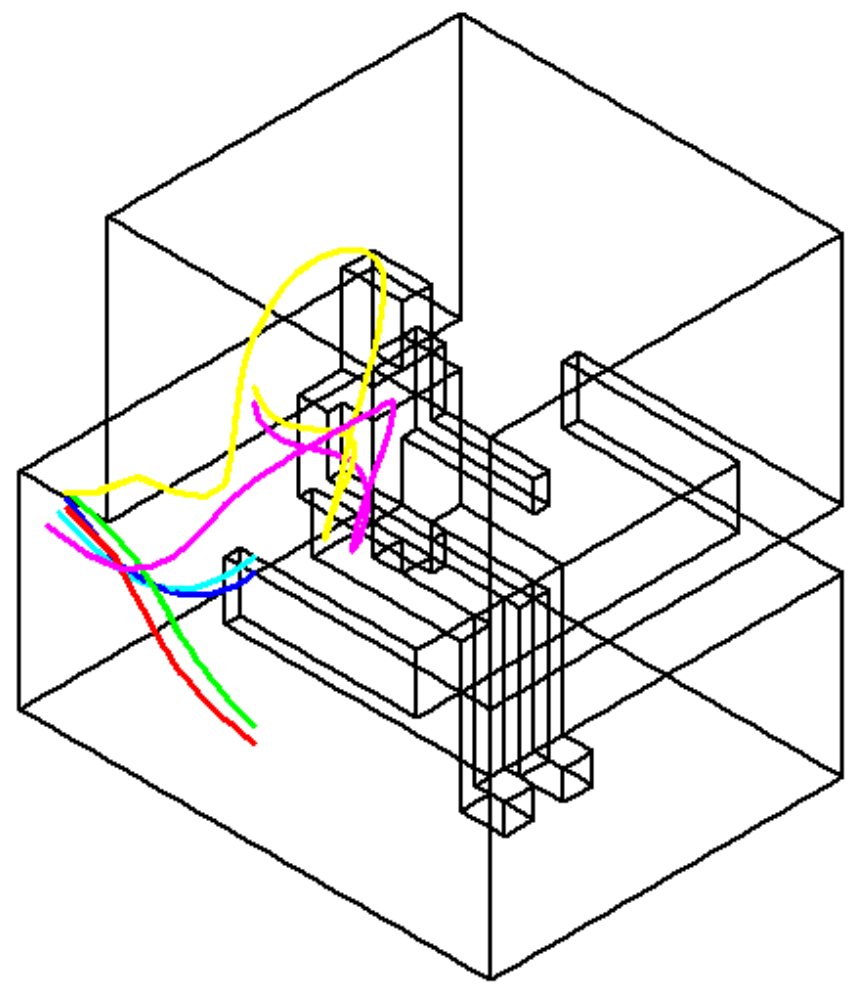

Figure 83. Typical flow lines released from the sensors and exiting through the outlet for the "cclearg" case. 
Table 42. Measured temperature data together with data from the CFD calculations.

\begin{tabular}{lcccccccccccccc}
\hline $\begin{array}{l}\text { Temp } \\
\text { erature } \\
\left({ }^{\circ} \mathrm{C}\right)\end{array}$ & $\begin{array}{c}\text { clearg } \\
\text { meas }\end{array}$ & $\begin{array}{c}\text { clearp } \\
\text { meas }\end{array}$ & $\begin{array}{c}\text { colop } \\
\text { meas }\end{array}$ & $\begin{array}{c}\text { reflg } \\
\text { meas }\end{array}$ & $\begin{array}{c}\text { clearg } \\
\text { calc }\end{array}$ & $\begin{array}{c}\text { clearp } \\
\text { calc }\end{array}$ & $\begin{array}{c}\text { colop } \\
\text { calc }\end{array}$ & $\begin{array}{c}\text { reflg } \\
\text { calc }\end{array}$ & $\begin{array}{c}\text { clearg } \\
\text { cfd }\end{array}$ & $\begin{array}{c}\text { clearp } \\
\text { cfd }\end{array}$ & $\begin{array}{c}\text { colop } \\
\text { cfd }\end{array}$ & $\begin{array}{c}\text { creflg } \\
\text { cfd }\end{array}$ & $\begin{array}{c}\text { calc } \\
\text { mean } \\
\text { diff. }\end{array}$ & $\begin{array}{c}\text { cfd } \\
\text { mean } \\
\text { diff. }\end{array}$ \\
\hline Defroster & 22.3 & 19.2 & 11.9 & 14.9 & 22.3 & 19.2 & 11.9 & 14.9 & 22.3 & 19.2 & 11.9 & 14.9 & 0.0 & 0.0 \\
Front Win. & 26.9 & 29.9 & 34.7 & 34.3 & 27.1 & 30.2 & 35.9 & 34.1 & 27.1 & 30.2 & 35.9 & 34.1 & 0.5 & 0.5 \\
Roof Win. & 27.3 & 30.5 & 37.0 & 33.9 & 27.1 & 30.2 & 35.9 & 34.1 & 27.1 & 30.2 & 35.9 & 34.1 & 0.5 & 0.5 \\
Air 0.1 & 25.7 & 21.4 & 22.1 & 22.9 & 25.7 & 21.4 & 22.1 & 22.9 & 26.3 & 22.9 & 23.6 & 24.3 & 0.0 & 1.2 \\
Air 0.6 & 26.1 & 20.5 & 16.1 & 16.4 & 26.1 & 20.5 & 20.1 & 20.4 & 26.3 & 23.6 & 24.7 & 25.2 & 0.0 & 3.2 \\
Air 1.1 & 25.1 & 27.4 & 21.9 & 23.8 & 25.1 & 27.4 & 21.9 & 23.8 & 25.9 & 22.7 & 23.7 & 24.3 & 0.0 & 1.9 \\
\hline
\end{tabular}

\section{Results from the 3:rd case}

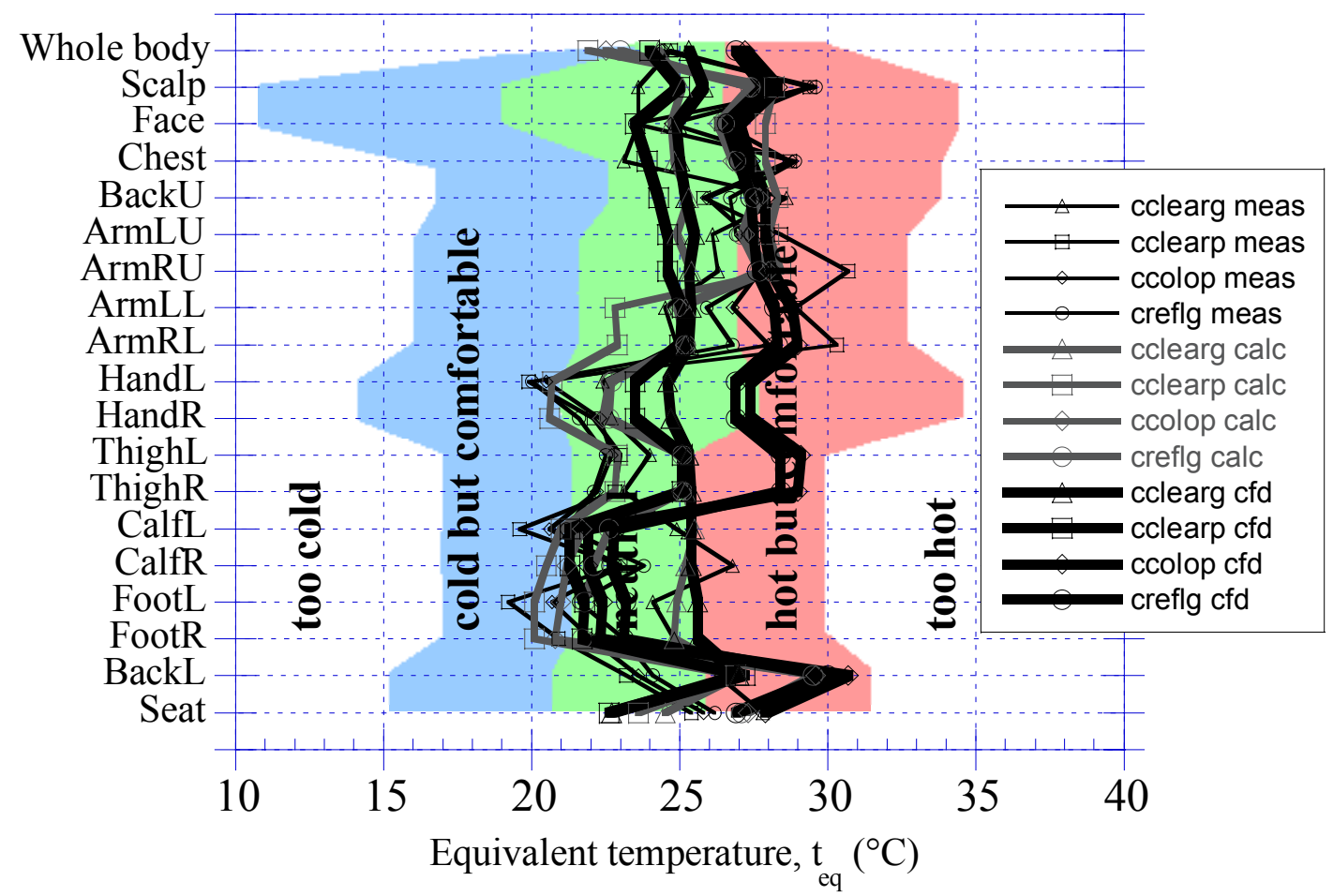

Figure 84. Data from the manikin clearly shows that high air velocities do not influence the scalp negatively in the case with high air temperature (cclearg). But in the cases with solar radiation (dotted line) the lover air temperature increases the heat loss from the shadowed scalp and decreases the heat loss from the chest caused by the sun.

All cases produce a quite high air velocity. That is acceptable in the case with no sun, but when the sun load increases and the temperature on the incoming air goes down the air streams that goes directly to the chest and head becomes significant. This can in the normal case be adjusted by the driver and/or his climate system during the exposure. 


\section{Summary the cabin simulator}

The third study was part of a project that investigated if the use of special glazing can improve the thermal climate in a vehicle cabin. The two main reasons for thermal asymmetries in the cabin are the windows and high cooling power required by the HVAC-system to obtain the right cab temperature. Severe radiant loads from the sun have to be compensated by low supply air temperatures to the cab. This situation considerably increases the thermal asymmetries and makes the situation very complex.

The calculated results from this study show relatively good agreement with the measurements made with MANIKIN2 in the real environment. The maximum heat loss deviations for the calculated data where found at the hands and the zones at the middle of the manikin. MANIKIN3 simulates the seat zone of the ventilated seat well, only the lower back zone gives a slightly higher value. CFD results with MANIKN3 produces higher equivalent temperatures at the middle zones due to higher simulated air temperatures around the manikin. The influence of the higher air velocity at the same level does not compensate for this. The mean difference in $t_{e q}$ is however also in this case less than the maximum $3.3^{\circ} \mathrm{C}$ (appendix A).

The simulated air velocities show good agreement in the high and low regions. All conditions produce a quite high air velocity. That is acceptable in the condition with no sun, but when the sun load increases and the temperature on the incoming air goes down the air streams that goes directly to the head and neck becomes significant. The driver and/or his climate system during the exposure can, in the ordinary situation, adjust this.

Table 43. Summary of calculation and simulation differences compared to measurements for the 3:rd case. ( $k-\varepsilon$ model runs within parenthesis.)

\begin{tabular}{|c|c|c|c|c|c|}
\hline $\begin{array}{l}\text { Heat loss } \\
\left(\mathrm{W} / \mathrm{m}^{2}\right) \\
\text { Zone }\end{array}$ & $\begin{array}{c}\text { case } 3 \\
\text { calc } \\
\text { mean diff. }\end{array}$ & $\begin{array}{c}\text { case } 3 \\
\text { cfd } \\
\text { mean diff. }\end{array}$ & $\begin{array}{l}\text { Equivalent } \\
\text { temperature } \\
\left({ }^{\circ} \mathrm{C}\right) \\
\text { Zone }\end{array}$ & $\begin{array}{c}\text { case } 3 \\
\text { calc } \\
\text { mean diff. }\end{array}$ & $\begin{array}{c}\text { case } 3 \\
\text { cfd } \\
\text { mean diff. }\end{array}$ \\
\hline Max & 19 & $45(27)$ & Max & 3.9 & $4.9(5.4)$ \\
\hline Mean & 9 & $13(15)$ & Mean & 1.7 & $2.5(3.1)$ \\
\hline Min & 1 & $1(5)$ & Min & 0.5 & 0.5 (1.4) \\
\hline
\end{tabular}




\section{Discussion and conclusions}

This research aims to complement difficult, time-consuming, expensive full-scale subjective and other experimental investigations with computer-based alternatives in order to determine human thermal comfort in different environments. Today CFD (Computational Fluid Dynamics) is applied to a vast range of climate situations. In the building and vehicle industries in particular, computers are increasingly being used to carry out all parts of the design process. Traditionally, the use of CFD has been thought of as a specialist or research application, but with the advanced capacity of modern personal computers it is now possible for architects and engineers to make realistic simulations on their desktops.

A systematic numerical evaluation of different systems is expected to give new ideas and guidelines for improved system solutions. The flexibility and rationality of computer methods and simulation tools will be useful in generalising results from one environment to the other. It is important to be able to explain the differences between good and bad system solutions and to inform target groups. There is a need for useful models for visualisation, design and quality assurance, and for results that are useful in building science and business.

Unfortunately, too few of the theories behind the simulations are currently available in the public domain. Many of the leading researchers and companies still use in-house codes for all or essential parts of the calculations. In this thesis, calculations and CFD simulations have been compared with measurements made in real, full-scale environments. Two commercial codes have been used, CFX ${ }^{\circledR}$ and Star $\mathrm{CD}^{\circledR}$. These codes allow implementation of special sub-routines for advanced boundary treatment. Effort has been put into reviewing and explaining the author's as well as other researchers' simulations and theories. New methods and codes have been developed. A virtual thermal manikin, MANIKIN3, has been constructed. The background theories and all codes used are fully available in this thesis for further development and evaluation.

Numerical and experimental methods need to be further developed. The computer should become an efficient tool for visualising complicated thermal comfort problems, developing new ventilation strategies or designing new systems. With more research and experience, the tools and methods for simulation of thermal environment conditions can be continuously improved. In the future, these kinds of calculations can enable engineers to make better predictions and early decisions in the design and construction process. It also opens possibilities to interpolate results from a reduced number of fullscale tests with manikins and/or subjects.

\section{Measurements, calculations and CFD}

Results from measurements with a full-scale thermal manikin, MANIKIN2, were compared to model calculations and CFD simulations. The heat loss and temperature of the manikin influenced the air movements around the body. Thermal interaction with 
walls, ventilation and the seat influenced the manikin's thermal situation. When this information was linked together with models for human thermal sensation, valuable knowledge about the thermal influence of the environment was obtained. This information was visualised in new "comfort zone diagrams", showing how an average human being in the same position would perceive the climate. These zones can be adapted to different types of clothing, in clothing-independent comfort zone diagrams, and can form a good basis for comparison of results between different manikins.

\section{Measurements}

For engineering purposes the "comfort" sensation has to be converted to and expressed in measurable, physical quantities. For the whole body sensation, this can be done with well-known standards with reasonable accuracy for relatively homogenous, indoor environments. However, in many cases the climate is far from uniform and considerable local thermal effects must be visualised and evaluated. This can readily be done using the concept of equivalent temperature $\left(t_{e q}\right)$. The major advantage of $t_{e q}$ is that it expresses the combined effects of thermal influences in a single figure, easy to interpret and explain. The variables behind $t_{e q}$, such as surface temperature or area, are scalars. Furthermore, as a result of the radiative heat transfer component, the full description of equivalent temperature contains not only its magnitude but also the direction, emanating from the interaction between the ambience and the sensor geometry used. Equivalent temperature can hence be defined as a vector. This fact can, in the future, be used to in the construction of more efficient computer simulations and also give better understanding to $t_{e q}$ measurement instrument differences, making result comparisons easier.

Scales, like the ASHRAE/PMV scale, have to be related to an acceptability scale in order to accomplish the same objective as with the MTV scale, where subject acceptability is included. By relating votes from the PMV or ASHRAE scale to an acceptability scale, a similar result can be achieved as with the MTV method. Consequently, the MTV scale is a good choice, especially when many local ratings have to be collected. The degree of thermal discomfort can also be expressed as "percentage dissatisfied". The construction of the MTV scale has made it possible for the subjects to show that the climate is acceptable. From the database of MTV, the PD (Percentage Dissatisfied: the percentage of subjects who find the thermal situation unacceptable) has been determined. Still, we cannot escape the fact that when persons are uncomfortable they tend to complain, so if satisfactory HVAC installations are to be designed, attention has to made to personal sensations of thermal comfort as well as possibilities for individual variation.

Equivalent temperature values obtained with different manikins in different test houses should be comparable during the same test conditions. To use climate evaluation limit lines is, as a consequence of methodological and individual variations, not the optimal solution. A more reasonable way to evaluate the thermal climate is to give a range of $t_{e q}$ values. When comfort zones are introduced, the differences between measurements with different manikins become marginal. This improvement establishes a more realistic base for the judgement of a complicated local thermal climate, and opens up for a general profile usable with different manikins, possibly also different 
methods, in various environments. However, more data from validation experiments with subjects and different methods will contribute to the development of this more general evaluation criterion.

Thermal manikin measurements of local climate disturbances with two different thermal manikins were found to be well correlated with the thermal sensation experienced by panels of subjects exposed to the same conditions. This study comprised $t_{e q}$ values from 10 to $35^{\circ} \mathrm{C}$ (MTV from -2 to +2 ). In another study, subjects were exposed to very warm to hot conditions with $t_{e q}$ values ranging from 20 to $45^{\circ} \mathrm{C}$ (LMV from -1 to +3 ). In this study the measurement of equivalent temperature was substituted for the surface temperature when zero or negative heat flux occurred at the warmest conditions. It is very encouraging for the proposed method that a good correlation was found between subjective and objective assessments in both studies.

The important calibration is carried out with the thermal manikin dressed in standard clothing. Clothing affects the values of the heat transfer coefficients. The less insulation clothing provides, the more sensitive the manikin segments will be to thermal influences. It is consequently very important to specify the clothing used, whenever data are compared from different investigations and manikins. This comparison can be facilitated with the use of "clothing-independent" comfort zone diagrams. It is therefore suggested that the comfort zone diagrams not only should be manikin/method independent but also clothing-independent. The concept of clothing independence assumes that the human being is equally sensitive to different heat losses independent of the insulation of the clothing worn. The new clothing-independent comfort zone diagrams have been constructed by calculating a mean acceptable heat loss. As expected, the comfort zone diagram with less clothing indicates increased sensitivity in all zones, except the normally unclothed face and hands. The opposite, decreased sensitivity, can be observed for the comfort zone diagram with increased clothing insulation.

In this thesis, studies of different seats have been carried out. In the case of comparison of seat insulation, similar manikins also seemed to be interchangeable. In spite of the different makes and sizes of the manikins, the total insulation results showed no difference when several conditions were compared. This conforms well to earlier findings of small differences between manikins of different makes and sizes.

Measurements of ordinary seats, like a net chair and a wooden stool, both gave a slightly decreased insulation, relative to the "No seat" condition. This can be explained by the fact that they act as cooling flanges and carry the heat out by their larger conductivity. A three-seat sofa had an increased insulation with occupants on both sides of the manikin compared to the manikin sitting alone in the sofa. The situation sofa sitting has often been overlooked in many cases using the ISO 7730 standard. Some calculations were made in order to predict a possible error due to increased insulation. The conditions were an operative temperature of $22{ }^{\circ} \mathrm{C}$, a relative humidity of $50 \%$ with an air velocity of $0.1 \mathrm{~m} / \mathrm{s}$ and a metabolism of $1.2 \mathrm{Met}$. This resulted in a PMV of 0.4 for the "No seat" condition. In order to get the same PMV in the occupied 3-seat sofa, the operative temperature would have to be $1.5^{\circ} \mathrm{C}$ lower.

Ventilation of the seat gave a decreased insulation relative to the "No seat" condition. This showed how ventilation made the seat transparent to heat. The fan worked as a transporter of heat through the seat. One way of showing the effect of increased seat 
ventilation is by reporting the temperature decrease on the seat fabric of between 0.4 and $2.6^{\circ} \mathrm{C}$. Another way of showing the effects is to study the heat losses from different zones during the different conditions. In between the two extreme conditions, the possible increase in ambient temperature with preserved heat loss was 1.5 to $1.9^{\circ} \mathrm{C}$. The heat loss was redistributed from zones in contact with the ambient air to zones with seat contact. Some kind of transfer effect, between zones in contact with the seat and those in contact with ambient air, can be the reason for correlation differences. Since ambient air temperature affects the cooling capacity of the seat ventilation, the influence on the larger portion of body surface is turned towards the ambience. Having in mind the human habit of accepting elevated seat zone temperatures also points to the conclusion that the heat loss changes from the seat zones have been suppressed. Since several subjects complained of difficulties when rating with the relatively coarse MTV scale perhaps "half steps" should be introduced for these types of studies.

\section{Calculations and CFD simulations}

Today CFD simulations have developed and a growing field of research is working to establish the methods for simulation of the human thermal environment. The continuous development of computer capacity has certainly improved the possibilities of use for this type of simulation. Taking this into account, there are still too many unexplained differences in the results within and between simulation methods, pointing out several limitations of the CFD methods currently available. This shows the need for a continued validation of CFD results with real life measurements of human beings.

As several measurements with full size manikins as well as human subjects had been performed, it was decided to test these data against computer simulations. A virtual MANIKIN3 of cubical shape was formed with the corresponding size, areas and number of zones as the real MANIKIN2. The modelling had steady state characteristics and was aimed at the assessment of human thermal comfort. Virtual calibrations were made for the situations when MANIKIN3 was clothed in the same summer and winter clothing that was used for the measurements with the physical MANIKIN2. This was done in order to get the right computational $h_{\text {teq }}$ for the three virtual cases. Results from virtual calibrations with summer (s) and winter (w) clothing showed very good results.

With unclothed calibrations made with MANIKIN2 in ambient temperatures reaching from 19 to $28^{\circ} \mathrm{C}$, a set of new heat transfer equations has been developed. These equations form the bases for the imitation of the heat transfer interface of a manikin. They are used in two ways: first, as ordinary equations for the calculation (calc) of local heat losses and equivalent temperatures with measured data as input; secondly, as dynamic boundaries for the virtual manikin with data from the CFD simulations (cfd) as input. These theories were validated with three well-defined test cases of increasing complexity.

All cases were first simulated with standard k- $\varepsilon$ turbulence modelling. Due to problems with grid adaptation, long calculation times as well as poor results, the turbulence model was changed to a zero-equation model. These simulations were found to be both faster and in better agreement with measured results.

In the first case study, a displacement ventilated empty room with air and wall temperatures close to $20^{\circ} \mathrm{C}$ was used. The calculated and simulated results from this 
study showed good agreement with the measurements made in the real environment. In homogeneous environments, as in this case, the simulations with MANIKIN3 made good predictions of how a human will perceive the thermal climate. The mean difference in $t_{e q}$ was $0.8^{\circ} \mathrm{C}$ (calc) and $1.2{ }^{\circ} \mathrm{C}(\mathrm{cfd})$, less than the maximum $3.3^{\circ} \mathrm{C}$ that in the worst case could be expected between calibrations with MANIKIN2. The calculated data produced smaller differences compared to data with CFD simulation input.

In the second case study, a mixing-ventilated office room equipped with displacement ventilation was evaluated. Calculated results showed good agreement with measurements made in the real environment. CFD simulations on the other hand produced generally lower equivalent temperatures, especially on nude body parts such as the face and hands, this due to a lower simulated air temperature. MANIKIN3 had no problem simulating the increased insulation of the seat zones supplied by the virtual office chair. The maximum heat loss deviations for the calculated data were found on the hands; the other zones had good agreement. The mean difference in $t_{e q}$ was $1.1^{\circ} \mathrm{C}$ (calc) and $1.8^{\circ} \mathrm{C}$ (cfd), also less than the MANIKIN2 maximum calibration deviation. The air speeds showed good agreement except for the office room with mixed ventilation (omix cfd) where higher values were simulated.

The third case study was a part of a project investigating the use of special glazing to improve the thermal climate in vehicle cabins. The calculated results from this study showed relatively good agreement with the measurements made with MANIKIN2 in the real environment. The maximum heat loss deviations for the calculated data were found on the hands and the zones at the middle of the manikin. MANIKIN3 simulated the seat zone of the ventilated seat well, only the lower back zone gave a slightly higher value. CFD results with MANIKN3 produced higher equivalent temperatures at the middle zones due to higher simulated air temperatures around the manikin. The influence of the higher air velocity at the same level did not compensate for this. The mean difference in $t_{e q}$ in this case was $1.7^{\circ} \mathrm{C}$ (calc) and $2.5^{\circ} \mathrm{C}(\mathrm{cfd})$. The simulated air velocities showed good agreement in the high and low regions. All conditions produced quite high air velocities.

The set of equations used in this thesis gave good agreement with real life measurements in the different environments. The use of input data from CFD calculations produced reasonable results, especially in the cases with relatively homogeneous climate (case 1 and 2), but still have to be further developed in order to be reliable in a preconstruction phase.

A remaining problem is the issue of how to create the right heat transfer from all types of wall boundaries to the fluid in the CFD simulations. In this thesis a number of ways have been recognised, but none provide a general answer to the question.

\section{Methods investigated in research are:}

- Using empirically derived coefficients.

- Adaptation of the node distance to empirical data.

- Adaptation of thermal conductivity and dynamic viscosity in the first grid cell

- Applying special near wall treatment with increased number of cells and low Reynolds-number turbulence modelling.

- In this thesis heat transfer coefficient calculation and virtual calibration together with a zero-equation turbulence model. 
However, none of these methods seems to provide a general and satisfactory solution to the problem. In this thesis a virtual calibration is used in order to even out the geometrical differences between MANIKIN2 and MANIKIN3. This virtual calibration offers a perfect fit between measured and calculated data and serves as a way to make a fuzzy thermal geometry for MANIKIN3.

Other factors like size, posture, positioning, number and location of zones and geometry also influence the measured or calculated $t_{e q}$. In homogenous environments, such as in case 1, the effects were less pronounced. In heterogeneous environments, such as in case 3 , where all climatic parameters were competing to increase or decrease the manikin heat loss, the effects were stronger. The magnitude seemed to be in the order that they have to be considered at least in heterogeneous conditions.

Another reason behind why the above factors caused deviations in measured $t_{e q}$ can be explained by looking at the heat transfer coefficients. MANIKIN2 had variations depending on the size and position of the zone. This was, despite virtual calibration, also seen with MANIKIN3, generating slightly different results. These methods were trying to predict the influence of the ambience with basically similar methods, but with different "eyes", in the sense of sensor geometry and direction.

It is promising that the calculations always produced smaller differences, proving the validity of the developed relationships.

\section{Future manikin research}

The cubical MANIKIN3 might be sufficient for most normal engineering calculations, but it is interesting from a research point of view to further develop the similarities between reality and the CFD model. Modern laser scanning technologies makes it possible to scan manikins with great detail and then convert them into CFD computational grids of different resolution. Cases can be run to find out what level of detail is necessary for sufficient, accurate results.

The point cloud shown in Figure 85 contains more than 35 thousand data points and has to undergo further noise and outlier removal as well as size reduction. Then a meshing of the point cloud data should be made, while preserving a sufficient level of detail in the high-curvature areas, to create an accurate 3D surface for CFD calculations. 


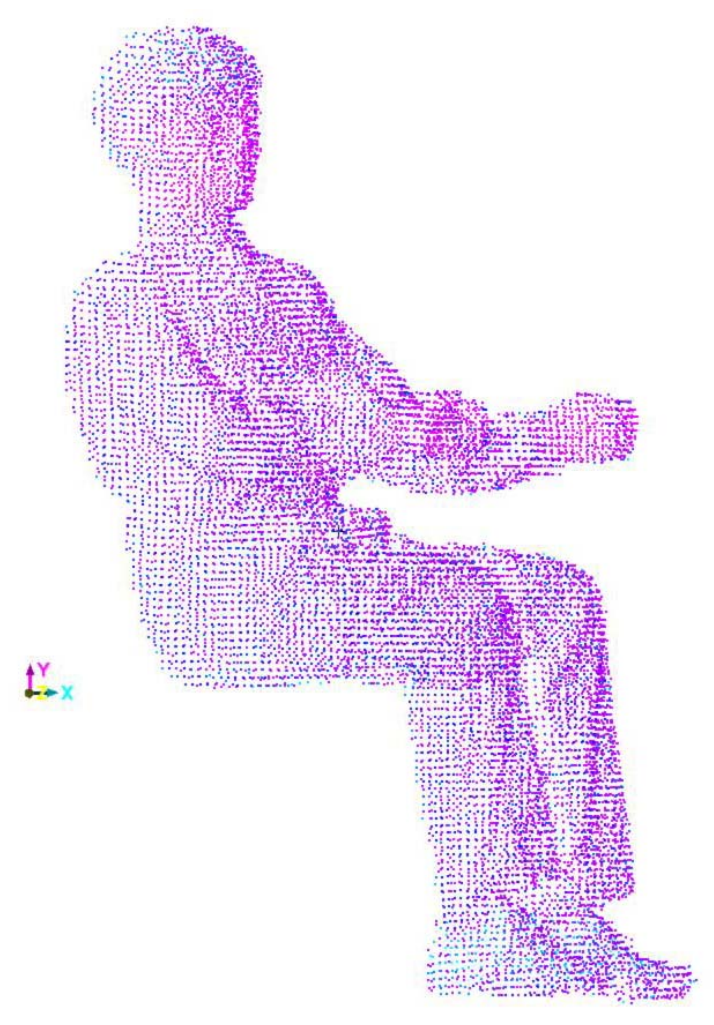

Figure 85. The first picture of MANIKIN4 made with methods for manikin shape reconstruction from point cloud data. Scanned with a CYRA Cyclone (Cyra Technologies Inc., www.cyra.com) at the Department of Technology and Built Environment, University of Gävle.

\section{CFD simulation research}

A remaining problem that requires more investigation is the issue of how to simulate the right heat transfer between all types of wall boundaries and the fluid. In this thesis a number of ways have been recognised, but none provides a general answer to the question. This has to be further addressed in future research, in order to make CFD modelling with better accuracy. A lot of effort is made today with DNS, LES and RANS calculations. This is very interesting from a research point of view, but research is also needed at the other end of the turbulence modelling scale.

\section{Continued seat research}

The seat is an important part of these investigations. More research is needed to study the theories behind seat comfort. We have to bear in mind that a normal seat covers around 20 to $25 \%$ of the human body. A natural continuation of the seat tests is a new study of the seat zones. The measurements should be performed in two different "temperature seats", with capabilities for seat surface temperatures sufficiently separated from the ambient temperature. Some type of simulated driving routine or work routine could also be used in order to come closer to a more realistic work situation. 


\section{Transient conditions}

It is increasingly important that research aiming to develop relationships for simulation of transient situations will be continued. This is certainly a demand from the vehicle industry and is also becoming more and more important in complicated indoor environments. The developments of thermal manikins able to handle fast thermal transient conditions have already started. Simulation models have been moving in this direction for decades. Still there is a need for the research community to carry out more tests with human subjects in order to investigate the correlation between subjective feelings and equivalent temperature. 


\section{Main findings and conclusions}

- For engineering purposes the "comfort" sensation has to be converted to and expressed in a measurable, physical quantity. In this thesis equivalent temperature $\left(t_{e q}\right)$ was chosen because the major advantage is that it expresses the combined effects of thermal influences in a single figure, easy to interpret and explain.

- Thermal manikin measurements of local climate disturbances with two different thermal manikins were found to be well correlated with the thermal sensation experienced by panels of subjects exposed to the same conditions.

- Equivalent temperature values obtained with different manikins in different test houses should be comparable during the same test conditions. A reasonable way to evaluate the thermal climate is to give a range of $t_{\text {eq }}$ values, a comfort zone.

- Thermal interaction with walls, ventilation and seat influence the manikin's thermal situation. When this information is linked with models for human thermal sensation, valuable knowledge about the thermal influence of the environment is obtained. This information was visualised in new "comfort zone diagrams", showing how an average human being in the same position would perceive the climate.

- The comfort zone diagram is a more realistic base for judgement of a complicated local thermal climate, and opens up for a general profile usable with different manikins and different methods in various environments. However, more data from validation experiments with subjects and different methods will contribute to the development of this more general evaluation criterion.

- The less insulation clothing provides, the more sensitive the manikin segments will be to thermal influences. Consequently, it is very important to specify the clothing used, whenever data are compared from different investigations and manikins. $A$ new idea is that comfort zones can be adapted to different types of clothing in "clothing-independent" comfort zone diagrams. It is therefore suggested that the comfort zone diagrams not only should be manikin/method independent but also clothing independent.

- As a result of the radiative heat transfer component, the full description of equivalent temperature contained not only its magnitude but also the direction, emanating from the interaction between the ambience and the sensor geometry used. Equivalent temperature can hence be defined as a vector. This fact can, in the future, be used to both make more efficient computer codes and also increase the understanding of $t_{e q}$ measurement instrument differences, facilitating comparison of results. 
- In this thesis, studies of different seats were carried out. In the case of comparison of seat insulation, similar manikins also seemed to be interchangeable. In spite of the different makes and sizes of the manikins the total insulation results showed no differences. This conforms well to earlier findings of none or small differences between manikins of different makes and sizes.

- The situation sofa sitting has often been overlooked in many cases using the ISO 7730 standard. Calculations were made in order to predict a possible error due to increased insulation. In order to get the same PMV in the occupied 3-seat sofa, the operative temperature would have to be $1.5^{\circ} \mathrm{C}$ lower.

- Ventilation of the seat gave a decreased insulation relative to the "No seat" condition. This showed how ventilation makes the seat transparent to heat. The fan worked as a transporter of heat through the seat. In between two extreme conditions the possible increase in ambient temperature with preserved heat loss was 1.5 to $1.9^{\circ} \mathrm{C}$. The heat loss was then redistributed from zones in contact with the ambient air to zones with seat contact.

- This kind of seat heat transfer effect can be the reason for some correlation differences. Since the ambient air temperature affects the cooling capacity of the seat ventilation, the influence on the larger portion of body surface is turned towards the ambience. Having in mind the human habit of accepting elevated seat zone temperatures also adds to the conclusion that the heat loss changes from the seat zones can be suppressed by heat loss changes from the ambience.

- The simulations in this research aim to supplement experimental investigations in order to determine human thermal comfort in different environments. New methods and codes have been developed. A virtual thermal manikin, MANIKIN3, has been constructed.

- With unclothed calibrations made with MANIKIN2 in ambient temperatures reaching from 19 to $28^{\circ} \mathrm{C}$, a set of new heat transfer equations has been developed. These equations form the bases for the imitation of the heat transfer interface of a manikin. They are used in two ways: first, as ordinary equations for calculation of local heat losses and equivalent temperatures with measured data as input; secondly, as dynamic boundaries for the virtual manikin with flow field data from the CFD simulations as input.

- In the first case study a displacement ventilated empty room with air and wall temperatures close to $20^{\circ} \mathrm{C}$ was used. The calculated and simulated results from this study showed very good agreement with the measurements made in the real environment. In a homogeneous environment, as in this case, the simulations with MANIKIN3 made good predictions of how a human will perceive the thermal climate. The calculated data produced slightly smaller differences compared to data with CFD simulation input. 
- In the second case a mixing-ventilated office room equipped with displacementventilation was evaluated. Calculated results showed good agreement with measurements made in the real environment. CFD simulations on the other hand produced generally lower equivalent temperatures, especially on unclothed body parts such as face and hands, this due to a lower simulated air temperature.

MANIKIN3 had no problem simulating the increased insulation of the seat zones supplied by the virtual office chair. The maximum heat loss deviations for the calculated data were found on the hands, the other zones had good agreement.

- The third case study was a part of a project investigating the use of special glazing to improve the heterogeneous thermal climate in vehicle cabins. The calculated results from this study showed relatively good agreement with the measurements made with MANIKIN2 in the real environment. The maximum heat loss deviations for the calculated data were found on the hands and the zones at the middle of the manikin. MANIKIN3 simulated the seat zone of the ventilated seat well, only the lower back zone gave a slightly higher value. CFD results with MANIKN3 produced higher equivalent temperatures at the middle zones due to higher simulated air temperatures around the manikin.

- All cases were first simulated with standard k- $\varepsilon$ turbulence modelling. Due to problems with grid adaptation, long calculation times as well as the poor results, the turbulence modelling was changed to a zero-equation model. The zero-equation model simulations were found to be both faster and show better agreement with measured results. 


\section{Original articles}

Below a list of articles, papers and reports that forms the basis for this work.

Peer reviewed scientific journal articles (2):

Nilsson HO (2004). Evaluation and visualisation of perceived thermal conditions. European Journal of Applied Physiology (Springer-Verlag, Germany): Special Issue 5I3M (in press).

Nilsson HO, Holmér I. (2003). Comfort climate evaluation with thermal manikin methods and computer simulation models. International journal of indoor air quality and climate (Munksgaard, Denmark): vol 13, pp 28-37.

International conference papers (26):

Håkan O. Nilsson and Ingvar Holmér. (2001) Comfort diagrams for improved evaluation of indoor climate. 4IMM, Fourth International Meeting on Thermal Manikins. Hosted by EMPA, Swiss Federal Laboratories for Materials Testing and Research, Switzerland, 27-28 September, 2001.

H. O. Nilsson and I Holmer, (2001) Evaluation and visualisation of thermal conditions in the modern work place. "Moving Thermal Comfort Standards into the 21st Century" at Cumberland Lodge, Windsor (near London), UK, 5th - 8th April 2001.

H. O. Nilsson, H. Anttonen, I. Holmér. (2000) Methods for cold protective clothing evaluation. "1st European Conference on Protective Clothing". Norra Latin, Stockholm, Arbete och Hälsa 2000:8, p179-182.

H. O. Nilsson, H Anttonen, I. Holmér. (2000) New algorithms for prediction of wind effects on cold protective clothing. "1st European Conference on Protective Clothing". Stockholm, Arbete och Hälsa 2000:8, p17-20.

Nilsson H, Holmér I., Holmberg S, Sandberg M, (2000). Thermal climate assessment in office environment - CFD calculations and thermal manikin measurements. Proceedings of the 7th International Conference on air distribution in rooms (ROOMVENT 2000)(Reading, UK): vol2, pp 90-95.

Nilsson H, Holmér I., Bohm M, Norén O, (1999). Definition and Theoretical Background of the Equivalent Temperature. Int. ATA Conf, 17-19 Nov(Florence, Italy): 1999A4082.

Nilsson H, Holmér I., Bohm M, Norén O, (1999). Effects on Thermal Comfort Using Special Glazing Comparison of CFD Calculations and Manikin Measurements. Int. ATA Conf, 17-19 Nov(Florence, Italy): 1999A4077.

Nilsson H, Holmér I., Holmberg S, (1999). Comparison between indoor environment measured with thermal manikin and computational fluid dynamics calculation. Proceedings of Indoor Air 99: The 9th International Conference on Indoor Air Quality and Climate(Edinburgh, UK): vol 2, pp 90-95.

Nilsson H (1999). The use of thermal manikin in the field. Proceedings of the third International Meeting on Thermal Manikin Testing, 3IMM (eds. Nilsson H, Holmér I)(arbetslivsinstitutet.se): Arbete \& Hälsa 2000:4 (ISSN 0346-7821) pp 59-65.

Nilsson H, Holmér I., Bohm M and Norén O, (1997). Equivalent temperature and thermal sensation Comparison with subjective responses. Proceedings of Comfort in the automotive industry - Recent development and achievements(Bologna, Italy): ATA vol 1, pp 157-162, 97A3018.

Nilsson H (1997). Analyses of two methods of calculating the total insulation. Proceedings of a European Seminar on Thermal Manikin Testing (eds. Nilsson H, Holmér I)(arbetslivsinstitutet.se): Arbetslivsrapport 1997:9 (ISSN 1401-2928) pp 17-22.

Nilsson, H., (1997), Prediction of motion effects from static manikin measurements. Proceedings of a European seminar on Thermal Manikin Testing, Solna, Arbetslivsinstitutet, Department of Ergonomics, 45-48. 
Nilsson, H. and Holmér, I., (1997), Development and clothing measurements methods with the thermal manikin TORE. Proceedings of Fifth Scandinavian Symposium on Protective Clothing, Elsinore, Danish Work Environment Fund, 30-35.

Nilsson H. (1997) Use of Manikin Data and Its Meaning. New Frontiers: First International Manikin Forum, Halifax, Nova Scotia, Canada. June 22-24.

Nilsson, H., Holmér, I., Ohlsson, G. and Anttonen, H., (1997), Clothing insulation at high wind speeds. In Problems with Cold Work, Kuklane, K., Holmér, I. and Ekeberg, C. (ed.) (National Institute for Working Life, Stockholm, Sweden), 63.

Nilsson H, Chen F, Holmér I. A (1996) Modified Hot Plate for Glove Contact Insulation Measurement. The 7th International Conference on Environmental Ergonomics. Jerusalem, Israel, 377-380.

Nilsson H and Holmér I. (1994) Changes in thermal insulation for seated persons. Second International Congress on Physiological Anthropology. Kiel, German Society of Physiological Anthropology 326-329.

Nilsson H, Holmér I. (1993). Impact of seat on thermal comfort. Proceedings of Indoor Air 93: The 6th International Conference on Indoor Air Quality and Climate(Helsinki, Finland): vol 6, pp 127-132, July $4-8$.

Nilsson HO, Gavhed D., Holmér I, (1992). Effect of step rate on clothing insulation-measurement with a moveable thermal manikin. Proceedings of the 5th International Conference on Environmental Ergonomics (Maastricht, the Netherlands): pp 174-175.

Nilsson H, Grahn S, Holmér I. (1992) Protection against cold - a method for evaluation of handwear. 4th Scandinavian Symposium on Protective Clothing Against Chemicals and Other Health Hazards (NOKOBETEF IV). Kittilä, Finland, 224-227.

Nilsson H, (1990) Holmér I, Smolander J, Rissanen S, Hirata K. Physiological and subjective responses to work in asbestos protective suits. International Conference on Environmental Ergonomics IV. Austin, USA, 162-164.

Nilsson H. (1989) Evaluation of sensors for skin humidity measurements during working conditions. Third scandinavian symposium on protective clothing against chemicals and other health risks. Gausdal, Defense Research Establishment 147-151.

\section{Project reports (3):}

Nilsson HO, Holmér I. (2002). Definitions and Measurements of Equivalent temperature. EQUIV Report No 1, European Commission Cost contract No SMT4 CT95-2017 (registrator@arbetslivsinstitutet.se): Dnr 2002-0213 National Institute for Working Life.

Nilsson HO, Zhou H, Melikov A, Gabay D (2002). Field Tests of Instruments for Measuring Equivalent Temperature. EQUIV Report No 5, European Commission Cost contract No SMT4 CT95-2017 (registrator@arbetslivsinstitutet.se): Dnr 2002-0214 National Institute for Working Life.

Nilsson H, Holmér I., Norén O., Bohm M. (1999). Klimatupplevelse med ventilerad stol. Arbetslivsrapport, 1999:2 (arbetslivsinstitutet.se): (in swedish). 


\section{References}

ASHRAE 55 (1992) Thermal environmental conditions for human occupancy. ASHRAE Standard, ashrae.org, 55-1992.

ASHRAE 62 (1989) Ventilation for acceptable indoor air quality. ASHRAE Standard, ashrae.org, 621989.

Bedford T (1936) The warmth factor in comfort at work. MRC Industrial Health Board Report HMSO, London, UK, vol 76.

Bedford T \& Warner C (1934) The globe thermometer in studies of heating and ventilation. Journal of Hygiene, UK, Ibid 34, pp 458-473.

Belding H (1949) Protection against dry cold. Physiology of heat regulation and the science of clothing, WB Saunders Company, pp 351-367.

Bischof W, Lund Madsen T \& Bánhidi L (1991) Physiological adaptation of thermal manikins. International Conference on Human-Environment System, Tokyo, Japan, pp 147-150.

Bjørn E (2000) Simulation of human respiration with breathing thermal manikin. Proceedings of the third International Meeting on Thermal Manikin Testing, 3IMM (eds. Nilsson H, Holmér I), Arbete \& Hälsa 2000:4, arbetslivsinstitutet.se, pp 78-82.

Bohm M (1999) Comparison of Instruments for Measurement of Equivalent Temperature in an Experimental Cab in a Climatic Chamber. EQUIV Report, Uppsala, Sweden, No 3.

Bohm M, Browén A, Norén O, Holmér I \& Nilsson H (1990) Evaluation of the thermal environment in tractor cabs. International Conference on Environmental Ergonomics IV, Austin, USA, pp 144-146.

Bohm M, Holmér I, Nilsson H \& Norén O (2002) Thermal effect of glazing in driver's cabs. JTIRapport, Uppsala, Sweden, no 305.

Brohus H (1997) Personal exposure to contaminant sources in ventilated rooms. Aalborg University, Aalborg, Denmark, Thesis.

Brohus H \& Nielsen P (1996) CFD Models of Persons Evaluated by Full-Scale Wind Channel Experiments. Proceedings of the 5th International conference on air distribution in rooms (ROOMVENT 96), Yokohama, Japan, pp 137- 144.

Brüel \& Kjær (1982) Thermal Comfort Meter Type 1212. Instruction Manual, Denmark, no 033-0699.

Bröde P \& Griefahn B (2003) Thermal comfort and heat radiation in car seats and moisture accumulation at the seat person interface. Proceedings of the 5th International meeting on thermal manikins and modelling, 5I3M, Strasbourg, France, CD cepa.c-strasbourg.fr.

Burke R, O`Neill F \& Stricker P (1994) The development of a heat pipe driven manikin with variable flow irrigated skin. Proceedings of the 6th International Conference on Environmental Ergonomics, Montebello, Canada, pp 196-197.

Burke R, Rugh J \& Farrington R (2003) ADAM - The Advanced Automotive Manikin. Proceedings of the 5th International meeting on thermal manikins and modelling, 5I3M, Strasbourg, France, CD cepa.c-strasbourg.fr.

Buxon A, Zhubrin S \& Daanen H (2003) The QinetiQ virtual manikin - Incorporation of a thermophysiological model. Proceedings of the 5th International meeting on thermal manikins and modelling, 5I3M, Strasbourg, France, CD cepa.c-strasbourg.fr.

CFX 5.4 (2000) CFX-5 User documentation. Ansys Inc., ansys.com, Manual.

Chen Q (1995) Prediction of Room Air Motion by Reynolds-Stress Models. Building and Environment, Pergamon Press, vol 31, no 3, pp 233-244.

Chen Q \& Xu W (1998) A zero-equation turbulence model for indoor airflow simulation. Energy and buildings, elsevier.com, no 28, pp 137-144.

de Dear R (1998) A global database of thermal comfort field experiments. ASHRAE Transactions, ashrae.org, vol 104 (1b), pp1141-1152.

de Dear R, Arens E \& Hui Z (1997) Convective and radiative heat transfer coefficients for individual human body segments. International Journal of Biometeorology, Springer, no 40, pp 141-156. 
de Dear R, Ring J \& Fanger P (1993) Thermal sensations resulting from suddden ambient changes. International journal of indoor air quality and climate, Munksgaard, vol 3, pp 181-192.

Dozen Y (1989) Studies of the heat and moisture transfer through clothing using a sweating thermal manikin. Thermal Physiology, Mercer JB ed, elsevier.com, pp 519-524, Excerpta Medica.

Dufton A (1929) The Eupatheostat. Journal of Scientific Instruments, UK, vol 6, pp 249-251.

Dufton A (1936) The equivalent temperature of a warmed room. JIHVE (Building Services: now the Journal of CIBSE), UK, vol 4, pp 227-229.

Elnäs S (1988) Thermal climate in confined spaces - Measurement and assessment using a thermal manikin. SAE-Technical Paper Series, sae.org, no 881111.

EQUIV (1999) Development of Standard Test Methods for Evaluation of Thermal Climate in Vehicles. Final report, jti.slu.se, EU-project SMT 4 CT95-2017.

Fan J (2003) New Functions and Applications of "Walter"-Sweating Fabric Manikin. Proceedings of the 5th International meeting on thermal manikins and modelling, 5I3M, Strasbourg, France, CD cepa.c-strasbourg.fr.

Fan J \& Keighley J (1991) An investigation on the effects of: Body motion, clothing design and environmental conditions on the clothing thermal insulation by using a fabric manikin. International Journal of clothing sciences and technology, MCB University Press, vol 3, no 5, pp 6-13.

Fanger P (1970) Thermal comfort. Danish Technical Press, Copenhagen, Denmark.

Fanger P \& Toftum J (2001) Thermal comfort in the future - Excellence and expectation. Moving Thermal Comfort Standards into the 21st Century, Windsor, UK, CD ROM Proceedings.

Fiala D, Lomas K \& Stohrer M (1999) A computer model of human thermoregulation for a wide range of environmental conditions: the passive system. Journal of Applied Physiology, jap.org, no 87(5), pp 1957-1972.

Fluent 5.4 (1998) FLUENT User's Guide. Fluent Inc., fluent.com, Manual.

Fung W \& Parssons K (1996) Some investigations into the relationship between car seat cover materials and thermal comfort using human subjects. Journal of coated fabrics, UK, vol 26, pp 147-176.

Gagge A, Fobelets A \& Berglund L (1986) A Standard Predictive Index of Human Response to the Thermal Environment. ASHRAE Transactions, ashrae.org, vol 92, part 2, pp 709-731.

Gagge A, Stolwijk A \& Nishi Y (1971) An effective temperature scale based on a simple model of human physiological regulatory response. ASHRAE Transactions, ashrae.org, vol 77, pp 247-257.

Gagge A, Stolwijk J \& Hardy J (1967) Comfort and thermal sensations and associated physiological responses at various ambient temperatures. Environmental Research, USA, vol 1, pp 1-20.

Gan G (1994) Numerical Method for a full Assessment of Indoor Thermal Comfort. International journal of indoor air quality and climate, Munksgaard, vol 4, no 3, pp 154-168.

Givoni B \& Goldman R (1971) Predicting metabolic energy cost. Journal of Applied Physiology, jap.org, vol 30, pp 429-433.

Goldman R (1983) Historical review of development in evaluating protective clothing with respect to physiological tolerance. Aspects médicaux et biophysiques des vetements de protection, Lyon-Bron, Centre de Recherches du Service de Santé des Armées, pp 169-174.

Han T, Huang L, Kelly S, Huizenga C \& Hui Z (2001) Virtual thermal comfort engineering. SAE 2001 World congress, Detroit, USA, 2001-01-0588.

Hatton A \& Awbi H (1996) Heat transfer from room surfaces. Proceedings of the 5th International conference on air distribution in rooms (ROOMVENT 96), Yokohama, Japan, vol 2, pp 395-402.

Hensel H (1979) Thermoreception and human comfort. Indoor Climate: (ed: PO Fanger and $O$ Valbjörn) Danish Building Research Institute, Copenhagen, Denmark, pp 425-440.

Hill L, Griffith O \& Flack M (1916) The measurement of the rate of heat loss at body temperature by convection, radiation and evaporation. Philosophical Transactions of the Royal Society, UK, vol 207, part B, pp 183-220.

Holand B (2000) Comfort temperatures for sleeping bags. Proceedings of the third International Meeting on Thermal Manikin Testing, 3IMM (eds. Nilsson H, Holmér I), arbetslivsinstitutet.se, pp 25-28. 
Holmer I (1999) Thermal manikins in research and standards. Proceedings of the third International Meeting on Thermal Manikin Testing, 3IMM (eds. Nilsson H, Holmér I), arbetslivsinstitutet.se, Arbete \& Hälsa 2000:4 (ISSN 0346-7821) pp 59-65.

Holmér I (1995) Heated manikins as a tool for evaluating clothing. Annals of Occupational Hygiene, vol 3, part 6, pp 809-818.

Holmér I, Nilsson H, Bohm M, Browén A \& Noren O (1992) Evaluation of thermal environment in automotive vehicles. 2nd ATA Int Conf. on Vehicle Comfort Ergonomic, Vibrational, Noice and Thermal Aspects, Italy, pp 295-302.

Houghten F \& Yagloglou C (1923) Determining equal comfort lines. Journal of ASHVE, USA, vol 29, pp 165-176.

Huizenga C, Hui Z \& Arens E (2001) A model of human physiology and comfort for assesing complex thermal environments. Building and Environment, elsevier.com, no 36, 691-699.

Humphreys M (1976) Field studies of thermal comfort compared and applied. Journal of Inst. Heat \& Vent. Eng., UK, no 44, pp 5-27.

Ichihara M, Saitou M, Nishimura M \& Tanabe S (1996) Measurement of convective and radiative heat transfer coefficients of standing human body by using a thermal manikin. Proceedings of Indoor Air 96: International Conference on Indoor Air Quality \& Climate, Nagoya, Japan, vol 2, pp 559-564.

ISO 7730 (1995) Moderate thermal environments - Determination of the PMV and PPD indices and specification of the conditions for thermal comfort. International Standardisation Organisation, iso.ch, EN-ISO-7730.

ISO 9920. (1993). Ergonomics - Estimation of the thermal characteristics of a clothing ensemble. Geneva: International Standards Organisation.

Johansson L \& Nilsson H (1987) En enkel termisk docka (in Swedish). Royal Institute of Technology, Stockholm, Sweden, A4-serien nr 134 (ISSN 0349-5388).

Kang (2002) An Occupant-Coupled CFD Model for Local WBGT Analysis in a Ventilated Enclosure. Journal of the Human-Environment System, Society of Human Environment System, vol 5, no 2, pp 79-86.

Kato S (1996) Numerical analysis of contaminant distribution around a human body. Proceedings of the 5th International conference on air distribution in rooms (ROOMVENT 96), Yokohama, Japan, vol. 2, pp 129-136.

Kohri I \& Moschida T (2002) Evaluation Method of Thermal Comfort in a Vehicle with a Dispersed Two-Node Model. Part 1 - Development of Dispersed Two-Node Model. Journal of HumanEnvironmental System, JHES, vol 6, no 1, pp 19-29.

Kohri I \& Moschida T (2003) Evaluation Method of Thermal Comfort in a Vehicle with a Dispersed Two-Node Model. Part 2 - Development of New Evaluation. Journal of Human-Environmental System, JHES, vol 6, no 2, pp 77-91.

Launder B \& Spalding D (1974) The numerical computation of turbulent flows. Computer Methods in Applied Mechanics and Engineering, North-Holland Publishing Company, vol 3, pp 269-289.

Lebbin P, Hosni M \& Gielda T (2003) Design and manufacturing of two thermal observation manikins for automobile applications. Proceedings of the 5th International meeting on thermal manikins and modelling, 5I3M, Strasbourg, France, CD cepa.c-strasbourg.fr.

Loomans M (1997) Design of and discussion on a thermal mannequin. Eindhoven University of Technology, Eindhoven, The Netherlands, FAGO-report 97.

Loomans M (1998) The mesurement and simulation of indoor airflow. Eindhoven University of Technology, Eindhoven, The Netherlands, Thesis.

Lotens W (1993) Heat transfer from humans wearing clothing. Technische Universitet Delft, Delft, The Nederlands, Thesis.

Madsen T, Olesen B \& Kristensen N (1984) Comparison between operative and equivalent temperature under typical indoor conditions. ASHRAE Transactions, ashrae.org, vol 90, part 1, pp 1077-1090.

Madsen T, Olesen B \& Reid K (1986) New methods for evaluation of the thermal environment in automotive vehicles. ASHRAE Transactions, ashrae.org, vol 92, part 1B, pp 38-54.

Madsen T, Soehrich E \& Popiolek Z (1992) Measurement of draught sensation by a new skin element. Thermal Insulation Laboratory, Technical University of Denmark, Copenhagen, Denmark, vol 8. 
Maué J, Wahl D \& Currle J (1997) Computation of the Thermal Environment in Passanger Compartments and Evaluation of Thermal Comfort. Proceedings of Comfort in the automotive industry - Recent development and achievements, Bologna, Italy, ATA vol 1, pp 147-155.

Mayer E \& Schwab R (1993) Evaluation of heat stress by an artificial skin. Proceedings of Indoor Air 93: The 6th International Conference on Indoor Air Quality and Climate, Helsinki, Finland, vol 6, pp 73-78.

McCullough E, Jones B \& Huck J (1985) A comprehensive data base for estimating clothing insulation. ASHRAE Transactions, ashrae.org, vol 91 (2A), pp 29-47.

McCullough E, Jones B \& Zbikowski P (1983) The effect of garment design on the thermal insulation values of clothing. ASHRAE Transactions, ashrae.org, vol 89.

McCullough E, Olesen B \& Hong S (1994) Thermal insulation provided by chairs. ASHRAE Transactions, ashrae.org, vol 100, part 1.

Meinander H (1992) Coppelius - a sweating thermal manikin for the assessment of functional clothing. Proceedings of Nokobetef IV: Quality and usage of protective clothing, Kittilä, Finland, pp 157-161.

Meinander H (2000) Extraction of data from sweating manikin tests. Proceedings of the third International Meeting on Thermal Manikin Testing, 3IMM (eds. Nilsson H, Holmér I), arbetslivsinstitutet.se, pp 96-99.

Melikov A (2003) Breathing thermal manikin for indoor environment assessment - Important characteristics and requirements. Proceedings of the 5th International meeting on thermal manikins and modelling, 5I3M, Strasbourg, France, CD cepa.c-strasbourg.fr.

Missenard A (1959) On thermally equivalent environments. JIHVE (Building Services: now the Journal of CIBSE), UK, vol 27, pp 231-237.

Morgan F (1998) Riemannian Geometry : a biginner's guide. A K Peters Ltd, Natick, USA, pp 88-93.

Murakami S \& Kato S (1989) Numerical and experimental study on room airflow - 3-D Predictions using thr k-e turbulence model. Building and Environment, Pergamon Press, vol 24, no 1, pp 85-97.

Murakami S, Kato S \& Zeng J (1997) Flow and Temperature Fields Around Human Body with Various Room Air Distribution: CFD Study on Computational Thermal Manikin - Part I. ASHRAE Transactions, ashrae.org, vol 103 part 1, pp 3-15.

Murakami S, Kato S \& Zeng J (1998) Numerical Simulation of Contaminant Distribution Around a Modelled Human Body: CFD Study on Computational Thermal Manikin - Part II. ASHRAE Transactions, ashrae.org, vol 104 part 2, pp 226-233.

Nicol J \& Humphreys M (2001) Adaptive thermal comfort and sustainable thermal standards for buildings. Moving Thermal Comfort Standards into the 21st Century, Windsor, UK, Proceedings Support CD ROM.

Nielsen P (1974) Flow in air conditioned rooms (model experiments and solution of the flow equation). Danish Technical University, Copenhagen, Denmark, Thesis.

Nielsen P (1998) The selection of turbulence models for prediction of room airflow. ASHRAE Transactions, ashrae.org, vol 104, part 1B, pp 1119-1127.

Nielsen P (2000) The Importance of a Thermal Manikin as Source and Obstacle in Full-Scale Experiments. Proceedings of the third International Meeting on Thermal Manikin Testing, 3IMM (eds. Nilsson H, Holmér I), arbetslivsinstitutet.se, pp 89-95.

Nielsen P, Restivo A \& Whitelaw J (1978) The velocity characteristics of ventilated rooms. Journal of Fluids Engineering, New York, USA, vol 100, pp 291-198.

Nilsson H, Gavhed D \& Holmér I (1992) Effect of step rate on clothing insulation-measurement with a moveable thermal manikin. Proceedings of the 5th International Conference on Environmental Ergonomics, Maastricht, the Netherlands, pp 174-175.

Nilsson H \& Holmér I (1993) Impact of seat on thermal comfort. Proceedings of Indoor Air 93: The 6th International Conference on Indoor Air Quality and Climate, Helsinki, Finland, vol 6, pp 127-132, July 4 - 8 .

Nilsson H, Holmér I, Bohm M \& Norén O (1997) Equivalent temperature and thermal sensation Comparison with subjective responses. Proceedings of Comfort in the automotive industry - Recent development and achievements, Bologna, Italy, ATA vol 1, pp 157-162, 97A3018. 
Nilsson H, Holmér I, Bohm M \& Norén O (1999a) Definition and Theoretical Background of the Equivalent Temperature. Int. ATA Conf, 17-19 Nov, Florence, Italy, 1999A4082.

Nilsson H, Holmér I, Bohm M \& Norén O (1999b) Effects on Thermal Comfort Using Special Glazing Comparison of CFD Calculations and Manikin Measurements. Int. ATA Conf, 17-19 Nov, Florence, Italy, 1999A4077.

Nilsson H, Holmér I \& Holmberg S (1999c) Comparison between indoor environment measured with thermal manikin and computational fluid dynamics calculation. Proceedings of Indoor Air 99: The 9th International Conference on Indoor Air Quality and Climate, Edinburgh, UK, vol 2, pp 90-95.

Nilsson H, Holmér I, Holmberg S \& Sandberg M (2000) Thermal climate assessment in office environment - CFD calculations and thermal manikin measurements. Proceedings of the 7 th International Conference on air distribution in rooms (ROOMVENT 2000), Reading, UK, vol2, pp 90-95.

Olesen B (1982) Effect of body posture and activity on the thermal insulation of clothing: Measurements by a movable thermal manikin. ASHRAE Transactions, ashrae.org, vol 88, part 2, pp 791-805.

Olesen B (1992) Evaluation of thermal comfort in vehicles during transient and steady state conditions. Proceedings of Comfort in the automotive industry - Recent development and achievements, Bologna, Italy, ATA vol 1, pp 359-369.

Olesen B \& Parssons K (2002) Introduction to thermal comfort standards and to the proposed new version of EN ISO 7730. Energy and Buildings, elsevier.com, no 34, pp 537-548.

Palazzetti M, Cisternino M \& Giordana M (1996) EVA3 - The virtual sensor thermal manikin. Fiat Research Center, Internal Document, Turin, Italy, DSP-960403, April 1996.

Parsons K (1993) Human thermal environments. Taylor \& Francis, UK, ISBN 0-7484-0041-9.

Piniec S, Thellier F, Duveau H \& Deffieux J (1997) MATHER - Model of local thermal sensations of a car driver. Proceedings of Comfort in the automotive industry - Recent development and achievements, Bologna, Italy, ATA vol 1, pp 109-117.

Rohles FJ \& Nevins R (1971) The nature of thermal comfort for sedentary man. ASHRAE Transactions, ashrae.org, vol 77 (1), pp 239-246.

SAE J2234 (1993) Equivalent temperature. Surface Vehicle Information Report, sae.org, J2234.

Schlichting H (1960) Boundary Layer Theory. 4th Edition, New York, USA, McGraw-Hill.

Schwab R, Conrad W \& Mayer E (1999) Correlation Between Objective and Subjective Measurements of Thermal Comfort. EQUIV Report No 4, Holtzkirchen, Germany, Fraunhofer-Institut für Bauphysik.

Schwab R \& Mayer E (1989) Grundlagenuntersuchung zum Einfluß der Sonneneinstrahlung auf die thermische Behaglichkeit in Kraftfahrzeugen - Grundlagen. FAT-Schriftenreihe, Holtzkirchen, Germany, Nr 81.

Schwab R \& Mayer E (1993) Einfluß der Sonneneinstrahlung auf die ther-mische Behaglichkeit in Kraftfahrzeugen. FAT-Schriftenreihe, Holtzkirchen, Germany, Nr 109.

Silva M (2002) Measurements of comfort in vehicles. Measurement Science and Technology, IOP Publishing Ltd, vol 13, pp R41-R60.

Silva M \& Coelho J (2002) Convection coefficients for the human body parts - Determined with a thermal mannequin. Proceedings of the 8th International Conference on air distribution in rooms (ROOMVENT 2002), Copenhagen, Denmark, pp 277-280.

Soltynski K (2000) Test research of a new generation thermal manikin. Proceedings of the third International Meeting on Thermal Manikin Testing, 3IMM (eds. Nilsson H, Holmér I), arbetslivsinstitutet.se, pp 18-22.

Srebric J, Chen Q \& Glicksman L (1999) Validation of a Zero-equation turbulence model for complex indoor airflow simulation. ASHRAE Transactions, ashrae.org, vol 105 (2), pp 414-426.

Star CD 3.1B (2001) User Guide and Methology. Computational Dynamics Limited, cd-adapco.com, Manual.

Stolwijk J (1971) Mathematical model of thermoregulation. Physiological and behavioral temperature regulation, ed Hardy JD, Gagge AP and Stolwijk AJ, Thomas Publisher, Springfield III, pp 703721. 
Sørensen D \& Nielsen P (2003) Quality control of computational fluid dynamics in indoor environments. International journal of indoor air quality and climate, Copenhagen, Denmark, vol 13, pp 2-17.

Tamura T \& Nomiyama I (1994) Effects of wet condition of skin surface on evaporative heat transfer through clothing. Second International Congress on Physiological Anthropology, Kiel, Germany, pp 334-337.

Tanabe S (2002) Numerical Comfort Simulator for Evaluating Thermal Environment. Proceedings of the 10th International Conference on Environmental Ergonomics, Fukuoka, Japan, pp 435-438.

Topp C, Hesselholt P, Trier M \& Nielsen P (2003a) Influence of Geometry of Thermal Manikins on Concentration Distribution and Personal Exposure. Proceedings of Healthy Buildings 2003, Singapore.

Topp C, Hesselholt P, Trier M \& Nielsen P (2003b) Influence of Geometry of Thermal Manikins on Room Airflow. Proceedings of Healthy Buildings 2003, Singapore.

Umbach K (1988) Physological tests and evaluations models for the optimization of the protective clothing. Environmental ergonomics, Mekjavic IB, Banister EW, Morrison JB eds, Taylor \& Francis, pp 139-161.

Wang X (1994) Thermal comfort and sensation under transient conditions. Royal Institute of Technology, Stockholm, Sweden, Thesis (ISSN 1100-8997).

Wang X \& Peterson F (1992) Estimating thermal transient comfort. ASHRAE Transactions, ashrae.org, vol 98, part 1.

Werner J \& Webb P (1993) Six-cylinder model of human thermoregulation for general use on personal computers. Annals of physiological anthropology, vol 12, no 3, pp 123134.

Vernon H \& Warner C (1932) The influence of humidity of the air on capacity for work at high temperatures. Journal Hygiene Cambridge, UK, vol 32, pp 431-462.

Winslow C \& Greenburg L (1935) The thermo-intergrator: a new instrument for the observation of thermal interchanges. Journal of Hygiene, UK, Ibid 7, pp 41.

Wissler E (1988) A review of human thermal models. Envoronmental Ergonomics B Mekjavic, E W Banister and J B Morrison (eds), Taylor \& Francis, pp 267-285.

Wyon D (1989) Use of thermal manikins in enviromental ergonomics. Scandinavian Journal of Work Environment and Health, Gävle, Sweden, no 15, suppl 1, pp 84-94.

Wyon D, Larsson S, Forsgren B \& Lundgren I (1989) Standard procedures for assessing vehicle climate with a thermal manikin. SAE-Technical Paper Series, sae.org, no 89004.

Wyon D, Lund Madsen T \& Tammela E (1986) Quantitative comparison of clothing insulation measurements using different thermal manikins. National Institute for Building Research (SIB), Gävle, Sweden.

Wyon D, Tennstedt C, Lundgren I \& Larsson S (1985) A new method for the detailed assessment of human heat balance in vehicles - Volvo's thermal manikin, Voltman. SAE-Technical Paper Series, sae.org, no 850042.

Zhai Z \& Cheng Q (2001) Strategies for coupling energy simulation and computational fluid dynamics programs. Proceedings of the 7th International IBPSA Conference, Rio de Janerio, Brazil, pp 59-63.

Åstrand P \& Rodahl K (1986) Textbook of Work Physiology. McGraw-Hill, New York, USA, 3rd edition. 


\section{Nomenclature}

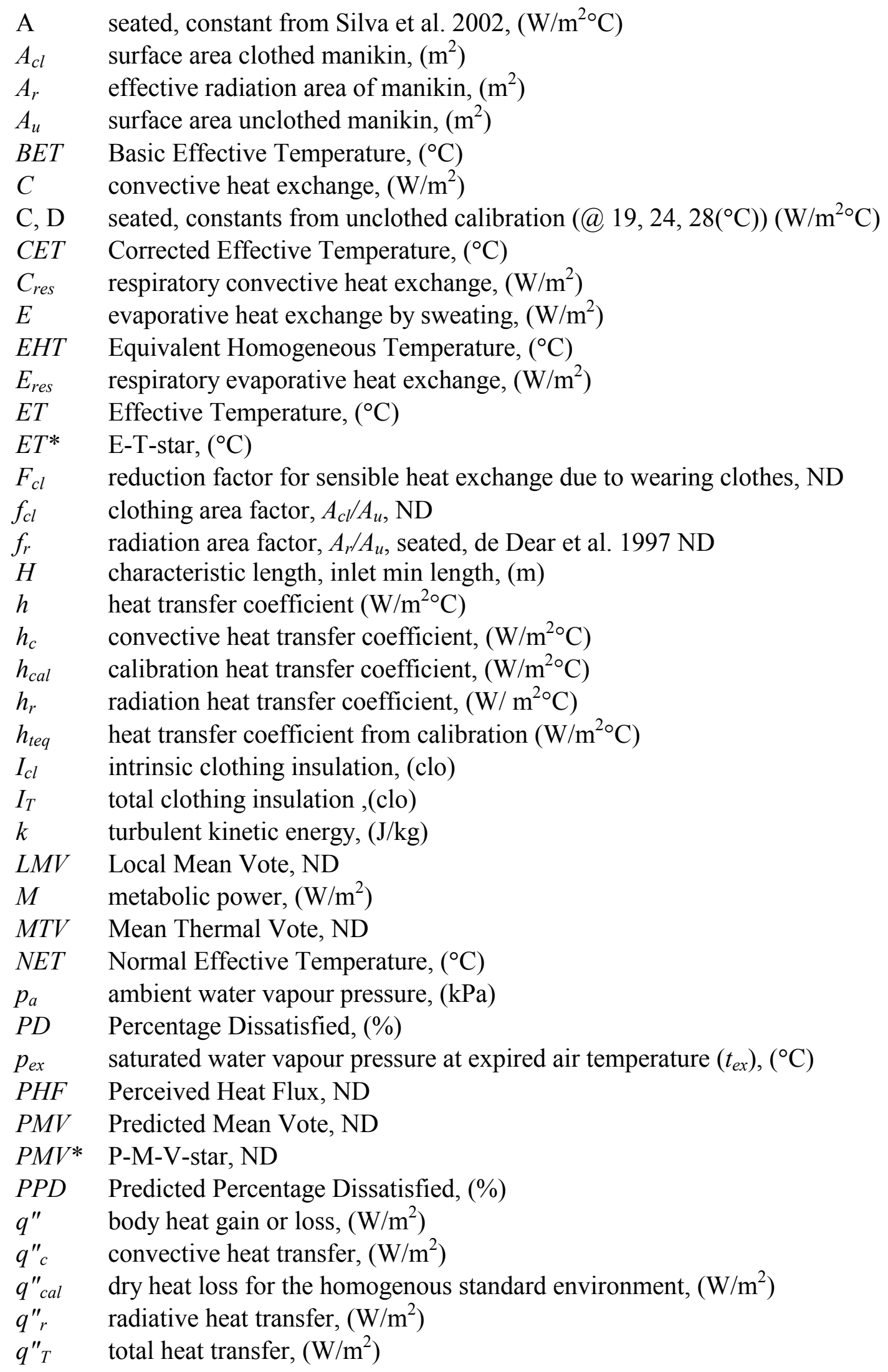


$\boldsymbol{q}^{\prime \prime}(\boldsymbol{r}) \quad$ vector valued heat loss, with direction $\hat{\boldsymbol{q}}^{\prime \prime}(\boldsymbol{r})$ and magnitude $\boldsymbol{q}^{\prime \prime}(\boldsymbol{r}),\left(\mathrm{W} / \mathrm{m}^{2}\right)$

$R \quad$ radiation heat exchange, $\left(\mathrm{W} / \mathrm{m}^{2}\right)$

$R_{c l} \quad$ intrinsic clothing insulation $\left(\mathrm{m}^{2}{ }^{\circ} \mathrm{C} / \mathrm{W}\right)$

$R_{T} \quad$ total clothing insulation $\left(\mathrm{m}^{2}{ }^{\circ} \mathrm{C} / \mathrm{W}\right)$

RST Resultant Surface Temperature, $\left({ }^{\circ} \mathrm{C}\right)$

$R T \quad$ Resultant Temperature, $\left({ }^{\circ} \mathrm{C}\right)$

$S \quad$ surface area $\left(\mathrm{m}^{2}\right)$

$S_{\phi} \quad$ source term

SET Standard Effective Temperature, $\left({ }^{\circ} \mathrm{C}\right)$

$t \quad$ time (s)

$t_{a} \quad$ ambient air temperature, $\left({ }^{\circ} \mathrm{C}\right)$

$t_{c l} \quad$ clothing surface temperature, $\left({ }^{\circ} \mathrm{C}\right)$

$t_{e q} \quad$ equivalent temperature $\left({ }^{\circ} \mathrm{C}\right)$

$t_{e q}$ (emp.) $\quad t_{e q}$ derived from the empirical equation, $\left({ }^{\circ} \mathrm{C}\right)$

$t_{e q}\left(p_{a}=\right.$ const. $) t_{e q}$ derived from $P M V$ with $v_{a}=0$ and constant $p_{a},\left({ }^{\circ} \mathrm{C}\right)$

$t_{e q}\left(v_{a}=0\right) \quad t_{e q}$ derived from $P M V$ with $v_{a}=0,\left({ }^{\circ} \mathrm{C}\right)$

$\tilde{\boldsymbol{t}}_{e q}(S)$ surface averaged equivalent temperature vector $\left({ }^{\circ} \mathrm{C}\right)$

$\boldsymbol{t}_{e q}(\boldsymbol{r}) \quad$ vector valued equivalent temperature, where $\boldsymbol{r}$ is the radius vector $\left({ }^{\circ} \mathrm{C}\right)$

$t_{e x} \quad$ expired air temperature, $\left({ }^{\circ} \mathrm{C}\right)$

$t_{g} \quad$ globe temperature, $\left({ }^{\circ} \mathrm{C}\right)$

$t_{o} \quad$ operative temperature, $\left({ }^{\circ} \mathrm{C}\right)$

$\bar{t}_{r} \quad$ mean radiant temperature, $\left({ }^{\circ} \mathrm{C}\right)$

$t_{s} \quad$ surface temperature, $\left({ }^{\circ} \mathrm{C}\right)$

$t_{s k} \quad$ skin temperature, $\left({ }^{\circ} \mathrm{C}\right)$

$\bar{t}_{s k} \quad$ mean skin temperature, $\left({ }^{\circ} \mathrm{C}\right)$

$u_{j} \quad$ fluid velocity component in direction $x_{j}(\mathrm{~m} / \mathrm{s})$

$u_{0} \quad$ characteristic velocity, inlet velocity $(\mathrm{m} / \mathrm{s})$

$W \quad$ effective mechanical power, $\left(\mathrm{W} / \mathrm{m}^{2}\right)$

$v_{a} \quad$ air velocity $(\mathrm{m} / \mathrm{s})$

$\Gamma_{\phi} \quad$ diffusion coefficient

$\varepsilon \quad$ the surface emissivity (0.95), ND

$\varepsilon_{a} \quad$ emissivity of ambience, ND

$\varepsilon_{s} \quad$ emissivity of sensor, ND

$\phi \quad$ represents any mean scalar variable

$\mu \quad$ molecular viscosity (Pa s)

$\mu_{t} \quad$ turbulent (eddy) viscosity (Pa s)

$v \quad$ kinematic viscosity $(v=\mu / \rho)\left(\mathrm{m}^{2} / \mathrm{s}\right)$

$\rho \quad$ fluid density $\left(\mathrm{kg} / \mathrm{m}^{3}\right)$

$\sigma \quad$ Stefan-Boltzmanns constant, $\left(\mathrm{W} / \mathrm{m}^{2} \mathrm{~K}^{4}\right)$ 


\section{Appendix A}

\section{Design and dimensions of the thermal MANIKIN2}

\section{The manikin shape and mould}

Since several problems where discovered, many related to the soft surface construction of MANIKIN1, but also the complicated operation of the four computers regulating the 36 zones. It was decided that a MANIKIN2 should be built. This second manikin was improved in many ways. In order to make this new manikin more robust and easier to use, only sitting position should be available.

There are always compromises, creating problems, with the making of multipurpose manikins, heavy skeletons, joints, zone division's etc. At the same time the regulation electronics as well as the computer program were modernised. The new system consisted of only one portable computer with two programs running simultaneously. This construction makes the system easy to use both in the lab as well as in the field environment. To be able to specially investigate seat comfort, the manikin had to undergo several zone modifications. The thighs were divided into three parts to be able to look at heat transfer solely through the seat zones as well as eliminating error of radiation between the thighs. Some of the torso zones was also modified in order to make it possible to have three temperature ambient air temperature sensors at all times, giving the manikin $33+3$ zones.

MANIKIN2 is made of plastic foam which makes it very light, only $16 \mathrm{~kg}$, and consequently easy to handle. The plastic foam mould for MANIKIN2 was purchased from HEATMAN AB (www.heatman.se, Box 4051, SE-791 04 Falun, Sweden). The manikin foam body was produced with two component hard plastic foam originally intended for making prostheses: 617H12 - PEDILEN ${ }^{\circledR}$ Rigid Foam 200 and hardener (www.ottobockus.com, Otto Bock Orthopedic Industry, Inc., 3000 Xenium Lane North, Minneapolis, MN 55441, USA).
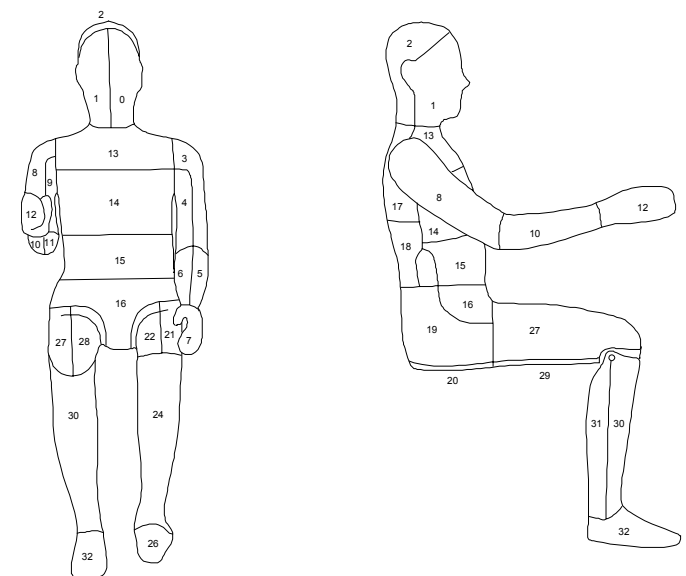

Figure A1. The 36 (0-35) zone divisions on the thermal MANIKIN2. 


\section{Heating wires}

The zone divisions where drawn onto the foam body, and the areas of each zone was measured. The area measurements were made with dense tape covering each zone. The tape has a certain mass per length, covering a zone with a tape and weighing the tape used, presents a possibility to calculate the zone areas.

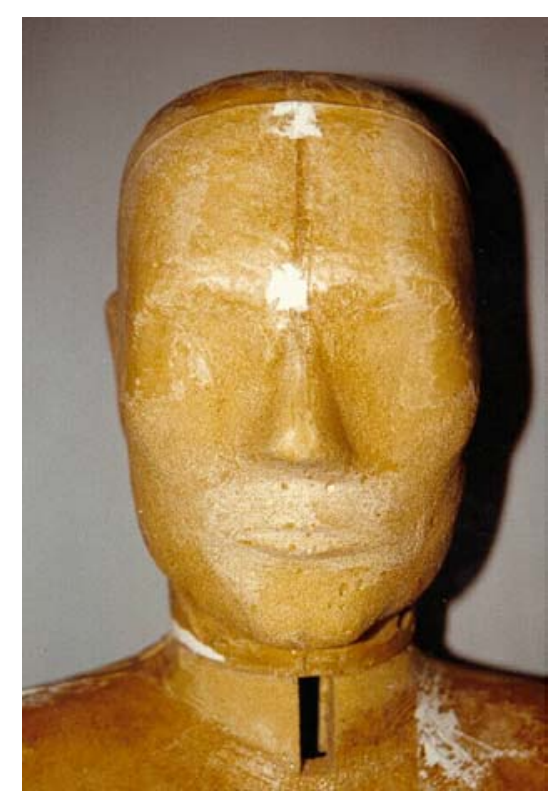

Figure A2. MANIKIN2 head directly from the mould.

When the areas are known, the resistance and length of the Kanthal ${ }^{\circledR}$ wire (www.clasohlson.se, Clas Ohlson AB, Riksväg 70, 79385 INSJÖN) with a resistance per unit length of 7, 11, 20 and 44 (Prod. nr: 49-211, 49-210, 49-208, 49-206,) $\Omega \mathrm{m}^{-1}$, could be calculated. This was facilitated by multiplying the area with the by the author invented, area density factor, $181 \mathrm{~m}^{-1}$. This factor ensures correct positioning and distribution of the wire over the surface.

In order to get the right resolution and consequently more accurate regulation for each zone, maximum power input was chosen to be 200,300 and $400 \mathrm{~W} / \mathrm{m}^{2}$ depending on the maximal heat loss of the zone. Taking into account internal radiation, clothed or unclothed etc. Voltage was decided to be 40 and $55 \mathrm{~V}$ to avoid higher voltage than the low voltage limit of $60 \mathrm{~V}$.

The heating wire was positioned tightly and evenly with about $0.5 \mathrm{~cm}$ distance between wires and fastened on the zone with a help of pins. Closer wiring gives more even heating of a zone. The material for covering the heating wires is normally liquid polyester plastic. But MANIKIN2 was sent to the prostheses manufacturer, Otto Bock Scandinavia AB (www.ottobock.se, Box 623, SE-60114 Norrköping, Sweden), for very even plastic covering in special vacuum machines. 


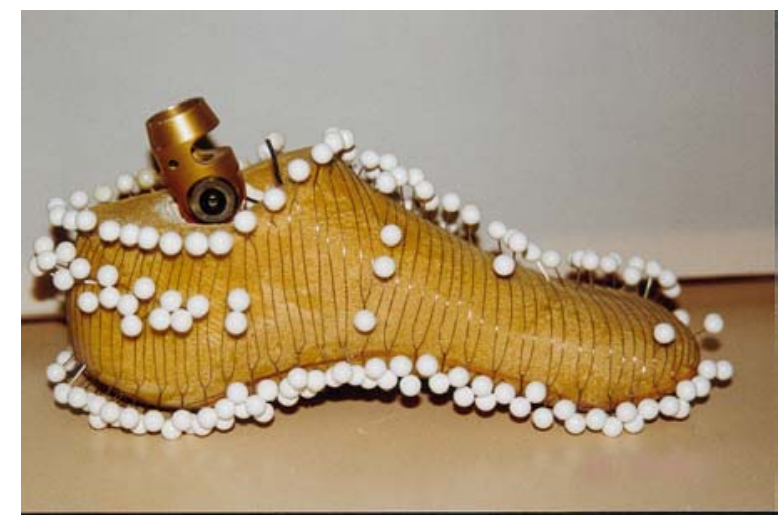

Figure A3. The left foot of MANIKIN2 showing the fixation of the heating wire with pins.

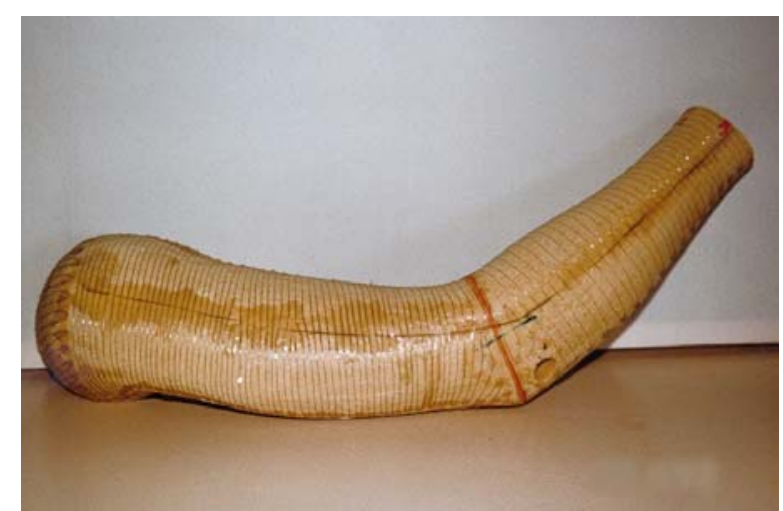

Figure A4. The ready made right arm of MANIKIN2 with the heating wires fixated with glue and the pins taken away, ready for plastic covering.

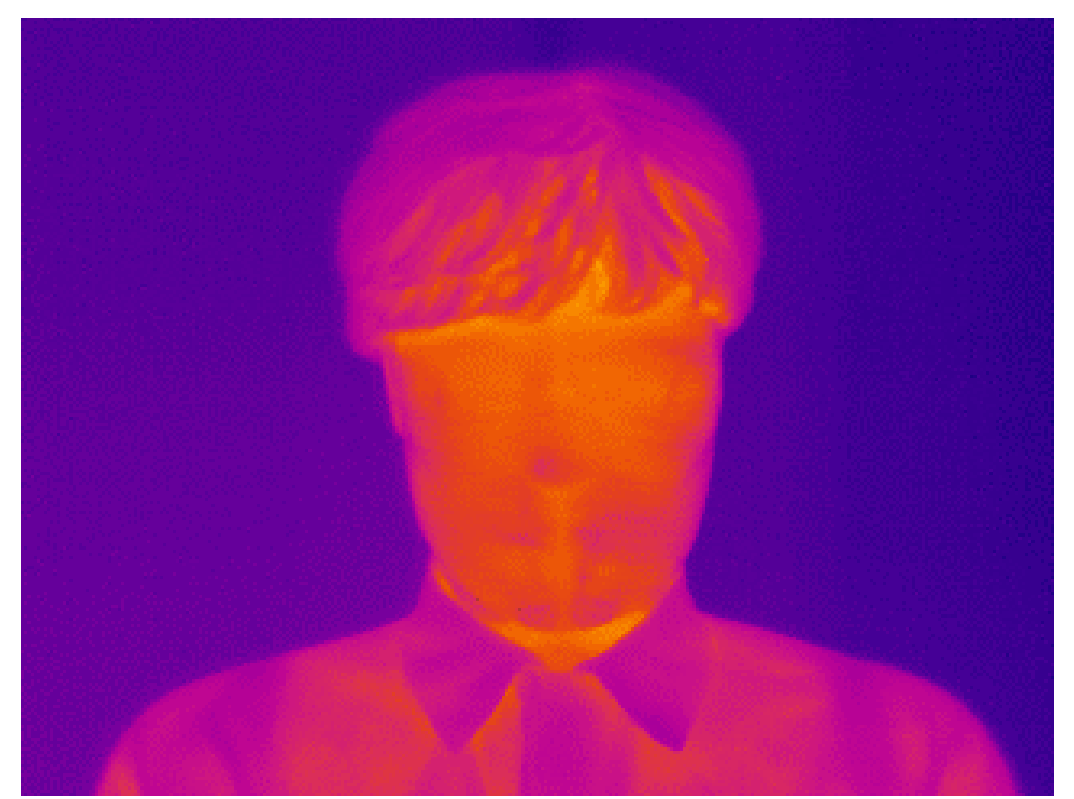

Figure A5. IR image (ThermaCAM PM 515, FLIR Systems AB, www.flir.se) of the heated face of MANIKIN2, showing the temperature distribution the hair, face and clothes when heated to $34^{\circ} \mathrm{C}$. 
Table A1. Zone resistance and wire length calculations for the manikin.

$\mathrm{L}=$ Left, $\mathrm{R}=$ Right, $\mathrm{U}=$ Upper, $\mathrm{L}=$ Lower, $\mathrm{O}=$ Outside, $\mathrm{I}=$ Inside, $\mathrm{B}=\mathrm{Back}, \mathrm{F}=$ Front

\begin{tabular}{|c|c|c|c|c|c|c|c|c|c|}
\hline Relationship & Area $\mathrm{m}^{2}$ & $\begin{array}{c}\text { Voltage } \\
\text { V } \\
\text { U }\end{array}$ & $\begin{array}{c}\text { Desired } \\
\text { power P/A } \\
\left(\mathrm{W} / \mathrm{m}^{2}\right) \\
\text { P/A }\end{array}$ & $\begin{array}{l}\text { Desired } \\
\text { length } L \\
(\mathrm{~m}) \\
\mathbf{L}=\mathbf{1 8 1 * \mathbf { A }}\end{array}$ & $\begin{array}{c}\text { Desired } \\
\text { resistance } \mathrm{R} \\
(\Omega) \\
\mathbf{R}=\mathbf{U}^{2} / \mathbf{P} * \mathbf{A}\end{array}$ & $\begin{array}{c}\text { Desired } \\
\text { wire } \\
(\Omega / \mathrm{m}) \\
\mathbf{R} / \mathbf{L}\end{array}$ & $\begin{array}{c}\text { Chosen } \\
\text { wire } \\
(\Omega / \mathrm{m}) \\
\text { R/L }\end{array}$ & $\begin{array}{l}\text { Measured } \\
\text { resistance R } \\
(\Omega) \\
\mathbf{R}\end{array}$ & $\begin{array}{c}\text { Max } \\
\text { power P } \\
(\mathrm{W}) \\
\mathbf{P}=\mathbf{U}^{2} / \mathbf{R}\end{array}$ \\
\hline FaceL & 0.0329 & 40 & 400 & 6.0 & 122 & 20 & 20 & 120 & 13.3 \\
\hline FaceR & 0.0325 & 40 & 400 & 5.9 & 123 & 21 & 20 & 118 & 13.6 \\
\hline Scalp & 0.0743 & 55 & 300 & 13.4 & 136 & 10 & 11 & 148 & 20.4 \\
\hline ArmLUO & 0.0507 & 55 & 300 & 9.2 & 199 & 22 & 20 & 201 & 15.0 \\
\hline ArmLUI & 0.0304 & 40 & 200 & 5.5 & 263 & 48 & 44 & 262 & 6.1 \\
\hline ArmLLO & 0.0275 & 40 & 300 & 5.0 & 194 & 39 & 44 & 196 & 8.2 \\
\hline ArmLLI & 0.0270 & 40 & 300 & 4.9 & 198 & 40 & 44 & 199 & 8.0 \\
\hline HandL & 0.0439 & 55 & 400 & 7.9 & 172 & 22 & 20 & 160 & 18.9 \\
\hline ArmRUO & 0.0509 & 55 & 300 & 9.2 & 198 & 22 & 20 & 197 & 15.4 \\
\hline ArmRUI & 0.0296 & 40 & 200 & 5.4 & 270 & 50 & 44 & 270 & 5.9 \\
\hline ArmRLO & 0.0303 & 40 & 300 & 5.5 & 176 & 32 & 44 & 176 & 9.1 \\
\hline ArmRLI & 0.0293 & 40 & 300 & 5.3 & 182 & 34 & 44 & 182 & 8.8 \\
\hline HandR & 0.0449 & 55 & 400 & 8.1 & 168 & 21 & 20 & 162 & 18.7 \\
\hline UChest & 0.0382 & 40 & 300 & 6.9 & 140 & 20 & 20 & 139 & 11.5 \\
\hline Chest & 0.0665 & 55 & 300 & 12.0 & 152 & 13 & 11 & 133 & 22.7 \\
\hline Abdom & 0.0423 & 40 & 300 & 7.7 & 126 & 16 & 20 & 154 & 10.4 \\
\hline LAbdom & 0.0368 & 40 & 200 & 6.7 & 217 & 33 & 44 & 296 & 5.4 \\
\hline UBack & 0.0754 & 55 & 300 & 13.6 & 134 & 10 & 11 & 151 & 20.0 \\
\hline MBack & 0.0635 & 40 & 200 & 11.5 & 126 & 11 & 11 & 127 & 12.6 \\
\hline LBack & 0.1153 & 55 & 200 & 20.9 & 131 & 6 & 7 & 147 & 20.6 \\
\hline Seat & 0.0422 & 40 & 200 & 7.6 & 190 & 25 & 20 & 154 & 10.4 \\
\hline ThigLO & 0.0576 & 55 & 300 & 10.4 & 175 & 17 & 20 & 211 & 14.3 \\
\hline ThigLI & 0.0618 & 55 & 200 & 11.2 & 245 & 22 & 20 & 224 & 13.5 \\
\hline ThigLB & 0.0293 & 40 & 200 & 5.3 & 273 & 51 & 44 & 237 & 6.8 \\
\hline CalfLF & 0.0536 & 55 & 300 & 9.7 & 188 & 19 & 20 & 195 & 15.5 \\
\hline CalfLB & 0.0481 & 55 & 300 & 8.7 & 210 & 24 & 20 & 175 & 17.3 \\
\hline FootL & 0.0526 & 55 & 300 & 9.5 & 192 & 20 & 20 & 191 & 15.8 \\
\hline ThigRO & 0.0574 & 55 & 300 & 10.4 & 176 & 17 & 20 & 209 & 14.5 \\
\hline ThigRI & 0.0585 & 55 & 200 & 10.6 & 259 & 24 & 20 & 212 & 14.3 \\
\hline ThigRB & 0.0306 & 40 & 200 & 5.5 & 261 & 47 & 44 & 246 & 6.5 \\
\hline CalfRF & 0.0562 & 55 & 300 & 10.2 & 179 & 18 & 20 & 205 & 14.8 \\
\hline CalfRB & 0.0490 & 55 & 300 & 8.9 & 206 & 23 & 20 & 179 & 16.9 \\
\hline FootR & 0.0528 & 55 & 300 & 9.6 & 191 & 20 & 20 & 192 & 15.8 \\
\hline \multicolumn{10}{|l|}{ Air0.1 } \\
\hline \multicolumn{10}{|l|}{ Air0.6 } \\
\hline \multicolumn{10}{|l|}{ Air1.1 } \\
\hline Total & 1.5919 & & & & & & & & 441 \\
\hline
\end{tabular}

\section{Temperature measuring wires}

The measuring wires were evenly distributed on each area. The measuring wires were covered with special tape $\operatorname{Scotch}^{\circledR}$ Tape by 3 M, Core series 2-0300, brand No. 396, Super Bond Film Tape (www.3m.com, 3M Svenska AB, SE-19189 Sollentuna). The length of the measuring wire was chosen so that it is possible to cover the areas of the zones evenly and it should give a resistance between 110 and $120 \Omega$, to fit into the window of the measurement transmitters.

Depending on the size of the zone two wire lengths were chosen, $\approx 68 \mathrm{~cm}$ and $\approx 270$ $\mathrm{cm}$. The wires are thin isolated Nickel resistance wires, brand name Resistherm ${ }^{\circledR}, \varnothing$ 
$0.10 \mathrm{~mm}, 42.3 \Omega / \mathrm{m} ; \varnothing 0.05 \mathrm{~mm}, 169.5 \Omega / \mathrm{m}$ made by Isabellenhütte

(www.isabellenhuette.de, Isabellenhütte Heusler GmbH KG, Eibacher Weg 3 - 5, D35683 Dillenburg, Germany). A small piece of shrinking tube was added to both connection ends to secure the thin wires.

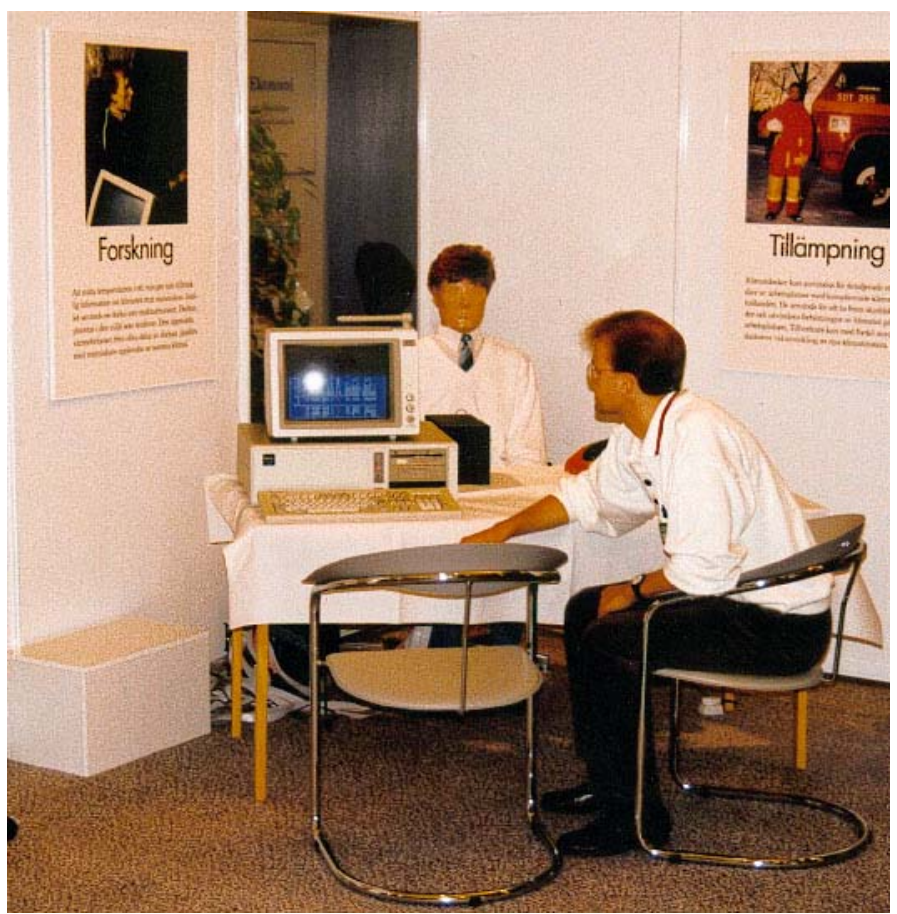

Figure A6. The complete MANIKIN2 at display at the Stockholm Technical Fair in October 1991. The author is operating the computer.

\section{Electronics and programs}

Now the manikin was connected to connected to model-box and further through computer-box to computer with a data collection program. The software consists of one resident regulation program and an acquisition program running on top.

\section{Layout for MANIKIN2}

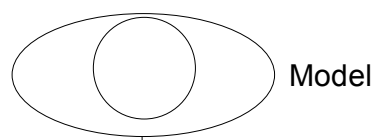

$(2 \mathrm{~m})$

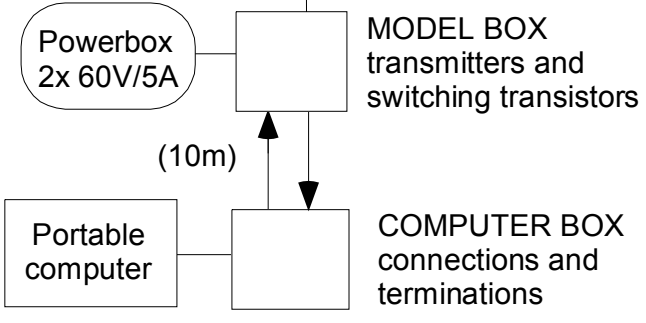

Figure A7. The technical layout of MANIKIN2. 
All connections, components and cables apply when possible to military specifications. The Powerbox laboratory power supplies gives long term performance and reliability combined with constant voltage or current operation; OUTPUT: load regulation $0.01 \%$ of Vmax, line regulation $0.01 \%$ of $\mathrm{Vmax}$, ripple \& noise $<1 \mathrm{mV} \mathrm{rms)}$

(www.powerbox.se, Powerbox, Box 148, SE-64622 Gnesta, Sweden)

The program is written with a configuration file that sets the number and properties of each channel and zone, this makes it possible to run different thermal models from 1 to 36 zones with the same program. These files contains all information about the model and sets the program functions at start:

$\begin{array}{ll}\text { Nr } & \text { the number of the computer channel } \\ \text { Text } & \text { channel identification } \\ \text { Area } & \text { the area of the zone } \\ \text { Res } & \text { the resistance of the zone } \\ \text { A } & \text { the calibration curve intercept } \\ \mathbf{B} & \text { the calibration curve slope } \\ \mathbf{R}^{2} & \text { the significance of the curve fit } \\ \text { Volt } & \text { the voltage for the zone } \\ \text { Con } & \text { the physical connection at the model box } \\ \alpha-\text {-surface } & \text { surface resistance between the heating and measuring wire } \\ \alpha-t_{\text {eq }} & \text { heat transfer coefficient for the } t_{\text {eq }} \text {-calibration } \\ \text { Set value } & \text { set value for the regulator } \\ \text { K-Power } & \text { constant power setting }\end{array}$

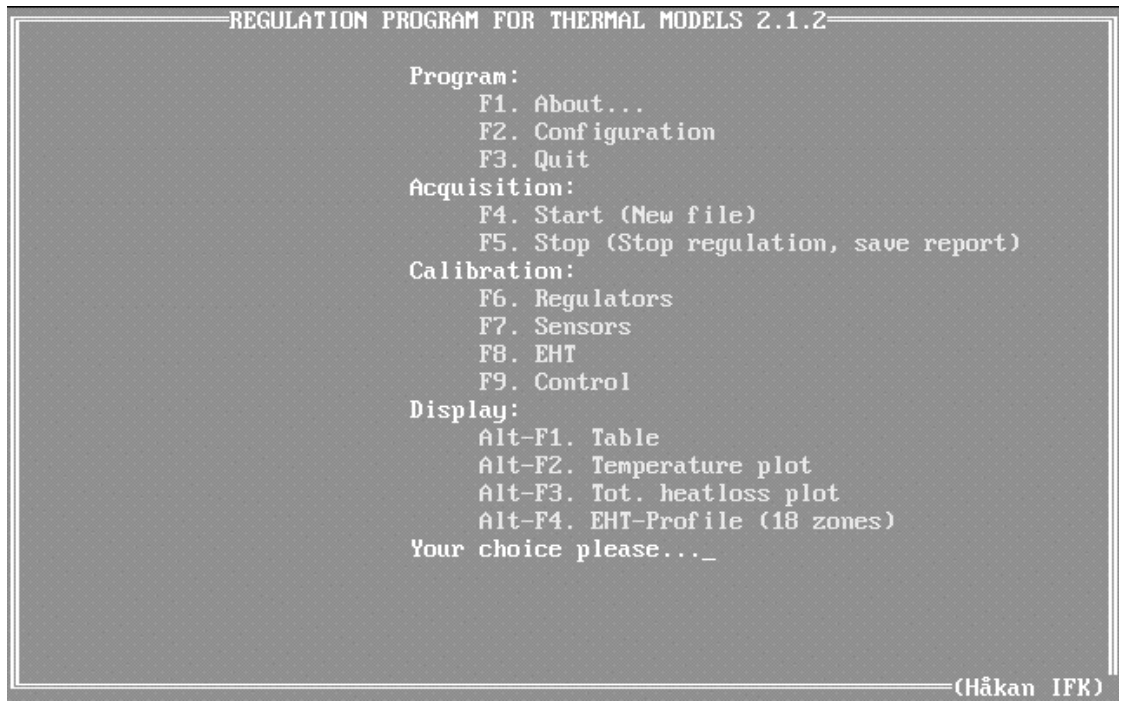

Figure A8. The regulation program main menu.

The program contains the following alternatives:
About ...
A short remark of the persons that have worked with the program
Configuration
Quit
Writing and editing configuration files (*.CHN)
Start
Quitting the program leaving the resident regulation part running.
Stop
Starts the acquisition of a new file continuously saved to disk
Regulators
Stops the acquisition and saves the data on disk for analyse
Gives the opportunity to change the regulation constants 
Sensors

EHT

Control

Table

Temperature plot

Tot. heat loss plot EHT-Profile (18 zones)
Calibration of resistance sensors for temperature measurement

Automatic $t_{e q}$ calibration

To check the temperature sensor connections

Shows all data from the last 10 seconds (and 10 minute average)

Shows the temperature data from the last 10 minutes

Shows the heat loss data from the last 10 minutes

Shows the $t_{e q}$-profile, with zones, from the last 10 minutes

To facilitate transport and field operation a special transportation box with build in calibration fans was built. The calibration part of the regulation program allows automatic overnight calibration with the three temperature sensors working as temperature control inside the box. The program is using a PID (Proportianal Integrating and Derivating) algorithm giving a fast an accurate regulation of any thermal model.

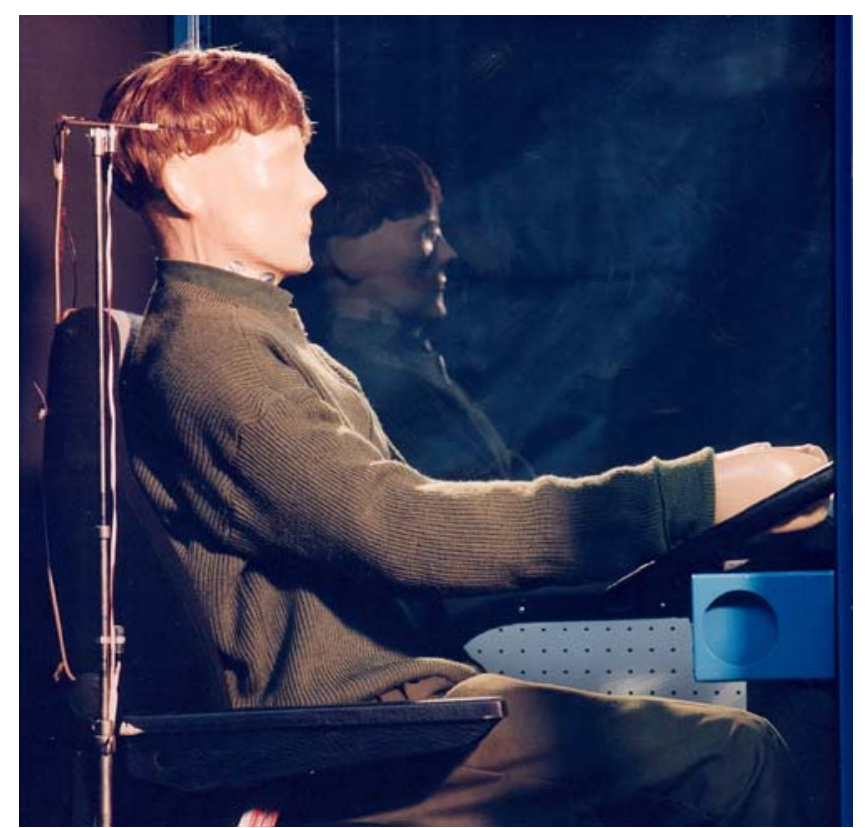

Figure A9. MANIKIN2 in winter clothing inside the cabin simulator with the sensor tree at the right side in the same way as MANIKIN3 in case 3.

\section{Accuracy, reproducability and repeatability}

The true $t_{e q}$ can not be determined, hence is accuracy difficult to estimate. The accuracy is depending on several factors: temperatures, size, position, direction, resolution etc. These factors can be defined as:

Accuracy refers to the ability to determine $t_{e q}$ in a known environment.

Repeatability refers to the largest difference between determinations carried out in the same environment with the same instrument and the same operator.

Reproducability refers to the largest difference between determinations when the measurement is reproduced. 


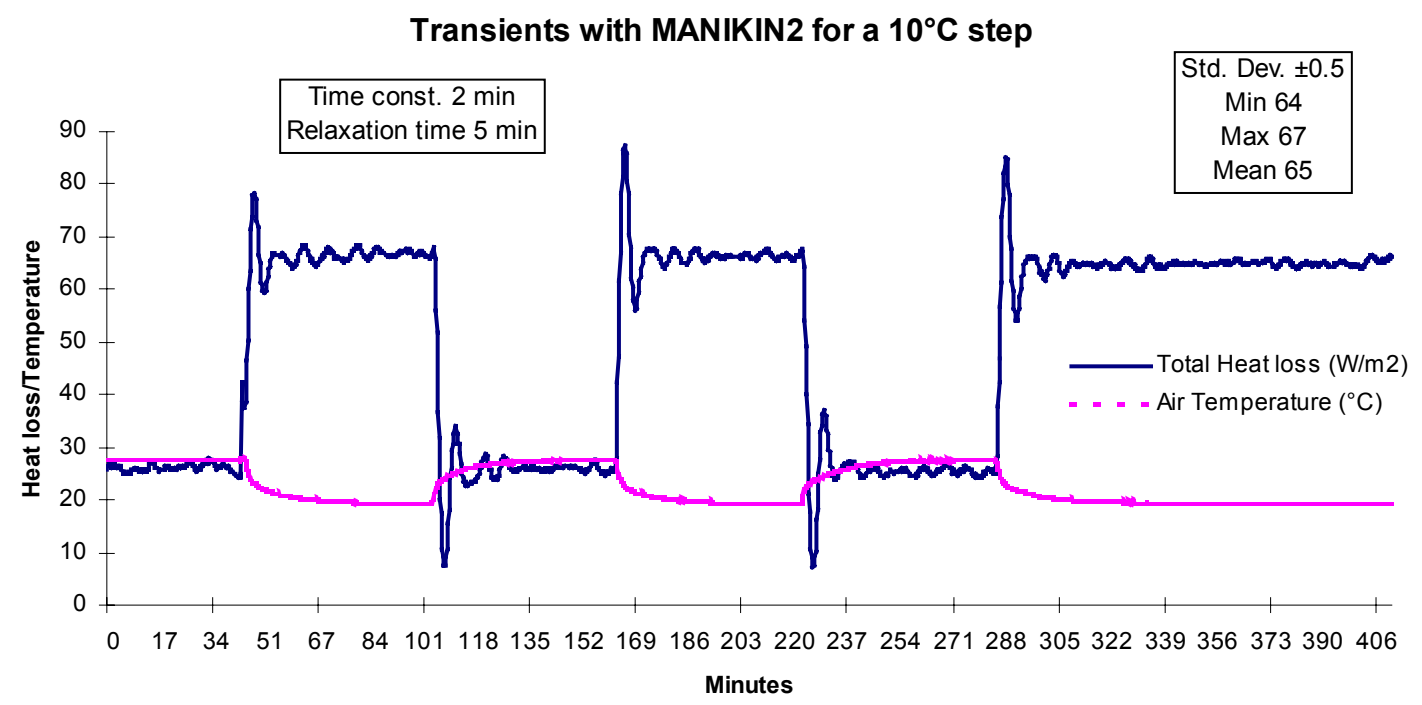

Figure A10. The regulation responses of MANIKIN2 after sevaral $10^{\circ} \mathrm{C}$ ambient temperature step changes. In constant the temeprature mode MANIKIN2 is a very quick and accurate instrument with a time constant of 2 minutes and a relaxation time of 5 minutes.

\section{Repeatability of heat transfer coefficients}

The repeatability of the heat transfer coefficients of MANIKIN2 was studied. This was accomplished by calculating the difference of the heat transfer coefficients from five calibrations originating from the years 1995 and 1998 (Table below). The results show that the mean standard deviation in heat transfer coefficients is $0.39 \mathrm{~W} / \mathrm{m}^{2} \mathrm{~K}(8 \%)$ for all zones. The inaccuracy of the calibration of the thermal manikins is mainly caused by the change of clothing position and environmental temperatures during transport. It is therefore essential to position the manikin in the same way during both calibration and measurements, and use the specially designed transport and calibration boxes to avoid damige during transit.

\section{The impact of repeatability on determination of equivalent temperature}

The repeatability on the determination of equivalent temperature by MANIKIN2 has also been estimated. The equivalent temperature for all 18 zones of MANIKN2 was calculated with the above min and max heat transfer coefficients at a surface set point of $34^{\circ} \mathrm{C}$ and the mean heat loss from the five calibrations. The difference in the equivalent temperature from the calculations is used as a measure of the repeatability. The results showed that the error introduced in the determination of equivalent temperature due to inaccuracy of the calibration was 1 to $3.3^{\circ} \mathrm{C}$ depending on the zone. 
Table A2. Heat transfer coefficients from five calibrations of MANIKIN2, originating from the years 1995 and 1998.

\begin{tabular}{|c|c|c|c|c|c|c|c|c|c|}
\hline YYMM & $\begin{array}{c}9511 \\
\left(\mathrm{~W} / \mathrm{m}^{2} \mathrm{~K}\right) \\
\mathrm{h}_{\mathrm{teq}}\end{array}$ & $\begin{array}{c}9602 \\
\left(\mathrm{~W} / \mathrm{m}^{2} \mathrm{~K}\right) \\
\mathrm{h}_{\mathrm{teq}}\end{array}$ & $\begin{array}{c}9701 \\
\left(\mathrm{~W} / \mathrm{m}^{2} \mathrm{~K}\right) \\
\mathrm{h}_{\mathrm{teq}}\end{array}$ & $\begin{array}{c}9710 \\
\left(\mathrm{~W} / \mathrm{m}^{2} \mathrm{~K}\right) \\
\mathrm{h}_{\mathrm{teq}}\end{array}$ & $\begin{array}{c}9801 \\
\left(\mathrm{~W} / \mathrm{m}^{2} \mathrm{~K}\right) \\
\mathrm{h}_{\mathrm{teq}}\end{array}$ & $\begin{array}{c}\text { StDev } \\
\left(\mathrm{W} / \mathrm{m}^{2} \mathrm{~K}\right) \\
\mathrm{h}_{\mathrm{teq}}\end{array}$ & $\begin{array}{l}\text { Diff. } \\
(\%) \\
\mathrm{h}_{\text {teq }}\end{array}$ & $\begin{array}{c}\text { Mean } \\
\text { HL } \\
\left(\mathrm{W} / \mathrm{m}^{2}\right) \\
\mathrm{q}^{\prime \prime}\end{array}$ & $\begin{array}{l}\text { Diff. } \\
\left({ }^{\circ} \mathrm{C}\right) \\
\mathrm{t}_{\mathrm{eq}}\end{array}$ \\
\hline Total & 4.94 & 4.83 & 4.83 & 4.30 & 4.53 & 0.26 & $6 \%$ & 54 & 1.3 \\
\hline Scalp & 3.54 & 3.57 & 3.73 & 3.09 & 3.33 & 0.25 & $7 \%$ & 40 & 1.7 \\
\hline Face & 8.38 & 8.03 & 8.41 & 7.61 & 7.55 & 0.41 & $5 \%$ & 93 & 1.2 \\
\hline Chest & 3.92 & 3.76 & 3.53 & 3.09 & 4.08 & 0.39 & $10 \%$ & 43 & 2.5 \\
\hline BackU & 5.35 & 5.35 & 5.02 & 4.94 & 5.69 & 0.30 & $6 \%$ & 61 & 1.3 \\
\hline ArmLU & 4.16 & 4.74 & 4.11 & 3.82 & 4.02 & 0.34 & $8 \%$ & 48 & 1.9 \\
\hline ArmRU & 4.29 & 4.39 & 3.67 & 4.32 & 4.30 & 0.30 & $7 \%$ & 48 & 1.6 \\
\hline ArmLL & 5.29 & 5.14 & 5.37 & 4.70 & 4.90 & 0.28 & $6 \%$ & 59 & 1.3 \\
\hline ArmRL & 4.99 & 5.10 & 5.09 & 4.87 & 4.58 & 0.22 & $4 \%$ & 57 & 1.0 \\
\hline HandL & 9.19 & 9.85 & 8.99 & 7.87 & 7.93 & 0.85 & $10 \%$ & 102 & 2.3 \\
\hline HandR & 8.66 & 9.06 & 9.39 & 7.79 & 7.86 & 0.71 & $8 \%$ & 99 & 1.9 \\
\hline ThighL & 5.01 & 4.74 & 4.94 & 4.63 & 4.48 & 0.22 & $5 \%$ & 55 & 1.1 \\
\hline ThighR & 4.92 & 4.73 & 5.10 & 4.52 & 4.35 & 0.30 & $6 \%$ & 55 & 1.5 \\
\hline CalfL & 5.12 & 4.80 & 5.12 & 4.52 & 4.88 & 0.25 & $5 \%$ & 57 & 1.2 \\
\hline CalfR & 5.28 & 4.70 & 5.22 & 4.27 & 4.61 & 0.43 & $9 \%$ & 56 & 2.1 \\
\hline FootL & 4.32 & 3.77 & 3.87 & 2.89 & 3.80 & 0.52 & $14 \%$ & 43 & 3.3 \\
\hline FootR & 4.52 & 3.97 & 4.25 & 3.18 & 3.83 & 0.50 & $13 \%$ & 46 & 3.0 \\
\hline BackL & 3.59 & 3.73 & 3.38 & 2.80 & 2.91 & 0.41 & $12 \%$ & 38 & 3.0 \\
\hline Seat & 5.01 & 4.29 & 4.79 & 4.54 & 3.91 & 0.43 & $10 \%$ & 52 & 2.2 \\
\hline Max & & & & & & 0.85 & $14 \%$ & & 3.3 \\
\hline Mean & & & & & & 0.39 & $8 \%$ & & 1.9 \\
\hline Min & & & & & & 0.22 & $4 \%$ & & 1.0 \\
\hline
\end{tabular}

\section{Field operation}

MANIKIN2 was built to operate during mobile conditions. In order to try this out, road tests have been carried out (Nilsson et al. 2002). The tests have been made in hard winter conditions. The objectives were to study the manikin method and to validate it during field conditions.

The road tests in winter climate was carried out in cooperation with Volvo Truck Corporation. The tests was performed in Volvo trucks at their test facilities in the north of Sweden in January. Ventilated seats from Be-Ge Industries was mounted at both driver and passenger position in the trucks. The truck corporation provided both vehicles and drivers. They also collected data concerning the weather and cabin conditions during the tests.

Different vehicles have different ventilation systems and their performance is not identical. The objective of this study was to gain field experience with with the manikin method for evaluation of the thermal environment in vehicles. The manikin method should be better adapted to asses the performances of various ventilation systems in different vehicles. Only evaluating the steady state condition is not enough for practical use. In real life, HVAC systems in vehicles, do never run under steady state conditions. Fluctuations of air temperature, air movement and radiation often exists. Methods for determination of equivalent temperature under transient conditions need to be further developed and studied. 


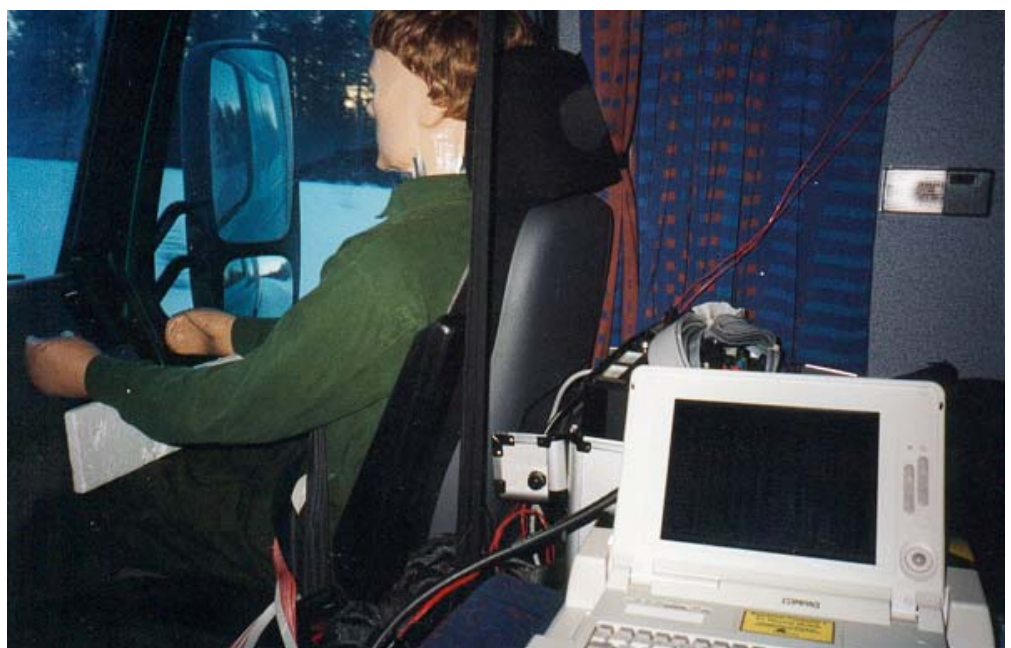

Figure A11. MANIKIN2 on the road inside the truck cabin during field tests in the north of Sweden.

An important task of these field tests was to evaluate the practical usability, robustness, sensitivity of the manikin method during active service conditions. Some of the experiences from these field tests were:

\section{Different regulation constants}

Experiments with different regulation constants for the manikin were made. With different constants it's obvious that the manikin can be either very fast or very slow. In sunny and transient conditions is it better to have a fast regulation to be able to detect quick changes. These constants differ from ordinary laboratory constants, were a very stable continuity is desired.

\section{Radio transmission}

Radio transmission could influence the instruments in the vehicle that is transmitting the radio message. Filtering for this can be made in the same fashion as filters for net frequencies already exists.

\section{Power requirements}

MANIKIN2 with equipment had a power cunsumption of around $250 \mathrm{~W}$ in stable conditions during the measurements. During the start-up phase the manikin could need up to double power. These requirement are minimised with preheating of the manikin and transport to the vehicle in a sleeping bag.

\section{Ventilated seats}

The ventilated seats was only tested once since the manikin did not have enough power to make the measurements. Both drivers complained and rated -2 on the MTV scale (much too cold). A separate series was made with the manikin to check the seats. It was noticed that, specially when coming in to the cabin from the cold, it was very unpleasant to use the ventilated seat in dry winter conditions. According to driver experience the ventilated seats could only be used during short periods of long driving. If you got in from the ambience at $-20^{\circ} \mathrm{C}$ the cooling power very quickly got intolerable. 


\section{Possible seat solution}

A possible solution to the problem should be to regulate the fan speed and heating of the seat from a only one temperature sensor in the seat. The regulation should be made so that in warm conditions only the fan worked and in cold conditions only the heater. In long driving periods the fan would go on shortly to lower the temperature slightly. 


\section{Appendix B}

\section{Design and dimensions of the virtual MANIKIN3}

Table B1. The commands for building the solid of the MANIKIN3 in CFX-Build.

\begin{tabular}{|c|c|c|c|c|c|c|c|c|c|}
\hline 1 & Scalp & $<0.1$ & 0.3 & 0.1 & $>1$ & 0 & 1 & -0.05 & ) \\
\hline 2 & Face & $<0.1$ & 0.2 & 0.1 & $>1$ & 0.1 & 1.1 & -0.05 & ) \\
\hline 3 & Chest & $<0.1$ & 0.45 & 0.3 & $>1$ & 0.1 & 0.55 & -0.15 & 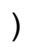 \\
\hline 4 & BackU & $<0.1$ & 0.15 & 0.3 & $>1$ & 0 & 0.85 & -0.15 & ) \\
\hline 5 & ArmLU & $<0.1$ & 0.3 & 0.05 & $>($ & 0 & 0.7 & -0.2 & ) \\
\hline 6 & ArmRU & $<0.1$ & 0.3 & 0.05 & $>1$ & 0 & 0.7 & 0.15 & , \\
\hline 7 & ArmLL & $<0.25$ & 0.1 & 0.05 & $>1$ & 0.1 & 0.7 & -0.2 & 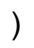 \\
\hline 8 & ArmRL & $<0.25$ & 0.1 & 0.05 & $>($ & 0.1 & 0.7 & 0.15 & , \\
\hline 9 & HandL & $<0.1$ & 0.1 & 0.05 & $>$ & 0.35 & 0.7 & -0.2 & 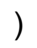 \\
\hline 10 & HandR & $<0.1$ & 0.1 & 0.05 & $>$ & 0.35 & 0.7 & 0.15 & 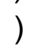 \\
\hline 11 & ThighL & $<0.35$ & 0.1 & 0.1 & $>1$ & 0.2 & 0.5 & -0.15 & 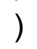 \\
\hline 12 & ThighR & $<0.35$ & 0.1 & 0.1 & : & 0.2 & 0.5 & 0.05 & 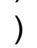 \\
\hline 13 & CalfL & $<0.05$ & 0.4 & 0.1 & 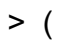 & 0.5 & 0.1 & -0.15 & ) \\
\hline 14 & CalfR & $<0.05$ & 0.4 & 0.1 & $>$ & 0.5 & 0.1 & 0.05 & ) \\
\hline 15 & FootL & $<0.15$ & 0.1 & 0.1 & $>1$ & 0.5 & 0 & -0.15 & ) \\
\hline 16 & FootR & $<0.15$ & 0.1 & 0.1 & $>1$ & 0.5 & 0 & 0.05 & ) \\
\hline 17 & BackL & $<0.1$ & 0.3 & 0.3 & $>1$ & 0 & 0.55 & -0.15 & ) \\
\hline 18 & Seat & $<0.2$ & 0.05 & 0.3 & $>($ & 0 & 0.5 & -0.15 & ) \\
\hline
\end{tabular}

Table B2. The commands for cutting out MANIKIN3 from the fluid in PROSTAR.

\begin{tabular}{|c|c|c|c|c|c|c|c|c|}
\hline 1 & Scalp & ige, & 0.025 & 0.175 & 0.975 & 1.325 & 0.175 & 0.325 \\
\hline 2 & Face & e, & 0.125 & 0.275 & 75 & 325 & .175 & 325 \\
\hline 3 & Chest & & 0.125 & 0.275 & 0.525 & 1.025 & 0.075 & 0.425 \\
\hline 4 & BackU & range, & 0.025 & 0.175 & 0.825 & 1.025 & .075 & 425 \\
\hline 5 & ArmLU & cset add grange, & 0.025 & 0.175 & 0.675 & 1.025 & 0.025 & 0.125 \\
\hline 6 & $\operatorname{rrmRU}$ & ange, & 0.025 & 0.175 & 0.675 & 1.025 & 0.375 & 0.475 \\
\hline 7 & ArmLL & cset add grange, & 0.125 & 0.425 & 0.675 & 0.825 & 0.025 & 0.125 \\
\hline 8 & ArmRL & cset & 0.125 & 0.425 & 0.675 & 0.825 & 0.375 & 0.475 \\
\hline 9 & & & & & & 0.825 & & 0.125 \\
\hline 10 & HandR & cse & 0.375 & 0.525 & 0.675 & 0.825 & 0.375 & 0.475 \\
\hline 11 & hL & & & 0.6 & & 25 & & 0.225 \\
\hline 12 & ThighR & cse & 0.225 & 0.625 & 0.475 & 0.625 & 0.275 & 0.425 \\
\hline 13 & $\mathrm{fL}$ & & 0.525 & 0.625 & & 0.525 & 0.075 & 0.225 \\
\hline 14 & CalfR & cset adc & 0.525 & 0.625 & 0.075 & 0.525 & 0.275 & 0.425 \\
\hline 15 & FootL & cset add grange, & 0.525 & 0.725 & -0.025 & 0.125 & 0.075 & 0.225 \\
\hline 16 & FootR & cset add grange, & 0.525 & 0.725 & -0.025 & 0.125 & 0.275 & 0.425 \\
\hline 17 & BackL & cset add grange, & 0.025 & 0.175 & 0.525 & 0.875 & 0.075 & 0.425 \\
\hline 18 & Seat & cset add grange, & 0.025 & 0.275 & 0.475 & 0.575 & 0.075 & 0.425 \\
\hline
\end{tabular}



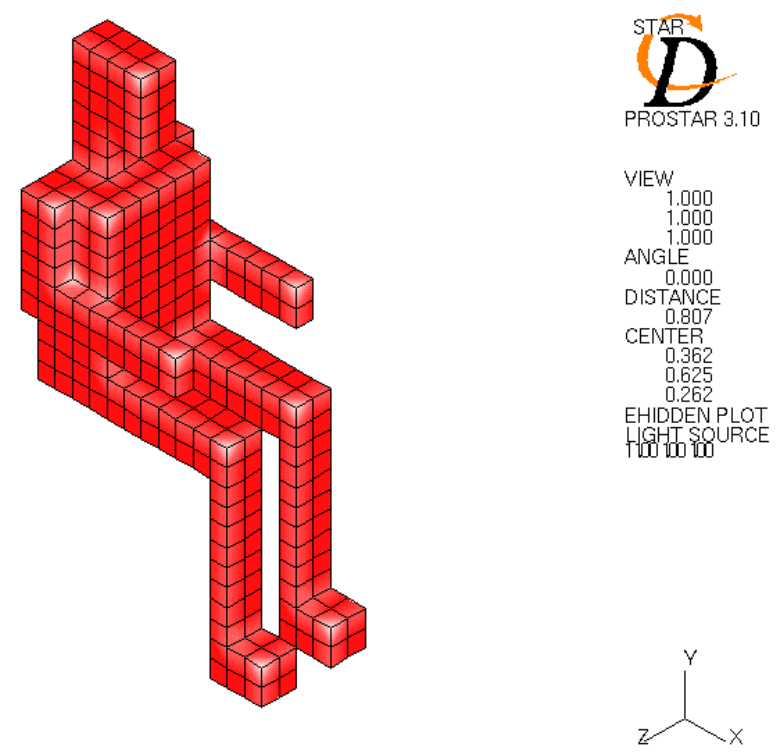

Figure B1. MANIKIN3 built with the code from any one of the tables above and reversed to a solid. (PROSTAR version 3.102.517, PRE/POST processor for STAR-CD)

\section{Surface temperature user subroutines}

As STAR-CD does permit introduction of modified boundary conditions during the flow field calculations. These functions are then introduced in the CFD calculations with the results of the flow field as input. The BCDEFW (Boundary Condition DEFinition Wall) subroutine enables the user to specify surface temperatures or heat transfer in an arbitrary manner. This function is called several times, for each manikin zone, during every iteration. In order to minimise the computational load the boundary layer surface temperature iterations are made in the POSDAT user subroutine that can be programmed to be called only once at every iteration step. The user subroutines are written so that a surface temperature file (tcl.dat) is updated by "posdat.f" every iteration. This file is then read by "bcdefw.f" whenever calculations of manikin boundaries are needed. The head DATA in "posdat.f" has to be updated according to the clothing and radiation situation. These two Fortran 77 files are then compiled before the first run of the STAR-CD code, using the SolvelOptions menu.

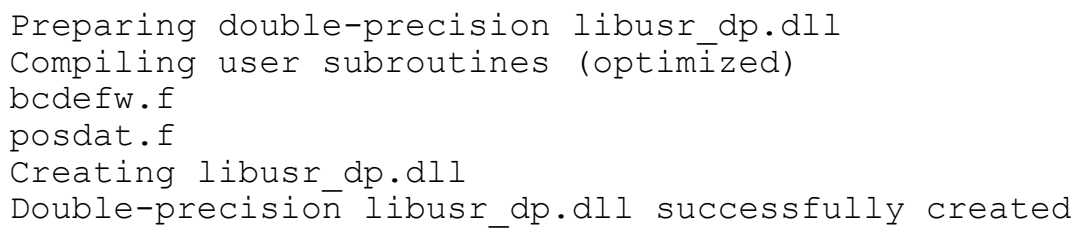

After the compilation is it necessary to check the outcome by viewing the file "stardll.out" for possible errors!

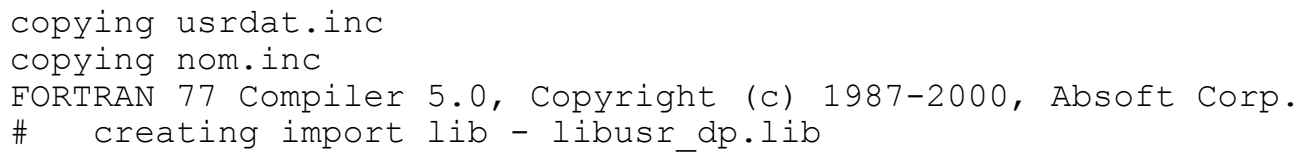




\section{Below the Fortran 77 code used for the "posdat.f" and "bcdefw.f" files. Copy the code and save the files in your "ufile" directory.}

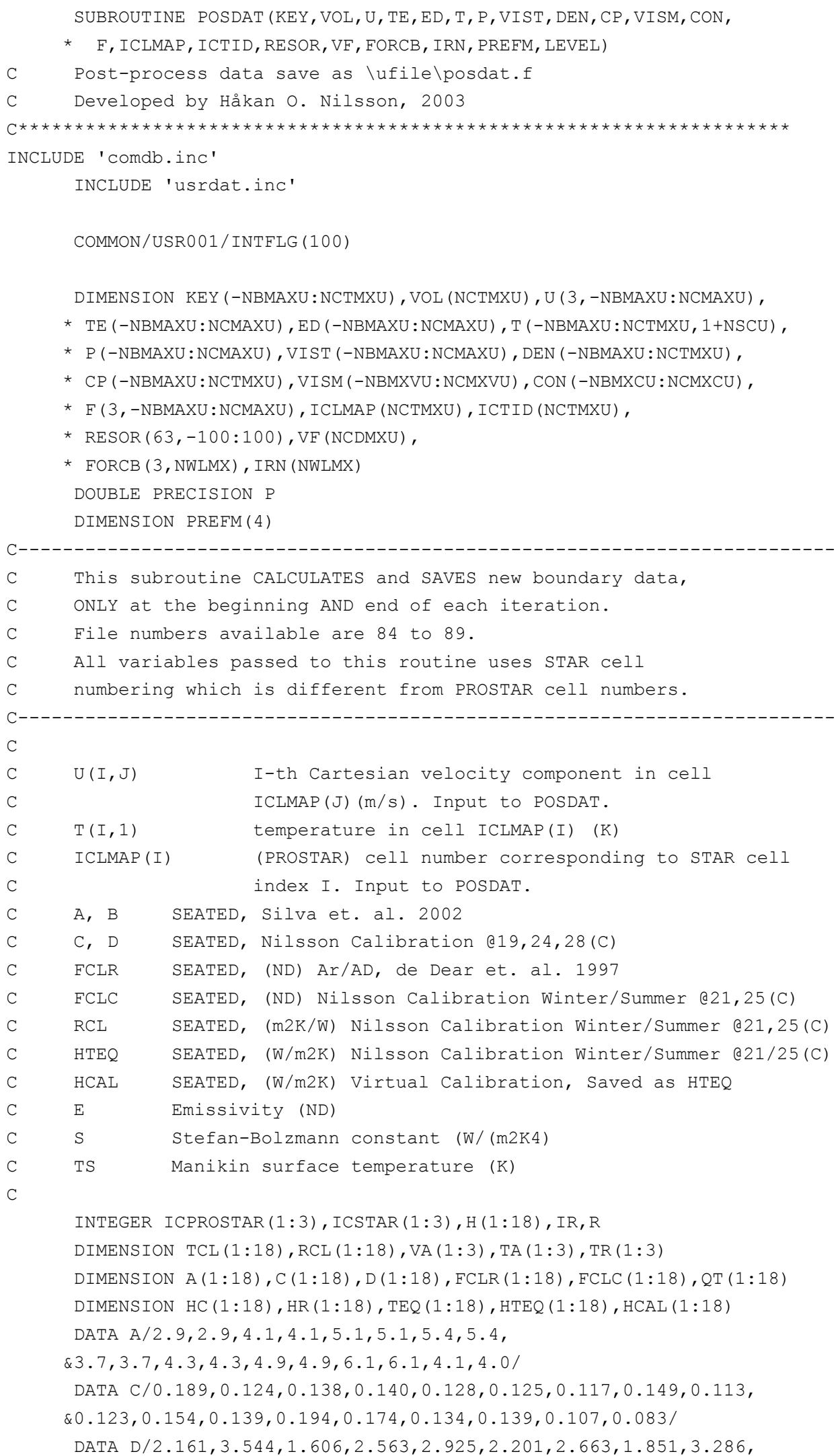


$\& 3.470,1.608,1.787,0.938,0.750,2.927,2.914,0.924,2.086 /$

DATA FCLR/0.66,0.66,0.58,0.78,0.82,0.82,0.88,0.88,0.66,

$\& 0.66,0.78,0.78,0.92,0.92,0.71,0.71,0.78,0.821$

C

C---- ICPROSTAR to ICSTAR conversion

DO $50 \mathrm{~J}=1,3$

DO $40 \mathrm{I}=1, \mathrm{NCELL}$

IF (ICLMAP (I) .EQ.ICPROSTAR (J)) THEN $\operatorname{ICSTAR}(J)=I$

ENDIF 


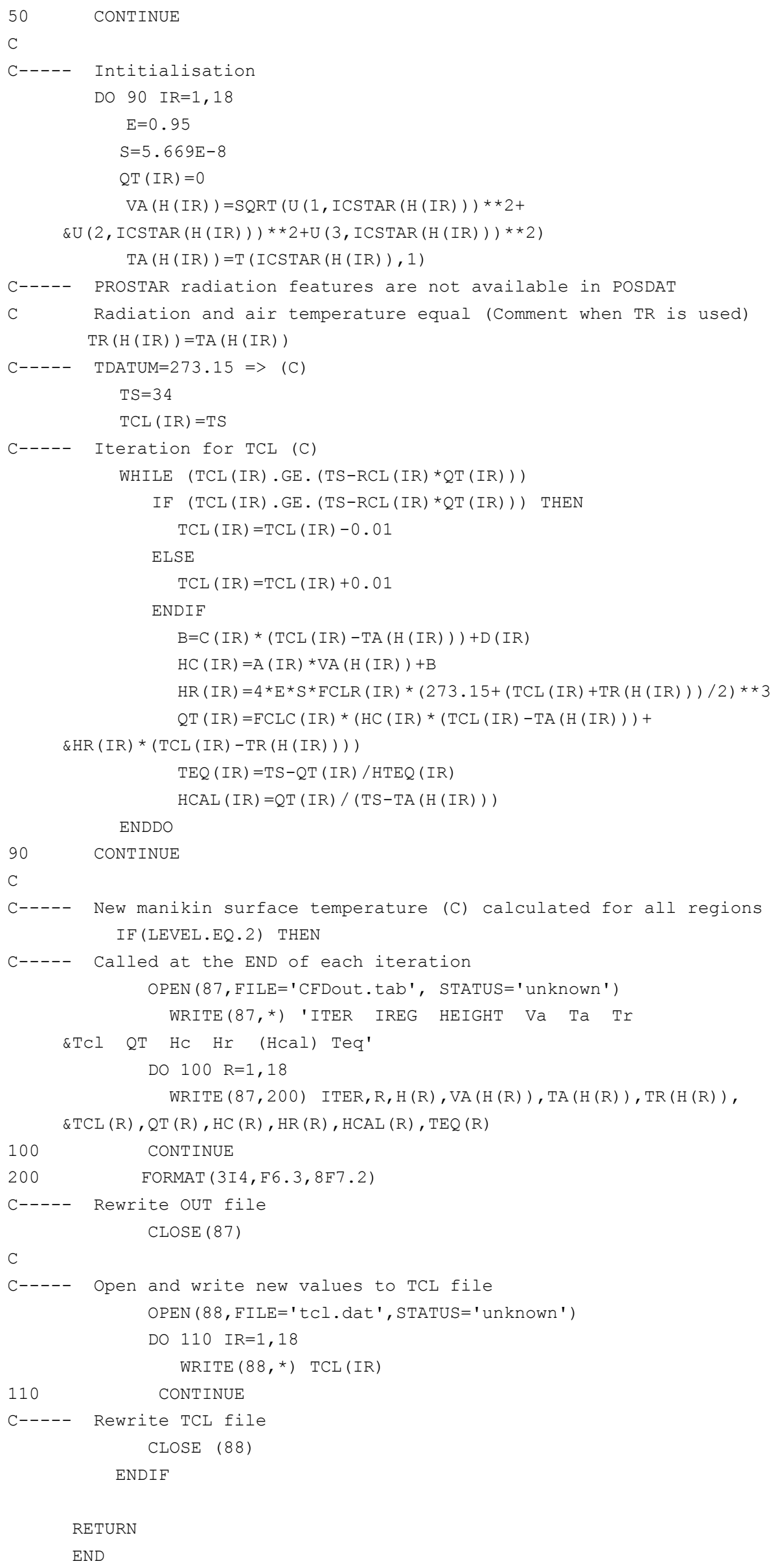




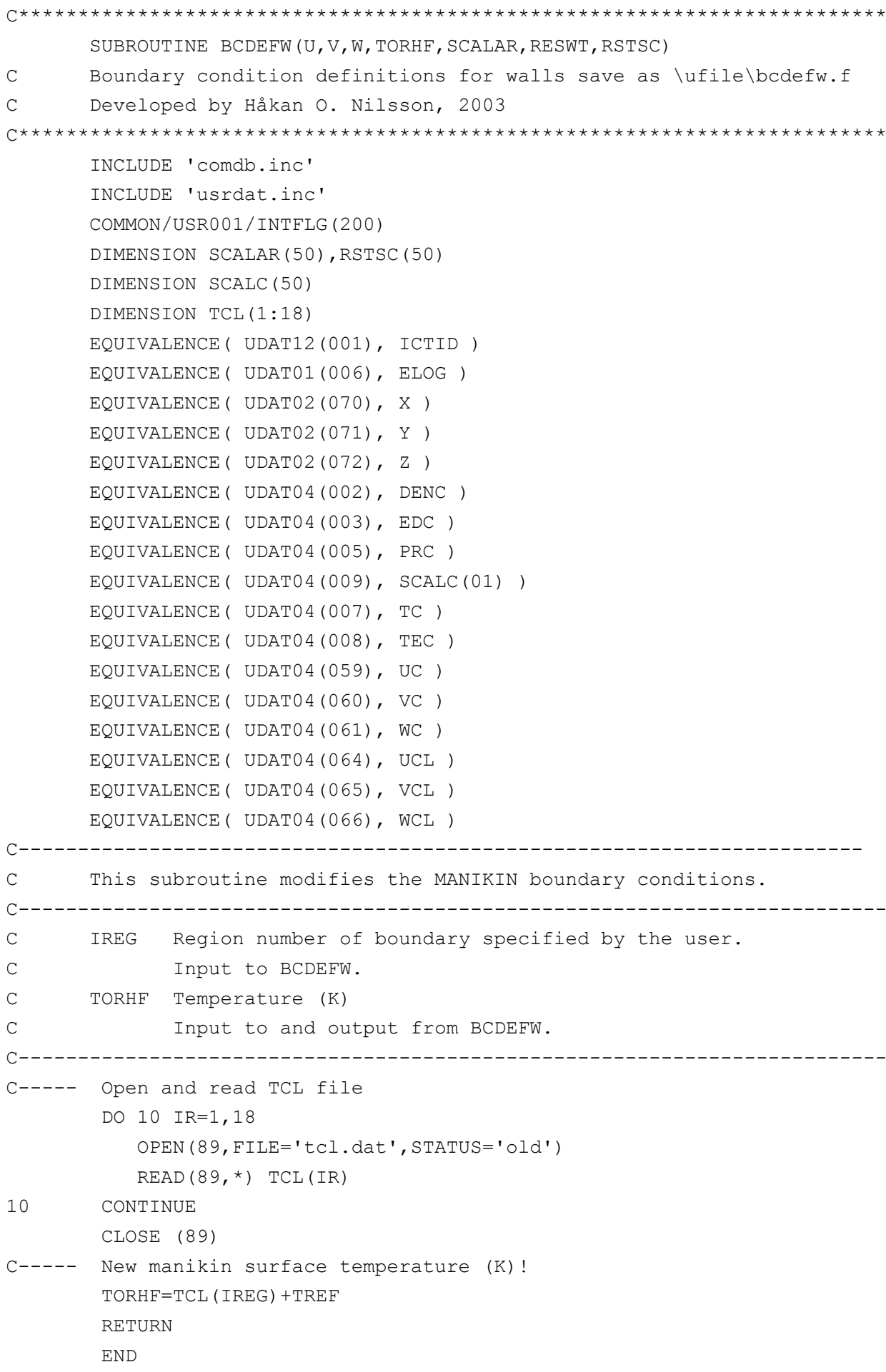




\section{Appendix C}

Geometries and problem specification summaries for the cases

Virtual calibration

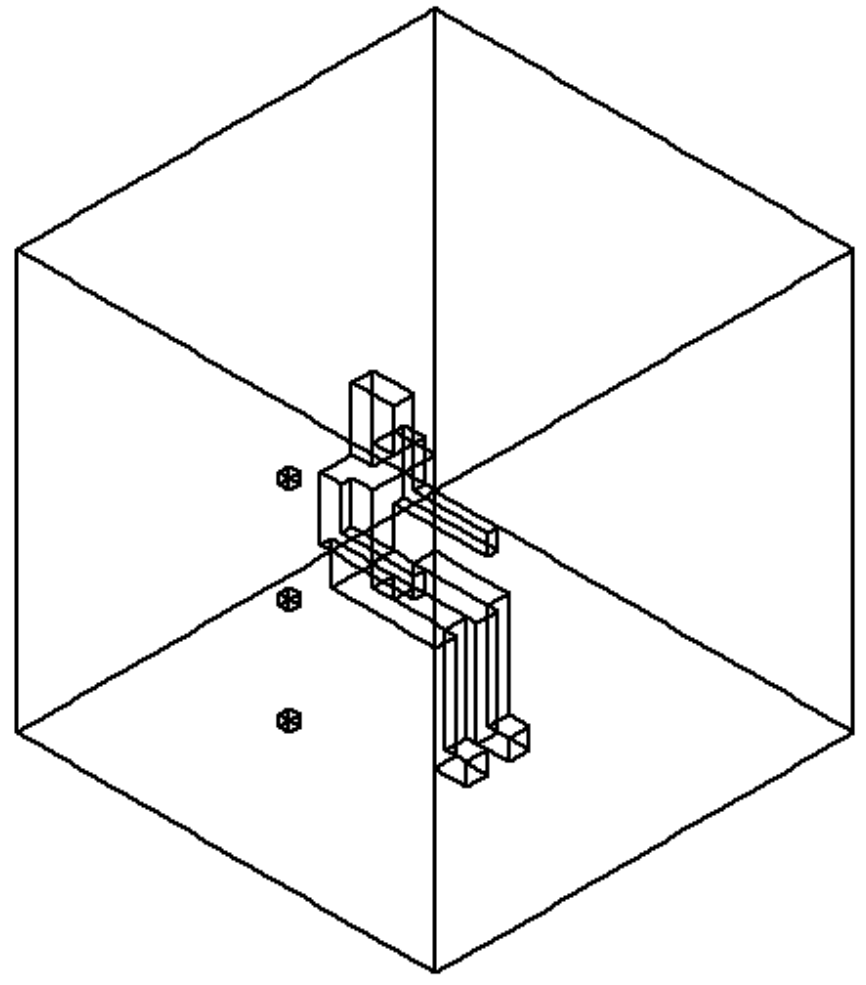

*** YOU ARE RUNNING 64 BIT PRECISION VERSION ***

$* * *$ GEOMETRICAL CALCULATIONS STARTED

*** GEOMETRICAL CALCULATIONS COMPLETED

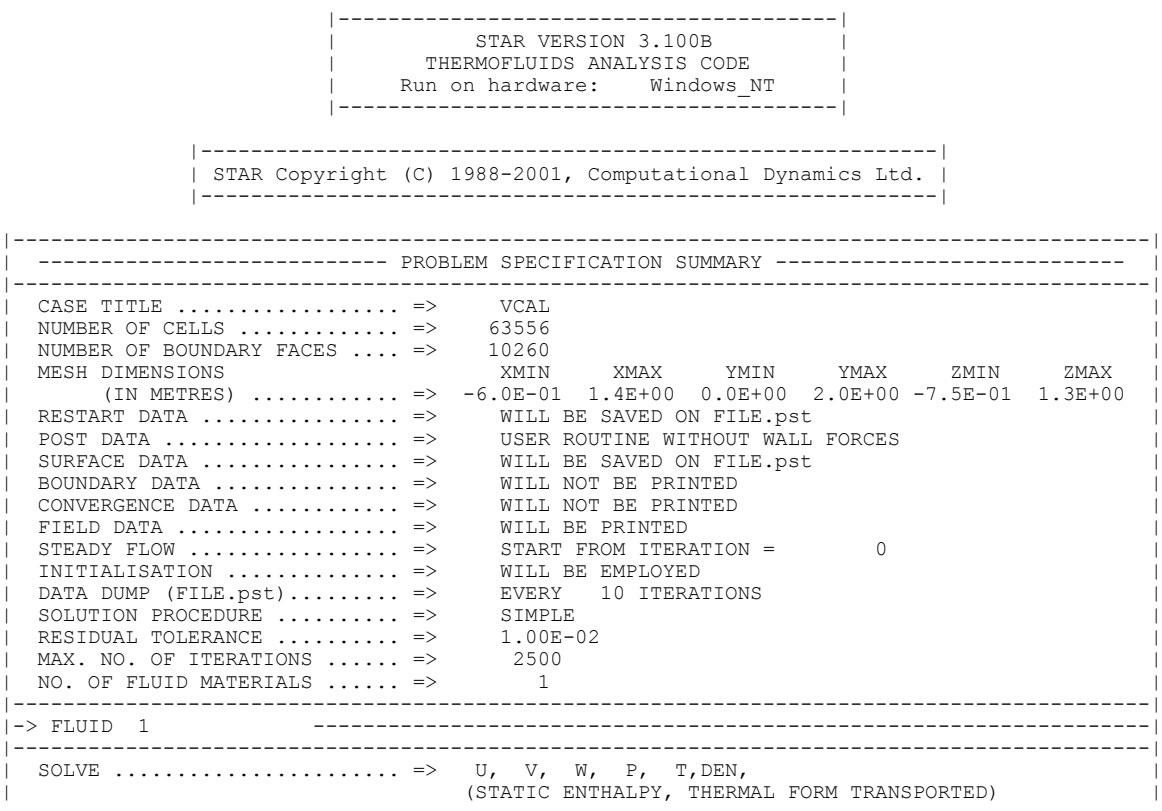




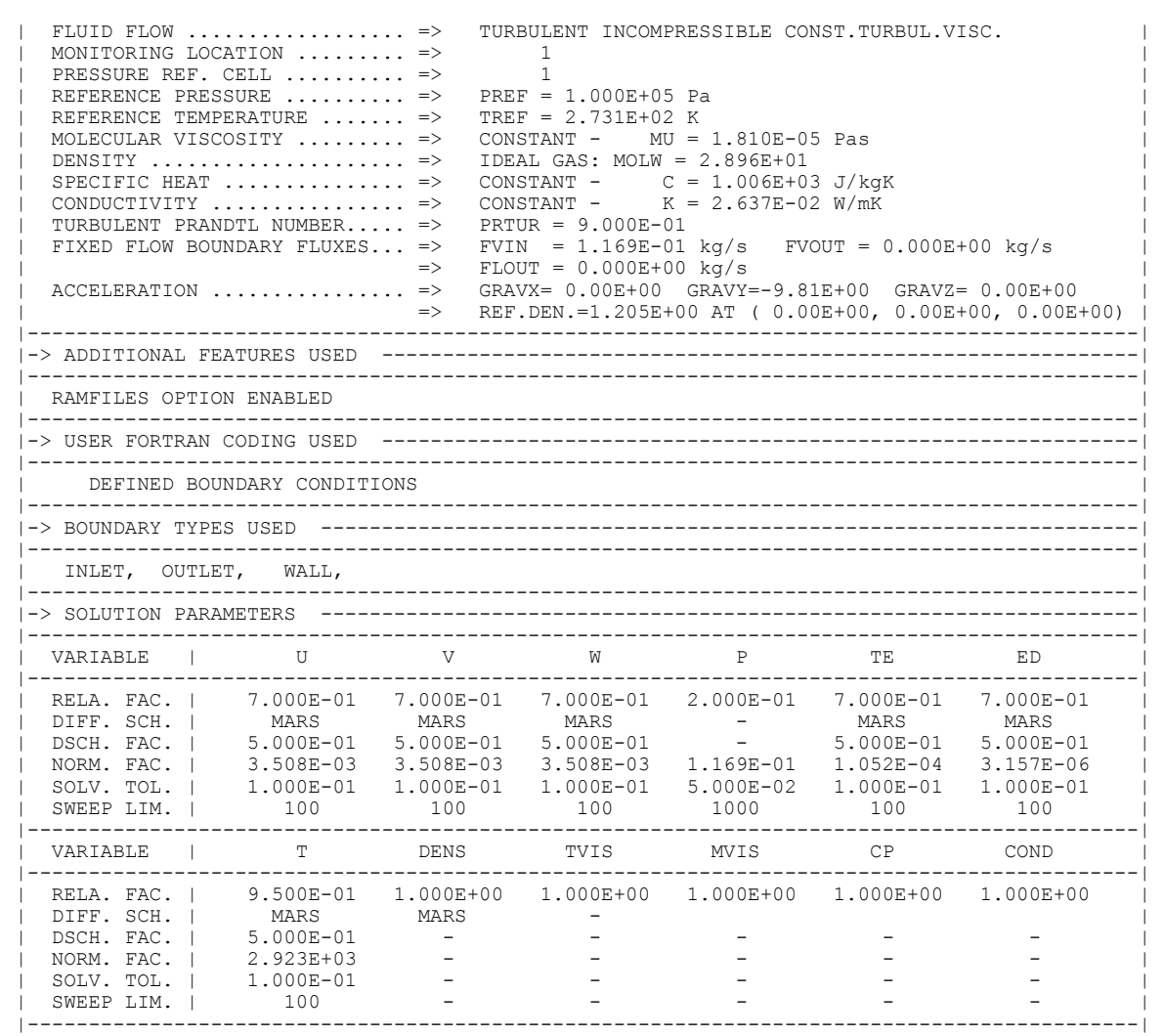

PROSTAR macro file, save as vcal.MAC

!Author Håkan O. Nilsson, 2003

clrm, reverse

pldi,off,all \$pldi,on,box \$wind,0,0,15,10 \$replot

vc3d, $-0.6,1.4,40,0,2,40,-0.75,1.25,40$

cset newset grange, $0.025,0.175,0.975,1.325,0.175,0.325$

cset add grange, $0.125,0.275,1.075,1.325,0.175,0.325$

cset add grange, $0.125,0.275,0.525,1.025,0.075,0.425$

cset add grange, $0.025,0.175,0.825,1.025,0.075,0.425$

cset add grange, $0.025,0.175,0.675,1.025,0.025,0.125$

cset add grange, $0.025,0.175,0.675,1.025,0.375,0.475$

cset add grange, $0.125,0.425,0.675,0.825,0.025,0.125$

cset add grange, $0.125,0.425,0.675,0.825,0.375,0.475$

cset add grange, $0.375,0.525,0.675,0.825,0.025,0.125$

cset add grange, $0.375,0.525,0.675,0.825,0.375,0.475$

cset add grange, $0.225,0.625,0.475,0.625,0.075,0.225$

cset add grange, $0.225,0.625,0.475,0.625,0.275,0.425$

cset add grange, $0.525,0.625,0.075,0.525,0.075,0.225$

cset add grange, $0.525,0.625,0.075,0.525,0.275,0.425$

cset add grange, $0.525,0.725,-0.025,0.125,0.075,0.225$

cset add grange, $0.525,0.725,-0.025,0.125,0.275,0.425$

cset add grange, $0.025,0.175,0.525,0.875,0.075,0.425$

cset add grange, $0.025,0.275,0.475,0.575,0.075,0.425$

cdel,cset

cplot 


\section{Case 1}

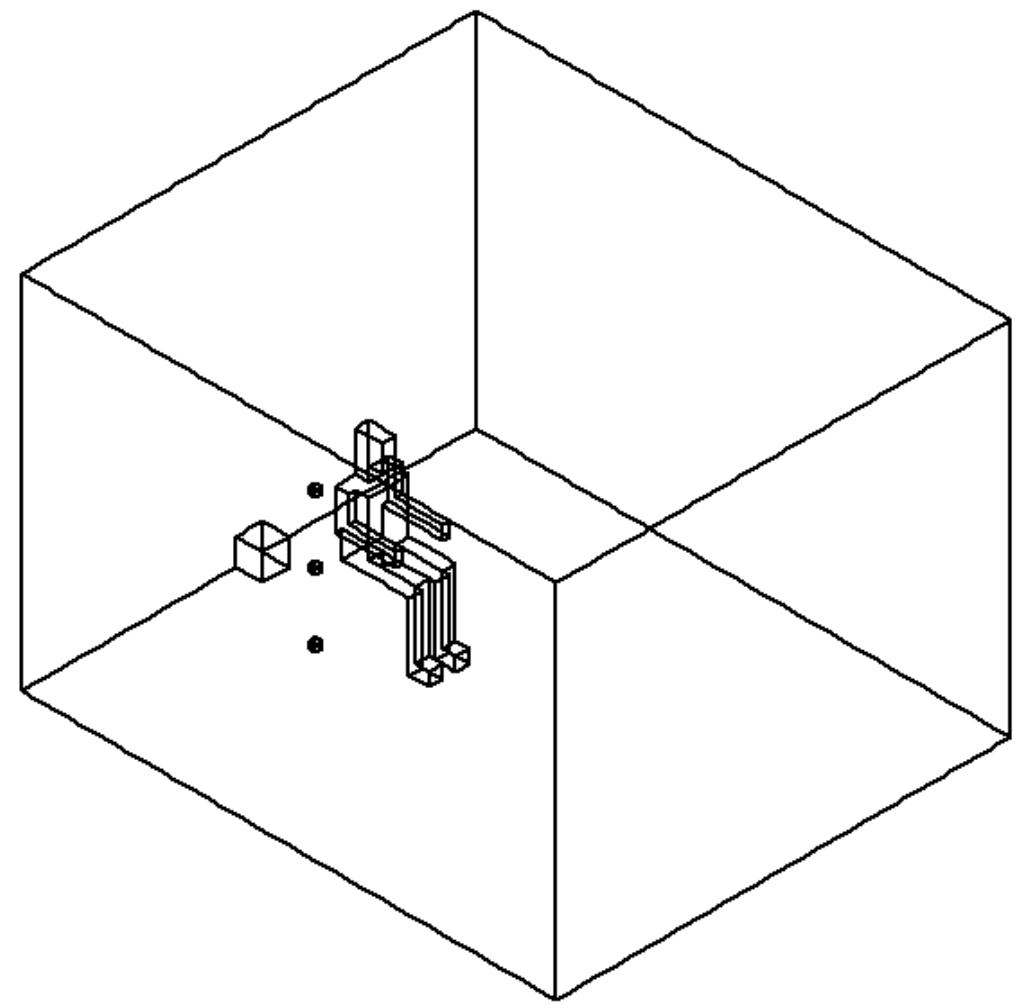

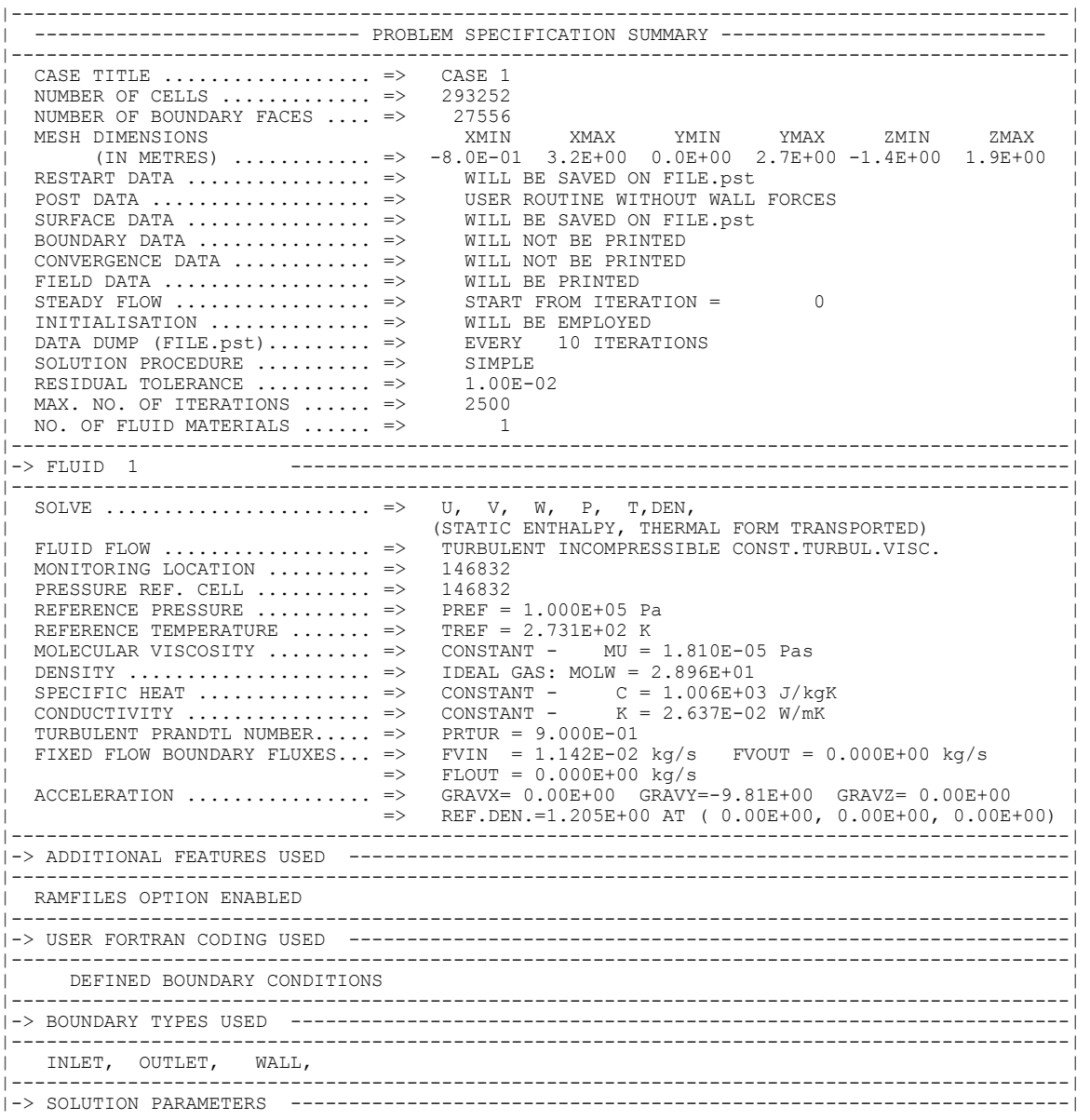




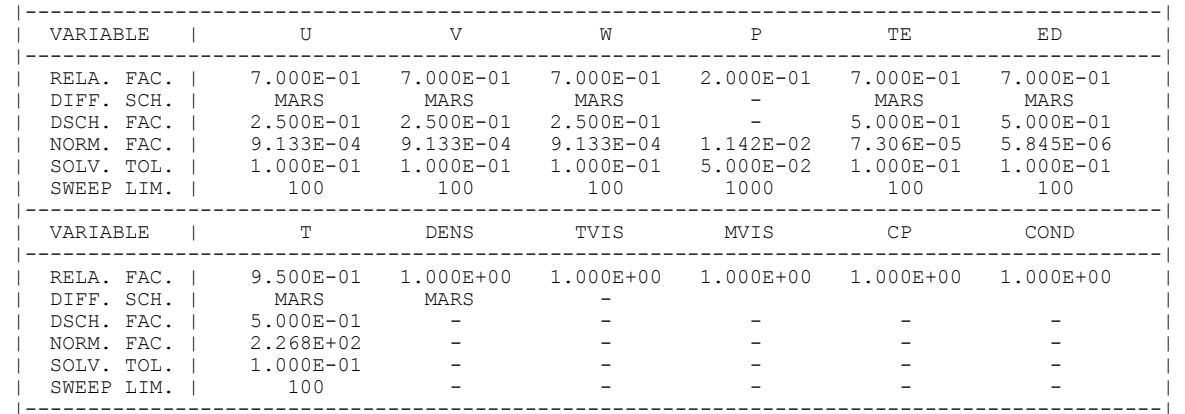

PROSTAR macro file, save as case1.MAC

!Author Håkan O. Nilsson, 2003

clrm, reverse

pldi,off,all \$pldi,on,box \$wind,0,0,15,10 \$replot

vc3d, $-0.8,3.2,80,0,2.7,54,-1.45,1.95,68$

cset newset grange, $0.025,0.175,0.975,1.325,0.175,0.325$

cset add grange, $0.125,0.275,1.075,1.325,0.175,0.325$

cset add grange, $0.125,0.275,0.525,1.025,0.075,0.425$

cset add grange, $0.025,0.175,0.825,1.025,0.075,0.425$

cset add grange, $0.025,0.175,0.675,1.025,0.025,0.125$

cset add grange, $0.025,0.175,0.675,1.025,0.375,0.475$

cset add grange, $0.125,0.425,0.675,0.825,0.025,0.125$

cset add grange, $0.125,0.425,0.675,0.825,0.375,0.475$

cset add grange, $0.375,0.525,0.675,0.825,0.025,0.125$

cset add grange, $0.375,0.525,0.675,0.825,0.375,0.475$

cset add grange, $0.225,0.625,0.475,0.625,0.075,0.225$

cset add grange, $0.225,0.625,0.475,0.625,0.275,0.425$

cset add grange, $0.525,0.625,0.075,0.525,0.075,0.225$

cset add grange, $0.525,0.625,0.075,0.525,0.275,0.425$

cset add grange, $0.525,0.725,-0.025,0.125,0.075,0.225$

cset add grange, $0.525,0.725,-0.025,0.125,0.275,0.425$

cset add grange, $0.025,0.175,0.525,0.875,0.075,0.425$

cset add grange, $0.025,0.275,0.475,0.575,0.075,0.425$

cset add grange, $-0.825,-0.575,-0.025,0.225,0.125,0.375$

cdel,cset

cplot 


\section{Case 2}

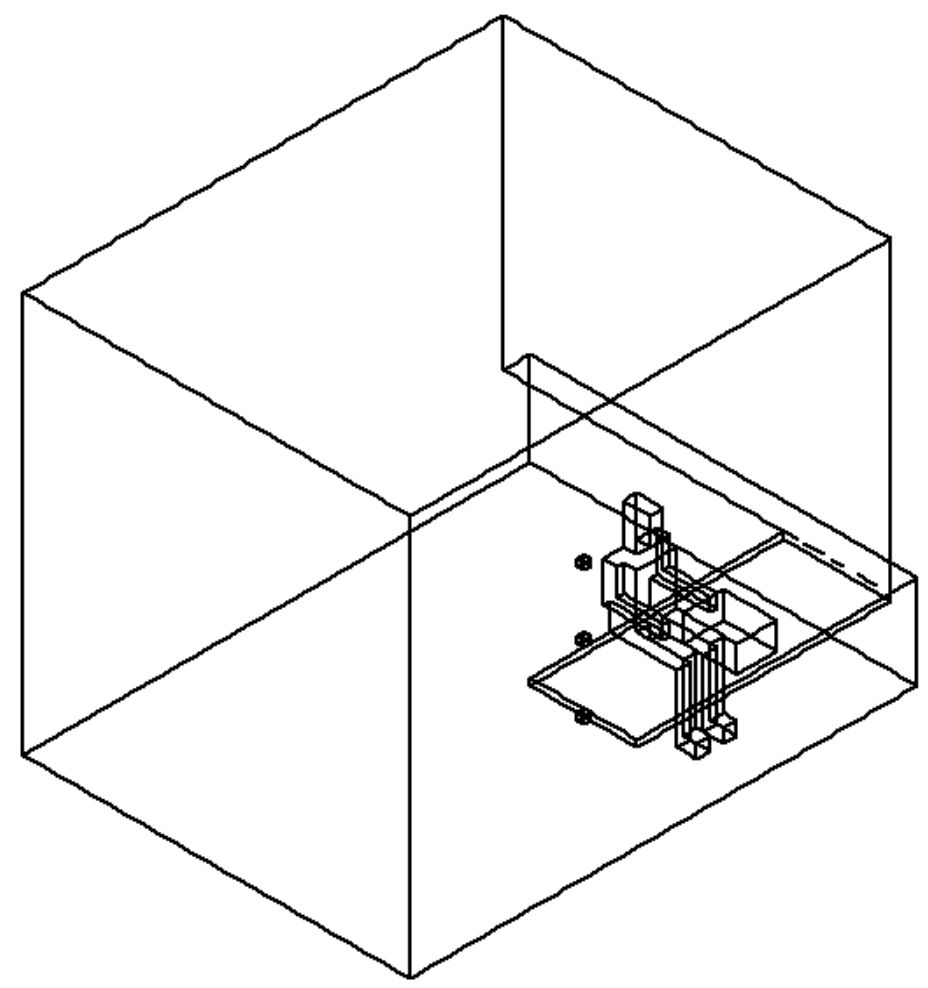

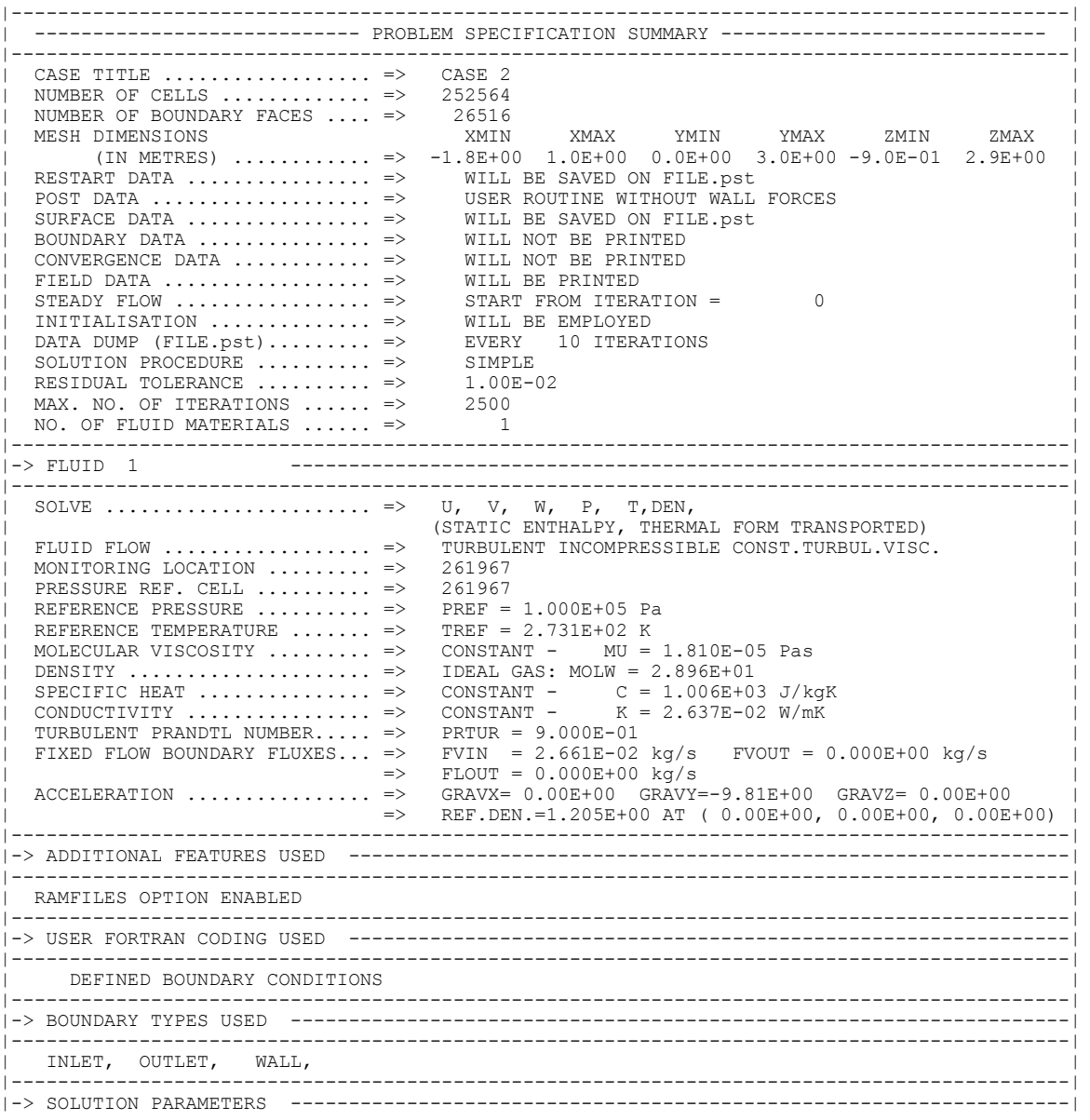




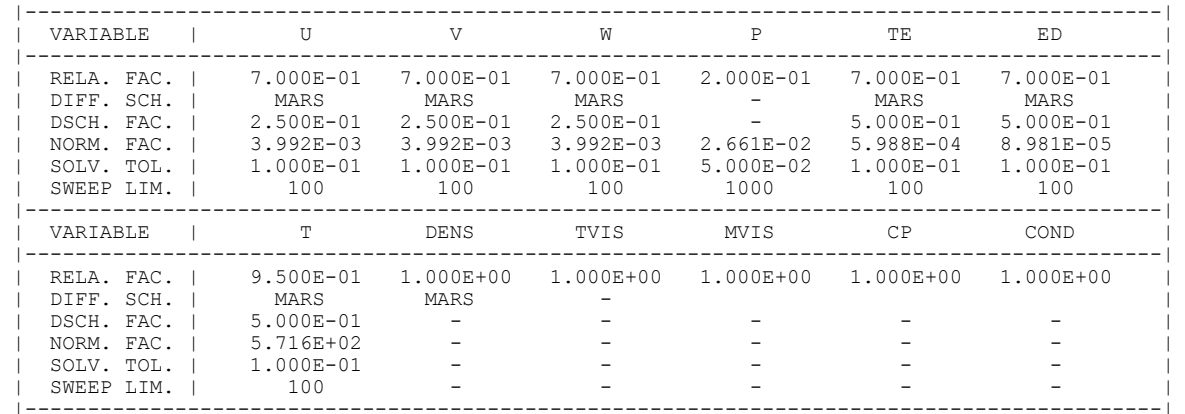

PROSTAR macro file, save as case2.MAC

!Author Håkan O. Nilsson, 2003

clrm, reverse

pldi,off,all \$pldi,on,box \$wind,0,0,15,10

vc3d,-1.85, $1.05,58,0,3,60,-0.90,2.9,76$

cset newset grange, $0.025,0.175,0.975,1.325,0.175,0.325$

cset add grange, $0.125,0.275,1.075,1.325,0.175,0.325$

cset add grange, $0.125,0.275,0.525,1.025,0.075,0.425$

cset add grange, $0.025,0.175,0.825,1.025,0.075,0.425$

cset add grange, $0.025,0.175,0.675,1.025,0.025,0.125$

cset add grange, $0.025,0.175,0.675,1.025,0.375,0.475$

cset add grange, $0.125,0.425,0.675,0.825,0.025,0.125$

cset add grange, $0.125,0.425,0.675,0.825,0.375,0.475$

cset add grange, $0.375,0.525,0.675,0.825,0.025,0.125$

cset add grange, $0.375,0.525,0.675,0.825,0.375,0.475$

cset add grange, $0.225,0.625,0.475,0.625,0.075,0.225$

cset add grange, $0.225,0.625,0.475,0.625,0.275,0.425$

cset add grange, $0.525,0.625,0.075,0.525,0.075,0.225$

cset add grange, $0.525,0.625,0.075,0.525,0.275,0.425$

cset add grange, $0.525,0.725,-0.025,0.125,0.075,0.225$

cset add grange, $0.525,0.725,-0.025,0.125,0.275,0.425$

cset add grange, $0.025,0.175,0.525,0.875,0.075,0.425$

cset add grange, $0.025,0.275,0.475,0.575,0.075,0.425$

cset add grange, $-1.875,1.075,0.675,3.025,-0.675,-0.925$

cset add grange, $0.225,1.075,0.625,0.725,1.225,-0.725$

cset add grange, $0.575,1.025,0.675,0.925,0.425,0.075$

cdel,cset

cplot 


\section{Case 3}

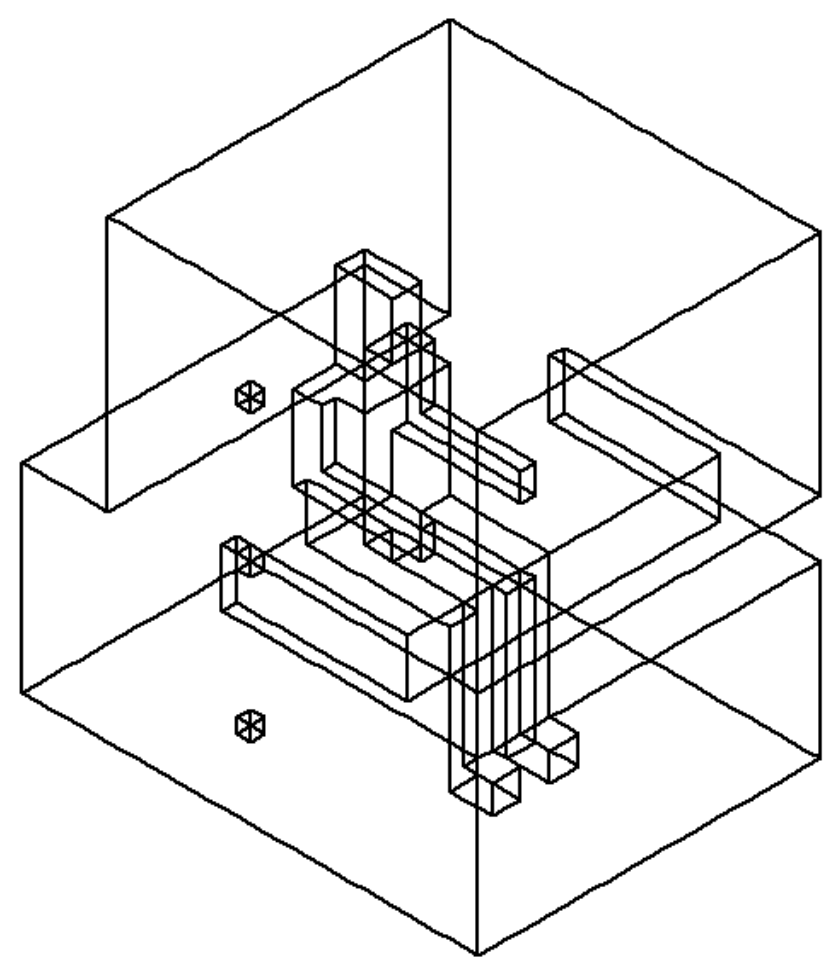

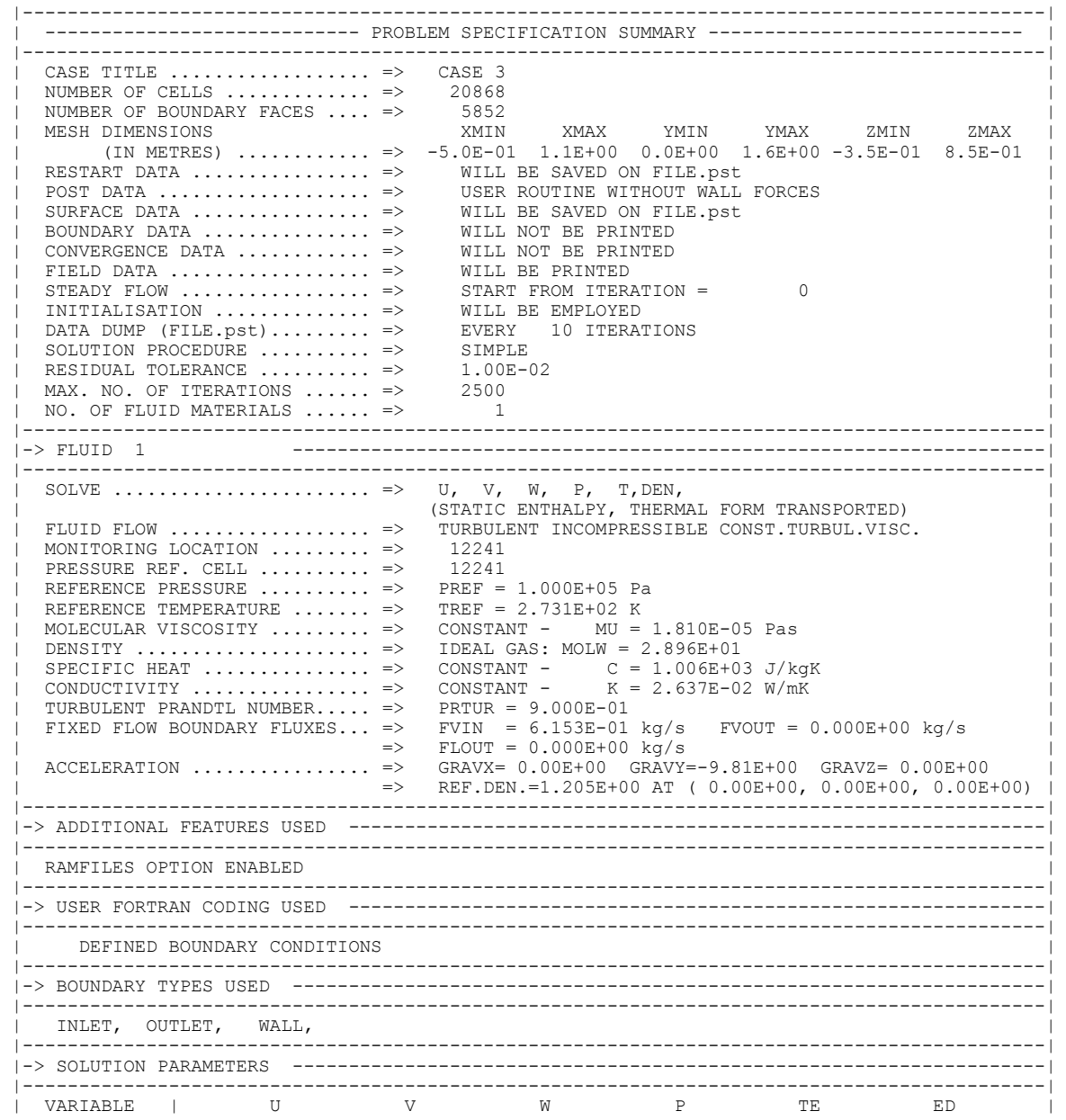




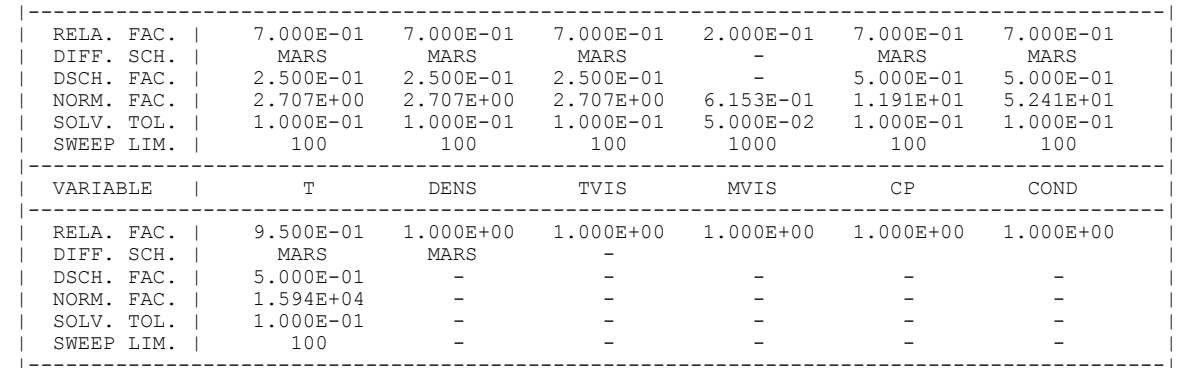

PROSTAR macro file, save as case3.MAC

!Author Håkan O. Nilsson, 2003

clrm, reverse

pldi,off,all \$pldi,on,box \$wind, $0,0,15,10$

vc3d,-0.5, 1.1,32,0,1.6,32,-0.35,0.85,24

cset newset grange, $0.025,0.175,0.975,1.325,0.175,0.325$

cset add grange, $0.125,0.275,1.075,1.325,0.175,0.325$

cset add grange, $0.125,0.275,0.525,1.025,0.075,0.425$

cset add grange, $0.025,0.175,0.825,1.025,0.075,0.425$

cset add grange, $0.025,0.175,0.675,1.025,0.025,0.125$

cset add grange, $0.025,0.175,0.675,1.025,0.375,0.475$

cset add grange, $0.125,0.425,0.675,0.825,0.025,0.125$

cset add grange, $0.125,0.425,0.675,0.825,0.375,0.475$

cset add grange, $0.375,0.525,0.675,0.825,0.025,0.125$

cset add grange, $0.375,0.525,0.675,0.825,0.375,0.475$

cset add grange, $0.225,0.625,0.475,0.625,0.075,0.225$

cset add grange, $0.225,0.625,0.475,0.625,0.275,0.425$

cset add grange, $0.525,0.625,0.075,0.525,0.075,0.225$

cset add grange, $0.525,0.625,0.075,0.525,0.275,0.425$

cset add grange, $0.525,0.725,-0.025,0.125,0.075,0.225$

cset add grange, $0.525,0.725,-0.025,0.125,0.275,0.425$

cset add grange, $0.025,0.175,0.525,0.875,0.075,0.425$

cset add grange, $0.025,0.275,0.475,0.575,0.075,0.425$

cset add grange, $-0.175,-0.525,0.675,1.625,-0.375,0.875$

cset add grange, $0.775,1.125,0.575,0.825,-0.375,0.875$

cset add grange, $0.175,0.825,0.575,0.825,-0.375,-0.275$

cset add grange, $0.175,0.825,0.575,0.825,0.775,0.875$

cdel,cset

cplot 


\section{Appendix D}

Linear regressions of MTV against equivalent temperature.

\section{Winter}

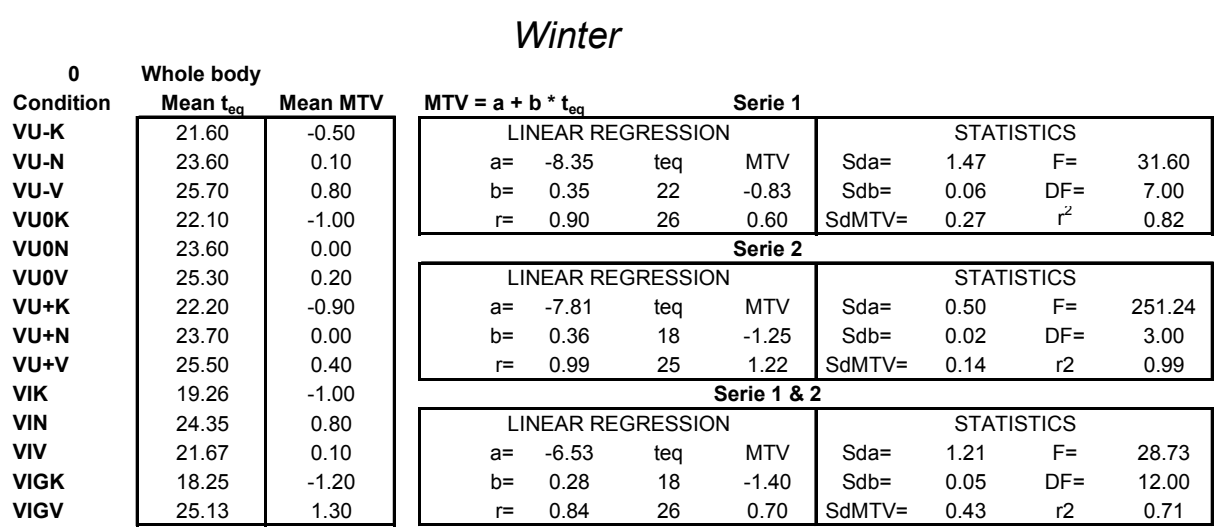

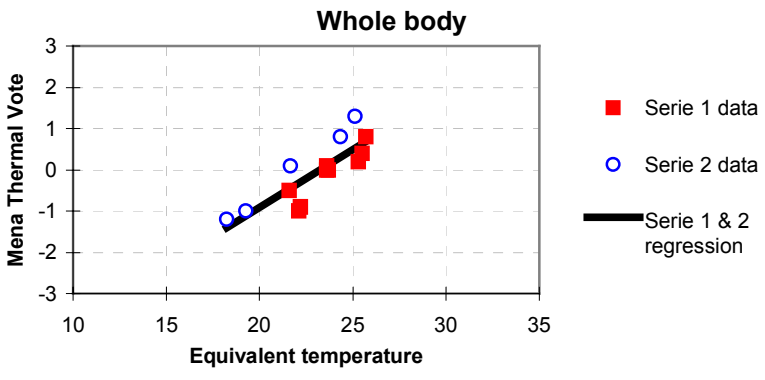

1 Scalp

\begin{tabular}{|c|c|c|c|c|c|c|c|c|c|c|}
\hline Condition & Mean $t_{e q}$ & Mean MTV & \multicolumn{4}{|c|}{ MTV $=a+b * t_{e q} \quad$ Serie 1} & \multirow{2}{*}{\multicolumn{4}{|c|}{ STATISTICS }} \\
\hline VU-K & 19.80 & -0.70 & \multicolumn{4}{|c|}{ LINEAR REGRESSION } & & & & \\
\hline VU-N & 21.20 & -0.30 & $a=$ & -4.49 & teq & MTV & $S d a=$ & 0.71 & $F=$ & 45.00 \\
\hline VU-V & 23.00 & 0.60 & $b=$ & 0.20 & 20 & -0.57 & $S d b=$ & 0.03 & $D F=$ & 7.00 \\
\hline VUOK & 21.90 & -0.40 & $r=$ & 0.93 & 29 & 1.24 & SdMTV= & 0.24 & $r^{2}$ & 0.87 \\
\hline VUON & 23.80 & 0.20 & \multicolumn{8}{|c|}{ Serie 2} \\
\hline vuov & 25.30 & 0.60 & \multicolumn{4}{|c|}{ LINEAR REGRESSION } & \multicolumn{4}{|c|}{ STATISTICS } \\
\hline VU+K & 25.00 & 0.40 & $a=$ & -3.23 & teq & MTV & Sda= & 0.57 & $F=$ & 35.01 \\
\hline $\mathrm{VU}+\mathrm{N}$ & 26.60 & 0.80 & $b=$ & 0.18 & 15 & -0.53 & $\mathrm{Sdb}=$ & 0.03 & $D F=$ & 3.00 \\
\hline vU+v & 28.90 & 1.10 & $r=$ & 0.96 & 23 & 0.84 & SdMTV= & 0.20 & $\mathrm{r} 2$ & 0.92 \\
\hline VIK & 15.79 & -0.60 & \multicolumn{8}{|c|}{ Serie $1 \& 2$} \\
\hline VIN & 20.73 & 0.30 & \multicolumn{4}{|c|}{ LINEAR REGRESSION } & \multicolumn{4}{|c|}{ STATISTICS } \\
\hline VIV & 18.79 & 0.30 & $a=$ & -2.37 & teq & MTV & Sda $=$ & 0.62 & $\mathrm{~F}=$ & 17.99 \\
\hline VIGK & 15.14 & -0.40 & $b=$ & 0.12 & 15 & -0.61 & $S d b=$ & 0.03 & $D F=$ & 12.00 \\
\hline VIGV & 22.81 & 0.90 & $r=$ & 0.77 & 29 & 1.00 & SdMTV= & 0.39 & r2 & 0.60 \\
\hline
\end{tabular}

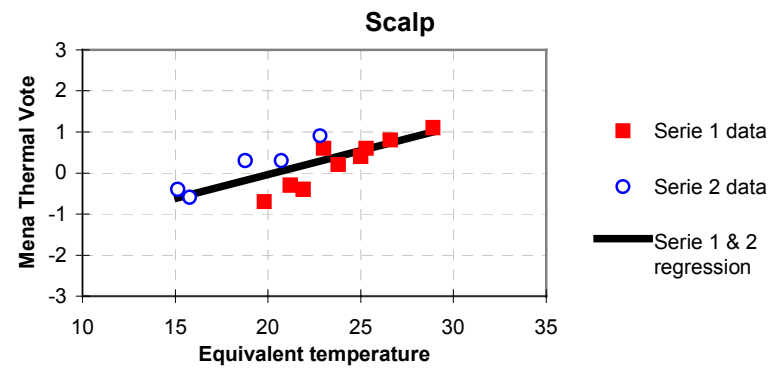


$2 \quad F a c$

\begin{tabular}{|c|c|c|c|c|c|c|c|c|c|c|}
\hline \multirow{2}{*}{$\begin{array}{l}\text { Condition } \\
\text { VU-K }\end{array}$} & \multirow{2}{*}{$\frac{\text { Mean } \mathbf{t}_{\mathrm{eq}}}{16.70}$} & \multirow{2}{*}{$\begin{array}{c}\text { Mean MTV } \\
-0.70\end{array}$} & \multicolumn{4}{|c|}{$M T V=a+b * t_{e q} \quad$ Serie 1} & \multirow{2}{*}{\multicolumn{4}{|c|}{ STATISTICS }} \\
\hline & & & \multicolumn{4}{|c|}{ LINEAR REGRESSION } & \multicolumn{2}{|c|}{ STATISTICS } & & \\
\hline VU-N & 18.70 & -0.30 & $a=$ & -4.08 & teq & MTV & Sda $=$ & 0.53 & $\mathrm{~F}=$ & 68.24 \\
\hline VU-V & 21.10 & 0.60 & $b=$ & 0.20 & 17 & -0.71 & $S d b=$ & 0.02 & $\mathrm{DF}=$ & 7.00 \\
\hline VUOK & 19.60 & -0.40 & $r=$ & 0.95 & 26 & 1.12 & SdMTV= & 0.20 & $r^{2}$ & 0.91 \\
\hline VUON & 21.60 & 0.20 & \multicolumn{8}{|c|}{ Serie 2} \\
\hline VuOV & 23.70 & 0.60 & \multicolumn{4}{|c|}{ LINEAR REGRESSION } & \multicolumn{4}{|c|}{ STATISTICS } \\
\hline VU+K & 22.30 & 0.40 & $a=$ & -2.84 & teq & MTV & Sda $=$ & 0.82 & $\mathrm{~F}=$ & 13.90 \\
\hline $\mathrm{VU}+\mathrm{N}$ & 24.10 & 0.80 & $b=$ & 0.16 & 15 & -0.52 & $S d b=$ & 0.04 & $\mathrm{DF}=$ & 3.00 \\
\hline $\mathrm{VU}+\mathrm{V}$ & 25.80 & 1.10 & $r=$ & 0.91 & 25 & 1.08 & SdMTV= & 0.33 & $\mathrm{r} 2$ & 0.82 \\
\hline VIK & 14.55 & -0.30 & \multicolumn{8}{|c|}{ Serie $1 \& 2$} \\
\hline VIN & 20.54 & 0.20 & \multicolumn{4}{|c|}{ LINEAR REGRESSION } & \multicolumn{4}{|c|}{ STATISTICS } \\
\hline VIV & 18.00 & 0.30 & $a=$ & -3.04 & teq & MTV & Sda $=$ & 0.51 & $\mathrm{~F}=$ & 42.16 \\
\hline VIGK & 16.46 & -0.60 & $b=$ & 0.16 & 15 & -0.73 & $S d b=$ & 0.02 & $\mathrm{DF}=$ & 12.00 \\
\hline VIGV & 24.65 & 1.20 & $r=$ & 0.88 & 26 & 1.05 & SdMTV= & 0.30 & $\mathrm{r} 2$ & 0.78 \\
\hline
\end{tabular}

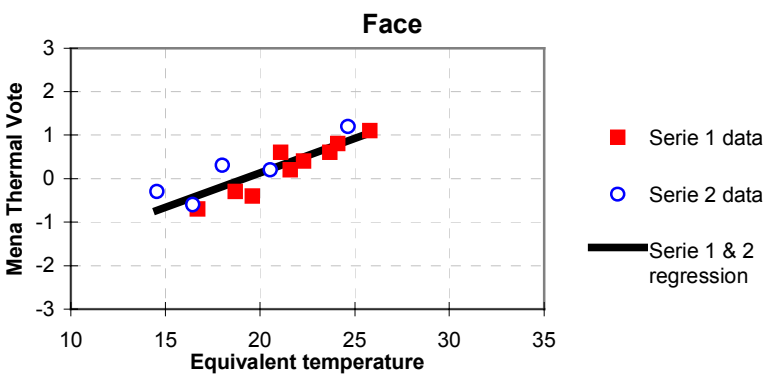

3 Chest

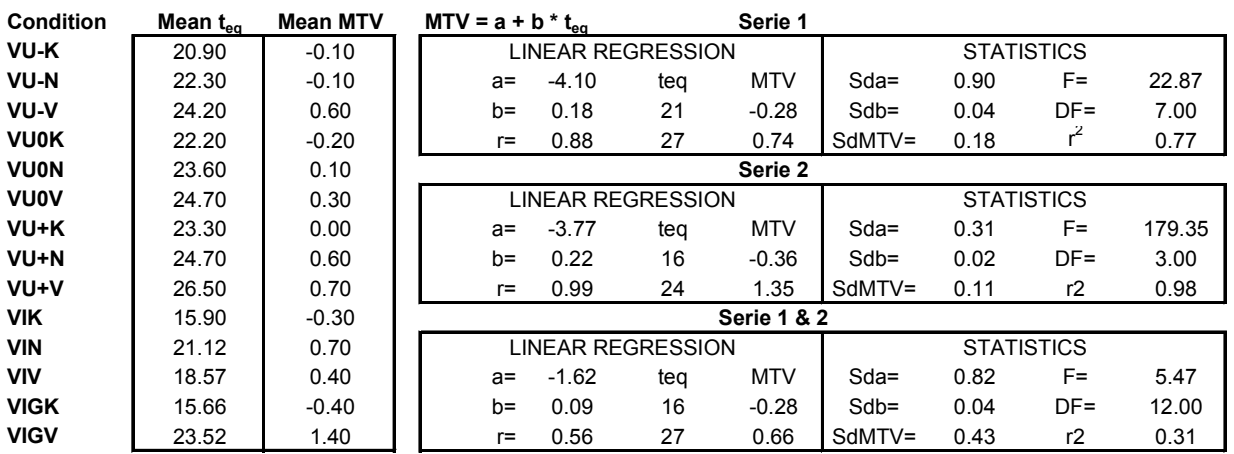

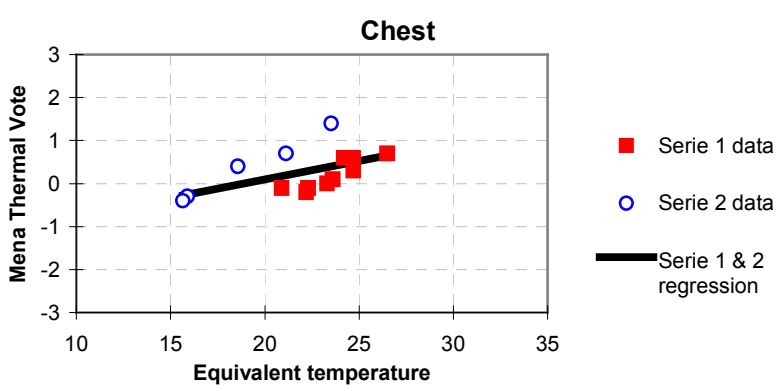


Up. Back

\begin{tabular}{|c|c|c|c|c|c|c|c|c|c|c|}
\hline Condition & Mean $t_{e q}$ & Mean MTV & MTV $=a$ & $0^{*} t_{e q}$ & & Serie 1 & & & & \\
\hline VU-K & 18.30 & -0.10 & & JEAR R & RESS & & & STA & TICS & \\
\hline VU-N & 19.80 & 0.00 & $a=$ & -1.87 & teq & MTV & Sda $=$ & 0.56 & $F=$ & 14.84 \\
\hline VU-V & 22.00 & 0.50 & $b=$ & 0.10 & 18 & -0.11 & $S d b=$ & 0.03 & $D F=$ & 7.00 \\
\hline VUOK & 20.50 & 0.00 & $r=$ & 0.82 & 26 & 0.61 & SdMTV $=$ & 0.18 & $r^{2}$ & 0.68 \\
\hline VUON & 22.90 & 0.30 & & & & Serie 2 & & & & \\
\hline VuOV & 25.40 & 0.40 & & JEAR R & RESS & & & STA & TICS & \\
\hline $\mathrm{VU}+\mathrm{K}$ & 22.30 & 0.10 & $a=$ & -5.03 & teq & MTV & Sda $=$ & 2.00 & $F=$ & 6.60 \\
\hline $\mathrm{VU}+\mathrm{N}$ & 23.80 & 0.70 & $b=$ & 0.21 & 21 & -0.69 & $S d b=$ & 0.08 & $D F=$ & 3.00 \\
\hline $\mathrm{VU}+\mathrm{V}$ & 25.70 & 0.60 & $r=$ & 0.83 & 27 & 0.66 & SdMTV $=$ & 0.40 & r2 & 0.69 \\
\hline VIK & 23.81 & -0.40 & & & & erie $1 \&$ & & & & \\
\hline VIN & 27.32 & 0.50 & & JEAR R & $\overline{R E S S}$ & & & STA & TICS & \\
\hline VIV & 25.39 & 0.10 & $a=$ & -1.95 & teq & MTV & Sda $=$ & 0.87 & $F=$ & 6.28 \\
\hline VIGK & 20.81 & -0.60 & $b=$ & 0.09 & 18 & -0.24 & $\mathrm{Sdb}=$ & 0.04 & $\mathrm{DF}=$ & 12.00 \\
\hline VIGV & 25.78 & 0.90 & $r=$ & 0.59 & 27 & 0.61 & SdMTV= & 0.36 & r2 & 0.34 \\
\hline
\end{tabular}

Up. Back

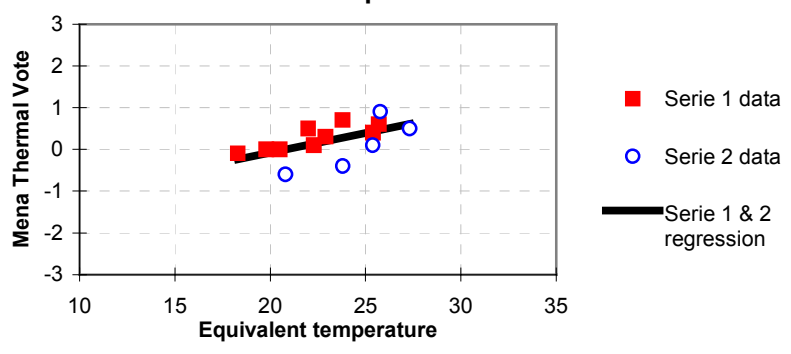

$5 \quad \mathrm{LU}$ arm

\begin{tabular}{|c|c|c|c|c|c|c|c|c|c|c|}
\hline \multirow{3}{*}{$\begin{array}{l}\text { Condition } \\
\text { VU-K } \\
\text { VU-N }\end{array}$} & \multirow{3}{*}{$\begin{array}{c}\text { Mean } \mathbf{t}_{\mathrm{eq}} \\
18.30 \\
19.60\end{array}$} & \multirow{3}{*}{$\begin{array}{c}\text { Mean MTV } \\
-0.10 \\
-0.10\end{array}$} & \multirow{2}{*}{\multicolumn{4}{|c|}{ MTV $=\mathbf{a}+\mathbf{b}^{*} \mathbf{t}_{\mathrm{eq}} \quad$ Serie $\mathbf{1}$}} & \multirow{2}{*}{\multicolumn{4}{|c|}{ STATISTICS }} \\
\hline & & & & & & & & & & \\
\hline & & & $a=$ & -3.70 & teq & MTV & $S d a=$ & 0.81 & $F=$ & 23.26 \\
\hline VU-V & 20.70 & 0.50 & $b=$ & 0.19 & 18 & -0.22 & $S d b=$ & 0.04 & $D F=$ & 7.00 \\
\hline VUOK & 18.70 & -0.20 & $r=$ & 0.88 & 23 & 0.58 & SdMTV= & 0.16 & $r^{2}$ & 0.77 \\
\hline VUON & 20.70 & 0.20 & \multicolumn{8}{|c|}{ Serie 2} \\
\hline Vuov & 22.50 & 0.50 & \multicolumn{4}{|c|}{ LINEAR REGRESSION } & \multicolumn{4}{|c|}{ STATISTICS } \\
\hline$V U+K$ & 20.10 & -0.10 & $a=$ & -3.72 & teq & MTV & $\mathrm{Sda}=$ & 0.48 & $\mathrm{~F}=$ & 65.40 \\
\hline $\mathrm{VU}+\mathrm{N}$ & 20.70 & 0.40 & $b=$ & 0.22 & 14 & -0.62 & $S d b=$ & 0.03 & $\mathrm{DF}=$ & 3.00 \\
\hline VU+V & 22.50 & 0.60 & $r=$ & 0.98 & 21 & 1.02 & SdMTV= & 0.17 & r2 & 0.96 \\
\hline VIK & 14.80 & -0.60 & \multicolumn{8}{|c|}{ Serie $1 \& 2$} \\
\hline VIN & 19.59 & 0.60 & \multicolumn{4}{|c|}{ LINEAR REGRESSION } & \multicolumn{4}{|c|}{ STATISTICS } \\
\hline VIV & 16.54 & 0.20 & $a=$ & -2.58 & teq & MTV & $\mathrm{Sda}=$ & 0.61 & $\mathrm{~F}=$ & 20.55 \\
\hline VIGK & 13.86 & -0.60 & $b=$ & 0.14 & 14 & -0.60 & $S d b=$ & 0.03 & $D F=$ & 12.00 \\
\hline VIGV & 21.24 & 1.00 & $r=$ & 0.79 & 23 & 0.62 & SdMTV= & 0.30 & r2 & 0.63 \\
\hline
\end{tabular}

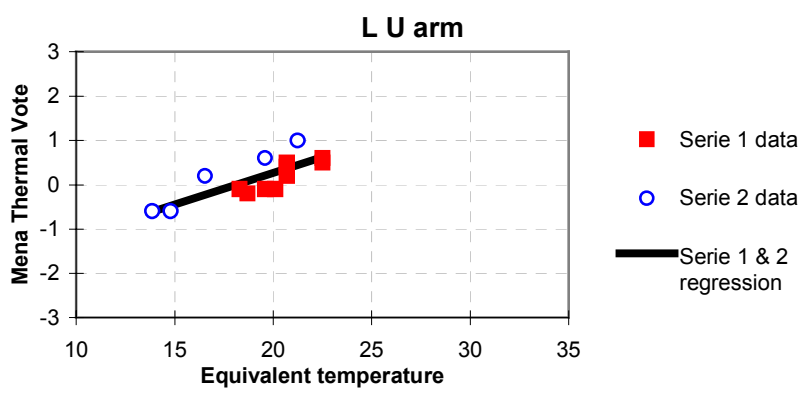


R U arm

\begin{tabular}{|c|c|c|c|c|c|c|c|c|c|c|}
\hline \multirow{3}{*}{$\begin{array}{l}\text { Condition } \\
\text { VU-K } \\
\text { VU-N }\end{array}$} & \multirow{3}{*}{$\begin{array}{c}\text { Mean } \mathbf{t}_{\mathrm{eq}} \\
20.60 \\
21.80\end{array}$} & \multirow{2}{*}{$\frac{\text { Mean MTV }}{-0.10}$} & \multirow{2}{*}{\multicolumn{4}{|c|}{ MTV $=a+b * t_{e q} \quad$ Serie 1}} & \multirow{2}{*}{\multicolumn{4}{|c|}{ STATISTICS }} \\
\hline & & & & & & LINEAR REGRESSION & & & & \\
\hline & & -0.10 & $a=$ & -4.05 & teq & MTV & $\mathrm{Sda}=$ & 1.09 & $\mathrm{~F}=$ & 15.24 \\
\hline VU-V & 23.60 & 0.50 & $b=$ & 0.19 & 21 & -0.22 & $S d b=$ & 0.05 & $\mathrm{DF}=$ & 7.00 \\
\hline VUOK & 22.40 & -0.20 & $r=$ & 0.83 & 25 & 0.62 & SdMTV= & 0.19 & $r^{2}$ & 0.69 \\
\hline VUON & 23.30 & 0.20 & \multicolumn{8}{|c|}{ Serie 2} \\
\hline Vuov & 25.10 & 0.50 & \multicolumn{4}{|c|}{ LINEAR REGRESSION } & \multicolumn{4}{|c|}{ STATISTICS } \\
\hline $\mathrm{VU}+\mathrm{K}$ & 21.80 & 0.00 & $a=$ & -3.62 & teq & MTV & $\mathrm{Sda}=$ & 0.58 & $\mathrm{~F}=$ & 42.78 \\
\hline $\mathrm{VU}+\mathrm{N}$ & 23.00 & 0.50 & $b=$ & 0.22 & 14 & -0.56 & $S d b=$ & 0.03 & $D F=$ & 3.00 \\
\hline VU+V & 24.50 & 0.60 & $r=$ & 0.97 & 22 & 1.06 & SdMTV= & 0.22 & r2 & 0.93 \\
\hline VIK & 14.10 & -0.60 & \multicolumn{8}{|c|}{ Serie $1 \& 2$} \\
\hline VIN & 19.61 & 0.60 & \multicolumn{4}{|c|}{ LINEAR REGRESSION } & \multicolumn{4}{|c|}{ STATISTICS } \\
\hline VIV & 16.15 & 0.20 & $a=$ & -1.65 & teq & MTV & $\mathrm{Sda}=$ & 0.63 & $\mathrm{~F}=$ & 8.59 \\
\hline VIGK & 14.36 & -0.70 & $b=$ & 0.09 & 14 & -0.42 & $\mathrm{Sdb}=$ & 0.03 & $\mathrm{DF}=$ & 12.00 \\
\hline VIGV & 21.58 & 1.00 & $r=$ & 0.65 & 25 & 0.54 & SdMTV= & 0.38 & r2 & 0.42 \\
\hline
\end{tabular}

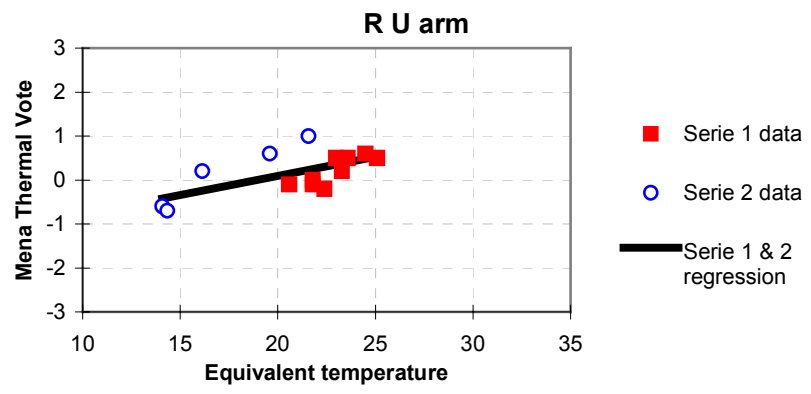

7 L L arm

\begin{tabular}{|c|c|c|c|c|c|c|c|c|c|c|}
\hline \multirow{3}{*}{$\begin{array}{l}\text { Condition } \\
\text { VU-K } \\
\text { VU-N }\end{array}$} & \multirow{3}{*}{$\begin{array}{c}\text { Mean } \mathrm{t}_{\mathrm{eq}} \\
20.20 \\
22.40\end{array}$} & \multirow{3}{*}{$\begin{array}{c}\text { Mean MTV } \\
-0.10 \\
0.20\end{array}$} & \multirow{2}{*}{\multicolumn{4}{|c|}{ MTV $=\mathbf{a}+\mathbf{b}^{*} \mathrm{t}_{\mathrm{eq}} \quad$ Serie 1}} & \multirow{2}{*}{\multicolumn{4}{|c|}{ STATISTICS }} \\
\hline & & & & & & & & & & \\
\hline & & & $a=$ & -3.20 & teq & MTV & $S d a=$ & 0.81 & $\mathrm{~F}=$ & 17.49 \\
\hline VU-V & 24.10 & 0.50 & $b=$ & 0.15 & 20 & -0.25 & $S d b=$ & 0.04 & $\mathrm{DF}=$ & 7.00 \\
\hline VUOK & 21.30 & -0.30 & $r=$ & 0.85 & 26 & 0.53 & SdMTV= & 0.18 & $r^{2}$ & 0.71 \\
\hline VUON & 23.40 & 0.40 & \multicolumn{8}{|c|}{ Serie 2} \\
\hline Vuov & 25.50 & 0.40 & \multicolumn{4}{|c|}{ LINEAR REGRESSION } & \multicolumn{4}{|c|}{ STATISTICS } \\
\hline $\mathrm{VU}+\mathrm{K}$ & 22.10 & -0.20 & $a=$ & -2.71 & teq & MTV & Sda $=$ & 0.77 & $F=$ & 15.46 \\
\hline $\mathrm{VU}+\mathrm{N}$ & 22.80 & 0.10 & $b=$ & 0.14 & 17 & -0.31 & $\mathrm{Sdb}=$ & 0.04 & $\mathrm{DF}=$ & 3.00 \\
\hline Vu+v & 25.40 & 0.50 & $r=$ & 0.92 & 25 & 0.93 & SdMTV= & 0.26 & r2 & 0.84 \\
\hline VIK & 16.80 & -0.30 & \multicolumn{8}{|c|}{ Serie $1 \& 2$} \\
\hline VIN & 23.51 & 0.70 & \multicolumn{4}{|c|}{ LINEAR REGRESSION } & \multicolumn{4}{|c|}{ STATISTICS } \\
\hline VIV & 20.11 & 0.50 & $a=$ & -2.31 & teq & MTV & $\mathrm{Sda}=$ & 0.63 & $F=$ & 16.22 \\
\hline VIGK & 18.11 & -0.40 & $b=$ & 0.11 & 17 & -0.41 & $\mathrm{Sdb}=$ & 0.03 & $\mathrm{DF}=$ & 12.00 \\
\hline VIGV & 25.48 & 0.80 & $r=$ & 0.76 & 26 & 0.57 & SdMTV $=$ & 0.27 & r2 & 0.57 \\
\hline
\end{tabular}

L L arm

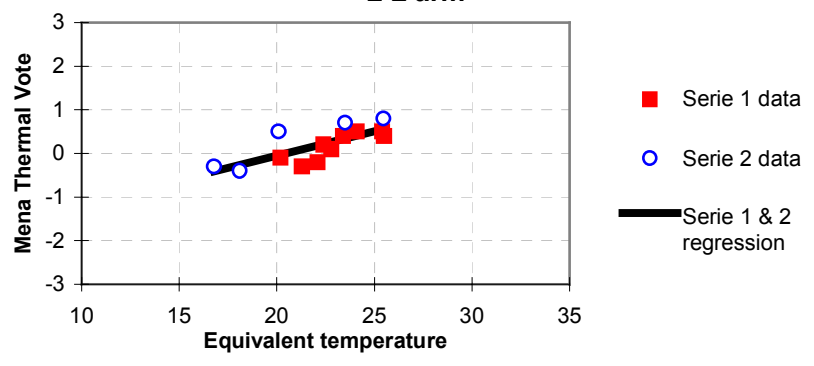


R L arm

\begin{tabular}{l|c|c|}
\multicolumn{1}{l}{$\begin{array}{l}\text { Condition } \\
\text { VU-K }\end{array}$} & \multicolumn{1}{c}{ Mean $\mathrm{t}_{\text {eq }}$} & \multicolumn{1}{c}{ Mean MTV } \\
\cline { 2 - 3 } VU-N & 21.70 & 0.00 \\
VU-V & 23.50 & 0.20 \\
VUOK & 24.90 & 0.50 \\
VUON & 23.00 & -0.20 \\
VUOV & 23.90 & 0.40 \\
VU+K & 25.70 & 0.40 \\
VU+N & 22.10 & 0.00 \\
VU+V & 23.70 & 0.20 \\
VIK & 25.30 & 0.50 \\
VIN & 16.60 & -0.30 \\
VIV & 21.88 & 0.70 \\
VIGK & 18.97 & 0.50 \\
VIGV & 18.11 & -0.50 \\
& 24.76 & 0.80 \\
\hline
\end{tabular}

$M T V=a+b * t_{e q}$

Serie 1

\begin{tabular}{|c|c|c|c|c|c|c|c|}
\hline \multicolumn{4}{|c|}{ LINEAR REGRESSION } & \multicolumn{4}{|c|}{ STATISTICS } \\
\hline$a=$ & -3.35 & teq & MTV & Sda $=$ & 0.91 & $F=$ & 15.43 \\
\hline$b=$ & 0.15 & 22 & -0.09 & $S d b=$ & 0.04 & $D F=$ & 7.00 \\
\hline$r=$ & 0.83 & 26 & 0.51 & SdMTV $=$ & 0.15 & $r^{2}$ & 0.69 \\
\hline \multicolumn{8}{|c|}{ Serie 2} \\
\hline \multicolumn{4}{|c|}{ LINEAR REGRESSION } & \multicolumn{4}{|c|}{ STATISTICS } \\
\hline$a=$ & -2.83 & teq & MTV & Sda $=$ & 1.19 & $\mathrm{~F}=$ & 6.85 \\
\hline$b=$ & 0.15 & 17 & -0.29 & $S d b=$ & 0.06 & $D F=$ & 3.00 \\
\hline$r=$ & 0.83 & 25 & 0.96 & SdMTV= & 0.38 & r2 & 0.70 \\
\hline \multicolumn{8}{|c|}{ Serie $1 \& 2$} \\
\hline \multicolumn{4}{|c|}{ LINEAR REGRESSION } & \multicolumn{4}{|c|}{ STATISTICS } \\
\hline$a=$ & -1.67 & teq & MTV & Sda $=$ & 0.71 & $F=$ & 7.18 \\
\hline$b=$ & 0.08 & 17 & -0.26 & $S d b=$ & 0.03 & $\mathrm{DF}=$ & 12.00 \\
\hline$r=$ & 0.61 & 26 & 0.50 & SdMTV $=$ & 0.32 & r2 & 0.37 \\
\hline
\end{tabular}

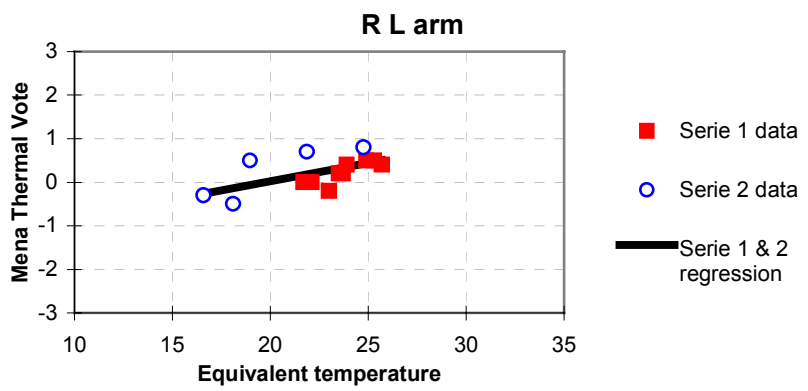

9 L hand

\begin{tabular}{|c|c|c|c|c|c|c|c|c|c|c|}
\hline \multirow{3}{*}{$\begin{array}{l}\text { Condition } \\
\text { VU-K } \\
\text { VU-N }\end{array}$} & \multirow{3}{*}{$\begin{array}{c}\text { Mean } t_{\text {eq }} \\
20.50 \\
22.70\end{array}$} & \multirow{3}{*}{$\begin{array}{c}\text { Mean MTV } \\
-0.70 \\
-0.30\end{array}$} & \multicolumn{4}{|c|}{ MTV $=a+b * t_{e q} \quad$ Serie 1} & \multirow{2}{*}{\multicolumn{4}{|c|}{ STATISTICS }} \\
\hline & & & \multicolumn{4}{|c|}{ LINEAR REGRESSION } & & & & \\
\hline & & & $a=$ & -3.84 & teq & MTV & Sda $=$ & 1.42 & $F=$ & 6.25 \\
\hline VU-V & 24.80 & 0.20 & $b=$ & 0.15 & 21 & -0.78 & $S d b=$ & 0.06 & $D F=$ & 7.00 \\
\hline VUOK & 22.80 & -0.60 & $r=$ & 0.69 & 26 & 0.08 & SdMTV= & 0.30 & $r^{2}$ & 0.47 \\
\hline VUON & 23.70 & 0.00 & \multicolumn{7}{|c|}{ Serie 2} & \\
\hline VuOV & 25.70 & 0.20 & \multicolumn{4}{|c|}{ LINEAR REGRESSION } & \multicolumn{4}{|c|}{ STATISTICS } \\
\hline VU+K & 23.20 & -0.80 & $a=$ & -2.58 & teq & MTV & Sda $=$ & 1.11 & $F=$ & 5.97 \\
\hline $\mathrm{VU}+\mathrm{N}$ & 24.50 & -0.50 & $b=$ & 0.14 & 15 & -0.48 & $S d b=$ & 0.06 & $\mathrm{DF}=$ & 3.00 \\
\hline Vu+V & 26.30 & -0.10 & $r=$ & 0.82 & 25 & 0.85 & SdMTV= & 0.41 & r2 & 0.67 \\
\hline VIK & 15.29 & -0.40 & \multicolumn{7}{|c|}{ Serie $1 \& 2$} & \\
\hline VIN & 20.74 & 0.50 & \multicolumn{4}{|c|}{ LINEAR REGRESSION } & \multicolumn{4}{|c|}{ STATISTICS } \\
\hline VIV & 19.01 & 0.40 & $a=$ & -1.07 & teq & MTV & Sda $=$ & 0.95 & $\mathrm{~F}=$ & 0.95 \\
\hline VIGK & 17.57 & -0.70 & $b=$ & 0.04 & 15 & -0.44 & $S d b=$ & 0.04 & $\mathrm{DF}=$ & 12.00 \\
\hline VIGV & 24.97 & 0.70 & $r=$ & 0.27 & 26 & 0.02 & SdMTV= & 0.49 & r2 & 0.07 \\
\hline
\end{tabular}

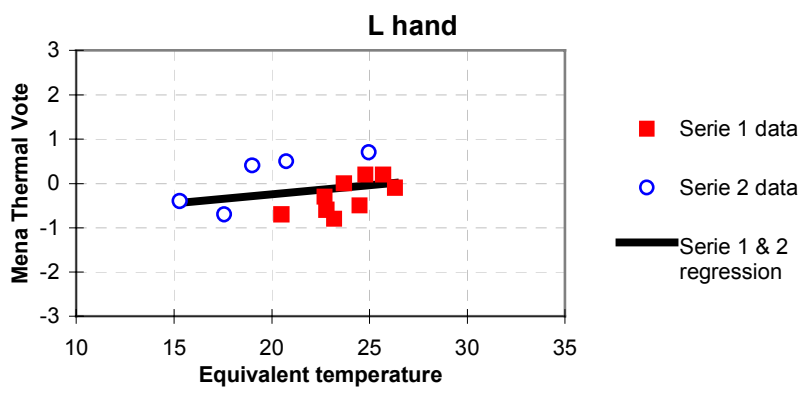




\begin{tabular}{l|c|c|}
\multicolumn{1}{l}{$\begin{array}{l}\text { Condition } \\
\text { VU-K }\end{array}$} & \multicolumn{1}{c}{ Mean $\mathrm{t}_{\text {eq }}$} & \multicolumn{1}{c}{ Mean MTV } \\
\cline { 2 - 3 } VU-N & 20.80 & -0.60 \\
VU-V & 22.70 & -0.30 \\
VUOK & 24.70 & 0.20 \\
VUON & 23.30 & -0.50 \\
VUOV & 25.50 & 0.00 \\
VU+K & 27.40 & 0.20 \\
VU+N & 23.80 & -0.70 \\
VU+V & 25.10 & -0.40 \\
VIK & 27.40 & -0.10 \\
VIN & 15.56 & -0.30 \\
VIV & 18.72 & 0.50 \\
VIGK & 17.69 & 0.40 \\
VIGV & 16.24 & -0.60 \\
& 23.66 & 0.70 \\
\hline
\end{tabular}

$M T V=a+b * t_{\text {eq }} \quad$ Serie 1

\begin{tabular}{|c|c|c|c|c|c|c|c|}
\hline \multicolumn{4}{|c|}{ LINEAR REGRESSION } & \multicolumn{4}{|c|}{ STATISTICS } \\
\hline$a=$ & -2.88 & teq & MTV & Sda= & 1.05 & $F=$ & 6.35 \\
\hline$b=$ & 0.11 & 21 & -0.64 & $S d b=$ & 0.04 & $D F=$ & 7.00 \\
\hline$r=$ & 0.69 & 27 & 0.07 & SdMTV= & 0.26 & $r^{2}$ & 0.48 \\
\hline \multicolumn{8}{|c|}{ Serie 2} \\
\hline \multicolumn{4}{|c|}{ LINEAR REGRESSION } & \multicolumn{4}{|c|}{ STATISTICS } \\
\hline$a=$ & -2.43 & teq & MTV & Sda $=$ & 1.13 & $F=$ & 5.29 \\
\hline$b=$ & 0.14 & 16 & -0.25 & $S d b=$ & 0.06 & $D F=$ & 3.00 \\
\hline$r=$ & 0.80 & 24 & 0.88 & SdMTV= & 0.39 & r2 & 0.64 \\
\hline \multicolumn{8}{|c|}{ Serie $1 \& 2$} \\
\hline \multicolumn{4}{|c|}{ LINEAR REGRESSION } & \multicolumn{4}{|c|}{ STATISTICS } \\
\hline$a=$ & -0.34 & teq & MTV & Sda= & 0.75 & $\mathrm{~F}=$ & 0.10 \\
\hline$b=$ & 0.01 & 16 & -0.18 & $S d b=$ & 0.03 & $D F=$ & 12.00 \\
\hline $\mathrm{r}=$ & 0.09 & 27 & -0.05 & SdMTV= & 0.47 & r2 & 0.01 \\
\hline
\end{tabular}

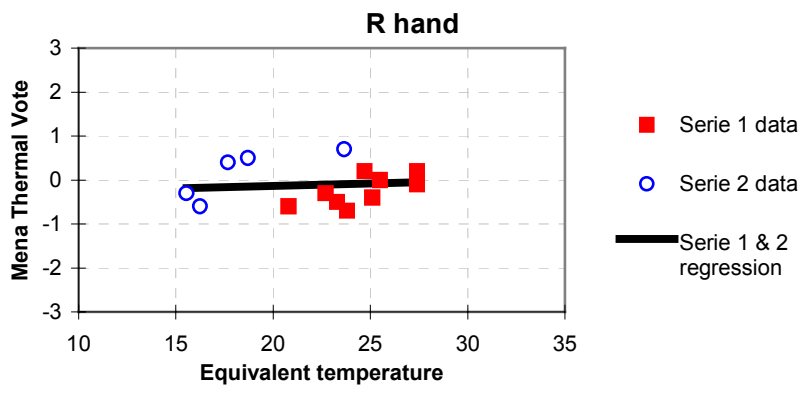

11 L thigh

\begin{tabular}{l|c|c|}
\multicolumn{1}{l}{$\begin{array}{l}\text { Condition } \\
\text { VU-K }\end{array}$} & \multicolumn{1}{c}{ Mean $t_{\text {eq }}$} & Mean MTV \\
\cline { 2 - 3 } VU-N & 23.00 & -0.90 \\
VU-V & 24.90 & -0.50 \\
VUOK & 26.60 & 0.10 \\
VUON & 22.90 & -1.50 \\
VUOV & 23.60 & -0.80 \\
VU+K & 25.50 & -0.60 \\
VU+N & 23.10 & -1.40 \\
VU+V & 24.60 & -1.10 \\
VIK & 26.10 & -0.90 \\
VIN & 22.66 & -0.80 \\
VIV & 26.58 & 0.40 \\
VIGK & 24.53 & 0.20 \\
VIGV & 21.61 & -1.00 \\
& 26.71 & 0.70 \\
\hline
\end{tabular}

$M T V=a+b * t_{\text {eq }}$

Serie 1

\begin{tabular}{cccc|cccc}
\multicolumn{3}{c|}{ LINEAR REGRESSION } & \multicolumn{4}{c}{ STATISTICS } \\
$\mathrm{a}=$ & -7.26 & teq & MTV & Sda $=$ & 2.09 & $\mathrm{~F}=$ & 9.44 \\
$\mathrm{~b}=$ & 0.26 & 23 & -1.26 & $\mathrm{Sdb}=$ & 0.09 & $\mathrm{DF}=$ & 7.00 \\
$\mathrm{r}=$ & 0.76 & 27 & -0.29 & SdMTV $=$ & 0.34 & $\mathrm{r}^{2}$ & 0.57
\end{tabular}

\begin{tabular}{|cccc|cccc|}
\hline \multicolumn{10}{|c|}{ Serie 2 } \\
\hline \multicolumn{10}{|c|}{ LINEAR REGRESSION } & \multicolumn{4}{c|}{ STATISTICS } \\
$\mathrm{a}=$ & -7.92 & teq & MTV & Sda $=$ & 1.12 & $\mathrm{~F}=$ & 49.47 \\
$\mathrm{~b}=$ & 0.32 & 22 & -1.00 & Sdb $=$ & 0.05 & DF $=$ & 3.00 \\
$\mathrm{r}=$ & 0.97 & 27 & 0.63 & SdMTV $=$ & 0.21 & r2 & 0.94 \\
\hline
\end{tabular}

\begin{tabular}{|cccc|cccc|}
\hline \multicolumn{10}{|c|}{ LINEAR REGRESSION } & \multicolumn{5}{c|}{ STATISTICS } \\
$\mathrm{a}=$ & -7.71 & teq & MTV & Sda $=$ & 1.96 & $\mathrm{~F}=$ & 13.22 \\
$\mathrm{~b}=$ & 0.29 & 22 & -1.41 & $\mathrm{Sdb}=$ & 0.08 & $\mathrm{DF}=$ & 12.00
\end{tabular}

\section{L thigh}

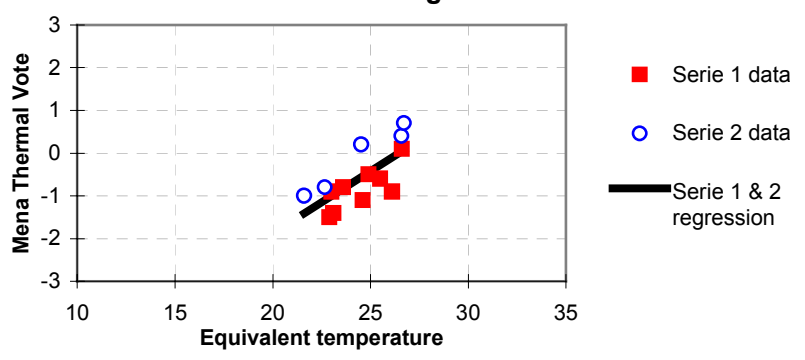


$2 \quad \mathrm{R}$ thigh

\begin{tabular}{l|c|c|}
\multicolumn{1}{l}{$\begin{array}{l}\text { Condition } \\
\text { VU-K }\end{array}$} & \multicolumn{1}{c}{ Mean $\mathrm{t}_{\text {eq }}$} & \multicolumn{1}{c}{ Mean MTV } \\
\cline { 2 - 3 } VU-N & 20.80 & -0.80 \\
VU-V & 23.20 & -0.30 \\
VUOK & 25.00 & 0.30 \\
VUON & 21.90 & -1.30 \\
VUOV & 23.20 & -0.70 \\
VU+K & 25.10 & -0.60 \\
VU+N & 21.20 & -1.30 \\
VU+V & 22.70 & -1.00 \\
VIK & 25.00 & -0.80 \\
VIN & 22.82 & -0.80 \\
VIV & 27.32 & 0.40 \\
VIGK & 24.91 & 0.20 \\
VIGV & 21.10 & -1.10 \\
& 27.62 & 0.70 \\
\hline
\end{tabular}

$M T V=a+b$ * $t_{e c}$

Serie 1

\begin{tabular}{|cccc|cccc|}
\hline \multicolumn{3}{|c|}{ LINEAR REGRESSION } & \multicolumn{4}{c|}{ STATISTICS } \\
$\mathrm{a}=$ & -5.15 & teq & MTV & Sda $=$ & 2.06 & $\mathrm{~F}=$ & 4.66 \\
$\mathrm{~b}=$ & 0.19 & 21 & -1.17 & Sdb $=$ & 0.09 & DF $=$ & 7.00 \\
$\mathrm{r}=$ & 0.63 & 25 & -0.34 & SdMTV $=$ & 0.41 & $\mathrm{r}^{2}$ & 0.40 \\
\hline \multicolumn{8}{|c}{}
\end{tabular}

\begin{tabular}{|c|c|c|c|c|c|c|c|}
\hline \multicolumn{4}{|c|}{ LINEAR REGRESSION } & \multicolumn{4}{|c|}{ STATISTICS } \\
\hline$a=$ & -6.81 & teq & MTV & Sda $=$ & 0.93 & $\mathrm{~F}=$ & 51.93 \\
\hline$b=$ & 0.27 & 21 & -1.11 & $S d b=$ & 0.04 & $D F=$ & 3.00 \\
\hline $\mathrm{r}=$ & 0.97 & 28 & 0.65 & SdMTV= & 0.21 & r2 & 0.95 \\
\hline \multicolumn{8}{|c|}{ Serie $1 \& 2$} \\
\hline \multicolumn{4}{|c|}{ LINEAR REGRESSION } & \multicolumn{4}{|c|}{ STATISTICS } \\
\hline$a=$ & -6.59 & teq & MTV & Sda $=$ & 1.08 & $F=$ & 31.90 \\
\hline$b=$ & 0.26 & 21 & -1.25 & $S d b=$ & 0.05 & $\mathrm{DF}=$ & 12.00 \\
\hline$r=$ & 0.85 & 28 & 0.50 & SdMTV $=$ & 0.36 & r2 & 0.73 \\
\hline
\end{tabular}

R thigh

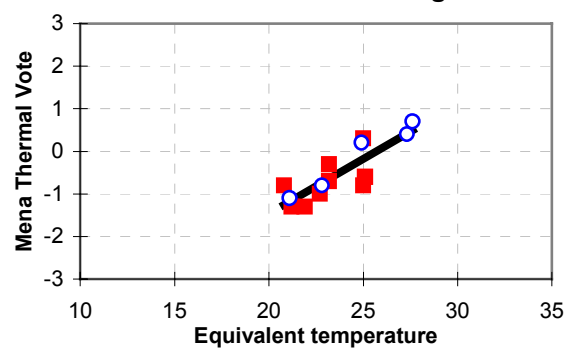

- Serie 1 data

- Serie 2 data

Serie 1 \& 2

regression

13 L calf

\begin{tabular}{|c|c|c|c|c|c|c|c|c|c|c|}
\hline \multirow{3}{*}{$\begin{array}{l}\text { Condition } \\
\text { VU-K } \\
\text { VU-N }\end{array}$} & \multirow{3}{*}{$\begin{array}{c}\text { Mean } t_{\text {eq }} \\
24.40 \\
27.30\end{array}$} & \multirow{3}{*}{$\begin{array}{c}\text { Mean MTV } \\
-0.30 \\
0.60\end{array}$} & \multirow{2}{*}{\multicolumn{4}{|c|}{ MTV $=a+b * t_{\text {eq }} \quad$ Serie 1}} & \multirow{2}{*}{\multicolumn{4}{|c|}{ STATISTICS }} \\
\hline & & & & & & & & & & \\
\hline & & & $a=$ & -9.93 & teq & MTV & Sda $=$ & 0.99 & $F=$ & 91.39 \\
\hline VU-V & 29.60 & 1.20 & $b=$ & 0.38 & 22 & -1.48 & $S d b=$ & 0.04 & $D F=$ & 7.00 \\
\hline VUOK & 22.80 & -1.40 & $r=$ & 0.96 & 30 & 1.29 & SdMTV= & 0.27 & $r^{2}$ & 0.93 \\
\hline VUON & 23.80 & -0.60 & \multicolumn{7}{|c|}{ Serie 2} & \\
\hline VUOV & 25.80 & -0.40 & \multicolumn{4}{|c|}{ LINEAR REGRESSION } & \multicolumn{4}{|c|}{ STATISTICS } \\
\hline $\mathrm{VU}+\mathrm{K}$ & 22.30 & -1.70 & $a=$ & -3.26 & teq & MTV & Sda $=$ & 0.37 & $\mathrm{~F}=$ & 63.12 \\
\hline $\mathrm{VU}+\mathrm{N}$ & 22.50 & -1.30 & $b=$ & 0.13 & 17 & -1.04 & $\mathrm{Sdb}=$ & 0.02 & $D F=$ & 3.00 \\
\hline VU+V & 24.40 & -1.00 & $r=$ & 0.98 & 26 & 0.15 & SdMTV= & 0.13 & r2 & 0.95 \\
\hline VIK & 19.09 & -0.90 & \multicolumn{8}{|c|}{ Serie $1 \& 2$} \\
\hline VIN & 25.98 & 0.20 & \multicolumn{4}{|c|}{ LINEAR REGRESSION } & \multicolumn{4}{|c|}{ STATISTICS } \\
\hline VIV & 22.39 & -0.40 & $a=$ & -4.82 & teq & MTV & Sda $=$ & 1.20 & $\mathrm{~F}=$ & 13.25 \\
\hline VIGK & 16.93 & -0.90 & $b=$ & 0.18 & 17 & -1.73 & $S d b=$ & 0.05 & $D F=$ & 12.00 \\
\hline VIGV & 25.33 & 0.10 & $r=$ & 0.72 & 30 & 0.58 & SdMTV= & 0.58 & r2 & 0.52 \\
\hline
\end{tabular}

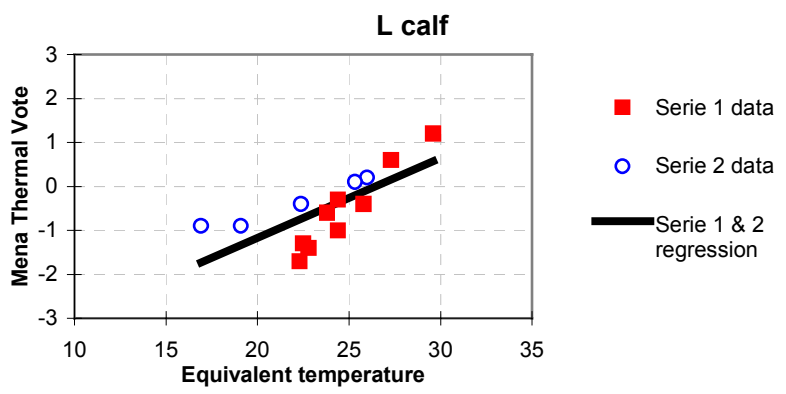


$4 \quad R$ calf

\begin{tabular}{l|c|c|}
\multicolumn{1}{l}{$\begin{array}{l}\text { Condition } \\
\text { VU-K }\end{array}$} & \multicolumn{1}{c}{ Mean $\mathrm{t}_{\text {eq }}$} & \multicolumn{1}{c}{ Mean MTV } \\
\cline { 2 - 3 } VU-N & 23.20 & -0.30 \\
VU-V & 25.80 & 0.50 \\
VUOK & 28.60 & 1.20 \\
VUON & 21.50 & -1.20 \\
VUOV & 23.00 & -0.50 \\
VU+K & 24.80 & -0.30 \\
VU+N & 19.80 & -1.50 \\
VU+V & 21.80 & -1.20 \\
VIK & 23.90 & -0.90 \\
VIN & 20.49 & -1.00 \\
VIV & 26.67 & 0.20 \\
VIGK & 23.03 & -0.40 \\
VIGV & 20.42 & -1.00 \\
& 25.77 & 0.10
\end{tabular}

$M T V=a+b * t_{e q} \quad$ Serie 1

\begin{tabular}{|cccc|cccc|}
\hline \multicolumn{3}{|c|}{ LINEAR REGRESSION } & \multicolumn{5}{c|}{ STATISTICS } \\
$\mathrm{a}=$ & -8.02 & teq & MTV & Sda $=$ & 0.93 & $\mathrm{~F}=$ & 67.29 \\
$\mathrm{~b}=$ & 0.32 & 20 & -1.68 & Sdb $=$ & 0.04 & DF $=$ & 7.00 \\
$\mathrm{r}=$ & 0.95 & 29 & 1.13 & SdMTV $=$ & 0.29 & $\mathrm{r}^{2}$ & 0.91 \\
\hline
\end{tabular}

\begin{tabular}{|c|c|c|c|c|c|c|c|}
\hline \multirow{2}{*}{\multicolumn{4}{|c|}{$\begin{array}{lr}\text { Serie } 2 \\
\text { LINEAR REGRESSION }\end{array}$}} & \multirow{2}{*}{\multicolumn{4}{|c|}{ STATISTICS }} \\
\hline & & & & & & & \\
\hline$a=$ & -5.02 & teq & MTV & Sda $=$ & 0.22 & $\mathrm{~F}=$ & 430.68 \\
\hline$b=$ & 0.20 & 20 & -0.98 & $S d b=$ & 0.01 & $\mathrm{DF}=$ & 3.00 \\
\hline$r=$ & 1.00 & 27 & 0.25 & SdMTV $=$ & 0.06 & r2 & 0.99 \\
\hline \multicolumn{8}{|c|}{ Serie $1 \& 2$} \\
\hline \multicolumn{4}{|c|}{ LINEAR REGRESSION } & \multicolumn{4}{|c|}{ STATISTICS } \\
\hline$a=$ & -6.82 & teq & MTV & Sda $=$ & 0.71 & $\mathrm{~F}=$ & 81.65 \\
\hline$b=$ & 0.27 & 20 & -1.45 & $\mathrm{Sdb}=$ & 0.03 & $D F=$ & 12.00 \\
\hline$r=$ & 0.93 & 29 & 0.94 & SdMTV $=$ & 0.28 & r2 & 0.87 \\
\hline
\end{tabular}

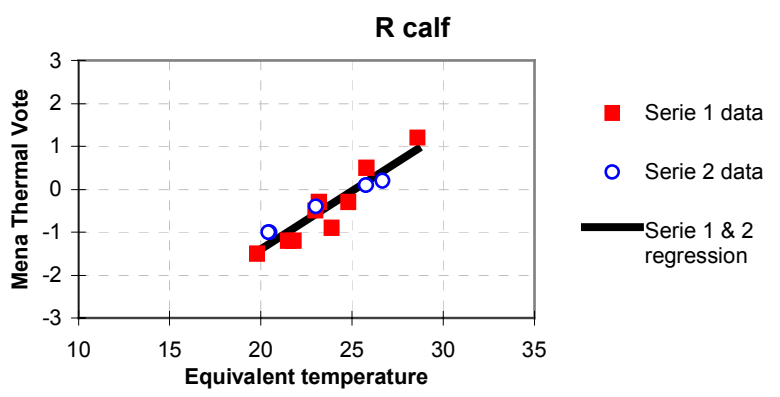

$15 L$ foot

\begin{tabular}{|c|c|c|c|c|c|c|c|c|c|c|}
\hline Condition & Mean $t_{\mathrm{eq}}$ & Mean MTV & MTV $=a+$ & ${ }^{*} t_{\mathrm{eq}}$ & & Serie 1 & & & & \\
\hline VU-K & 26.10 & 0.10 & & EAR R & RESS & & & STA & IICS & \\
\hline VU-N & 28.10 & 0.60 & $a=$ & -7.14 & teq & MTV & Sda= & 0.50 & $\mathrm{~F}=$ & 196.67 \\
\hline VU-V & 29.90 & 1.20 & $b=$ & 0.28 & 20 & -1.55 & $S d b=$ & 0.02 & $\mathrm{DF}=$ & 7.00 \\
\hline VUOK & 23.40 & -0.80 & $r=$ & 0.98 & 30 & 1.17 & SdMTV= & 0.17 & $r^{2}$ & 0.97 \\
\hline VUON & 25.10 & 0.00 & & & & Serie 2 & & & & \\
\hline vuov & 26.10 & 0.30 & & EAR R & RESS & & & STA & IICS & \\
\hline VU+K & 20.10 & -1.40 & $a=$ & -3.61 & teq & MTV & Sda= & 1.26 & $\mathrm{~F}=$ & 9.67 \\
\hline VU+N & 22.50 & -0.90 & $b=$ & 0.19 & 16 & -0.51 & $S d b=$ & 0.06 & $\mathrm{DF}=$ & 3.00 \\
\hline VU+V & 23.80 & -0.80 & $r=$ & 0.87 & 24 & 1.02 & SdMTV= & 0.45 & r2 & 0.76 \\
\hline VIK & 17.63 & -0.60 & & & & erie $1 \&$ & & & & \\
\hline VIN & 24.14 & 0.80 & & JEAR R & RESS & & & STA & IICS & \\
\hline VIV & 19.19 & 0.70 & $a=$ & -2.68 & teq & MTV & Sda $=$ & 1.21 & $F=$ & 4.96 \\
\hline VIGK & 16.14 & -0.60 & $b=$ & 0.11 & 16 & -0.84 & $S d b=$ & 0.05 & $\mathrm{DF}=$ & 12.00 \\
\hline VIGV & 23.73 & 1.00 & $r=$ & 0.54 & 30 & 0.73 & SdMTV= & 0.72 & r2 & 0.29 \\
\hline
\end{tabular}

$L$ foot

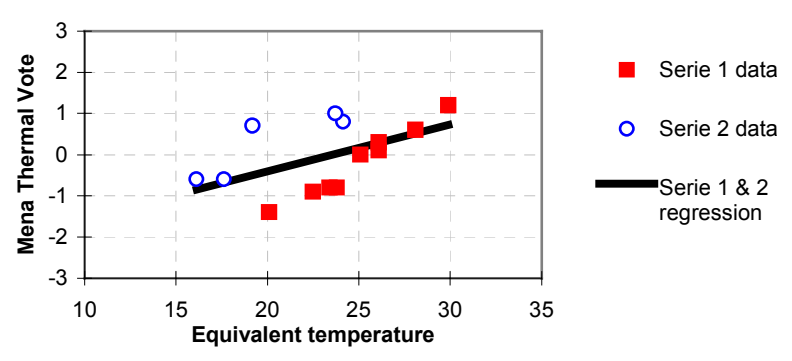


Mean MTV

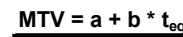

Serie 1

VU-N

VU-V

VUOK

VUON

VUOV

$\mathrm{VU}+\mathrm{K}$

$\mathrm{VU}+\mathrm{N}$

VU+V

VIK

VIN

VIV

VIGK

VIGV

\begin{tabular}{|c|c|}
\hline 25.90 & 0.00 \\
29.20 & 0.50 \\
31.80 & 1.20 \\
22.80 & -0.70 \\
23.70 & 0.00 \\
25.20 & 0.40 \\
19.60 & -1.20 \\
21.80 & -0.70 \\
23.50 & -0.80 \\
18.01 & -0.50 \\
24.12 & 0.80 \\
20.43 & 0.80 \\
15.71 & -0.50 \\
22.53 & 1.00 \\
\hline
\end{tabular}

\begin{tabular}{|cccc|cccc|}
\hline \multicolumn{3}{|c|}{ LINEAR REGRESSION } & \multicolumn{4}{c|}{ STATISTICS } \\
$\mathrm{a}=$ & -4.90 & teq & MTV & Sda $=$ & 0.68 & $\mathrm{~F}=$ & 49.28 \\
$\mathrm{~b}=$ & 0.19 & 20 & -1.15 & Sdb $=$ & 0.03 & DF $=$ & 7.00 \\
$\mathrm{r}=$ & 0.94 & 32 & 1.19 & SdMTV $=$ & 0.29 & $\mathrm{r}^{2}$ & 0.88 \\
\hline \multicolumn{8}{|c}{}
\end{tabular}

\begin{tabular}{|c|c|c|c|c|c|c|c|}
\hline \multicolumn{4}{|c|}{$\begin{array}{rr}\text { Serie 2 } \\
\end{array}$} & \multirow{2}{*}{\multicolumn{4}{|c|}{ STATISTICS }} \\
\hline \multicolumn{4}{|c|}{ LINEAR REGRESSION } & & & & \\
\hline$a=$ & -3.66 & teq & MTV & $\mathrm{Sda}=$ & 1.20 & $\mathrm{~F}=$ & 11.25 \\
\hline$b=$ & 0.20 & 16 & -0.56 & $S d b=$ & 0.06 & $\mathrm{DF}=$ & 3.00 \\
\hline $\mathrm{r}=$ & 0.89 & 24 & 1.10 & SdMTV $=$ & 0.40 & r2 & 0.79 \\
\hline \multicolumn{7}{|c|}{ Serie $1 \& 2$} & \\
\hline \multicolumn{4}{|c|}{ LINEAR REGRESSION } & \multicolumn{4}{|c|}{ STATISTICS } \\
\hline$a=$ & -2.38 & teq & MTV & Sda $=$ & 1.03 & $\mathrm{~F}=$ & 5.62 \\
\hline$b=$ & 0.10 & 16 & -0.75 & $S d b=$ & 0.04 & $\mathrm{DF}=$ & 12.00 \\
\hline$r=$ & 0.56 & 32 & 0.92 & SdMTV $=$ & 0.66 & r2 & 0.32 \\
\hline
\end{tabular}

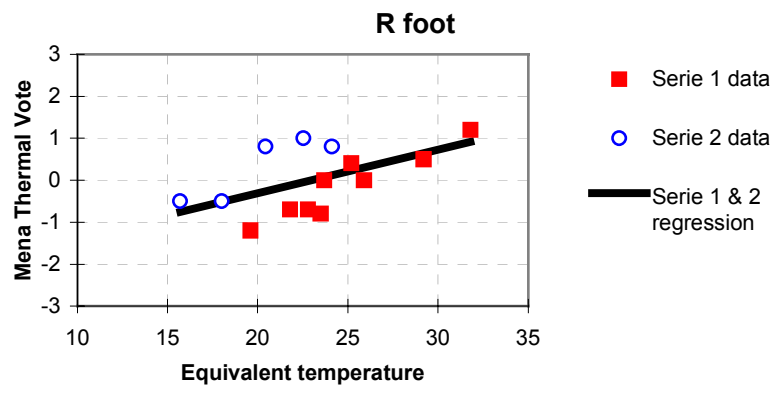

17 Lo. back

\begin{tabular}{|c|c|c|c|c|c|c|c|c|c|c|}
\hline \multirow{2}{*}{$\begin{array}{l}\text { Condition } \\
\text { VU-K }\end{array}$} & Mean $t_{\mathrm{eq}}$ & Mean MTV & \multirow{2}{*}{\multicolumn{4}{|c|}{ MTV $=a+b * t_{\text {eq }} \quad$ Serie 1}} & \multirow{2}{*}{\multicolumn{4}{|c|}{ STATISTICS }} \\
\hline & & & & & & & & & & \\
\hline VU-N & & & $a=$ & & teq & MTV & Sda $=$ & \multicolumn{3}{|c|}{$F=$} \\
\hline VU-V & & & $b=$ & & & & $\mathrm{Sdb}=$ & & $\mathrm{DF}=$ & \\
\hline VUOK & & & $r=$ & & & & SdMTV= & & $r^{2}$ & \\
\hline VUON & & & \multicolumn{8}{|c|}{ Serie 2} \\
\hline VuOV & & & \multicolumn{4}{|c|}{ LINEAR REGRESSION } & \multicolumn{4}{|c|}{ STATISTICS } \\
\hline VU+K & & & $a=$ & -0.68 & teq & MTV & Sda= & 0.82 & $\mathrm{~F}=$ & 3.41 \\
\hline $\mathbf{V U}+\mathbf{N}$ & & & $b=$ & 0.06 & 17 & 0.38 & $S d b=$ & 0.03 & $\mathrm{DF}=$ & 3.00 \\
\hline VU+V & & & $r=$ & 0.73 & 29 & 1.12 & SdMTV= & 0.29 & r2 & 0.53 \\
\hline VIK & 25.12 & 0.60 & \multicolumn{8}{|c|}{ Serie $1 \& 2$} \\
\hline VIN & 28.60 & 1.10 & \multicolumn{4}{|c|}{ LINEAR REGRESSION } & \multicolumn{4}{|c|}{ STATISTICS } \\
\hline VIV & 25.18 & 0.90 & $a=$ & -0.68 & teq & MTV & Sda $=$ & 0.82 & $\mathrm{~F}=$ & 3.41 \\
\hline VIGK & 16.91 & 0.30 & $b=$ & 0.06 & 17 & 0.38 & $S d b=$ & 0.03 & $\mathrm{DF}=$ & 3.00 \\
\hline VIGV & 23.54 & 1.20 & $r=$ & 0.73 & 29 & 1.12 & SdMTV= & 0.29 & r2 & 0.53 \\
\hline
\end{tabular}

\section{Lo. Back}

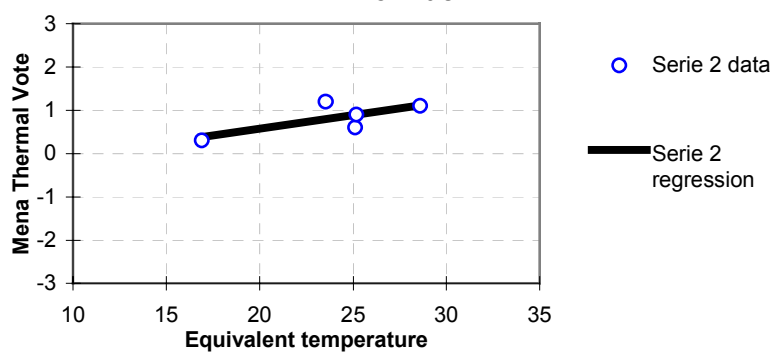


18 Seat

\begin{tabular}{|c|c|c|c|c|c|c|c|c|c|c|}
\hline \multirow{2}{*}{$\begin{array}{l}\text { Condition } \\
\text { VU-K }\end{array}$} & Mean $t_{e q}$ & Mean MTV & \multirow{2}{*}{\multicolumn{4}{|c|}{ MTV $=a+b * t_{\text {eq }} \quad$ Serie 1}} & \multirow{2}{*}{\multicolumn{4}{|c|}{ STATISTICS }} \\
\hline & & & & & & & & & & \\
\hline VU-N & & & $a=$ & & teq & MTV & $\mathrm{Sda}=$ & & $\mathrm{F}=$ & \\
\hline VU-V & & & $b=$ & & & & $S d b=$ & & $\mathrm{DF}=$ & \\
\hline VUOK & & & $r=$ & & & & SdMTV= & & $r^{2}$ & \\
\hline VUON & & & \multicolumn{8}{|c|}{ Serie 2} \\
\hline Vuov & & & \multicolumn{4}{|c|}{ LINEAR REGRESSION } & \multicolumn{4}{|c|}{ STATISTICS } \\
\hline VU+K & & & $a=$ & -0.57 & teq & MTV & Sda $=$ & 1.87 & $F=$ & 0.71 \\
\hline $\mathrm{VU}+\mathrm{N}$ & & & $b=$ & 0.05 & 26 & 0.77 & $S d b=$ & 0.06 & $\mathrm{DF}=$ & 3.00 \\
\hline VU+V & & & $r=$ & 0.44 & 32 & 1.11 & SdMTV= & 0.33 & r2 & 0.19 \\
\hline VIK & 31.78 & 0.80 & \multicolumn{8}{|c|}{ Serie $1 \& 2$} \\
\hline VIN & 32.04 & 1.20 & \multicolumn{4}{|c|}{ LINEAR REGRESSION } & \multicolumn{4}{|c|}{ STATISTICS } \\
\hline VIV & 31.34 & 1.00 & $a=$ & -0.57 & teq & MTV & Sda $=$ & 1.87 & $F=$ & 0.71 \\
\hline VIGK & 25.71 & 0.60 & $b=$ & 0.05 & 26 & 0.77 & $S d b=$ & 0.06 & $D F=$ & 3.00 \\
\hline VIGV & 29.25 & 1.40 & $r=$ & 0.44 & 32 & 1.11 & SdMTV= & 0.33 & r2 & 0.19 \\
\hline
\end{tabular}

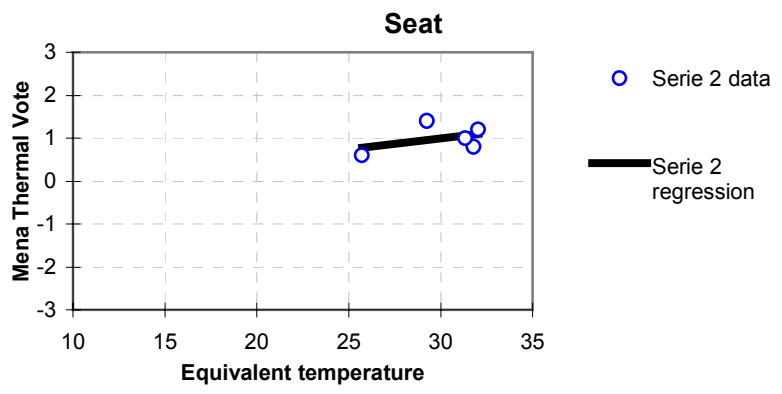

\section{Summer}

Summer

\begin{tabular}{|c|c|c|c|c|c|c|c|c|c|c|}
\hline $\begin{array}{c}0 \\
\text { Condition }\end{array}$ & $\begin{array}{l}\text { Whole body } \\
\text { Mean } t_{\text {eq }}\end{array}$ & Mean MTV & MTV $=a+$ & ${ }^{*} t_{e q}$ & & Serie 1 & & & & \\
\hline S00K & 21.66 & -1.50 & & IEAR R & RESS & & & $\overline{\text { STA }}$ & IICS & \\
\hline $\mathrm{S} 00 \mathrm{~N}$ & 23.65 & -0.80 & $a=$ & -9.49 & teq & MTV & Sda $=$ & 1.32 & $F=$ & 46.00 \\
\hline Soov & 25.09 & 0.10 & $b=$ & 0.36 & 22 & -1.63 & $S d b=$ & 0.05 & $\mathrm{DF}=$ & 9.00 \\
\hline soox & 26.90 & 0.80 & $r=$ & 0.91 & 28 & 0.56 & SdMTV= & 0.31 & $r^{2}$ & 0.84 \\
\hline SHOK & 22.77 & -1.20 & \multicolumn{8}{|c|}{ Serie 2} \\
\hline SHON & 24.23 & -1.00 & \multicolumn{4}{|c|}{ LINEAR REGRESSION } & \multicolumn{4}{|c|}{ STATISTICS } \\
\hline SHOV & 25.73 & -0.50 & $a=$ & -6.90 & teq & MTV & Sda $=$ & 1.32 & $F=$ & 29.12 \\
\hline SMOK & 22.89 & -1.30 & $b=$ & 0.29 & 20 & -1.08 & $S d b=$ & 0.05 & $\mathrm{DF}=$ & 3.00 \\
\hline SMON & 24.73 & -0.70 & $r=$ & 0.95 & 29 & 1.36 & SdMTV= & 0.35 & r2 & 0.91 \\
\hline SMOV & 25.95 & -0.30 & \multicolumn{8}{|c|}{ Serie 1\& 2} \\
\hline SM0X & 27.71 & 0.40 & \multicolumn{4}{|c|}{ LINEAR REGRESSION } & \multicolumn{4}{|c|}{ STATISTICS } \\
\hline SOK & 22.74 & -0.20 & $a=$ & -8.34 & teq & MTV & $\mathrm{Sda}=$ & 1.29 & $\mathrm{~F}=$ & 38.79 \\
\hline SON & 25.95 & 0.60 & $b=$ & 0.32 & 20 & -1.73 & $S d b=$ & 0.05 & $\mathrm{DF}=$ & 14.00 \\
\hline sov & 28.89 & 1.60 & $r=$ & 0.86 & 29 & 1.04 & SdMTV= & 0.46 & r2 & 0.73 \\
\hline SOGK & 20.36 & -1.10 & & & & & & & & \\
\hline SOGV & 26.29 & 0.10 & & & & & & & & \\
\hline
\end{tabular}

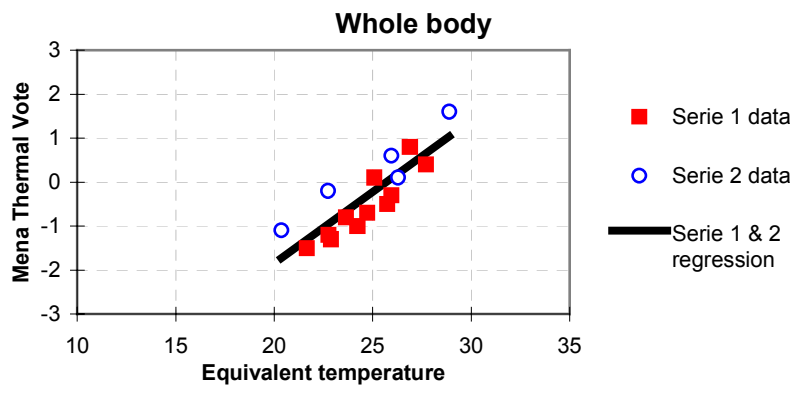


Scalp

\begin{tabular}{l|c|c|}
\multicolumn{1}{l}{$\begin{array}{l}\text { Condition } \\
\text { SOOK }\end{array}$} & \multicolumn{1}{c}{ Mean $\mathrm{t}_{\mathrm{eq}}$} & \multicolumn{1}{c}{ Mean MTV } \\
\cline { 2 - 3 } SOON & 15.51 & -1.10 \\
SOOV & 18.69 & -0.70 \\
SOOX & 21.71 & -0.30 \\
SHOK & 25.12 & 0.60 \\
SHON & 10.42 & -1.00 \\
SHOV & 13.28 & -0.90 \\
SMOK & 15.53 & -0.60 \\
SMON & 8.53 & -1.00 \\
SMOV & 11.43 & -0.70 \\
SMOX & 13.49 & -0.60 \\
SOK & 16.64 & -0.30 \\
SON & 19.92 & 0.00 \\
SOV & 24.28 & 0.20 \\
SOGK & 26.52 & 1.20 \\
SOGV & 18.82 & -0.40 \\
& 20.97 & 0.10 \\
\hline
\end{tabular}

$M T V=a+b * t_{e q} \quad$ Serie 1

\begin{tabular}{|c|c|c|c|c|c|c|c|}
\hline \multicolumn{4}{|c|}{ LINEAR REGRESSION } & \multicolumn{4}{|c|}{ STATISTICS } \\
\hline$a=$ & -1.83 & teq & MTV & Sda $=$ & 0.30 & $F=$ & 18.18 \\
\hline$b=$ & 0.08 & 9 & -1.15 & $S d b=$ & 0.02 & $D F=$ & 9.00 \\
\hline$r=$ & 0.82 & 25 & 0.17 & SdMTV= & 0.29 & $r^{2}$ & 0.67 \\
\hline \multicolumn{8}{|c|}{ Serie 2} \\
\hline \multicolumn{4}{|c|}{ LINEAR REGRESSION } & \multicolumn{4}{|c|}{ STATISTICS } \\
\hline$a=$ & -3.51 & teq & MTV & Sda $=$ & 0.98 & $\mathrm{~F}=$ & 14.83 \\
\hline$b=$ & 0.17 & 19 & -0.33 & $S d b=$ & 0.04 & $D F=$ & 3.00 \\
\hline$r=$ & 0.91 & 27 & 0.97 & SdMTV $=$ & 0.28 & r2 & 0.83 \\
\hline \multicolumn{8}{|c|}{ Serie $1 \& 2$} \\
\hline \multicolumn{4}{|c|}{ LINEAR REGRESSION } & \multicolumn{4}{|c|}{ STATISTICS } \\
\hline$a=$ & -2.16 & teq & MTV & Sda $=$ & 0.28 & $F=$ & 47.31 \\
\hline$b=$ & 0.10 & 9 & -1.28 & $S d b=$ & 0.02 & $D F=$ & 14.00 \\
\hline$r=$ & 0.88 & 27 & 0.58 & SdMTV $=$ & 0.31 & r2 & 0.77 \\
\hline
\end{tabular}

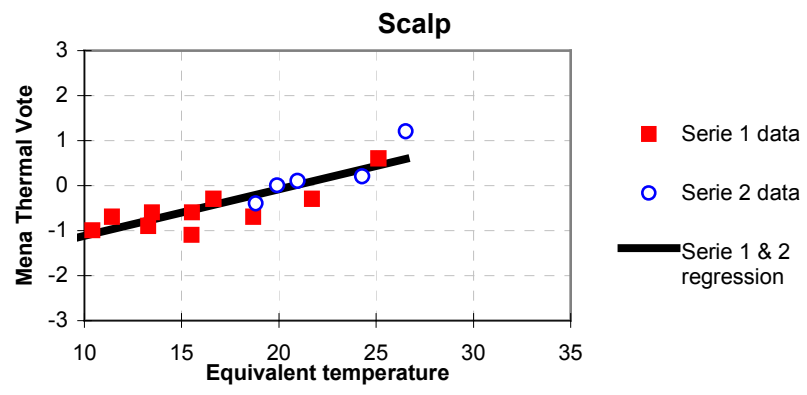

2 Face

\begin{tabular}{|c|c|c|c|c|c|c|c|c|c|c|}
\hline \multirow{3}{*}{$\begin{array}{l}\text { Condition } \\
\text { S00K } \\
\text { S00N }\end{array}$} & \multirow{3}{*}{$\begin{array}{c}\text { Mean } \mathbf{t}_{\mathrm{eq}} \\
18.80 \\
21.05\end{array}$} & \multirow{3}{*}{$\begin{array}{c}\text { Mean MTV } \\
-1.10 \\
-0.70\end{array}$} & \multirow{2}{*}{\multicolumn{4}{|c|}{$\mathbf{M T V}=\mathbf{a}+\mathbf{b}^{*} \mathbf{t}_{\mathrm{eq}} \quad$ Serie $\mathbf{1}$}} & \multirow{2}{*}{\multicolumn{4}{|c|}{ STATISTICS }} \\
\hline & & & & & & & & & & \\
\hline & & & $a=$ & -2.90 & teq & MTV & Sda $=$ & 0.44 & $F=$ & 27.89 \\
\hline soov & 23.49 & -0.30 & $b=$ & 0.12 & 15 & -1.18 & $\mathrm{Sdb}=$ & 0.02 & $\mathrm{DF}=$ & 9.00 \\
\hline soox & 26.12 & 0.60 & $r=$ & 0.87 & 26 & 0.21 & SdMTV= & 0.25 & $r^{2}$ & 0.76 \\
\hline SHOK & 15.19 & -1.00 & \multicolumn{8}{|c|}{ Serie 2} \\
\hline SHON & 17.21 & -0.90 & \multicolumn{4}{|c|}{ LINEAR REGRESSION } & \multicolumn{4}{|c|}{ STATISTICS } \\
\hline SHOV & 19.54 & -0.60 & $a=$ & -4.96 & teq & MTV & Sda $=$ & 0.75 & $F=$ & 50.40 \\
\hline SMOK & 14.51 & -1.00 & $b=$ & 0.22 & 20 & -0.55 & $S d b=$ & 0.03 & $\mathrm{DF}=$ & 3.00 \\
\hline SMON & 16.93 & -0.70 & $r=$ & 0.97 & 29 & 1.41 & SdMTV= & 0.22 & r2 & 0.94 \\
\hline SMOV & 18.57 & -0.60 & \multicolumn{8}{|c|}{ Serie 1 \& 2} \\
\hline SMOX & 21.52 & -0.30 & \multicolumn{4}{|c|}{ LINEAR REGRESSION } & \multicolumn{4}{|c|}{ STATISTICS } \\
\hline sok & 21.71 & 0.00 & $a=$ & -3.74 & teq & MTV & Sda $=$ & 0.41 & $\mathrm{~F}=$ & 72.93 \\
\hline SON & 25.79 & 0.50 & $b=$ & 0.17 & 15 & -1.34 & $S d b=$ & 0.02 & $\mathrm{DF}=$ & 14.00 \\
\hline sov & 28.67 & 1.60 & $r=$ & 0.92 & 29 & 1.00 & SdMTV= & 0.30 & r2 & 0.84 \\
\hline SOGK & 19.83 & -0.50 & & & & & & & & \\
\hline
\end{tabular}

Face

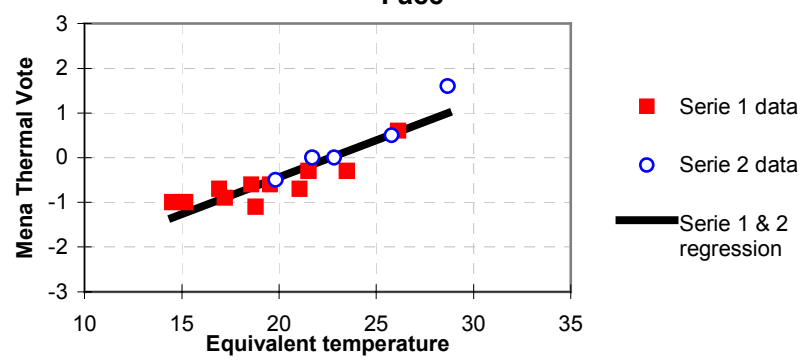




\begin{tabular}{lc|c|}
\multicolumn{1}{l}{$\begin{array}{l}\text { Condition } \\
\text { SOOK }\end{array}$} & \multicolumn{1}{c}{ Mean $\mathrm{t}_{\mathrm{eq}}$} & Mean MTV \\
\cline { 2 - 3 } SOON & 18.36 & -1.30 \\
SOOV & 20.66 & -0.40 \\
SO0X & 22.43 & -0.30 \\
SHOK & 24.67 & 0.70 \\
SHON & 22.04 & -0.40 \\
SHOV & 24.05 & -0.10 \\
SMOK & 25.75 & 0.40 \\
SMON & 24.69 & 0.30 \\
SMOV & 27.09 & 0.50 \\
SMOX & 28.66 & 0.50 \\
SOK & 30.57 & 1.00 \\
SON & 24.71 & -0.30 \\
SOV & 29.44 & 0.80 \\
SOGK & 31.49 & 1.60 \\
SOGV & 20.29 & -1.00 \\
& 30.96 & 0.40 \\
\hline
\end{tabular}

$M T V=a+b * t_{e q} \quad$ Serie 1

\begin{tabular}{|c|c|c|c|c|c|c|c|}
\hline \multicolumn{4}{|c|}{ LINEAR REGRESSION } & \multicolumn{4}{|c|}{ STATISTICS } \\
\hline$a=$ & -4.08 & teq & MTV & $S d a=$ & 0.59 & $F=$ & 50.26 \\
\hline$b=$ & 0.17 & 18 & -0.95 & $S d b=$ & 0.02 & $D F=$ & 9.00 \\
\hline$r=$ & 0.92 & 31 & 1.12 & SdMTV $=$ & 0.27 & $r^{2}$ & 0.85 \\
\hline \multicolumn{8}{|c|}{ Serie 2} \\
\hline \multicolumn{4}{|c|}{ LINEAR REGRESSION } & \multicolumn{4}{|c|}{ STATISTICS } \\
\hline$a=$ & -4.96 & teq & MTV & $S d a=$ & 1.33 & $F=$ & 16.09 \\
\hline$b=$ & 0.19 & 20 & -1.06 & $S d b=$ & 0.05 & $D F=$ & 3.00 \\
\hline$r=$ & 0.92 & 31 & 1.09 & SdMTV $=$ & 0.46 & r2 & 0.84 \\
\hline \multicolumn{8}{|c|}{ Serie 1\&2 } \\
\hline \multicolumn{4}{|c|}{ LINEAR REGRESSION } & \multicolumn{4}{|c|}{ STATISTICS } \\
\hline$a=$ & -4.08 & teq & MTV & Sda $=$ & 0.55 & $F=$ & 60.39 \\
\hline$b=$ & 0.17 & 18 & -1.02 & $S d b=$ & 0.02 & $D F=$ & 14.00 \\
\hline$r=$ & 0.90 & 31 & 1.17 & SdMTV $=$ & 0.34 & r2 & 0.81 \\
\hline
\end{tabular}

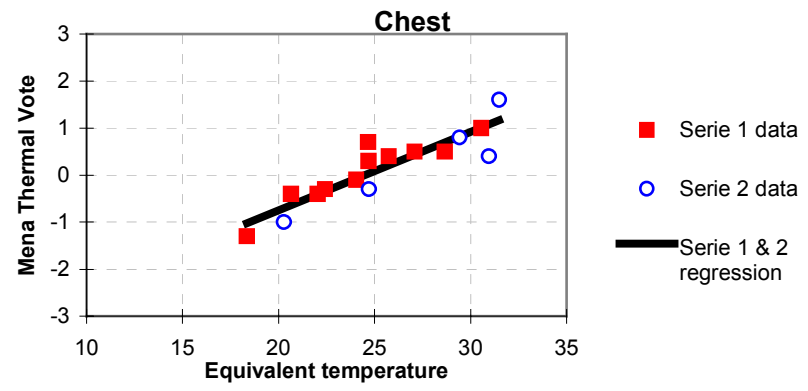

$4 \quad$ Up. Back

\begin{tabular}{|c|c|c|c|c|c|c|c|c|c|c|}
\hline \multirow{3}{*}{$\begin{array}{l}\text { Condition } \\
\text { SOOK } \\
\text { SOON }\end{array}$} & \multirow{3}{*}{$\begin{array}{c}\text { Mean } \mathbf{t}_{\mathrm{eq}} \\
19.24 \\
22.37\end{array}$} & \multirow{2}{*}{$\begin{array}{c}\text { Mean MTV } \\
-1.20\end{array}$} & \multicolumn{4}{|c|}{ MTV $=a+b * t_{e q}$} & \multirow{2}{*}{\multicolumn{4}{|c|}{ STATISTICS }} \\
\hline & & & \multicolumn{4}{|c|}{ LINEAR REGRESSION } & \multicolumn{3}{|c|}{ STATISTICS } & \\
\hline & & -0.90 & $a=$ & -3.52 & teq & MTV & Sda $=$ & 0.53 & $\mathrm{~F}=$ & 24.67 \\
\hline Soov & 23.37 & -0.40 & $b=$ & 0.14 & 13 & -1.68 & $\mathrm{Sdb}=$ & 0.03 & $\mathrm{DF}=$ & 9.00 \\
\hline soox & 25.72 & 0.60 & $r=$ & 0.86 & 26 & 0.03 & SdMTV $=$ & 0.33 & $r^{2}$ & 0.73 \\
\hline SHOK & 15.76 & -1.00 & \multirow{2}{*}{\multicolumn{4}{|c|}{$\begin{array}{l}\text { Serie 2 } \\
\text { LINEAR REGRESSION }\end{array}$}} & & & & \\
\hline SHON & 17.36 & -1.40 & & & & & \multicolumn{4}{|c|}{ STATISTICS } \\
\hline SHOV & 19.05 & -1.00 & $a=$ & -6.03 & teq & MTV & $\mathrm{Sda}=$ & 2.09 & $\mathrm{~F}=$ & 8.37 \\
\hline SMOK & 13.35 & -1.50 & $b=$ & 0.23 & 22 & -0.91 & $S d b=$ & 0.08 & $\mathrm{DF}=$ & 3.00 \\
\hline SMON & 15.37 & -1.50 & $r=$ & 0.86 & 29 & 0.67 & SdMTV= & 0.44 & r2 & 0.74 \\
\hline SMOV & 16.90 & -1.00 & \multirow{2}{*}{\multicolumn{4}{|c|}{$\begin{array}{l}\text { Serie 1 \& } \\
\text { LINEAR REGRESSION }\end{array}$}} & & & & \\
\hline SMOX & 18.99 & -0.80 & & & & & \multicolumn{4}{|c|}{ STATISTICS } \\
\hline SOK & 24.03 & -0.50 & $a=$ & -3.65 & teq & MTV & $\mathrm{Sda}=$ & 0.43 & $\mathrm{~F}=$ & 52.32 \\
\hline SON & 26.58 & 0.00 & $b=$ & 0.14 & 13 & -1.73 & $\mathrm{Sdb}=$ & 0.02 & $\mathrm{DF}=$ & 14.00 \\
\hline sov & 28.66 & 1.20 & $r=$ & 0.89 & 29 & 0.46 & SdMTV= & 0.36 & r2 & 0.79 \\
\hline SOGK & 21.90 & -0.70 & & & & & & & & \\
\hline SOGV & 27.33 & -0.10 & & & & & & & & \\
\hline
\end{tabular}

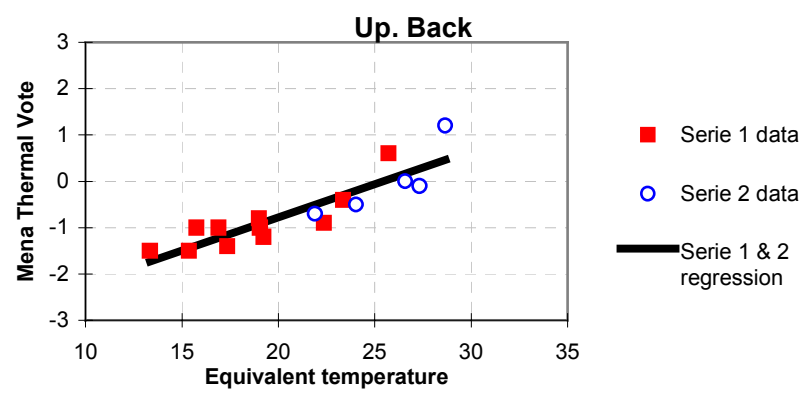


L U arm

\begin{tabular}{l|c|c|}
\multicolumn{1}{l}{ Condition } & \multicolumn{1}{c}{ Mean teq $_{\text {eq }}$} & \multicolumn{1}{c}{ Mean MTV } \\
\cline { 2 - 3 } SO0K & 19.81 & -1.40 \\
SO0N & 22.26 & -0.90 \\
SOOV & 24.57 & -0.40 \\
SO0X & 26.85 & 0.50 \\
SHOK & 20.37 & -0.80 \\
SHON & 22.23 & -1.00 \\
SHOV & 24.04 & -0.60 \\
SMOK & 22.71 & -1.20 \\
SMON & 25.23 & -0.90 \\
SMOV & 24.75 & -0.40 \\
SMOX & 27.29 & 0.30 \\
SOK & 22.28 & -0.50 \\
SON & 26.04 & 0.50 \\
SOV & 28.56 & 1.10 \\
SOGK & 21.24 & -1.10 \\
SOGV & 24.68 & 0.20 \\
& &
\end{tabular}

$M T V=a+b * t_{\text {eq }} \quad$ Serie 1

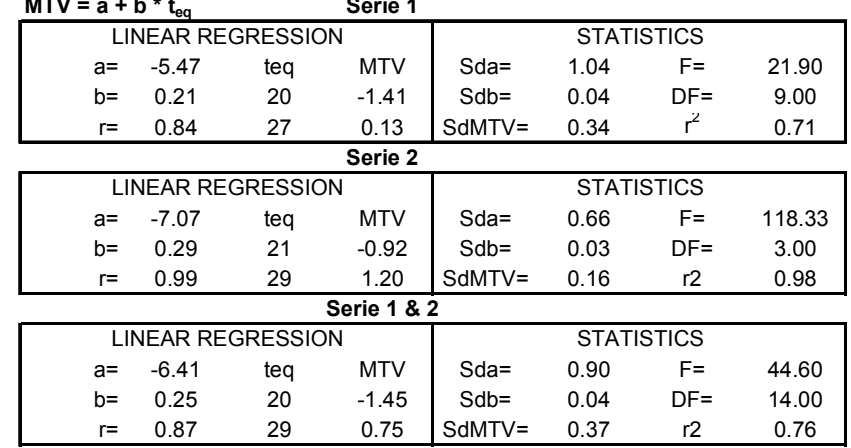

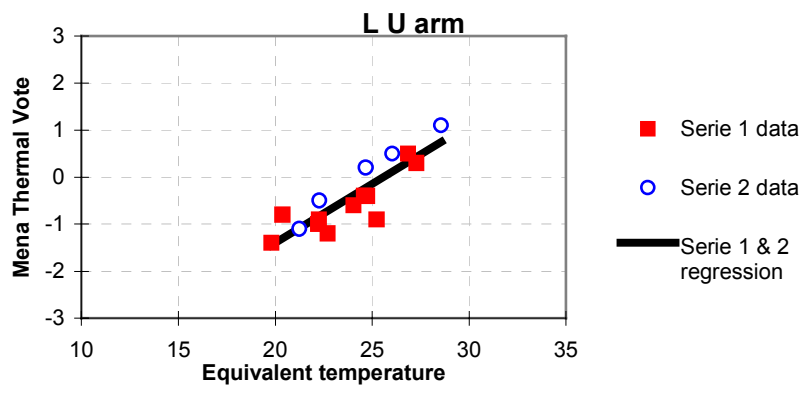

$6 \quad R U$ arm

\begin{tabular}{|c|c|c|c|c|c|c|c|c|c|c|}
\hline \multirow{3}{*}{$\begin{array}{l}\text { Condition } \\
\text { S00K } \\
\text { S00N }\end{array}$} & \multirow{3}{*}{$\begin{array}{c}\text { Mean } t_{\text {eq }} \\
18.23 \\
21.28\end{array}$} & \multirow{3}{*}{$\begin{array}{c}\text { Mean MTV } \\
-1.40 \\
-0.90\end{array}$} & \multirow{2}{*}{\multicolumn{4}{|c|}{$\mathbf{M T V}=\mathbf{a}+\mathbf{b} * \mathbf{t}_{\mathrm{eq}} \quad$ Serie $\mathbf{1}$}} & \multirow{2}{*}{\multicolumn{4}{|c|}{ STATISTICS }} \\
\hline & & & & & & & & & & \\
\hline & & & $a=$ & -3.42 & teq & MTV & Sda $=$ & 0.66 & $F=$ & 18.40 \\
\hline soov & 23.57 & -0.20 & $b=$ & 0.14 & 15 & -1.33 & $\mathrm{Sdb}=$ & 0.03 & $\mathrm{DF}=$ & 9.00 \\
\hline Soox & 25.77 & 0.50 & $r=$ & 0.82 & 26 & 0.25 & SdMTV= & 0.35 & $r^{2}$ & 0.67 \\
\hline SHOK & 15.90 & -0.80 & \multicolumn{8}{|c|}{ Serie 2} \\
\hline SHON & 17.69 & -1.10 & \multicolumn{4}{|c|}{ LINEAR REGRESSION } & \multicolumn{4}{|c|}{ STATISTICS } \\
\hline SHOV & 19.61 & -0.60 & $a=$ & -6.96 & teq & MTV & Sda $=$ & 0.87 & $F=$ & 65.43 \\
\hline SMOK & 14.65 & -1.20 & $b=$ & 0.27 & 22 & -1.04 & $\mathrm{Sdb}=$ & 0.03 & $\mathrm{DF}=$ & 3.00 \\
\hline SMON & 17.09 & -1.00 & $r=$ & 0.98 & 29 & 0.88 & SdMTV= & 0.19 & r2 & 0.96 \\
\hline SMOV & 18.61 & -0.50 & \multicolumn{8}{|c|}{ Serie $1 \& 2$} \\
\hline SMOX & 21.72 & 0.10 & \multicolumn{4}{|c|}{ LINEAR REGRESSION } & \multicolumn{4}{|c|}{ STATISTICS } \\
\hline SOK & 23.58 & -0.50 & $a=$ & -3.50 & teq & MTV & Sda $=$ & 0.49 & $F=$ & 40.43 \\
\hline SON & 27.47 & 0.30 & $b=$ & 0.14 & 15 & -1.40 & $\mathrm{Sdb}=$ & 0.02 & $\mathrm{DF}=$ & 14.00 \\
\hline sov & 28.68 & 1.10 & $r=$ & 0.86 & 29 & 0.61 & SdMTV= & 0.37 & r2 & 0.74 \\
\hline SOGK & 21.65 & -1.00 & & & & & & & & \\
\hline SOGV & 26.29 & 0.20 & & & & & & & & \\
\hline
\end{tabular}

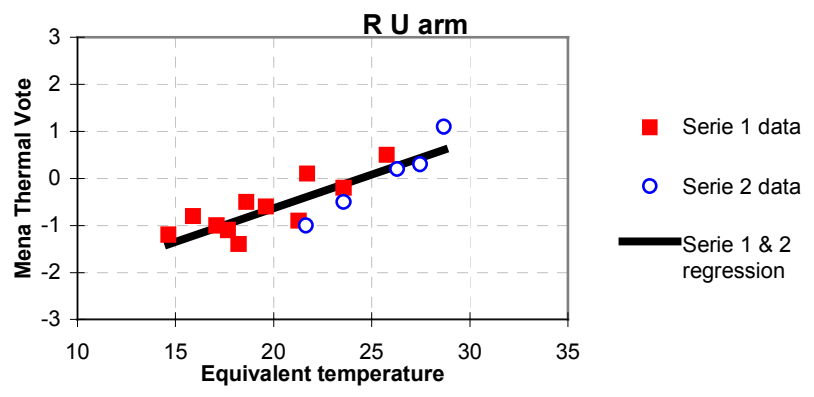


L L arm

\begin{tabular}{l|c|c|}
\multicolumn{1}{l}{$\begin{array}{l}\text { Condition } \\
\text { SO0K }\end{array}$} & \multicolumn{1}{c}{ Mean teq $_{\text {eq }}$} & \multicolumn{1}{c}{ Mean MTV } \\
\cline { 2 - 3 } SO0N & 18.01 & -0.90 \\
SOOV & 20.10 & -0.30 \\
SO0X & 21.91 & -0.10 \\
SHOK & 24.25 & 0.60 \\
SHON & 20.05 & -0.50 \\
SHOV & 21.73 & -0.30 \\
SMOK & 23.30 & -0.10 \\
SMON & 20.73 & -0.30 \\
SMOV & 22.90 & 0.20 \\
SMOX & 24.27 & -0.20 \\
SOK & 26.56 & 0.33 \\
SON & 20.26 & -0.50 \\
SOV & 25.13 & 0.30 \\
SOGK & 28.29 & 1.30 \\
SOGV & 17.07 & -1.10 \\
& 21.57 & -0.10 \\
\hline
\end{tabular}

$M T V=a+b$ * $t_{\text {eq }} \quad$ Serie 1

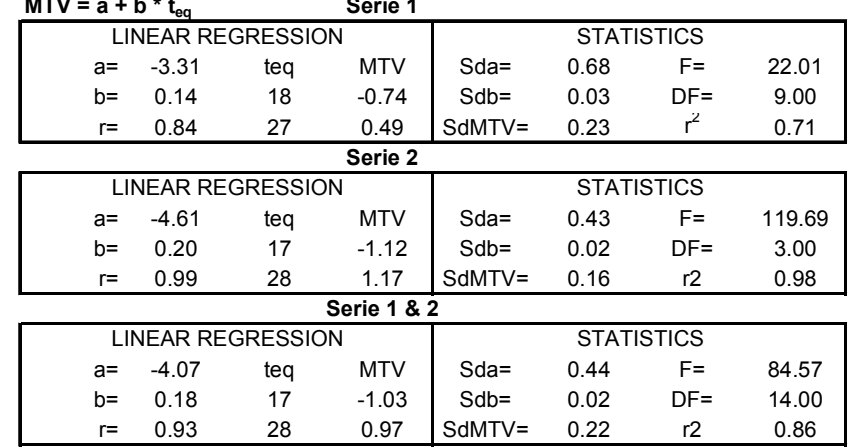

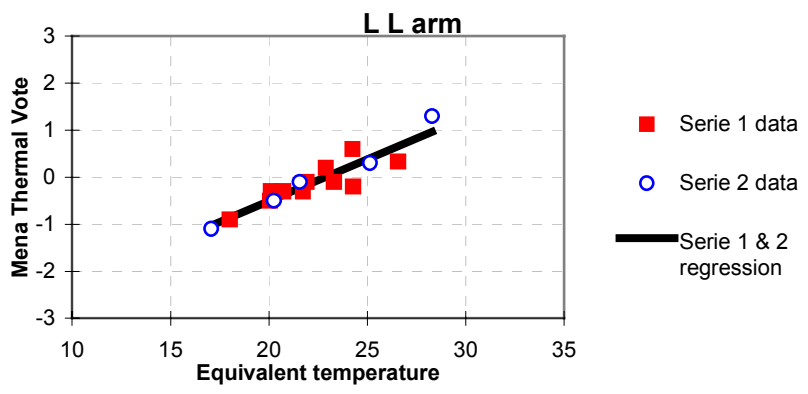

$8 \quad R$ L arm

\begin{tabular}{|c|c|c|c|c|c|c|c|c|c|c|}
\hline \multirow{3}{*}{$\begin{array}{l}\text { Condition } \\
\text { S0OK } \\
\text { S0ON }\end{array}$} & \multirow{3}{*}{$\begin{array}{c}\text { Mean } \mathbf{t}_{\mathrm{eq}} \\
17.60 \\
19.64\end{array}$} & \multirow{3}{*}{$\begin{array}{c}\text { Mean MTV } \\
-0.90 \\
-0.40\end{array}$} & \multirow{2}{*}{\multicolumn{4}{|c|}{$\mathbf{M T V}=\mathbf{a}+\mathbf{b} * \mathbf{t}_{\mathrm{eq}} \quad$ Serie $\mathbf{1}$}} & \multirow{2}{*}{\multicolumn{4}{|c|}{ STATISTICS }} \\
\hline & & & & & & & & & & \\
\hline & & & $a=$ & -2.71 & teq & MTV & Sda $=$ & 0.80 & $\mathrm{~F}=$ & 10.17 \\
\hline soov & 20.38 & 0.00 & $b=$ & 0.12 & 18 & -0.65 & $S d b=$ & 0.04 & $D F=$ & 9.00 \\
\hline soox & 22.87 & 0.60 & $r=$ & 0.73 & 26 & 0.34 & SdMTV= & 0.28 & $r^{2}$ & 0.53 \\
\hline SHOK & 19.60 & -0.40 & \multicolumn{8}{|c|}{ Serie 2} \\
\hline SHON & 21.53 & -0.30 & \multicolumn{4}{|c|}{ LINEAR REGRESSION } & \multicolumn{4}{|c|}{ STATISTICS } \\
\hline SHOV & 23.39 & -0.10 & $a=$ & -5.29 & teq & MTV & Sda $=$ & 1.08 & $F=$ & 24.72 \\
\hline SMOK & 20.36 & -0.40 & $b=$ & 0.21 & 20 & -1.09 & $S d b=$ & 0.04 & $D F=$ & 3.00 \\
\hline SMON & 22.82 & -0.10 & $r=$ & 0.94 & 29 & 0.95 & SdMTV= & 0.32 & r2 & 0.89 \\
\hline SMOV & 23.65 & -0.20 & \multicolumn{8}{|c|}{ Serie $1 \& 2$} \\
\hline SMox & 26.05 & 0.20 & \multicolumn{4}{|c|}{ LINEAR REGRESSION } & \multicolumn{4}{|c|}{ STATISTICS } \\
\hline sok & 22.73 & -0.50 & $a=$ & -3.27 & teq & MTV & Sda $=$ & 0.62 & $\mathrm{~F}=$ & 26.14 \\
\hline SON & 26.85 & 0.30 & $b=$ & 0.14 & 18 & -0.82 & $\mathrm{Sdb}=$ & 0.03 & $D F=$ & 14.00 \\
\hline sov & 29.25 & 1.30 & $r=$ & 0.81 & 29 & 0.80 & SdMTV= & 0.33 & r2 & 0.65 \\
\hline SOGK & 19.68 & -0.90 & & & & & & & & \\
\hline
\end{tabular}

R L arm

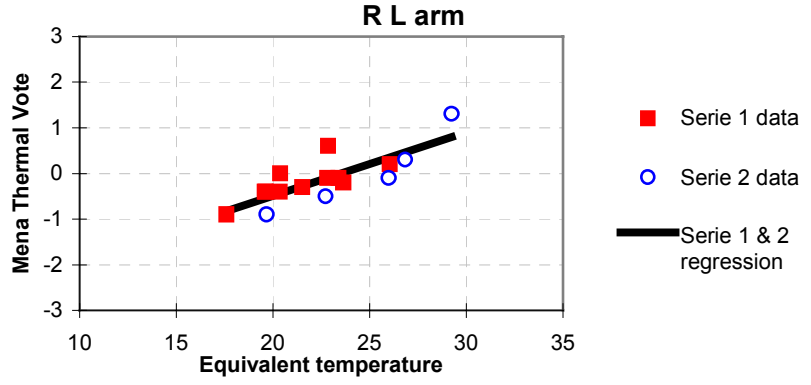


L hand

\begin{tabular}{l|c|c|}
\multicolumn{1}{l}{$\begin{array}{l}\text { Condition } \\
\text { SO0K }\end{array}$} & \multicolumn{1}{c}{ Mean teq $_{\text {eq }}$} & \multicolumn{1}{c}{ Mean MTV } \\
\cline { 2 - 3 } SOON & 22.15 & -0.60 \\
SOOV & 23.05 & -0.50 \\
SO0X & 24.63 & 0.00 \\
SHOK & 26.20 & 0.40 \\
SHON & 28.29 & -0.60 \\
SHOV & 29.87 & 0.20 \\
SMOK & 31.53 & 0.30 \\
SMON & 30.32 & 0.30 \\
SMOV & 32.44 & 0.70 \\
SMOX & 33.84 & 0.60 \\
SOK & 34.93 & 0.89 \\
SON & 23.63 & -0.10 \\
SOV & 26.97 & 0.67 \\
SOGK & 29.72 & 1.30 \\
SOGV & 19.80 & -0.90 \\
& 26.19 & 0.20 \\
\hline
\end{tabular}

$M T V=a+b * t_{e q} \quad$ Serie 1

\begin{tabular}{|cccc|cccc|}
\hline \multicolumn{3}{|c|}{ LINEAR REGRESSION } & \multicolumn{5}{c|}{ STATISTICS } \\
$\mathrm{a}=$ & -2.68 & teq & MTV & Sda $=$ & 0.67 & F $=$ & 18.20 \\
$\mathrm{~b}=$ & 0.10 & 22 & -0.50 & Sdb $=$ & 0.02 & DF $=$ & 9.00 \\
$\mathrm{r}=$ & 0.82 & 35 & 0.75 & SdMTV $=$ & 0.32 & $\mathrm{r}^{2}$ & 0.67 \\
\hline \multicolumn{8}{|c}{ S }
\end{tabular}

\begin{tabular}{|c|c|c|c|c|c|c|c|}
\hline \multicolumn{4}{|c|}{ LINEAR REGRESSION } & \multicolumn{4}{|c|}{ STATISTICS } \\
\hline$a=$ & -5.26 & teq & MTV & $S d a=$ & 0.53 & $F=$ & 110.67 \\
\hline$b=$ & 0.22 & 20 & -0.95 & $S d b=$ & 0.02 & $D F=$ & 3.00 \\
\hline$r=$ & 0.99 & 30 & 1.20 & SdMTV= & 0.15 & r2 & 0.97 \\
\hline \multicolumn{8}{|c|}{ Serie $1 \& 2$} \\
\hline \multicolumn{4}{|c|}{ LINEAR REGRESSION } & \multicolumn{4}{|c|}{ STATISTICS } \\
\hline$a=$ & -2.68 & teq & MTV & Sda $=$ & 0.68 & $F=$ & 17.87 \\
\hline$b=$ & 0.10 & 20 & -0.64 & $S d b=$ & 0.02 & $D F=$ & 14.00 \\
\hline$r=$ & 0.75 & 35 & 0.92 & SdMTV $=$ & 0.41 & r2 & 0.56 \\
\hline
\end{tabular}

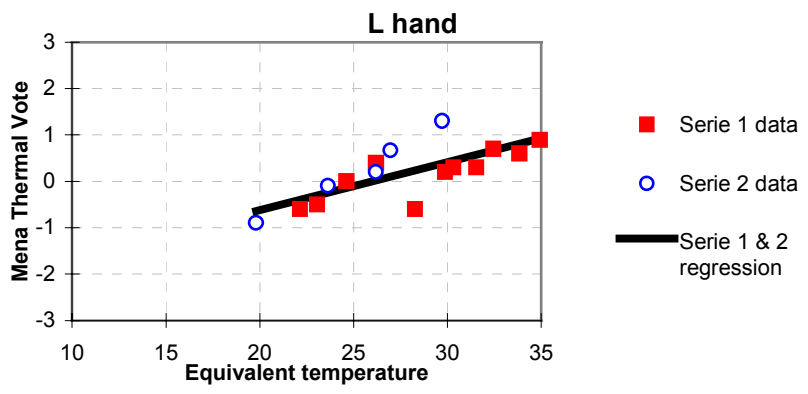

$10 \quad \mathrm{R}$ hand

\begin{tabular}{l|c|c|} 
Condition & \multicolumn{1}{c}{ Mean $t_{\text {eq }}$} & Mean MTV \\
\cline { 2 - 3 } SOOK & 19.79 & -0.60 \\
SOON & 21.03 & -0.40 \\
SOOV & 22.05 & 0.00 \\
S00X & 23.99 & 0.40 \\
SHOK & 22.25 & -0.50 \\
SHON & 24.34 & 0.30 \\
SHOV & 26.06 & 0.40 \\
SMOK & 24.03 & 0.40 \\
SMON & 26.03 & 0.70 \\
SMOV & 27.29 & 0.70 \\
SMOX & 29.19 & 0.80 \\
SOK & 22.18 & -0.10 \\
SON & 26.62 & 0.67 \\
SOV & 29.39 & 1.30 \\
SOGK & 18.13 & -0.70 \\
SOGV & 27.20 & 0.30 \\
& \multicolumn{2}{|}{}
\end{tabular}

$M T V=a+b * t_{e q} \quad$ Serie 1

\begin{tabular}{|cccc|cccc|}
\hline \multicolumn{3}{|c|}{ LINEAR REGRESSION } & \multicolumn{4}{c|}{ STATISTICS } \\
$\mathrm{a}=$ & -3.77 & teq & MTV & Sda $=$ & 0.57 & $\mathrm{~F}=$ & 49.07 \\
$\mathrm{~b}=$ & 0.16 & 20 & -0.52 & Sdb $=$ & 0.02 & DF $=$ & 9.00 \\
$\mathrm{r}=$ & 0.92 & 29 & 1.02 & SdMTV $=$ & 0.21 & $\mathrm{r}^{2}$ & 0.85 \\
\hline
\end{tabular}

\begin{tabular}{|cccc|cccc|}
\hline \multicolumn{10}{|c|}{ Serie 2 } \\
\hline \multicolumn{10}{|c|}{ LINEAR REGRESSION } & \multicolumn{4}{c|}{ STATISTICS } \\
$\mathrm{a}=$ & -3.64 & teq & MTV & Sda $=$ & 0.76 & $\mathrm{~F}=$ & 27.23 \\
$\mathrm{~b}=$ & 0.16 & 18 & -0.75 & Sdb $=$ & 0.03 & DF $=$ & 3.00 \\
$\mathrm{r}=$ & 0.95 & 29 & 1.04 & SdMTV $=$ & 0.28 & $\mathrm{r} 2$ & 0.90 \\
\hline
\end{tabular}

$r=0.95 \quad 29$

Serie 1 \& 2

\begin{tabular}{|cccc|cccc|}
\hline \multicolumn{10}{|c|}{ LINEAR REGRESSION } & \multicolumn{5}{c|}{ STATISTICS } \\
$\mathrm{a}=$ & -3.71 & teq & MTV & Sda $=$ & 0.41 & $\mathrm{~F}=$ & 95.48 \\
$\mathrm{~b}=$ & 0.16 & 18 & -0.78 & $\mathrm{Sdb}=$ & 0.02 & $\mathrm{DF}=$ & 14.00 \\
$\mathrm{r}=$ & 0.93 & 29 & 1.05 & $\mathrm{SdMTV}=$ & 0.21 & $\mathrm{r} 2$ & 0.87 \\
\hline
\end{tabular}

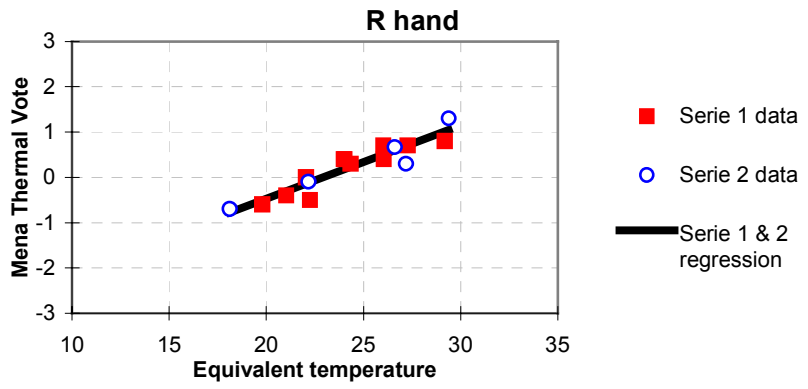


L thigh

\begin{tabular}{l|c|c|}
\multicolumn{1}{l}{$\begin{array}{l}\text { Condition } \\
\text { SOOK }\end{array}$} & \multicolumn{1}{c}{ Mean $\mathrm{t}_{\mathrm{eq}}$} & \multicolumn{1}{c}{ Mean MTV } \\
\cline { 2 - 3 } SOON & 21.81 & -1.40 \\
SOOV & 23.51 & -0.80 \\
S00X & 24.85 & -0.50 \\
SHOK & 26.48 & 0.30 \\
SHON & 27.33 & -0.80 \\
SHOV & 28.41 & 0.30 \\
SMOK & 30.55 & 1.00 \\
SMON & 28.23 & 0.80 \\
SMOV & 29.75 & 1.30 \\
SMOX & 30.29 & 1.10 \\
SOK & 31.41 & 1.30 \\
SON & 24.54 & 0.20 \\
SOV & 26.82 & 1.20 \\
SOGK & 30.38 & 1.80 \\
SOGV & 21.42 & -0.90 \\
& 29.30 & 0.80
\end{tabular}

$M T V=a+b * t_{\text {eq }} \quad$ Serie 1

\begin{tabular}{|cccc|cccc|}
\hline \multicolumn{3}{c|}{ LINEAR REGRESSION } & \multicolumn{4}{c|}{ STATISTICS } \\
$\mathrm{a}=$ & -7.64 & teq & MTV & Sda $=$ & 1.15 & $\mathrm{~F}=$ & 47.62 \\
$\mathrm{~b}=$ & 0.29 & 22 & -1.40 & $\mathrm{Sdb}=$ & 0.04 & DF $=$ & 9.00 \\
$\mathrm{r}=$ & 0.92 & 31 & 1.35 & SdMTV $=$ & 0.40 & $\mathrm{r}^{2}$ & 0.84 \\
\hline
\end{tabular}

\begin{tabular}{|c|c|c|c|c|c|c|c|}
\hline \multicolumn{4}{|c|}{ LINEAR REGRESSION } & \multicolumn{4}{|c|}{ STATISTICS } \\
\hline$a=$ & -6.33 & teq & MTV & Sda $=$ & 1.68 & $F=$ & 17.45 \\
\hline$b=$ & 0.26 & 21 & -0.71 & $S d b=$ & 0.06 & $\mathrm{DF}=$ & 3.00 \\
\hline$r=$ & 0.92 & 30 & 1.64 & SdMTV= & 0.46 & r2 & 0.85 \\
\hline \multicolumn{8}{|c|}{ Serie $1 \& 2$} \\
\hline \multicolumn{4}{|c|}{ LINEAR REGRESSION } & \multicolumn{4}{|c|}{ STATISTICS } \\
\hline$a=$ & -6.78 & teq & MTV & $\mathrm{Sda}=$ & 1.13 & $\mathrm{~F}=$ & 40.40 \\
\hline$b=$ & 0.26 & 21 & -1.16 & $S d b=$ & 0.04 & $D F=$ & 14.00 \\
\hline$r=$ & 0.86 & 31 & 1.46 & SdMTV= & 0.51 & r2 & 0.74 \\
\hline
\end{tabular}

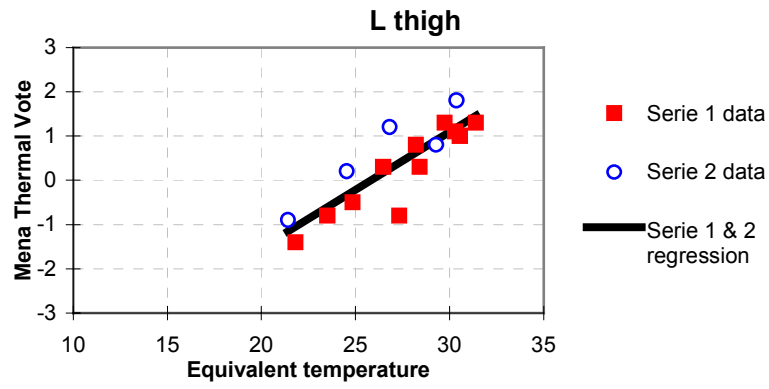

\begin{tabular}{lc|c|}
\multicolumn{1}{c}{$\begin{array}{c}12 \\
\text { Condition }\end{array}$} & \multicolumn{1}{c}{$\begin{array}{c}\text { R thigh } \\
\text { Mean } \mathbf{t}_{\text {eq }}\end{array}$} & \multicolumn{1}{c}{ Mean MTV } \\
\cline { 2 - 3 } SOOK & 21.00 & -0.90 \\
SOON & 22.91 & -0.50 \\
SOOV & 24.58 & -0.20 \\
SOOX & 26.63 & 0.40 \\
SHOK & 26.11 & -0.70 \\
SHON & 27.49 & 0.20 \\
SHOV & 28.59 & 1.00 \\
SMOK & 26.15 & 0.80 \\
SMON & 27.35 & 1.30 \\
SMOV & 28.13 & 1.10 \\
SMOX & 29.67 & 1.30 \\
SOK & 24.73 & 0.20 \\
SON & 27.03 & 1.20 \\
SOV & 31.02 & 1.80 \\
SOGK & 20.38 & -0.90 \\
SOGV & 30.22 & 0.80 \\
\hline
\end{tabular}

MTV $=a+b$ * $t_{\text {eq }} \quad$ Serie 1

\begin{tabular}{|cccc|cccc|}
\hline \multicolumn{3}{|c|}{ LINEAR REGRESSION } & \multicolumn{4}{c|}{ STATISTICS } \\
$\mathrm{a}=$ & -6.70 & teq & MTV & Sda $=$ & 1.54 & F $=$ & 20.98 \\
$\mathrm{~b}=$ & 0.27 & 21 & -1.06 & Sdb $=$ & 0.06 & DF $=$ & 9.00 \\
$\mathrm{r}=$ & 0.84 & 30 & 1.27 & SdMTV $=$ & 0.47 & $\mathrm{r}^{2}$ & 0.70 \\
\hline
\end{tabular}

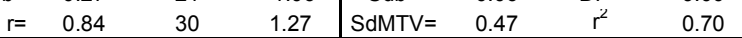

\begin{tabular}{|cccc|cccc|}
\hline \multicolumn{10}{|c|}{ Serie 2 } \\
\hline \multicolumn{10}{|c|}{ LINEAR REGRESSION } & \multicolumn{4}{c|}{ STATISTICS } \\
$\mathrm{a}=$ & -5.20 & teq & MTV & Sda $=$ & 1.48 & $\mathrm{~F}=$ & 15.73 \\
$\mathrm{~b}=$ & 0.22 & 20 & -0.75 & $\mathrm{Sdb}=$ & 0.06 & $\mathrm{DF}=$ & 3.00 \\
$\mathrm{r}=$ & 0.92 & 31 & 1.57 & SdMTV $=$ & 0.48 & $\mathrm{r} 2$ & 0.84 \\
\hline
\end{tabular}

\begin{tabular}{|cccc|cccc|}
\hline \multicolumn{10}{|c|}{ Serie 1 \& 2 } \\
\hline \multicolumn{10}{|c|}{ LINEAR REGRESSION } & \multicolumn{5}{c|}{ STATISTICS } \\
$\mathrm{a}=$ & -5.99 & teq & MTV & Sda $=$ & 1.01 & F $=$ & 40.53 \\
$\mathrm{~b}=$ & 0.24 & 20 & -1.03 & Sdb $=$ & 0.04 & DF $=$ & 14.00 \\
\hline & 0.86 & 31 & 1.56 & SdMTV & 0.45 & 2 & 0.74
\end{tabular}

$\begin{array}{ll}30.22 & 0.80\end{array}$

R thigh

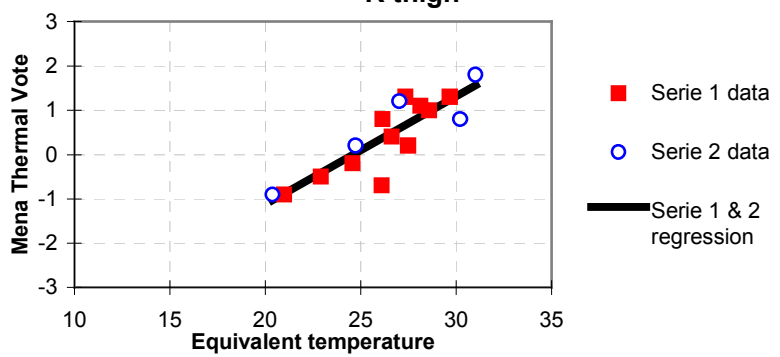


L calf

\begin{tabular}{l|c|c|}
\multicolumn{1}{l}{$\begin{array}{l}\text { Condition } \\
\text { SO0K }\end{array}$} & \multicolumn{1}{c}{ Mean teq $_{\text {eq }}$} & \multicolumn{1}{c}{ Mean MTV } \\
\cline { 2 - 3 } SO0N & 20.35 & -1.30 \\
SOOV & 23.59 & -1.10 \\
SO0X & 25.53 & 0.00 \\
SHOK & 27.80 & 0.60 \\
SHON & 20.78 & -1.30 \\
SHOV & 23.00 & -0.70 \\
SMOK & 24.53 & -0.50 \\
SMON & 19.85 & -1.00 \\
SMOV & 21.94 & -0.30 \\
SMOX & 24.07 & -0.40 \\
SOK & 26.37 & 0.00 \\
SON & 17.44 & -0.40 \\
SOV & 21.25 & 0.10 \\
SOGK & 25.77 & 1.10 \\
SOGV & 18.01 & -1.30 \\
& 20.24 & -0.40 \\
\hline
\end{tabular}

$M T V=a+b * t_{e q} \quad$ Serie 1

\begin{tabular}{|cccc|cccc|}
\hline \multicolumn{3}{c|}{ LINEAR REGRESSION } & \multicolumn{4}{c|}{ STATISTICS } \\
$\mathrm{a}=$ & -5.36 & teq & MTV & Sda $=$ & 0.92 & $\mathrm{~F}=$ & 27.62 \\
$\mathrm{~b}=$ & 0.21 & 20 & -1.28 & $\mathrm{Sdb}=$ & 0.04 & $\mathrm{DF}=$ & 9.00 \\
$\mathrm{r}=$ & 0.87 & 28 & 0.35 & SdMTV $=$ & 0.32 & $\mathrm{r}^{2}$ & 0.75 \\
\hline
\end{tabular}

\begin{tabular}{|c|c|c|c|c|c|c|c|}
\hline \multicolumn{4}{|c|}{ LINEAR REGRESSION } & \multirow{2}{*}{\multicolumn{4}{|c|}{ STATISTICS }} \\
\hline $\mathrm{L}$ & IEAR R & 5 & ATY & & & & \\
\hline & -0.01 & teq & IVIV & $S d b=$ & 1.31 & $\mathrm{PE}=$ & 13.09 \\
\hline$b=$ & 0.24 & 17 & -0.92 & $\mathrm{Sdb}=$ & 0.07 & $\mathrm{DF}=$ & 3.00 \\
\hline$r=$ & 0.90 & 26 & 1.07 & SdMTV= & 0.44 & r2 & 0.81 \\
\hline \multicolumn{8}{|c|}{ Serie 1\& 2} \\
\hline \multicolumn{4}{|c|}{ LINEAR REGRESSION } & \multicolumn{4}{|c|}{ STATISTICS } \\
\hline$a=$ & -3.74 & teq & MTV & Sda $=$ & 1.06 & $F=$ & 9.89 \\
\hline$b=$ & 0.15 & 17 & -1.18 & $S d b=$ & 0.05 & $D F=$ & 14.00 \\
\hline$r=$ & 0.64 & 28 & 0.34 & SdMTV $=$ & 0.55 & r2 & 0.41 \\
\hline
\end{tabular}

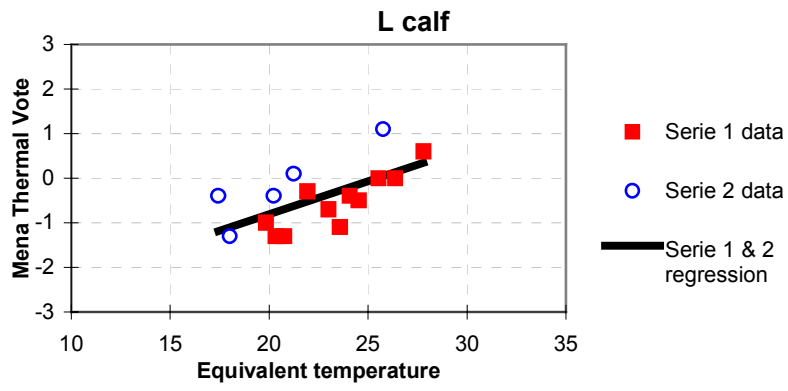

$14 \quad R$ calf

\begin{tabular}{|c|c|c|c|c|c|c|c|c|c|c|}
\hline \multirow{3}{*}{$\begin{array}{l}\text { Condition } \\
\text { SOOK } \\
\text { SOON }\end{array}$} & \multirow{3}{*}{$\begin{array}{c}\text { Mean } \mathbf{t}_{\mathrm{eq}} \\
22.60 \\
25.12\end{array}$} & \multirow{3}{*}{\begin{tabular}{c|} 
Mean MTV \\
-0.70 \\
-0.50
\end{tabular}} & \multicolumn{4}{|c|}{$M T V=a+b * t_{e q} \quad$ Serie 1} & \multirow{2}{*}{\multicolumn{4}{|c|}{ STATISTICS }} \\
\hline & & & \multicolumn{4}{|c|}{ LINEAR REGRESSION } & & & & \\
\hline & & & $a=$ & -4.66 & teq & MTV & $S d a=$ & 0.86 & $\mathrm{~F}=$ & 25.38 \\
\hline soov & 26.31 & 0.30 & $b=$ & 0.18 & 20 & -1.12 & $S d b=$ & 0.04 & $\mathrm{DF}=$ & 9.00 \\
\hline soox & 28.11 & 0.60 & $r=$ & 0.86 & 28 & 0.39 & SdMTV= & 0.27 & $r^{2}$ & 0.74 \\
\hline SHOK & 21.85 & -1.00 & \multicolumn{8}{|c|}{ Serie 2} \\
\hline SHON & 23.07 & -0.70 & \multicolumn{4}{|c|}{ LINEAR REGRESSION } & \multicolumn{4}{|c|}{ STATISTICS } \\
\hline SHOV & 24.49 & -0.40 & $a=$ & -6.97 & teq & MTV & $S d a=$ & 1.37 & $F=$ & 24.86 \\
\hline SMOK & 19.68 & -1.00 & $b=$ & 0.30 & 20 & -0.97 & $S d b=$ & 0.06 & $\mathrm{DF}=$ & 3.00 \\
\hline SMON & 21.87 & -0.20 & $r=$ & 0.94 & 27 & 1.06 & SdMTV= & 0.33 & r2 & 0.89 \\
\hline SMOV & 23.59 & -0.40 & \multicolumn{8}{|c|}{ Serie $1 \& 2$} \\
\hline SMOX & 25.78 & -0.10 & \multicolumn{4}{|c|}{ LINEAR REGRESSION } & \multicolumn{4}{|c|}{ STATISTICS } \\
\hline soK & 20.23 & -0.60 & $a=$ & -5.14 & teq & MTV & $S d a=$ & 0.88 & $F=$ & 30.30 \\
\hline SON & 23.38 & 0.10 & $b=$ & 0.20 & 20 & -1.11 & $S d b=$ & 0.04 & $\mathrm{DF}=$ & 14.00 \\
\hline sov & 27.10 & 1.10 & $r=$ & 0.83 & 28 & 0.62 & SdMTV= & 0.36 & r2 & 0.68 \\
\hline SOGK & 20.61 & -1.20 & & & & & & & & \\
\hline SOGV & 22.89 & -0.40 & & & & & & & & \\
\hline
\end{tabular}

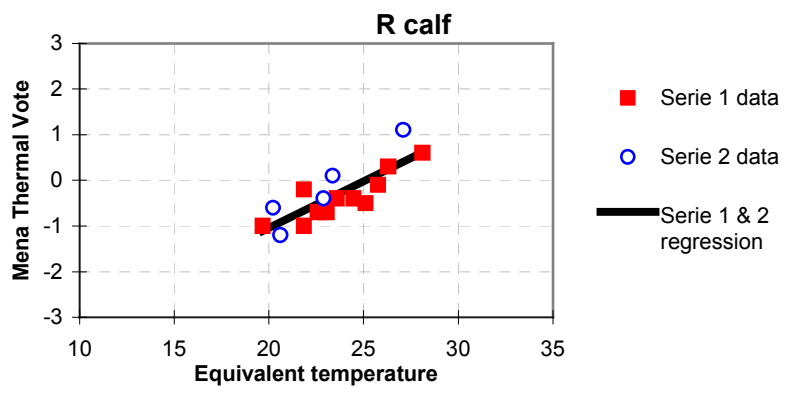


$L$ foot

\begin{tabular}{l|c|c|}
\multicolumn{1}{l}{$\begin{array}{l}\text { Condition } \\
\text { SO0K }\end{array}$} & \multicolumn{1}{c}{ Mean teq $_{\text {eq }}$} & \multicolumn{1}{c}{ Mean MTV } \\
\cline { 2 - 3 } SOON & 22.62 & -0.90 \\
SOOV & 25.08 & -0.70 \\
SO0X & 25.75 & 0.30 \\
SHOK & 27.17 & 1.00 \\
SHON & 20.93 & -0.90 \\
SHOV & 22.02 & -0.20 \\
SMOK & 23.17 & 0.30 \\
SMON & 19.16 & -0.60 \\
SMOV & 20.89 & 0.00 \\
SMOX & 22.99 & 0.10 \\
SOK & 24.67 & 0.50 \\
SON & 17.46 & 0.40 \\
SOV & 20.36 & 1.00 \\
SOGK & 24.58 & 2.00 \\
SOGV & 17.82 & -0.90 \\
& 18.52 & 0.20 \\
\hline
\end{tabular}

$M T V=a+b$ * $t_{\text {eq }} \quad$ Serie 1

\begin{tabular}{|cccc|cccc|}
\hline \multicolumn{3}{c|}{ LINEAR REGRESSION } & \multicolumn{4}{c|}{ STATISTICS } \\
$\mathrm{a}=$ & -3.90 & teq & MTV & Sda $=$ & 1.57 & $\mathrm{~F}=$ & 5.92 \\
$\mathrm{~b}=$ & 0.16 & 19 & -0.75 & $\mathrm{Sdb}=$ & 0.07 & $\mathrm{DF}=$ & 9.00 \\
$\mathrm{r}=$ & 0.63 & 27 & 0.56 & SdMTV $=$ & 0.51 & $\mathrm{r}^{2}$ & 0.40 \\
\hline
\end{tabular}

\begin{tabular}{|c|c|c|c|c|c|c|c|}
\hline \multicolumn{4}{|r|}{ Serie 2} & \multicolumn{4}{|c|}{ 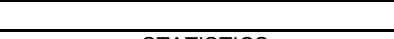 } \\
\hline \multicolumn{4}{|c|}{ LINEAR REGRESSION } & \multicolumn{4}{|c|}{ STATISTICS } \\
\hline$a=$ & -5.72 & teq & MTV & Sda $=$ & 2.07 & $F=$ & 9.29 \\
\hline$b=$ & 0.32 & 17 & -0.19 & $S d b=$ & 0.10 & $\mathrm{DF}=$ & 3.00 \\
\hline$r=$ & 0.87 & 25 & 2.07 & SdMTV= & 0.61 & r2 & 0.76 \\
\hline \multicolumn{8}{|c|}{ Serie 1\&2 } \\
\hline \multicolumn{4}{|c|}{ LINEAR REGRESSION } & \multicolumn{4}{|c|}{ STATISTICS } \\
\hline$a=$ & -2.05 & teq & MTV & Sda $=$ & 1.53 & $\mathrm{~F}=$ & 2.02 \\
\hline$b=$ & 0.10 & 17 & -0.35 & $\mathrm{Sdb}=$ & 0.07 & $\mathrm{DF}=$ & 14.00 \\
\hline$r=$ & 0.36 & 27 & 0.60 & SdMTV= & 0.78 & r2 & 0.13 \\
\hline
\end{tabular}

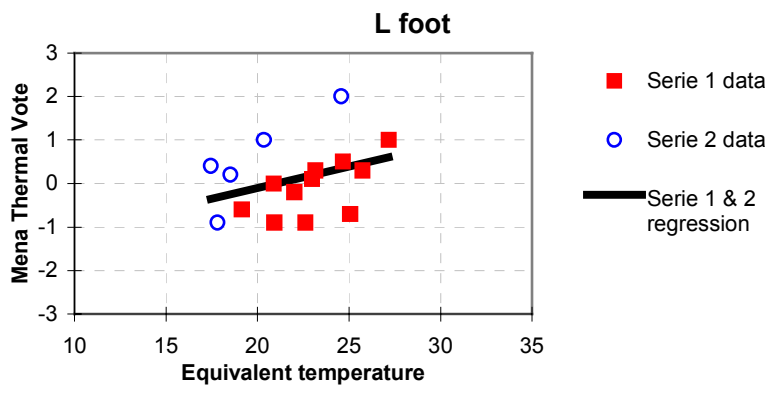

$16 \quad \mathrm{R}$ foot

\begin{tabular}{l|c|c|}
\multicolumn{1}{c}{ Condition } & \multicolumn{1}{c}{ Mean teq $_{\text {eq }}$} & \multicolumn{1}{c}{ Mean MTV } \\
\cline { 2 - 3 } SOOK & 22.62 & -0.50 \\
SOON & 25.08 & -0.20 \\
SOOV & 25.75 & 0.50 \\
SO0X & 27.17 & 1.00 \\
SHOK & 20.93 & -0.70 \\
SHON & 22.02 & -0.20 \\
SHOV & 23.17 & 0.30 \\
SMOK & 19.16 & -0.70 \\
SMON & 20.89 & 0.00 \\
SMOV & 22.99 & 0.20 \\
SMOX & 24.67 & 0.50 \\
SOK & 18.49 & 0.40 \\
SON & 21.81 & 1.00 \\
SOV & 25.29 & 2.00 \\
SOGK & 19.05 & -0.80 \\
SOGV & 20.10 & 0.20 \\
\hline
\end{tabular}

$M T V=a+b * t_{e q} \quad$ Serie 1

\begin{tabular}{|cccc|cccc|}
\hline \multicolumn{3}{|c|}{ LINEAR REGRESSION } & \multicolumn{4}{c|}{ STATISTICS } \\
$\mathrm{a}=$ & -4.29 & teq & MTV & Sda $=$ & 1.02 & $\mathrm{~F}=$ & 17.94 \\
$\mathrm{~b}=$ & 0.19 & 19 & -0.72 & Sdb $=$ & 0.04 & DF $=$ & 9.00 \\
$\mathrm{r}=$ & 0.82 & 27 & 0.77 & SdMTV $=$ & 0.33 & $\mathrm{r}^{2}$ & 0.67 \\
\hline
\end{tabular}

\begin{tabular}{|cccc|cccc|}
\hline \multicolumn{10}{|c|}{ Serie 2 } \\
\hline \multicolumn{10}{|c|}{ LINEAR REGRESSION } & \multicolumn{4}{c|}{ STATISTICS } \\
$\mathrm{a}=$ & -6.37 & teq & MTV & Sda $=$ & 2.21 & $\mathrm{~F}=$ & 9.94 \\
$\mathrm{~b}=$ & 0.33 & 18 & -0.25 & Sdb $=$ & 0.10 & DF $=$ & 3.00 \\
$\mathrm{r}=$ & 0.88 & 25 & 2.00 & SdMTV $=$ & 0.57 & $\mathrm{r} 2$ & 0.77 \\
\hline
\end{tabular}

Serie 1 \& 2

\begin{tabular}{|cccc|cccc|}
\hline \multicolumn{3}{|c|}{ LINEAR REGRESSION } & \multicolumn{4}{c|}{ STATISTICS } \\
$\mathrm{a}=$ & -3.38 & teq & MTV & Sda $=$ & 1.41 & $\mathrm{~F}=$ & 6.48 \\
$\mathrm{~b}=$ & 0.16 & 18 & -0.44 & $\mathrm{Sdb}=$ & 0.06 & $\mathrm{DF}=$ & 14.00 \\
$\mathrm{r}=$ & 0.56 & 27 & 0.94 & $\mathrm{SdMTV}=$ & 0.63 & $\mathrm{r} 2$ & 0.32 \\
\hline
\end{tabular}

$R$ foot

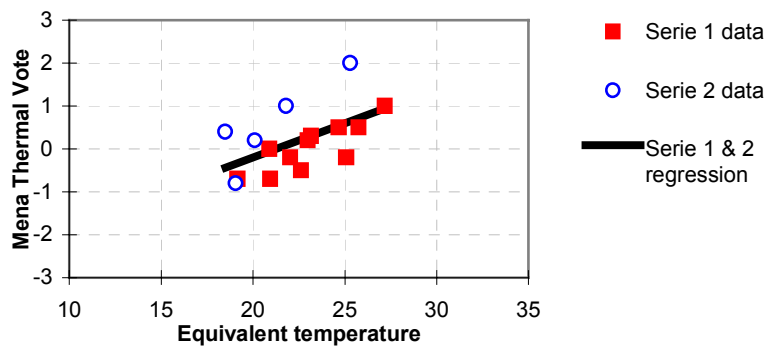


Lo. back

Condition Mean t

SoOK

SOON

Soov

SOOX

SHOK

SHON

SHOV

SMOK

SMON

SMOV

SMOX

SOK

SON

sov

SOGK

Mean $\mathrm{t}_{\mathrm{eq}}$ Mean MTV

$M T V=a+b * t_{e q}$

Serie 1

\begin{tabular}{|c|c|}
\hline & \\
& \\
& \\
& \\
& \\
& \\
& \\
& \\
26.33 & 0.60 \\
27.67 & 0.70 \\
28.99 & 1.60 \\
22.64 & -0.10 \\
30.50 & 0.80 \\
\hline
\end{tabular}

\begin{tabular}{|lc|cc|}
\hline \multicolumn{2}{|c|}{ LINEAR REGRESSION } & \multicolumn{2}{c|}{ STATISTICS } \\
$\mathrm{a}=$ & teq MTV & $\mathrm{Sda}=$ & $\mathrm{F}=$ \\
$\mathrm{b}=$ & $\mathrm{Sdb}=$ & $\mathrm{DF}=$ \\
$\mathrm{r}=$ & & SdMTV $=$ & $\mathrm{r}^{2}$ \\
\hline
\end{tabular}

\begin{tabular}{|cccc|cccc|}
\hline \multicolumn{10}{|c|}{ SINEAR REGRESSION } & \multicolumn{4}{c|}{ STATISTICS } \\
$\mathrm{a}=$ & -3.59 & teq & MTV & Sda $=$ & 1.99 & $\mathrm{~F}=$ & 4.72 \\
$\mathrm{~b}=$ & 0.16 & 23 & -0.01 & Sdb $=$ & 0.07 & DF $=$ & 3.00 \\
$\mathrm{r}=$ & 0.78 & 30 & 1.24 & SdMTV $=$ & 0.44 & $\mathrm{r} 2$ & 0.61 \\
\hline
\end{tabular}

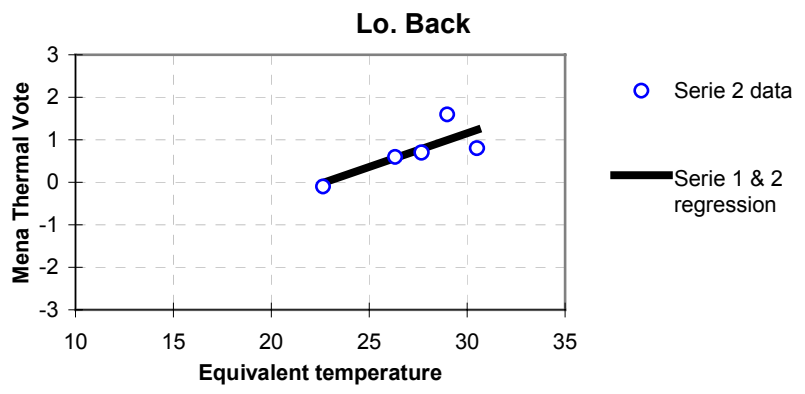

18 Seat

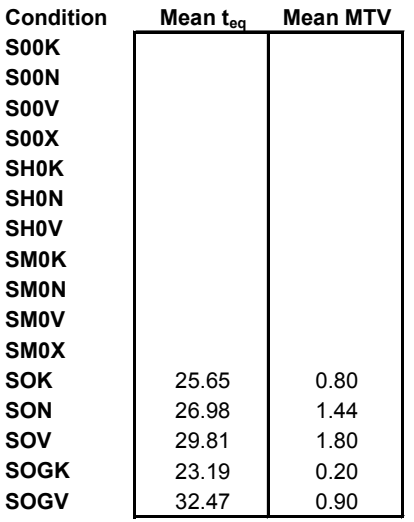

$\operatorname{MTV}=a+b$ * $t_{\text {eq }} \quad$ Serie 1

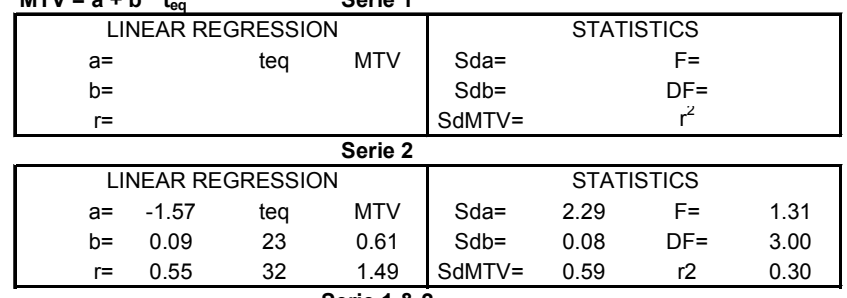

\begin{tabular}{|cccc|cccc|}
\hline \multicolumn{10}{|c|}{ Serie 1 \& 2 } & \multicolumn{4}{c|}{ STATISTICS } \\
$\mathrm{a}=$ & -1.57 & teq & MTV & Sda $=$ & 2.29 & $\mathrm{~F}=$ & 1.31 \\
$\mathrm{~b}=$ & 0.09 & 23 & 0.61 & $\mathrm{Sdb}=$ & 0.08 & $\mathrm{DF}=$ & 3.00 \\
$\mathrm{r}=$ & 0.55 & 32 & 1.49 & $\mathrm{SdMTV}=$ & 0.59 & $\mathrm{r} 2$ & 0.30 \\
\hline
\end{tabular}

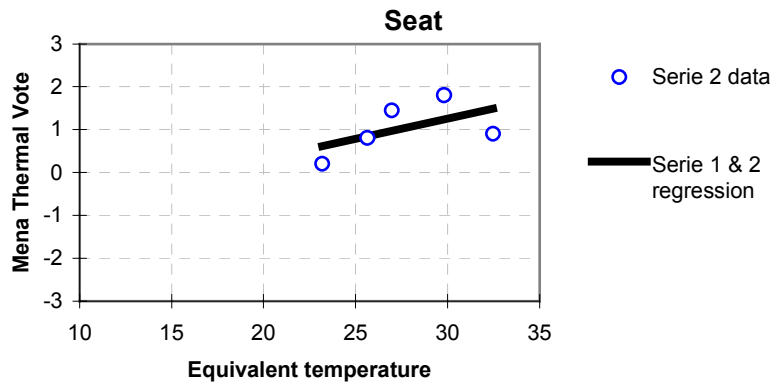




\section{Ventilated seat}

\section{Ventilated seat}

$0 \quad$ Whole body

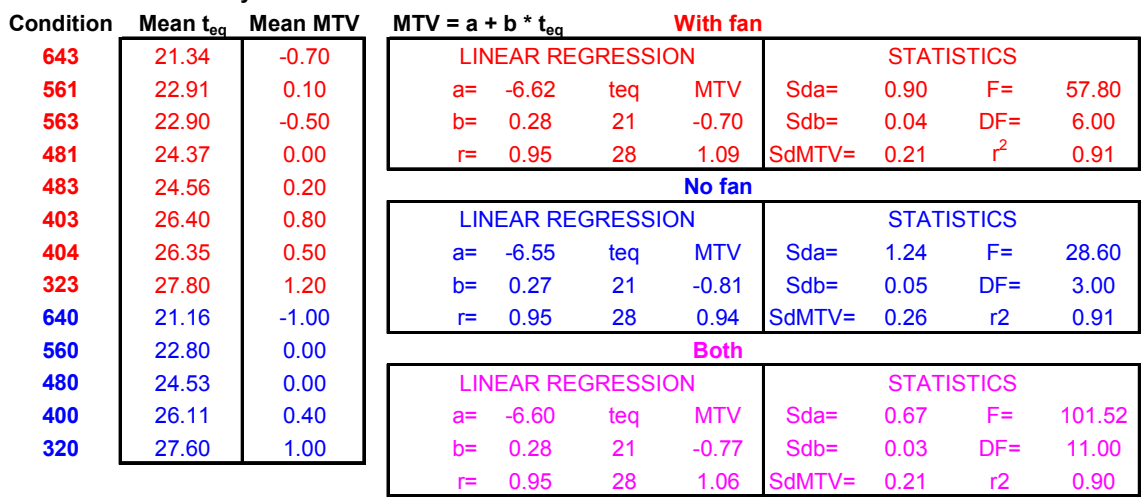

\section{Whole body}

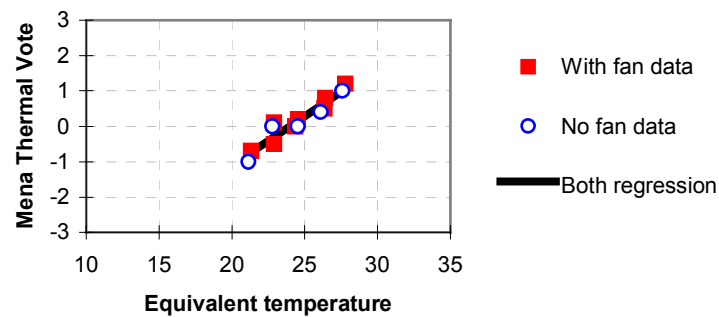

Chest, stomach

\begin{tabular}{|c|c|c|c|c|c|c|c|c|c|c|}
\hline \multirow{3}{*}{$\begin{array}{c}\text { Condition } \\
643\end{array}$} & \\
\hline & Mean $t_{\text {eq }}$ & Mean MTV & \multirow{2}{*}{\multicolumn{4}{|c|}{$\begin{array}{c}\mathbf{M T V}=\mathbf{a}+\mathbf{b}{ }^{*} \mathbf{t}_{\mathbf{e q}} \quad \text { With fan } \\
\text { LINEAR REGRESSION }\end{array}$}} & \multirow{2}{*}{\multicolumn{4}{|c|}{ STATISTICS }} \\
\hline & 20.68 & -0.60 & & & & & & & & \\
\hline 561 & 21.82 & 0.00 & $a=$ & -5.32 & teq & MTV & Sda $=$ & 0.95 & $\mathrm{~F}=$ & 32.79 \\
\hline 563 & 22.11 & -0.40 & $b=$ & 0.23 & 21 & -0.60 & $S d b=$ & 0.04 & $\mathrm{DF}=$ & 6.00 \\
\hline 481 & 23.07 & -0.30 & $r=$ & 0.92 & 27 & 0.90 & SdMTV= & 0.25 & $r^{2}$ & 0.85 \\
\hline 483 & 23.79 & 0.10 & \multicolumn{7}{|c|}{ No fan } & \\
\hline 403 & 25.80 & 0.60 & \multicolumn{4}{|c|}{ LINEAR REGRESSION } & \multicolumn{4}{|c|}{ STATISTICS } \\
\hline 404 & 26.43 & 0.40 & $a=$ & -5.27 & teq & MTV & Sda $=$ & 1.21 & $F=$ & 19.24 \\
\hline 323 & 27.26 & 1.20 & $b=$ & 0.24 & 19 & -0.90 & $\mathrm{Sdb}=$ & 0.05 & $\mathrm{DF}=$ & 3.00 \\
\hline 640 & 18.59 & -1.10 & $r=$ & 0.93 & 26 & 0.86 & SdMTV= & 0.32 & r2 & 0.87 \\
\hline 560 & 20.64 & -0.10 & \multicolumn{8}{|c|}{ Both } \\
\hline 480 & 22.46 & 0.10 & \multicolumn{4}{|c|}{ LINEAR REGRESSION } & \multicolumn{4}{|c|}{ STATISTICS } \\
\hline 400 & 24.39 & 0.10 & $a=$ & -5.05 & teq & MTV & Sda $=$ & 0.70 & $\mathrm{~F}=$ & 53.73 \\
\hline 320 & 26.06 & 1.00 & $b=$ & 0.22 & 19 & -0.96 & $S d b=$ & 0.03 & $\mathrm{DF}=$ & 11.00 \\
\hline & & & $r=$ & 0.91 & 27 & 0.94 & SdMTV= & 0.27 & r2 & 0.83 \\
\hline
\end{tabular}

Chest, stomach

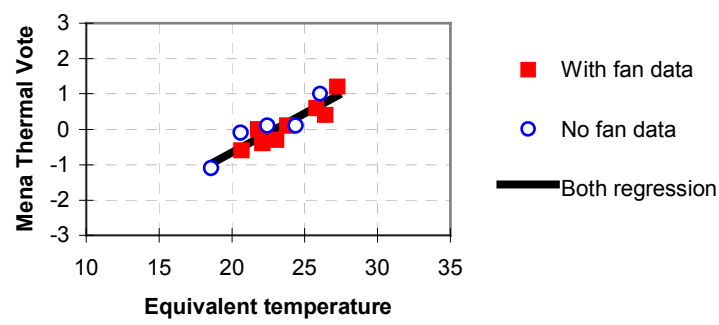


Back, lower

\begin{tabular}{|c|c|c|c|c|c|c|c|c|c|c|}
\hline Condition & Mean $t_{e q}$ & Mean MTV & \multicolumn{4}{|c|}{ MTV $=a+b^{*} t_{\text {eq }} \quad$ With fan } & \\
\hline 643 & 21.52 & 0.00 & \multicolumn{4}{|c|}{ LINEAR REGRESSION } & \multicolumn{2}{|c|}{ STATISTICS } & & \\
\hline 561 & 24.97 & 0.50 & $a=$ & -3.90 & teq & MTV & Sda $=$ & 0.96 & $\mathrm{~F}=$ & 21.17 \\
\hline 563 & 23.12 & 0.10 & $b=$ & 0.18 & 22 & -0.13 & $S d b=$ & 0.04 & $D F=$ & 6.00 \\
\hline 481 & 26.20 & 0.30 & $r=$ & 0.88 & 28 & 1.00 & SdMTV= & 0.21 & $r^{2}$ & 0.78 \\
\hline 483 & 24.86 & 0.50 & \multicolumn{7}{|c|}{ No fan } & \\
\hline 403 & 26.53 & 0.70 & \multicolumn{4}{|c|}{ LINEAR REGRESSION } & \multicolumn{4}{|c|}{ STATISTICS } \\
\hline 404 & 26.76 & 0.80 & $a=$ & -15.46 & teq & MTV & Sda $=$ & 2.48 & $\mathrm{~F}=$ & 42.12 \\
\hline 323 & 27.96 & 1.30 & $b=$ & 0.54 & 29 & -0.02 & $\mathrm{Sdb}=$ & 0.08 & $\mathrm{DF}=$ & 3.00 \\
\hline 640 & 28.56 & -0.10 & $r=$ & 0.97 & 31 & 1.35 & SdMTV= & 0.18 & r2 & 0.93 \\
\hline 560 & 28.81 & 0.30 & \multirow{2}{*}{\multicolumn{4}{|c|}{$\begin{array}{l}\text { Both } \\
\text { LINEAR REGRESSION }\end{array}$}} & & & & \\
\hline 480 & 29.74 & 0.50 & & & & & \multicolumn{4}{|c|}{ STATISTICS } \\
\hline 400 & 30.55 & 0.90 & $a=$ & -1.99 & teq & MTV & Sda $=$ & 1.12 & $F=$ & 5.22 \\
\hline \multirow[t]{2}{*}{320} & 31.10 & 1.50 & $b=$ & 0.09 & 22 & 0.05 & $S d b=$ & 0.04 & $\mathrm{DF}=$ & 11.00 \\
\hline & & & $r=$ & 0.57 & 31 & 0.95 & SdMTV= & 0.41 & r2 & 0.32 \\
\hline
\end{tabular}

\section{Back, lower}

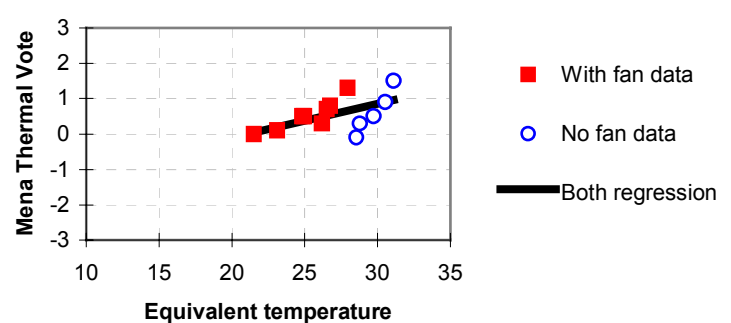

\section{Warm conditions}

\begin{tabular}{r|c|c|}
\multicolumn{3}{c}{ Head } \\
Condition & $\mathbf{t}_{\mathrm{eq}}$ & \multicolumn{1}{c}{ LMV } \\
\cline { 3 - 4 } $\mathbf{1}$ & 36.70 & 1.80 \\
$\mathbf{2}$ & 38.60 & 2.60 \\
$\mathbf{3}$ & 35.70 & 1.40 \\
$\mathbf{4}$ & 23.10 & -0.10 \\
$\mathbf{5}$ & 27.50 & 0.20 \\
\cline { 2 - 3 } & &
\end{tabular}

Warm conditions

LMV $=\mathbf{a}+\mathbf{b}$ * teq
\begin{tabular}{|cccc|cccc|}
\hline \multicolumn{2}{|c|}{ LINEAR REGRESSION } & \multicolumn{4}{c|}{ STATISTICS } \\
$\mathrm{a}=$ & -4.06 & teq & LMV & Sda $=$ & 0.86 & $\mathrm{~F}=$ & 38.75 \\
$\mathrm{~b}=$ & 0.16 & 23 & -0.31 & $\mathrm{Sdb}=$ & 0.03 & $\mathrm{DF}=$ & 3.00 \\
$\mathrm{r}=$ & 0.96 & 39 & 2.20 & SdLMV $=$ & 0.35 & $\mathrm{r}^{2}$ & 0.93 \\
\hline
\end{tabular}

Head

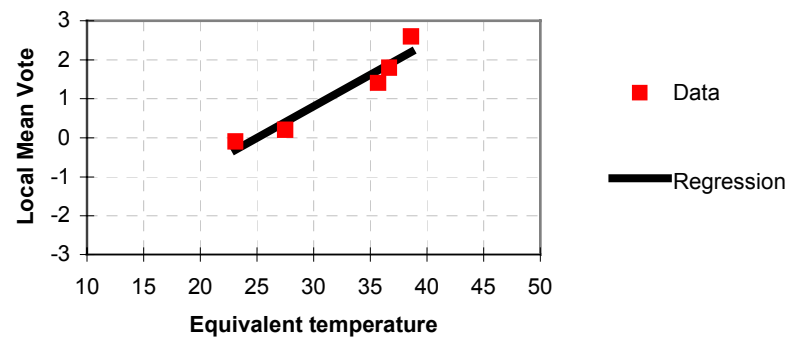




\begin{tabular}{|c|c|c|}
\hline \multicolumn{3}{|c|}{ Chest } \\
\hline Condition & $t_{e q}$ & LMV \\
\hline 1 & 37.20 & 1.80 \\
\hline 2 & 45.30 & 2.30 \\
\hline 3 & 39.60 & 1.20 \\
\hline 4 & 21.50 & -0.10 \\
\hline 5 & 25.20 & 0.20 \\
\hline
\end{tabular}

LMV $=\mathbf{a}+\mathbf{~} \mathbf{b}$ * teq
\begin{tabular}{|cccc|cccc|}
\hline \multicolumn{2}{|c|}{ LINEAR REGRESSION } & \multicolumn{4}{|c|}{ STATISTICS } \\
$\mathrm{a}=$ & -2.21 & teq & LMV & Sda $=$ & 0.60 & $\mathrm{~F}=$ & 31.79 \\
$\mathrm{~b}=$ & 0.10 & 22 & -0.12 & $\mathrm{Sdb}=$ & 0.02 & DF $=$ & 3.00 \\
$\mathrm{r}=$ & 0.96 & 45 & 2.20 & SdLMV $=$ & 0.35 & $\mathrm{r}^{2}$ & 0.91 \\
\hline
\end{tabular}

\section{Chest}

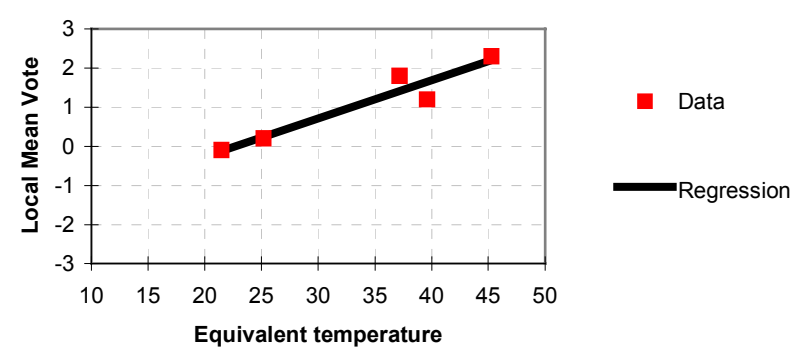

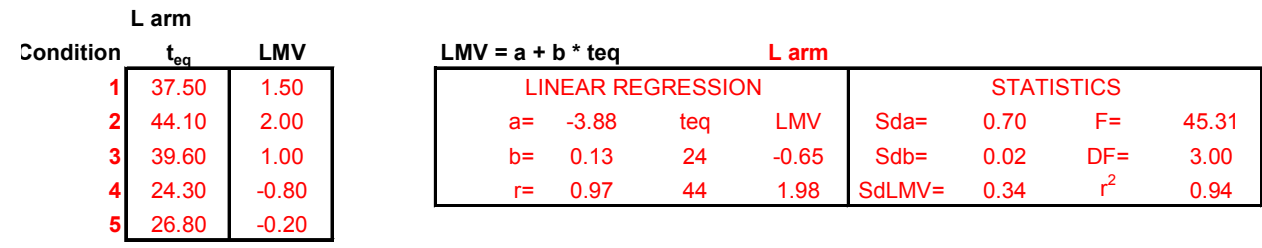

L arm

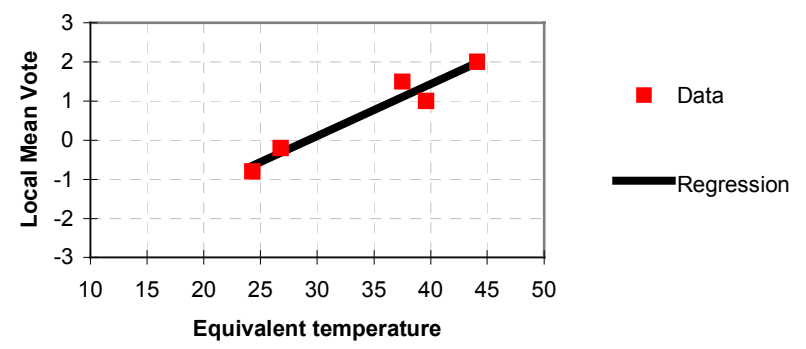

\begin{tabular}{|c|c|c|}
\hline \multicolumn{3}{|c|}{$R$ arm } \\
\hline Condition & $t_{e q}$ & LMV \\
\hline 1 & 38.40 & 1.50 \\
\hline 2 & 46.60 & 2.00 \\
\hline 3 & 42.90 & 1.00 \\
\hline 4 & 24.20 & -1.00 \\
\hline 5 & 28.40 & -0.30 \\
\hline
\end{tabular}

LMV $=\mathbf{a}+\mathbf{b}$ * teq
\begin{tabular}{|cccc|cccc|}
\hline \multicolumn{2}{c|}{$\mathbf{L I N E A R}$ REGRESSION } & \multicolumn{4}{c|}{ STATISTICS } \\
$\mathrm{a}=$ & -3.88 & teq & LMV & Sda $=$ & 0.87 & $\mathrm{~F}=$ & 28.79 \\
$\mathrm{~b}=$ & 0.13 & 24 & -0.85 & $\mathrm{Sdb}=$ & 0.02 & $\mathrm{DF}=$ & 3.00 \\
$\mathrm{r}=$ & 0.95 & 47 & 1.96 & SdLMV $=$ & 0.44 & $\mathrm{r}^{2}$ & 0.91 \\
\hline
\end{tabular}

R arm

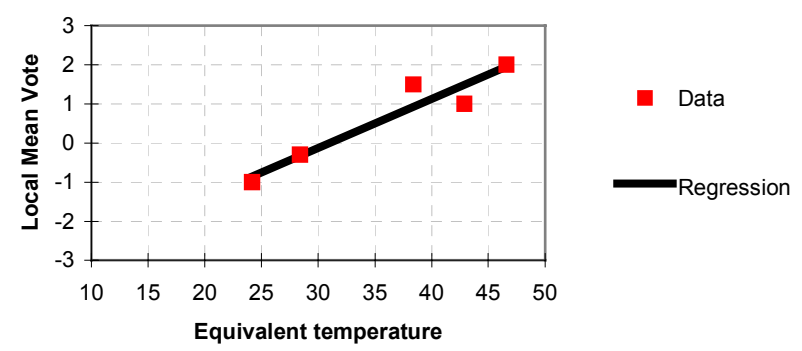




\begin{tabular}{|c|c|c|}
\hline \multicolumn{3}{|c|}{ L leg } \\
\hline Condition & $t_{\text {eq }}$ & LMV \\
\hline 1 & 39.70 & 1.60 \\
\hline 2 & 39.70 & 1.90 \\
\hline 3 & 39.10 & 1.20 \\
\hline 4 & 24,40 & -0.40 \\
\hline 5 & 27.50 & -0.20 \\
\hline
\end{tabular}

LMV $\mathbf{=} \mathbf{a}+\mathbf{~} \mathbf{b}$ * teq
\begin{tabular}{|cccc|cccc|}
\hline \multicolumn{2}{|c|}{ LINEAR REGRESSION } & & \multicolumn{4}{c|}{ STATISTICS } \\
$\mathrm{a}=$ & -3.85 & teq & LMV & Sda $=$ & 0.62 & $\mathrm{~F}=$ & 58.70 \\
$\mathrm{~b}=$ & 0.14 & 24 & -0.51 & Sdb $=$ & 0.02 & DF $=$ & 3.00 \\
$\mathrm{r}=$ & 0.98 & 40 & 1.59 & SdLMV $=$ & 0.27 & $\mathrm{r}^{2}$ & 0.95 \\
\hline
\end{tabular}

\section{L leg}

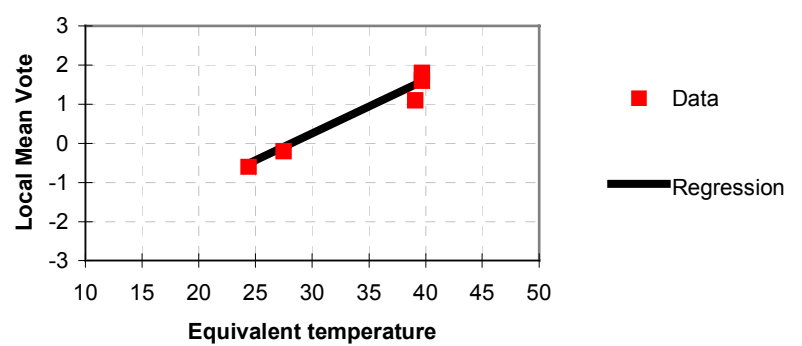

\begin{tabular}{rc|c}
\multicolumn{3}{c}{$\mathbf{R}$ leg } \\
Eondition & $\mathbf{t}_{\text {eq }}$ & \multicolumn{1}{c}{ LMV } \\
\cline { 3 - 4 } $\mathbf{1}$ & 40.80 & 1.60 \\
$\mathbf{2}$ & 40.60 & 1.80 \\
$\mathbf{3}$ & 40.30 & 1.10 \\
$\mathbf{4}$ & 26.80 & -0.60 \\
$\mathbf{5}$ & 30.40 & -0.20 \\
\cline { 3 - 4 } & &
\end{tabular}

LMV $=\mathbf{a}+\mathbf{b}$ * teq
\begin{tabular}{|cccc|cccc|}
\hline \multicolumn{3}{|c|}{ LINEAR REGRESSION } & & \multicolumn{4}{c|}{ STATISTICS } \\
$\mathrm{a}=$ & -4.90 & teq & LMV & Sda $=$ & 0.76 & $\mathrm{~F}=$ & 57.09 \\
$\mathrm{~b}=$ & 0.16 & 27 & -0.68 & $\mathrm{Sdb}=$ & 0.02 & DF $=$ & 3.00 \\
$\mathrm{r}=$ & 0.97 & 41 & 1.53 & SdLMV $=$ & 0.28 & $\mathrm{r}^{2}$ & 0.95 \\
\hline
\end{tabular}

\section{R leg}

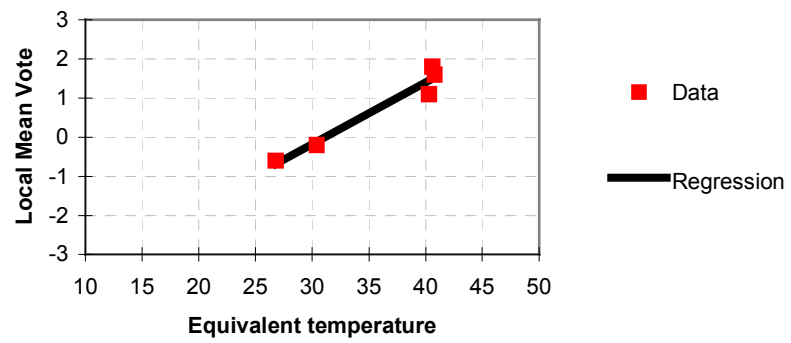

